Amundson, C. L., P. L. Flint, R. A. Stehn, R. M. Platte, H. M. Wilson, W. W. Larned and J. B. Fischer. 2019. Spatio-temporal population change of Arctic-breeding waterbirds on the Arctic Coastal Plain of Alaska. Avian Conservation and Ecology 14(1):18. https://doi.org/10.5751/ ACE-01383-140118

Copyright (C) 2019 by the author(s). Published here under license by the Resilience Alliance.

Research Paper

\title{
Spatio-temporal population change of Arctic-breeding waterbirds on the Arctic Coastal Plain of Alaska
}

Courtney L. Amundson ${ }^{1}$, Paul L. Flint ${ }^{2}$, Robert A. Stehn ${ }^{3}$, Robert M. Platte ${ }^{3}$, Heather M. Wilson ${ }^{4}$, William W. Larned ${ }^{3}$ and Julian B. Fischer ${ }^{4}$

${ }^{1}$ U.S. Geological Survey, Alaska Science Center, ${ }^{2}$ U.S. Geological Survey, Alaska Science Center, Anchorage, Alaska, ${ }^{3}$ U.S. Fish and Wildlife Service, Anchorage, Alaska (retired), ${ }^{4}$ U.S. Fish and Wildlife Service, Anchorage, Alaska

\begin{abstract}
Rapid physical changes that are occurring in the Arctic are primary drivers of landscape change and thus may drive population dynamics of Arctic-breeding birds. Despite the importance of this region to breeding and molting waterbirds, lack of a comprehensive analysis of historic data has hindered quantifying avian population change. We estimated distribution, abundance, and spatially explicit population trend of 20 breeding waterbird species using 25 years (1992-2016) of aerial survey data collected on the Arctic Coastal Plain (ACP), Alaska. The ACP is an extensive wetland complex on Alaska's North Slope that supports millions of breeding waterbirds and includes portions of the National Petroleum Reserve-Alaska and the Arctic National Wildlife Refuge. We summarized annual counts into approximately $6-\mathrm{km}$ by $6-\mathrm{km}$ grid cells and analyzed data with generalized linear mixed models that accounted for survey timing and spatio-temporal autocorrelation. Geese and swans were most abundant along the coast between Admiralty Bay and Prudhoe Bay. Sea ducks, generalist predators (i.e., jaeger, gulls, terns), and loons were most abundant between Utqiagivik and Point Lay, Alaska. Important areas for most species included the coastal fringe near Teshekpuk Lake, the Colville River Delta, and Admiralty Bay. The National Petroleum Reserve-Alaska was an important area for all species examined. Conversely, density on the coastal plain of the Arctic National Wildlife Refuge was greater than average for $20 \%$ of species. Annual population growth rates over the 25-year survey period were variable: 13 increased (range: $1.4 \%-13.8 \%$ ), one decreased (-3.4\%), and six were stable. However, even species with no overall population trend had areas of changing population size, suggesting localized conditions affected waterbird distributions on the ACP. Our results can be used to better inform land use decisions, improve monitoring of waterbird populations, and increase understanding of avian response to ecological change in the Arctic.
\end{abstract}

\section{Changements spatio-temporels chez des populations d'oiseaux aquatiques nichant dans la Plaine côtière arctique de l'Alaska}

RÉSUMÉ. Les changements physiques rapides qui se produisent dans l'Arctique figurent parmi les premiers facteurs responsables des changements qui surviennent dans le paysage, et pourraient donc déterminer la dynamique des populations d'oiseaux nichant dans l'Arctique. Malgré l'importance de cette région pour la nidification et la mue des oiseaux aquatiques, l'absence d'une analyse complète des données historiques a freiné la quantification des changements advenus dans les populations d'oiseaux. Nous avons estimé la répartition, l'abondance et la tendance des populations de façon explicite spatialement chez 20 espèces d'oiseaux aquatiques au moyen de 25 années (1992-2016) de données issues de relevés aériens menés sur la Plaine côtière arctique (PCA), en Alaska. La PCA est un vaste complexe de milieux humides dans la région de North Slope en Alaska et supporte des millions d'oiseaux aquatiques nicheurs; elle comprend des portions de la National Petroleum Reserve - Alaska et de l'Arctic National Wildlife Refuge. Nous avons compilé les dénombrements annuels à l'échelle de cellules de grille de $6 \mathrm{~km}$ par $6 \mathrm{~km}$ environ et analysé les données au moyen de modèles linéaires généralisés à effets mixtes qui tenaient compte de la durée d'inventaire et de l'autocorrélation spatio-temporelle. Les oies et les cygnes étaient le plus abondants le long de la côte entre la baie Admiralty et la baie Prudhoe. Les canards de mer, les prédateurs généralistes (c.-à-d. les labbes, goélands et sternes) et les plongeons étaient le plus abondants entre Utqiaǵvik et Point Lay. Les secteurs importants pour la plupart des espèces comprenaient la bordure côtière près du lac Teshekpuk, le delta de la rivière Colville et la baie Admiralty. La National Petroleum Reserve - Alaska était un endroit important pour toutes les espèces examinées. À l'opposé, la densité calculée sur la plaine côtière de l'Arctic National Wildlife Refuge était supérieure à la moyenne pour $20 \%$ des espèces. Les taux annuels de croissance des populations durant les 25 années d'inventaire étaient variables : 13 ont augmenté (étendue : 1,4\%-13,8\%), une a diminué $(-3,4 \%)$ et six étaient stables. Cependant, même chez les espèces globalement stables, la taille de population avait changé dans certains secteurs, indiquant que les conditions locales influaient sur la répartition des oiseaux aquatiques sur la PCA. Nos résultats peuvent servir à éclairer la prise de décision quant à l'utilisation des terres, à améliorer le suivi des populations d'oiseaux aquatiques et à accroître la compréhension de la réaction des oiseaux face aux changements écologiques dans l'Arctique.

Key Words: abundance; aerial survey; Arctic National Wildlife Refuge; eiders; geese; important areas; loons; National Petroleum Reserve; population change; sea ducks; spatial autocorrelation; waterfowl 


\section{INTRODUCTION}

Several studies indicate that the distribution and abundance of some avian populations are changing rapidly in the Arctic (Hines et al. 2003, Alisauskas et al. 2011, Van Hemert et al. 2015, Wilson et al. 2018). Over a similar period, northern Alaska has experienced warming temperatures, changes in the timing, duration, and warmth of growing seasons, loss of sea ice, permafrost degradation, changes in biogeochemical fluxes, and a net loss in terrestrial surface water (Hinzman et al. 2005, Kittel et al. 2011, Serreze and Stroeve 2015, Van Hemert et al. 2015, Gustine et al. 2017). These changes have the potential to alter terrestrial, marine, and lacustrine food abundance and availability for birds, which in turn may affect avian productivity, survival, and associated population growth. Further, climate-driven environmental impacts to birds will likely vary by trophic level (Van Hemert et al. 2015). For example, terrestrial herbivores may benefit from increased vegetation quality (Tape et al. 2013) or biomass, whereas birds that eat invertebrates (hereafter invertivores), e.g., sea ducks and shorebirds, may experience either increased or reduced food availability because of a phenological mismatch or change in invertebrate populations. Hines et al. (2003) found avian herbivore populations in the central Canadian Arctic increased substantially from the mid-1970s to the mid-1990s, but populations of King Eider (Somateria spectabilis) and Long-tailed Duck (Clangula hyemalis) declined during that same period. These inconsistencies suggest climate change may be acting on marine- and terrestrial-dependent bird populations differently, but few studies have examined broad scale avian population trends in the Arctic (though see Bart et al. 2013).

In addition to changing climate, several of the world's largest oil and gas deposits lie north of the Arctic Circle (Brownfield et al. 2012) and active oil and gas exploration and production are underway (Harsem et al. 2015, Hayes 2015). Natural resource extraction may directly affect avian populations by reducing productivity and avian density through increased predator abundance around human developments and disturbance or avoidance of areas surrounding development (Liebezeit and Zack 2008, Northrup and Wittemyer 2013, but see Meixell and Flint 2017). Additionally, oil and gas development activities and site use (especially roads) may have indirect environmental effects by exacerbating vegetation change, e.g., compaction, subsidence, and dust, altering hydrological cycles, e.g., snowmelt and ice road construction, and increasing the frequency and extent of thermokarst events (Natural Resource Council 2003, MyersSmith et al. 2006). Inferring causative factors affecting Arcticbreeding bird populations is complicated because nearly all are migratory and therefore subject to stressors in a variety of distant migration, staging, and wintering areas. In addition, although some species show high fidelity to nesting and molting areas, some fraction of young birds are capable of dispersing in response to changing environmental conditions (Flint et al. 2014). As such, population change may be the result of birth/death processes either in the Arctic or elsewhere, and/or local immigration/ emigration driven by landscape change.

Like elsewhere in the Arctic, the Arctic Coastal Plain (ACP), an extensive wetland complex along the northern coast of Alaska, is experiencing both increased interest in oil resources and rapid environmental change (Kittel et al. 2011, BLM 2018). The ACP includes North America's largest oil field (Prudhoe Bay and associated fields), much of the National Petroleum ReserveAlaska (NPR-A), and the 1002 area of the Arctic National Wildlife Refuge (ANWR). The ACP is also an important breeding area for millions of migratory wetland-dependent birds (Brown et al. 2007, Bart et al. 2013). Understanding abundance and population trends across the ACP is necessary to inform oil and gas leasing decisions, identify important areas for species, and identify hotspots of population change to focus subsequent research into causal mechanisms. Two studies previously summarized waterbird aerial survey data on the ACP and reported overall abundance and trends of species (Wilson et al. 2018) and east-west patterns in abundance (Bart et al. 2013), but spatially explicit estimates of abundance, trends, and relative importance remain largely unknown. Here, we estimated spatially explicit relative density and population trends for 20 species of breeding waterfowl and waterbirds across the ACP using 19922016 aerial observation index data collected by the U.S. Fish and Wildlife Service (USFWS) on the ACP Aerial Breeding Waterfowl Survey (Stehn et al. 2013). Our study expands previous efforts by accounting for survey timing and spatial covariance in estimates, and mapping density, population trends, and important areas across the ACP. Our objectives include (1) provide estimates of abundance and trends for each species across the ACP, the NPRA, and the ANWR; (2) provide spatially explicit density and trend maps for each species; and (3) provide maps of the relative importance of an area to each species and guild. Our results can inform site-specific development activities and leasing decisions made by public and private land managers and provide insight into how birds may be responding to changes in the environment.

\section{METHODS}

\section{Study area and species}

The ACP covers approximately $90,000 \mathrm{~km}^{2}$ bordering the Chukchi and Southern Beaufort Seas from Point Lay, Alaska to the Canadian border just east of the Kongakut River Delta (Fig. 1, upper). It is underlain by continuous permafrost and is vegetated by sedge, graminoid, dwarf shrub, and/or moss tundra communities. The ACP landscape is treeless, topographically flat ( $<100 \mathrm{~m}$ in elevation) and dotted by millions of shallow thermokarst lakes. This wetland mosaic hosts greater populations of breeding waterbirds ( $>5$ million) than any other Arctic region in the world (Bart et al. 2013). It is also home to over 10,000 human residents in small, scattered villages (Vadapalli et al. 2015). It has several known oil rich deposits including the greater Prudhoe Bay and Colville River Delta area and the potential for more oil and gas exploration in the NPR-A and coastal plain of the ANWR, e.g., 1002 area.

We evaluated 20 waterbird species that were consistently surveyed on the ACP and represent a suite of ecological niches (e.g., marine and terrestrial) and trophic levels. Specifically, we examined longterm distribution and abundance information for large-bodied avian herbivores including the following: Lesser Snow Goose (Chen caerulescens caerulescens), Greater White-fronted Goose (Anser albifrons frontalis), Black Brant (Branta berniclanigricans), Cackling Goose (B. hutchinsii taverneri), and Tundra Swan (Cygnus columbianus); aquatic omnivores including Northern Pintail (Anas acuta), and Scaup (Aythya spp., Lesser and Greater combined); invertivores including Steller's Eider (Polysticta 
Fig. 1. Arctic Coastal Plain, Alaska, survey boundary, human settlements (red points), and relevant federal land management units including (a) the National Petroleum Reserve-Alaska (NPR-A), the Teshekpuk Lake Special Area (TLSA), and Arctic National Wildlife Refuge (ANWR) and (b) transect locations for the early and later-flown surveys. Survey transects were flown in a four-year rotation.

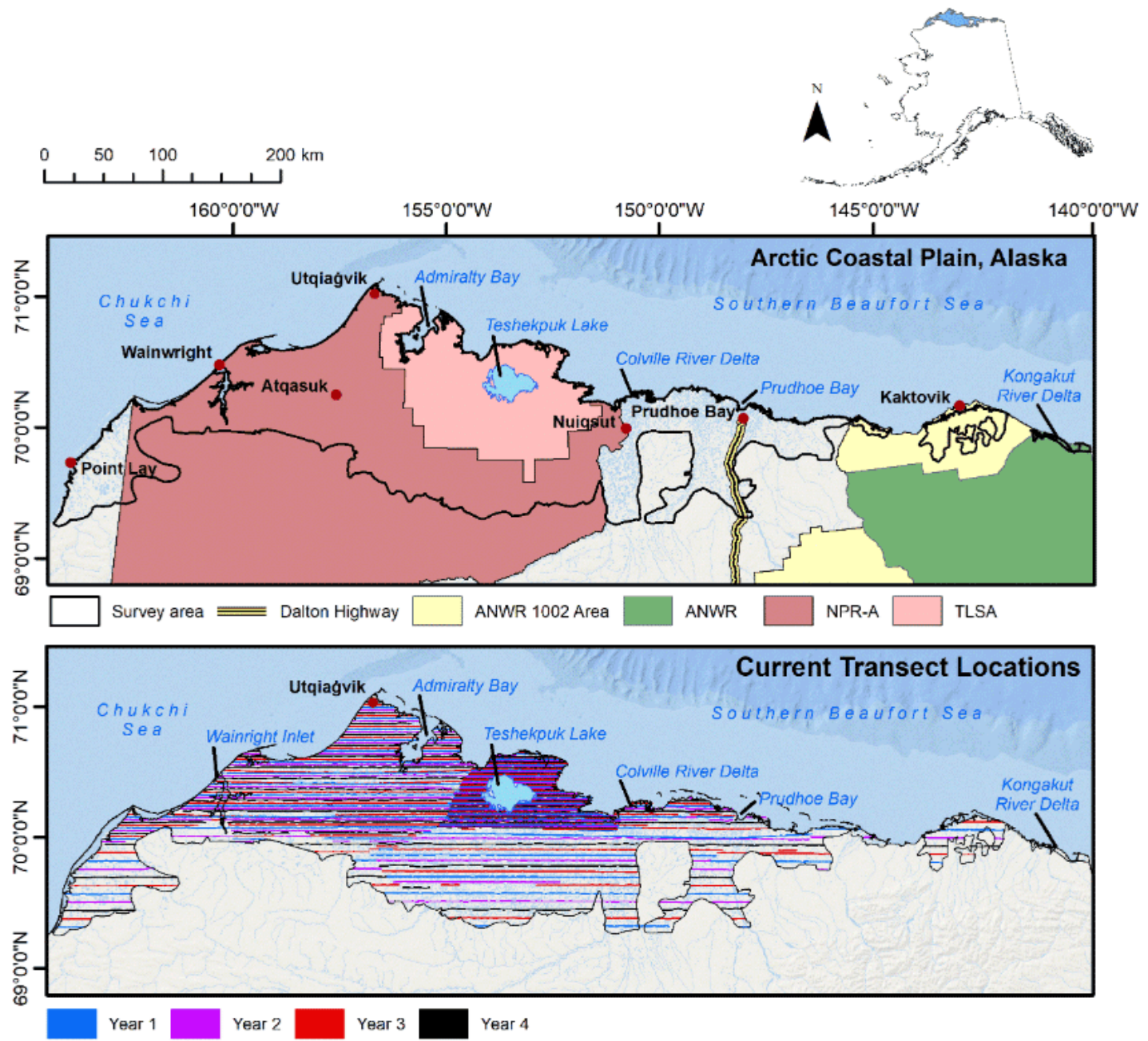

stelleri), Spectacled Eider (Somateria fischeri), King Eider, Whitewinged Scoter (Melanitta fusca), and Long-tailed Duck; piscivores including Red-breasted Merganser (Mergus serrator), Red-throated Loon (Gavia stellata), Pacific Loon (G. pacifica), and Yellow-billed Loon ( $G$. adamsii); and generalist predators including Jaeger (Stercorarius spp., Pomarine, Parasitic, and Long-tailed combined), Sabine's Gull (Xema sabini), Glaucous Gull (Larus hyperboreus), and Arctic Tern (Sterna paradisaea) that feed primarily on lemmings (Jaeger), fish, aquatic invertebrates, insects, eggs, and young birds during the breeding season (Wiley and Lee 1998, 1999, 2000, Day et al. 2001, Hatch 2002, Weiser and Gilchrist 2012).

\section{Aerial survey}

The ACP Aerial Breeding Waterfowl Survey began in 1986 and is flown annually by the USFWS in a fixed-wing aircraft with a pilot-observer and passenger-observer. The survey consists of systematic east-west transects flown $38 \mathrm{~m}$ above the ground at about $175 \mathrm{~km} / \mathrm{hr}$. Procedures generally follow the standard protocol developed for the North American Breeding Waterfowl Survey (USFWS and Canadian Wildlife Service 1987). Observers count birds within $200 \mathrm{~m}$ on each side of the plane for a combined 400-m wide strip-transect. The aircraft's GPS location was downloaded each time an observer records an observation. The aircraft flightpath is also recorded by GPS location every 5 seconds. Observation location accuracy, stratum boundaries, transect placement, and survey timing have changed considerably through time. We analyzed data 1992-2016 because prior to 1992 observation locations were only summarized into $25.7-\mathrm{km}$ (16mile) segments, therefore, we could not accurately assign these observations to grid cells.

From 1992 to 2006, the USFWS conducted two surveys annually: (1) an early-season survey in the northern portion of the ACP initiated around 10 June that covered approximately $1558 \mathrm{~km}^{2}$ 
and averaged 8.6 days duration, and (2) an extensive survey across the ACP started around 24 June that covered approximately $1183 \mathrm{~km}^{2}$, and averaged 7.3 days duration. In 2007, the late-flown survey was eliminated and the USFWS devised a new survey design conducted for the entire ACP in early June (average start date: 9 June; area surveyed: $1815 \mathrm{~km}^{2}$ ) with four strata flown at varying sampling intensities based on waterfowl density and management concern (Fig. 1 in Wilson et al. 2018). Transects were flown in a 4-year rotating panel design and spacing between surveyed transects in a given year for the low, medium, and high density strata and the high interest stratum around Teshekpuk Lake was approximately $28 \mathrm{~km}, 18$ $\mathrm{km}, 9 \mathrm{~km}$, and $5 \mathrm{~km}$, respectively (Fig. 1, lower). Stratum number and boundaries changed through time and were based on previous estimates of bird density and physiographic characteristics, e.g., wetland density (Stehn et al. 2013). The high interest area represents the core of the BLM designated Teshekpuk Lake Special Area, which is an extremely important area for breeding waterfowl and area with high potential for containing hydrocarbon deposits (Derksen et al. 1981, BLM 2018). The southern boundary of the ACP survey area is defined by both elevation and the associated abrupt change in wetland density.

These surveys generate count data, comprising observations of a species along each transect during a specific survey (early or late) in each year. Although pilots attempted to replicate the same flight line for a given transect, actual transect locations varied between the early and late survey and among years because of weather, e.g., patchy fog requiring a deviation from the established transect, and imprecision in navigating lines. Furthermore, most observations are of flying birds that are likely responding to aircraft noise flushing them from their habitat. Because of the uncertainty surrounding on-the-ground locations of birds and inconsistency in the placement and survey frequency of transects, we created $6 \mathrm{~km}$ by $6 \mathrm{~km}$ grid cells across the survey area and assigned specific observations to the appropriate cell so we could include spatially explicit covariates and temporal trends. This grid size was defined to remain consistent with previous summaries of the data (Bart et al. 2013) and maintain sufficient replication of flight-line sections within most cells. Grid cells overlapping the ACP boundary or Teshekpuk Lake were clipped to onshore area resulting in several cells $<36 \mathrm{~km}^{2}$. We determined the area surveyed per cell-year-survey from the area of a 400-m diameter buffer superimposed on the transect flightpath that fell within each grid cell during each survey.

Observations represent an index to true abundance rather than an estimate of the actual population size. Waterbird distribution and detectability may change as the season progresses because of behaviors associated with nesting and incubation. Thus, the timing of a survey in a given year likely influences indices of abundance. Moreover, some waterbirds adjust their timing of breeding relative to local seasonal phenology at specific breeding locations (McKinnon et al. 2012, Liebezeit et al. 2014), making calendar date a poor measure of timing of breeding. We calculated a covariate to assess the influence of annual phenology by subtracting the date of predicted onset of the growing season in each cell-year from the survey date in each cell-year-survey to estimate the number of days a cell was surveyed before or after the onset of spring green-up for each cell-year-survey. We obtained spatially explicit (approximately 5 $\mathrm{km} \times 2.5 \mathrm{~km}$ resolution) start-of-season-time (SOST) data for our time series from vegetation phenology indices derived from MODIS satellite imagery (Zhang et al. 2003, Didan et al. 2015). For cell-year-surveys with $>1$ unique SOST or survey day value, we calculated weighted mean SOST and survey date for each cell. We weighted averaged SOST by the area covered by each value in each year, and weighted survey date by the proportion of the area surveyed each day in each cell-year-survey. Beyond seasonal variation in detection probability, we assume that detection rate of a species is consistent for the combined counting ability of the pilot and observer across years and space.

\section{Model assumptions and data considerations}

We made several assumptions when including data. First, counts per area surveyed within a cell are representative of the density of birds in that cell. We only included counts during a survey if $\geq 0.4 \mathrm{~km}^{2}$ of a cell was surveyed during a single year-survey to ensure counts were representative of the entire cell. This value represents flying a single transect approximately $2 \mathrm{~km}$ within a cell. We chose this cutoff based on preliminary analyses that suggested counts were correlated within cells through time when at least this amount of area was covered. Second, consistent with USFWS survey protocol (Wilson et al. 2018), we doubled single observations for ducks to account for unseen paired females on nests (hereafter, indicated pairs). We also doubled single observations for dark geese (Greater White-fronted Goose, Cackling Goose, and Black Brant). Other species were presumed visible enough to see nesting females, e.g., loons, Lesser Snow Geese, or are known to have male-skewed sex ratios, e.g., Scaup. We excluded observations $>2$ birds, including only pairs or indicated pairs, in analyses to minimize inclusion of presettled flocks of birds or flocks of failed breeders that have moved from original breeding areas, e.g., Black Brant (Taylor 1995, Lewis et al. 2010). Therefore, although counts are restricted to single birds and pairs, we define counts as the number of breeding birds and as an index to their total breeding populations across the ACP.

\section{Model structure}

We accounted for the hierarchical nature of the counts through space and time by modeling abundance using negative binomial or zero-inflated negative binomial (ZINB) mixed-effects generalized linear models combined with a nonlinear spatiotemporal smoothing parameter, i.e., general additive mixed model, implemented in a Bayesian framework. Every cell was not surveyed in every year; thus, we used a spatio-temporal smoother in lieu of traditional random effects that allowed for autocorrelated variation in counts through space and time. Each species was not observed in all cells either because the habitat was unsuitable or because of insufficient sampling effort or imperfect detection of birds. Consequently, we included only cells where a species had ever been observed in analyses, i.e., we removed structural zeros (Martin et al. 2005). Despite removing structural zeros, data contained a high proportion of zero counts (range: 0.47-0.95). Therefore, for each species, we first attempted to fit the negative binomial distribution, and if models did not accurately reflect the true proportion of zeros in the data as determined by comparing Monte Carlo realizations of count data based on model parameter estimates to the observed proportion of zero counts in the data (Appendix 1), we refit models using the ZINB distribution. 


\section{Abundance and trend}

For each species, we modeled the likelihood as the sum of birds observed in spatial grid cells $c$ during a specific year $y$ and survey $s$ either as a generalized linear model using (1) the negative binomial realization of $\lambda$ where $\operatorname{Count}_{\mathrm{c}, \mathrm{t}, \mathrm{s}} \sim \operatorname{NegBin}\left(\mathrm{p}_{\mathrm{c}, \mathrm{t}, \mathrm{s}}, \mathrm{r}_{\mathrm{c}, \mathrm{t}}\right), r$ is an overdispersion term per year-cell and $\mathrm{p}_{\mathrm{c}, \mathrm{t}, \mathrm{s}}=\mathrm{r}_{\mathrm{c}, \mathrm{t}} /\left(\mathrm{r}_{\mathrm{c}, \mathrm{t}}+\lambda_{\mathrm{c}, \mathrm{t}, \mathrm{s}}\right)$ or (2) as a two-part model where the binomial realization $z$ that a count was zero in a given cell-year-survey was a Bernoulli draw with probability $\Psi$. Nonzero counts were then a negative binomial realization of the expected count $\lambda$. For the ZINB distribution, $\lambda_{\mathrm{c}, \mathrm{t}, \mathrm{s}}=$ Density $_{\mathrm{c}, \mathrm{t}, \mathrm{s}} \times$ area surveyed $_{\mathrm{c}, \mathrm{t}, \mathrm{s}} \times\left(1-\mathrm{z}_{\mathrm{c}, \mathrm{t, \textrm {s }}}\right)$, whereas the negative binomial distribution did not include the $z$ parameter. Density $\left(\mathrm{km}^{2}\right)$ per cell-year-survey is then a log-linear function of random spatial and temporal variation (CELL and $\mathrm{Y}_{0}$, respectively), a spatio-temporal smooth term (ST; described below), late survey replicate offset (SURV), and log-linear and log-quadratic forms of survey date adjusted for the onset of spring in year $t$ (A.DOY). Similarly, $\Psi$ was a logit-linear function of random spatial (Z.CELL) and temporal variation $\left(Z . Y_{0}\right)$, late survey replicate offset (Z.SURV), and logit-linear and logitquadratic forms of survey date adjusted for the onset of spring in year $t$ (Z.DOY). Across species, the proportion of a cell surveyed varied during each year-survey. Therefore, we weighted the variance of the dispersion parameter $r$ where $\log \left(\mathrm{r}_{\mathrm{c}, \mathrm{t}, \mathrm{s}}\right)=\log$ $\left(\mathrm{r}^{\prime}-\mathrm{S} . \mathrm{PROP}_{\mathrm{ctt.s}}\right)$; S.PROP is the normalized relative proportion of a cell surveyed and $\mathrm{r}^{\prime}$ is the overdispersion value at the mean cell proportion surveyed. We then derived annual indices of abundance based on model parameters for the average A.DOY and the early-flown survey. Total abundance in year $t$ was the sum of cell-specific expected abundances in year $t$. We calculated overall and cell-specific population change as the geometric mean rate of change of predicted abundance (i.e., averaging over random temporal variation, $Y_{n}$ : Sauer et al. 2014).

$$
\Delta \hat{N} \%=100 \times\left(\frac{\hat{N}_{2016}}{\hat{N}_{1992}}\right)^{\frac{1}{2016-1992}}
$$

but also report Ricker intrinsic rates of population growth $g_{t}=$ $\log \left(\hat{N}_{\mathrm{t}+1} / \hat{N}_{\mathrm{t}}\right)$; Dennis and Taper (1994) in the supplemental material.

\section{Spatio-temporal smooth}

Waterbird observations could be correlated in space and time because of environmental conditions (e.g., habitat), life-history traits (e.g., coloniality), and population trends that may vary spatially. Permafrost characteristics, soil type, and topography vary east to west on the ACP leading to shifts in habitat at similar latitudes (Fresco et al. 2015). Further, elevation, climate, and habitat vary with proximity to the coast (Fresco et al. 2015). Last, changes in climate varied spatially over the period considered (Appendix 2). To capture dependence in observations in space and time, we modeled spatio-temporal autocorrelation in survey data (ST) by fitting low rank, i.e., reduced dimension, thin plate spline smoothers for space and time parameters (Wood 2003, Conn et al. 2015, Hefley et al. 2017). We transformed longitude (Long) and distance to the coast (Dcoast; highly correlated with latitude, $r=-0.7$, and more biologically meaningful) using penalized thin plate splines into a 2-dimensional plane (SP) with $k$ inflection points (knots) using a full tensor product smooth $(t e)$. Inflection points were spaced approximately every $30 \mathrm{~km}$ distance from the coast and $60 \mathrm{~km}$ longitude across the range of each species. This corresponds to gradients of habitat composition and climate and is consistent with previous distribution maps that showed some species changed more rapidly as a function of distance to the coast than longitude (Larned et al. 2011). We set the minimum number of inflection points in the tensor product to 3 in either dimension to ensure a sufficient number to compute a smooth for species with narrow distributions ( $k$ range: $6-32$ ). We applied a 1 -dimensional nonlinear smooth $(s)$ to year (YR; $k$ $=5$ ) (temporal model), and then created a tensor interaction $(t i)$ term between SP and YR using the same number of $k$ as in lower order smooths. The spatio-temporal model was then $\mathrm{ST}=s(\mathrm{YR})$ $+t e($ Long, Dcoast $)+t i$ (Long, Dcoast, YR). For ZINB models, we applied the spatio-temporal smooth to the negative binomial model component, i.e., counts, and applied additive, random spatial and temporal effects to presence/absence within a cell.

We conducted analyses in JAGS ver. 4.1.0 (Plummer 2003) called remotely from the package jagsUI (Kellner 2018) in $\mathrm{R}$ version 3.4.2 (R Core Team 2018, example code available in Appendix 3). We obtained smoothing coefficients and code for spatio-temporal effects using the jagam function in the $m g c v$ package (Wood et al. 2016). We specified vague normal prior distributions with mean 0 and precision (i.e., $\left.1 / \sigma^{2}\right) \tau=0.001$ for fixed effects while random effects had a common mean $(\mu)$ and precision $\tau=0.001$. The hyperprior on $\mu$ was then normally distributed with mean 0 and $\tau=0.001$. We assigned uniform priors to variances ranging from 0 to 1000 (Kéry 2010). We analyzed each species independently using 20,000 iterations conducted across 5 chains, discarding the first half and retaining every 3 rd iteration to reduce output file size. We assessed model convergence by examining effective sample size, ensuring the Gelman-Rubin potential scale reduction parameter was < 1.1 (Gelman and Rubin 1992), and visually inspecting density plots of posterior distributions of each parameter. We evaluated model fit using posterior predictive checks, i.e., Bayesian P-values (Royle and Dorazio 2008), Bayesian P-values from $0.2-0.8$, with 0.5 as the optimal value, suggest an acceptable fit to the data. We summarized predictive ability of models by calculating mean square error for each iteration and summarizing results. We report relative breeding bird density per $\mathrm{km}^{2}$ by dividing predicted abundance by the area surveyed per cell-year-survey, and mean parameter estimates with 95\% credible limits (CL) throughout. We used a statistical significance criterion of whether $95 \%$ credible intervals overlapped zero for fixed effects and comparisons of interest.

\section{Population change in space and time}

We evaluated spatially explicit population change in three ways. First, we mapped the geometric mean rate of change in predicted abundance per cell for cells with $\geq 3$ non-zero counts over the 25 year period. We restricted maps to these cells so that trends within cells were representative of the cell and not solely an artifact of smoothing. Second, we summarized density and trends for the entire ACP, as well as the NPR-A and the ANWR. Third, we evaluated whether population growth rate varied over the survey period by conducting a rolling-window analysis where we estimated the geometric mean rate of change over successive 10- 
year periods beginning each year 1992-2007. We then plotted population growth in each period relative to the long-term average for each species. We evaluated potential density-dependence in populations by plotting predicted Ricker intrinsic rate of population growth in year $t$ against predicted population size $\hat{N}_{\mathrm{t}}$.

\section{Important areas}

We derived indices of relative importance (RI) across the study period for individual species for each cell by calculating the standardized deviance of annual cell density to annual mean density and then averaging these deviances through time for each cell RI $\mathrm{c}_{\mathrm{c}}=\operatorname{mean}\left[\left(\right.\right.$ density $_{\mathrm{c}, \mathrm{t}}-$ density $\left._{\mathrm{t}}\right) /$ density $\left._{\mathrm{t}}\right]$.

Calculating ratios by year ensured overall importance of a cell was independent of any temporal trend in population size. Index values equal to zero represented cells that had mean density equal to overall density. We calculated RI for each iteration of the posterior distribution of predicted density to obtain error estimates. We then classified cells as important $=1$, average $=0$, or unimportant $=-1$ by comparing whether $95 \%$ credible intervals around standardized deviance overlapped zero. We further summarized relative importance of each cell by guild and all analyzed species by summing importance scores, i.e., $-1,0,1$, across groups. We assigned cells never occupied by a species to $\mathrm{RI}=-1$. This approach to estimating relative importance has two advantages: (1) using model-predicted density estimates and their associated error accounts for unequal survey effort and area surveyed among cell-years, and (2) each species had one value, ensuring more abundant species were not over-represented in maps of relative importance at broader taxonomic scales.

\section{RESULTS}

Our data set included 98,610 observations and 329,546 indicated breeding birds of the 20 species we evaluated. At least one species was observed in 1774 approximately $36 \mathrm{~km}^{2}$ cells $($ mean $=32.08$ $\mathrm{km}^{2}$; range $1.02-36 \mathrm{~km}^{2}$ ) that encompassed $56,904 \mathrm{~km}^{2}$ of the $57,336 \mathrm{~km}^{2}$ ACP survey area (99.2\%; Wilson et al. 2018). The earliest year surveyed per cell ranged from 1992 to 2011, but this distribution was highly skewed with most sampling first occurring 1992-1995 (Fig. 2a). Cells were surveyed 1-24 times each (mean $=13$, mode $=19$; Fig. $2 \mathrm{~b})$. The NPR-A was surveyed more intensely (mean $=15$ times; range $=1-24)$ than the ANWR (mean $=6$ times; range $=1-15$ ). The mean onset of phenological spring was 11-12 June (DOY = 162.3; SD = 6.14; range $=149-193)$, but the onset of spring advanced approximately 9 days between 1992 and 2016 (Appendix 2). The early-flown survey was conducted on average $0.6(\mathrm{SD}=6.12)$ days after the predicted onset of spring (range: -26-25) while the average late-flown survey was conducted $14(\mathrm{SD}=6.59)$ days after the predicted onset of spring (range: -15 37). In general, survey dates were better aligned with the SOST spring phenology index at higher latitudes closer to the coast (Fig. 3).

\section{Model results}

Posterior predictive plots and values suggested models converged for all species after issues were addressed for several species. We modeled 17 species using the negative binomial distribution and the remaining three species using the ZINB distribution (Appendix 1). We averaged predicted density over surveys for Scaup and Yellow-billed Loon because they nest frequently in the southern extent of the ACP, a region that was not surveyed during the early-flown survey 1992-2006. This led to a strongly positive later survey effect on abundance, which when applied to predictions for the early survey across the entire ACP, led to very low predicted abundance in early years. In general, breeding bird abundance declined with phenologically adjusted survey date and the probability a count was zero increased with relative survey date for the three species modeled using the ZINB distribution suggesting breeding pair abundance (or possibly their detection rate) decreased throughout the season (Table A4.1). Nevertheless, Tundra Swan, Scaup, Jaeger, and Pacific Loon showed an increase in abundance with relative survey date, i.e., positive A.DOY coefficients (Table A4.1). In general, predictive ability of models was consistent with the modeled distribution used for each species but was lower for Lesser Snow Goose than other species, possibly because of their highly clustered distribution, i.e., occurring in discrete colonies, and relatively recent colonization of the ACP (Tables A4.4-A4.24).

Total abundance varied by species (Fig. 4, Appendix 4) and Greater White-fronted Goose, Northern Pintail, Long-tailed Duck, and to a lesser extent, King Eider, Arctic Tern, and Redthroated Loon showed negative associations between population size and growth (Fig. 5). Predicted density varied among administrative units (Fig. 6, Table A4.2). Jaeger, Tundra Swan, Red-throated Loon, and Cackling Goose averaged higher density in the ANWR. Greater White-fronted Goose, Pacific Loon, and Sabine's Gull averaged lower densities in the ANWR. Further, White-winged Scoter, Yellow-billed Loon, and Steller's Eider were never observed in the ANWR.

Densities of 10 species were greatest near the coast including all goose species, Tundra Swan, Northern Pintail, Steller's and Spectacled Eider, and Red-throated Loon (Appendix 5). In contrast, White-winged Scoter, Scaup, and Red-breasted Merganser showed an affinity for the southern extent of the ACP (Appendix 5).

Population trends varied widely by species; 13 increased, 1 declined, and 6 were stable over the study period (Fig. 4). Lesser Snow Goose showed the largest annual growth rate $(13.8 \% ; 95 \%$ CL: 9.0, 18.4\%) while Red-throated Loon declined at 3.4\% per year (95\% CL: -5.6, -1.3\%; Table A4.2). Several species showed some evidence of variable rates of growth over the study period; over the entire study period Arctic Tern population trend was positive, but most recent 10-year windows, i.e., > 2002, suggest populations may be declining (Fig. 7). Similarly, several species showed a peak in population growth from the late 1990s to early 2000s, with decreases in recent years, e.g., Greater White-fronted Goose, Sabine's Gull, Glaucous Gull.

Trends were generally similar among administrative units (Fig. 8, Table A4.2), but lower sampling intensity in the ANWR led to greater uncertainty in estimates with 13 of 17 species present in the ANWR showing stable populations (Fig. 8). Several species showed declines in portions of their range including: Long-tailed Duck to the east and around Teshekpuk Lake, Red-throated Loon to the south and west of their range, Spectacled Eider along the coast south of Point Lay, and Jaeger around Utqiagivik and Admiralty Bay (Appendix 6). Red-throated Loon was the only species that declined throughout its range. Pacific Loon, Yellowbilled Loon, Tundra Swan, and Arctic Tern showed hot spots of 
Fig. 2. Arctic Coastal Plain Breeding Waterfowl Survey effort, 1992-2016 within approximately $36 \mathrm{~km}^{2}$ cells including the first year surveyed (a) and the number of times surveyed (b).

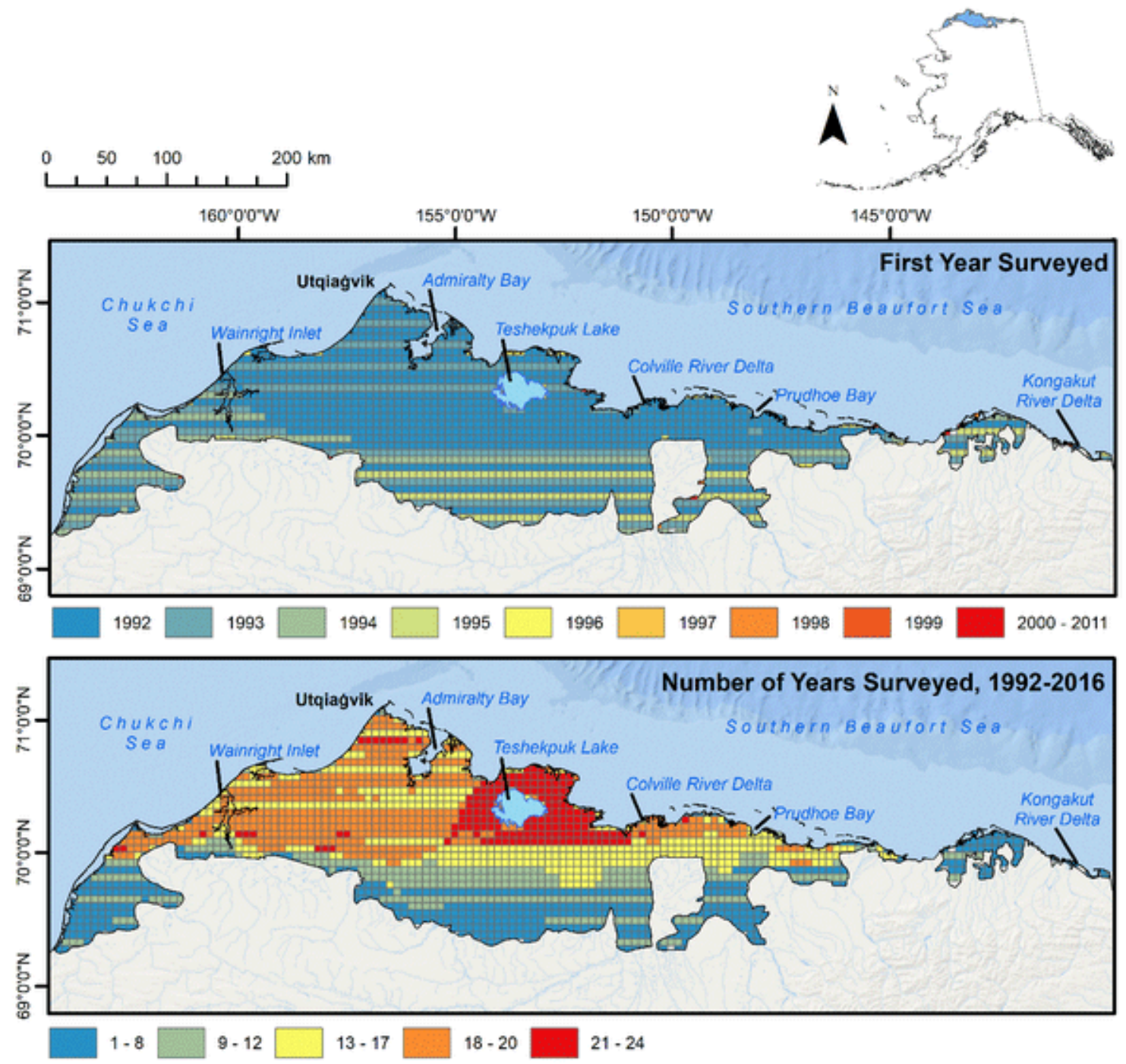

growth around Atqasuk and generally faster growth in the western portions of their ranges. Goose populations grew fastest near the coast and especially near the Colville River Delta. There was only a single cell in which Steller's Eider were observed $>2$ times and the trend on this cell was stable. Spectacled Eider numbers were stable throughout much of their core range but increased between Admiralty Bay and Teshekpuk Lake. Long-tailed Ducks were very close to an overall negative population trend (Fig. 4, Table A4.2), but were stable throughout the core of their range between Point Lay and Utqiaġvik (Appendix 5 and 6).

The NPR-A was the most important area for waterbirds in our study. Important areas, cells with higher than average density for a species, also occurred around Point Lay, south of Admiralty Bay, in the vicinity of Atqasuk, near Teshekpuk Lake, and the western edge of the Colville River Delta (Fig. 9). Areas of relative importance varied among guilds (Appendix 7) and species (Appendix 8). Nearshore areas between Admiralty Bay and
Prudhoe Bay were most important for herbivores, whereas the NPR-A south of Admiralty Bay was important to generalist predators and piscivores. The western ACP near Point Lay was important to piscivores, invertivores, and to a lesser extent, herbivores. Areas between Wainwright Inlet and Utqiagivik, Alaska were important to invertivores, as was the eastern shore of Teshekpuk Lake to Prudhoe Bay. The ANWR wetland area is small and was less important than other areas but was moderately important for generalist predators. Similar to guild level results, important areas for individual species were highly variable but provide useful information for land managers (Appendix 8).

\section{DISCUSSION}

Understanding spatial and temporal patterns of abundance and distributions of wildlife populations is essential for making informed management decisions. Further, making predictions regarding future change requires understanding the processes that 
Fig. 3. The mean relative date surveyed (days since initiation of spring growing season) within $36 \mathrm{~km}^{2}$ cells during the Arctic Coastal Plain Breeding Waterfowl Survey, Alaska, 1992-2016. We subtracted the predicted onset of spring in each cell-year (based on vegetation indices derived from satellite imagery) from the mean date surveyed within a year.

\section{Mean Relative Day Surveyed 1992-2016}

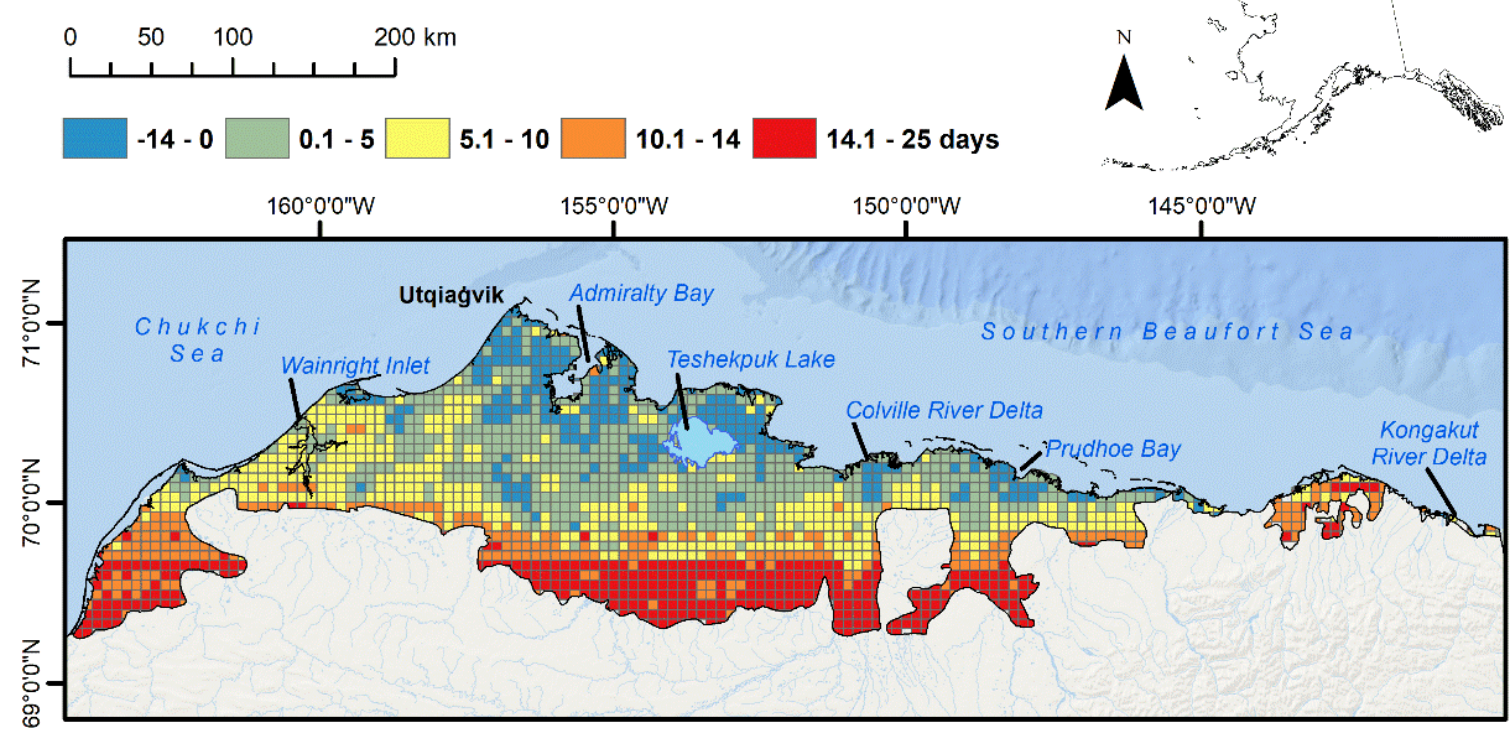

inherently drive population change. Our study represents one of the first steps in evaluating patterns of avian population change across the ACP landscape by describing spatially explicit population trends of waterbirds. Our results support earlier studies that found the ACP supports large breeding waterbird populations (Bart et al. 2013). Furthermore, most species increased or were stable over the study period, except for Redthroated Loon and possibly, Long-tailed Duck, which appeared to experience long-term population declines. Within species, patterns of density and population change were heterogeneous across the ACP, and some stable species experienced areas of local decline.

\section{Abundance and important areas}

Our indices of abundance are generally consistent with other analyses of data from the ACP breeding waterfowl survey (Wilson et al. 2018). Steller's Eider abundance estimates, however, are higher than previous reports because we included data from the later-timed survey that may have included non- or failed-nesting birds that were not incorporated in other studies (Bart et al. 2013, Dunham and Grand 2017, Wilson et al. 2018). The NPR-A contained areas of high density for most species (Appendix 5), which is consistent with Bart et al. (2013) who showed highest density in similar areas. Bart et al. (2013) concluded the ANWR had the lowest waterbird densities in the ACP based on aerial and ground survey data from 1992-2010. Conversely, we found greater than average densities of four species within the 1002 area of the ANWR. Densities in the ANWR were high for Cackling Goose, Tundra Swan, Jaeger, and Red-throated Loon (Fig. 6), although the wetland area and total population sizes were small compared to the NPR-A. Because surveys occurred relatively late in the
ANWR (Fig. 3), compensating for late survey timing caused estimates of density to be higher compared to Bart et al. (2013). The area also had relatively low survey effort (Fig. 2b), increasing uncertainty in estimates.

Important waterbird areas were concentrated between Utqiagivik and Prudhoe Bay (Fig. 9), especially for waterfowl (Appendix 7, 8), specifically, within the Teshekpuk Lake Special Area in the NPR-A, which has been identified as the most important breeding area for waterbirds on the ACP (Liebezeit et al. 2011, Andres et al. 2012) and is also an important molting area for Arctic-breeding geese (Derksen et al. 1982, Flint et al. 2008, Shults and Dau 2016). Previous work suggests the area just south of Utqiagivik, Alaska supports high densities $\left(>0.75\right.$ birds per $\left.\mathrm{km}^{2}\right)$ of breeding Steller's, spectacled, and King Eiders at local scales (Obritschkewitsch and Ritchie 2017). Our results show relatively high densities of eiders in this area, but our importance index did not identify these areas as hotspots. Thus, important areas for these and possibly other species may occur at spatial scales smaller than we evaluated or were not identified because of relatively uniform densities throughout their range or high variance within cell predictions. Further, because our index relies on model-based estimates, estimates with greater precision, e.g., surveyed in more years, could be more likely to demonstrate importance (or lack thereof), as such, estimates of importance from the southern ACP and the ANWR, which was surveyed less intensely over our study period (Fig. 2b) should be interpreted with caution.

\section{Population trends}

Most waterbird populations on the ACP increased, which is generally consistent with other published studies of breeding population trends in the ACP and elsewhere (Appendix 9). All 
Fig. 4. Predicted breeding bird abundance of 20 waterbird species surveyed 1992-2016 across the Arctic Coastal Plain, Alaska. Gray points represent predicted abundance, black lines are smoothed trends, and shaded regions represent 95\% credible intervals. We present plots in taxonomic order (Chesser et al. 2018) for Lesser Snow Goose (Chen caerulescens caerulescens), Greater White-fronted Goose (Anser albifrons frontalis), Black Brant (Branta bernicla nigricans), Cackling Goose (B. hutchinsii taverneri), Tundra Swan (Cygnus columbianus), Northern Pintail (Anas acuta), Scaup (Aythya spp., Lesser and Greater combined), Steller's Eider (Polysticta stelleri), Spectacled Eider (Somateria fischeri), King Eider (Somateria spectabilis), White-winged Scoter (Melanitta fusca), Long-tailed Duck (Clangula hyemalis), Red-breasted Merganser (Mergus serrator), Jaeger (Stercorarius spp., Pomarine, Parasitic, and Long-tailed combined), Sabine's Gull (Xema sabini), Glaucous Gull (Larus hyperboreus), Arctic Tern (Sterna paradisaea), Red-throated Loon (Gavia stellata), Pacific Loon ( $G$. pacifica), and Yellow-billed Loon (G. adamsii).
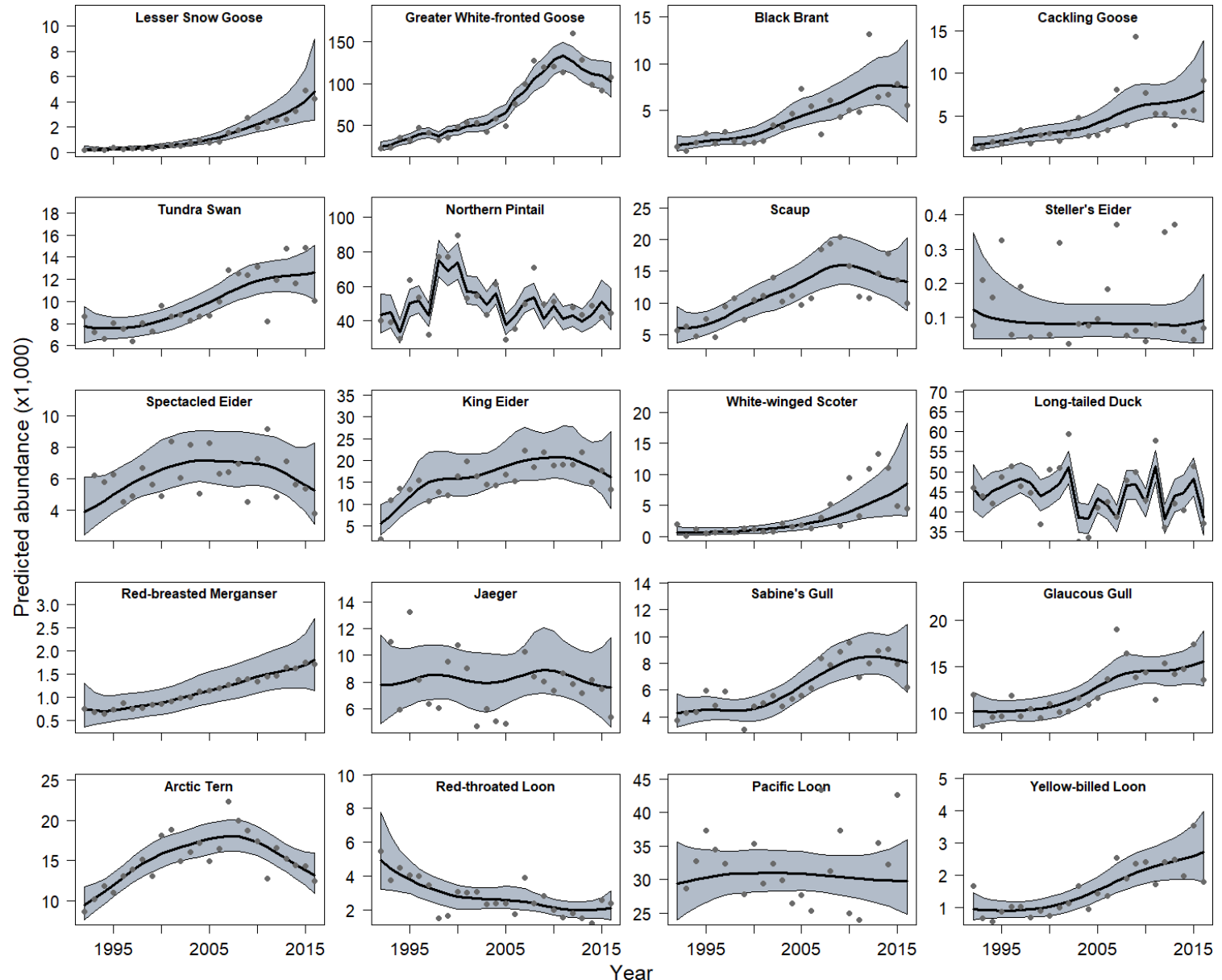

goose and swan populations increased on the ACP, especially in the NPR-A near Teshekpuk Lake (Fig. 4). Further, only Greater White-fronted Geese showed slowing of population growth in recent years (Fig. 5), suggesting patterns of increase for geese and swans may continue.

We observed several notable contrasts to other published population trends for some species. The population trends for Scaup $(3.3 \%$; $95 \%$ CL: $0.9 \%, 6.2 \%)$ and Northern Pintail $(0.1 \%$; 95\% CL: $-1.4 \%, 2 \%$ ) differ from the broader continental pattern of gradual decline (USFWS 2018). Part of this divergence is that the ACP Scaup population consists mostly of Greater Scaup, and areas where Scaup comprise mostly Greater Scaup are experiencing less drastic declines than areas occupied by Lesser
Scaup (Kessel et al. 2002, Ross et al. 2015). Additionally, Northern Pintail were previously shown to overfly the Midcontinent prairies in dry years when numbers in the Arctic would increase (Henny 1973, Derksen and Eldridge 1980, Nicolai et al. 2005). As such, the numbers seen in the Arctic are not necessarily independent of habitat conditions elsewhere.

Multiple sea duck species have declined globally, but most species in the Pacific Flyway were stable or increasing through 2011 (Flint 2013). We found similarly mixed results among sea ducks in our study. White-winged Scoters increased $>10 \%$ per year, but the ACP represents the northern edge of their breeding range and the little information available on population trends suggests declines in some areas along their southern extent (Brown and Fredrickson 
Fig. 5. Plots of the intrinsic rate of population growth $(r=\log (\mathrm{Nt}+1 / \mathrm{Nt}))$ relative to population size for 20 species of waterbirds surveyed during the Arctic Coastal Plain Breeding Waterfowl Survey, Alaska, 1992-2016. Black lines represent smoothed trends in population growth, shaded regions represent $95 \%$ credible intervals around annual estimates of $r$, and blue horizontal bars represent the $95 \%$ credible intervals around mean intrinsic growth rate. Trends in population growth rate relative to population size are indicative of density dependence in population growth. We present plots in taxonomic order (Chesser et al. 2018) for Lesser Snow Goose (Chen caerulescens caerulescens), Greater White-fronted Goose (Anser albifrons frontalis), Black Brant (Branta bernicla nigricans), Cackling Goose (B. hutchinsii taverneri), Tundra Swan (Cygnus columbianus), Northern Pintail (Anas acuta), Scaup (Aythya spp., Lesser and Greater combined), Steller's Eider (Polysticta stelleri), Spectacled Eider (Somateria fischeri), King Eider (Somateria spectabilis), White-winged Scoter (Melanitta fusca), Long-tailed Duck (Clangula hyemalis), Red-breasted Merganser (Mergus serrator), Jaeger (Stercorarius spp., Pomarine, Parasitic, and Long-tailed combined), Sabine's Gull (Xema sabini), Glaucous Gull (Larus hyperboreus), Arctic Tern (Sterna paradisaea), Red-throated Loon (Gavia stellata), Pacific Loon (G. pacifica), and Yellow-billed Loon (G. adamsii).
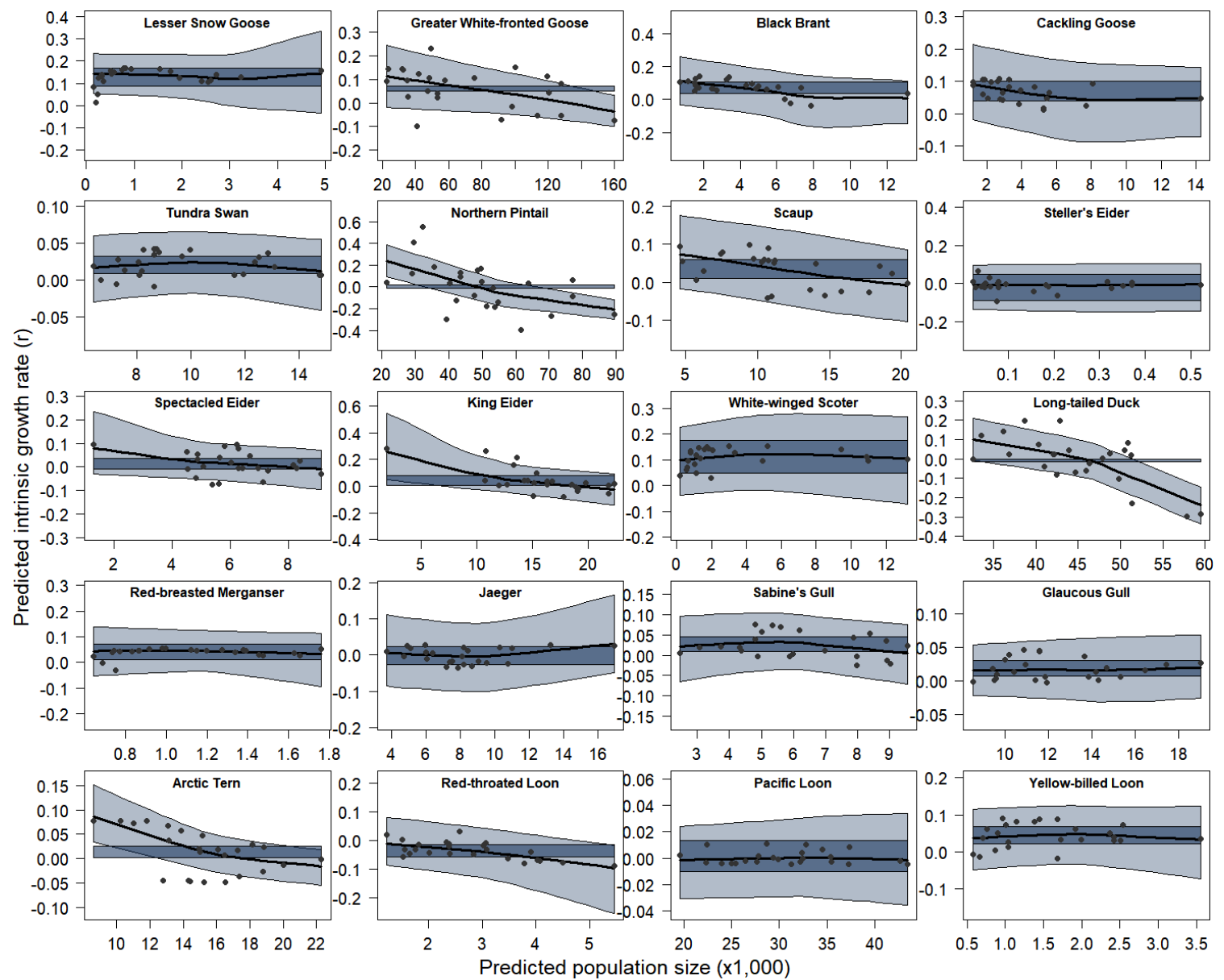

2019). Therefore, our results may demonstrate range expansion of scoters northward; however, issues with scoter data may have affected our results. Surveys likely underestimated abundance in early years. White-winged Scoters are present only in the southern ACP which, prior to 2007 , was surveyed once late relative to seasonal phenology and at low intensity. Surveys may have missed breeding scoters during those years if late surveys coincided with nesting and incubation; scoters initiate nests early in the season and exhibit secretive behavior during nesting resulting in low detection rate of birds (Safine and Lindberg 2008). Further, previous summaries of these data also suggested scoter identification to species is difficult and identification may have changed or improved over time or among crews (Stehn et al. 2013). Therefore, we urge caution in interpreting trends for this species. King Eiders initially increased during our study but have stabilized in recent years (Fig. 4). Conversely, King Eiders decreased substantially on the North Slope from the 1970s to 1990s (Suydam et al. 2000) and a population model suggests a stable or slowly declining population in Alaska driven mostly by adult and duckling survival (Bentzen and Powell 2012).

The three loon species present on the ACP show the full range of population processes. The numerically dominant Pacific Loon appears stable, whereas the less numerous Red-throated and 
Fig. 6. Predicted density (km2) of 20 waterbird species surveyed 1992-2016 across the entire Arctic Coastal Plain, Alaska (ACP; blue), in the National Petroleum Reserve-Alaska (NPR-A; gray), and in the Arctic National Wildlife Refuge (ANWR; yellow). Error bars represent 95\% credible intervals. We present plots in taxonomic order (Chesser et al. 2018) for Lesser Snow Goose (Chen caerulescens caerulescens), Greater Whitefronted Goose (Anser albifrons frontalis), Black Brant (Branta bernicla nigricans), Cackling Goose (B. hutchinsii taverneri), Tundra Swan (Cygnus columbianus), Northern Pintail (Anas acuta), Scaup (Aythya spp., Lesser and Greater combined), Steller's Eider (Polysticta stelleri), Spectacled Eider (Somateria fischeri), King Eider (Somateria spectabilis), White-winged Scoter (Melanitta fusca), Long-tailed Duck (Clangula hyemalis), Redbreasted Merganser (Mergus serrator), Jaeger (Stercorarius spp., Pomarine, Parasitic, and Long-tailed combined), Sabine's Gull (Xema sabini), Glaucous Gull (Larus hyperboreus), Arctic Tern (Sterna paradisaea), Red-throated Loon (Gavia stellata), Pacific Loon (G. pacifica), and Yellow-billed Loon (G. adamsii).
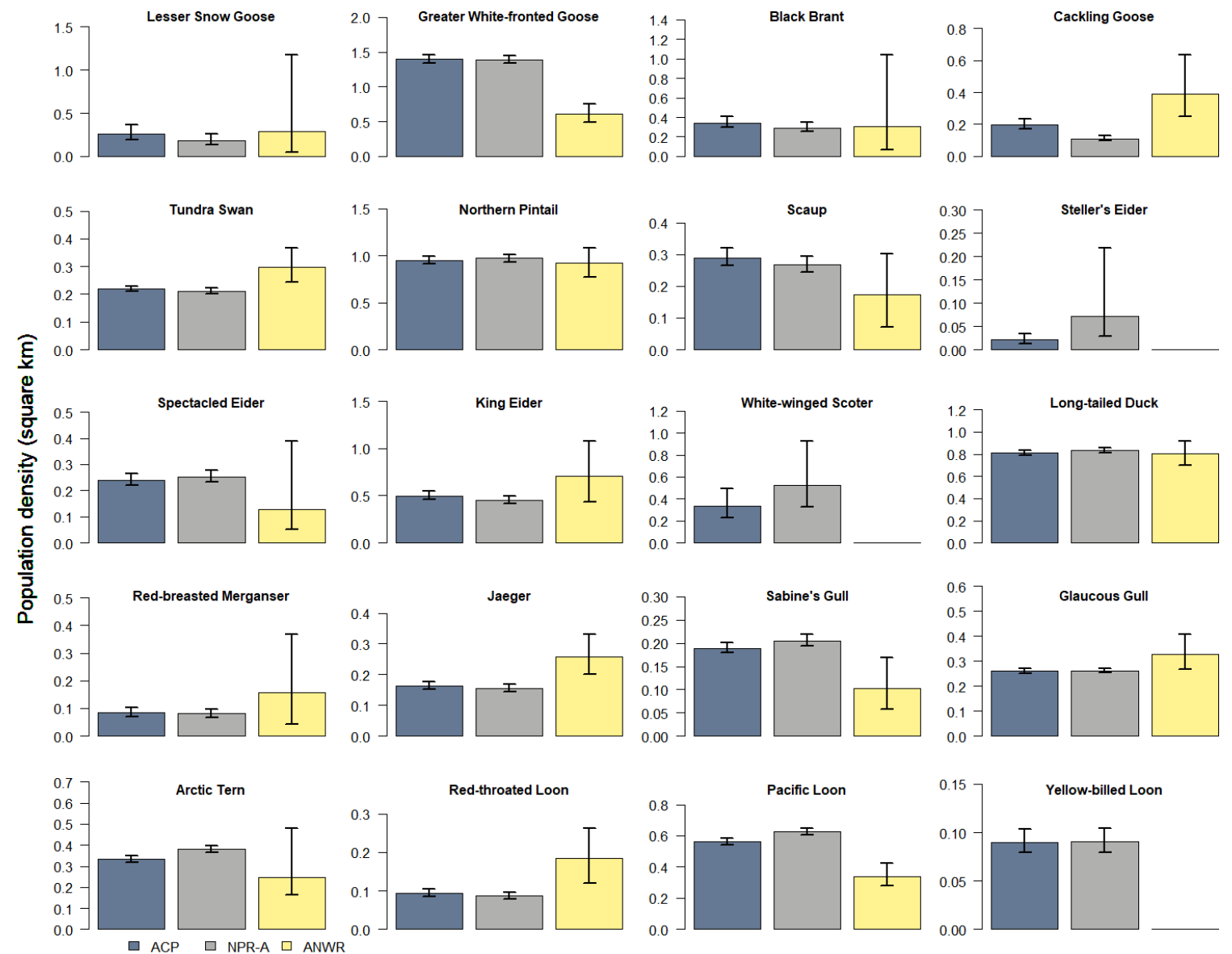

Administrative unit

Yellow-billed Loons demonstrate declining and increasing trends, respectively. Red-throated Loons were the only species to show a substantial rate of decline $\left(-3.4 \% \mathrm{yr}^{-1}\right)$ in our study, which is contrary to stable populations reported from recent data summaries for this survey $\left(-0.5 \% \mathrm{yr}^{-1}, 95 \% \mathrm{CL}:-1.8 \%, 0.08 \% \mathrm{yr}^{-1}\right.$; Wilson et al. 2018) and the Yukon-Kuskokwim Delta Aerial Breeding Waterfowl Survey $(0.0 \%, 90 \%$ CL: $-5 \%, 10 \%$; Swaim 2017). Nevertheless, our results show a slowing of population decline through the study period (Fig. 7), which is consistent with trends on the ACP and other parts of the state (Mallek and Groves 2011, Swaim 2017, Wilson et al. 2018). These loon species have substantially different foraging behaviors and diets as well as migration and wintering habits. Therefore, variation in population trajectory for sympatric species could be driven by local forage availability (Rizzolo 2017) or factors outside the ACP.

Glaucous Gulls are increasing on the ACP whereas some populations have declined in the Canadian Arctic (Gaston et al. 2012, Petersen et al. 2015). These important predators of eggs and chicks have shown similar population growth patterns to cooccurring waterbirds (Barry and Barry 1990, Gilchrist and Robertson 1999). Previous studies have found positive associations between nesting gulls and Common Eiders (Somateria mollissima) at high latitudes (Götmark and Ahlund 1988, Robertson and Chaulk 2017). Similarly, mean cell-specific density of Glaucous Gulls was positively associated with densities 
Fig. 7. Moving window analysis of 10-year population trends for 20 waterbird species surveyed during the Arctic Coastal Plain Breeding Waterfowl Survey, Alaska, 1992-2016. We summarized the geometric mean rate of change in population size for 10-year periods beginning in 1992, i.e., 16 time periods beginning 1992 to 2007. Black lines are loess-smoothed (span $=1$, degree $=1$ ) fits of population trend through time periods. Polygons are loess-smoothed $95 \%$ credible intervals of each decadal trend, and blue polygons represent the $95 \%$ credible interval of the overall population trend (i.e., across all 25 years of the survey). Changes in population trend through time are indicative of increasing or slowing population growth relative to time period. We present plots in taxonomic order (Chesser et al. 2018) for Lesser Snow Goose (Chen caerulescens caerulescens), Greater White-fronted Goose (Anser albifrons frontalis), Black Brant (Branta bernicla nigricans), Cackling Goose (B. hutchinsii taverneri), Tundra Swan (Cygnus columbianus), Northern Pintail (Anas acuta), Scaup (Aythya spp., Lesser and Greater combined), Steller's Eider (Polysticta stelleri), Spectacled Eider (Somateria fischeri), King Eider (Somateria spectabilis), White-winged Scoter (Melanitta fusca), Long-tailed Duck (Clangula hyemalis), Red-breasted Merganser (Mergus serrator), Jaeger (Stercorarius spp., Pomarine, Parasitic, and Long-tailed combined), Sabine's Gull (Xema sabini), Glaucous Gull (Larus hyperboreus), Arctic Tern (Sterna paradisaea), Red-throated Loon (Gavia stellata), Pacific Loon (G. pacifica), and Yellow-billed Loon (G. adamsii).
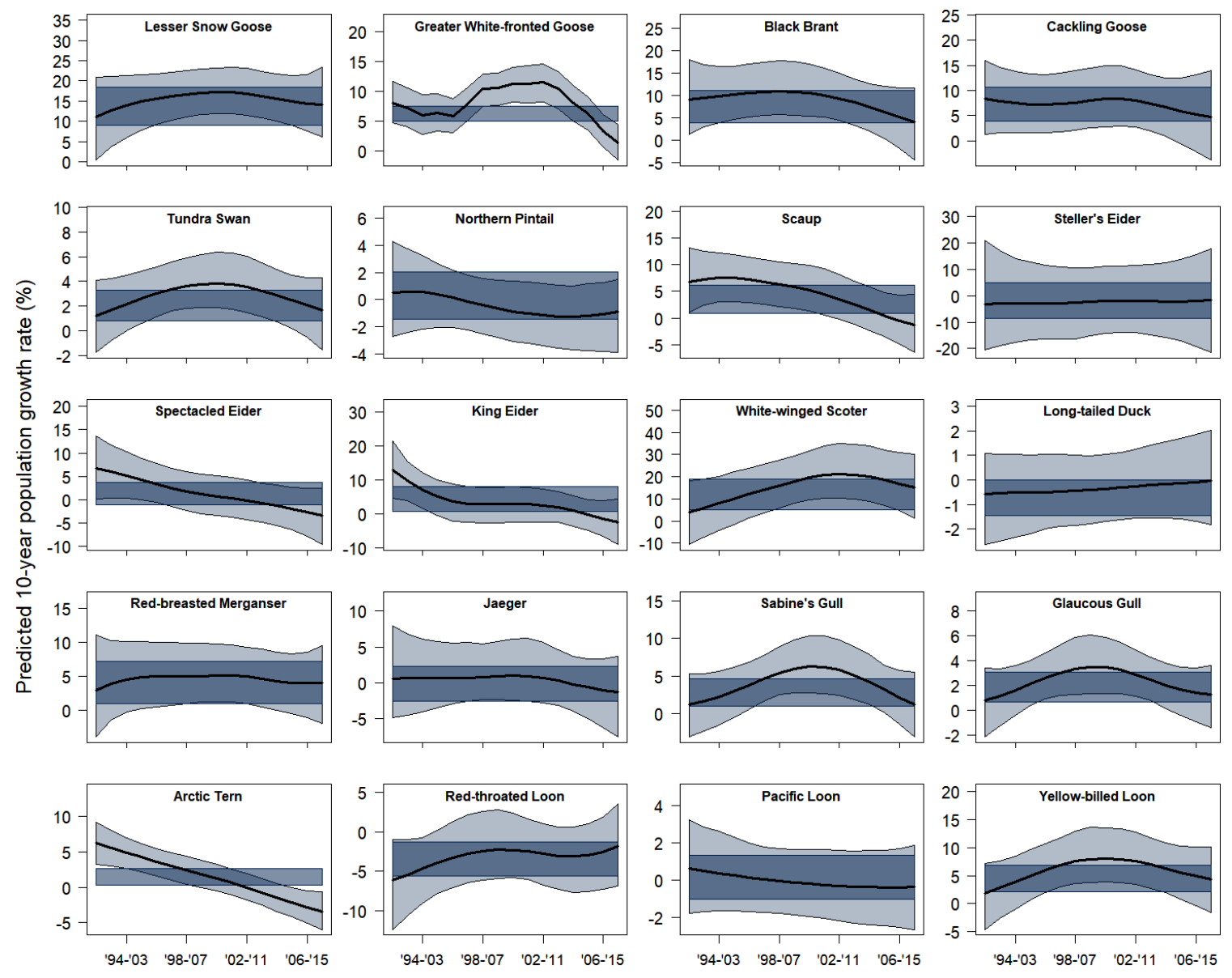

10-year window
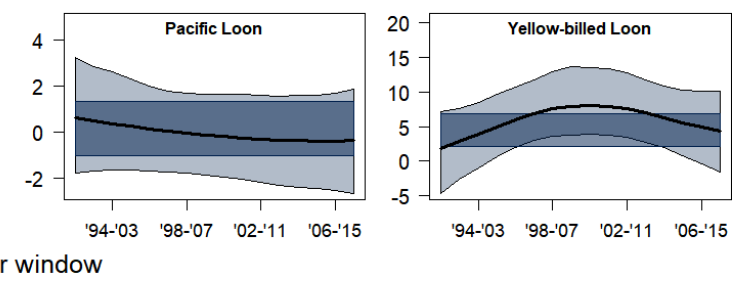

of Spectacled Eider, Sabine's Gull, Red-throated Loon, Greater White-fronted Goose, Tundra Swan, and Northern Pintail in our study (Table A4.3).

Sabine's Gull and Arctic Tern populations were positively associated with each other $(r=0.57)$ and both species increased slowly during the study period. Concordantly, populations of Sabine's Gulls appear to be increasing elsewhere in Alaska, i.e., Yukon-Kuskokwim Delta (Swaim 2017) while Arctic Tern population trends may be declining in Arctic Canada (Gilchrist and Robertson 1999, Maftei et al. 2015). Our results further suggest both populations have showed slowed growth or declines over the most recent 10 years (Fig. 7), with Arctic Terns potentially experiencing density-dependent growth (Fig. 5).

Almost all species we examined showed spatial variation in population trend. For most broadly distributed species, population trends varied latitudinally, with five species increasing faster toward the coast, and four species showing the opposite trend (Appendix 6). Concurrently, trends in the onset of 
Fig. 8. Predicted population trend of 20 waterbird species surveyed 1992-2016 across the Arctic Coastal Plain, Alaska (ACP; blue), in the National Petroleum Reserve-Alaska (NPR-A; gray), and in the Arctic National Wildlife Refuge (ANWR; yellow). Error bars represent 95\% credible intervals. We present plots in taxonomic order (Chesser et al. 2018) for Lesser Snow Goose (Chen caerulescens caerulescens), Greater White-fronted Goose (Anser albifrons frontalis), Black Brant (Branta bernicla nigricans), Cackling Goose (B. hutchinsii taverneri), Tundra Swan (Cygnus columbianus), Northern Pintail (Anas acuta), Scaup (Aythya spp., Lesser and Greater combined), Steller's Eider (Polysticta stelleri), Spectacled Eider (Somateria fischeri), King Eider (Somateria spectabilis), White-winged Scoter (Melanitta fusca), Long-tailed Duck (Clangula hyemalis), Redbreasted Merganser (Mergus serrator), Jaeger (Stercorarius spp., Pomarine, Parasitic, and Long-tailed combined), Sabine's Gull (Xema sabini), Glaucous Gull (Larus hyperboreus), Arctic Tern (Sterna paradisaea), Red-throated Loon (Gavia stellata), Pacific Loon (G. pacifica), and Yellow-billed Loon (G. adamsii).
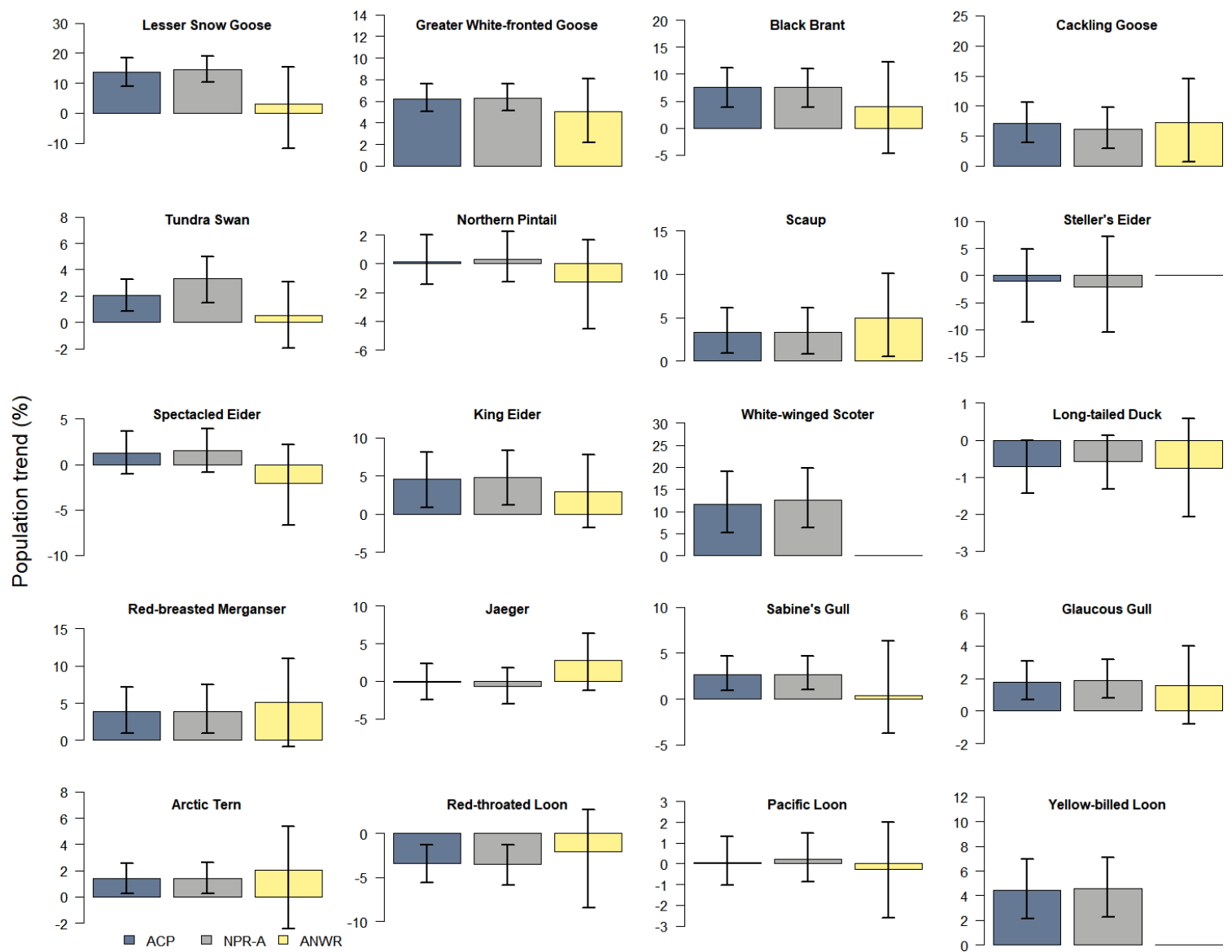

Administrative unit

phenological spring over our study period were stronger in nearshore areas compared to interior areas, especially near the Chukchi Sea coast in northwestern ACP(Figure A2.2). This area, one of the latest areas to warm, has shown the largest change in timing of green-up. These patterns suggest waterbirds may be responding differently to earlier springs and the magnitude and distribution of change in the arrival of spring on the ACP. Further research is needed to assess mechanisms underlying observed patterns of change in our study.

\section{Study considerations}

Our study employed several methods to address potential issues in data collection including spatio-temporal smoothing to account for intermittent sampling in space and time, weighting variances by area surveyed per cell-year-survey, adjusting predictions for phenologically adjusted survey date, and using an offset for the later-survey to account for some variability in detection rate among observers.

Survey timing relative to the onset of spring was important for $85 \%$ of species examined and varied in direction; the number of observations decreased throughout the season for all but four species (Table A4.1). Our results were consistent with trends in survey timing from previous summaries of these data (Larned et al. 2008, 2009) but showed somewhat stronger associations for some species. Therefore, our results confirm the need to control and/or adjust for survey timing relative to breeding status when monitoring abundance of nesting birds with aerial surveys. 
Fig. 9. The relative importance of approximately $36 \mathrm{~km}^{2}$ cells $(6 \mathrm{~km}$ by $6 \mathrm{~km})$ to 20 waterbird species observed during the Arctic Coastal Plain Breeding Waterfowl Survey, Alaska, 1992-2016. We define relative importance as the density of each species divided by their overall mean density in each year; values of $-1,0$, and 1 denote areas that are unimportant, average, or important for each species. We summed values across species to estimate overall importance of a cell. Overall importance includes (in taxonomic order; Chesser et al. 2018) Lesser Snow Goose (Chen caerulescens caerulescens), Greater White-fronted Goose (Anser albifrons frontalis), Black Brant (Branta bernicla nigricans), Cackling Goose (B. hutchinsii taverneri), Tundra Swan (Cygnus columbianus), Northern Pintail (Anas acuta), Scaup (Aythya spp., Lesser and Greater combined), Steller's Eider (Polysticta stelleri), Spectacled Eider (Somateria fischeri), King Eider (Somateria spectabilis), White-winged Scoter (Melanitta fusca), Long-tailed Duck (Clangula hyemalis), Red-breasted Merganser (Mergus serrator), Jaeger (Stercorarius spp., Pomarine, Parasitic, and Long-tailed combined), Sabine's Gull (Xema sabini), Glaucous Gull (Larus hyperboreus), Arctic Tern (Sterna paradisaea), Red-throated Loon (Gavia stellata), Pacific Loon ( $G$. pacifica), and Yellow-billed Loon (G. adamsii).

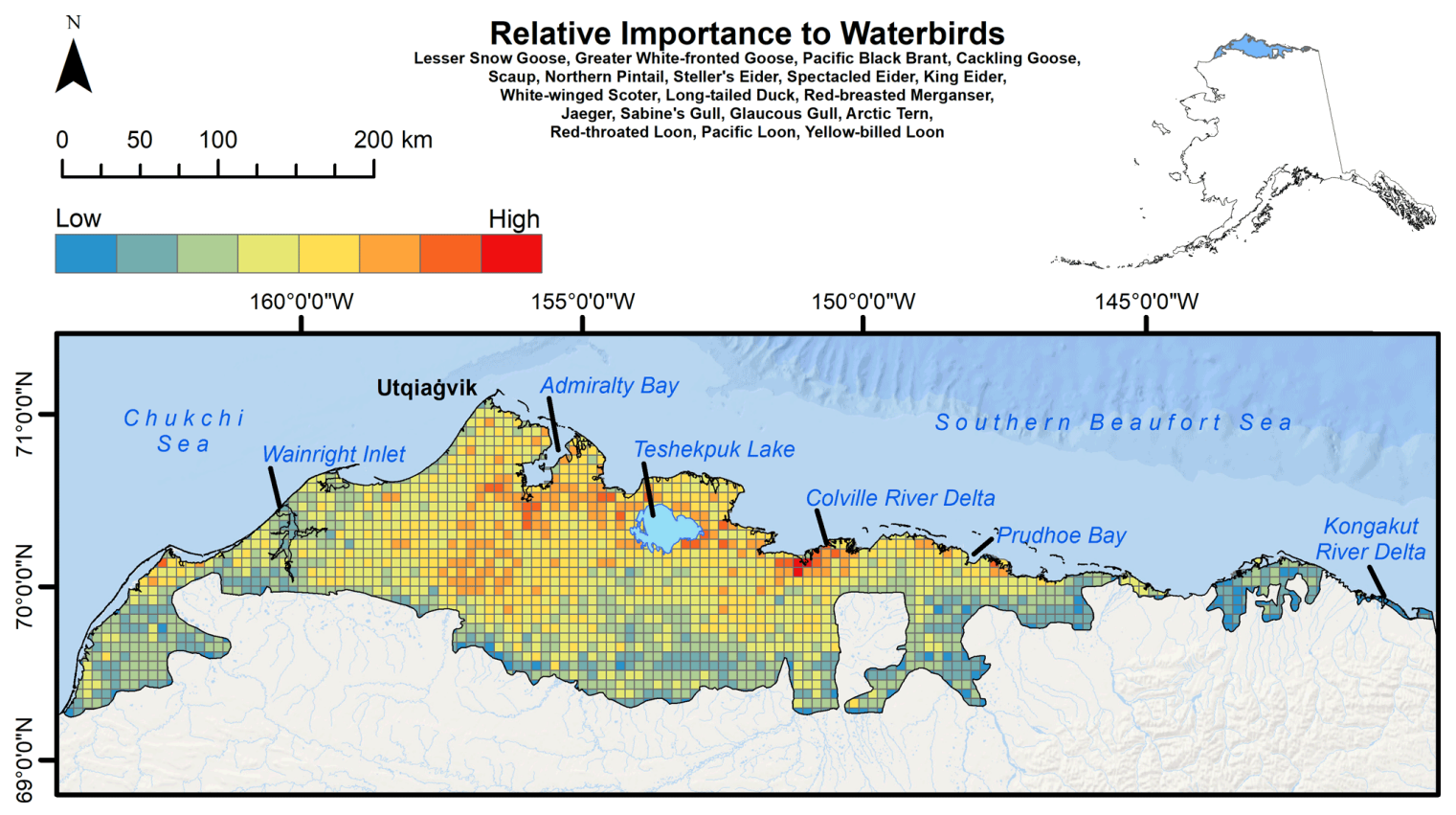

We were unable to formally account for imperfect detection of birds in our study, and as such, assumed that pilot-observer pairs had similar detection among years. To refine this assumption, we included an effect of the latter survey to account for potential observer-pilot pair effects within a year. In general, detection probability may vary by species, group size, time of day, habitat type, flight conditions (e.g., weather, sun glare), season date, observer, or aircraft (Cook and Jacobson 1979, Tracey et al. 2005, Pearse et al. 2008, Koneff et al. 2008, Sauer et al. 2014). Further, some birds may have been missed because planes flushed them outside the 400-m survey strip, and an unknown proportion of birds are not detected. Although study design can minimize some of these effects, detection probability during aerial surveys is imperfect and may bias abundance indices if they vary through time and space (Koneff et al. 2008). Recent work on the ACP suggests detection probability for single and pair observations for a subset of species examined in our study had detection probabilities from $0.41-0.91$ and varied by group size (H. Wilson, USFWS, unpublished data). Similar aerial surveys of waterfowl often incorporate a double-sampling protocol to adjust counts for imperfect detection (Smith 1995). Such methods are logistically intractable in remote, roadless areas of the Arctic, but methods that incorporate photo or video imagery (Buckland et al. 2012), or an additional observer in the plane during surveys, i.e., double-observer (Koneff et al. 2008) may provide valuable ways to include detection probability in abundance indices in the Arctic.

Despite accounting for several sources of potential error, our inference was somewhat limited by sampling constraints. Inconsistent sampling effort in space and time may have masked some spatial patterns of density, occupancy, and core use at the spatial scale we used $(6 \mathrm{~km}$ by $6 \mathrm{~km})$. Increasing the spatial scale examined may illuminate additional patterns at the expense of sampling intensity within each sampling unit, i.e., less area surveyed per cell-year. Further, sampling effort was relatively low in the ANWR and the southern ACP and often these areas were surveyed phenologically late, leading to less power to detect population change and possibly lower or higher detection of some species. For example, Yellow-billed Loon maps suggest relatively high density in the south-central portion of the ACP (Appendix 5), despite low density of large lakes necessary for breeding loon pairs (North and Ryan 1989). Upon further examination, these cells were surveyed infrequently and contained $<3$ nonzero counts 
across the study period. Thus, uncertainty in density for these cells is high, but not captured in density maps (cell-specific density SEs are available from the corresponding author). We removed cells with $<3$ nonzero counts across the study period from trend maps to avoid making conclusions regarding population trend in areas with low sampling effort but did not apply that criterion to density maps to illustrate the full distribution of each species on the ACP. Regardless, increased sampling effort in these areas is needed to improve estimates and resulting maps. Third, we attempted to reduce counts of nonbreeding birds or birds temporarily in an area before dispersing to breeding locations by only including counts of pairs or indicated pairs. This approach may underestimate population size to some extent for many species, particularly colonial species because recording of pairs versus flocks may have been inconsistent among observers and through time. For example, an observation of birds may have been recorded as a flock of eight or four pairs, only the latter of which would have been included in our analysis. Because we only included individuals or pairs, our indices of abundance may be lower than similar studies and trends conservative for some colonial species like Lesser Snow Geese (see Burgess et al. 2017, Wilson et al. 2018). We recommend observers create standardized protocol to ensure breeding status of groups $>2$ are consistently recorded.

Because Arctic waterbirds are migratory, population regulation could occur at other times in their annual cycle. Changes in survival or body condition, e.g., for capital breeders, during the nonbreeding season may influence abundance or breeding success the following year. If so, the resulting change on the ACP is more likely to be uniform and widespread than spatially restricted, a pattern that emerged for several species including Pacific Loon, Northern Pintail, and Long-tailed Duck (Appendix 6). Lifehistory traits make some species more or less susceptible to environmental conditions in the Arctic (Saalfeld and Lanctot 2017). For example, all the species we evaluated are site faithful to breeding areas, but 17 species, i.e., all but Scaup, Red-breasted Merganser, and Northern Pintail, also exhibit delayed reproduction. That is, for most of these species, surviving young may not return to breeding grounds for two to five years and breeding propensity of adults is relatively low and variable (Sibley 2009, Elphick 2016). Therefore, effects of local breeding conditions on reproduction, survival, and dispersal may result in a lagged response in terms of population change. During that time, survival rates of nonbreeding adults will vary based on conditions away from breeding areas, thus masking the effects of local breeding-area conditions on population growth. Population models that incorporate data estimating vital rates such as survival or productivity could help detangle the source, e.g., breeding or nonbreeding areas, and mechanisms underlying observed change for these species.

\section{Future directions}

Although changes to physical environmental drivers are documented and modeled well into the future, responses of avian populations are mediated through complex interactions among life-history traits, environmental conditions, and trophic interactions that are not homogeneous across the landscape or the annual cycle. Because of this, accurate prediction of future population dynamics is difficult (Van Hemert et al. 2015). Our study is the first step to identifying important areas for breeding waterbirds on the ACP and areas exhibiting rapid population change based on long-term, comprehensive survey data of multiple species.

Because the ACP landscape is changing as a result of climatedriven environmental change and anthropogenic disturbance, future research is necessary to identify drivers of observed patterns of change, quantify their effects, and forecast population change into the future. Logical next steps include (1) examining patterns in abundance and trends relative to vegetation and wetland composition to identify environmental characteristics associated with avian density and population change in the Arctic, (2) examining population trends relative to life history traits and nonbreeding ecology that may identify traits or areas outside the Arctic influencing population growth, (3) assessing abundance and trends relative to existing oil and gas infrastructure to inform future leasing decisions, and (4) identifying areas of landscape change, e.g., thermokarst events or vegetation change, in the Arctic to determine wildlife responses and predict how populations will continue to change into the future.

Responses to this article can be read online at: http://www.ace-eco.org/issues/responses.php/1383

\section{Acknowledgments:}

We thank pilots and biologists of the USFWS Migratory Bird Management Division including E. Taylor, pilots E. Mallek and R. King, and numerous right-side observers, especially C. Dau, for contributing to data collection. D. Nigro and others at the Bureau of Land Management provided survey support and valuable comments on earlier drafts. B. Ritchie and others from Alaska Biological Resources, Inc. shared valuable information to assist survey timing. M. Swaim provided data assistance. R. Taylor provided statistical insight. J. Pearce, and two anonymous reviewers provided valuable comments on earlier drafts. The USGS provided funding through the Changing Arctic Ecosystems Initiative and Wildlife Program of the USGS Ecosystem Mission Area, and provided high-powered computing support through the USGS Core Science Analytics, Synthesis, and Libraries Advanced Research Computing group. Any use of trade, product, or firm names in this publication is for descriptive purposes only and does not imply endorsement by the U.S. government.

\section{LITERATURE CITED}

Alisauskas, R. T., R. F. Rockwell, K. W. Dufour, E. G. Cooch, G. Zimmerman, K. L. Drake, J. O. Leafloor, T. J. Moser, and E. T. Reed. 2011. Harvest, survival, and abundance of midcontinent Lesser Snow Geese relative to population reduction efforts. Wildlife Monographs 179:1-42. https://doi.org/10.1002/wmon.5

Andres, B. A., P. A. Smith, R. I. G. Morrison, C. L. Gratto-Trevor, S. C. Brown, and C. A. Friis. 2012. Population estimates of North American shorebirds, 2012. Wader Study Group Bulletin 119:178-194. 
Barry, S. J., and T. W. Barry. 1990. Food habits of Glaucous Gulls in the Beaufort Sea. Arctic 43:43-49. https://doi.org/10.14430/ $\operatorname{arctic} 1589$

Bart, J., R. M. Platte, B. Andres, S. Brown, J. A. Johnson, and W. Larned. 2013. Importance of the National Petroleum ReserveAlaska for aquatic birds. Conservation Biology 27:1304-1312. https://doi.org/10.1111/cobi.12133

Bentzen, R. L., and A. N. Powell. 2012. Population dynamics of King Eiders breeding in northern Alaska. Journal of Wildlife Management 76:1011-1020. https://doi.org/10.1002/jwmg.335

Brown, P. W., and L. H. Fredrickson. 2019. White-winged Scoter (Melanitta fusca), version 1.1. In P. G. Rodewald, editor. The birds of North America. Cornell Lab of Ornithology, Ithaca, New York, USA. https://doi.org/10.2173/bna.whwsco4.01.1

Brown, S., J. Bart, R. B. Lanctot, J. A. Johnson, S. Kendall, D. Payer, and J. Johnson. 2007. Shorebird abundance and distribution on the Coastal Plain of the Arctic National Wildlife Refuge. Condor 109:1-14. https://doi.org/10.1650/0010-5422 (2007)109[1:SAADOT]2.0.CO;2

Brownfield, M. E., R. R. Charpentier, T. A. Cook, D. L. Gautier, D. K. Higley, M. A. Kirschbaum, T. R. Klett, J. K. Pitman, R. M. Pollastro, C. J. Schenk, M. E. Tennyson, C. J. Wandrey, and K. J. Whidden. 2012. An estimate of undiscovered conventional oil and gas resources. Fact Sheet. U. S. Geological Survey, Denver, Colorado, USA.

Buckland, S. T., M. L. Burt, E. A. Rexstad, M. Mellor, A. E. Williams, and R. Woodward. 2012. Aerial surveys of seabirds: the advent of digital methods. Journal of Applied Ecology 49:960-967. https://doi.org/10.1111/j.1365-2664.2012.02150.x

Bureau of Land Management (BLM). 2018. Draft supplemental environmental impact statement for the Alpine Satellite Development Plan for the proposed Greater Moose's Tooth Two Development Project. U. S. Department of the Interior, Anchorage, Alaska, USA.

Burgess, R. M., R. J. Ritchie, B. T. Person, R. S. Suydam, J. E. Shook, A. K. Prichard, and T. Obritschkewitsch. 2017. Rapid growth of a nesting colony of Lesser Snow Geese (Chen caerulescens caerulescens) on the Ikpikpuk River Delta, North Slope, Alaska, USA. Waterbirds 40:11-23. https://doi. org/10.1675/063.040.0103

Chesser, R. T., K. J. Burns, C. Cicero, J. L. Dunn, A. W. Kratter, I. J. Lovette, P. C. Rasmussen, J. V. Remsen, Jr., D. F. Stotz, B. M. Winger, and K. Winker. 2018. Fifty-ninth supplement to the American Ornithological Society's check-list of North American birds. Auk 135:798-813. https://doi.org/10.1642/AUK-18-62.1

Conn, P. B., D. S. Johnson, J. M. Ver Hoef, M. B. Hooten, J. M. London, and P. L. Boveng. 2015. Using spatiotemporal statistical models to estimate animal abundance and infer ecological dynamics from survey counts. Ecological Monographs 85:235-252. https://doi.org/10.1890/14-0959.1

Cook, D. R., and J. O. Jacobson. 1979. A design for estimating visibility bias in aerial surveys. Biometrics 35:735-742. https://doi. org/10.2307/2530104
Day, R. H., I. J. Stenhouse, and H. G. Gilchrist. 2001. Sabine's Gull (Xema sabini), version 2.0. In A. F. Poole and F. B. Gill, editors. The birds of North America. Cornell Lab of Ornithology, Ithaca, New York, USA. https://doi.org/10.2173/bna.593

Dennis, B., and M. L. Taper. 1994. Density dependence in time series observations of natural populations; estimation and testing. Ecological Monographs 64:205-224. https://doi.org/10.2307/2937041

Derksen, D. V., and W. D. Eldridge. 1980. Drought-displacement of pintails to the Arctic Coastal Plain, Alaska. Journal of Wildlife Management 44:224-229. https://doi.org/10.2307/3808374

Derksen, D. V., W. D. Eldridge, and M. W. Weller. 1982. Habitat ecology of Pacific Black Brant and other geese moulting near Teshekpuk Lake, Alaska. Wildfowl 33:39-57.

Derksen, D. V., T. C. Rothe, and W. D. Eldridge. 1981. Use of wetland habitats in the National Petroleum Reserve-Alaska. Resource Publication 141, U.S. Department of the Interior, Fish and Wildlife Service, Washington, D.C., USA.

Didan, K., A. B. Munoz, T. Miura, X. Zhang, M. Friedl, J. Gray, W. Van, J. Czapla-myers, S. D. Bennett, C. Jenkerson, T. Maiersperger, and D. Meyer. 2015. Multi-sensor vegetation index and phenology earth science data records: algorithm theoretical basis document and user guide. University of Arizona, Tuscon, Arizona, USA.

Dunham, K., and J. B. Grand. 2017. Evaluating models of population process in a threatened population of Steller's Eiders: a retrospective approach. Ecosphere 8:e01720. https://doi. org/10.1002/ecs 2.1720

Elphick, J. 2016. Birds: a complete guide to their biology and behavior. Firefly Books, Richmond Hill, Ontario, Canada.

Flint, P. L. 2013. Changes in size and trends of North American sea duck populations associated with North Pacific oceanic regime shifts. Marine Biology 160:59-65. https://doi.org/10.1007/ s00227-012-2062-y

Flint, P. L., E. J. Mallek, R. J. King, J. A. Schmutz, K. S. Bollinger, and D. V. Derksen. 2008. Changes in abundance and spatial distribution of geese molting near Teshekpuk Lake, Alaska: interspecific competition or ecological change? Polar Biology 31:549-556. https://doi.org/10.1007/s00300-007-0386-8

Flint, P. L., B. W. Meixell, and E. J. Mallek. 2014. High fidelity does not preclude colonization: range expansion of molting Black Brant on the Arctic coast of Alaska. Journal of Field Ornithology 85:75-83. https://doi.org/10.1111/jofo. 12051

Fresco, N., A. Floyd, M. Lindgren, A. Bennett, L. Krutikov, and S. Marchenko. 2015. Abiotic change agents. Section C in E. J. Trammell, M. L. Carlson, N. Fresco, T. Gotthardt, M. L. McTeague, and D. Vadapalli, editors. North slope rapid ecoregional assessment. Prepared for the Bureau of Land Management, U. S. Department of the Interior, Anchorage, Alaska, USA.

Gaston, A. J., M. L. Mallory, and H. G. Gilchrist. 2012. Populations and trends of Canadian Arctic seabirds. Polar Biology 35:1221-1232. https://doi.org/10.1007/s00300-012-1168-5 
Gelman, A., and D. Rubin. 1992. Inference from iterative simulation using multiple sequences. Statistical Science 7:457-472. https://doi.org/10.1214/ss/1177011136

Gilchrist, H. G., and G. J. Robertson. 1999. Population trends of gulls and Arctic Terns nesting in the Belcher Islands, Nunavut. Arctic 52:325-331. https://doi.org/10.14430/arctic939

Götmark, F., and M. Ahlund. 1988. Nest predation and nest site selection among eiders Somateria mollissima: the influence of gulls. Ibis 130:111-123. https://doi.org/10.1111/j.1474-919X.1988. tb00961.x

Gustine, D., P. Barboza, L. Adams, B. Griffith, R. Cameron, and K. Whitten. 2017. Advancing the match-mismatch framework for large herbivores in the Arctic: evaluating the evidence for a trophic mismatch in caribou. PLoS ONE 12:e171807. https://doi. org/10.1371/journal.pone.0171807

Harsem, Ø., K. Heen, J. M. P. Rodrigues, and T. Vassdal. 2015. Oil exploration and sea ice projections in the Arctic. Polar Record 51:91-106. https://doi.org/10.1017/S0032247413000624

Hatch, J. J. 2002. Arctic Tern (Sterna paradisaea), version 2.0. In A. F. Poole and F. B. Gill, editors. The birds of North America. Cornell Lab of Ornithology, Ithaca, New York, USA. https://doi. org/10.2173/bna.707

Hayes, M. 2015. Cracking the ice on arctic oil and gas exploration: assessing the environmental and financial feasibility of arctic oil and natural gas exploration. Thesis. Duke University, North Carolina, USA.

Hefley, T. J., K. M. Broms, B. M. Brost, F. E. Buderman, S. L. Kay, H. R. Scharf, J. R. Tipton, P. J. Williams, and M. B. Hooten. 2017. The basis function approach for modeling autocorrelation in ecological data. Ecology 98:632-646. https://doi.org/10.1002/ ecy. 1674

Henny, C. J. 1973. Drought displaced movement of North American pintails into Siberia. Journal of Wildlife Management 37:23-29. https://doi.org/10.2307/3799734

Hines, J. E., M. F. Kay, and M. O. Wiebe. 2003. Aerial surveys of Greater White-fronted Geese Anser albifrons frontalis and other waterfowl in the Rasmussen Lowlands of the Central Canadian Arctic. Wildfowl 54:185-201.

Hinzman, L. D., N. D. Bettez, W. R. Bolton, F. S. Chapin, M. B. Dyurgerov, C. L. Fastie, B. Griffith, R. D. Hollister, A. Hope, H. P. Huntington, A. M. Jensen, G. J. Jia, T. Jorgenson, D. L. Kane, D. R. Klein, G. Kofinas, A. H. Lynch, A. H. Lloyd, A. D. McGuire, F. E. Nelson, W. C. Oechel, T. E. Osterkamp, C. H. Racine, V. E. Romanovsky, R. S. Stone, D. A. Stow, M. Sturm, C. E. Tweedie, G. L. Vourlitis, M. D. Walker, D. A. Walker, P. J. Webber, J. M. Welker, K. S. Winker, and K. Yoshikawa. 2005. Evidence and implications of recent climate change in northern Alaska and other Arctic regions. Climatic Change 72:251-298. https://doi.org/10.1007/s10584-005-5352-2

Kellner, K. 2018. JagsUI: a wrapper around rjags to streamline JAGS analyses. R package version 1.5.0. [online] URL: https:// cran.r-project.org/web/packages/jagsUI/index.html

Kéry M. 2010. Introduction to WinBUGS for ecologists. Academic Press, New York, New York, USA.
Kessel, B., D. A. Rocque, and J. S. Barclay. 2002. Greater Scaup (Aythya marila), version 2.0. In A. F. Poole and F. B. Gill, editors. The birds of North America. Cornell Lab of Ornithology, Ithaca, New York, USA. https://doi.org/10.2173/bna.650

Kittel, T. G. F., B. B. Baker, J. V. Higgins, and J. C. Haney. 2011. Climate vulnerability of ecosystems and landscapes on Alaska's North Slope. Regional Environmental Change 11:249-264. https:// doi.org/10.1007/s10113-010-0180-y

Koneff, M. D., J. A. Royle, M. C. Otto, J. S. Wortham, and J. K. Bidwell. 2008. A double-observer method to estimate detection rate during aerial waterfowl surveys. Journal of Wildlife Management 72:1641-1649.

Larned, W. W., R. M. Platte and R. A. Stehn. 2009. Waterfowl breeding population survey, Arctic Coastal Plain, Alaska, 2008. U. S. Fish and Wildlife Service, Region 7, Division of Migratory Bird Management, Anchorage, Alaska, USA.

Larned, W., R. A. Stehn, and R. M. Platte. 2011. Waterfowl breeding population survey, Arctic Coastal Plain, Alaska 2010. U. S. Fish and Wildlife Service, Region 7, Division of Migratory Birds, Anchorage, Alaska, USA.

Larned, W. W., R. A. Stehn, and R. M. Platte. 2008. Waterfowl breeding population survey, Arctic Coastal Plain, Alaska, 2007. U. S. Fish and Wildlife Service, Region 7, Division of Migratory Bird Management, Anchorage, Alaska, USA.

Lewis, T. L., P. L. Flint, J. A. Schmutz, and D. V. Derksen. 2010. Temporal and spatial shifts in habitat use by Black Brant immediately following flightless molt. Wilson Journal of Ornithology 122:484-493. https://doi.org/10.1676/09-114.1

Liebezeit, J. R., K. E. B. Gurney, M. Budde, S. Zack, and D. Ward. 2014. Phenological advancement in Arctic bird species: relative importance of snow melt and ecological factors. Polar Biology 37:1309-1320. https://doi.org/10.1007/s00300-014-1522-x

Liebezeit, J. R., G. C. White, and S. Zack. 2011. Breeding ecology of birds at Teshekpuk Lake: a key habitat site on the Arctic Coastal Plain of Alaska. Arctic 64:32-44. https://doi. org/10.14430/arctic4078

Liebezeit, J. R., and S. Zack. 2008. Point counts underestimate the importance of Arctic foxes as avian nest predators: evidence from remote video cameras in Arctic Alaskan oil fields. Arctic 61:153-161. https://doi.org/10.14430/arctic32

Maftei, M., S. E. Davis, and M. L. Mallory. 2015. Assessing regional populations of ground-nesting marine birds in the Canadian High Arctic. Polar Research 34. https://doi. org/10.3402/polar.v34.25055

Mallek, E. J., and D. J. Groves. 2011. Alaska-Yukon waterfowl breeding population survey. U.S. Fish and Wildlife Service, Region 7, Division of Migratory Birds, Fairbanks and Juneau, Alaska, USA.

Martin, T. G., B. A. Wintle, J. R. Rhodes, P. M. Kuhnert, S. A. Field, S. J. Low-Choy, A. J. Tyre, and H. P. Possingham. 2005. Zero tolerance ecology: improving ecological inference by modelling the source of zero observations. Ecology Letters 8:1235-1246. https://doi.org/10.1111/j.1461-0248.2005.00826.x 
McKinnon, L., M. Picotin, E. Bolduc, C. Julliet, and J. Bêty. 2012. Timing of breeding, peak food availability, and effects of mismatch on chick growth in birds nesting in the High Arctic. Canadian Journal of Zoology 90:961-971. https://doi.org/10.1139/ z2012-064

Meixell, B. W., and P. L. Flint. 2017. Effects of industrial and investigator disturbance on Arctic-nesting geese. Journal of Wildlife Management 81:1372-1385. https://doi.org/10.1002/ jwmg.21312

Myers-Smith, I. H., B. K. Arnesen, R. M. Thompson, and F. S. Chapin. 2006. Cumulative impacts on Alaskan Arctic tundra of a quarter century of road dust. Ecoscience 13:503-510.

National Research Council. 2003. Cumulative environmental effects of oil and gas activities on Alaska's North Slope. National Academies Press, Washington, D.C., USA. https://doi. org/10.17226/10639

Nicolai, C. A., P. L. Flint, and M. L. Wege. 2005. Annual survival and site fidelity of Northern Pintails banded on the YukonKuskokwim Delta, Alaska. Journal of Wildlife Management 69:1202-1210.

North, M. R., and M. R. Ryan. 1989. Characteristics of lakes and nest sites used by Yellow-billed Loons in Arctic Alaska. Journal of Field Ornithology 60:296-304.

Northrup, J. M., and G. Wittemyer. 2013. Characterising the impacts of emerging energy development on wildlife, with an eye towards mitigation. Ecology Letters 16:112-125. https://doi. org/10.1111/ele.12009

Obritschkewitsch, T., and R. J. Ritchie. 2017. Steller's Eider surveys near Utqiagivik, Alaska, 2016. U.S. Bureau of Land Management and U.S. Fish and Wildlife Service, Fairbanks, Alaska, USA.

Pearse, A. T., P. D. Gerard, S. J. Dinsmore, R. M. Kaminski, and K. J. Reinecke. 2008. Estimation and correction of visibility bias in aerial surveys of wintering ducks. Journal of Wildlife Management 72:808-813. https://doi.org/10.2193/2007-274

Petersen, A., D. B. Irons, H. G. Gilchrist, G. J. Robertson, D. Boertmann, H. Strøm, M. Gavrilo, Y. Artukhin, D. S. Clausen, K. J. Kuletz, and M. L. Mallory. 2015. The status of Glaucous Gulls Larus hyperboreus in the circumpolar Arctic. Arctic 68:107-120. https://doi.org/10.14430/arctic4462

Plummer, M. 2003. JAGS: a program for analysis of Bayesian graphical models using Gibbs sampling. Proceedings of the $3 r d$ International Workshop on Distributed Statistical Computing (DSC 2003). March 20-22, Vienna, Austria. ISSN 1609-395X. [online] URL: https://www.r-project.org/conferences/DSC-2003/ Drafts/Plummer.pdf

R Core Team. 2018. R: a language and environment for statistical computing. R Foundation for Statistical Computing, Vienna, Austria. [online] URL: http://www.R-project.org/

Rizzolo, D. 2017. Contrasting diet, growth, and energy provisioning in loons breeding sympatrically in the Arctic. Dissertation, University of Alaska, Fairbanks, USA.
Robertson, G. J., and K. G. Chaulk. 2017. Common Eider and large gull nesting associations in coastal Labrador. Arctic Science 3:689-697. https://doi.org/10.1139/as-2017-0012

Ross, B. E., M. B. Hooten, J.-M. DeVink, and D. N. Koons. 2015. Combined effects of climate, predation, and density dependence on Greater and Lesser Scaup population dynamics. Ecological Applications 25:1606-1617. https://doi.org/10.1890/14-0582.1

Royle, J. A., and R. M. Dorazio. 2008. Hierarchical modeling and inference in ecology: the analysis of data from populations, metapopulations and communities. Elsevier/Academic Press, New York, New York, USA.

Saalfeld, S. T., and R. B. Lanctot. 2017. Multispecies comparisons of adaptability to climate change: a role for life-history characteristics? Ecology and Evolution 7:10492-10502. https://doi. org/10.1002/ece3.3517

Safine, D. E., and M. S. Lindberg. 2008. Nest habitat selection of White-winged Scoters on Yukon Flats, Alaska. Wilson Journal of Ornithology 120:582-593. https://doi.org/10.1676/06-157.1

Sauer, J. R., G. S. Zimmerman, J. D. Klimstra, and W. A. Link. 2014. Hierarchical model analysis of the Atlantic Flyway Breeding Waterfowl Survey. Journal of Wildlife Management 78:1050-1059. https://doi.org/10.1002/jwmg.748

Serreze, M. C., and J. Stroeve. 2015. Arctic sea ice trends, variability and implications for seasonal ice forecasting. Philosophical Transactions of the Royal Society A: Mathematical, Physical and Engineering Sciences 373(2045). https://doi. org/10.1098/rsta.2014.0159

Shults, B. S., and C. P. Dau. 2016. Abundance and distribution of molting geese in the Teshekpuk Lake area, July 2015. U.S. Fish and Wildlife Service, Region 7, Division of Migratory Birds, Anchorage, Alaska, USA.

Sibley, D. A. 2009. The Sibley guide to bird life and behavior. Knopf/ Doubleday, New York, New York, USA.

Smith, G. W. 1995. A critical review of the aerial and ground surveys of breeding waterfowl in North America. Biological Science Report 5, National Biological Service, Washington, D.C., USA.

Stehn, R. A., W. W. Larned, and R. M. Platte. 2013. Analysis of aerial survey indices monitoring waterbird populations of the Arctic Coastal Plain, Alaska, 1986-2012. U.S. Fish and Wildlife Service, Region 7, Division of Migratory Birds, Anchorage, Alaska, USA.

Suydam, R. S., D. L. Dickson, J. B. Fadely, and L. T. Quakenbush. 2000. Population declines of King and Common Eiders of the Beaufort Sea. Condor 102:219-222.

Swaim, M. A. 2017. Abundance and Trend of Waterbird Populations on the Yukon-Kuskokwim Delta, Alaska, 1988-2016. U.S. Fish and Wildlife Service, Region 7, Migratory Birds, Anchorage, Alaska, USA.

Tape, K. D., P. L. Flint, B. W. Meixell, and B. V Gaglioti. 2013. Inundation, sedimentation, and subsidence creates goose habitat along the Arctic coast of Alaska. Environmental Research Letters 8:45031. https://doi.org/10.1088/1748-9326/8/4/045031 
Taylor, E. J. 1995. Molt of Black Brant (Branta bernicla nigricans) on the Arctic Coastal Plain, Alaska. Auk 112:904-919. https://doi. org/10.2307/4089022

Tracey, J. P., P. J. Fleming, and G. J. Melville. 2005. Does variable probability of detection compromise the use of indices in aerial surveys of medium-sized mammals? Wildlife Research 32:245-252. https://doi.org/10.1071/wr03126

U.S. Fish and Wildlife Service (USFWS). 2018. Waterfowl population status, 2018. Department of the Interior, Washington, D.C., USA.

U.S. Fish and Wildlife Service (USFWS), and Canadian Wildlife Service. 1987. Standard operating procedures for aerial breeding ground population and habitat surveys in North America. Department of Interior and Canadian Wildlife Service, Laurel, Maryland, USA.

Vadapalli, D., S. Martin, S. Pathan, C. Kennedy, L. Fritz, V. deCarli, and R. Frazier. 2015. Anthropogenic change agents. Pages E1-E99 in E. J. Trammell, M. L. Carlson, N. Fresco, T. Gotthardt, M. L. McTeague, and D. Vadapalli, editors. North Slope rapid ecoregional assessment. Prepared for the Bureau of Land Management, U.S. Department of the Interior, Anchorage, Alaska, USA.

Van Hemert, C., P. L. Flint, M. S. Udevitz, J. C. Koch, T. C. Atwood, K. L. Oakley, and J. M. Pearce. 2015. Forecasting wildlife response to rapid warming in the Alaskan Arctic. BioScience 65:718-728. https://doi.org/10.1093/biosci/biv069

Weiser, E., and H. G. Gilchrist. 2012. Glaucous Gull (Larus hyperboreus), version 2.0. In A. F. Poole, editor. The birds of North America. Cornell Lab of Ornithology, Ithaca, New York, USA. https://doi.org/10.2173/bna.573

Wiley, R. H., and D. S. Lee. 1998. Long-tailed Jaeger (Stercorarius longicaudus), version 2.0. In A. F. Poole and F. B. Gill, editors. The birds of North America. Cornell Lab of Ornithology, Ithaca, New York, USA. https://doi.org/10.2173/bna.365

Wiley, R. H., and D. S. Lee. 1999. Parasitic Jaeger (Stercorarius parasiticus), version 2.0. In A. F. Poole and F. B. Gill, editor. The birds of North America. Cornell Lab of Ornithology, Ithaca, New York, USA. https://doi.org/10.2173/bna.445

Wiley, R. H., and D. S. Lee. 2000. Pomarine Jaeger (Stercorarius pomarinus), version 2.0. In A. F. Poole and F. B. Gill, editor. The birds of North America. Cornell Lab of Ornithology, Ithaca, New York, USA. https://doi.org/10.2173/bna.483

Wilson, H. M., W. W. Larned, and M. A. Swaim. 2018. Aerial survey indices of waterbird populations on the Arctic Coastal Plain, 1986-2017. U.S. Fish and Wildlife Service, Region 7, Migratory Birds, Anchorage, Alaska, USA.
Wood, S. N. 2003. Thin plate regression splines. Journal of the Royal Statistical Society: Series B: Statistical Methodology 65:95-114. https://doi.org/10.1111/1467-9868.00374

Wood, S. N., N. Pya, and B. Säfken. 2016. Smoothing parameter and model selection for general smooth models. Journal of the American Statistical Association 111:1548-1563. https://doi. org/10.1080/01621459.2016.1180986

Zhang, X., M. A. Friedl, C. B. Schaaf, A. H. Strahler, J. C. F. Hodges, F. Gao, B. C. Reed, and A. Huete. 2003. Monitoring vegetation phenology using MODIS. Remote Sensing of Environment 84:471-475. https://doi.org/10.1016/S0034-4257(02) 00135-9
Editor-in-Chief: Ryan Norris Subject Editor: Alexander L.Bond
Sponsored by the Society of Canadian Ornithologists and Bird Studies Canada Parrainée par la Société des ornithologistes du Canada et Études d'oiseaux Canada

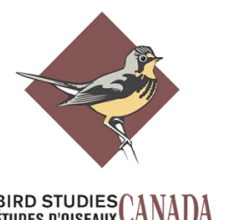


Appendix 1: Zero-inflation

\section{Spatio-temporal population change of arctic-breeding waterbirds}

We evaluated the ability of our model structure to accurately represent the number of zeros in the data by deriving 3,000 realizations of count data using Monte Carlo simulations of converged model parameter estimates and associated error and comparing the resulting proportion of zeros in the realized counts with the proportion of zeros observed in the real data (Figure 1). Results suggest negative binomial models were sufficient for most species, but underestimated the proportion of zeros for Greater White-fronted Goose, Northern Pintail, and Long-tailed Duck by to 0.02 to 0.05 . These species were the most abundant and broadly distributed in our study and thus, higher mean abundance may have prevented the level of overdispersion necessary in the negative binomial distribution to accurately reflect the number of zeros in the data. Further examination of the spatio-temporal trends in bias showed no pattern in bias through time; estimates were generally consistently lower for realized data (Figure 2). Patterns in space showed that locations of disagreement were generally peripheral to high density areas (Figure 3). The negative binomial Northern Pintail model could adequately predict the number of zeros in the early survey replicate, but not the later survey (Figure 4). As a result of this analysis we reran the three biased species using a zero-inflated negative binomial distribution (ZINB) (see main text). 
Figure A1.1. Proportion of zero counts from the data and 3,000 realizations of count data from four species of waterbirds counted on the Arctic Coastal Plain, Alaska 1992-2016. Species include: Lesser Snow Goose (SNGO), Greater White-fronted Goose (GWFG), Black Brant (BLBR), Cackling Goose (CAGO), Tundra Swan (TUSW), Northern Pintail (NOPI), Scaup (Lesser and Greater combined) (SCAU), Steller's Eider (STEI), Spectacled Eider (SPEI), King Eider (KIEI), White-winged Scoter (WWSC), Long-tailed Duck (LTDU), Red-breasted Merganser (RBME), Jaeger (Pomarine, Parasitic, and Long-tailed combined) (JAEG), Sabine's Gull (SAGU), Glaucous Gull (GLGU), Arctic Tern (ARTE), Red-throated Loon (RTLO), Pacific Loon (PALO), and Yellow-billed Loon (YBLO). We report the distribution of realized values and the actual proportion of zeros as a dashed line.
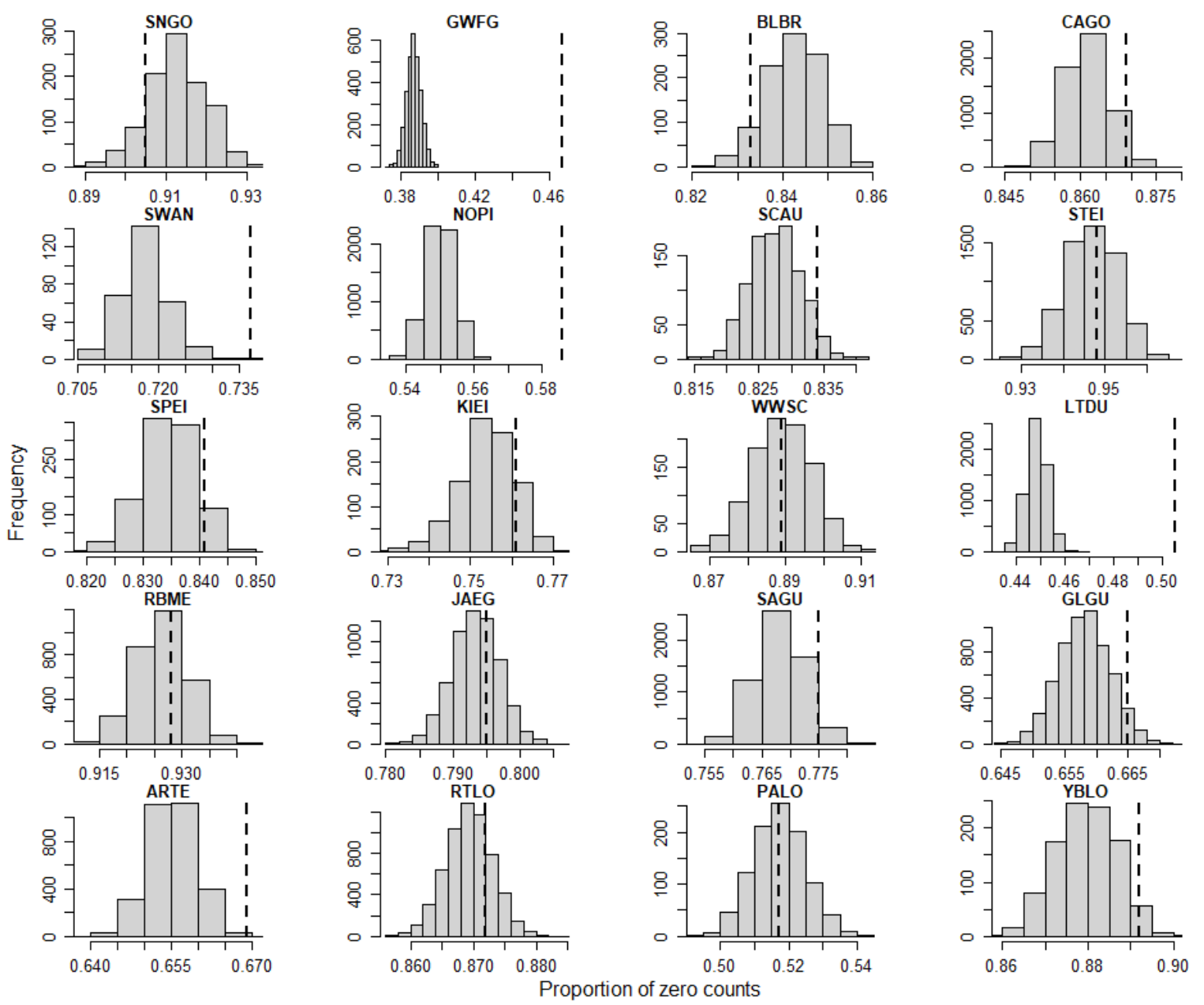
Figure A1.2. Annual mean proportion of zero counts from the data and 3,000 realizations of count data from three species of waterbirds counted on the Arctic Coastal Plain, Alaska 19922016 (year 1 = 1992) including Greater White-fronted Goose (GWFG), Northern Pintail (NOPI), and Long-tailed Duck (LTDU). Black points denote mean actual data and red points denote mean realizations.
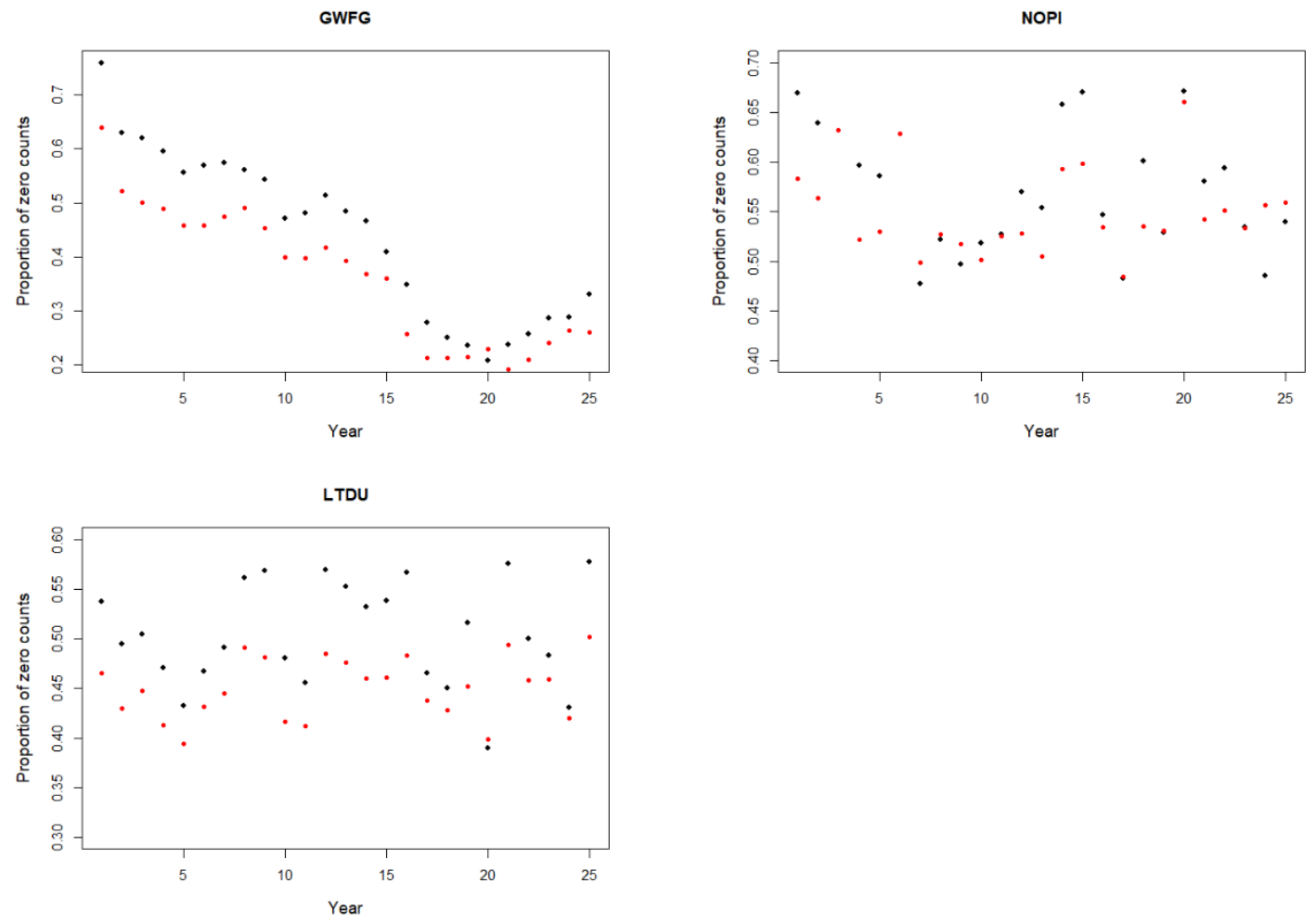
Figure A1.3. Proportion of zero counts from the data and 3,000 realizations of count data from three species of waterbirds counted on the Arctic Coastal Plain, Alaska 1992-2016 including Greater White-fronted Goose (GWFG), Northern Pintail (NOPI), and Long-tailed Duck (LTDU). Purple denotes areas where realized data and actual data both predicted non-zero counts, tealblue areas are areas of disagreement between realized and actual data (i.e., only one group predicted a zero count), and yellow denotes areas where both realized and actual data resulted in zero counts.
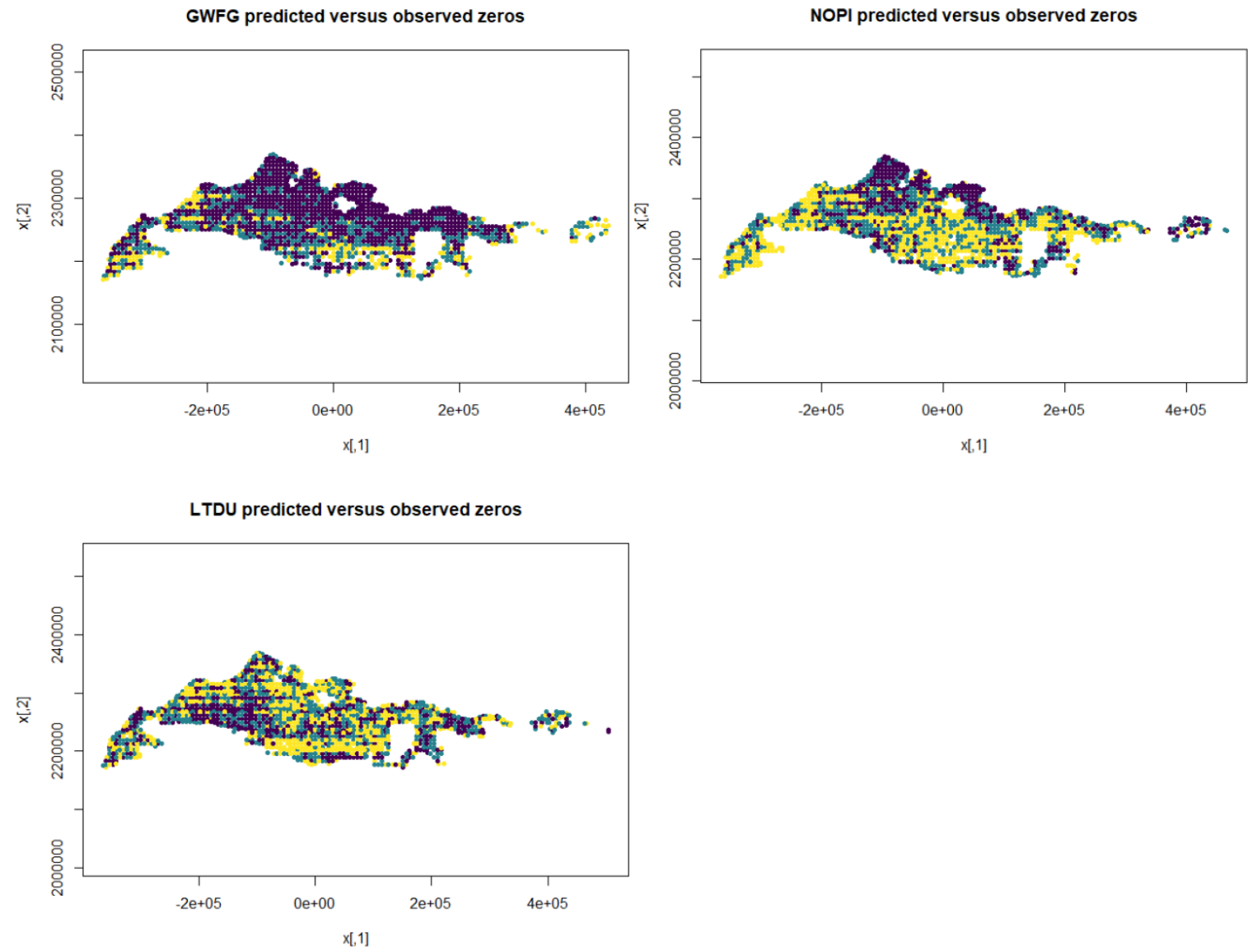
Figure A1.4. Proportion of zero counts from the data and 3,000 realizations of count data from three species of waterbirds counted on the Arctic Coastal Plain, Alaska 1992-2016 including Greater White-fronted Goose (GWFG), Northern Pintail (NOPI), and Long-tailed Duck (LTDU). We report the distribution of realized values and the actual proportion of zeros as a dashed line for each survey replicate (early, late).
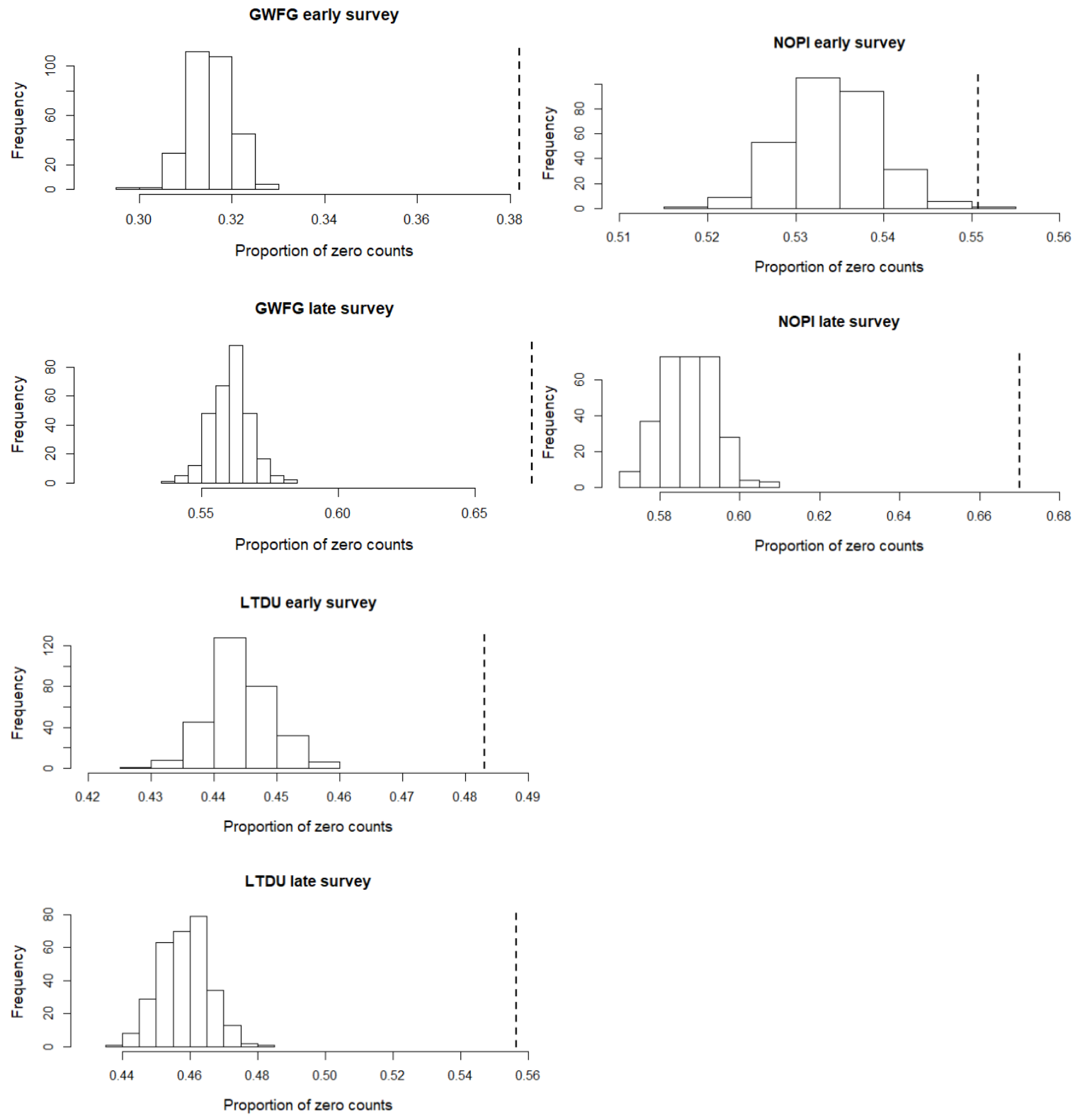
Appendix 2. Spring phenology

\section{Spatio-temporal population change of arctic-breeding waterbirds}

We obtained data on the predicted start of the growing season (start of season time, SOST; day of the year) from vegetation indices derived from MODIS satellite imagery from 1992 to 2016 (Zhang et al. 2003, Didan et al. 2016). Data are available at approximately $2.5 \mathrm{~km}$ by $5 \mathrm{~km}$ spatial resolution across the Arctic Coastal Plain, Alaska (ACP). We summarized SOST by cellyear by calculating the mean, weighted by area covering each cell for cells with $>1$ value. Some SOST values are not well predicted along the coast and particularly in river deltas subject to spring flooding. Therefore, we removed SOST values in cells that were < 122 (2 or 3 May) or > 228 (16 or $17 \mathrm{Aug}$ ) and imputed values for those cell-years by interpolating values from the 40 nearest neighbors in each year weighted by the inverse-weighted distance to the target cell. In most cases, this represented cells $<3$ cell-widths or $17 \mathrm{~km}$ from the target cell boundary (i.e., on the diagonal). The days chosen as cutoffs represented natural break-points in the data. We used the knnimputation function in the R package DMwR (Torgo 2015, R Core Team 2018) to impute omitted values.

We evaluated trends in spring phenology through space and time in two ways. First, we evaluated a generalized additive model (GAM; Wood 2017) that incorporated spatial autocorrelation and an additive temporal trend to estimate changes in SOST through time. Second, we evaluated a space by year interaction to examine spatial patterns of temporal change in SOST. We implemented GAMs using the gam function in the R package mgcv (Wood 2018). We obtained confidence intervals around differences in SOST between 1992 and 2016 using Monte Carlo simulations. In this appendix, we provide $\mathrm{R}$ code and results to facilitate replication of our analyses.

\section{RESULTS}

Model selection results supported an interaction between space and time, and a smoothed spatial component (Table A2.1). The most supported model explained $72.6 \%$ of the deviance in the data. Predicted SOST occurred, on average, 9 days earlier in 2016 (day 158, SE = 0.065; 7 June) than in 1992 (day 167, SE $=0.065 ; 16$ June) (Figure A2.1). The predicted difference in SOST from 1992 to 2016 varied through space (Figure S2), and all cells showed SOST decreasing through time (range: 5.32 to 15.67 days earlier) with $95 \%$ confidence intervals (CI) that did not overlap zero (upper 95\% CI range among cells: 4.37 to 14.49 days earlier). 
Table A2.1. Model selection results from four generalized additive model structures evaluated that examined effects of space (cellX, cellY) and time (year) on the start of spring green up (SOST) across the Arctic Coastal Plain, Alaska, 1992-2016. Models varied in complexity and smooth parameters for the spatial component; either (cellX, cellY) was modeled using a smooth $(\mathrm{s}())$ or a full tensor product $(\operatorname{te}(())$. The value with the lowest AIC value was used for subsequent model predictions.

\begin{tabular}{llll}
\hline Model & AAIC & AIC & df \\
\hline SOST s(year) & 51,393 & $299,122.4$ & 6.00 \\
SOST te(cellX, cellY) + s(year) & $2,861.9$ & $250,591.3$ & 28.49 \\
SOST s(cellX, cellY) + s(year) & $1,549.2$ & $249,278.6$ & 34.70 \\
SOST te(cellX, cellY) + s(year) + ti(cellX, cellY, year) & 1,378 & $249,107.4$ & 86.71 \\
SOST s (cellX, cellY) + s(year) + ti(cellX, cellY, year) & 0 & $247,729.4$ & 92.13 \\
\hline
\end{tabular}


Figure A2.1. Predicted start of the growing season (day) from 1992 to 2016 for $1,9146 \mathrm{~km}$ by 6 $\mathrm{km}$ cells across the Arctic Coastal Plain, Alaska. We modeled the effect of space and time on start of growing season day using generalized additive models that apply a non-linear smooth to spatio-temporal effects. The gray shaded region denotes $95 \%$ confidence interval around predictions. The lower plot is identical, but includes semi-transparent data values across cells for each year (in blue).
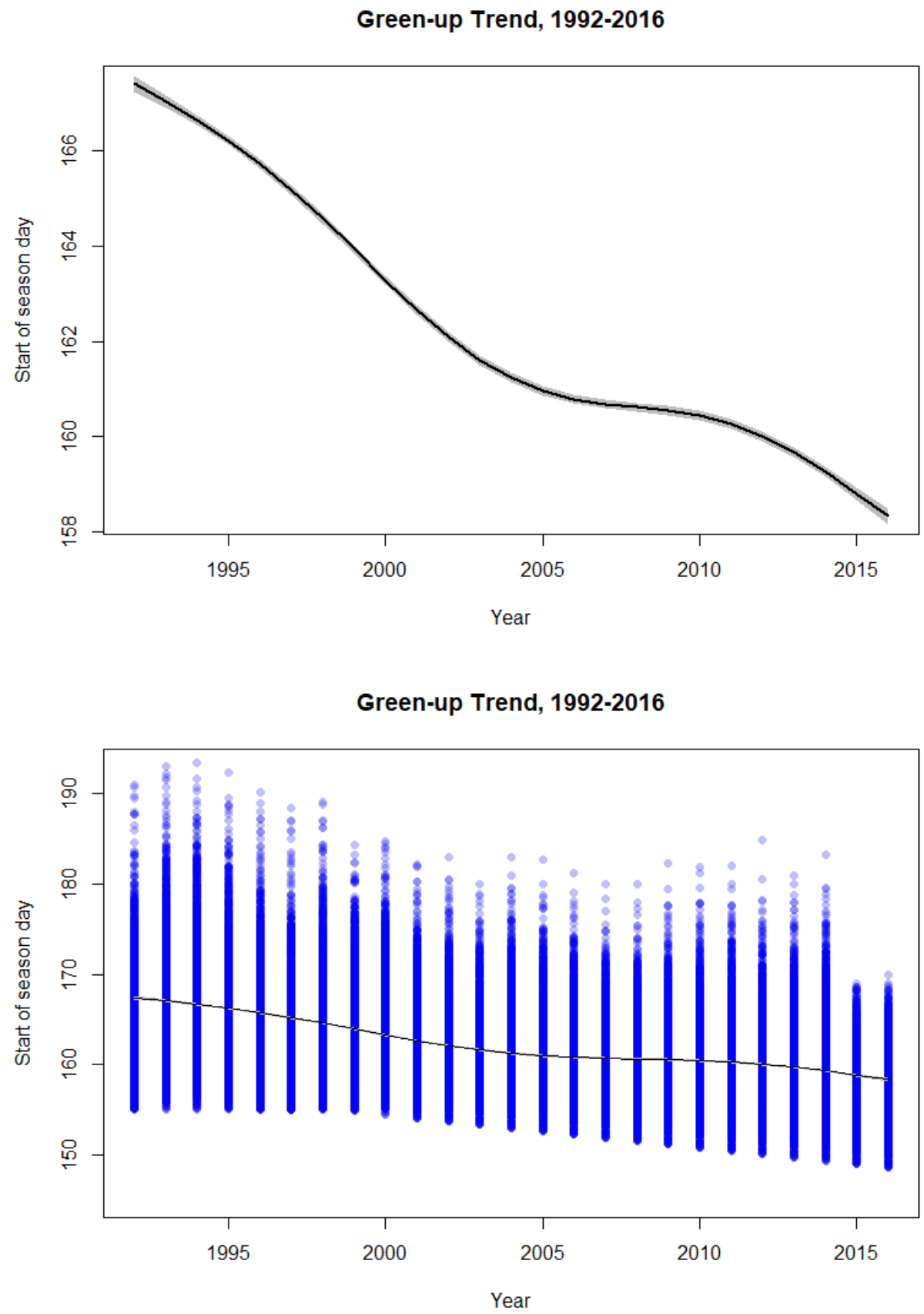
Figure A2.2. The change (in days) in the start of spring growing season (day of year) between 1992 and 2016 within approximately $36 \mathrm{~km}^{2}$ cells $(6 \mathrm{~km}$ by $6 \mathrm{~km}$ ) across the Arctic Coastal Plain, Alaska. We modeled the effect of space and time on start of growing season day using generalized additive models that apply a non-linear smooth to spatio-temporal effects, then subtracted cell-specific values in 1992 from 2016. Negative values denote a decreasing trend in the start of spring (i.e., start of the growing season occurred earlier in recent years).

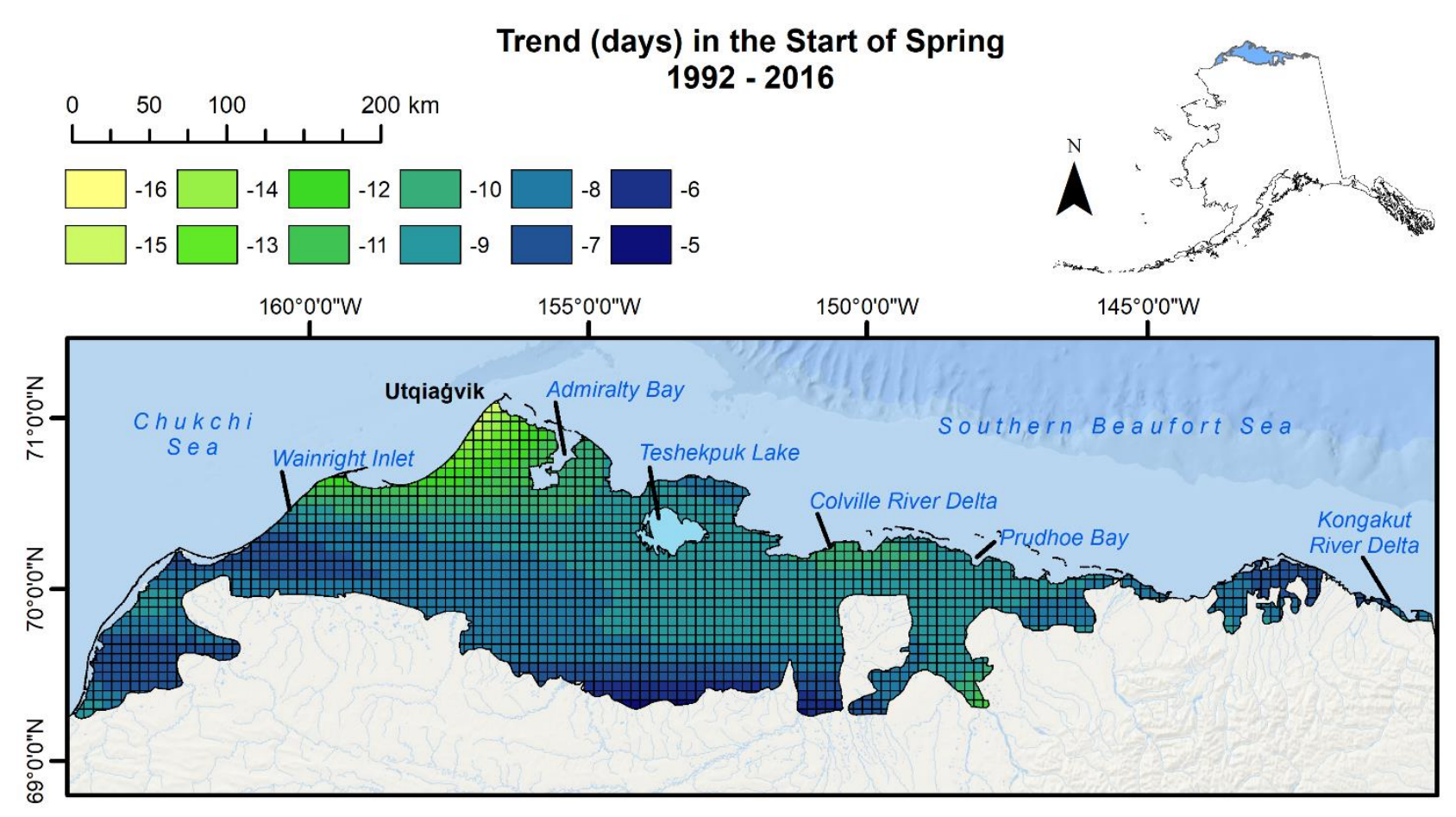




\section{LITERATURE CITED}

Didan, K., A. B. Munoz, T. Miura, J. Tsend-Ayush, X. Zhang, M. Friedl, J. Gray, W. Van Leeuwen, J. Czapla-Myers, C. Jenkerson, T. Maiersperger, D. Meyer. 2016. Multi-sensor vegetation index and phenology Earth science data records: algorithm theoretical basis document and user guide, ver. 4.0. Available: https://vip.arizona.edu/documents/VIP_ESDRs_ATBD_UsersGuide_06_28_2016_Final. pdf (5 MAR 2018)

Torgo, L. 2015. Package DMwR, Available: https://cran.rproject.org/web/packages/DMwR/DMwR.pdf (5 MAR 2018).

Wood, S. N. 2018. Package mgcv, Available: https://cran.rproject.org/web/packages/mgcv/mgcv.pdf (5 MAR 2018).

Wood, S. N. 2017. Generalized Additive Models: An Introduction with R (2nd edition). Chapman and Hall/CRC Press.

Zhang, X., M. A. Friedl, C. B. Schaaf, A. H. Strahler, J. C. F. Hodges, F. Gao, B. C. Reed, and A. Huete. 2003. Monitoring vegetation phenology using MODIS. Remote Sensing of Environment 84:471-475. 


\section{$R$ code for regression and plots.}

library(fields)

library(mgcv)

library(plyr)

library(rgdal)

library(sp)

\# load dataset of SOST values by year

sst<-read.csv(" /acp_sost.csv",header=T) \# data available from corresponding author, camundson@usgs.gov

\#quick and dirty plot of SOST through space, averaged across years

quilt.plot(sst\$cellX,sst\$cellY,sst\$sost)

\# look at simple to more complex models

\# limit $\mathrm{k}$ to 5 for year to avoid overfit - meaningless curves

$\mathrm{m} 0<$-gam(sost $\sim \mathrm{s}($ year,k=5), method="REML",data=sst) \# year only

$\mathrm{m} 1<$-gam(sost $\sim$ te $($ cellX,cellY)+s(year,k=5), method="REML",data=sst $)$ \# additive year, full tensor spatial effect

$\mathrm{m} 2<$-gam(sost $\sim \mathrm{s}($ cellX,cellY)+s(year,k=5), method="REML",data=sst) \# additive year, smoothed spatial effect

$\mathrm{m} 3<$-gam(sost $\sim \mathrm{s}($ cellX, cellY $)+\mathrm{s}($ year,k=5) +ti(cellX,cellY,year), method="REML",data=sst) \# interactive year, smoothed spatial effect

$\mathrm{m} 4<-$ gam $($ sost $\sim$ te $($ cellX, cellY $)+\mathrm{s}($ year,k=5) +ti(cellX,cellY,year), method="REML",data=sst $)$ \# interactive year, tensor spatial effect

\# model selection

$\mathrm{AIC}(\mathrm{m} 0, \mathrm{~m} 1, \mathrm{~m} 2, \mathrm{~m} 3, \mathrm{~m} 4)$

\# Examine model results

$\operatorname{summary}(\mathrm{m} 4)$

$\mathrm{m} 4$

$\operatorname{plot}(\mathrm{m} 4, \mathrm{scheme}=2)$

\# m3 fits best

vis.gam $(\mathrm{m} 3)$

fitted<-predict $(\mathrm{m} 3, \mathrm{sst}, \mathrm{se}=\mathrm{T})$

str(fitted)

plot(as.factor(sst\$year),fitted\$fit)

newd<-data.frame (cellX=sst\$cellX[1],cellY=sst\$cellY[1],year=seq $(1992,2016))$

\# Predictions averaged over space

preds <-predict $(\mathrm{m} 3$, newdata=newd, exclude $=c(" t i($ cellX, cellY,year)","s(cellX,cellY)"), se.fit=TRUE)

str(preds) 
fit<-preds $\$$ fit

fit.up95<-fit-1.96*preds\$se.fit

fit.low $95<-$ fit $+1.96 *$ preds $\$$ se.fit

plot(newd\$year, fit, type="n", lwd=3, \#ylim=c(145,193), main="Green-up Trend, 1992-2016",xlab="Year",

ylab="Start of season day")

\#Include data points or not, if so, including ylim argument above

\#points(sst\$year,sst\$sost, pch=16, col=rgb(0, 0, 1, 0.25))

\# For the confidence grey polygon

polygon(c(newd\$year, rev(newd\$year)),

c(fit.low95,rev(fit.up95)), col="gray",

border=NA)

lines(newd\$year, fit, lwd=1)

\# Monte Carlo variance around difference in SOST from 1992 to 2016 for each cell

res<-double()

nsim<-1000\# pick a large number here, but TAKES AWHILE

system.time(for ( $\mathrm{n}$ in 1:nsim) \{

tmp=rnorm(length(sst\$year),fitted $\$$ fit,fitted\$se.fit)

res=rbind(res,data.frame $($ cellX $=$ sst $\$$ cellX, cellY=sst $\$$ cellY,year=sst $\$ y e a r, p r d=t m p))$

\})

yr2016<-subset $($ res, year $==2016)$

yr1992<-subset(res, year==1992)

diff $<-y r 2016 \$$ prd-yr1992\$prd

diff.df<-data.frame(cellX=yr2016\$cellX,cellY=yr2016\$cellY,diff)

diff.df\$char.loc<-as.factor(paste(diff.df\$cellX,diff.df\$cellY,sep="_"))

df<-ddply(diff.df,.(char.loc), summarize,mndiff=mean(diff), se=sd(diff),lci=quantile(diff,0.025), cellX=mean $($ cellX), cellY $=$ mean $($ cellY $), \mathrm{uci}=$ quantile $(\operatorname{diff}, 0.975)$ )

$\operatorname{str}(\mathrm{df})$

summary(df)

quilt.plot(df\$cellX,df\$cellY,(df\$mndiff*-1),nx=147,ny=34)

\# convert into ESRI shapefile for mapping

proj<-("+proj=aea +lat_1 $=55+$ lat_2 $=65+$ lat_0 $=50+$ lon_0 $=-154+\mathrm{x} \_0=0+\mathrm{y} \_0=0$

+datum=NAD83 +units=m +no_defs +ellps=GRS80 +towgs84=0,0,0")

spat.sost<-SpatialPointsDataFrame(cbind(df\$cellX,df\$cellY),df,proj4string=CRS(proj))

writeOGR(obj=spat.sost, dsn="D:/acp_predshapefiles",layer="acp_SOSTpreddiff",

driver="ESRI Shapefile") 
Appendix 3: Jags model code

\section{Spatio-temporal population change of arctic-breeding waterbirds}

Example model notation for a negative binomial generalized linear mixed model implemented in jags (Plummer 2003) through R (R Core Team 2018) to evaluate spatially-explicit abundance and trends of waterbirds on the Arctic Coastal Plain, Alaska.

Plummer, M. 2003. JAGS: A program for analysis of Bayesian graphical models using Gibbs sampling. Proceedings of the 3rd International Workshop on Distributed Statistical Computing (DSC 2003), March 20-22, Vienna, Austria. ISSN 1609-395X. https://www.r-project.org/conferences/DSC-2003/Drafts/Plummer.pdf (14 March 2018).

R Core Team. 2018. R: A language and environment for statistical computing. Vienna, Austria: R Foundation for Statistical Computing. http://www.R-project.org/ (14 March 2018).

Wood, S. N. 2018. Package mgcv, Available: https://cran.rproject.org/web/packages/mgcv/mgcv.pdf (5 MAR 2018).

Data objects include:

ncells $=$ number of unique approximately $6 \mathrm{~km}$ by $6 \mathrm{~km}$ cells

ncellyrrep = number of unique cell-year-surveys (counts)

nyears $=$ number of years

repyrs $=$ specific years with a later survey replicate

z.repyrs $=$ specific years without a later survey replicate

REP $=$ survey ID ( 0 for early survey, 1 for later survey)

year $=$ numeric year $(1: 25)$

cell $=$ numeric cell ID (1:ncells)

cellyr $=$ unique cell-yr ID (length: ncells*nyears)

$\mathrm{S}=$ smoothing parameters from jagam() in mgcv R package

zero $=$ matrix of zeros from jagam() in mgcv $\mathrm{R}$ package

p.surv $=$ proportion of the cell surveyed per cell-year-survey (to weight uncertainty in estimates)

a.surv $=$ area surveyed per cell-year-survey (to adjust counts to density per $\mathrm{km}^{2}$ )

sost $=$ normalized start of season day $($ mean $=0, \mathrm{SD}=1)$

counts $=$ observed counts per cell-year-survey

model \{

\# Covariate priors

b.spring dnorm $(0,0.001)$

b.spring2 dnorm $(0,0.001)$

for (y in 1:nyears) \{

b0[y] dnorm(0,tau.yr) \# year intercepts

\}

tau.yr<-pow(sd.yr,-2)

sd.yr $\sim \operatorname{dunif}(0,5)$

mn.b0<-mean $(\mathrm{b} 0[]) \#$ average year effect for smoothed predictions 


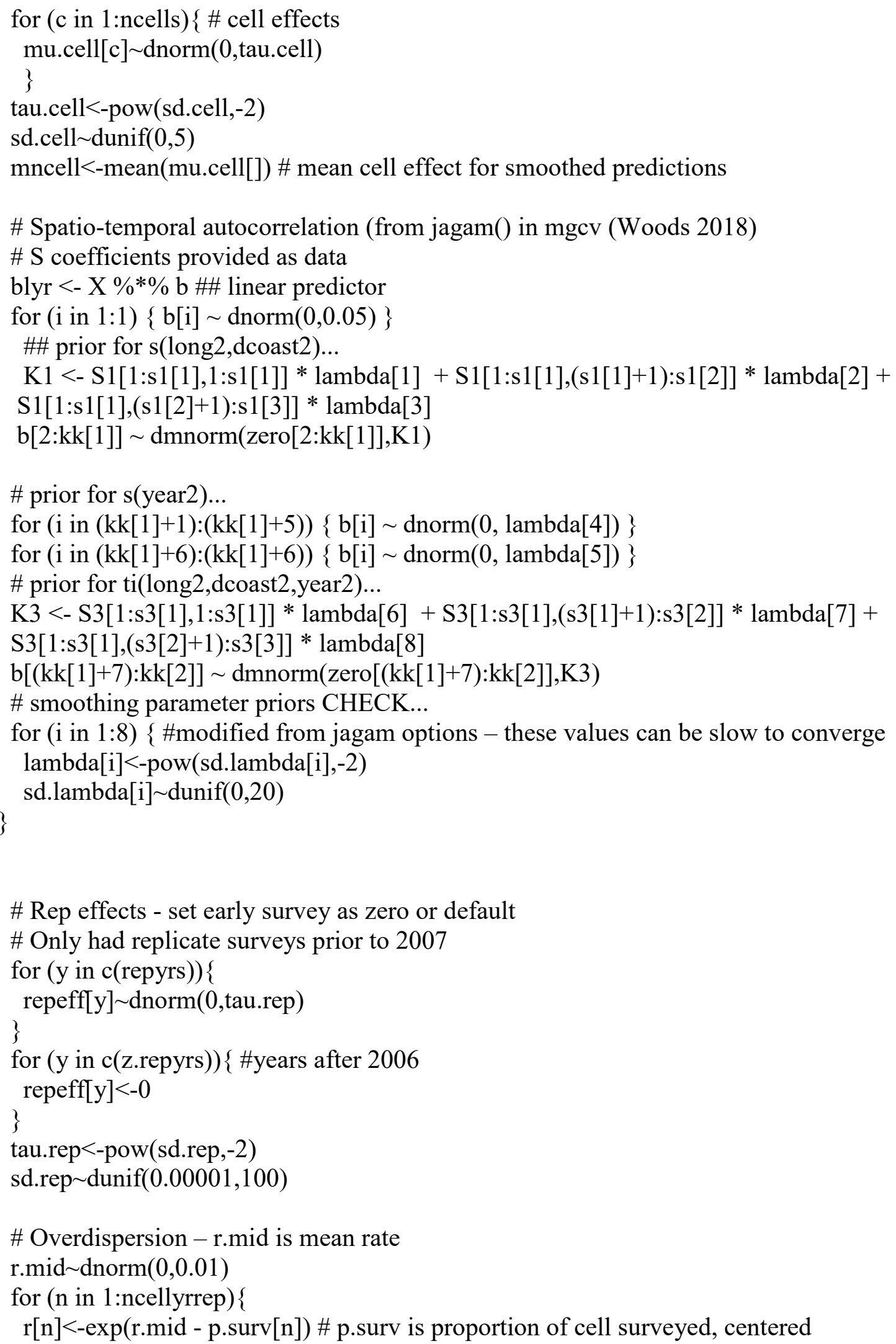




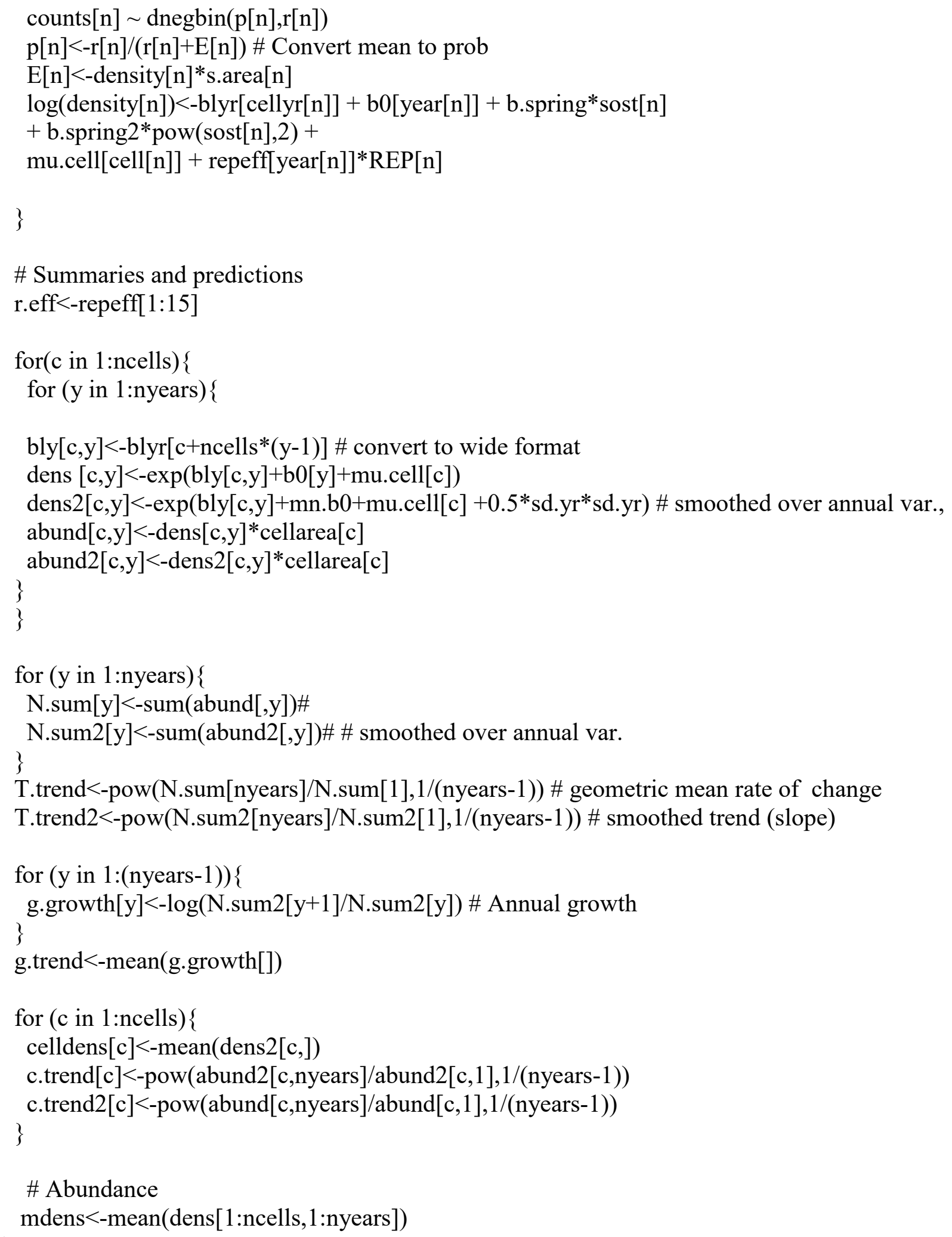


Appendix 4. Summary tables

\section{Spatio-temporal population change of arctic-breeding waterbirds}

We provide summary tables supplemental to figures provided in the main text including: distribution and fixed effect summaries (A4.1), estimates of relative density and trend estimates (95\% CI) (A4.3), correlations in density among species through space (A4.3), and speciesspecific model coefficients (A4.4- A4.24). Original data are available from the U.S. Fish and Wildlife Service, Region 7, Migratory Bird Management (https://www.fws.gov/alaska/mbsp/mbm/waterfowl/waterfowl.htm). Additional model output can be obtained from the corresponding author (C. Amundson, camundson[at]usgs.gov).

Chesser, R. T., K. J. Burns, C. Cicero, J. L. Dunn, A. W. Kratter, I. J. Lovette, P. C. Rasmussen, J. V. Remsen, Jr., J. D. Rising, D. F. Stotz, and K. Winker. 2018. Fifty-ninth supplement to the American Ornithological Society's check-list of North American birds. The Auk: Ornithological Advances 135:798-813. 
Table A4.1. Results for models of abundance and population trend for 20 waterbird species observed during aerial surveys on the Arctic Coastal Plain, Alaska, 1992-2016. We present the observed proportion of zeros per cell (Ozero; across survey replicates and years) and distribution (negative binomial, NB or zero-inflated NB, ZINB) used for the analysis. We evaluated log-linear and log-quadratic survey date relative to the onset of spring on counts (A.DOY and A.DOY ${ }^{2}$ ) and presence (ZINB binomial component) where applicable (Z.DOY and Z.DOY ${ }^{2}$, and an offset for the later survey replicate (1992-2006; Later Survey) for counts and zero inflation model component (Z Later Survey). We denote positive (+) or negative (-) coefficients that had $95 \%$ CIs that did not overlap zero for each variable except Later Survey, where we list the percentage of years where the effect of the later survey was positive or negative with $95 \%$ CIs that did not overlap zero (e.g., $+50 \%,-10 \%$ would indicate abundance was substantially higher during the later survey in $50 \%$ of years and lower during the later survey in $10 \%$ of years). Scaup and Yellow-billed Loon estimates were pooled between survey replicates and White-winged Scoter were not observed during the later survey (shaded).

\begin{tabular}{|c|c|c|c|c|c|c|c|c|c|c|}
\hline Species & Ozero & Distribution & A.DOY & A.DOY ${ }^{2}$ & Z.DOY & Z.DOY ${ }^{2}$ & \multicolumn{2}{|c|}{ Later Survey } & \multicolumn{2}{|c|}{ Z Later Survey } \\
\hline $\begin{array}{l}\text { Lesser Snow Goose (Anser } \\
\text { caerulescens caerulescens) }\end{array}$ & 0.905 & NB & - & & & & $+(0 \%)$ & $-(0 \%)$ & & \\
\hline $\begin{array}{l}\text { Greater White-fronted Goose } \\
\text { (Anser alibfrons) }\end{array}$ & 0.467 & ZINB & - & & + & + & $+(13.3 \%)$ & $-(53.3 \%)$ & $+(13.3 \%)$ & $-(0 \%)$ \\
\hline $\begin{array}{l}\text { Black Brant (Branta bernicla } \\
\text { nigricans) }\end{array}$ & 0.833 & NB & & & & & $+(13.3 \%)$ & $-(26.7 \%)$ & & \\
\hline $\begin{array}{l}\text { Cackling Boose (Branta } \\
\text { hutchinsii) }\end{array}$ & 0.869 & NB & - & & & & $+(33.3 \%)$ & $-(0 \%)$ & & \\
\hline $\begin{array}{l}\text { Tundra Swan (Cygnus } \\
\text { columbianus) }\end{array}$ & 0.737 & NB & + & & & & $+(20 \%)$ & $-(0 \%)$ & & \\
\hline $\begin{array}{l}\text { Northern Pintail (Anser } \\
\text { acuta) }\end{array}$ & 0.586 & ZINB & & & + & + & $+(33.3 \%)$ & $-(26.7 \%)$ & $+(40 \%)$ & $-(13.3 \%)$ \\
\hline $\begin{array}{l}\text { Scaup (Lesser and Greater } \\
\text { combined) (Aythya sp.) }\end{array}$ & 0.834 & NB & + & - & & & & & & \\
\hline $\begin{array}{l}\text { Steller's Eider (Polysticta } \\
\text { stelleri) }\end{array}$ & 0.948 & NB & & & & & $+(40 \%)$ & $-(0 \%)$ & & \\
\hline $\begin{array}{l}\text { Spectacled Eider (Somateria } \\
\text { fischeri) }\end{array}$ & 0.841 & NB & - & - & & & $+(0 \%)$ & $-(100 \%)$ & & \\
\hline
\end{tabular}




\begin{tabular}{|c|c|c|c|c|c|c|c|c|c|c|}
\hline $\begin{array}{l}\text { King Eider (Somateria } \\
\text { spectabilis) }\end{array}$ & 0.761 & NB & - & - & & & $+(0 \%)$ & $-(100 \%)$ & & \\
\hline $\begin{array}{l}\text { White-winged Scoter } \\
\text { (Melanitta perspicillata) }\end{array}$ & 0.889 & NB & - & - & & & & & & \\
\hline $\begin{array}{l}\text { Long-tailed Duck (Clangula } \\
\text { hyemalis) }\end{array}$ & 0.505 & ZINB & & & + & + & $+(46.7 \%)$ & $-(6.7 \%)$ & $+(26.7 \%)$ & $-(6.7 \%)$ \\
\hline $\begin{array}{l}\text { Red-breasted Merganser } \\
\text { (Mergus serrator) }\end{array}$ & 0.928 & NB & & & & & $+(26.7 \%)$ & $-(6.7 \%)$ & & \\
\hline $\begin{array}{l}\text { Jaeger (Pomarine, Parasitic } \\
\text { and Long-tailed combined) } \\
\text { (Stercorarius } \text { sp.) }\end{array}$ & 0.795 & NB & + & & & & $+(0 \%)$ & $-(66.7 \%)$ & & \\
\hline Sabine's Gull (Xema sabini) & 0.775 & NB & - & & & & $+(0 \%)$ & $-(33.3 \%)$ & & \\
\hline $\begin{array}{l}\text { Glaucous Gull (Larus } \\
\text { hyperboreus) }\end{array}$ & 0.665 & NB & - & & & & $+(0 \%)$ & $-(0 \%)$ & & \\
\hline $\begin{array}{l}\text { Arctic Tern (Sterna } \\
\text { paradisaea) }\end{array}$ & 0.669 & NB & - & & & & $+(13.3 \%)$ & $-(26.7 \%)$ & & \\
\hline $\begin{array}{l}\text { Red-throated Loon (Gavia } \\
\text { stellata) }\end{array}$ & 0.872 & NB & - & & & & $+(53.3 \%)$ & $-(26.7 \%)$ & & \\
\hline Pacific Loon (Gavia pacifica) & 0.517 & NB & + & & & & $+(6.7 \%)$ & $-(73.3 \%)$ & & \\
\hline $\begin{array}{l}\text { Yellow-billed Loon (Gavia } \\
\text { adamsii) }\end{array}$ & 0.892 & NB & - & - & & & & & & \\
\hline
\end{tabular}


Table A4.2. Mean density $\left(\mathrm{km}^{2}\right)$ and population trend $( \pm 95 \%$ CIs; $\lambda=1.0$ denotes a stable population; percent change $=(\lambda-1.0) * 100)$, and the number of approximately $36 \mathrm{~km}^{2}$ cells occupied $(n)$ for 20 waterbird species surveyed 1992-2016 on the Arctic Coastal Plain, Alaska (ACP). Estimates are also specified for the National Petroleum Reserve - Alaska (NPR-A), and the Arctic National Wildlife Refuge (ANWR). See also Figures 6 and 8 in the main text for visualizations of these results. Species are presented in taxonomic order (Chesser et al. 2018).

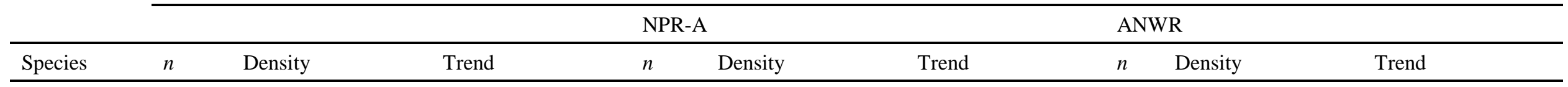

Lesser Snow

Goose (Anser

caerulescens

caerulescens
$0.261(0.191,0.365)$

caerulescens)

Greater

White-

fronted

Goose (Anser

alibfrons)

1,58

1,584

$1.399(1.347,1.458)$

$1.062(1.05,1.076)$

1,128

$1.394(1.343,1.451)$

$1.063(1.051,1.076) \quad 37$

$0.612(0.49,0.757)$

$1.05(1.022,1.081)$

Black Brant

(Branta

bernicla)

Cackling

Goose

(Branta

665

415

$0.342(0.295,0.404) \quad 1.076(1.038,1.111) \quad 313$

$0.293(0.254,0.346)$

$1.076(1.039,1.11) \quad 3$

$0.305(0.066,1.037)$

$1.04(0.953,1.122)$

hutchinsii)

665

\footnotetext{
$0.198(0.172,0.236) \quad 1.071(1.039,1.107) \quad 394$
}

$0.111(0.097,0.132) \quad 1.061(1.029,1.098)$

23

$0.391(0.251,0.633) \quad 1.072(1.006,1.146)$ 
Tundra Swan

(Cygnus

columbianus)

$1,363 \quad 0.219(0.21,0.229) \quad 1.021(1.008,1.033) \quad 995$

Northern

Pintail

(Anser acuta)

1,628

$0.947(0.91,0.989)$

$1.001(0.986,1.02)$

1,156

$0.972(0.934,1.014)$

$1.003(0.987,1.022) \quad 56$

$0.92(0.771,1.078)$

$0.988(0.955,1.016)$

Scaup

(Lesser and

Greater

combined)

(Aythya sp.)
$1,199 \quad 0.29(0.264,0.319) \quad 1.033(1.009,1.062) \quad 904$
$0.268(0.245,0.295)$

$1.033(1.008,1.061) \quad 18$
$0.174(0.072,0.302) \quad 1.05(1.005,1.101)$

Steller's

Eider

(Polysticta

stelleri)

11

$0.021(0.013,0.034)$

$0.99(0.914,1.048)$

110

$0.022(0.013,0.034) \quad 0.993(0.917,1.051) \quad 0$

Spectacled

Eider

760

$0.24(0.221,0.263)$

$1.013(0.989,1.037) \quad 612$

$0.253(0.233,0.278) \quad 1.015(0.991,1.039) \quad 3$

$0.129(0.051,0.388) \quad 0.98(0.933,1.022)$ 
King Eider

(Somateria spectabilis)

White-

winged

Scoter

(Melanitta

240

$0.334(0.226,0.49)$

$1.116(1.051,1.191) \quad 189$

$0.325(0.213,0.493)$

$1.122(1.054,1.2)$

0

perspicillata)

Long-tailed

Duck

(Clangula

$0.803(0.702,0.914) \quad 0.992(0.979,1.006)$
Red-breasted

Merganser

(Mergus

$0.085(0.069,0.103) \quad 1.038(1.009,1.072) \quad 308$
$0.082(0.068,0.098) \quad 1.039(1.009,1.075) \quad 1$
$0.157(0.044,0.366) \quad 1.051(0.991,1.11)$

serrator) 
Glaucous

Gull (Larus

hyperboreus)

Arctic Tern

(Sterna 
Pacific Loon

(Gavia

1,600

$0.561(0.542,0.582) \quad 1.001(0.99,1.013)$

1,146

$0.627(0.606,0.65)$

$1.002(0.991,1.015) \quad 51$

$0.338(0.277,0.425) \quad 0.997(0.974,1.02)$
Yellow-

billed Loon

(Gavia

500

$0.09(0.079,0.103)$

$1.044(1.021,1.069) \quad 452$

$0.091(0.079,0.105) \quad 1.046(1.022,1.071) \quad 0$ 
Table A4.3. Correlations among cell-specific (i.e., averaged across years) predicted density for 20 waterbird species observed during aerial surveys on the Arctic Coastal Plain, Alaska, 1992-2016. We assumed species without observations in a surveyed cell had density $=0$. Correlations $>0.4$ are in bold text. Species are listed in taxonomic order using standardized 4-letter codes (Chesser et al. 2018) and include Lesser Snow Goose (LSGO), Greater White-fronted Goose (GWFG), Black Brant (BLBR), Cackling goose (CACG), Tundra Swan (TUSW), Northern Pintail (NOPI), Scaup (Lesser and Greater combined) (SCAU), Steller's Eider (STEI), Spectacled Eider (SPEI), King Eider (KIEI), White-winged Scoter (WWSC), Longtailed Duck (LTDU), Red-breasted Merganser (RBME), Jaeger (Pomarine, Parasitic, and Long-tailed combined) (JAEG), Sabine's Gull (SAGU), Glaucous Gull (GLGU), Arctic Tern (ARTE), Red-throated Loon (RTLO), Pacific Loon (PALO), and Yellow-billed Loon (YBLO).

\begin{tabular}{|c|c|c|c|c|c|c|c|c|c|c|c|c|c|c|c|c|c|c|c|c|}
\hline & LSGO & GWFG & BLBR & CACG & TUSW & NOPI & SCAU & STEI & SPEI & KIEI & WWSC & LTDU & RBME & JAEG & SAGU & GLGU & ARTE & RTLO & PALO & YBLO \\
\hline LSGO & 1.000 & 0.155 & 0.246 & 0.048 & 0.169 & 0.150 & -0.056 & 0.011 & 0.082 & 0.036 & -0.026 & 0.007 & -0.002 & -0.034 & 0.058 & 0.155 & -0.032 & 0.091 & -0.018 & 0.002 \\
\hline GWFG & & 1.000 & 0.451 & 0.155 & 0.577 & 0.496 & -0.189 & 0.109 & 0.483 & 0.415 & -0.162 & 0.293 & 0.038 & 0.019 & 0.362 & 0.525 & 0.073 & 0.340 & 0.337 & 0.108 \\
\hline BLBR & & & 1.000 & 0.150 & 0.351 & 0.430 & -0.145 & 0.069 & 0.255 & 0.196 & -0.077 & 0.036 & -0.024 & -0.041 & 0.210 & 0.315 & -0.064 & 0.241 & 0.022 & -0.022 \\
\hline CACG & & & & 1.000 & 0.168 & 0.092 & 0.053 & -0.032 & -0.056 & 0.253 & 0.078 & -0.013 & 0.000 & -0.007 & -0.126 & 0.085 & -0.158 & 0.048 & -0.007 & -0.093 \\
\hline TUSW & & & & & 1.000 & 0.433 & -0.100 & 0.127 & 0.395 & 0.323 & -0.124 & 0.262 & 0.025 & 0.052 & 0.342 & 0.541 & 0.114 & 0.385 & 0.348 & 0.138 \\
\hline NOPI & & & & & & 1.000 & -0.139 & 0.218 & 0.508 & 0.185 & -0.083 & 0.329 & 0.016 & 0.177 & 0.294 & 0.487 & -0.017 & 0.317 & 0.203 & -0.029 \\
\hline SCAU & & & & & & & 1.000 & -0.070 & -0.309 & -0.212 & 0.406 & -0.042 & 0.170 & 0.088 & -0.081 & -0.187 & 0.191 & -0.165 & 0.006 & 0.159 \\
\hline STEI & & & & & & & & 1.000 & 0.226 & 0.074 & -0.048 & 0.092 & -0.019 & 0.051 & 0.100 & 0.206 & 0.009 & 0.112 & 0.090 & -0.029 \\
\hline SPEI & & & & & & & & & 1.000 & 0.263 & -0.171 & 0.426 & -0.089 & 0.065 & 0.351 & 0.467 & -0.053 & 0.271 & 0.432 & -0.043 \\
\hline KIEI & & & & & & & & & & 1.000 & -0.123 & 0.229 & 0.006 & -0.038 & 0.285 & 0.250 & 0.086 & 0.132 & 0.382 & -0.011 \\
\hline WWSC & & & & & & & & & & & 1.000 & -0.024 & 0.047 & 0.075 & -0.114 & -0.139 & 0.011 & -0.123 & -0.012 & 0.052 \\
\hline LTDU & & & & & & & & & & & & 1.000 & 0.067 & 0.266 & 0.197 & 0.306 & 0.129 & 0.231 & 0.469 & 0.018 \\
\hline RBME & & & & & & & & & & & & & 1.000 & 0.033 & 0.147 & 0.070 & 0.322 & 0.050 & 0.138 & 0.149 \\
\hline JAEG & & & & & & & & & & & & & & 1.000 & 0.021 & 0.100 & 0.054 & 0.065 & 0.067 & 0.010 \\
\hline SAGU & & & & & & & & & & & & & & & 1.000 & 0.400 & 0.567 & 0.137 & 0.565 & 0.231 \\
\hline GLGU & & & & & & & & & & & & & & & & 1.000 & 0.204 & 0.447 & 0.367 & 0.150 \\
\hline ARTE & & & & & & & & & & & & & & & & & 1.000 & 0.079 & 0.472 & 0.461 \\
\hline RTLO & & & & & & & & & & & & & & & & & & 1.000 & 0.117 & 0.096 \\
\hline PALO & & & & & & & & & & & & & & & & & & & 1.000 & 0.198 \\
\hline YBLO & & & & & & & & & & & & & & & & & & & & 1.000 \\
\hline
\end{tabular}


Tables A4.4 - A4.24. Bayesian generalized linear mixed model (Negative Binomial or zero-inflated Negative Binomial, ZINB) output for 20 species of waterbirds observed during aerial surveys 1992-2016 on the Arctic Coastal Plain, Alaska and a key to derived and model parameters summarized (Table A4.4). We provide summaries including mean, SE, and several quantiles of the posterior distribution for model and derived parameters. We present model results for Lesser Snow Goose, Greater White-fronted Goose, Black Brant, Cackling Goose, Tundra Swan, Northern Pintail, Scaup (Lesser and Greater combined), Steller's Eider, Spectacled Eider, King Eider, White-winged Scoter, Long-tailed Duck, Red-breasted Merganser, Jaeger (Pomarine, Parasitic, and Long-tailed combined), Sabine's Gull, Glaucous Gull, Arctic Tern, Red-throated Loon, Pacific Loon, and Yellow-billed Loon.

Table A4.4. Key to derived and model parameters estimated from Bayesian generalized linear mixed models for 20 waterbird species across the Arctic Coastal Plain, Alaska, 1992-2016. Tables for specific species follow.

\begin{tabular}{|c|c|}
\hline Parameter & Definition \\
\hline MSE & $\begin{array}{l}\text { Mean squared error in counts per cell-year-survey where MSE = sum of squared residuals } \\
\text { divided by the total number of counts (across cells, years, and surveys) }\end{array}$ \\
\hline MSE.CV & $=$ MSE divided by the mean observed count (across cells, years, and surveys) \\
\hline SSE & Sum of squared error across cell-year-surveys \\
\hline Trend & Population trend where $1.0=$ stable population (i.e., lambda) \\
\hline R.trend & Mean Ricker intrinsic population growth rate across years (R.growth $\left.=\log \left(\widehat{N}_{\mathrm{t}+1} / \widehat{N}_{\mathrm{t}}\right)\right)$ \\
\hline M.dens & Mean density $\left(\mathrm{km}^{2}\right)$ \\
\hline M.y0 & Mean of annual random effect \\
\hline M.yz & Mean of annual random effect on binomial portion of ZINB model ( 4 species only) \\
\hline M.blyr & Mean of spatio-temporal smooth terms \\
\hline M.cell & Mean of spatial random effect \\
\hline M.psi & Mean probability a count per cell-year-survey is zero \\
\hline b.ADOY & DOY coefficient (linear) \\
\hline b.ADOY.2 & DOY coefficient (quadratic) \\
\hline z.ADOY & DOY coefficient (linear) on binomial portion of ZINB model (4 species only) \\
\hline Z.ADOY.2 & DOY coefficient (quadratic) on binomial portion of ZINB model (4 species only) \\
\hline SD.surv & Standard deviation of survey offset \\
\hline SD.surv.z & Standard deviation of survey offset on binomial portion of ZINB model ( 4 species only) \\
\hline SD.y0 & Standard deviation of annual random effect \\
\hline SD.yz & $\begin{array}{l}\text { Standard deviation of annual random effect on binomial portion of ZINB model ( } 4 \text { species } \\
\text { only) }\end{array}$ \\
\hline R.mid & The log overdispersion term (r) for the average area surveyed (Negative Binomial) \\
\hline SD.cell & Standard deviation of spatial random effect \\
\hline SD.cell.z & $\begin{array}{l}\text { Standard deviation of spatial random effect on binomial portion of ZINB model ( } 4 \text { species } \\
\text { only) } \\
\text { SD of variance terms for multinomial spatio-temporal smooth for term x (see Appendix 2: }\end{array}$ \\
\hline SD.lam $[\mathrm{x}]$ & Jags code) \\
\hline S.eff $[x]$ & Survey offset coefficient for year $\mathrm{x}$ where $\mathrm{x}=1=1992$ \\
\hline S.eff.z[x] & $\begin{array}{l}\text { Survey offset coefficient for year } x \text { where } x=1=1992 \text { on binomial portion of ZINB model } \\
\text { ( } 4 \text { species only) }\end{array}$ \\
\hline $\mathrm{y} 0[\mathrm{x}]$ & Annual random effect coefficient for year $\mathrm{x}$ where $\mathrm{x}=1=1992$ \\
\hline $\mathrm{yz}[\mathrm{x}]$ & $\begin{array}{l}\text { Annual random effect coefficient for year } \mathrm{x} \text { where } \mathrm{x}=1=1992 \text { on binomial portion of ZINB } \\
\text { model ( } 4 \text { species only) }\end{array}$ \\
\hline
\end{tabular}


N.sum[x] Total population size in year $\mathrm{x}$ where $\mathrm{x}=1=1992$

Total population size averaged over annual random effect (y0) in year $\mathrm{x}$ where $\mathrm{x}=1=1992$,

N.sum2[x] useful for trends

Ricker's intrinsic rate of population growth for year $\mathrm{x}$ where $\mathrm{x}=1=1992$. $($ R.growth $=$

R.growth[x] $\left.\log \left(\widehat{N}_{\mathrm{t}+1} / \widehat{N}_{\mathrm{t}}\right)\right)$

Deviance Model deviance 
Tables A4.5. Bayesian generalized linear mixed model output for Lesser Snow Goose observed during aerial surveys 1992-2016 on the Arctic Coastal Plain, Alaska. We provide summaries including mean, SE, and several quantiles of the posterior distribution for model and derived parameters. See Table A4.4 for a key to parameter names.

\begin{tabular}{|c|c|c|c|c|c|c|c|}
\hline Parameter & Mean & SD & $2.50 \%$ & $25 \%$ & $50 \%$ & $75 \%$ & $97.50 \%$ \\
\hline MSE & 22.604 & 4.474 & 17.561 & 20.709 & 21.832 & 23.228 & 32.618 \\
\hline MSE.CV & 45.868 & 9.080 & 35.634 & 42.022 & 44.301 & 47.134 & 66.189 \\
\hline SSE & $92,699.05$ & $18,349.84$ & $72,016.64$ & $84,927.16$ & $89,532.26$ & $95,257.41$ & $133,767.29$ \\
\hline Trend & 1.138 & 0.024 & 1.090 & 1.122 & 1.138 & 1.153 & 1.184 \\
\hline R.trend & 0.129 & 0.021 & 0.086 & 0.115 & 0.129 & 0.142 & 0.169 \\
\hline M.dens & 0.261 & 0.045 & 0.191 & 0.230 & 0.255 & 0.285 & 0.365 \\
\hline M.y0 & -0.001 & 0.068 & -0.141 & -0.038 & -0.001 & 0.036 & 0.137 \\
\hline M.blyr & -2.830 & 0.150 & -3.136 & -2.929 & -2.827 & -2.728 & -2.543 \\
\hline M.cell & -0.002 & 0.075 & -0.145 & -0.052 & -0.002 & 0.048 & 0.151 \\
\hline R.mid & -1.939 & 0.096 & -2.127 & -2.004 & -1.938 & -1.874 & -1.754 \\
\hline b.ADOY & -0.245 & 0.104 & -0.456 & -0.314 & -0.245 & -0.174 & -0.040 \\
\hline b.ADOY.2 & -0.034 & 0.067 & -0.167 & -0.078 & -0.034 & 0.010 & 0.095 \\
\hline SD.surv & 0.485 & 0.305 & 0.025 & 0.250 & 0.463 & 0.680 & 1.141 \\
\hline SD.y0 & 0.318 & 0.132 & 0.063 & 0.229 & 0.314 & 0.404 & 0.598 \\
\hline SD.cell & 1.022 & 0.095 & 0.845 & 0.955 & 1.019 & 1.084 & 1.214 \\
\hline SD.lam[1] & 0.335 & 0.134 & 0.129 & 0.247 & 0.311 & 0.405 & 0.666 \\
\hline SD.lam[2] & 3.737 & 3.183 & 0.133 & 1.030 & 3.103 & 5.396 & 12.057 \\
\hline SD.lam[3] & 1.764 & 2.544 & 0.012 & 0.450 & 0.882 & 1.808 & 11.000 \\
\hline SD.lam[4] & 1.338 & 1.583 & 0.046 & 0.355 & 0.819 & 1.683 & 5.877 \\
\hline SD.lam[5] & 8.188 & 3.644 & 1.840 & 5.251 & 8.052 & 11.182 & 14.621 \\
\hline SD.lam[6] & 4.837 & 1.393 & 2.656 & 3.742 & 4.718 & 5.752 & 7.889 \\
\hline SD.lam[7] & 4.212 & 1.840 & 2.073 & 2.814 & 3.653 & 5.220 & 8.748 \\
\hline SD.lam[8] & 1.323 & 2.826 & 0.014 & 0.136 & 0.362 & 0.975 & 13.287 \\
\hline S.eff[1] & -0.017 & 0.463 & -0.990 & -0.252 & -0.007 & 0.205 & 0.947 \\
\hline S.eff[2] & 0.236 & 0.451 & -0.545 & -0.033 & 0.141 & 0.479 & 1.317 \\
\hline S.eff[3] & 0.000 & 0.000 & 0.000 & 0.000 & 0.000 & 0.000 & 0.000 \\
\hline S.eff[4] & -0.149 & 0.439 & -1.181 & -0.357 & -0.071 & 0.086 & 0.656 \\
\hline S.eff[5] & 0.060 & 0.426 & -0.806 & -0.160 & 0.026 & 0.273 & 1.007 \\
\hline S.eff[6] & -0.019 & 0.438 & -0.950 & -0.237 & -0.006 & 0.200 & 0.886 \\
\hline S.eff[7] & -0.204 & 0.494 & -1.407 & -0.434 & -0.107 & 0.062 & 0.646 \\
\hline S.eff[8] & -0.075 & 0.460 & -1.097 & -0.292 & -0.032 & 0.154 & 0.832 \\
\hline S.eff[9] & -0.031 & 0.399 & -0.890 & -0.240 & -0.015 & 0.166 & 0.809 \\
\hline S.eff[10] & 0.561 & 0.484 & -0.104 & 0.145 & 0.498 & 0.886 & 1.612 \\
\hline S.eff[11] & 0.154 & 0.401 & -0.575 & -0.068 & 0.084 & 0.371 & 1.085 \\
\hline S.eff[12] & 0.495 & 0.518 & -0.192 & 0.069 & 0.383 & 0.817 & 1.697 \\
\hline S.eff[13] & 0.214 & 0.367 & -0.402 & -0.021 & 0.145 & 0.425 & 1.052 \\
\hline S.eff[14] & 0.246 & 0.378 & -0.365 & -0.005 & 0.171 & 0.464 & 1.113 \\
\hline S.eff[15] & 0.301 & 0.442 & -0.392 & -0.001 & 0.206 & 0.549 & 1.360 \\
\hline $\mathrm{y} 0[1]$ & -0.056 & 0.301 & -0.698 & -0.222 & -0.040 & 0.117 & 0.539 \\
\hline
\end{tabular}




\begin{tabular}{|c|c|c|c|c|c|c|c|}
\hline $\mathrm{y} 0[2]$ & 0.077 & 0.280 & -0.449 & -0.096 & 0.056 & 0.244 & 0.680 \\
\hline $\mathrm{y} 0[3]$ & -0.363 & 0.342 & -1.147 & -0.565 & -0.316 & -0.109 & 0.155 \\
\hline $\mathrm{y} 0[4]$ & 0.387 & 0.308 & -0.102 & 0.156 & 0.354 & 0.589 & 1.057 \\
\hline $\mathrm{y} 0[5]$ & -0.017 & 0.268 & -0.575 & -0.176 & -0.012 & 0.146 & 0.523 \\
\hline $\mathrm{y} 0[6]$ & 0.071 & 0.271 & -0.461 & -0.093 & 0.053 & 0.230 & 0.648 \\
\hline $\mathrm{y} 0[7]$ & -0.070 & 0.266 & -0.634 & -0.230 & -0.056 & 0.086 & 0.454 \\
\hline $\mathrm{y} 0[8]$ & -0.195 & 0.285 & -0.828 & -0.367 & -0.166 & -0.004 & 0.313 \\
\hline y0[9] & 0.159 & 0.252 & -0.300 & -0.009 & 0.136 & 0.307 & 0.706 \\
\hline y0[10] & 0.113 & 0.258 & -0.388 & -0.045 & 0.097 & 0.268 & 0.660 \\
\hline y0[11] & -0.168 & 0.268 & -0.749 & -0.326 & -0.140 & 0.003 & 0.313 \\
\hline y0[12] & 0.058 & 0.245 & -0.435 & -0.090 & 0.045 & 0.211 & 0.564 \\
\hline y0[13] & 0.139 & 0.237 & -0.303 & -0.016 & 0.117 & 0.285 & 0.652 \\
\hline y0[14] & -0.211 & 0.260 & -0.778 & -0.368 & -0.185 & -0.029 & 0.236 \\
\hline y0[15] & -0.353 & 0.301 & -1.018 & -0.542 & -0.319 & -0.131 & 0.126 \\
\hline y0[16] & 0.130 & 0.251 & -0.336 & -0.031 & 0.109 & 0.286 & 0.661 \\
\hline y0[17] & 0.101 & 0.244 & -0.364 & -0.055 & 0.083 & 0.252 & 0.615 \\
\hline y0[18] & 0.367 & 0.277 & -0.083 & 0.159 & 0.347 & 0.549 & 0.948 \\
\hline y0[19] & -0.093 & 0.236 & -0.594 & -0.237 & -0.078 & 0.058 & 0.357 \\
\hline y0[20] & -0.008 & 0.243 & -0.501 & -0.152 & -0.008 & 0.140 & 0.475 \\
\hline y0[21] & -0.062 & 0.230 & -0.543 & -0.202 & -0.051 & 0.080 & 0.394 \\
\hline y0[22] & -0.152 & 0.252 & -0.693 & -0.304 & -0.132 & 0.010 & 0.321 \\
\hline y0[23] & -0.049 & 0.241 & -0.550 & -0.197 & -0.041 & 0.099 & 0.421 \\
\hline y0[24] & 0.241 & 0.260 & -0.203 & 0.058 & 0.214 & 0.401 & 0.820 \\
\hline y0[25] & -0.073 & 0.253 & -0.603 & -0.222 & -0.057 & 0.078 & 0.433 \\
\hline N.sum[1] & 208.972 & 111.098 & 75.386 & 137.961 & 185.895 & 252.791 & 473.301 \\
\hline N.sum[2] & 233.246 & 99.564 & 104.520 & 167.617 & 212.812 & 275.394 & 479.625 \\
\hline N.sum[3] & 160.396 & 70.744 & 57.667 & 110.695 & 149.744 & 196.950 & 327.577 \\
\hline N.sum[4] & 355.639 & 125.718 & 184.366 & 265.874 & 331.469 & 419.002 & 666.218 \\
\hline N.sum[5] & 263.358 & 87.784 & 130.422 & 203.780 & 250.993 & 307.990 & 472.491 \\
\hline N.sum[6] & 324.219 & 103.523 & 167.644 & 252.990 & 308.878 & 378.067 & 565.085 \\
\hline N.sum[7] & 322.933 & 104.710 & 154.849 & 250.136 & 310.814 & 381.369 & 567.267 \\
\hline N.sum[8] & 327.271 & 107.825 & 152.824 & 251.195 & 317.131 & 389.655 & 565.744 \\
\hline N.sum[9] & 527.395 & 150.975 & 301.995 & 423.060 & 503.153 & 604.419 & 886.814 \\
\hline N.sum[10] & 584.155 & 174.399 & 314.650 & 464.505 & 559.542 & 669.525 & $1,005.052$ \\
\hline N.sum[11] & 512.814 & 152.909 & 253.412 & 406.540 & 500.466 & 602.781 & 850.817 \\
\hline N.sum[12] & 746.333 & 209.915 & 410.590 & 603.686 & 717.222 & 860.435 & $1,239.404$ \\
\hline N.sum[13] & 943.249 & 239.192 & 570.725 & 778.085 & 909.985 & $1,071.860$ & $1,502.823$ \\
\hline N.sum[14] & 791.813 & 221.865 & 410.428 & 633.556 & 775.322 & 932.441 & $1,260.674$ \\
\hline N.sum[15] & 826.014 & 271.162 & 371.159 & 626.518 & 800.220 & $1,002.615$ & $1,401.030$ \\
\hline N.sum[16] & $1,539.473$ & 371.400 & 961.305 & $1,280.456$ & $1,489.047$ & 1,741.279 & $2,418.309$ \\
\hline N.sum[17] & $1,767.188$ & 456.225 & $1,071.940$ & $1,448.488$ & $1,704.815$ & $2,000.571$ & $2,844.608$ \\
\hline N.sum[18] & $2,710.759$ & 798.175 & $1,573.341$ & $2,141.525$ & $2,569.622$ & $3,113.541$ & $4,684.410$ \\
\hline N.sum[19] & $1,952.500$ & 501.198 & $1,123.121$ & $1,600.436$ & $1,902.330$ & $2,250.010$ & $3,077.620$ \\
\hline N.sum[20] & $2,410.123$ & 662.726 & $1,352.348$ & $1,943.974$ & $2,334.742$ & 2,778.937 & $3,955.874$ \\
\hline
\end{tabular}




\begin{tabular}{|c|c|c|c|c|c|c|c|}
\hline N.sum[21] & $2,542.630$ & 661.714 & $1,468.554$ & $2,085.219$ & $2,471.117$ & $2,929.791$ & $4,041.300$ \\
\hline N.sum[22] & $2,603.758$ & 737.510 & $1,428.028$ & $2,072.305$ & $2,523.094$ & $3,043.980$ & $4,287.417$ \\
\hline N.sum[23] & 228.773 & 928.588 & $1,788.921$ & $2,583.622$ & $3,113.951$ & $3,722.803$ & $5,427.221$ \\
\hline N.sum[24] & 916.596 & $1,471.401$ & $2,818.322$ & $3,878.505$ & $4,659.174$ & $5,663.156$ & $8,387.687$ \\
\hline N.sum[25] &, 237.147 & $1,606.882$ & $2,324.629$ & $3,300.992$ & $3,976.527$ & $4,805.850$ & $7,720.085$ \\
\hline N.sum2[1] & 229.215 & 111.143 & 98.128 & 159.460 & 206.193 & 269.796 & 501.888 \\
\hline N.sum2[2] & 225.041 & 85.168 & 114.034 & 169.748 & 208.492 & 260.836 & 429.096 \\
\hline N.sum2[3] & 232.570 & 73.022 & 131.586 & 184.104 & 219.992 & 266.035 & 402.806 \\
\hline N.sum2[4] & 250.238 & 68.511 & 151.411 & 204.129 & 240.375 & 283.185 & 409.105 \\
\hline N.sum2[5] & 277.112 & 68.237 & 174.234 & 230.942 & 267.316 & 311.681 & 436.368 \\
\hline N.sum2[6] & 312.628 & 70.793 & 201.995 & 264.670 & 303.591 & 351.154 & 476.826 \\
\hline N.sum2[7] & 356.293 & 76.344 & 234.907 & 304.538 & 346.623 & 397.329 & 529.060 \\
\hline N.sum2[8] & 407.795 & 84.533 & 273.421 & 350.544 & 397.504 & 452.179 & 596.384 \\
\hline N.sum2[9] & 468.032 & 93.237 & 319.483 & 405.346 & 457.737 & 516.638 & 684.134 \\
\hline N.sum2[10] & 539.251 & 101.456 & 373.892 & 471.425 & 529.138 & 594.182 & 772.760 \\
\hline N.sum2[11] & 624.956 & 111.543 & 436.461 & 549.194 & 614.444 & 687.504 & 873.601 \\
\hline N.sum2[12] & 729.224 & 126.024 & 511.215 & 643.536 & 718.618 & 801.415 & $1,006.430$ \\
\hline N.sum2[13] & 855.921 & 144.720 & 605.836 & 758.438 & 844.389 & 938.799 & $1,175.729$ \\
\hline N.sum2[14] & $1,008.949$ & 166.180 & 724.596 & 898.038 & 993.976 & $1,102.579$ & $1,378.614$ \\
\hline N.sum2[15] & $1,193.012$ & 191.669 & 869.459 & $1,063.459$ & $1,173.730$ & $1,301.751$ & $1,616.959$ \\
\hline N.sum2[16] & $1,411.525$ & 226.079 & $1,040.252$ & $1,256.477$ & $1,386.996$ & $1,534.937$ & $1,917.186$ \\
\hline N.sum2[17] & $1,663.355$ & 273.392 & $1,218.242$ & $1,476.655$ & $1,630.950$ & $1,813.999$ & $2,284.468$ \\
\hline N.sum2[18] & $1,940.816$ & 330.833 & $1,408.426$ & $1,715.769$ & $1,901.874$ & $2,124.318$ & $2,692.870$ \\
\hline N.sum2[19] & $2,231.149$ & 393.282 & $1,598.685$ & $1,965.521$ & $2,185.321$ & $2,454.370$ & $3,126.684$ \\
\hline N.sum2[20] & $2,523.684$ & 461.467 & $1,780.213$ & $2,207.855$ & $2,467.117$ & $2,778.455$ & $3,584.631$ \\
\hline N.sum2[21] & $2,823.607$ & 540.987 & $1,962.624$ & $2,452.198$ & $2,757.768$ & $3,115.709$ & 3.917 \\
\hline N.sum2[22] & $3,150.725$ & 642.060 & $2,130.775$ & $2,709.463$ & $3,063.669$ & $3,500.931$ & $4,648.086$ \\
\hline N.sum2[23] & $3,540.944$ & 794.458 & $2,306.198$ & $2,991.857$ & $3,431.415$ & $3,962.398$ & $5,432.216$ \\
\hline N.sum2[24] & $4,056.858$ & $1,058.947$ & $2,447.911$ & $3,329.127$ & $3,905.421$ & $4,588.657$ & $6,591.331$ \\
\hline N.sum2[25] & $4,838.564$ & $1,798.831$ & $2,559.406$ & $3,748.488$ & $4,525.469$ & $5,498.897$ & $8,949.755$ \\
\hline R.growth[1] & 0.012 & 0.112 & -0.221 & -0.054 & 0.021 & 0.083 & 0.218 \\
\hline R.growth[2] & 0.050 & 0.091 & -0.140 & -0.004 & 0.054 & 0.106 & 0.223 \\
\hline R.growth[3] & 0.083 & 0.071 & -0.068 & 0.041 & 0.085 & 0.126 & 0.222 \\
\hline R.growth[4] & 0.108 & 0.058 & -0.014 & 0.073 & 0.109 & 0.144 & 0.223 \\
\hline R.growth[5] & 0.124 & 0.054 & 0.015 & 0.093 & 0.125 & 0.155 & 0.234 \\
\hline R.growth[6] & 0.133 & 0.051 & 0.030 & 0.104 & 0.134 & 0.161 & 0.234 \\
\hline R.growth[7] & 0.136 & 0.046 & 0.039 & 0.112 & 0.138 & 0.162 & 0.228 \\
\hline R.growth[8] & 0.139 & 0.041 & 0.048 & 0.117 & 0.141 & 0.164 & 0.220 \\
\hline R.growth[9] & 0.143 & 0.042 & 0.053 & 0.121 & 0.146 & 0.169 & 0.222 \\
\hline R.growth[10] & 0.149 & 0.044 & 0.050 & 0.128 & 0.151 & 0.174 & 0.231 \\
\hline R.growth[11] & 0.155 & 0.043 & 0.058 & 0.134 & 0.156 & 0.179 & 0.240 \\
\hline R.growth[12] & 0.161 & 0.039 & 0.080 & 0.139 & 0.160 & 0.182 & 0.243 \\
\hline R.growth[13] & 0.165 & 0.037 & 0.096 & 0.143 & 0.163 & 0.184 & 0.249 \\
\hline R.growth[14] & 0.168 & 0.041 & 0.099 & 0.144 & 0.164 & 0.188 & 0.262 \\
\hline
\end{tabular}




\begin{tabular}{lrrrrrrr} 
R.growth[15] & 0.168 & 0.043 & 0.095 & 0.142 & 0.164 & 0.189 & 0.268 \\
R.growth[16] & 0.164 & 0.042 & 0.090 & 0.138 & 0.160 & 0.185 & 0.259 \\
R.growth[17] & 0.153 & 0.039 & 0.081 & 0.129 & 0.151 & 0.175 & 0.240 \\
R.growth[18] & 0.138 & 0.040 & 0.058 & 0.115 & 0.138 & 0.161 & 0.225 \\
R.growth[19] & 0.122 & 0.043 & 0.031 & 0.099 & 0.123 & 0.147 & 0.206 \\
R.growth[20] & 0.111 & 0.045 & 0.013 & 0.086 & 0.113 & 0.139 & 0.197 \\
R.growth[21] & 0.107 & 0.049 & 0.001 & 0.079 & 0.110 & 0.139 & 0.202 \\
R.growth[22] & 0.113 & 0.058 & -0.013 & 0.082 & 0.116 & 0.149 & 0.221 \\
R.growth[23] & 0.128 & 0.072 & -0.023 & 0.092 & 0.130 & 0.167 & 0.264 \\
R.growth[24] & 0.156 & 0.110 & -0.016 & 0.108 & 0.148 & 0.193 & 0.362 \\
Deviance & $4,006.236$ & 21.424 & $3,965.660$ & $3,991.568$ & $4,005.504$ & $4,020.136$ & $4,050.861$ \\
\hline
\end{tabular}


Tables A4.6. Bayesian generalized linear mixed model output for Greater White-fronted Goose observed during aerial surveys 1992-2016 on the Arctic Coastal Plain, Alaska. We provide summaries including mean, $\mathrm{SE}$, and several quantiles of the posterior distribution for model and derived parameters. See Table A4.4 for a key to parameter names.

\begin{tabular}{|c|c|c|c|c|c|c|c|}
\hline Parameter & Mean & SD & $2.50 \%$ & $25 \%$ & $50 \%$ & $75 \%$ & $97.50 \%$ \\
\hline MSE & 18.645 & 0.163 & 18.335 & 18.531 & 18.641 & 18.755 & 18.971 \\
\hline MSE.CV & 5.660 & 0.050 & 5.565 & 5.625 & 5.658 & 5.693 & 5.759 \\
\hline SSE & $477,076.3$ & $4,179.0$ & $469,126.9$ & $474,153.2$ & $476,954.8$ & $479,896.5$ & $485,413.3$ \\
\hline Trend & 1.062 & 0.006 & 1.050 & 1.057 & 1.062 & 1.066 & 1.076 \\
\hline R.trend & 0.060 & 0.006 & 0.049 & 0.056 & 0.060 & 0.064 & 0.073 \\
\hline M.dens & 1.399 & 0.028 & 1.347 & 1.380 & 1.398 & 1.418 & 1.458 \\
\hline M.y0 & 0.000 & 0.034 & -0.071 & -0.021 & 0.001 & 0.022 & 0.065 \\
\hline M.yz & -0.653 & 0.034 & -0.720 & -0.677 & -0.654 & -0.630 & -0.586 \\
\hline M.blyr & 0.490 & 0.038 & 0.420 & 0.465 & 0.489 & 0.514 & 0.570 \\
\hline M.cell & 0.000 & 0.007 & -0.013 & -0.005 & 0.000 & 0.005 & 0.013 \\
\hline M.psi & 0.399 & 0.003 & 0.393 & 0.397 & 0.399 & 0.402 & 0.406 \\
\hline R.mid & 1.656 & 0.027 & 1.604 & 1.638 & 1.656 & 1.674 & 1.709 \\
\hline b.ADOY & -0.064 & 0.017 & -0.097 & -0.075 & -0.064 & -0.052 & -0.030 \\
\hline b.ADOY.2 & -0.005 & 0.009 & -0.022 & -0.011 & -0.005 & 0.001 & 0.013 \\
\hline z.ADOY & 0.430 & 0.030 & 0.370 & 0.410 & 0.430 & 0.450 & 0.489 \\
\hline z.ADOY.2 & 0.078 & 0.017 & 0.045 & 0.066 & 0.077 & 0.089 & 0.111 \\
\hline SD.surv & 0.318 & 0.073 & 0.210 & 0.267 & 0.307 & 0.357 & 0.491 \\
\hline SD.surv.z & 0.235 & 0.079 & 0.104 & 0.182 & 0.228 & 0.280 & 0.416 \\
\hline SD.y0 & 0.169 & 0.032 & 0.119 & 0.147 & 0.166 & 0.187 & 0.242 \\
\hline SD.yz & 0.973 & 0.149 & 0.727 & 0.866 & 0.957 & 1.063 & 1.307 \\
\hline SD.cell & 0.265 & 0.010 & 0.246 & 0.258 & 0.265 & 0.272 & 0.285 \\
\hline SD.cell.z & 0.780 & 0.028 & 0.724 & 0.761 & 0.780 & 0.799 & 0.835 \\
\hline SD.lam[1] & 0.480 & 0.086 & 0.350 & 0.421 & 0.468 & 0.525 & 0.684 \\
\hline SD.lam[2] & 0.851 & 0.316 & 0.481 & 0.645 & 0.770 & 0.958 & 1.733 \\
\hline SD.lam[3] & 0.588 & 0.833 & 0.009 & 0.129 & 0.341 & 0.727 & 2.695 \\
\hline SD.lam[4] & 2.135 & 1.432 & 0.574 & 1.247 & 1.792 & 2.567 & 6.264 \\
\hline SD.lam[5] & 5.607 & 5.309 & 0.125 & 1.339 & 3.687 & 8.605 & 18.557 \\
\hline SD.lam[6] & 1.228 & 0.238 & 0.876 & 1.063 & 1.191 & 1.353 & 1.791 \\
\hline SD.lam[7] & 0.917 & 0.343 & 0.384 & 0.624 & 0.907 & 1.158 & 1.617 \\
\hline SD.lam[8] & 0.723 & 1.793 & 0.007 & 0.076 & 0.189 & 0.496 & 6.369 \\
\hline S.eff[1] & -0.129 & 0.114 & -0.357 & -0.205 & -0.127 & -0.052 & 0.091 \\
\hline S.eff[2] & -0.084 & 0.089 & -0.254 & -0.144 & -0.085 & -0.025 & 0.090 \\
\hline S.eff[3] & -0.340 & 0.087 & -0.513 & -0.399 & -0.338 & -0.280 & -0.169 \\
\hline S.eff[4] & -0.170 & 0.079 & -0.328 & -0.223 & -0.169 & -0.116 & -0.016 \\
\hline S.eff[5] & -0.624 & 0.094 & -0.812 & -0.688 & -0.625 & -0.559 & -0.443 \\
\hline S.eff[6] & -0.219 & 0.088 & -0.394 & -0.278 & -0.218 & -0.159 & -0.046 \\
\hline S.eff[7] & -0.049 & 0.083 & -0.213 & -0.105 & -0.050 & 0.006 & 0.114 \\
\hline S.eff[8] & -0.089 & 0.099 & -0.283 & -0.156 & -0.089 & -0.022 & 0.105 \\
\hline S.eff[9] & -0.382 & 0.084 & -0.547 & -0.439 & -0.381 & -0.325 & -0.219 \\
\hline
\end{tabular}




\begin{tabular}{|c|c|c|c|c|c|c|c|}
\hline S.eff[10] & -0.194 & 0.067 & -0.330 & -0.239 & -0.193 & -0.148 & -0.061 \\
\hline S.eff[11] & -0.124 & 0.067 & -0.253 & -0.168 & -0.125 & -0.079 & 0.010 \\
\hline S.eff[12] & 0.215 & 0.069 & 0.081 & 0.169 & 0.216 & 0.261 & 0.348 \\
\hline S.eff[13] & -0.234 & 0.069 & -0.369 & -0.281 & -0.234 & -0.188 & -0.098 \\
\hline S.eff[14] & 0.297 & 0.059 & 0.181 & 0.258 & 0.298 & 0.337 & 0.412 \\
\hline S.eff[15] & -0.421 & 0.066 & -0.551 & -0.465 & -0.420 & -0.376 & -0.293 \\
\hline S.eff.z[1] & 0.041 & 0.146 & -0.240 & -0.055 & 0.035 & 0.135 & 0.337 \\
\hline S.eff.z[2] & -0.016 & 0.139 & -0.287 & -0.107 & -0.018 & 0.071 & 0.264 \\
\hline S.eff.z[3] & 0.222 & 0.145 & -0.040 & 0.119 & 0.215 & 0.317 & 0.525 \\
\hline S.eff.z[4] & -0.196 & 0.140 & -0.480 & -0.287 & -0.194 & -0.101 & 0.066 \\
\hline S.eff.z[5] & 0.133 & 0.146 & -0.139 & 0.033 & 0.129 & 0.229 & 0.432 \\
\hline S.eff.z[6] & -0.044 & 0.137 & -0.314 & -0.134 & -0.043 & 0.045 & 0.222 \\
\hline S.eff.z[7] & -0.034 & 0.134 & -0.294 & -0.122 & -0.036 & 0.056 & 0.236 \\
\hline S.eff.z[8] & 0.203 & 0.157 & -0.080 & 0.092 & 0.195 & 0.305 & 0.527 \\
\hline S.eff.z[9] & 0.083 & 0.139 & -0.190 & -0.010 & 0.080 & 0.172 & 0.364 \\
\hline S.eff.z[10] & 0.155 & 0.141 & -0.107 & 0.058 & 0.151 & 0.247 & 0.440 \\
\hline S.eff.z[11] & 0.345 & 0.157 & 0.059 & 0.234 & 0.339 & 0.448 & 0.675 \\
\hline S.eff.z[12] & -0.085 & 0.136 & -0.358 & -0.171 & -0.082 & 0.004 & 0.180 \\
\hline S.eff.z[13] & 0.289 & 0.149 & 0.014 & 0.184 & 0.282 & 0.388 & 0.595 \\
\hline S.eff.z[14] & -0.124 & 0.130 & -0.385 & -0.210 & -0.122 & -0.035 & 0.121 \\
\hline S.eff.z[15] & 0.137 & 0.142 & -0.131 & 0.040 & 0.129 & 0.229 & 0.427 \\
\hline $\mathrm{y} 0[1]$ & -0.053 & 0.113 & -0.280 & -0.124 & -0.053 & 0.018 & 0.173 \\
\hline $\mathrm{y} 0[2]$ & -0.115 & 0.091 & -0.301 & -0.174 & -0.113 & -0.055 & 0.062 \\
\hline $\mathrm{y} 0[3]$ & 0.143 & 0.085 & -0.025 & 0.086 & 0.145 & 0.199 & 0.313 \\
\hline $\mathrm{y} 0[4]$ & -0.026 & 0.090 & -0.201 & -0.088 & -0.026 & 0.036 & 0.150 \\
\hline $\mathrm{y} 0[5]$ & 0.220 & 0.090 & 0.049 & 0.157 & 0.220 & 0.283 & 0.391 \\
\hline $\mathrm{y} 0[6]$ & 0.015 & 0.084 & -0.149 & -0.044 & 0.016 & 0.076 & 0.174 \\
\hline y0[7] & -0.129 & 0.077 & -0.281 & -0.181 & -0.128 & -0.078 & 0.019 \\
\hline y0[8] & -0.177 & 0.075 & -0.326 & -0.228 & -0.175 & -0.126 & -0.038 \\
\hline y0[9] & 0.116 & 0.076 & -0.033 & 0.065 & 0.116 & 0.167 & 0.264 \\
\hline y0[10] & 0.104 & 0.084 & -0.065 & 0.047 & 0.104 & 0.162 & 0.265 \\
\hline y0[11] & 0.079 & 0.090 & -0.100 & 0.019 & 0.079 & 0.142 & 0.251 \\
\hline y0[12] & -0.199 & 0.094 & -0.395 & -0.259 & -0.196 & -0.134 & -0.026 \\
\hline y0[13] & 0.000 & 0.089 & -0.182 & -0.057 & 0.004 & 0.063 & 0.163 \\
\hline y0[14] & -0.260 & 0.081 & -0.417 & -0.314 & -0.258 & -0.206 & -0.104 \\
\hline y0[15] & -0.057 & 0.073 & -0.199 & -0.106 & -0.057 & -0.009 & 0.090 \\
\hline y0[16] & 0.119 & 0.073 & -0.019 & 0.069 & 0.118 & 0.165 & 0.270 \\
\hline y0[17] & 0.212 & 0.077 & 0.074 & 0.157 & 0.207 & 0.265 & 0.371 \\
\hline y0[18] & 0.059 & 0.079 & -0.080 & 0.003 & 0.054 & 0.109 & 0.225 \\
\hline y0[19] & -0.042 & 0.077 & -0.171 & -0.097 & -0.048 & 0.006 & 0.123 \\
\hline y0[20] & -0.147 & 0.075 & -0.281 & -0.200 & -0.151 & -0.099 & 0.014 \\
\hline y0[21] & 0.250 & 0.077 & 0.105 & 0.197 & 0.248 & 0.299 & 0.412 \\
\hline y0[22] & 0.100 & 0.079 & -0.053 & 0.047 & 0.101 & 0.151 & 0.252 \\
\hline y0[23] & -0.110 & 0.079 & -0.262 & -0.162 & -0.108 & -0.056 & 0.041 \\
\hline
\end{tabular}




\begin{tabular}{|c|c|c|c|c|c|c|c|}
\hline $\mathrm{y} 0[24]$ & -0.160 & 0.088 & -0.335 & -0.217 & -0.159 & -0.103 & 0.014 \\
\hline $\mathrm{y} 0[25]$ & 0.064 & 0.111 & -0.156 & -0.011 & 0.066 & 0.139 & 0.274 \\
\hline $\mathrm{yz}[1]$ & 0.534 & 0.111 & 0.311 & 0.459 & 0.535 & 0.609 & 0.752 \\
\hline$y z[2]$ & 0.444 & 0.106 & 0.237 & 0.371 & 0.446 & 0.518 & 0.646 \\
\hline$y z[3]$ & 0.243 & 0.096 & 0.053 & 0.177 & 0.244 & 0.309 & 0.428 \\
\hline$y z[4]$ & 0.126 & 0.092 & -0.052 & 0.064 & 0.125 & 0.187 & 0.308 \\
\hline$y z[5]$ & -0.127 & 0.091 & -0.308 & -0.190 & -0.126 & -0.066 & 0.051 \\
\hline$y z[6]$ & -0.202 & 0.093 & -0.385 & -0.266 & -0.202 & -0.139 & -0.018 \\
\hline$y z[7]$ & 0.068 & 0.105 & -0.137 & -0.003 & 0.070 & 0.142 & 0.268 \\
\hline$y z[8]$ & -0.282 & 0.107 & -0.496 & -0.354 & -0.281 & -0.207 & -0.078 \\
\hline $\mathrm{yz}[9]$ & -0.339 & 0.100 & -0.536 & -0.406 & -0.337 & -0.272 & -0.143 \\
\hline yz[10] & -0.611 & 0.105 & -0.824 & -0.683 & -0.610 & -0.538 & -0.414 \\
\hline yz[11] & -0.566 & 0.114 & -0.796 & -0.642 & -0.564 & -0.488 & -0.349 \\
\hline yz[12] & -0.495 & 0.109 & -0.706 & -0.569 & -0.496 & -0.421 & -0.288 \\
\hline yz[13] & -0.612 & 0.102 & -0.814 & -0.680 & -0.611 & -0.543 & -0.416 \\
\hline yz[14] & -0.567 & 0.100 & -0.768 & -0.635 & -0.567 & -0.501 & -0.374 \\
\hline yz[15] & -0.976 & 0.109 & -1.193 & -1.051 & -0.975 & -0.900 & -0.773 \\
\hline yz[16] & -0.938 & 0.097 & -1.132 & -1.001 & -0.936 & -0.874 & -0.754 \\
\hline yz[17] & -1.176 & 0.101 & -1.379 & -1.243 & -1.175 & -1.109 & -0.979 \\
\hline yz[18] & -1.225 & 0.106 & -1.438 & -1.296 & -1.225 & -1.154 & -1.019 \\
\hline yz[19] & -1.622 & 0.115 & -1.856 & -1.698 & -1.620 & -1.545 & -1.404 \\
\hline$y z[20]$ & -1.833 & 0.132 & -2.101 & -1.919 & -1.830 & -1.741 & -1.585 \\
\hline yz[21] & -1.544 & 0.107 & -1.756 & -1.613 & -1.541 & -1.471 & -1.339 \\
\hline yz[22] & -1.263 & 0.108 & -1.480 & -1.335 & -1.261 & -1.190 & -1.057 \\
\hline$y z[23]$ & -1.140 & 0.108 & -1.355 & -1.212 & -1.137 & -1.067 & -0.934 \\
\hline$y z[24]$ & -1.208 & 0.114 & -1.438 & -1.284 & -1.206 & -1.130 & -0.995 \\
\hline$y z[25]$ & -1.022 & 0.107 & -1.235 & -1.093 & -1.019 & -0.950 & -0.813 \\
\hline N.sum[1] & $22,619.89$ & $1,665.47$ & $19,431.96$ & $21,494.66$ & $22,596.40$ & $23,693.32$ & $26,037.44$ \\
\hline N.sum[2] & $23,317.91$ & $1,544.49$ & $20,387.31$ & $22,264.87$ & $23,271.25$ & $24,340.73$ & $26,424.92$ \\
\hline N.sum[3] & $34,836.27$ & $1,983.92$ & $31,123.69$ & $33,472.41$ & $34,799.96$ & $36,156.78$ & $38,867.06$ \\
\hline N.sum[4] & $32,289.24$ & $1,649.12$ & $29,144.62$ & $31,170.17$ & $32,263.19$ & $33,393.22$ & $35,666.35$ \\
\hline N.sum[5] & $47,463.28$ & $2,183.45$ & $43,231.30$ & $45,995.82$ & $47,430.76$ & $48,932.88$ & $51,762.38$ \\
\hline N.sum[6] & $40,700.03$ & $1,894.12$ & $37,097.35$ & $39,408.11$ & $40,642.83$ & $41,973.52$ & $44,511.60$ \\
\hline N.sum[7] & $31,925.62$ & $1,862.37$ & $28,398.61$ & $30,641.48$ & $31,872.76$ & $33,163.60$ & $35,719.98$ \\
\hline N.sum[8] & $35,181.56$ & $1,783.44$ & $31,839.29$ & $33,945.36$ & $35,147.36$ & $36,337.56$ & $38,830.53$ \\
\hline N.sum[9] & $48,421.23$ & $2,286.01$ & $44,076.17$ & $46,834.67$ & $48,394.25$ & $49,966.80$ & $53,003.08$ \\
\hline N.sum[10] & $53,239.89$ & $2,307.20$ & $48,891.58$ & $51,631.50$ & $53,207.21$ & $54,786.87$ & $57,882.88$ \\
\hline N.sum[11] & $53,106.02$ & $2,546.29$ & $48,260.58$ & $51,368.09$ & $53,025.83$ & $54,781.87$ & $58,343.84$ \\
\hline N.sum[12] & $41,831.50$ & $2,004.39$ & $38,030.19$ & $40,460.85$ & $41,806.19$ & $43,150.30$ & $45,890.32$ \\
\hline N.sum[13] & $57,816.75$ & $2,524.00$ & $52,954.12$ & $56,079.32$ & $57,792.64$ & $59,491.17$ & $62,952.01$ \\
\hline N.sum[14] & $49,013.71$ & $2,160.13$ & $44,859.48$ & $47,541.91$ & $49,001.69$ & $50,455.77$ & $53,404.22$ \\
\hline N.sum[15] & $75,591.65$ & $3,044.42$ & $69,792.41$ & $73,517.56$ & $75,533.02$ & $77,614.80$ & $81,696.41$ \\
\hline N.sum[16] & $100,076.88$ & $3,448.45$ & $93,498.10$ & $97,703.83$ & $100,030.47$ & $102,367.47$ & $107,035.29$ \\
\hline N.sum[17] & $127,940.51$ & $4,038.96$ & $120,321.10$ & $125,152.05$ & $127,935.97$ & $130,676.93$ & $135,939.04$ \\
\hline
\end{tabular}




\begin{tabular}{|c|c|c|c|c|c|c|c|}
\hline N.sum[18] & $119,290.48$ & $3,983.56$ & $111,677.00$ & $116,580.09$ & $119,229.57$ & $121,978.01$ & $127,150.16$ \\
\hline N.sum[19] & $120,661.03$ & $3,645.59$ & $113,781.15$ & $118,160.68$ & $120,601.38$ & $123,155.35$ & $127,897.88$ \\
\hline N.sum[20] & $113,355.57$ & $3,383.87$ & $106,785.97$ & $111,058.59$ & $113,346.09$ & $115,591.23$ & $119,980.75$ \\
\hline N.sum[21] & $160,080.75$ & $4,627.53$ & $151,051.52$ & $156,936.01$ & $160,077.50$ & $163,171.07$ & $169,184.35$ \\
\hline N.sum[22] & $128,006.76$ & $4,227.73$ & $119,902.82$ & $125,147.98$ & $127,932.43$ & $130,831.73$ & $136,383.55$ \\
\hline N.sum[23] & $98,307.28$ & $3,479.65$ & $91,700.51$ & $95,979.19$ & $98,217.93$ & $100,603.38$ & $105,335.20$ \\
\hline N.sum[24] & $91,980.11$ & $3,183.96$ & $85,858.97$ & $89,840.46$ & $91,975.16$ & $94,100.70$ & $98,231.72$ \\
\hline N.sum[25] & $107,324.00$ & $3,604.12$ & $100,347.99$ & $104,886.78$ & $107,274.48$ & $109,751.63$ & $114,462.20$ \\
\hline N.sum2[1] & $24,317.36$ & $2,876.15$ & $19,075.30$ & $22,312.90$ & $24,172.16$ & $26,168.11$ & $30,297.71$ \\
\hline N.sum2[2] & $26,616.19$ & $2,522.73$ & $21,987.91$ & $24,864.69$ & $26,531.45$ & $28,223.94$ & $31,933.22$ \\
\hline N.sum2[3] & $30,705.37$ & $2,563.40$ & $25,876.06$ & $28,934.59$ & $30,589.41$ & $32,393.78$ & $35,967.73$ \\
\hline N.sum2[4] & $33,737.13$ & $2,891.19$ & $28,607.81$ & $31,632.46$ & $33,608.95$ & $35,641.06$ & $39,688.74$ \\
\hline N.sum2[5] & $38,763.29$ & $3,195.83$ & $32,905.59$ & $36,435.55$ & $38,712.64$ & $40,841.98$ & $45,352.38$ \\
\hline N.sum2[6] & $40,762.89$ & $3,037.48$ & $35,262.83$ & $38,638.53$ & $40,673.51$ & $42,724.93$ & $47,101.86$ \\
\hline N.sum2[7] & $36,913.60$ & $2,616.43$ & $32,142.36$ & $35,116.62$ & $36,797.44$ & $38,554.83$ & $42,484.07$ \\
\hline N.sum2[8] & $42,691.77$ & $2,997.57$ & $37,368.76$ & $40,630.82$ & $42,519.24$ & $44,596.43$ & $48,833.79$ \\
\hline N.sum2[9] & $43,861.82$ & $3,217.85$ & $38,141.41$ & $41,597.43$ & $43,669.68$ & $45,942.49$ & $50,368.05$ \\
\hline N.sum2[10] & $48,832.60$ & $3,987.68$ & $41,661.12$ & $45,929.89$ & $48,770.03$ & $51,467.37$ & $56,969.34$ \\
\hline N.sum2[11] & $49,952.15$ & $4,466.97$ & $42,020.72$ & $46,839.76$ & $49,670.45$ & $52,800.96$ & $59,424.16$ \\
\hline N.sum2[12] & $51,966.83$ & $4,732.86$ & $43,768.66$ & $48,754.34$ & $51,615.64$ & $54,857.70$ & $62,385.51$ \\
\hline N.sum2[13] & $58,823.35$ & $5,008.70$ & $50,214.89$ & $55,381.59$ & $58,357.70$ & $61,785.49$ & $69,949.60$ \\
\hline N.sum2[14] & $64,623.05$ & $4,793.13$ & $55,848.24$ & $61,266.41$ & $64,373.34$ & $67,726.06$ & $74,832.98$ \\
\hline N.sum2[15] & $81,324.46$ & $5,504.55$ & $70,593.46$ & $77,649.58$ & $81,185.68$ & $85,020.75$ & $92,343.61$ \\
\hline N.sum2[16] & $90,400.85$ & $6,203.65$ & $78,366.13$ & $86,158.55$ & $90,377.00$ & $94,532.79$ & $102,909.24$ \\
\hline N.sum2[17] & $105,341.81$ & $7,736.75$ & $90,706.44$ & $99,837.86$ & $105,417.94$ & $110,655.36$ & $120,759.65$ \\
\hline N.sum2[18] & $114,397.83$ & $8,566.36$ & $98,083.92$ & $108,346.39$ & $114,613.16$ & $120,094.69$ & $131,312.08$ \\
\hline N.sum2[19] & $127,969.73$ & $8,914.58$ & $110,594.79$ & $121,862.23$ & $128,532.10$ & $134,174.13$ & $144,265.91$ \\
\hline N.sum2[20] & $133,521.07$ & $8,676.74$ & $116,164.71$ & $127,472.51$ & $133,878.99$ & $139,768.01$ & $149,466.82$ \\
\hline N.sum2[21] & $126,811.70$ & $8,405.23$ & $111,086.94$ & $120,876.60$ & $126,752.73$ & $132,348.61$ & $143,832.51$ \\
\hline N.sum2[22] & $117,813.70$ & $8,051.67$ & $103,070.10$ & $112,201.50$ & $117,532.99$ & $122,945.07$ & $134,691.78$ \\
\hline N.sum2[23] & $111,570.85$ & $7,782.59$ & $97,090.83$ & $106,200.89$ & $111,260.24$ & $116,573.86$ & $127,962.19$ \\
\hline N.sum2[24] & $109,883.73$ & $8,817.43$ & $93,616.24$ & $103,885.30$ & $109,366.16$ & $115,629.96$ & $127,742.00$ \\
\hline N.sum2[25] & $102,746.25$ & $10,884.76$ & $83,462.18$ & $95,223.96$ & $101,980.38$ & $109,762.87$ & $125,504.22$ \\
\hline R.growth[1] & 0.093 & 0.089 & -0.076 & 0.031 & 0.091 & 0.152 & 0.272 \\
\hline R.growth[2] & 0.144 & 0.075 & 0.000 & 0.093 & 0.143 & 0.194 & 0.292 \\
\hline R.growth[3] & 0.094 & 0.064 & -0.030 & 0.050 & 0.095 & 0.137 & 0.221 \\
\hline R.growth[4] & 0.139 & 0.056 & 0.030 & 0.101 & 0.139 & 0.177 & 0.249 \\
\hline R.growth[5] & 0.051 & 0.054 & -0.054 & 0.014 & 0.050 & 0.087 & 0.157 \\
\hline R.growth[6] & -0.099 & 0.062 & -0.220 & -0.141 & -0.098 & -0.057 & 0.022 \\
\hline R.growth[7] & 0.145 & 0.063 & 0.023 & 0.103 & 0.145 & 0.189 & 0.269 \\
\hline R.growth[8] & 0.027 & 0.058 & -0.087 & -0.013 & 0.027 & 0.067 & 0.141 \\
\hline R.growth[9] & 0.107 & 0.053 & 0.004 & 0.070 & 0.107 & 0.142 & 0.213 \\
\hline R.growth[10] & 0.022 & 0.051 & -0.079 & -0.012 & 0.022 & 0.056 & 0.124 \\
\hline R.growth[11] & 0.039 & 0.055 & -0.069 & 0.004 & 0.040 & 0.076 & 0.146 \\
\hline
\end{tabular}




\begin{tabular}{lrrrrrrr} 
R.growth[12] & 0.124 & 0.051 & 0.025 & 0.090 & 0.125 & 0.159 & 0.223 \\
R.growth[13] & 0.095 & 0.052 & -0.007 & 0.060 & 0.095 & 0.130 & 0.196 \\
R.growth[14] & 0.230 & 0.052 & 0.127 & 0.195 & 0.231 & 0.266 & 0.332 \\
R.growth[15] & 0.106 & 0.047 & 0.010 & 0.074 & 0.107 & 0.139 & 0.195 \\
R.growth[16] & 0.153 & 0.042 & 0.069 & 0.125 & 0.153 & 0.181 & 0.232 \\
R.growth[17] & 0.082 & 0.038 & 0.009 & 0.056 & 0.082 & 0.108 & 0.155 \\
R.growth[18] & 0.112 & 0.037 & 0.039 & 0.087 & 0.112 & 0.137 & 0.189 \\
R.growth[19] & 0.043 & 0.037 & -0.030 & 0.017 & 0.042 & 0.069 & 0.114 \\
R.growth[20] & -0.052 & 0.037 & -0.124 & -0.077 & -0.052 & -0.026 & 0.020 \\
R.growth[21] & -0.074 & 0.037 & -0.145 & -0.099 & -0.074 & -0.049 & 0.000 \\
R.growth[22] & -0.055 & 0.041 & -0.133 & -0.083 & -0.055 & -0.027 & 0.028 \\
R.growth[23] & -0.016 & 0.048 & -0.109 & -0.049 & -0.016 & 0.015 & 0.083 \\
R.growth[24] & -0.070 & 0.054 & -0.172 & -0.106 & -0.070 & -0.034 & 0.041 \\
Deviance & $76,451.111$ & 216.173 & $76,038.905$ & $76,303.493$ & $76,447.001$ & $76,595.265$ & $76,876.707$ \\
\hline
\end{tabular}


Tables A4.7. Bayesian generalized linear mixed model output for Black Brant observed during aerial surveys 1992-2016 on the Arctic Coastal Plain, Alaska. We provide summaries including mean, SE, and several quantiles of the posterior distribution for model and derived parameters. See Table A4.4 for a key to parameter names.

\begin{tabular}{|c|c|c|c|c|c|c|c|}
\hline Parameter & Mean & SD & $2.50 \%$ & $25 \%$ & $50 \%$ & $75 \%$ & $97.50 \%$ \\
\hline MSE & 5.109 & 0.334 & 4.715 & 4.896 & 5.037 & 5.231 & 5.940 \\
\hline MSE.CV & 7.286 & 0.477 & 6.724 & 6.982 & 7.182 & 7.460 & 8.471 \\
\hline SSE & $45,288.433$ & $2,964.508$ & $41,797.094$ & $43,398.073$ & $44,645.135$ & $46,368.405$ & $52,656.516$ \\
\hline Trend & 1.076 & 0.018 & 1.038 & 1.065 & 1.076 & 1.088 & 1.111 \\
\hline R.trend & 0.073 & 0.017 & 0.037 & 0.063 & 0.073 & 0.084 & 0.105 \\
\hline M.dens & 0.342 & 0.028 & 0.295 & 0.323 & 0.339 & 0.357 & 0.404 \\
\hline M.y0 & -0.001 & 0.085 & -0.174 & -0.055 & 0.000 & 0.057 & 0.164 \\
\hline M.blyr & -1.936 & 0.102 & -2.134 & -2.006 & -1.936 & -1.869 & -1.733 \\
\hline M.cell & 0.001 & 0.039 & -0.075 & -0.025 & 0.002 & 0.027 & 0.077 \\
\hline R.mid & -1.394 & 0.050 & -1.493 & -1.427 & -1.395 & -1.361 & -1.298 \\
\hline b.ADOY & -0.083 & 0.058 & -0.196 & -0.123 & -0.084 & -0.044 & 0.033 \\
\hline b.ADOY.2 & 0.047 & 0.028 & -0.007 & 0.028 & 0.047 & 0.067 & 0.102 \\
\hline SD.surv & 0.874 & 0.219 & 0.540 & 0.721 & 0.847 & 0.990 & 1.400 \\
\hline SD.y0 & 0.419 & 0.088 & 0.271 & 0.358 & 0.409 & 0.470 & 0.614 \\
\hline SD.cell & 0.783 & 0.047 & 0.696 & 0.751 & 0.782 & 0.814 & 0.879 \\
\hline SD.lam[1] & 0.422 & 0.105 & 0.264 & 0.348 & 0.405 & 0.477 & 0.669 \\
\hline SD.lam[2] & 5.261 & 3.335 & 1.448 & 2.696 & 4.150 & 7.106 & 13.507 \\
\hline SD.lam[3] & 2.704 & 2.353 & 0.520 & 1.208 & 2.000 & 3.309 & 10.394 \\
\hline SD.lam[4] & 2.800 & 2.817 & 0.089 & 0.863 & 1.840 & 3.697 & 10.929 \\
\hline SD.lam[5] & 6.105 & 4.080 & 0.288 & 2.712 & 5.353 & 9.137 & 14.366 \\
\hline SD.lam[6] & 4.474 & 1.360 & 2.648 & 3.454 & 4.166 & 5.263 & 7.718 \\
\hline SD.lam[7] & 3.944 & 2.066 & 0.750 & 2.381 & 3.845 & 5.317 & 8.396 \\
\hline SD.lam[8] & 0.842 & 1.375 & 0.006 & 0.097 & 0.297 & 0.841 & 4.797 \\
\hline S.eff[1] & -0.685 & 0.412 & -1.502 & -0.964 & -0.683 & -0.404 & 0.101 \\
\hline S.eff[2] & 1.137 & 0.333 & 0.501 & 0.911 & 1.133 & 1.356 & 1.811 \\
\hline S.eff[3] & -0.111 & 0.347 & -0.799 & -0.342 & -0.111 & 0.118 & 0.573 \\
\hline S.eff[4] & -0.389 & 0.312 & -1.004 & -0.597 & -0.388 & -0.179 & 0.209 \\
\hline S.eff[5] & 0.250 & 0.322 & -0.397 & 0.038 & 0.254 & 0.462 & 0.873 \\
\hline S.eff[6] & -0.384 & 0.317 & -1.032 & -0.595 & -0.380 & -0.167 & 0.218 \\
\hline S.eff[7] & -0.544 & 0.363 & -1.267 & -0.786 & -0.538 & -0.294 & 0.160 \\
\hline S.eff[8] & -0.393 & 0.367 & -1.113 & -0.633 & -0.397 & -0.138 & 0.314 \\
\hline S.eff[9] & -0.987 & 0.404 & -1.799 & -1.255 & -0.978 & -0.710 & -0.225 \\
\hline S.eff[10] & 1.052 & 0.290 & 0.494 & 0.854 & 1.052 & 1.244 & 1.626 \\
\hline S.eff[11] & -1.057 & 0.339 & -1.733 & -1.282 & -1.051 & -0.825 & -0.412 \\
\hline S.eff[12] & -0.508 & 0.309 & -1.117 & -0.712 & -0.502 & -0.302 & 0.097 \\
\hline S.eff[13] & -0.728 & 0.292 & -1.297 & -0.926 & -0.728 & -0.535 & -0.153 \\
\hline S.eff[14] & -1.069 & 0.282 & -1.627 & -1.258 & -1.066 & -0.880 & -0.510 \\
\hline S.eff[15] & -0.577 & 0.302 & -1.172 & -0.778 & -0.573 & -0.375 & 0.020 \\
\hline $\mathrm{y} 0[1]$ & 0.012 & 0.316 & -0.633 & -0.189 & 0.019 & 0.219 & 0.620 \\
\hline
\end{tabular}




\begin{tabular}{|c|c|c|c|c|c|c|c|}
\hline $\mathrm{y} 0[2]$ & -0.603 & 0.284 & -1.186 & -0.788 & -0.594 & -0.403 & -0.068 \\
\hline $\mathrm{y} 0[3]$ & 0.090 & 0.243 & -0.386 & -0.073 & 0.089 & 0.252 & 0.567 \\
\hline $\mathrm{y} 0[4]$ & 0.477 & 0.243 & 0.001 & 0.317 & 0.478 & 0.640 & 0.960 \\
\hline $\mathrm{y} 0[5]$ & -0.093 & 0.233 & -0.541 & -0.252 & -0.096 & 0.060 & 0.371 \\
\hline y0[6] & 0.452 & 0.222 & 0.026 & 0.303 & 0.449 & 0.595 & 0.902 \\
\hline $\mathrm{y} 0[7]$ & -0.063 & 0.221 & -0.496 & -0.212 & -0.065 & 0.086 & 0.375 \\
\hline $\mathrm{y} 0[8]$ & -0.279 & 0.233 & -0.726 & -0.438 & -0.280 & -0.121 & 0.177 \\
\hline $\mathrm{y} 0[9]$ & -0.329 & 0.236 & -0.782 & -0.488 & -0.331 & -0.175 & 0.154 \\
\hline y0[10] & -0.335 & 0.228 & -0.772 & -0.490 & -0.338 & -0.182 & 0.114 \\
\hline $\mathrm{y} 0[11]$ & 0.173 & 0.202 & -0.224 & 0.038 & 0.170 & 0.305 & 0.576 \\
\hline y0[12] & -0.007 & 0.208 & -0.419 & -0.149 & -0.007 & 0.130 & 0.394 \\
\hline y0[13] & 0.232 & 0.214 & -0.194 & 0.090 & 0.236 & 0.379 & 0.635 \\
\hline y0[14] & 0.593 & 0.215 & 0.158 & 0.450 & 0.599 & 0.735 & 1.009 \\
\hline y0[15] & 0.228 & 0.209 & -0.193 & 0.089 & 0.232 & 0.371 & 0.622 \\
\hline y0[16] & -0.629 & 0.224 & -1.094 & -0.774 & -0.621 & -0.477 & -0.212 \\
\hline y0[17] & 0.209 & 0.205 & -0.198 & 0.078 & 0.212 & 0.347 & 0.602 \\
\hline y0[18] & -0.215 & 0.211 & -0.644 & -0.353 & -0.211 & -0.073 & 0.184 \\
\hline y0[19] & -0.155 & 0.197 & -0.554 & -0.285 & -0.151 & -0.020 & 0.225 \\
\hline y0[20] & -0.267 & 0.209 & -0.688 & -0.402 & -0.262 & -0.126 & 0.142 \\
\hline $\mathrm{y} 0[21]$ & 0.664 & 0.212 & 0.246 & 0.524 & 0.665 & 0.802 & 1.084 \\
\hline y0[22] & -0.089 & 0.216 & -0.522 & -0.232 & -0.086 & 0.058 & 0.326 \\
\hline y0[23] & -0.042 & 0.219 & -0.476 & -0.187 & -0.042 & 0.103 & 0.389 \\
\hline y0[24] & 0.136 & 0.244 & -0.324 & -0.033 & 0.130 & 0.296 & 0.631 \\
\hline $\mathrm{y} 0[25]$ & -0.178 & 0.306 & -0.766 & -0.387 & -0.182 & 0.025 & 0.427 \\
\hline N.sum[1] & $1,161.571$ & 246.365 & 746.727 & 983.481 & $1,136.566$ & $1,310.178$ & $1,714.362$ \\
\hline N.sum[2] & 707.435 & 169.303 & 429.858 & 585.852 & 689.462 & 810.072 & $1,088.080$ \\
\hline N.sum[3] & $1,557.136$ & 305.761 & $1,049.305$ & $1,338.963$ & $1,525.775$ & $1,742.049$ & $2,229.511$ \\
\hline N.sum[4] & $2,497.151$ & 458.861 & $1,718.823$ & $2,172.248$ & $2,454.419$ & $2,776.807$ & $3,535.241$ \\
\hline N.sum[5] & $1,512.431$ & 302.109 & $1,015.254$ & $1,299.599$ & $1,478.951$ & $1,688.796$ & $2,201.896$ \\
\hline N.sum[6] & $2,740.343$ & 468.902 & $1,949.992$ & $2,402.628$ & $2,696.665$ & $3,024.752$ & $3,763.044$ \\
\hline N.sum[7] & $1,737.852$ & 338.513 & $1,164.424$ & $1,498.683$ & $1,702.920$ & $1,942.460$ & $2,510.773$ \\
\hline N.sum[8] & $1,504.295$ & 281.407 & $1,028.444$ & $1,302.928$ & $1,483.209$ & $1,683.424$ & $2,108.064$ \\
\hline N.sum[9] & $1,580.074$ & 289.271 & $1,084.501$ & $1,377.512$ & $1,552.855$ & $1,759.409$ & $2,216.252$ \\
\hline N.sum[10] & $1,780.491$ & 332.340 & $1,217.962$ & $1,546.690$ & $1,751.005$ & $1,980.020$ & $2,528.945$ \\
\hline N.sum[11] & $3,391.266$ & 570.034 & $2,426.401$ & $2,988.406$ & $3,336.215$ & $3,733.003$ & $4,659.878$ \\
\hline N.sum[12] & $3,247.886$ & 527.023 & $2,360.704$ & $2,876.206$ & $3,197.873$ & $3,563.402$ & $4,423.730$ \\
\hline N.sum[13] & $4,639.958$ & 695.782 & $3,417.455$ & $4,152.900$ & $4,581.761$ & $5,078.172$ & $6,149.400$ \\
\hline N.sum[14] & $7,307.714$ & $1,090.482$ & $5,446.175$ & $6,532.302$ & $7,224.264$ & $7,958.880$ & $9,725.800$ \\
\hline N.sum[15] & $5,465.135$ & 861.225 & $4,010.664$ & $4,855.968$ & $5,387.600$ & $5,999.193$ & $7,351.849$ \\
\hline N.sum[16] & $2,477.678$ & 447.943 & $1,719.376$ & $2,151.916$ & $2,437.563$ & $2,757.122$ & $3,463.495$ \\
\hline N.sum[17] & $6,089.127$ & 903.344 & $4,516.744$ & $5,463.057$ & $6,011.980$ & $6,645.669$ & $8,049.763$ \\
\hline N.sum[18] & $4,310.889$ & 693.655 & $3,113.197$ & $3,821.115$ & $4,248.434$ & $4,740.903$ & $5,872.498$ \\
\hline N.sum[19] & $4,981.419$ & 758.955 & $3,673.898$ & $4,453.295$ & $4,924.511$ & $5,455.683$ & $6,643.984$ \\
\hline N.sum[20] & $4,852.610$ & 811.537 & $3,441.541$ & $4,276.053$ & $4,785.096$ & $5,348.143$ & $6,635.961$ \\
\hline
\end{tabular}




\begin{tabular}{|c|c|c|c|c|c|c|c|}
\hline N.sum[21] & $13,120.009$ & $2,020.998$ & $9,679.822$ & $11,712.914$ & $12,953.973$ & $14,316.703$ & $17,568.495$ \\
\hline N.sum[22] & $6,413.754$ & $1,026.690$ & $4,662.382$ & $5,680.232$ & $6,331.414$ & $7,045.242$ & $8,702.649$ \\
\hline N.sum[23] & $6,735.924$ & $1,089.455$ & $4,868.601$ & $5,976.518$ & $6,634.911$ & $7,400.330$ & $9,162.250$ \\
\hline N.sum[24] & $7,843.351$ & $1,249.242$ & $5,722.430$ & $6,954.970$ & $7,740.243$ & $8,597.290$ & $10,569.641$ \\
\hline N.sum[25] & $5,517.404$ & 865.745 & $4,076.881$ & $4,892.081$ & $5,449.759$ & $6,053.841$ & $7,417.515$ \\
\hline N.sum2[1] & $1,291.788$ & 424.131 & 660.509 & $1,002.353$ & $1,234.545$ & $1,507.160$ & $2,296.292$ \\
\hline N.sum2[2] & $1,413.559$ & 334.085 & 883.738 & $1,180.767$ & $1,368.410$ & $1,601.214$ & $2,192.043$ \\
\hline N.sum2[3] & $1,559.443$ & 313.731 & $1,059.660$ & $1,337.320$ & $1,521.348$ & $1,740.779$ & $2,260.487$ \\
\hline N.sum2[4] & $1,700.154$ & 340.500 & $1,186.793$ & $1,459.341$ & $1,646.145$ & $1,881.044$ & $2,499.481$ \\
\hline N.sum2[5] & $1,812.363$ & 332.885 & $1,286.253$ & $1,583.563$ & $1,771.683$ & $1,991.391$ & $2,612.905$ \\
\hline N.sum2[6] & $1,906.673$ & 308.679 & $1,357.046$ & $1,695.225$ & $1,883.167$ & $2,098.142$ & $2,574.492$ \\
\hline N.sum2[7] & $2,017.133$ & 331.685 & $1,389.966$ & $1,792.243$ & $2,008.119$ & $2,230.186$ & $2,703.365$ \\
\hline N.sum2[8] & $2,175.197$ & 385.023 & $1,416.303$ & $1,919.181$ & $2,185.020$ & $2,427.357$ & $2,940.814$ \\
\hline N.sum2[9] & $2,403.168$ & 425.656 & $1,536.376$ & $2,142.280$ & $2,416.714$ & $2,668.277$ & $3,245.436$ \\
\hline N.sum2[10] & $2,715.672$ & 440.978 & $1,864.736$ & $2,444.152$ & $2,699.633$ & $2,971.122$ & $3,660.444$ \\
\hline N.sum2[11] & $3,115.041$ & 464.233 & $2,311.500$ & $2,807.886$ & $3,064.500$ & $3,365.200$ & $4,184.758$ \\
\hline N.sum2[12] & $3,576.069$ & 559.072 & $2,716.601$ & $3,185.262$ & $3,494.660$ & $3,885.540$ & $4,898.631$ \\
\hline N.sum2[13] & $4,037.613$ & 692.389 & $3,038.162$ & $3,543.192$ & $3,908.798$ & $4,395.608$ & $5,739.584$ \\
\hline N.sum2[14] & $4,435.907$ & 758.243 & $3,350.599$ & $3,879.876$ & $4,298.030$ & $4,849.564$ & $6,275.350$ \\
\hline N.sum2[15] & $4,762.476$ & 764.028 & $3,643.865$ & $4,209.299$ & $4,633.556$ & $5,179.198$ & $6,522.541$ \\
\hline N.sum2[16] & $5,068.757$ & 803.052 & $3,837.290$ & $4,512.625$ & 4,946.706 & $5,491.939$ & $6,963.717$ \\
\hline N.sum2[17] & $5,417.893$ & 881.192 & $3,973.332$ & $4,825.080$ & $5,303.719$ & $5,898.738$ & $7,532.642$ \\
\hline N.sum2[18] & $5,848.546$ & 938.813 & $4,310.466$ & $5,224.607$ & $5,730.753$ & $6,360.790$ & $8,020.989$ \\
\hline N.sum2[19] & $6,362.558$ & 959.118 & $4,852.668$ & $5,709.846$ & $6,219.322$ & $6,860.403$ & $8,607.619$ \\
\hline N.sum2[20] & $6,913.555$ & $1,010.496$ & $5,298.951$ & $6,211.315$ & $6,767.201$ & $7,482.563$ & $9,233.291$ \\
\hline N.sum2[21] & 7,399.106 & $1,155.107$ & $5,531.618$ & $6,586.194$ & $7,245.937$ & $8,081.545$ & $10,060.460$ \\
\hline N.sum2[22] & $7,684.671$ & $1,279.384$ & $5,657.284$ & $6,790.656$ & $7,515.729$ & $8,415.302$ & $10,650.417$ \\
\hline N.sum2[23] & $7,712.988$ & $1,355.874$ & $5,456.383$ & $6,770.772$ & $7,579.912$ & $8,526.529$ & $10,751.468$ \\
\hline N.sum2[24] & $7,584.398$ & $1,668.271$ & $4,780.796$ & $6,396.677$ & $7,416.339$ & $8,586.258$ & $11,269.754$ \\
\hline N.sum2[25] & $7,470.188$ & $2,278.475$ & $3,735.034$ & $5,808.424$ & $7,210.873$ & $8,873.527$ & $12,498.883$ \\
\hline R.growth[1] & 0.113 & 0.132 & -0.119 & 0.042 & 0.090 & 0.163 & 0.458 \\
\hline R.growth[2] & 0.105 & 0.109 & -0.097 & 0.047 & 0.089 & 0.151 & 0.373 \\
\hline R.growth[3] & 0.087 & 0.072 & -0.062 & 0.049 & 0.082 & 0.125 & 0.241 \\
\hline R.growth[4] & 0.067 & 0.056 & -0.054 & 0.035 & 0.069 & 0.102 & 0.175 \\
\hline R.growth[5] & 0.054 & 0.073 & -0.141 & 0.025 & 0.068 & 0.099 & 0.164 \\
\hline R.growth[6] & 0.056 & 0.080 & -0.176 & 0.032 & 0.072 & 0.102 & 0.174 \\
\hline R.growth[7] & 0.073 & 0.066 & -0.107 & 0.051 & 0.081 & 0.109 & 0.183 \\
\hline R.growth[8] & 0.100 & 0.049 & 0.004 & 0.070 & 0.096 & 0.127 & 0.201 \\
\hline R.growth[9] & 0.125 & 0.059 & 0.039 & 0.082 & 0.114 & 0.157 & 0.269 \\
\hline R.growth[10] & 0.140 & 0.076 & 0.041 & 0.085 & 0.120 & 0.173 & 0.339 \\
\hline R.growth[11] & 0.137 & 0.075 & 0.037 & 0.085 & 0.117 & 0.171 & 0.331 \\
\hline R.growth[12] & 0.119 & 0.058 & 0.020 & 0.082 & 0.107 & 0.150 & 0.258 \\
\hline R.growth[13] & 0.094 & 0.048 & -0.009 & 0.069 & 0.092 & 0.120 & 0.196 \\
\hline R.growth[14] & 0.073 & 0.058 & -0.069 & 0.048 & 0.082 & 0.107 & 0.170 \\
\hline
\end{tabular}




\begin{tabular}{lrrrrrrr} 
R.growth[15] & 0.063 & 0.064 & -0.106 & 0.038 & 0.076 & 0.101 & 0.160 \\
R.growth[16] & 0.066 & 0.054 & -0.070 & 0.040 & 0.074 & 0.098 & 0.158 \\
R.growth[17] & 0.077 & 0.042 & -0.012 & 0.053 & 0.078 & 0.102 & 0.169 \\
R.growth[18] & 0.086 & 0.052 & -0.014 & 0.055 & 0.083 & 0.113 & 0.202 \\
R.growth[19] & 0.084 & 0.065 & -0.025 & 0.044 & 0.076 & 0.109 & 0.252 \\
R.growth[20] & 0.066 & 0.062 & -0.044 & 0.028 & 0.063 & 0.096 & 0.216 \\
R.growth[21] & 0.036 & 0.055 & -0.084 & 0.002 & 0.041 & 0.075 & 0.130 \\
R.growth[22] & 0.002 & 0.074 & -0.158 & -0.048 & 0.008 & 0.060 & 0.121 \\
R.growth[23] & -0.026 & 0.107 & -0.268 & -0.092 & -0.008 & 0.058 & 0.133 \\
R.growth[24] & -0.038 & 0.127 & -0.338 & -0.112 & -0.012 & 0.060 & 0.143 \\
Deviance & $14,698.145$ & 31.598 & $14,638.032$ & $14,676.298$ & $14,697.791$ & $14,719.310$ & $14,760.839$ \\
\hline
\end{tabular}


Tables A4.8. Bayesian generalized linear mixed model output for Cackling Goose observed during aerial surveys 1992-2016 on the Arctic Coastal Plain, Alaska. We provide summaries including mean, SE, and several quantiles of the posterior distribution for model and derived parameters. See Table A4.4 for a key to parameter names.

\begin{tabular}{|c|c|c|c|c|c|c|c|}
\hline Parameter & Mean & SD & $2.50 \%$ & $25 \%$ & $50 \%$ & $75 \%$ & $97.50 \%$ \\
\hline MSE & 1.205 & 0.021 & 1.173 & 1.190 & 1.201 & 1.215 & 1.258 \\
\hline MSE.CV & 3.291 & 0.059 & 3.206 & 3.251 & 3.281 & 3.320 & 3.437 \\
\hline SSE & $14,244.512$ & 253.735 & $13,876.920$ & $14,069.817$ & $14,199.475$ & $14,368.720$ & $14,876.995$ \\
\hline Trend & 1.071 & 0.017 & 1.039 & 1.058 & 1.070 & 1.082 & 1.107 \\
\hline R.trend & 0.068 & 0.016 & 0.038 & 0.057 & 0.067 & 0.079 & 0.101 \\
\hline M.dens & 0.198 & 0.016 & 0.172 & 0.186 & 0.196 & 0.207 & 0.236 \\
\hline M.y0 & 0.003 & 0.082 & -0.153 & -0.051 & 0.003 & 0.058 & 0.168 \\
\hline M.blyr & -2.265 & 0.097 & -2.456 & -2.331 & -2.265 & -2.200 & -2.076 \\
\hline M.cell & 0.000 & 0.006 & -0.012 & -0.003 & 0.000 & 0.003 & 0.012 \\
\hline R.mid & -1.622 & 0.049 & -1.718 & -1.655 & -1.623 & -1.589 & -1.526 \\
\hline b.ADOY & -0.131 & 0.059 & -0.247 & -0.170 & -0.133 & -0.092 & -0.013 \\
\hline b.ADOY.2 & 0.015 & 0.029 & -0.043 & -0.005 & 0.015 & 0.034 & 0.070 \\
\hline SD.surv & 0.756 & 0.185 & 0.465 & 0.626 & 0.731 & 0.861 & 1.184 \\
\hline SD.y0 & 0.411 & 0.083 & 0.276 & 0.353 & 0.402 & 0.458 & 0.600 \\
\hline SD.cell & 0.136 & 0.067 & 0.013 & 0.096 & 0.143 & 0.184 & 0.259 \\
\hline SD.lam[1] & 0.675 & 0.195 & 0.352 & 0.525 & 0.664 & 0.804 & 1.089 \\
\hline SD.lam[2] & 1.706 & 0.718 & 0.721 & 1.241 & 1.575 & 2.016 & 3.492 \\
\hline SD.lam[3] & 4.716 & 2.907 & 1.308 & 2.547 & 3.892 & 6.091 & 12.470 \\
\hline SD.lam[4] & 2.365 & 1.939 & 0.241 & 1.114 & 1.822 & 3.024 & 7.695 \\
\hline SD.lam[5] & 5.700 & 4.087 & 0.248 & 2.249 & 4.893 & 8.673 & 14.206 \\
\hline SD.lam[6] & 5.699 & 1.424 & 3.400 & 4.597 & 5.601 & 6.616 & 8.740 \\
\hline SD.lam[7] & 3.089 & 0.874 & 1.791 & 2.422 & 2.981 & 3.610 & 5.091 \\
\hline SD.lam[8] & 0.876 & 1.369 & 0.013 & 0.160 & 0.448 & 0.978 & 5.051 \\
\hline S.eff[1] & 0.471 & 0.333 & -0.165 & 0.246 & 0.468 & 0.686 & 1.141 \\
\hline S.eff[2] & 0.570 & 0.306 & -0.026 & 0.360 & 0.566 & 0.773 & 1.168 \\
\hline S.eff[3] & -0.339 & 0.307 & -0.929 & -0.550 & -0.340 & -0.128 & 0.257 \\
\hline S.eff[4] & -0.199 & 0.305 & -0.813 & -0.402 & -0.194 & 0.008 & 0.397 \\
\hline S.eff[5] & 0.379 & 0.280 & -0.160 & 0.190 & 0.384 & 0.563 & 0.936 \\
\hline S.eff[6] & -0.406 & 0.298 & -0.999 & -0.604 & -0.404 & -0.205 & 0.172 \\
\hline S.eff[7] & 0.638 & 0.290 & 0.074 & 0.441 & 0.636 & 0.837 & 1.213 \\
\hline S.eff[8] & 0.490 & 0.286 & -0.064 & 0.300 & 0.487 & 0.686 & 1.056 \\
\hline S.eff[9] & 1.058 & 0.261 & 0.556 & 0.881 & 1.057 & 1.236 & 1.571 \\
\hline S.eff[10] & 1.551 & 0.266 & 1.034 & 1.370 & 1.549 & 1.732 & 2.070 \\
\hline S.eff[11] & -0.021 & 0.265 & -0.539 & -0.196 & -0.023 & 0.156 & 0.507 \\
\hline S.eff[12] & 0.191 & 0.250 & -0.305 & 0.025 & 0.194 & 0.354 & 0.693 \\
\hline S.eff[13] & 0.566 & 0.257 & 0.069 & 0.393 & 0.562 & 0.736 & 1.083 \\
\hline S.eff[14] & 0.707 & 0.262 & 0.204 & 0.528 & 0.706 & 0.884 & 1.218 \\
\hline S.eff[15] & 0.115 & 0.277 & -0.422 & -0.076 & 0.114 & 0.308 & 0.664 \\
\hline $\mathrm{y} 0[1]$ & -0.145 & 0.299 & -0.740 & -0.345 & -0.142 & 0.051 & 0.443 \\
\hline
\end{tabular}




\begin{tabular}{|c|c|c|c|c|c|c|c|}
\hline $\mathrm{y} 0[2]$ & -0.201 & 0.262 & -0.719 & -0.372 & -0.199 & -0.030 & 0.307 \\
\hline $\mathrm{y} 0[3]$ & 0.137 & 0.230 & -0.325 & -0.013 & 0.137 & 0.286 & 0.583 \\
\hline $\mathrm{y} 0[4]$ & -0.031 & 0.216 & -0.460 & -0.173 & -0.031 & 0.114 & 0.390 \\
\hline $\mathrm{y} 0[5]$ & 0.106 & 0.210 & -0.307 & -0.033 & 0.107 & 0.243 & 0.519 \\
\hline $\mathrm{y} 0[6]$ & 0.380 & 0.214 & -0.034 & 0.233 & 0.376 & 0.518 & 0.815 \\
\hline y0[7] & -0.322 & 0.221 & -0.756 & -0.469 & -0.316 & -0.173 & 0.099 \\
\hline $\mathrm{y} 0[8]$ & 0.066 & 0.210 & -0.352 & -0.071 & 0.065 & 0.204 & 0.484 \\
\hline y0[9] & 0.073 & 0.204 & -0.333 & -0.059 & 0.074 & 0.209 & 0.467 \\
\hline y0[10] & -0.323 & 0.223 & -0.775 & -0.466 & -0.319 & -0.175 & 0.110 \\
\hline y0[11] & -0.007 & 0.213 & -0.420 & -0.148 & -0.008 & 0.136 & 0.414 \\
\hline y0[12] & 0.409 & 0.207 & 0.006 & 0.271 & 0.404 & 0.551 & 0.819 \\
\hline y0[13] & -0.261 & 0.212 & -0.688 & -0.399 & -0.260 & -0.118 & 0.144 \\
\hline y0[14] & -0.322 & 0.216 & -0.761 & -0.467 & -0.319 & -0.178 & 0.098 \\
\hline y0[15] & -0.242 & 0.218 & -0.681 & -0.387 & -0.236 & -0.094 & 0.176 \\
\hline y0[16] & 0.552 & 0.218 & 0.127 & 0.407 & 0.546 & 0.698 & 0.986 \\
\hline y0[17] & -0.268 & 0.222 & -0.699 & -0.416 & -0.268 & -0.117 & 0.161 \\
\hline y0[18] & 0.950 & 0.219 & 0.521 & 0.801 & 0.952 & 1.096 & 1.374 \\
\hline y0[19] & 0.285 & 0.213 & -0.131 & 0.141 & 0.285 & 0.427 & 0.705 \\
\hline y0[20] & -0.118 & 0.204 & -0.522 & -0.254 & -0.117 & 0.019 & 0.280 \\
\hline y0[21] & -0.132 & 0.201 & -0.522 & -0.267 & -0.132 & 0.001 & 0.265 \\
\hline y0[22] & -0.457 & 0.230 & -0.917 & -0.610 & -0.453 & -0.308 & -0.006 \\
\hline y0[23] & -0.144 & 0.241 & -0.628 & -0.302 & -0.141 & 0.018 & 0.321 \\
\hline y0[24] & -0.163 & 0.262 & -0.696 & -0.330 & -0.156 & 0.009 & 0.348 \\
\hline y0[25] & 0.265 & 0.289 & -0.299 & 0.077 & 0.263 & 0.447 & 0.829 \\
\hline N.sum[1] & $1,197.009$ & 274.911 & 741.132 & $1,003.481$ & $1,170.959$ & $1,363.952$ & $1,804.871$ \\
\hline N.sum[2] & $1,231.886$ & 269.595 & 780.213 & $1,042.951$ & $1,205.798$ & $1,395.593$ & $1,827.096$ \\
\hline N.sum[3] & $1,891.633$ & 356.401 & $1,297.918$ & $1,644.677$ & $1,852.710$ & $2,105.407$ & $2,686.190$ \\
\hline N.sum[4] & $1,771.472$ & 307.560 & $1,245.217$ & $1,550.631$ & $1,744.265$ & $1,962.412$ & $2,448.511$ \\
\hline N.sum[5] & $2,254.721$ & 374.526 & $1,614.057$ & $1,989.156$ & $2,221.550$ & $2,485.878$ & $3,083.420$ \\
\hline N.sum[6] & $3,273.636$ & 562.366 & $2,344.390$ & $2,875.904$ & $3,216.969$ & $3,619.749$ & $4,544.996$ \\
\hline N.sum[7] & $1,772.275$ & 364.164 & $1,144.665$ & $1,511.823$ & $1,738.056$ & $2,003.598$ & $2,565.307$ \\
\hline N.sum[8] & $2,758.971$ & 495.378 & $1,929.914$ & $2,410.591$ & $2,714.165$ & $3,052.992$ & $3,850.440$ \\
\hline N.sum[9] & $2,898.658$ & 461.934 & $2,082.819$ & $2,575.923$ & $2,864.750$ & $3,190.485$ & $3,892.000$ \\
\hline N.sum[10] & $2,042.592$ & 389.441 & $1,390.790$ & $1,762.454$ & $2,004.581$ & $2,286.787$ & $2,896.083$ \\
\hline N.sum[11] & $2,931.180$ & 507.818 & $2,065.443$ & $2,576.800$ & $2,890.204$ & $3,235.619$ & $4,027.338$ \\
\hline N.sum[12] & $4,720.159$ & 713.192 & $3,503.862$ & $4,221.009$ & $4,667.547$ & $5,165.336$ & $6,263.824$ \\
\hline N.sum[13] & $2,635.118$ & 459.664 & $1,848.919$ & $2,313.704$ & $2,598.178$ & $2,917.507$ & $3,625.556$ \\
\hline N.sum[14] & $2,740.033$ & 498.945 & $1,892.840$ & $2,383.953$ & $2,690.043$ & $3,038.000$ & $3,842.514$ \\
\hline N.sum[15] & $3,304.982$ & 580.893 & $2,331.080$ & $2,890.856$ & $3,258.611$ & $3,642.664$ & $4,603.270$ \\
\hline N.sum[16] & $8,097.405$ & $1,244.868$ & $5,955.347$ & $7,223.840$ & 7,989.376 & $8,857.332$ & $10,908.051$ \\
\hline N.sum[17] & $3,929.011$ & 674.406 & $2,789.670$ & $3,446.921$ & $3,863.187$ & $4,347.527$ & $5,434.097$ \\
\hline N.sum[18] & $14,250.479$ & $2,307.297$ & $10,386.909$ & $12,638.479$ & $14,043.733$ & $15,600.550$ & $19,432.121$ \\
\hline N.sum[19] & $7,688.024$ & $1,366.306$ & $5,447.021$ & $6,715.941$ & $7,543.831$ & $8,490.750$ & $10,822.097$ \\
\hline N.sum[20] & $5,257.705$ & 925.118 & $3,678.716$ & $4,612.471$ & $5,161.850$ & $5,819.140$ & $7,287.879$ \\
\hline
\end{tabular}




\begin{tabular}{|c|c|c|c|c|c|c|c|}
\hline N.sum[21] & $5,238.584$ & 849.769 & $3,781.475$ & $4,642.823$ & $5,168.831$ & $5,764.664$ & $7,083.582$ \\
\hline N.sum[22] & $3,862.034$ & 736.273 & $2,627.132$ & $3,347.605$ & $3,794.498$ & $4,296.146$ & $5,514.797$ \\
\hline N.sum[23] & $5,444.255$ & $1,063.009$ & $3,683.757$ & $4,694.112$ & $5,339.878$ & $6,081.933$ & $7,834.042$ \\
\hline N.sum[24] & $5,597.682$ & $1,073.219$ & $3,844.972$ & $4,829.256$ & $5,487.802$ & $6,229.470$ & $7,986.722$ \\
\hline N.sum[25] &, 125.532 & $1,603.690$ & $6,443.447$ & 7,979.203 & $8,977.600$ & $10,088.507$ & $12,724.318$ \\
\hline N.sum2[1] & $1,536.896$ & 437.815 & 849.026 & $1,224.035$ & $1,480.104$ & $1,791.730$ & $2,536.424$ \\
\hline N.sum2[2] & $1,653.529$ & 378.752 & $1,041.094$ & $1,387.512$ & $1,605.662$ & $1,874.674$ & $2,507.067$ \\
\hline N.sum2[3] & $1,809.340$ & 349.309 & $1,228.028$ & $1,562.146$ & $1,773.589$ & $2,014.792$ & $2,585.770$ \\
\hline N.sum2[4] & $2,002.678$ & 349.863 & $1,411.379$ & $1,757.122$ & $1,967.850$ & $2,207.555$ & $2,773.985$ \\
\hline N.sum2[5] & $2,222.277$ & 364.857 & $1,602.569$ & $1,969.988$ & $2,189.910$ & $2,437.160$ & $3,053.268$ \\
\hline N.sum2[6] & $2,447.873$ & 381.435 & $1,800.808$ & $2,184.525$ & $2,414.488$ & $2,672.372$ & $3,304.500$ \\
\hline N.sum2[7] & $2,653.317$ & 401.253 & $1,961.117$ & $2,377.396$ & $2,613.235$ & $2,888.687$ & $3,548.803$ \\
\hline N.sum2[8] & $2,817.869$ & 426.703 & $2,076.551$ & $2,525.065$ & $2,779.382$ & $3,068.369$ & $3,775.047$ \\
\hline N.sum2[9] & $2,948.828$ & 452.877 & $2,164.492$ & $2,640.334$ & $2,903.194$ & $3,224.426$ & $3,957.167$ \\
\hline N.sum2[10] & $3,073.258$ & 478.800 & $2,235.944$ & $2,742.708$ & $3,028.389$ & $3,364.752$ & $4,123.671$ \\
\hline N.sum2[11] & $3,226.487$ & 511.071 & $2,313.748$ & $2,879.666$ & $3,183.620$ & $3,537.775$ & $4,345.841$ \\
\hline N.sum2[12] & $3,440.734$ & 551.696 & $2,467.575$ & $3,061.152$ & $3,394.848$ & $3,775.119$ & $4,664.272$ \\
\hline N.sum2[13] & $3,737.857$ & 598.555 & $2,724.995$ & $3,312.959$ & $3,676.507$ & $4,101.625$ & $5,053.504$ \\
\hline N.sum2[14] & $4,127.745$ & 660.447 & $3,041.800$ & $3,660.384$ & $4,058.873$ & $4,515.068$ & $5,608.913$ \\
\hline N.sum2[15] & $4,602.401$ & 762.040 & $3,378.375$ & $4,063.486$ & $4,528.402$ & $5,035.900$ & $6,376.874$ \\
\hline N.sum2[16] & $5,128.247$ & 909.942 & $3,682.030$ & $4,489.267$ & $5,009.820$ & $5,626.116$ & $7,305.010$ \\
\hline N.sum2[17] & $5,639.115$ & $1,049.534$ & $4,007.424$ & $4,907.280$ & $5,489.094$ & $6,209.403$ & $8,193.987$ \\
\hline N.sum2[18] & $6,054.952$ & $1,099.488$ & $4,337.287$ & $5,280.857$ & $5,910.966$ & $6,662.090$ & $8,679.132$ \\
\hline N.sum2[19] & $6,324.257$ & $1,044.365$ & $4,636.470$ & $5,602.052$ & $6,190.788$ & $6,923.208$ & $8,792.588$ \\
\hline N.sum2[20] & $6,458.213$ & 974.062 & $4,850.582$ & $5,768.688$ & $6,342.265$ & $7,033.458$ & $8,663.293$ \\
\hline N.sum2[21] & $6,538.938$ & $1,003.521$ & $4,878.268$ & $5,832.828$ & $6,437.031$ & $7,121.674$ & $8,771.662$ \\
\hline N.sum2[22] & $6,660.996$ & $1,159.890$ & $4,769.209$ & $5,867.763$ & $6,532.382$ & $7,308.253$ & $9,215.499$ \\
\hline N.sum2[23] & $6,899.975$ & $1,437.266$ & $4,677.139$ & $5,923.687$ & $6,705.085$ & $7,673.332$ & $10,155.917$ \\
\hline N.sum2[24] & $7,308.553$ & $1,870.751$ & $4,521.141$ & $6,034.876$ & 7,019.499 & $8,259.267$ & $11,587.113$ \\
\hline N.sum2[25] & 7,916.612 & $2,512.635$ & $4,265.219$ & $6,201.705$ & $7,487.916$ & $9,181.813$ & $13,827.668$ \\
\hline R.growth[1] & 0.087 & 0.086 & -0.084 & 0.033 & 0.085 & 0.139 & 0.273 \\
\hline R.growth[2] & 0.097 & 0.077 & -0.050 & 0.047 & 0.094 & 0.146 & 0.264 \\
\hline R.growth[3] & 0.105 & 0.063 & -0.013 & 0.060 & 0.103 & 0.146 & 0.236 \\
\hline R.growth[4] & 0.106 & 0.052 & 0.009 & 0.069 & 0.105 & 0.140 & 0.210 \\
\hline R.growth[5] & 0.098 & 0.050 & 0.009 & 0.063 & 0.095 & 0.131 & 0.200 \\
\hline R.growth[6] & 0.081 & 0.051 & -0.012 & 0.047 & 0.078 & 0.112 & 0.192 \\
\hline R.growth[7] & 0.060 & 0.050 & -0.041 & 0.029 & 0.059 & 0.090 & 0.163 \\
\hline R.growth[8] & 0.045 & 0.048 & -0.059 & 0.017 & 0.046 & 0.077 & 0.137 \\
\hline R.growth[9] & 0.041 & 0.049 & -0.068 & 0.013 & 0.044 & 0.073 & 0.132 \\
\hline R.growth[10] & 0.048 & 0.050 & -0.061 & 0.019 & 0.049 & 0.079 & 0.150 \\
\hline R.growth[11] & 0.064 & 0.049 & -0.033 & 0.035 & 0.062 & 0.093 & 0.171 \\
\hline R.growth[12] & 0.083 & 0.049 & -0.007 & 0.050 & 0.079 & 0.110 & 0.198 \\
\hline R.growth[13] & 0.099 & 0.055 & 0.008 & 0.062 & 0.091 & 0.130 & 0.227 \\
\hline R.growth[14] & 0.108 & 0.060 & 0.012 & 0.067 & 0.099 & 0.141 & 0.248 \\
\hline
\end{tabular}




\begin{tabular}{lrrrrrrr} 
R.growth[15] & 0.106 & 0.057 & 0.010 & 0.068 & 0.098 & 0.138 & 0.242 \\
R.growth[16] & 0.094 & 0.048 & 0.005 & 0.063 & 0.091 & 0.121 & 0.204 \\
R.growth[17] & 0.072 & 0.044 & -0.023 & 0.046 & 0.073 & 0.099 & 0.160 \\
R.growth[18] & 0.046 & 0.052 & -0.069 & 0.014 & 0.049 & 0.082 & 0.140 \\
R.growth[19] & 0.023 & 0.062 & -0.117 & -0.015 & 0.029 & 0.067 & 0.129 \\
R.growth[20] & 0.012 & 0.066 & -0.128 & -0.029 & 0.018 & 0.058 & 0.126 \\
R.growth[21] & 0.015 & 0.065 & -0.118 & -0.027 & 0.018 & 0.060 & 0.134 \\
R.growth[22] & 0.029 & 0.068 & -0.115 & -0.014 & 0.031 & 0.077 & 0.153 \\
R.growth[23] & 0.048 & 0.076 & -0.111 & -0.003 & 0.049 & 0.103 & 0.184 \\
R.growth[24] & 0.064 & 0.083 & -0.107 & 0.008 & 0.066 & 0.123 & 0.217 \\
Deviance & $14,949.248$ & 28.521 & $14,891.212$ & $14,929.886$ & $14,950.263$ & $14,970.318$ & $14,999.424$ \\
\hline
\end{tabular}


Tables A4.9. Bayesian generalized linear mixed model output for Tundra Swan observed during aerial surveys 1992-2016 on the Arctic Coastal Plain, Alaska. We provide summaries including mean, SE, and several quantiles of the posterior distribution for model and derived parameters. See Table A4.4 for a key to parameter names.

\begin{tabular}{|c|c|c|c|c|c|c|c|}
\hline Parameter & Mean & SD & $2.50 \%$ & $25 \%$ & $50 \%$ & $75 \%$ & $97.50 \%$ \\
\hline MSE & 1.233 & 0.025 & 1.187 & 1.216 & 1.233 & 1.250 & 1.282 \\
\hline MSE.CV & 2.568 & 0.051 & 2.471 & 2.532 & 2.568 & 2.603 & 2.670 \\
\hline SSE & $29,075.0$ & 582.0 & $27,977.9$ & $28,666.7$ & $29,070.5$ & $29,464.3$ & $30,226.2$ \\
\hline Trend & 1.028 & 0.009 & 1.010 & 1.023 & 1.029 & 1.034 & 1.045 \\
\hline R.trend & 0.028 & 0.009 & 0.010 & 0.023 & 0.028 & 0.034 & 0.044 \\
\hline M.dens & 0.215 & 0.005 & 0.205 & 0.211 & 0.214 & 0.218 & 0.227 \\
\hline М.y0 & -0.005 & 0.045 & -0.094 & -0.038 & -0.005 & 0.026 & 0.081 \\
\hline M.yz & 0.126 & 0.049 & 0.028 & 0.093 & 0.126 & 0.158 & 0.221 \\
\hline M.blyr & -0.888 & 0.051 & -0.989 & -0.923 & -0.889 & -0.854 & -0.784 \\
\hline M.cell & 0.000 & 0.007 & -0.013 & -0.005 & 0.000 & 0.004 & 0.013 \\
\hline M.psi & 0.546 & 0.006 & 0.533 & 0.541 & 0.546 & 0.550 & 0.558 \\
\hline R.mid & 7.501 & 1.166 & 5.818 & 6.662 & 7.297 & 8.127 & 10.373 \\
\hline b.ADOY & 0.070 & 0.021 & 0.028 & 0.057 & 0.070 & 0.085 & 0.110 \\
\hline b.ADOY.2 & 0.003 & 0.013 & -0.023 & -0.006 & 0.003 & 0.012 & 0.029 \\
\hline z.ADOY & 0.143 & 0.047 & 0.052 & 0.111 & 0.142 & 0.175 & 0.238 \\
\hline z.ADOY.2 & 0.026 & 0.022 & -0.017 & 0.011 & 0.027 & 0.040 & 0.066 \\
\hline SD.surv & 0.112 & 0.054 & 0.015 & 0.075 & 0.109 & 0.145 & 0.233 \\
\hline SD.surv.z & 0.485 & 0.139 & 0.260 & 0.388 & 0.471 & 0.565 & 0.805 \\
\hline SD.y0 & 0.241 & 0.043 & 0.171 & 0.210 & 0.236 & 0.265 & 0.337 \\
\hline SD.yz & 0.771 & 0.139 & 0.546 & 0.673 & 0.756 & 0.851 & 1.086 \\
\hline SD.cell & 0.252 & 0.019 & 0.216 & 0.240 & 0.252 & 0.265 & 0.289 \\
\hline SD.cell.z & 0.615 & 0.034 & 0.551 & 0.592 & 0.614 & 0.638 & 0.681 \\
\hline SD.lam[1] & 0.252 & 0.057 & 0.158 & 0.210 & 0.246 & 0.288 & 0.380 \\
\hline SD.lam[2] & 1.220 & 0.468 & 0.638 & 0.906 & 1.119 & 1.412 & 2.390 \\
\hline SD.lam[3] & 0.517 & 0.660 & 0.018 & 0.111 & 0.292 & 0.678 & 2.249 \\
\hline SD.lam[4] & 0.422 & 0.439 & 0.009 & 0.123 & 0.278 & 0.572 & 1.634 \\
\hline SD.lam[5] & 4.947 & 5.187 & 0.070 & 0.924 & 2.857 & 7.549 & 18.359 \\
\hline SD.lam[6] & 1.712 & 0.638 & 0.879 & 1.215 & 1.546 & 2.095 & 3.185 \\
\hline SD.lam[7] & 0.683 & 0.273 & 0.348 & 0.491 & 0.611 & 0.816 & 1.389 \\
\hline SD.lam[8] & 0.183 & 0.281 & 0.006 & 0.042 & 0.089 & 0.199 & 0.981 \\
\hline S.eff[1] & -0.054 & 0.085 & -0.237 & -0.106 & -0.046 & 0.003 & 0.100 \\
\hline S.eff[2] & 0.047 & 0.092 & -0.118 & -0.012 & 0.036 & 0.104 & 0.249 \\
\hline S.eff[3] & 0.077 & 0.097 & -0.083 & 0.007 & 0.063 & 0.137 & 0.293 \\
\hline S.eff[4] & -0.038 & 0.084 & -0.221 & -0.089 & -0.030 & 0.015 & 0.121 \\
\hline S.eff[5] & -0.001 & 0.086 & -0.177 & -0.052 & -0.001 & 0.050 & 0.183 \\
\hline S.eff[6] & -0.030 & 0.087 & -0.210 & -0.081 & -0.024 & 0.023 & 0.135 \\
\hline S.eff[7] & 0.166 & 0.117 & -0.012 & 0.074 & 0.157 & 0.245 & 0.410 \\
\hline S.eff[8] & 0.077 & 0.099 & -0.088 & 0.007 & 0.063 & 0.136 & 0.299 \\
\hline S.eff[9] & 0.063 & 0.087 & -0.085 & 0.002 & 0.052 & 0.116 & 0.256 \\
\hline
\end{tabular}




\begin{tabular}{|c|c|c|c|c|c|c|c|}
\hline S.eff[10] & 0.013 & 0.082 & -0.146 & -0.036 & 0.010 & 0.061 & 0.186 \\
\hline S.eff[11] & -0.029 & 0.085 & -0.211 & -0.080 & -0.022 & 0.023 & 0.130 \\
\hline S.eff[12] & 0.013 & 0.085 & -0.159 & -0.037 & 0.009 & 0.062 & 0.190 \\
\hline S.eff[13] & -0.070 & 0.094 & -0.280 & -0.127 & -0.058 & -0.004 & 0.092 \\
\hline S.eff[14] & 0.003 & 0.085 & -0.167 & -0.047 & 0.002 & 0.052 & 0.182 \\
\hline S.eff[15] & -0.079 & 0.090 & -0.285 & -0.133 & -0.068 & -0.014 & 0.074 \\
\hline S.eff.z[1] & 0.076 & 0.188 & -0.286 & -0.053 & 0.077 & 0.205 & 0.454 \\
\hline S.eff.z[2] & 0.020 & 0.200 & -0.375 & -0.112 & 0.022 & 0.154 & 0.408 \\
\hline S.eff.z[3] & -0.140 & 0.201 & -0.535 & -0.274 & -0.137 & -0.005 & 0.245 \\
\hline S.eff.z[4] & -0.515 & 0.227 & -0.982 & -0.664 & -0.511 & -0.360 & -0.083 \\
\hline S.eff.z[5] & -0.423 & 0.211 & -0.850 & -0.565 & -0.418 & -0.279 & -0.018 \\
\hline S.eff.z[6] & -0.872 & 0.232 & -1.315 & -1.032 & -0.873 & -0.714 & -0.423 \\
\hline S.eff.z[7] & -0.312 & 0.216 & -0.738 & -0.458 & -0.306 & -0.161 & 0.094 \\
\hline S.eff.z[8] & -0.932 & 0.269 & -1.463 & -1.105 & -0.928 & -0.750 & -0.413 \\
\hline S.eff.z[9] & -0.250 & 0.197 & -0.644 & -0.382 & -0.245 & -0.114 & 0.121 \\
\hline S.eff.z[10] & -0.328 & 0.200 & -0.730 & -0.461 & -0.325 & -0.195 & 0.056 \\
\hline S.eff.z[11] & 0.065 & 0.194 & -0.313 & -0.064 & 0.067 & 0.198 & 0.443 \\
\hline S.eff.z[12] & -0.118 & 0.203 & -0.522 & -0.255 & -0.115 & 0.019 & 0.275 \\
\hline S.eff.z[13] & -0.059 & 0.218 & -0.494 & -0.203 & -0.054 & 0.092 & 0.354 \\
\hline S.eff.z[14] & -0.078 & 0.201 & -0.482 & -0.213 & -0.075 & 0.058 & 0.311 \\
\hline S.eff.z[15] & -0.101 & 0.200 & -0.506 & -0.231 & -0.096 & 0.038 & 0.271 \\
\hline $\mathrm{y} 0[1]$ & 0.149 & 0.137 & -0.122 & 0.059 & 0.149 & 0.239 & 0.416 \\
\hline $\mathrm{y} 0[2]$ & 0.029 & 0.128 & -0.215 & -0.055 & 0.024 & 0.112 & 0.294 \\
\hline $\mathrm{y} 0[3]$ & -0.071 & 0.116 & -0.294 & -0.151 & -0.070 & 0.006 & 0.160 \\
\hline $\mathrm{y} 0[4]$ & -0.026 & 0.107 & -0.233 & -0.097 & -0.028 & 0.046 & 0.185 \\
\hline $\mathrm{y} 0[5]$ & -0.031 & 0.097 & -0.220 & -0.097 & -0.032 & 0.036 & 0.159 \\
\hline $\mathrm{y} 0[6]$ & -0.097 & 0.102 & -0.294 & -0.168 & -0.097 & -0.027 & 0.106 \\
\hline $\mathrm{y} 0[7]$ & -0.110 & 0.101 & -0.313 & -0.179 & -0.108 & -0.040 & 0.085 \\
\hline $\mathrm{y} 0[8]$ & -0.069 & 0.101 & -0.269 & -0.137 & -0.067 & -0.003 & 0.127 \\
\hline $\mathrm{y} 0[9]$ & 0.196 & 0.095 & 0.008 & 0.135 & 0.197 & 0.258 & 0.381 \\
\hline y0[10] & 0.044 & 0.095 & -0.140 & -0.019 & 0.044 & 0.108 & 0.230 \\
\hline y0[11] & 0.004 & 0.095 & -0.179 & -0.058 & 0.004 & 0.066 & 0.188 \\
\hline $\mathrm{y} 0[12]$ & -0.017 & 0.098 & -0.209 & -0.083 & -0.018 & 0.051 & 0.170 \\
\hline y0[13] & -0.122 & 0.097 & -0.316 & -0.187 & -0.120 & -0.057 & 0.067 \\
\hline y0[14] & -0.053 & 0.094 & -0.238 & -0.115 & -0.053 & 0.010 & 0.127 \\
\hline y0[15] & 0.048 & 0.095 & -0.146 & -0.014 & 0.048 & 0.110 & 0.236 \\
\hline y0[16] & 0.092 & 0.093 & -0.094 & 0.030 & 0.094 & 0.155 & 0.274 \\
\hline y0[17] & 0.035 & 0.092 & -0.147 & -0.025 & 0.036 & 0.095 & 0.212 \\
\hline y0[18] & 0.135 & 0.094 & -0.052 & 0.072 & 0.137 & 0.198 & 0.317 \\
\hline y0[19] & 0.275 & 0.091 & 0.101 & 0.213 & 0.273 & 0.333 & 0.462 \\
\hline y0[20] & -0.716 & 0.109 & -0.933 & -0.790 & -0.714 & -0.642 & -0.508 \\
\hline y0[21] & 0.101 & 0.097 & -0.090 & 0.036 & 0.101 & 0.163 & 0.299 \\
\hline y0[22] & 0.282 & 0.098 & 0.096 & 0.217 & 0.281 & 0.346 & 0.481 \\
\hline $\mathrm{y} 0[23]$ & 0.062 & 0.109 & -0.151 & -0.012 & 0.061 & 0.134 & 0.278 \\
\hline
\end{tabular}




\begin{tabular}{|c|c|c|c|c|c|c|c|}
\hline $\mathrm{y} 0[24]$ & 0.157 & 0.117 & -0.063 & 0.079 & 0.152 & 0.233 & 0.401 \\
\hline $\mathrm{y} 0[25]$ & -0.434 & 0.139 & -0.700 & -0.529 & -0.437 & -0.342 & -0.153 \\
\hline$y z[1]$ & 0.719 & 0.137 & 0.448 & 0.629 & 0.720 & 0.812 & 0.987 \\
\hline$y z[2]$ & 1.000 & 0.155 & 0.700 & 0.897 & 0.996 & 1.105 & 1.305 \\
\hline$y z[3]$ & 0.973 & 0.160 & 0.658 & 0.867 & 0.973 & 1.081 & 1.283 \\
\hline$y z[4]$ & 0.694 & 0.150 & 0.389 & 0.599 & 0.697 & 0.796 & 0.980 \\
\hline$y z[5]$ & 0.720 & 0.150 & 0.418 & 0.622 & 0.721 & 0.820 & 1.008 \\
\hline$y z[6]$ & 1.000 & 0.141 & 0.716 & 0.904 & 1.005 & 1.095 & 1.269 \\
\hline$y z[7]$ & 0.462 & 0.159 & 0.149 & 0.353 & 0.465 & 0.570 & 0.775 \\
\hline$y z[8]$ & 0.782 & 0.160 & 0.459 & 0.677 & 0.788 & 0.892 & 1.088 \\
\hline$y z[9]$ & 0.530 & 0.139 & 0.257 & 0.436 & 0.532 & 0.625 & 0.801 \\
\hline yz[10] & 0.487 & 0.143 & 0.202 & 0.391 & 0.488 & 0.583 & 0.767 \\
\hline$y z[11]$ & 0.376 & 0.150 & 0.081 & 0.277 & 0.378 & 0.480 & 0.663 \\
\hline yz[12] & 0.455 & 0.150 & 0.152 & 0.356 & 0.457 & 0.561 & 0.745 \\
\hline$y z[13]$ & 0.141 & 0.157 & -0.173 & 0.037 & 0.145 & 0.249 & 0.439 \\
\hline yz[14] & 0.223 & 0.147 & -0.075 & 0.124 & 0.228 & 0.326 & 0.492 \\
\hline$y z[15]$ & 0.223 & 0.146 & -0.070 & 0.129 & 0.223 & 0.322 & 0.499 \\
\hline yz[16] & -0.392 & 0.149 & -0.696 & -0.491 & -0.388 & -0.290 & -0.108 \\
\hline yz[17] & -0.415 & 0.154 & -0.735 & -0.515 & -0.410 & -0.310 & -0.127 \\
\hline yz[18] & -0.148 & 0.143 & -0.446 & -0.242 & -0.143 & -0.051 & 0.121 \\
\hline yz[19] & 0.012 & 0.124 & -0.241 & -0.069 & 0.015 & 0.095 & 0.252 \\
\hline$y z[20]$ & -1.591 & 0.384 & -2.456 & -1.813 & -1.554 & -1.321 & -0.964 \\
\hline$y z[21]$ & -0.193 & 0.145 & -0.486 & -0.290 & -0.192 & -0.092 & 0.081 \\
\hline$y z[22]$ & -0.303 & 0.137 & -0.580 & -0.393 & -0.298 & -0.213 & -0.046 \\
\hline$y z[23]$ & -0.175 & 0.151 & -0.486 & -0.273 & -0.169 & -0.073 & 0.109 \\
\hline$y z[24]$ & -0.732 & 0.166 & -1.076 & -0.841 & -0.727 & -0.617 & -0.422 \\
\hline$y z[25]$ & -1.707 & 0.392 & -2.572 & -1.943 & -1.675 & -1.430 & -1.029 \\
\hline N.sum[1] & $9,062.702$ & 802.689 & $7,594.652$ & $8,498.810$ & $9,035.348$ & $9,599.416$ & $10,693.938$ \\
\hline N.sum[2] & $6,619.313$ & 672.635 & $5,417.359$ & $6,152.321$ & $6,581.970$ & 7,048.910 & $8,071.699$ \\
\hline N.sum[3] & $5,993.213$ & 581.757 & $4,896.278$ & $5,590.561$ & $5,987.802$ & $6,372.754$ & $7,177.347$ \\
\hline N.sum[4] & $7,344.975$ & 600.027 & $6,233.188$ & $6,924.638$ & $7,331.941$ & $7,745.211$ & $8,561.062$ \\
\hline N.sum[5] & $7,100.264$ & 578.593 & $6,012.004$ & $6,702.328$ & $7,085.432$ & $7,480.047$ & $8,296.474$ \\
\hline N.sum[6] & $5,514.306$ & 477.631 & $4,596.908$ & $5,187.596$ & $5,503.634$ & $5,826.762$ & $6,490.367$ \\
\hline N.sum[7] & $7,451.536$ & 662.138 & $6,234.664$ & $6,990.713$ & $7,417.958$ & $7,881.533$ & $8,818.825$ \\
\hline N.sum[8] & $6,393.619$ & 571.429 & $5,332.103$ & $6,002.104$ & $6,373.154$ & $6,759.984$ & $7,571.904$ \\
\hline N.sum[9] & $9,615.439$ & 756.035 & $8,184.736$ & $9,089.789$ & $9,603.355$ & $10,119.718$ & $11,120.239$ \\
\hline N.sum[10] & $8,422.652$ & 647.582 & $7,202.416$ & $7,978.637$ & $8,406.846$ & $8,850.694$ & $9,735.005$ \\
\hline N.sum[11] & $8,562.961$ & 655.400 & $7,331.332$ & $8,102.101$ & $8,540.264$ & $8,998.288$ & $9,911.866$ \\
\hline N.sum[12] & $8,005.924$ & 635.957 & $6,808.144$ & $7,580.967$ & $7,984.272$ & $8,409.293$ & $9,344.120$ \\
\hline N.sum[13] & $8,439.724$ & 596.967 & $7,319.112$ & $8,025.480$ & $8,426.925$ & $8,845.923$ & $9,629.686$ \\
\hline N.sum[14] & $8,652.319$ & 602.535 & $7,521.641$ & $8,243.010$ & $8,634.988$ & $9,042.935$ & $9,915.882$ \\
\hline N.sum[15] & $9,522.701$ & 677.751 & $8,234.200$ & $9,059.988$ & $9,499.364$ & $9,977.150$ & $10,873.309$ \\
\hline N.sum[16] & $12,928.169$ & 738.555 & $11,548.648$ & $12,408.368$ & $12,904.317$ & $13,420.114$ & $14,440.748$ \\
\hline N.sum[17] & $12,231.403$ & 679.116 & $10,940.503$ & $11,767.789$ & $12,225.151$ & $12,674.640$ & $13,591.044$ \\
\hline
\end{tabular}




\begin{tabular}{|c|c|c|c|c|c|c|c|}
\hline N.sum[18] & $12,076.309$ & 711.798 & $10,742.928$ & $11,589.680$ & $12,062.096$ & $12,541.902$ & $13,508.523$ \\
\hline N.sum[19] & $12,849.562$ & 765.637 & $11,377.403$ & $12,330.351$ & $12,833.016$ & $13,349.869$ & $14,413.190$ \\
\hline N.sum[20] & $7,646.627$ & 474.269 & $6,720.970$ & $7,329.369$ & $7,633.697$ & $7,967.750$ & $8,594.022$ \\
\hline N.sum[21] & $11,594.939$ & 674.213 & $10,287.035$ & $11,135.708$ & $11,579.005$ & $12,045.925$ & $12,942.677$ \\
\hline N.sum[22] & $14,390.029$ & 796.353 & $12,842.745$ & $13,860.079$ & $14,377.573$ & $14,918.289$ & $15,974.611$ \\
\hline N.sum[23] & $10,853.432$ & 650.290 & $9,588.322$ & $10,417.711$ & $10,841.753$ & $11,292.418$ & $12,142.897$ \\
\hline N.sum[24] & $14,460.125$ & 773.942 & $12,971.681$ & $13,934.475$ & $14,447.170$ & $14,962.781$ & $16,030.909$ \\
\hline N.sum[25] & $9,848.960$ & 592.451 & $8,742.283$ & $9,449.698$ & $9,839.051$ & $10,233.325$ & $11,066.174$ \\
\hline N.sum2[1] & $8,052.840$ & $1,184.225$ & $6,054.463$ & $7,240.225$ & $7,945.616$ & $8,750.770$ & $10,718.029$ \\
\hline N.sum2[2] & $6,616.219$ & 903.028 & $5,033.983$ & $5,978.470$ & $6,570.629$ & $7,168.767$ & $8,579.679$ \\
\hline N.sum2[3] & $6,614.334$ & 833.169 & $5,157.279$ & $6,022.585$ & $6,564.946$ & $7,137.587$ & $8,367.118$ \\
\hline N.sum2[4] & $7,747.361$ & 880.947 & $6,174.100$ & $7,124.434$ & $7,700.532$ & $8,303.011$ & $9,623.462$ \\
\hline N.sum2[5] & $7,522.012$ & 782.523 & $6,083.278$ & $6,991.089$ & $7,494.917$ & $8,022.549$ & $9,165.009$ \\
\hline N.sum2[6] & $6,236.477$ & 656.942 & $5,056.088$ & $5,766.614$ & $6,209.883$ & $6,650.460$ & $7,621.955$ \\
\hline N.sum2[7] & $8,533.615$ & 834.004 & $7,006.962$ & $7,944.179$ & $8,495.851$ & $9,076.800$ & $10,250.841$ \\
\hline N.sum2[8] & $7,033.834$ & 748.908 & $5,639.090$ & $6,525.130$ & $6,999.076$ & $7,500.029$ & $8,622.069$ \\
\hline N.sum2[9] & $8,104.482$ & 730.181 & $6,742.244$ & $7,605.504$ & $8,099.542$ & $8,579.843$ & $9,580.586$ \\
\hline N.sum2[10] & $8,266.569$ & 766.943 & $6,837.261$ & $7,741.545$ & $8,244.414$ & $8,773.096$ & $9,812.630$ \\
\hline N.sum2[11] & $8,747.616$ & 805.456 & $7,243.454$ & $8,187.462$ & $8,717.238$ & $9,260.197$ & $10,418.652$ \\
\hline N.sum2[12] & $8,356.008$ & 778.982 & $6,927.086$ & $7,829.945$ & $8,324.107$ & $8,850.204$ & $9,980.049$ \\
\hline N.sum2[13] & $9,785.861$ & 870.486 & $8,173.093$ & $9,180.931$ & $9,750.410$ & $10,341.078$ & $11,604.454$ \\
\hline N.sum2[14] & $9,361.813$ & 818.455 & $7,883.853$ & $8,793.832$ & $9,318.119$ & $9,880.964$ & $11,077.898$ \\
\hline N.sum2[15] & $9,314.560$ & 824.040 & $7,830.489$ & $8,752.031$ & $9,262.955$ & $9,814.540$ & $11,105.709$ \\
\hline N.sum2[16] & $12,095.581$ & 933.366 & $10,463.474$ & $11,452.337$ & $12,015.958$ & $12,669.378$ & $14,103.910$ \\
\hline N.sum2[17] & $12,116.275$ & 921.407 & $10,465.534$ & $11,471.230$ & $12,049.926$ & $12,697.827$ & $14,093.767$ \\
\hline N.sum2[18] & $10,827.537$ & 880.553 & $9,259.495$ & $10,208.536$ & $10,770.567$ & $11,385.586$ & $12,722.934$ \\
\hline N.sum2[19] & $10,015.446$ & 800.744 & $8,569.848$ & $9,474.450$ & $9,969.905$ & $10,512.199$ & $11,750.915$ \\
\hline N.sum2[20] & $16,060.895$ & $1,416.504$ & $13,497.639$ & $15,071.280$ & $15,982.369$ & $16,959.684$ & $19,019.682$ \\
\hline N.sum2[21] & $10,756.208$ & 906.835 & $9,100.400$ & $10,123.257$ & $10,718.215$ & $11,332.511$ & $12,619.997$ \\
\hline N.sum2[22] & $11,143.161$ & 951.905 & $9,380.877$ & $10,493.776$ & $11,092.491$ & $11,751.749$ & $13,127.686$ \\
\hline N.sum2[23] & $10,486.650$ & $1,032.031$ & $8,643.551$ & $9,756.890$ & $10,427.419$ & $11,152.225$ & $12,646.466$ \\
\hline N.sum2[24] & $12,712.918$ & $1,329.236$ & $10,175.855$ & $11,844.841$ & $12,674.053$ & $13,539.768$ & $15,452.994$ \\
\hline N.sum2[25] & $15,670.239$ & $1,942.444$ & $12,077.055$ & $14,336.264$ & $15,611.182$ & $16,861.274$ & $19,754.398$ \\
\hline R.growth[1] & -0.195 & 0.133 & -0.457 & -0.286 & -0.193 & -0.105 & 0.059 \\
\hline R.growth[2] & 0.001 & 0.127 & -0.246 & -0.085 & 0.002 & 0.089 & 0.244 \\
\hline R.growth[3] & 0.160 & 0.121 & -0.079 & 0.078 & 0.158 & 0.244 & 0.396 \\
\hline R.growth[4] & -0.029 & 0.116 & -0.260 & -0.104 & -0.029 & 0.047 & 0.200 \\
\hline R.growth[5] & -0.188 & 0.122 & -0.424 & -0.270 & -0.188 & -0.107 & 0.062 \\
\hline R.growth[6] & 0.314 & 0.117 & 0.082 & 0.238 & 0.316 & 0.395 & 0.539 \\
\hline R.growth[7] & -0.194 & 0.116 & -0.431 & -0.271 & -0.193 & -0.113 & 0.027 \\
\hline R.growth[8] & 0.143 & 0.117 & -0.085 & 0.065 & 0.142 & 0.220 & 0.376 \\
\hline R.growth[9] & 0.020 & 0.107 & -0.192 & -0.052 & 0.020 & 0.091 & 0.233 \\
\hline R.growth[10] & 0.057 & 0.109 & -0.157 & -0.017 & 0.057 & 0.130 & 0.278 \\
\hline R.growth[11] & -0.046 & 0.113 & -0.268 & -0.122 & -0.044 & 0.031 & 0.170 \\
\hline
\end{tabular}




\begin{tabular}{lrrrrrrr} 
R.growth[12] & 0.158 & 0.113 & -0.066 & 0.083 & 0.160 & 0.235 & 0.380 \\
R.growth[13] & -0.044 & 0.105 & -0.250 & -0.114 & -0.044 & 0.025 & 0.161 \\
R.growth[14] & -0.005 & 0.101 & -0.206 & -0.072 & -0.005 & 0.063 & 0.190 \\
R.growth[15] & 0.262 & 0.090 & 0.087 & 0.201 & 0.261 & 0.323 & 0.440 \\
R.growth[16] & 0.002 & 0.079 & -0.151 & -0.053 & 0.001 & 0.056 & 0.158 \\
R.growth[17] & -0.113 & 0.083 & -0.272 & -0.170 & -0.114 & -0.055 & 0.052 \\
R.growth[18] & -0.078 & 0.083 & -0.239 & -0.135 & -0.078 & -0.023 & 0.090 \\
R.growth[19] & 0.472 & 0.086 & 0.302 & 0.413 & 0.472 & 0.530 & 0.638 \\
R.growth[20] & -0.401 & 0.087 & -0.569 & -0.459 & -0.400 & -0.342 & -0.230 \\
R.growth[21] & 0.035 & 0.083 & -0.129 & -0.020 & 0.035 & 0.091 & 0.199 \\
R.growth[22] & -0.062 & 0.082 & -0.222 & -0.117 & -0.063 & -0.008 & 0.101 \\
R.growth[23] & 0.192 & 0.085 & 0.024 & 0.137 & 0.193 & 0.249 & 0.356 \\
R.growth[24] & 0.207 & 0.080 & 0.050 & 0.153 & 0.208 & 0.262 & 0.362 \\
Deviance & $27,380.102$ & 218.923 & $26,963.061$ & $27,232.155$ & $27,374.916$ & $27,523.178$ & $27,818.065$ \\
\hline
\end{tabular}


Tables A4.10. Bayesian generalized linear mixed model output for Northern Pintail observed during aerial surveys 1992-2016 on the Arctic Coastal Plain, Alaska. We provide summaries including mean, SE, and several quantiles of the posterior distribution for model and derived parameters. See Table A4.4 for a key to parameter names.

\begin{tabular}{|c|c|c|c|c|c|c|c|}
\hline Parameter & Mean & SD & $2.50 \%$ & $25 \%$ & $50 \%$ & $75 \%$ & $97.50 \%$ \\
\hline MSE & 20.785 & 0.303 & 20.214 & 20.576 & 20.772 & 20.985 & 21.411 \\
\hline MSE.CV & 9.790 & 0.143 & 9.521 & 9.692 & 9.784 & 9.885 & 10.085 \\
\hline SSE & $537,606.2$ & $7,827.4$ & $522,839.5$ & $532,186.6$ & $537,266.7$ & $542,784.5$ & $553,807.0$ \\
\hline Trend & 1.001 & 0.008 & 0.986 & 0.996 & 1.001 & 1.006 & 1.020 \\
\hline R.trend & 0.001 & 0.008 & -0.014 & -0.004 & 0.001 & 0.006 & 0.020 \\
\hline M.dens & 0.947 & 0.020 & 0.910 & 0.933 & 0.946 & 0.959 & 0.989 \\
\hline M.y0 & -0.005 & 0.043 & -0.093 & -0.033 & -0.006 & 0.023 & 0.078 \\
\hline M.yz & -0.014 & 0.026 & -0.066 & -0.031 & -0.014 & 0.004 & 0.038 \\
\hline M.blyr & 0.482 & 0.047 & 0.389 & 0.451 & 0.483 & 0.513 & 0.578 \\
\hline M.cell & 0.000 & 0.007 & -0.015 & -0.005 & 0.000 & 0.005 & 0.014 \\
\hline M.psi & 0.526 & 0.004 & 0.519 & 0.524 & 0.526 & 0.529 & 0.533 \\
\hline R.mid & 1.253 & 0.030 & 1.195 & 1.232 & 1.253 & 1.273 & 1.310 \\
\hline b.ADOY & 0.003 & 0.018 & -0.032 & -0.009 & 0.003 & 0.016 & 0.039 \\
\hline b.ADOY.2 & -0.015 & 0.008 & -0.032 & -0.021 & -0.015 & -0.010 & 0.001 \\
\hline z.ADOY & 0.172 & 0.028 & 0.119 & 0.153 & 0.172 & 0.191 & 0.227 \\
\hline z.ADOY.2 & 0.057 & 0.014 & 0.029 & 0.048 & 0.057 & 0.067 & 0.084 \\
\hline SD.surv & 0.247 & 0.057 & 0.160 & 0.207 & 0.240 & 0.278 & 0.381 \\
\hline SD.surv.z & 0.621 & 0.139 & 0.412 & 0.522 & 0.599 & 0.696 & 0.946 \\
\hline SD.y0 & 0.228 & 0.040 & 0.164 & 0.199 & 0.223 & 0.251 & 0.319 \\
\hline SD.yz & 0.432 & 0.068 & 0.322 & 0.385 & 0.424 & 0.471 & 0.591 \\
\hline SD.cell & 0.301 & 0.012 & 0.278 & 0.293 & 0.301 & 0.309 & 0.324 \\
\hline SD.cell.z & 0.445 & 0.022 & 0.401 & 0.429 & 0.444 & 0.460 & 0.489 \\
\hline SD.lam[1] & 0.144 & 0.032 & 0.090 & 0.120 & 0.142 & 0.165 & 0.211 \\
\hline SD.lam[2] & 1.301 & 0.550 & 0.662 & 0.939 & 1.172 & 1.506 & 2.740 \\
\hline SD.lam[3] & 0.999 & 1.050 & 0.126 & 0.387 & 0.663 & 1.210 & 4.152 \\
\hline SD.lam[4] & 0.593 & 0.506 & 0.024 & 0.230 & 0.474 & 0.806 & 1.939 \\
\hline SD.lam[5] & 5.559 & 5.320 & 0.156 & 1.243 & 3.549 & 8.605 & 18.419 \\
\hline SD.lam[6] & 1.393 & 0.475 & 0.811 & 1.043 & 1.244 & 1.657 & 2.546 \\
\hline SD.lam[7] & 1.827 & 0.457 & 1.166 & 1.512 & 1.751 & 2.061 & 2.962 \\
\hline SD.lam[8] & 0.536 & 1.112 & 0.022 & 0.101 & 0.193 & 0.442 & 4.259 \\
\hline S.eff[1] & -0.007 & 0.086 & -0.174 & -0.065 & -0.007 & 0.050 & 0.163 \\
\hline S.eff[2] & 0.145 & 0.079 & -0.011 & 0.091 & 0.145 & 0.197 & 0.297 \\
\hline S.eff[3] & -0.035 & 0.092 & -0.216 & -0.097 & -0.036 & 0.028 & 0.146 \\
\hline S.eff[4] & -0.168 & 0.075 & -0.317 & -0.218 & -0.167 & -0.117 & -0.023 \\
\hline S.eff[5] & 0.001 & 0.073 & -0.140 & -0.048 & 0.001 & 0.049 & 0.143 \\
\hline S.eff[6] & 0.143 & 0.104 & -0.062 & 0.072 & 0.144 & 0.213 & 0.346 \\
\hline S.eff[7] & 0.051 & 0.074 & -0.096 & 0.002 & 0.051 & 0.101 & 0.193 \\
\hline S.eff[8] & -0.228 & 0.092 & -0.413 & -0.291 & -0.228 & -0.165 & -0.050 \\
\hline S.eff[9] & -0.577 & 0.086 & -0.748 & -0.634 & -0.576 & -0.519 & -0.412 \\
\hline
\end{tabular}




\begin{tabular}{|c|c|c|c|c|c|c|c|}
\hline S.eff[10] & 0.140 & 0.067 & 0.009 & 0.095 & 0.140 & 0.184 & 0.272 \\
\hline S.eff[11] & -0.160 & 0.069 & -0.299 & -0.206 & -0.161 & -0.114 & -0.024 \\
\hline S.eff[12] & 0.261 & 0.072 & 0.119 & 0.213 & 0.262 & 0.310 & 0.402 \\
\hline S.eff[13] & 0.173 & 0.071 & 0.033 & 0.125 & 0.174 & 0.221 & 0.312 \\
\hline S.eff[14] & 0.176 & 0.083 & 0.015 & 0.121 & 0.176 & 0.231 & 0.339 \\
\hline S.eff[15] & 0.178 & 0.088 & 0.007 & 0.117 & 0.178 & 0.237 & 0.355 \\
\hline S.eff.z[1] & -0.229 & 0.146 & -0.514 & -0.327 & -0.230 & -0.132 & 0.058 \\
\hline S.eff.z[2] & 0.197 & 0.140 & -0.070 & 0.100 & 0.195 & 0.290 & 0.473 \\
\hline S.eff.z[3] & 0.175 & 0.146 & -0.109 & 0.077 & 0.173 & 0.273 & 0.462 \\
\hline S.eff.z[4] & 0.153 & 0.136 & -0.110 & 0.060 & 0.153 & 0.245 & 0.425 \\
\hline S.eff.z[5] & 0.115 & 0.136 & -0.147 & 0.021 & 0.114 & 0.208 & 0.383 \\
\hline S.eff.z[6] & 0.727 & 0.162 & 0.407 & 0.620 & 0.725 & 0.836 & 1.045 \\
\hline S.eff.z[7] & 1.044 & 0.148 & 0.762 & 0.945 & 1.041 & 1.143 & 1.342 \\
\hline S.eff.z[8] & 1.079 & 0.164 & 0.756 & 0.968 & 1.080 & 1.189 & 1.405 \\
\hline S.eff.z[9] & 0.966 & 0.161 & 0.648 & 0.853 & 0.966 & 1.075 & 1.280 \\
\hline S.eff.z[10] & -0.130 & 0.140 & -0.402 & -0.222 & -0.131 & -0.039 & 0.146 \\
\hline S.eff.z[11] & -0.063 & 0.145 & -0.343 & -0.162 & -0.065 & 0.035 & 0.221 \\
\hline S.eff.z[12] & -0.404 & 0.139 & -0.672 & -0.498 & -0.402 & -0.307 & -0.138 \\
\hline S.eff.z[13] & 0.310 & 0.135 & 0.044 & 0.218 & 0.306 & 0.400 & 0.585 \\
\hline S.eff.z[14] & -0.555 & 0.144 & -0.840 & -0.652 & -0.555 & -0.455 & -0.277 \\
\hline S.eff.z[15] & 0.288 & 0.145 & 0.004 & 0.187 & 0.287 & 0.386 & 0.577 \\
\hline $\mathrm{y} 0[1]$ & -0.055 & 0.137 & -0.327 & -0.146 & -0.055 & 0.036 & 0.220 \\
\hline $\mathrm{y} 0[2]$ & -0.114 & 0.117 & -0.340 & -0.192 & -0.115 & -0.036 & 0.116 \\
\hline $\mathrm{y} 0[3]$ & -0.095 & 0.106 & -0.295 & -0.167 & -0.097 & -0.025 & 0.116 \\
\hline $\mathrm{y} 0[4]$ & 0.259 & 0.094 & 0.080 & 0.195 & 0.255 & 0.320 & 0.456 \\
\hline $\mathrm{y} 0[5]$ & 0.060 & 0.091 & -0.112 & -0.003 & 0.057 & 0.118 & 0.249 \\
\hline $\mathrm{y} 0[6]$ & -0.273 & 0.094 & -0.460 & -0.335 & -0.275 & -0.211 & -0.089 \\
\hline y0[7] & 0.047 & 0.089 & -0.133 & -0.012 & 0.047 & 0.107 & 0.220 \\
\hline y0[8] & 0.128 & 0.086 & -0.049 & 0.073 & 0.128 & 0.185 & 0.297 \\
\hline y0[9] & 0.218 & 0.085 & 0.038 & 0.166 & 0.220 & 0.275 & 0.379 \\
\hline y0[10] & -0.049 & 0.084 & -0.220 & -0.102 & -0.046 & 0.006 & 0.111 \\
\hline y0[11] & -0.013 & 0.081 & -0.174 & -0.065 & -0.010 & 0.040 & 0.142 \\
\hline y0[12] & -0.104 & 0.079 & -0.263 & -0.155 & -0.102 & -0.050 & 0.046 \\
\hline y0[13] & 0.115 & 0.074 & -0.035 & 0.068 & 0.119 & 0.167 & 0.253 \\
\hline y0[14] & -0.243 & 0.083 & -0.416 & -0.295 & -0.242 & -0.187 & -0.085 \\
\hline y0[15] & -0.160 & 0.081 & -0.330 & -0.214 & -0.157 & -0.105 & -0.009 \\
\hline y0[16] & -0.011 & 0.077 & -0.165 & -0.062 & -0.010 & 0.040 & 0.139 \\
\hline y0[17] & 0.294 & 0.074 & 0.141 & 0.248 & 0.295 & 0.343 & 0.439 \\
\hline y0[18] & 0.205 & 0.079 & 0.048 & 0.153 & 0.206 & 0.256 & 0.359 \\
\hline y0[19] & 0.070 & 0.080 & -0.087 & 0.017 & 0.069 & 0.121 & 0.228 \\
\hline y0[20] & -0.629 & 0.095 & -0.822 & -0.691 & -0.628 & -0.565 & -0.449 \\
\hline y0[21] & 0.128 & 0.087 & -0.045 & 0.071 & 0.128 & 0.185 & 0.297 \\
\hline y0[22] & 0.111 & 0.095 & -0.085 & 0.051 & 0.112 & 0.174 & 0.299 \\
\hline y0[23] & 0.131 & 0.103 & -0.076 & 0.066 & 0.133 & 0.200 & 0.324 \\
\hline
\end{tabular}




\begin{tabular}{|c|c|c|c|c|c|c|c|}
\hline y0[24] & -0.162 & 0.116 & -0.405 & -0.233 & -0.158 & -0.085 & 0.059 \\
\hline $\mathrm{y} 0[25]$ & 0.009 & 0.137 & -0.278 & -0.076 & 0.012 & 0.095 & 0.275 \\
\hline$y z[1]$ & 0.385 & 0.099 & 0.186 & 0.318 & 0.385 & 0.452 & 0.579 \\
\hline$y z[2]$ & 0.330 & 0.097 & 0.137 & 0.266 & 0.330 & 0.395 & 0.519 \\
\hline$y z[3]$ & 0.836 & 0.092 & 0.655 & 0.774 & 0.836 & 0.901 & 1.018 \\
\hline$y z[4]$ & 0.137 & 0.081 & -0.019 & 0.083 & 0.136 & 0.192 & 0.295 \\
\hline$y z[5]$ & 0.087 & 0.081 & -0.072 & 0.032 & 0.087 & 0.141 & 0.247 \\
\hline$y z[6]$ & 0.441 & 0.086 & 0.275 & 0.380 & 0.440 & 0.499 & 0.609 \\
\hline$y z[7]$ & -0.861 & 0.105 & -1.069 & -0.931 & -0.860 & -0.789 & -0.658 \\
\hline$y z[8]$ & -0.604 & 0.093 & -0.788 & -0.666 & -0.604 & -0.541 & -0.420 \\
\hline$y z[9]$ & -0.820 & 0.101 & -1.021 & -0.889 & -0.818 & -0.751 & -0.625 \\
\hline yz[10] & -0.147 & 0.094 & -0.331 & -0.210 & -0.148 & -0.082 & 0.035 \\
\hline$y z[11]$ & -0.152 & 0.098 & -0.346 & -0.217 & -0.151 & -0.088 & 0.038 \\
\hline yz[12] & 0.128 & 0.091 & -0.050 & 0.065 & 0.128 & 0.189 & 0.305 \\
\hline$y z[13]$ & -0.183 & 0.088 & -0.358 & -0.243 & -0.184 & -0.123 & -0.014 \\
\hline yz[14] & 0.560 & 0.094 & 0.380 & 0.496 & 0.560 & 0.622 & 0.749 \\
\hline yz[15] & 0.308 & 0.096 & 0.118 & 0.243 & 0.309 & 0.373 & 0.497 \\
\hline$y z[16]$ & -0.117 & 0.086 & -0.286 & -0.175 & -0.118 & -0.058 & 0.050 \\
\hline yz[17] & -0.274 & 0.079 & -0.432 & -0.327 & -0.275 & -0.219 & -0.121 \\
\hline yz[18] & 0.248 & 0.083 & 0.085 & 0.192 & 0.249 & 0.304 & 0.413 \\
\hline yz[19] & -0.151 & 0.085 & -0.319 & -0.208 & -0.152 & -0.095 & 0.020 \\
\hline$y z[20]$ & 0.169 & 0.108 & -0.044 & 0.098 & 0.170 & 0.242 & 0.378 \\
\hline$y z[21]$ & 0.038 & 0.084 & -0.127 & -0.018 & 0.038 & 0.095 & 0.201 \\
\hline$y z[22]$ & 0.151 & 0.088 & -0.019 & 0.090 & 0.151 & 0.210 & 0.326 \\
\hline$y z[23]$ & -0.093 & 0.088 & -0.270 & -0.152 & -0.092 & -0.034 & 0.076 \\
\hline$y z[24]$ & -0.516 & 0.105 & -0.728 & -0.586 & -0.514 & -0.445 & -0.317 \\
\hline$y z[25]$ & -0.239 & 0.097 & -0.431 & -0.302 & -0.241 & -0.173 & -0.052 \\
\hline N.sum[1] & $40,309.578$ & $2,934.233$ & $34,769.930$ & $38,290.338$ & $40,244.833$ & $42,241.941$ & $46,163.868$ \\
\hline N.sum[2] & $39,211.263$ & $2,743.532$ & $34,061.484$ & $37,343.948$ & $39,159.183$ & $41,009.514$ & $44,777.926$ \\
\hline N.sum[3] & $29,639.349$ & $2,254.819$ & $25,428.058$ & $28,083.777$ & $29,539.578$ & $31,123.075$ & $34,253.008$ \\
\hline N.sum[4] & $63,723.862$ & $3,525.095$ & $57,131.924$ & $61,313.672$ & $63,638.870$ & $66,085.308$ & $70,820.333$ \\
\hline N.sum[5] & $53,864.986$ & $2,850.370$ & $48,596.325$ & $51,905.894$ & $53,823.058$ & $55,698.447$ & $59,787.047$ \\
\hline N.sum[6] & $32,166.409$ & $2,117.144$ & $28,274.816$ & $30,694.332$ & $32,086.176$ & $33,541.214$ & $36,582.380$ \\
\hline N.sum[7] & $77,042.972$ & $3,884.563$ & $69,774.312$ & $74,367.685$ & $76,969.843$ & $79,624.974$ & $84,785.169$ \\
\hline N.sum[8] & $76,900.515$ & $3,734.443$ & $69,735.347$ & $74,392.518$ & $76,791.639$ & $79,431.336$ & $84,365.319$ \\
\hline N.sum[9] & $89,535.344$ & $3,963.113$ & $81,960.849$ & $86,837.682$ & $89,485.222$ & $92,183.261$ & $97,344.526$ \\
\hline N.sum[10] & $53,260.477$ & $2,941.709$ & $47,680.782$ & $51,238.385$ & $53,224.723$ & $55,142.012$ & $59,250.430$ \\
\hline N.sum[11] & $54,769.515$ & $3,166.888$ & $48,795.181$ & $52,641.291$ & $54,706.339$ & $56,838.915$ & $61,230.991$ \\
\hline N.sum[12] & $43,402.886$ & $2,665.193$ & $38,453.175$ & $41,603.958$ & $43,282.518$ & $45,159.986$ & $48,869.930$ \\
\hline N.sum[13] & $61,469.623$ & $3,314.709$ & $55,248.995$ & $59,202.391$ & $61,373.045$ & $63,635.296$ & $68,111.350$ \\
\hline N.sum[14] & $28,962.226$ & $2,124.813$ & $24,966.492$ & $27,508.499$ & $28,876.139$ & $30,367.571$ & $33,325.402$ \\
\hline N.sum[15] & $35,568.565$ & $2,475.718$ & $31,005.864$ & $33,816.608$ & $35,499.334$ & $37,172.837$ & $40,644.135$ \\
\hline N.sum[16] & $49,767.103$ & $2,626.690$ & $44,812.516$ & $47,958.333$ & $49,701.872$ & $51,530.417$ & $55,027.921$ \\
\hline N.sum[17] & $70,708.535$ & $3,307.781$ & $64,485.112$ & $68,389.926$ & $70,625.897$ & $72,891.845$ & $77,446.522$ \\
\hline
\end{tabular}


N.sum[18]

N.sum[19]

N.sum[20]

N.sum[21]

N.sum[22]

N.sum[23]

N.sum[24]

N.sum[25]

N.sum2[1]

N.sum2[2]

N.sum2[3]

N.sum2[4]

N.sum2[5]

N.sum2[6]

N.sum2[7]

N.sum2[8]

N.sum2[9]

N.sum2[10]

N.sum2[11]

N.sum2[12]

N.sum2[13]

N.sum2[14]

N.sum2[15]

N.sum2[16]

N.sum2[17]

N.sum2[18]

N.sum2[19]

N.sum2[20]

N.sum2[21]

N.sum2[22]

N.sum2[23]

N.sum2[24]

N.sum2[25]

R.growth[1]

R.growth[2]

R.growth[3]

R.growth[4]

R.growth[5]

R.growth[6]

R.growth[7]

R.growth[8]

R.growth[9]

R.growth[10]

R.growth[11]
49,675.989

$51,350.558$

$21,482.052$

$47,676.462$

$43,416.385$

$48,713.523$

42,295.302

$44,370.366$

$43,760.245$

$45,071.903$

$33,373.347$

$50,322.550$

$51,920.826$

$43,225.484$

$75,201.722$

$69,207.054$

$73,658.592$

$57,208.349$

$56,753.284$

49,228.061

$56,003.663$

$37,736.730$

$42,667.734$

$51,472.317$

$53,878.770$

41,381.977

$48,984.990$

41,212.680

$42,936.881$

$39,769.409$

$43,784.871$

$51,020.365$

$45,243.053$

0.032

$-0.300$

0.413

0.032

$-0.183$

0.554

$-0.083$

0.062

$-0.253$

$-0.008$

$-0.142$
$2,947.349$

$2,725.684$

$1,447.272$

$2,592.937$

$2,637.332$

$2,707.916$

$2,122.689$

$2,315.458$

$5,671.777$

$4,985.432$

$3,402.567$

$4,130.842$

$4,003.743$

$3,404.935$

$5,268.443$

$4,937.648$

$5,282.886$

$4,168.291$

$4,114.800$

$3,479.342$

$3,603.090$

$2,925.823$

$3,184.378$

$3,549.190$

$3,614.645$

$3,042.848$

$3,468.077$

$3,435.424$

$3,347.385$

$3,478.468$

$4,180.198$

$5,576.709$

$5,969.529$

0.081

0.078

0.072

0.057

0.065

0.059

0.043

0.044

0.051

0.059

0.065
$44,145.478$

$46,269.040$

$18,763.845$

$42,746.134$

$38,464.615$

$43,593.603$

$38,209.662$

$40,010.621$

$32,826.193$

$35,742.255$

$27,135.709$

$42,406.057$

$44,523.231$

$36,863.451$

$65,800.250$

$60,412.756$

$64,386.487$

$49,826.116$

$49,457.671$

$43,209.988$

$49,682.195$

$32,285.568$

$36,985.563$

$44,944.035$

$47,389.743$

$35,652.587$

$42,522.405$

$34,857.176$

$36,855.693$

$33,504.710$

$36,427.648$

$41,332.147$

$35,023.974$

$-0.124$

$-0.451$

0.273

$-0.078$

$-0.309$

0.441

$-0.168$

$-0.023$

$-0.356$

$-0.125$

$-0.269$
$47,644.426$

$49,489.612$

$20,490.246$

$45,915.072$

$41,579.342$

$46,906.429$

$40,842.713$

$42,780.474$

$39,943.286$

$41,644.240$

$31,053.785$

$47,541.249$

$49,290.320$

$40,843.951$

$71,554.029$

$65,802.603$

$70,053.424$

$54,371.049$

$53,967.946$

$46,829.821$

$53,593.531$

$35,758.141$

$40,495.718$

$49,077.083$

$51,497.204$

$39,367.791$

$46,678.060$

$38,861.591$

$40,677.556$

$37,385.079$

$40,996.651$

$47,248.243$

$41,206.244$

$-0.023$

$-0.352$

0.364

$-0.007$

$-0.228$

0.513

$-0.112$

0.033

$-0.287$

$-0.048$

$-0.186$
$49,608.828$

$51,263.100$

$21,426.694$

$47,628.745$

$43,379.841$

$48,636.771$

$42,234.354$

$44,286.075$

$43,655.747$

$44,894.639$

$33,241.614$

$50,214.213$

$51,836.899$

$43,072.693$

$74,889.570$

$68,884.815$

$73,230.424$

$56,912.863$

$56,419.873$

$48,961.725$

$55,764.652$

$37,616.146$

$42,443.832$

$51,244.559$

$53,634.160$

$41,264.639$

$48,859.266$

$41,077.049$

$42,764.886$

$39,575.868$

$43,460.150$

$50,656.665$

$44,847.755$

0.031

$-0.300$

0.413

0.032

$-0.183$

0.554

$-0.084$

0.062

$-0.253$

$-0.008$

$-0.142$

Appendix 4 -Summary Tables 37

$51,604.352 \quad 55,615.693$

$53,155.827 \quad 56,886.989$

$22,446.512 \quad 24,427.615$

$49,346.043 \quad 53,046.170$

$45,137.189 \quad 48,786.774$

$50,496.318 \quad 54,178.198$

$43,718.378 \quad 46,606.668$

$45,913.536 \quad 49,079.309$

$47,408.378 \quad 55,596.635$

$48,376.134 \quad 55,235.976$

$35,564.415 \quad 40,466.909$

$53,009.800 \quad 58,638.178$

$54,446.750 \quad 60,269.033$

$45,471.459 \quad 50,202.441$

$78,536.884 \quad 86,549.815$

$72,336.967 \quad 79,742.305$

$76,744.984 \quad 85,440.959$

$59,738.988 \quad 66,271.876$

$59,221.453 \quad 65,807.901$

$51,332.583 \quad 56,842.008$

$58,094.056 \quad 64,042.670$ 


\begin{tabular}{lrrrrrrr} 
R.growth[12] & 0.129 & 0.062 & 0.010 & 0.088 & 0.129 & 0.170 & 0.253 \\
R.growth[13] & -0.396 & 0.069 & -0.533 & -0.442 & -0.396 & -0.347 & -0.265 \\
R.growth[14] & 0.123 & 0.077 & -0.028 & 0.072 & 0.123 & 0.175 & 0.274 \\
R.growth[15] & 0.188 & 0.065 & 0.061 & 0.144 & 0.187 & 0.231 & 0.318 \\
R.growth[16] & 0.046 & 0.051 & -0.055 & 0.011 & 0.046 & 0.081 & 0.145 \\
R.growth[17] & -0.264 & 0.055 & -0.375 & -0.301 & -0.264 & -0.227 & -0.157 \\
R.growth[18] & 0.169 & 0.059 & 0.054 & 0.128 & 0.170 & 0.208 & 0.285 \\
R.growth[19] & -0.174 & 0.069 & -0.309 & -0.220 & -0.173 & -0.127 & -0.041 \\
R.growth[20] & 0.041 & 0.070 & -0.095 & -0.007 & 0.042 & 0.089 & 0.178 \\
R.growth[21] & -0.077 & 0.063 & -0.200 & -0.120 & -0.077 & -0.035 & 0.046 \\
R.growth[22] & 0.096 & 0.064 & -0.027 & 0.052 & 0.095 & 0.138 & 0.221 \\
R.growth[23] & 0.152 & 0.060 & 0.036 & 0.112 & 0.151 & 0.191 & 0.272 \\
R.growth[24] & -0.123 & 0.062 & -0.245 & -0.165 & -0.124 & -0.081 & -0.002 \\
Deviance & $60,463.066$ & 216.207 & $60,039.635$ & $60,316.825$ & $60,462.408$ & $60,608.505$ & $60,892.418$ \\
\hline
\end{tabular}


Tables A4.11. Bayesian generalized linear mixed model output for Scaup (Lesser and Greater combined) observed during aerial surveys 1992-2016 on the Arctic Coastal Plain, Alaska. We provide summaries including mean, SE, and several quantiles of the posterior distribution for model and derived parameters. See Table A4.4 for a key to parameter names.

\begin{tabular}{|c|c|c|c|c|c|c|c|}
\hline Parameter & Mean & SD & $2.5 \%$ & $25.0 \%$ & $50.0 \%$ & $75.0 \%$ & $97.5 \%$ \\
\hline MSE & 1.407 & 0.018 & 1.379 & 1.395 & 1.405 & 1.417 & 1.448 \\
\hline MSE.CV & 3.325 & 0.042 & 3.258 & 3.295 & 3.319 & 3.347 & 3.422 \\
\hline SSE & $26,916.555$ & 340.643 & $26,380.023$ & $26,678.647$ & $26,871.243$ & $27,094.859$ & $27,704.235$ \\
\hline Trend & 1.033 & 0.013 & 1.009 & 1.024 & 1.033 & 1.042 & 1.062 \\
\hline R.trend & 0.033 & 0.013 & 0.009 & 0.024 & 0.032 & 0.041 & 0.060 \\
\hline M.dens & 0.290 & 0.014 & 0.264 & 0.280 & 0.288 & 0.299 & 0.319 \\
\hline M.y0 & -0.004 & 0.057 & -0.126 & -0.040 & -0.002 & 0.035 & 0.103 \\
\hline M.blyr & -1.736 & 0.065 & -1.858 & -1.780 & -1.739 & -1.693 & -1.599 \\
\hline M.cell & 0.000 & 0.012 & -0.023 & -0.008 & 0.000 & 0.008 & 0.023 \\
\hline R.mid & -1.364 & 0.035 & -1.431 & -1.388 & -1.364 & -1.341 & -1.297 \\
\hline b.ADOY & 0.186 & 0.034 & 0.120 & 0.163 & 0.186 & 0.209 & 0.251 \\
\hline b.ADOY.2 & -0.091 & 0.022 & -0.135 & -0.106 & -0.091 & -0.076 & -0.046 \\
\hline SD.y0 & 0.278 & 0.056 & 0.187 & 0.238 & 0.272 & 0.312 & 0.403 \\
\hline SD.cell & 0.406 & 0.032 & 0.344 & 0.385 & 0.407 & 0.427 & 0.467 \\
\hline SD.lam[1] & 0.654 & 0.134 & 0.435 & 0.561 & 0.640 & 0.731 & 0.964 \\
\hline SD.lam[2] & 0.761 & 0.368 & 0.170 & 0.492 & 0.755 & 0.977 & 1.573 \\
\hline SD.lam[3] & 4.091 & 2.340 & 0.570 & 2.372 & 3.770 & 5.575 & 9.231 \\
\hline SD.lam[4] & 2.203 & 1.420 & 0.651 & 1.234 & 1.800 & 2.726 & 6.078 \\
\hline SD.lam[5] & 4.509 & 2.741 & 0.258 & 2.203 & 4.231 & 6.666 & 9.599 \\
\hline SD.lam[6] & 0.931 & 0.253 & 0.453 & 0.796 & 0.966 & 1.103 & 1.382 \\
\hline SD.lam[7] & 3.653 & 1.424 & 1.618 & 2.694 & 3.368 & 4.316 & 7.391 \\
\hline SD.lam[8] & 0.823 & 1.202 & 0.018 & 0.191 & 0.415 & 0.887 & 4.875 \\
\hline $\mathrm{y} 0[1]$ & -0.022 & 0.212 & -0.448 & -0.161 & -0.019 & 0.114 & 0.395 \\
\hline $\mathrm{y} 0[2]$ & 0.071 & 0.177 & -0.282 & -0.044 & 0.068 & 0.187 & 0.418 \\
\hline $\mathrm{y} 0[3]$ & -0.233 & 0.164 & -0.565 & -0.340 & -0.231 & -0.121 & 0.082 \\
\hline $\mathrm{y} 0[4]$ & 0.176 & 0.145 & -0.109 & 0.081 & 0.176 & 0.273 & 0.462 \\
\hline $\mathrm{y} 0[5]$ & -0.401 & 0.146 & -0.706 & -0.491 & -0.398 & -0.304 & -0.121 \\
\hline $\mathrm{y} 0[6]$ & 0.225 & 0.139 & -0.047 & 0.133 & 0.226 & 0.317 & 0.499 \\
\hline y0[7] & 0.257 & 0.140 & -0.013 & 0.164 & 0.256 & 0.349 & 0.536 \\
\hline y0[8] & -0.202 & 0.143 & -0.489 & -0.299 & -0.202 & -0.104 & 0.074 \\
\hline y0[9] & 0.064 & 0.141 & -0.217 & -0.028 & 0.064 & 0.158 & 0.342 \\
\hline y0[10] & 0.073 & 0.136 & -0.195 & -0.016 & 0.071 & 0.163 & 0.342 \\
\hline y0[11] & 0.251 & 0.140 & -0.019 & 0.157 & 0.250 & 0.342 & 0.533 \\
\hline y0[12] & -0.111 & 0.142 & -0.396 & -0.204 & -0.111 & -0.017 & 0.170 \\
\hline y0[13] & -0.078 & 0.141 & -0.361 & -0.169 & -0.075 & 0.016 & 0.196 \\
\hline y0[14] & -0.277 & 0.142 & -0.565 & -0.373 & -0.274 & -0.180 & 0.002 \\
\hline y0[15] & -0.238 & 0.143 & -0.534 & -0.330 & -0.234 & -0.141 & 0.024 \\
\hline y0[16] & 0.247 & 0.144 & -0.040 & 0.155 & 0.248 & 0.342 & 0.527 \\
\hline y0[17] & 0.252 & 0.141 & -0.036 & 0.160 & 0.252 & 0.347 & 0.524 \\
\hline
\end{tabular}




\begin{tabular}{|c|c|c|c|c|c|c|c|}
\hline y0[18] & 0.281 & 0.142 & 0.002 & 0.185 & 0.279 & 0.373 & 0.560 \\
\hline y0[19] & 0.029 & 0.142 & -0.257 & -0.064 & 0.032 & 0.125 & 0.302 \\
\hline y0[20] & -0.311 & 0.150 & -0.612 & -0.406 & -0.308 & -0.211 & -0.023 \\
\hline $\mathrm{y} 0[21]$ & -0.300 & 0.150 & -0.606 & -0.397 & -0.296 & -0.196 & -0.019 \\
\hline $0[22]$ & 0.058 & 0.157 & -0.256 & -0.049 & 0.061 & 0.164 & 0.362 \\
\hline $\mathrm{y} 0[23]$ & 0.283 & 0.158 & -0.023 & 0.178 & 0.282 & 0.385 & 0.591 \\
\hline y0[24] & 0.049 & 0.175 & -0.307 & -0.064 & 0.050 & 0.164 & 0.389 \\
\hline y0[25] & -0.243 & 0.211 & -0.671 & -0.379 & -0.239 & -0.100 & 0.167 \\
\hline N.sum[1] & $5,733.259$ & $1,084.284$ & $3,902.876$ & $4,942.205$ & $5,660.059$ & $6,418.158$ & $8,033.230$ \\
\hline N.sum[2] & $6,283.336$ & 942.429 & $4,638.500$ & $5,612.289$ & $6,216.526$ & $6,879.710$ & $8,273.559$ \\
\hline N.sum[3] & $4,771.086$ & 695.348 & $3,540.073$ & $4,283.919$ & $4,727.463$ & $5,198.117$ & $6,279.478$ \\
\hline N.sum[4] & $7,561.278$ & 867.460 & $6,005.329$ & $6,944.564$ & $7,518.896$ & $8,125.403$ & $9,352.710$ \\
\hline N.sum[5] & $4,599.588$ & 560.950 & $3,607.380$ & $4,205.001$ & $4,555.490$ & $4,945.401$ & $5,803.541$ \\
\hline N.sum[6] & $9,437.905$ & $1,029.069$ & $7,645.223$ & $8,706.791$ & $9,365.966$ & $10,081.136$ & $11,660.248$ \\
\hline N.sum[7] & $10,747.302$ & $1,221.726$ & $8,592.668$ & $9,867.537$ & $10,675.322$ & $11,518.786$ & $13,346.396$ \\
\hline I.sum[8] & $7,431.290$ & 896.425 & $5,817.617$ & $6,799.371$ & $7,380.724$ & 7,992.777 & $9,349.424$ \\
\hline N.sum[9] & $10,452.199$ & $1,193.743$ & $8,280.612$ & $9,621.940$ & $10,382.098$ & $11,211.191$ & $12,999.502$ \\
\hline N.sum[10] & $11,176.595$ & $1,110.882$ & $9,131.846$ & $10,401.640$ & $11,107.007$ & $11,904.957$ & $13,523.081$ \\
\hline N.sum[11] & $14,050.040$ & $1,370.484$ & $11,525.989$ & $13,080.246$ & $14,003.628$ & $14,933.588$ & $16,888.582$ \\
\hline N.sum[12] & $10,286.939$ & $1,077.689$ & $8,356.675$ & $9,532.862$ & $10,231.616$ & $10,974.828$ & $12,511.794$ \\
\hline N.sum[13] & $11,211.690$ & $1,182.973$ & $9,064.367$ & $10,393.899$ & $11,146.510$ & $11,978.214$ & $13,700.621$ \\
\hline N.sum[14] & $9,740.166$ & $1,024.912$ & $7,870.548$ & $9,025.319$ & $9,692.718$ & $10,408.466$ & $11,919.338$ \\
\hline N.sum[15] & $10,762.230$ & $1,132.885$ & $8,695.843$ & $9,979.060$ & $10,701.620$ & $11,490.951$ & $13,096.176$ \\
\hline N.sum[16] & $18,495.580$ & $1,910.164$ & $14,985.721$ & $17,191.273$ & $18,384.709$ & $19,695.781$ & $22,560.789$ \\
\hline N.sum[17] & $19,379.656$ & $1,815.616$ & $16,132.834$ & $18,111.531$ & $19,257.605$ & $20,542.856$ & $23,192.412$ \\
\hline .sum[18] & $20,425.972$ & $2,251.762$ & $16,460.973$ & $18,844.270$ & $20,241.942$ & $21,833.201$ & $25,272.504$ \\
\hline N.sum[19] & $15,841.544$ & $1,778.943$ & $12,690.581$ & $14,582.727$ & $15,739.645$ & $16,967.603$ & $19,673.812$ \\
\hline N.sum[20] & $11,011.774$ & $1,373.959$ & $8,582.707$ & $10,037.118$ & $10,935.997$ & $11,860.280$ & $13,994.350$ \\
\hline N.sum[21] & $10,699.127$ & $1,155.611$ & $8,630.493$ & $9,899.046$ & $10,636.263$ & $11,419.810$ & $13,120.604$ \\
\hline N.sum[22] & $14,705.559$ & $1,853.188$ & $11,440.868$ & $13,440.320$ & $14,570.657$ & $15,841.136$ & $18,681.442$ \\
\hline N.sum[23] & $17,740.812$ & $2,132.476$ & $14,026.897$ & $16,228.481$ & $17,589.052$ & $19,123.113$ & $22,348.671$ \\
\hline N.sum[24] & $13,674.144$ & $1,752.790$ & $10,589.440$ & $12,407.067$ & $13,560.722$ & $14,777.808$ & $17,399.755$ \\
\hline N.sum[25] & $9,993.187$ & $1,136.971$ & $7,965.502$ & $9,195.227$ & $9,928.365$ & $10,704.129$ & $12,427.456$ \\
\hline N.sum2[1] & $6,138.315$ & $1,463.764$ & $3,694.563$ & $5,107.104$ & $6,004.825$ & $7,000.460$ & $9,417.534$ \\
\hline N.sum2[2] & $6,107.734$ & $1,140.233$ & $4,145.620$ & $5,310.260$ & $6,021.528$ & $6,791.954$ & $8,578.748$ \\
\hline N.sum2[3] & $6,251.571$ & 965.260 & $4,569.568$ & $5,575.972$ & $6,176.619$ & $6,845.626$ & $8,356.509$ \\
\hline N.sum2[4] & $6,591.891$ & 918.113 & $5,021.247$ & $5,961.160$ & $6,515.082$ & $7,125.244$ & $8,665.033$ \\
\hline N.sum2[5] & $7,126.695$ & 933.863 & $5,574.650$ & $6,483.162$ & $7,037.449$ & $7,661.184$ & $9,223.113$ \\
\hline N.sum2[6] & $7,824.986$ & 969.226 & $6,246.886$ & $7,149.368$ & $7,717.762$ & $8,378.358$ & $9,938.729$ \\
\hline N.sum2[7] & $8,622.594$ & $1,024.841$ & $6,928.281$ & $7,885.833$ & $8,508.782$ & $9,245.590$ & $10,888.517$ \\
\hline N.sum2[8] & $9,430.202$ & $1,099.723$ & $7,497.008$ & $8,647.490$ & $9,318.411$ & $10,107.037$ & $11,845.243$ \\
\hline N.sum2[9] & $10,163.659$ & $1,166.745$ & $8,056.196$ & $9,371.651$ & $10,077.800$ & $10,887.402$ & $12,712.813$ \\
\hline N.sum2[10] & $10,794.503$ & $1,221.308$ & $8,507.182$ & $9,985.110$ & $10,742.239$ & $11,544.850$ & $13,396.359$ \\
\hline N.sum2[11] & $11,360.427$ & $1,293.248$ & $8,865.663$ & $10,509.638$ & $11,322.872$ & $12,183.806$ & $14,026.329$ \\
\hline
\end{tabular}




\begin{tabular}{|c|c|c|c|c|c|c|c|}
\hline N.sum2[12] & $11,933.862$ & $1,381.495$ & $9,365.716$ & $10,999.905$ & $11,880.392$ & $12,832.752$ & 7.973 \\
\hline N.sum2[13] & $12,579.724$ & $1,442.164$ & $10,004.307$ & $11,581.030$ & $12,506.532$ & $13,491.826$ & $15,513.457$ \\
\hline N.sum2[14] & $3,328.973$ & $1,470.650$ & $10,767.845$ & $12,308.923$ & $13,218.311$ & $14,232.893$ & $16,501.125$ \\
\hline N.sum2[15] & $4,166.038$ & $1,551.928$ & $11,505.944$ & $13,091.725$ & $14,026.034$ & $15,091.233$ & $17,613.818$ \\
\hline N.sum2[16] & $14,999.556$ & $1,737.104$ & $12,146.391$ & $13,816.603$ & $14,806.784$ & $16,000.112$ & $19,017.231$ \\
\hline N.sum2[17] & $15,668.924$ & $1,910.842$ & $12,613.531$ & $14,344.223$ & $15,413.194$ & $16,716.621$ & 04.358 \\
\hline N.sum2[18] & $16,013.284$ & $1,944.916$ & $12,956.282$ & $14,651.489$ & $15,767.002$ & $17,092.438$ & $20,551.945$ \\
\hline N.sum2[19] & $15,961.650$ & $1,876.266$ & $12,983.340$ & $14,651.027$ & $15,735.447$ & $16,995.583$ & $20,228.317$ \\
\hline J.sum2[20] & $15,569.633$ & $1,823.031$ & $12,630.012$ & $14,317.892$ & $15,350.170$ & $16,569.874$ & $19,673.733$ \\
\hline N.sum2[21] & $14,990.255$ & $1,803.336$ & $11,995.916$ & $13,756.038$ & $14,793.759$ & $16,037.961$ & 29.298 \\
\hline N.sum2[22] & $14,386.997$ & $1,778.917$ & $11,387.552$ & $13,173.196$ & $14,203.825$ & $15,427.983$ & 20.705 \\
\hline N.sum2[23] & $13,886.257$ & $1,838.628$ & $10,866.502$ & $12,627.427$ & $13,673.491$ & $14,934.748$ & 07.277 \\
\hline [.sum2[24] & $13,561.310$ & $2,186.130$ & $9,990.111$ & $12,027.746$ & $13,298.175$ & $14,831.080$ & 02.016 \\
\hline J.sum2[25] & $13,425.403$ & $2,895.506$ & $8,792.191$ & $11,350.162$ & $13,051.759$ & $15,167.428$ & $20,273.778$ \\
\hline .growth[1] & 0.006 & 0.075 & -0.131 & -0.046 & 0.003 & 0.055 & 0.163 \\
\hline R.growth[2] & 0.029 & 0.065 & -0.091 & -0.017 & 0.026 & 0.071 & 0.169 \\
\hline R.growth[3] & 0.055 & 0.052 & -0.040 & 0.020 & 0.053 & 0.089 & 0.168 \\
\hline R.growth[4] & 0.079 & 0.042 & -0.001 & 0.051 & 0.078 & 0.106 & 0.168 \\
\hline R.growth[5] & 0.094 & 0.040 & 0.022 & 0.067 & 0.092 & 0.120 & 0.177 \\
\hline R.growth[6] & 0.098 & 0.040 & 0.024 & 0.070 & 0.095 & 0.123 & 0.182 \\
\hline R.growth[7] & 0.090 & 0.038 & 0.012 & 0.064 & 0.089 & 0.115 & 0.166 \\
\hline R.growth[8] & 0.075 & 0.038 & -0.010 & 0.053 & 0.077 & 0.100 & 0.145 \\
\hline R.growth[9] & 0.060 & 0.040 & -0.026 & 0.037 & 0.063 & 0.087 & 0.133 \\
\hline R.growth[10] & 0.051 & 0.041 & -0.036 & 0.026 & 0.054 & 0.078 & 0.127 \\
\hline R.growth[11] & 0.049 & 0.039 & -0.036 & 0.025 & 0.052 & 0.074 & 0.121 \\
\hline R.growth[12] & 0.053 & 0.037 & -0.023 & 0.030 & 0.053 & 0.076 & 0.126 \\
\hline R.growth[13] & 0.058 & 0.041 & -0.014 & 0.032 & 0.054 & 0.080 & 0.154 \\
\hline .growth[14] & 0.061 & 0.045 & -0.016 & 0.032 & 0.055 & 0.086 & 0.170 \\
\hline growth[15] & 0.057 & 0.044 & -0.022 & 0.027 & 0.052 & 0.082 & 0.157 \\
\hline R.growth[16] & 0.043 & 0.038 & -0.027 & 0.017 & 0.041 & 0.067 & 0.126 \\
\hline R.growth[17] & 0.022 & 0.036 & -0.049 & -0.002 & 0.022 & 0.044 & 0.096 \\
\hline growth[18] & -0.003 & 0.041 & -0.095 & -0.027 & 0.000 & 0.024 & 0.073 \\
\hline R.growth[19] & -0.025 & 0.045 & -0.129 & -0.050 & -0.020 & 0.004 & 0.057 \\
\hline R.growth[20] & -0.038 & 0.043 & -0.129 & -0.066 & -0.036 & -0.009 & 0.044 \\
\hline R.growth[21] & -0.042 & 0.042 & -0.123 & -0.070 & -0.042 & -0.012 & 0.040 \\
\hline R.growth[22] & -0.036 & 0.053 & -0.140 & -0.072 & -0.035 & 0.001 & 0.065 \\
\hline R.growth[23] & -0.028 & 0.069 & -0.157 & -0.074 & -0.027 & 0.017 & 0.111 \\
\hline R.growth[24] & -0.020 & 0.079 & -0.166 & -0.073 & -0.020 & 0.029 & 0.143 \\
\hline Deviance & $27,378.215$ & 46.038 & $27,290.988$ & $27,347.274$ & $27,376.845$ & $27,409.028$ & $27,472.053$ \\
\hline
\end{tabular}


Tables A4.12. Bayesian generalized linear mixed model output for Steller's Eider observed during aerial surveys 1992-2016 on the Arctic Coastal Plain, Alaska. We provide summaries including mean, SE, and several quantiles of the posterior distribution for model and derived parameters. See Table A4.4 for a key to parameter names.

\begin{tabular}{|c|c|c|c|c|c|c|c|}
\hline Parameter & Mean & SD & $2.5 \%$ & $25 \%$ & $50 \%$ & $75 \%$ & $97.5 \%$ \\
\hline MSE & 0.797 & 4.214 & 0.465 & 0.502 & 0.561 & 0.698 & 2.006 \\
\hline MSE.CV & 5.853 & 30.958 & 3.414 & 3.687 & 4.119 & 5.130 & 14.735 \\
\hline SSE & $2,025.019$ & $10,711.573$ & $1,181.088$ & $1,275.764$ & $1,425.054$ & $1,774.969$ & $5,098.459$ \\
\hline Trend & 0.990 & 0.033 & 0.914 & 0.973 & 0.994 & 1.009 & 1.048 \\
\hline R.trend & -0.011 & 0.034 & -0.090 & -0.027 & -0.006 & 0.009 & 0.047 \\
\hline M.dens & 0.021 & 0.005 & 0.013 & 0.018 & 0.021 & 0.024 & 0.034 \\
\hline М.y0 & -0.020 & 0.268 & -0.562 & -0.190 & -0.019 & 0.146 & 0.517 \\
\hline M.blyr & -4.145 & 0.370 & -4.953 & -4.376 & -4.120 & -3.891 & -3.487 \\
\hline M.cell & 0.000 & 0.027 & -0.059 & -0.013 & 0.000 & 0.012 & 0.058 \\
\hline b.ADOY & -2.675 & 0.150 & -2.971 & -2.778 & -2.676 & -2.575 & -2.378 \\
\hline b.ADOY.2 & -0.068 & 0.215 & -0.488 & -0.212 & -0.068 & 0.077 & 0.358 \\
\hline SD.surv & 0.017 & 0.099 & -0.176 & -0.048 & 0.017 & 0.084 & 0.211 \\
\hline Sd.y0 & 2.844 & 0.962 & 1.429 & 2.185 & 2.694 & 3.340 & 5.129 \\
\hline SD.e & 1.333 & 0.336 & 0.782 & 1.093 & 1.293 & 1.530 & 2.097 \\
\hline SD.cell & 0.258 & 0.151 & 0.028 & 0.140 & 0.238 & 0.360 & 0.586 \\
\hline S.eff[1] & 2.963 & 1.326 & 0.553 & 2.062 & 2.896 & 3.788 & 5.753 \\
\hline S.eff[2] & 2.298 & 0.905 & 0.586 & 1.669 & 2.273 & 2.899 & 4.135 \\
\hline S.eff[3] & 2.826 & 0.985 & 0.961 & 2.152 & 2.794 & 3.470 & 4.823 \\
\hline S.eff[4] & 1.213 & 0.885 & -0.483 & 0.607 & 1.190 & 1.793 & 3.005 \\
\hline S.eff[5] & 3.337 & 1.210 & 1.128 & 2.522 & 3.277 & 4.105 & 5.864 \\
\hline S.eff[6] & 1.548 & 1.028 & -0.374 & 0.841 & 1.526 & 2.226 & 3.637 \\
\hline S.eff[7] & 3.492 & 1.217 & 1.272 & 2.663 & 3.435 & 4.260 & 6.041 \\
\hline S.eff[8] & 1.598 & 0.954 & -0.207 & 0.934 & 1.581 & 2.235 & 3.522 \\
\hline S.eff[9] & 2.633 & 1.277 & 0.358 & 1.750 & 2.553 & 3.433 & 5.357 \\
\hline S.eff[10] & 0.279 & 0.999 & -1.632 & -0.392 & 0.253 & 0.928 & 2.334 \\
\hline S.eff[11] & 0.000 & 0.000 & 0.000 & 0.000 & 0.000 & 0.000 & 0.000 \\
\hline S.eff[12] & 0.000 & 0.000 & 0.000 & 0.000 & 0.000 & 0.000 & 0.000 \\
\hline S.eff[13] & 0.000 & 0.000 & 0.000 & 0.000 & 0.000 & 0.000 & 0.000 \\
\hline S.eff[14] & 0.632 & 1.240 & -1.772 & -0.183 & 0.617 & 1.434 & 3.127 \\
\hline S.eff[15] & 0.000 & 0.000 & 0.000 & 0.000 & 0.000 & 0.000 & 0.000 \\
\hline y0[1] & -0.828 & 0.991 & -2.972 & -1.439 & -0.764 & -0.156 & 0.931 \\
\hline y0[2] & 0.524 & 0.758 & -0.926 & 0.006 & 0.515 & 1.026 & 2.046 \\
\hline y0[3] & 0.336 & 0.739 & -1.113 & -0.150 & 0.327 & 0.822 & 1.798 \\
\hline y0[4] & 1.179 & 0.672 & -0.079 & 0.723 & 1.156 & 1.615 & 2.542 \\
\hline y0[5] & -0.964 & 0.913 & -2.911 & -1.530 & -0.912 & -0.359 & 0.679 \\
\hline y0[6] & 0.685 & 0.662 & -0.572 & 0.230 & 0.671 & 1.115 & 2.035 \\
\hline y0[7] & -1.085 & 0.927 & -3.089 & -1.643 & -1.039 & -0.468 & 0.590 \\
\hline y0[8] & 1.736 & 0.635 & 0.566 & 1.295 & 1.714 & 2.153 & 3.048 \\
\hline y0[9] & -0.872 & 0.936 & -2.899 & -1.429 & -0.805 & -0.236 & 0.809 \\
\hline $\mathrm{y} 0[10]$ & 1.272 & 0.621 & 0.109 & 0.854 & 1.245 & 1.665 & 2.569 \\
\hline $\mathrm{y} 0[11]$ & -1.696 & 0.983 & -3.853 & -2.283 & -1.605 & -1.019 & -0.020 \\
\hline
\end{tabular}




\begin{tabular}{|c|c|c|c|c|c|c|c|}
\hline $\mathrm{y} 0[12]$ & -0.179 & 0.672 & -1.517 & -0.620 & -0.180 & 0.270 & 1.132 \\
\hline y0[13] & -0.232 & 0.673 & -1.567 & -0.679 & -0.236 & 0.217 & 1.087 \\
\hline y0[14] & -0.031 & 0.688 & -1.360 & -0.493 & -0.040 & 0.419 & 1.328 \\
\hline $\mathrm{y} 0[15]$ & 0.717 & 0.577 & -0.380 & 0.328 & 0.704 & 1.087 & 1.907 \\
\hline y0[16] & 1.406 & 0.627 & 0.267 & 0.975 & 1.373 & 1.809 & 2.731 \\
\hline y0[17] & -0.822 & 0.799 & -2.445 & -1.332 & -0.802 & -0.282 & 0.695 \\
\hline y0[18] & -0.540 & 0.791 & -2.150 & -1.052 & -0.523 & -0.009 & 0.970 \\
\hline y0[19] & -1.458 & 1.028 & -3.728 & -2.058 & -1.380 & -0.747 & 0.296 \\
\hline y0[20] & -0.246 & 0.806 & -1.875 & -0.772 & -0.235 & 0.296 & 1.293 \\
\hline y0[21] & 1.426 & 0.631 & 0.249 & 0.992 & 1.402 & 1.836 & 2.746 \\
\hline y0[22] & 1.487 & 0.654 & 0.274 & 1.043 & 1.454 & 1.906 & 2.854 \\
\hline y0[23] & -0.497 & 0.802 & -2.112 & -1.021 & -0.488 & 0.032 & 1.066 \\
\hline y0[24] & -1.327 & 1.068 & -3.700 & -1.952 & -1.242 & -0.608 & 0.533 \\
\hline $\mathrm{y} 0[25]$ & -0.500 & 0.846 & -2.209 & -1.040 & -0.494 & 0.052 & 1.155 \\
\hline SD.lam[1] & 0.180 & 0.084 & 0.063 & 0.121 & 0.165 & 0.223 & 0.392 \\
\hline SD.lam[2] & 5.329 & 3.684 & 0.417 & 2.445 & 4.587 & 7.615 & 13.789 \\
\hline SD.lam[3] & 2.209 & 2.796 & 0.087 & 0.643 & 1.292 & 2.389 & 12.445 \\
\hline SD.lam[4] & 2.943 & 3.038 & 0.084 & 0.805 & 1.867 & 3.945 & 11.732 \\
\hline SD.lam[5] & 6.226 & 4.253 & 0.268 & 2.502 & 5.592 & 9.603 & 14.390 \\
\hline SD.lam[6] & 2.592 & 0.697 & 1.468 & 2.116 & 2.534 & 2.978 & 4.164 \\
\hline SD.lam[7] & 5.975 & 3.439 & 1.105 & 3.163 & 5.173 & 8.472 & 13.403 \\
\hline SD.lam[8] & 1.263 & 1.646 & 0.073 & 0.392 & 0.730 & 1.416 & 5.844 \\
\hline N.sum[1] & 74.483 & 88.005 & 4.783 & 23.949 & 48.843 & 92.881 & 296.063 \\
\hline N.sum[2] & 208.323 & 171.755 & 39.367 & 97.358 & 160.443 & 261.958 & 666.054 \\
\hline N.sum[3] & 156.930 & 118.081 & 32.492 & 79.321 & 124.501 & 198.305 & 482.167 \\
\hline N.sum[4] & 325.585 & 209.719 & 96.964 & 189.903 & 272.575 & 400.274 & 856.967 \\
\hline N.sum[5] & 49.016 & 52.039 & 3.808 & 17.438 & 33.918 & 62.283 & 184.709 \\
\hline N.sum[6] & 188.522 & 115.949 & 55.459 & 112.186 & 160.016 & 231.536 & 484.586 \\
\hline N.sum[7] & 42.047 & 44.833 & 3.193 & 14.765 & 29.076 & 53.725 & 161.470 \\
\hline N.sum[8] & 521.011 & 317.343 & 160.036 & 311.113 & 441.565 & 636.264 & $1,348.181$ \\
\hline N.sum[9] & 49.597 & 48.968 & 3.809 & 18.456 & 35.428 & 63.788 & 181.175 \\
\hline N.sum[10] & 317.718 & 178.590 & 108.030 & 198.822 & 276.263 & 386.563 & 780.880 \\
\hline N.sum[11] & 22.462 & 21.909 & 1.486 & 7.904 & 16.000 & 29.703 & 80.074 \\
\hline N.sum[12] & 79.353 & 50.824 & 19.636 & 45.111 & 67.948 & 99.819 & 204.238 \\
\hline N.sum[13] & 76.508 & 49.210 & 18.445 & 42.323 & 64.537 & 96.709 & 204.190 \\
\hline N.sum[14] & 94.801 & 65.880 & 22.847 & 51.490 & 78.390 & 118.199 & 267.169 \\
\hline N.sum[15] & 182.403 & 90.623 & 67.660 & 120.997 & 162.709 & 221.625 & 408.738 \\
\hline N.sum[16] & 371.788 & 218.101 & 127.074 & 228.680 & 318.121 & 453.470 & 943.826 \\
\hline N.sum[17] & 46.652 & 40.009 & 6.658 & 21.013 & 35.699 & 59.617 & 150.072 \\
\hline N.sum[18] & 60.305 & 50.735 & 9.285 & 27.945 & 47.288 & 76.456 & 188.668 \\
\hline N.sum[19] & 28.768 & 29.827 & 1.638 & 9.648 & 19.735 & 37.830 & 108.032 \\
\hline N.sum[20] & 78.904 & 68.187 & 11.970 & 35.794 & 60.512 & 99.458 & 258.186 \\
\hline N.sum[21] & 350.883 & 202.372 & 118.326 & 218.347 & 305.137 & 428.564 & 861.537 \\
\hline N.sum[22] & 370.849 & 225.345 & 123.952 & 225.533 & 315.660 & 448.755 & 942.342 \\
\hline N.sum[23] & 58.535 & 50.807 & 9.328 & 27.487 & 45.409 & 73.450 & 183.890 \\
\hline N.sum[24] & 33.947 & 39.326 & 1.777 & 10.267 & 22.058 & 42.678 & 136.710 \\
\hline
\end{tabular}




\begin{tabular}{|c|c|c|c|c|c|c|c|}
\hline N.sum[25] & 68.352 & 68.921 & 8.943 & 28.933 & 49.811 & 83.805 & 243.545 \\
\hline N.sum2[1] & 121.211 & 93.739 & 37.508 & 72.555 & 97.095 & 138.902 & 347.420 \\
\hline N.sum2[2] & 106.551 & 67.791 & 36.544 & 68.010 & 89.333 & 123.176 & 282.468 \\
\hline N.sum2[3] & 97.786 & 54.867 & 36.225 & 65.131 & 84.244 & 113.743 & 240.618 \\
\hline N.sum2[4] & 92.382 & 47.040 & 36.758 & 63.596 & 81.444 & 107.796 & 215.489 \\
\hline N.sum2[5] & 88.765 & 40.863 & 37.553 & 62.950 & 79.905 & 104.042 & 193.557 \\
\hline N.sum2[6] & 86.050 & 35.691 & 38.579 & 62.618 & 79.064 & 100.906 & 176.806 \\
\hline N.sum2[7] & 83.815 & 31.754 & 39.229 & 62.454 & 78.050 & 98.423 & 163.807 \\
\hline N.sum2[8] & 82.003 & 29.012 & 39.584 & 62.411 & 77.138 & 95.939 & 153.174 \\
\hline N.sum2[9] & 80.858 & 27.201 & 39.922 & 62.556 & 76.711 & 94.326 & 146.031 \\
\hline N.sum2[10] & 80.511 & 25.986 & 40.748 & 63.207 & 76.827 & 93.547 & 140.730 \\
\hline N.sum2[11] & 80.864 & 25.175 & 41.998 & 64.134 & 77.577 & 93.798 & 138.649 \\
\hline N.sum2[12] & 81.587 & 24.734 & 42.666 & 65.038 & 78.470 & 94.566 & 138.486 \\
\hline N.sum2[13] & 82.169 & 24.576 & 43.568 & 65.504 & 79.247 & 95.411 & 138.367 \\
\hline N.sum2[14] & 82.223 & 24.580 & 43.512 & 65.636 & 79.219 & 95.493 & 139.190 \\
\hline N.sum2[15] & 81.926 & 24.852 & 43.099 & 65.246 & 78.919 & 94.879 & 137.838 \\
\hline N.sum2[16] & 81.521 & 25.358 & 42.798 & 64.611 & 78.331 & 94.577 & 138.076 \\
\hline N.sum2[17] & 81.062 & 25.813 & 41.624 & 63.813 & 77.629 & 94.532 & 138.538 \\
\hline N.sum2[18] & 80.435 & 26.013 & 40.139 & 63.105 & 76.966 & 94.207 & 138.673 \\
\hline N.sum2[19] & 79.464 & 26.000 & 38.268 & 61.873 & 76.256 & 93.542 & 138.084 \\
\hline N.sum2[20] & 78.129 & 26.003 & 36.068 & 60.316 & 75.137 & 92.630 & 137.066 \\
\hline N.sum2[21] & 76.913 & 26.580 & 33.161 & 58.638 & 74.076 & 91.845 & 136.581 \\
\hline N.sum2[22] & 76.554 & 28.378 & 30.148 & 57.138 & 73.415 & 92.324 & 140.012 \\
\hline N.sum2[23] & 77.928 & 32.232 & 27.679 & 56.421 & 73.889 & 94.370 & 151.228 \\
\hline N.sum2[24] & 82.202 & 40.245 & 25.744 & 57.110 & 76.124 & 99.600 & 176.656 \\
\hline N.sum2[25] & 91.218 & 58.141 & 23.906 & 58.976 & 81.004 & 108.782 & 225.957 \\
\hline R.growth[1] & -0.094 & 0.104 & -0.323 & -0.144 & -0.080 & -0.031 & 0.079 \\
\hline R.growth[2] & -0.065 & 0.084 & -0.245 & -0.105 & -0.056 & -0.018 & 0.087 \\
\hline R.growth[3] & -0.041 & 0.069 & -0.186 & -0.074 & -0.036 & -0.006 & 0.090 \\
\hline R.growth[4] & -0.026 & 0.062 & -0.158 & -0.055 & -0.022 & 0.004 & 0.093 \\
\hline R.growth[5] & -0.019 & 0.062 & -0.151 & -0.045 & -0.015 & 0.010 & 0.097 \\
\hline R.growth[6] & -0.017 & 0.065 & -0.153 & -0.041 & -0.011 & 0.013 & 0.098 \\
\hline R.growth[7] & -0.015 & 0.063 & -0.151 & -0.040 & -0.009 & 0.015 & 0.094 \\
\hline R.growth[8] & -0.009 & 0.059 & -0.139 & -0.035 & -0.004 & 0.021 & 0.096 \\
\hline R.growth[9] & 0.000 & 0.056 & -0.124 & -0.027 & 0.002 & 0.030 & 0.106 \\
\hline R.growth[10] & 0.007 & 0.055 & -0.111 & -0.020 & 0.007 & 0.037 & 0.117 \\
\hline R.growth[11] & 0.011 & 0.055 & -0.103 & -0.017 & 0.010 & 0.040 & 0.123 \\
\hline R.growth[12] & 0.008 & 0.054 & -0.100 & -0.019 & 0.008 & 0.035 & 0.116 \\
\hline R.growth[13] & 0.001 & 0.054 & -0.110 & -0.026 & 0.003 & 0.028 & 0.106 \\
\hline R.growth[14] & -0.004 & 0.055 & -0.121 & -0.033 & -0.001 & 0.024 & 0.101 \\
\hline R.growth[15] & -0.007 & 0.055 & -0.125 & -0.036 & -0.002 & 0.022 & 0.100 \\
\hline R.growth[16] & -0.008 & 0.054 & -0.127 & -0.036 & -0.003 & 0.021 & 0.097 \\
\hline R.growth[17] & -0.010 & 0.055 & -0.134 & -0.035 & -0.004 & 0.019 & 0.090 \\
\hline R.growth[18] & -0.015 & 0.058 & -0.148 & -0.040 & -0.008 & 0.016 & 0.087 \\
\hline R.growth[19] & -0.020 & 0.065 & -0.165 & -0.048 & -0.013 & 0.015 & 0.089 \\
\hline R.growth[20] & -0.021 & 0.070 & -0.178 & -0.052 & -0.013 & 0.016 & 0.100 \\
\hline
\end{tabular}




\begin{tabular}{lrrrrrrr} 
R.growth[21] & -0.014 & 0.075 & -0.182 & -0.046 & -0.007 & 0.024 & 0.122 \\
R.growth[22] & 0.004 & 0.082 & -0.167 & -0.031 & 0.006 & 0.039 & 0.164 \\
R.growth[23] & 0.031 & 0.097 & -0.149 & -0.014 & 0.021 & 0.070 & 0.251 \\
R.growth[24] & 0.064 & 0.123 & -0.135 & 0.000 & 0.040 & 0.116 & 0.367 \\
Deviance & $1,420.778$ & 13.449 & $1,395.640$ & $1,411.556$ & $1,420.474$ & $1,429.634$ & $1,448.337$ \\
\hline
\end{tabular}


Tables A4.13. Bayesian generalized linear mixed model output for Spectacled Eider observed during aerial surveys 1992-2016 on the Arctic Coastal Plain, Alaska. We provide summaries including mean, SE, and several quantiles of the posterior distribution for model and derived parameters. See Table A4.4 for a key to parameter names.

\begin{tabular}{|c|c|c|c|c|c|c|c|}
\hline Parameter & Mean & SD & $2.50 \%$ & $25 \%$ & $50 \%$ & $75 \%$ & $97.50 \%$ \\
\hline MSE & 1.384 & 0.008 & 1.369 & 1.378 & 1.384 & 1.389 & 1.401 \\
\hline MSE.CV & 3.032 & 0.018 & 2.998 & 3.019 & 3.031 & 3.043 & 3.070 \\
\hline SSE & $22,294.243$ & 134.986 & $22,048.779$ & $22,202.710$ & $22,289.728$ & $22,380.015$ & $22,574.510$ \\
\hline Trend & 1.013 & 0.012 & 0.989 & 1.005 & 1.013 & 1.021 & 1.037 \\
\hline R.trend & 0.013 & 0.012 & -0.011 & 0.005 & 0.013 & 0.021 & 0.036 \\
\hline M.dens & 0.240 & 0.011 & 0.221 & 0.232 & 0.239 & 0.246 & 0.263 \\
\hline М.y0 & -0.002 & 0.074 & -0.155 & -0.049 & -0.001 & 0.046 & 0.142 \\
\hline M.blyr & -1.687 & 0.083 & -1.848 & -1.741 & -1.688 & -1.633 & -1.519 \\
\hline M.cell & 0.000 & 0.013 & -0.026 & -0.008 & 0.000 & 0.008 & 0.024 \\
\hline R.mid & -1.499 & 0.038 & -1.574 & -1.525 & -1.499 & -1.474 & -1.426 \\
\hline b.ADOY & -0.400 & 0.046 & -0.489 & -0.431 & -0.400 & -0.370 & -0.309 \\
\hline b.ADOY.2 & -0.084 & 0.025 & -0.132 & -0.101 & -0.084 & -0.067 & -0.036 \\
\hline SD.surv & 2.518 & 0.583 & 1.663 & 2.113 & 2.421 & 2.815 & 3.930 \\
\hline SD.y0 & 0.366 & 0.073 & 0.249 & 0.316 & 0.358 & 0.407 & 0.531 \\
\hline SD.cell & 0.343 & 0.042 & 0.262 & 0.315 & 0.343 & 0.371 & 0.424 \\
\hline SD.lam[1] & 0.210 & 0.065 & 0.109 & 0.164 & 0.204 & 0.247 & 0.363 \\
\hline SD.lam[2] & 0.742 & 0.561 & 0.075 & 0.371 & 0.612 & 0.963 & 2.183 \\
\hline SD.lam[3] & 1.814 & 1.552 & 0.061 & 0.845 & 1.414 & 2.282 & 6.233 \\
\hline SD.lam[4] & 1.841 & 1.699 & 0.126 & 0.779 & 1.357 & 2.345 & 6.381 \\
\hline SD.lam[5] & 4.683 & 4.042 & 0.140 & 1.307 & 3.431 & 7.211 & 14.014 \\
\hline SD.lam[6] & 1.729 & 0.472 & 1.010 & 1.399 & 1.642 & 1.987 & 2.874 \\
\hline SD.lam[7] & 1.643 & 0.621 & 0.894 & 1.224 & 1.493 & 1.859 & 3.303 \\
\hline SD.lam[8] & 1.178 & 1.891 & 0.102 & 0.226 & 0.415 & 0.954 & 7.425 \\
\hline S.eff[1] & 0.000 & 0.000 & 0.000 & 0.000 & 0.000 & 0.000 & 0.000 \\
\hline S.eff[2] & -1.596 & 0.290 & -2.174 & -1.791 & -1.592 & -1.398 & -1.045 \\
\hline S.eff[3] & -2.666 & 0.459 & -3.628 & -2.961 & -2.650 & -2.347 & -1.819 \\
\hline S.eff[4] & -2.543 & 0.452 & -3.496 & -2.833 & -2.519 & -2.234 & -1.714 \\
\hline S.eff[5] & -2.615 & 0.543 & -3.781 & -2.953 & -2.582 & -2.240 & -1.647 \\
\hline S.eff[6] & -2.400 & 0.537 & -3.561 & -2.735 & -2.364 & -2.025 & -1.441 \\
\hline S.eff[7] & -2.014 & 0.357 & -2.720 & -2.252 & -2.005 & -1.769 & -1.349 \\
\hline S.eff[8] & -3.028 & 0.723 & -4.648 & -3.462 & -2.965 & -2.524 & -1.792 \\
\hline S.eff[9] & 0.000 & 0.000 & 0.000 & 0.000 & 0.000 & 0.000 & 0.000 \\
\hline S.eff[10] & -2.252 & 0.361 & -2.989 & -2.489 & -2.239 & -2.007 & -1.590 \\
\hline S.eff[11] & -1.808 & 0.334 & -2.485 & -2.025 & -1.798 & -1.578 & -1.185 \\
\hline S.eff[12] & -2.872 & 0.488 & -3.909 & -3.184 & -2.843 & -2.532 & -1.998 \\
\hline S.eff[13] & -1.417 & 0.329 & -2.073 & -1.638 & -1.412 & -1.191 & -0.781 \\
\hline S.eff[14] & -0.496 & 0.225 & -0.933 & -0.648 & -0.497 & -0.347 & -0.053 \\
\hline S.eff[15] & -2.100 & 0.413 & -2.964 & -2.369 & -2.084 & -1.815 & -1.327 \\
\hline $\mathrm{y} 0[1]$ & -1.001 & 0.279 & -1.582 & -1.182 & -0.993 & -0.812 & -0.471 \\
\hline
\end{tabular}




\begin{tabular}{|c|c|c|c|c|c|c|c|}
\hline $\mathrm{y} 0[2]$ & 0.472 & 0.205 & 0.076 & 0.334 & 0.470 & 0.606 & 0.879 \\
\hline $\mathrm{y} 0[3]$ & 0.308 & 0.178 & -0.041 & 0.190 & 0.306 & 0.422 & 0.669 \\
\hline $\mathrm{y} 0[4]$ & 0.300 & 0.171 & -0.033 & 0.185 & 0.302 & 0.410 & 0.644 \\
\hline $\mathrm{y} 0[5]$ & -0.104 & 0.167 & -0.438 & -0.213 & -0.104 & 0.007 & 0.227 \\
\hline $\mathrm{y} 0[6]$ & -0.091 & 0.165 & -0.416 & -0.201 & -0.088 & 0.020 & 0.234 \\
\hline $\mathrm{y} 0[7]$ & 0.170 & 0.160 & -0.143 & 0.063 & 0.168 & 0.276 & 0.488 \\
\hline $\mathrm{y} 0[8]$ & -0.044 & 0.166 & -0.373 & -0.155 & -0.045 & 0.066 & 0.288 \\
\hline $\mathrm{y} 0[9]$ & -0.226 & 0.164 & -0.552 & -0.333 & -0.225 & -0.119 & 0.099 \\
\hline y0[10] & 0.282 & 0.171 & -0.049 & 0.169 & 0.279 & 0.393 & 0.618 \\
\hline $\mathrm{y} 0[11]$ & -0.076 & 0.170 & -0.409 & -0.185 & -0.078 & 0.035 & 0.259 \\
\hline $\mathrm{y} 0[12]$ & 0.212 & 0.165 & -0.112 & 0.105 & 0.211 & 0.320 & 0.536 \\
\hline y0[13] & -0.278 & 0.167 & -0.607 & -0.389 & -0.277 & -0.167 & 0.050 \\
\hline y0[14] & 0.214 & 0.165 & -0.112 & 0.104 & 0.215 & 0.325 & 0.539 \\
\hline y0[15] & -0.056 & 0.171 & -0.394 & -0.169 & -0.054 & 0.055 & 0.280 \\
\hline y0[16] & -0.028 & 0.173 & -0.363 & -0.144 & -0.026 & 0.084 & 0.319 \\
\hline y0[17] & 0.058 & 0.172 & -0.275 & -0.059 & 0.056 & 0.170 & 0.406 \\
\hline y0[18] & -0.370 & 0.174 & -0.716 & -0.486 & -0.368 & -0.250 & -0.036 \\
\hline y0[19] & 0.115 & 0.169 & -0.221 & 0.004 & 0.114 & 0.226 & 0.449 \\
\hline $\mathrm{y} 0[20]$ & 0.358 & 0.171 & 0.033 & 0.243 & 0.355 & 0.471 & 0.699 \\
\hline $\mathrm{y} 0[21]$ & -0.254 & 0.176 & -0.617 & -0.369 & -0.250 & -0.133 & 0.080 \\
\hline $\mathrm{y} 0[22]$ & 0.187 & 0.180 & -0.166 & 0.065 & 0.189 & 0.308 & 0.540 \\
\hline y0[23] & 0.008 & 0.189 & -0.369 & -0.111 & 0.013 & 0.134 & 0.382 \\
\hline y0[24] & 0.040 & 0.213 & -0.391 & -0.097 & 0.044 & 0.183 & 0.452 \\
\hline $\mathrm{y} 0[25]$ & -0.236 & 0.260 & -0.739 & -0.411 & -0.236 & -0.062 & 0.277 \\
\hline N.sum[1] & $1,306.441$ & 239.804 & 889.543 & $1,136.518$ & $1,289.277$ & $1,454.706$ & $1,822.540$ \\
\hline N.sum[2] & $6,220.748$ & 826.944 & $4,791.346$ & $5,637.981$ & $6,155.970$ & $6,739.890$ & $8,026.123$ \\
\hline N.sum[3] & $5,794.599$ & 731.112 & $4,488.303$ & $5,296.061$ & $5,742.299$ & $6,239.878$ & $7,377.983$ \\
\hline N.sum[4] & $6,266.733$ & 755.915 & $4,915.413$ & $5,743.401$ & $6,220.866$ & $6,744.032$ & $7,899.678$ \\
\hline N.sum[5] & $4,515.740$ & 558.613 & $3,535.191$ & $4,120.376$ & $4,488.665$ & $4,861.424$ & $5,701.685$ \\
\hline N.sum[6] & $4,880.396$ & 606.633 & $3,818.036$ & $4,459.648$ & $4,843.874$ & $5,251.720$ & $6,204.212$ \\
\hline N.sum[7] & $6,681.235$ & 816.976 & $5,219.851$ & $6,099.648$ & $6,625.490$ & $7,196.739$ & $8,441.926$ \\
\hline N.sum[8] & $5,636.467$ & 676.269 & $4,437.192$ & $5,167.937$ & $5,601.833$ & $6,051.173$ & $7,102.669$ \\
\hline N.sum[9] & $4,878.395$ & 513.238 & $3,965.137$ & $4,522.862$ & $4,846.182$ & $5,207.552$ & $5,955.671$ \\
\hline N.sum[10] & $8,384.157$ & 961.053 & $6,667.408$ & $7,713.092$ & $8,319.733$ & $8,994.871$ & $10,443.756$ \\
\hline N.sum[11] & $6,024.741$ & 724.197 & $4,743.082$ & $5,515.497$ & $5,981.722$ & $6,475.258$ & $7,549.461$ \\
\hline N.sum[12] & $8,174.383$ & 915.970 & $6,516.159$ & $7,541.254$ & $8,117.417$ & $8,761.406$ & $10,103.628$ \\
\hline N.sum[13] & $5,053.376$ & 594.498 & $3,979.909$ & $4,637.422$ & $5,008.437$ & $5,424.855$ & $6,358.427$ \\
\hline N.sum[14] & $8,268.534$ & 926.177 & $6,597.735$ & $7,625.712$ & $8,217.729$ & $8,843.602$ & $10,227.324$ \\
\hline N.sum[15] & $6,282.190$ & 740.465 & $4,963.111$ & $5,763.671$ & $6,227.807$ & $6,753.220$ & $7,839.074$ \\
\hline N.sum[16] & $6,427.371$ & 771.094 & $5,034.742$ & $5,888.869$ & $6,389.633$ & $6,920.274$ & $8,090.601$ \\
\hline N.sum[17] & $6,961.254$ & 802.651 & $5,542.469$ & $6,400.564$ & $6,918.284$ & $7,463.475$ & $8,651.052$ \\
\hline N.sum[18] & $4,518.719$ & 550.324 & $3,551.103$ & $4,133.399$ & $4,479.848$ & $4,867.960$ & $5,663.028$ \\
\hline N.sum[19] & $7,278.680$ & 835.608 & $5,810.839$ & $6,686.926$ & 7,229.954 & $7,804.082$ & $9,088.236$ \\
\hline N.sum[20] & $9,133.760$ & $1,090.452$ & $7,236.085$ & $8,365.849$ & $9,044.367$ & $9,816.709$ & $11,519.431$ \\
\hline
\end{tabular}




\begin{tabular}{|c|c|c|c|c|c|c|c|}
\hline N.sum[21] & $4,806.432$ & 620.884 & $3,701.123$ & $4,376.707$ & $4,772.351$ & $5,191.962$ & $6,147.513$ \\
\hline N.sum[22] & $7,117.502$ & 876.524 & $5,553.824$ & $6,510.722$ & $7,054.053$ & $7,669.828$ & $8,951.931$ \\
\hline N.sum[23] & $5,590.052$ & 699.895 & $4,364.923$ & $5,102.777$ & $5,534.600$ & $6,029.207$ & 7,093.992 \\
\hline N.sum[24] & $5,368.398$ & 663.170 & $4,173.659$ & $4,909.371$ & $5,323.673$ & $5,795.402$ & $6,812.432$ \\
\hline N.sum[25] & $3,784.157$ & 482.179 & $2,923.208$ & $3,449.204$ & $3,751.882$ & $4,082.881$ & $4,806.787$ \\
\hline N.sum2[1] & $3,857.117$ & 966.898 & $2,370.522$ & $3,162.010$ & $3,712.198$ & $4,412.273$ & $6,109.938$ \\
\hline N.sum2[2] & $4,193.426$ & 823.151 & $2,915.312$ & $3,602.053$ & $4,083.442$ & $4,673.253$ & $6,082.768$ \\
\hline N.sum2[3] & $4,577.375$ & 724.219 & $3,402.584$ & $4,058.641$ & $4,496.862$ & $5,014.167$ & $6,208.686$ \\
\hline N.sum2[4] & $4,978.946$ & 681.940 & $3,827.203$ & $4,492.033$ & $4,917.423$ & $5,383.830$ & $6,479.323$ \\
\hline N.sum2[5] & $5,364.048$ & 660.932 & $4,206.545$ & $4,906.655$ & $5,314.973$ & $5,762.195$ & $6,819.379$ \\
\hline N.sum2[6] & $5,713.592$ & 651.162 & $4,595.133$ & $5,266.142$ & $5,651.760$ & $6,104.680$ & $7,149.244$ \\
\hline N.sum2[7] & $6,026.105$ & 684.786 & $4,878.231$ & $5,562.337$ & $5,951.221$ & $6,419.885$ & $7,593.638$ \\
\hline N.sum2[8] & $6,306.326$ & 757.913 & $5,053.308$ & $5,783.768$ & $6,222.001$ & $6,731.605$ & $8,056.158$ \\
\hline N.sum2[9] & $6,557.593$ & 821.489 & $5,204.713$ & $5,990.221$ & $6,463.715$ & $7,022.699$ & $8,460.295$ \\
\hline N.sum2[10] & $6,776.925$ & 843.146 & $5,396.750$ & $6,187.875$ & $6,683.234$ & $7,255.130$ & $8,692.663$ \\
\hline N.sum2[11] & $6,955.486$ & 837.243 & $5,581.192$ & $6,369.269$ & $6,862.447$ & $7,433.804$ & $8,891.213$ \\
\hline N.sum2[12] & $7,080.848$ & 835.854 & $5,745.530$ & $6,493.188$ & $6,983.755$ & $7,554.024$ & $9,009.954$ \\
\hline N.sum2[13] & $7,143.561$ & 841.496 & $5,805.975$ & $6,541.549$ & $7,052.748$ & $7,623.175$ & $9,046.116$ \\
\hline N.sum2[14] & $7,148.315$ & 844.572 & $5,722.535$ & $6,554.696$ & $7,064.321$ & $7,651.255$ & $9,044.505$ \\
\hline N.sum2[15] & $7,116.737$ & 855.153 & $5,634.881$ & $6,525.333$ & $7,046.942$ & $7,635.278$ & $8,995.645$ \\
\hline N.sum2[16] & $7,075.248$ & 873.486 & $5,520.490$ & $6,481.718$ & $7,009.717$ & $7,584.656$ & $8,996.143$ \\
\hline N.sum2[17] & $7,038.933$ & 874.936 & $5,482.006$ & $6,449.238$ & $6,956.160$ & $7,545.517$ & $9,012.560$ \\
\hline N.sum2[18] & $7,003.891$ & 847.217 & $5,550.186$ & $6,434.310$ & $6,921.932$ & $7,478.370$ & $8,949.003$ \\
\hline N.sum2[19] & $6,947.285$ & 818.243 & $5,553.455$ & $6,392.410$ & $6,863.060$ & $7,426.546$ & $8,825.883$ \\
\hline N.sum2[20] & $6,833.311$ & 823.749 & $5,470.293$ & $6,269.692$ & $6,736.939$ & $7,288.998$ & $8,732.435$ \\
\hline N.sum2[21] & $6,630.079$ & 844.171 & $5,267.267$ & $6,056.264$ & $6,519.977$ & $7,076.913$ & $8,629.255$ \\
\hline N.sum2[22] & $6,326.662$ & 846.052 & $4,920.026$ & $5,752.352$ & $6,229.770$ & $6,782.443$ & $8,323.533$ \\
\hline N.sum2[23] & $5,953.057$ & 886.856 & $4,471.946$ & $5,349.140$ & $5,869.766$ & $6,446.380$ & $8,018.514$ \\
\hline N.sum2[24] & $5,572.386$ & $1,052.823$ & $3,865.314$ & $4,833.023$ & $5,456.081$ & $6,181.025$ & $7,991.584$ \\
\hline N.sum2[25] & $5,244.079$ & $1,314.445$ & $3,090.717$ & $4,295.160$ & $5,098.599$ & $6,009.270$ & $8,260.382$ \\
\hline R.growth[1] & 0.095 & 0.075 & -0.025 & 0.041 & 0.088 & 0.139 & 0.271 \\
\hline R.growth[2] & 0.094 & 0.067 & -0.016 & 0.045 & 0.089 & 0.134 & 0.245 \\
\hline R.growth[3] & 0.087 & 0.053 & -0.007 & 0.050 & 0.085 & 0.122 & 0.198 \\
\hline R.growth[4] & 0.076 & 0.044 & -0.004 & 0.046 & 0.075 & 0.106 & 0.162 \\
\hline R.growth[5] & 0.064 & 0.044 & -0.017 & 0.035 & 0.062 & 0.093 & 0.149 \\
\hline R.growth[6] & 0.053 & 0.045 & -0.040 & 0.027 & 0.053 & 0.081 & 0.142 \\
\hline R.growth[7] & 0.045 & 0.041 & -0.039 & 0.020 & 0.045 & 0.069 & 0.128 \\
\hline R.growth[8] & 0.038 & 0.035 & -0.032 & 0.015 & 0.038 & 0.061 & 0.110 \\
\hline R.growth[9] & 0.033 & 0.035 & -0.032 & 0.010 & 0.032 & 0.054 & 0.105 \\
\hline R.growth[10] & 0.026 & 0.038 & -0.051 & 0.004 & 0.025 & 0.048 & 0.108 \\
\hline R.growth[11] & 0.018 & 0.041 & -0.071 & -0.002 & 0.018 & 0.041 & 0.103 \\
\hline R.growth[12] & 0.009 & 0.040 & -0.084 & -0.011 & 0.011 & 0.031 & 0.086 \\
\hline R.growth[13] & 0.001 & 0.040 & -0.093 & -0.020 & 0.004 & 0.024 & 0.081 \\
\hline R.growth[14] & -0.005 & 0.040 & -0.099 & -0.025 & -0.001 & 0.018 & 0.074 \\
\hline
\end{tabular}




\begin{tabular}{lrrrrrrr} 
R.growth[15] & -0.006 & 0.038 & -0.094 & -0.025 & -0.004 & 0.016 & 0.066 \\
R.growth[16] & -0.005 & 0.036 & -0.079 & -0.026 & -0.005 & 0.016 & 0.067 \\
R.growth[17] & -0.005 & 0.037 & -0.071 & -0.028 & -0.007 & 0.015 & 0.084 \\
R.growth[18] & -0.008 & 0.042 & -0.080 & -0.035 & -0.012 & 0.013 & 0.098 \\
R.growth[19] & -0.017 & 0.045 & -0.096 & -0.046 & -0.020 & 0.007 & 0.088 \\
R.growth[20] & -0.031 & 0.042 & -0.115 & -0.059 & -0.030 & -0.004 & 0.051 \\
R.growth[21] & -0.048 & 0.045 & -0.141 & -0.078 & -0.046 & -0.015 & 0.032 \\
R.growth[22] & -0.063 & 0.059 & -0.198 & -0.100 & -0.058 & -0.019 & 0.037 \\
R.growth[23] & -0.073 & 0.077 & -0.257 & -0.117 & -0.063 & -0.016 & 0.047 \\
R.growth[24] & -0.074 & 0.087 & -0.288 & -0.123 & -0.062 & -0.010 & 0.057 \\
Deviance & $23,906.684$ & 40.847 & $23,827.955$ & $23,879.346$ & $23,906.461$ & $23,934.024$ & $23,987.716$ \\
\hline
\end{tabular}


Tables A4.14. Bayesian generalized linear mixed model output for King Eider observed during aerial surveys 1992-2016 on the Arctic Coastal Plain, Alaska. We provide summaries including mean, SE, and several quantiles of the posterior distribution for model and derived parameters. See Table A4.4 for a key to parameter names.

\begin{tabular}{|c|c|c|c|c|c|c|c|}
\hline Parameter & Mean & SD & $2.50 \%$ & $25 \%$ & $50 \%$ & $75 \%$ & $97.50 \%$ \\
\hline MSE & 3.411 & 0.059 & 3.318 & 3.372 & 3.404 & 3.442 & 3.548 \\
\hline MSE.CV & 3.856 & 0.067 & 3.750 & 3.811 & 3.847 & 3.890 & 4.010 \\
\hline SSE & $66,377.412$ & $1,150.781$ & $64,565.109$ & $65,606.901$ & $66,229.082$ & $66,977.262$ & $69,041.198$ \\
\hline Trend & 1.046 & 0.019 & 1.009 & 1.032 & 1.046 & 1.061 & 1.081 \\
\hline R.trend & 0.045 & 0.019 & 0.009 & 0.031 & 0.045 & 0.059 & 0.078 \\
\hline M.dens & 0.497 & 0.022 & 0.460 & 0.482 & 0.494 & 0.509 & 0.545 \\
\hline M.y0 & 0.001 & 0.071 & -0.142 & -0.045 & 0.000 & 0.047 & 0.143 \\
\hline M.blyr & -1.140 & 0.078 & -1.290 & -1.192 & -1.140 & -1.089 & -0.986 \\
\hline M.cell & 0.000 & 0.016 & -0.032 & -0.011 & 0.000 & 0.011 & 0.032 \\
\hline R.mid & -0.983 & 0.029 & -1.040 & -1.003 & -0.983 & -0.963 & -0.927 \\
\hline b.ADOY & -0.129 & 0.043 & -0.211 & -0.158 & -0.130 & -0.101 & -0.045 \\
\hline b.ADOY.2 & -0.083 & 0.020 & -0.123 & -0.097 & -0.084 & -0.070 & -0.045 \\
\hline SD.surv & 1.556 & 0.343 & 1.043 & 1.318 & 1.500 & 1.736 & 2.394 \\
\hline SD.y0 & 0.347 & 0.070 & 0.235 & 0.297 & 0.337 & 0.388 & 0.508 \\
\hline SD.cell & 0.525 & 0.025 & 0.477 & 0.508 & 0.525 & 0.543 & 0.575 \\
\hline SD.lam[1] & 1.246 & 0.232 & 0.872 & 1.083 & 1.218 & 1.384 & 1.777 \\
\hline SD.lam[2] & 2.389 & 0.708 & 1.450 & 1.901 & 2.246 & 2.709 & 4.170 \\
\hline SD.lam[3] & 1.865 & 2.404 & 0.040 & 0.328 & 0.913 & 2.443 & 9.126 \\
\hline SD.lam[4] & 3.669 & 2.616 & 0.602 & 1.777 & 3.015 & 4.753 & 10.902 \\
\hline SD.lam[5] & 7.446 & 4.126 & 0.454 & 4.050 & 7.382 & 10.826 & 14.590 \\
\hline SD.lam[6] & 0.984 & 0.487 & 0.325 & 0.697 & 0.864 & 1.139 & 2.298 \\
\hline SD.lam[7] & 1.594 & 1.030 & 0.472 & 0.894 & 1.348 & 1.906 & 4.358 \\
\hline SD.lam[8] & 0.616 & 1.146 & 0.013 & 0.108 & 0.246 & 0.557 & 4.249 \\
\hline S.eff[1] & -0.893 & 0.366 & -1.630 & -1.134 & -0.884 & -0.638 & -0.191 \\
\hline S.eff[2] & -1.228 & 0.212 & -1.645 & -1.370 & -1.224 & -1.085 & -0.814 \\
\hline S.eff[3] & -1.866 & 0.236 & -2.341 & -2.019 & -1.861 & -1.703 & -1.416 \\
\hline S.eff[4] & -2.394 & 0.281 & -2.951 & -2.581 & -2.391 & -2.205 & -1.855 \\
\hline S.eff[5] & -1.971 & 0.247 & -2.463 & -2.137 & -1.967 & -1.806 & -1.499 \\
\hline S.eff[6] & -1.267 & 0.251 & -1.764 & -1.433 & -1.261 & -1.101 & -0.779 \\
\hline S.eff[7] & -1.080 & 0.209 & -1.492 & -1.220 & -1.079 & -0.938 & -0.679 \\
\hline S.eff[8] & -1.308 & 0.246 & -1.786 & -1.472 & -1.305 & -1.143 & -0.829 \\
\hline S.eff[9] & -2.208 & 0.263 & -2.731 & -2.380 & -2.203 & -2.029 & -1.693 \\
\hline S.eff[10] & -0.779 & 0.173 & -1.123 & -0.894 & -0.780 & -0.661 & -0.444 \\
\hline S.eff[11] & -1.296 & 0.184 & -1.665 & -1.419 & -1.294 & -1.170 & -0.939 \\
\hline S.eff[12] & -0.543 & 0.176 & -0.888 & -0.662 & -0.542 & -0.425 & -0.200 \\
\hline S.eff[13] & -1.000 & 0.190 & -1.377 & -1.126 & -0.999 & -0.868 & -0.630 \\
\hline S.eff[14] & -0.635 & 0.170 & -0.971 & -0.749 & -0.633 & -0.522 & -0.301 \\
\hline S.eff[15] & -0.785 & 0.187 & -1.144 & -0.913 & -0.784 & -0.655 & -0.427 \\
\hline $\mathrm{y} 0[1]$ & -0.972 & 0.326 & -1.632 & -1.194 & -0.964 & -0.745 & -0.364 \\
\hline
\end{tabular}




\begin{tabular}{|c|c|c|c|c|c|c|c|}
\hline $\mathrm{y} 0[2]$ & 0.478 & 0.221 & 0.044 & 0.329 & 0.477 & 0.630 & 0.896 \\
\hline $\mathrm{y} 0[3]$ & 0.447 & 0.175 & 0.102 & 0.330 & 0.449 & 0.564 & 0.795 \\
\hline $\mathrm{y} 0[4]$ & 0.210 & 0.184 & -0.145 & 0.083 & 0.210 & 0.336 & 0.567 \\
\hline $\mathrm{y} 0[5]$ & 0.199 & 0.199 & -0.207 & 0.067 & 0.209 & 0.338 & 0.562 \\
\hline $\mathrm{y} 0[6]$ & -0.259 & 0.204 & -0.686 & -0.386 & -0.249 & -0.122 & 0.118 \\
\hline $\mathrm{y} 0[7]$ & -0.136 & 0.183 & -0.509 & -0.251 & -0.133 & -0.015 & 0.221 \\
\hline $\mathrm{y} 0[8]$ & -0.208 & 0.176 & -0.563 & -0.318 & -0.206 & -0.092 & 0.132 \\
\hline $\mathrm{y} 0[9]$ & 0.101 & 0.173 & -0.239 & -0.013 & 0.097 & 0.208 & 0.456 \\
\hline y0[10] & 0.280 & 0.170 & -0.036 & 0.167 & 0.272 & 0.385 & 0.638 \\
\hline y0[11] & 0.059 & 0.164 & -0.255 & -0.050 & 0.053 & 0.164 & 0.396 \\
\hline y0[12] & -0.094 & 0.155 & -0.396 & -0.197 & -0.095 & 0.008 & 0.210 \\
\hline y0[13] & -0.158 & 0.150 & -0.460 & -0.257 & -0.157 & -0.055 & 0.136 \\
\hline y0[14] & -0.042 & 0.151 & -0.345 & -0.140 & -0.039 & 0.059 & 0.257 \\
\hline y0[15] & -0.180 & 0.161 & -0.515 & -0.282 & -0.174 & -0.072 & 0.125 \\
\hline y0[16] & 0.182 & 0.161 & -0.146 & 0.079 & 0.184 & 0.288 & 0.497 \\
\hline y0[17] & -0.025 & 0.164 & -0.342 & -0.135 & -0.026 & 0.082 & 0.301 \\
\hline y0[18] & 0.126 & 0.159 & -0.169 & 0.016 & 0.120 & 0.227 & 0.460 \\
\hline y0[19] & -0.025 & 0.163 & -0.336 & -0.135 & -0.027 & 0.083 & 0.296 \\
\hline y0[20] & -0.009 & 0.173 & -0.358 & -0.125 & -0.006 & 0.105 & 0.327 \\
\hline y0[21] & 0.006 & 0.179 & -0.363 & -0.109 & 0.008 & 0.122 & 0.365 \\
\hline $\mathrm{y} 0[22]$ & 0.183 & 0.172 & -0.154 & 0.071 & 0.178 & 0.294 & 0.541 \\
\hline y0[23] & -0.133 & 0.168 & -0.473 & -0.241 & -0.132 & -0.021 & 0.200 \\
\hline y0[24] & 0.106 & 0.197 & -0.299 & -0.022 & 0.111 & 0.238 & 0.485 \\
\hline $\mathrm{y} 0[25]$ & -0.104 & 0.267 & -0.627 & -0.285 & -0.113 & 0.065 & 0.435 \\
\hline N.sum[1] & $1,927.028$ & 338.362 & $1,336.795$ & $1,693.044$ & $1,907.307$ & $2,137.283$ & $2,662.535$ \\
\hline N.sum[2] & $10,790.505$ & $1,229.516$ & $8,633.971$ & $9,917.648$ & $10,711.414$ & $11,563.961$ & $13,427.118$ \\
\hline N.sum[3] & $13,579.140$ & $1,355.589$ & $11,127.002$ & $12,621.061$ & $13,511.121$ & $14,471.476$ & $16,446.824$ \\
\hline N.sum[4] & $13,299.813$ & $1,295.410$ & $10,992.075$ & $12,396.592$ & $13,224.553$ & $14,128.935$ & $16,039.057$ \\
\hline N.sum[5] & $15,388.054$ & $1,391.868$ & $12,860.507$ & $14,399.795$ & $15,336.579$ & $16,290.128$ & $18,303.501$ \\
\hline N.sum[6] & $10,729.592$ & $1,087.021$ & $8,823.794$ & $9,968.114$ & $10,656.486$ & $11,423.622$ & $13,082.260$ \\
\hline N.sum[7] & $12,693.419$ & $1,349.997$ & $10,226.998$ & $11,734.888$ & $12,652.316$ & $13,537.504$ & $15,533.170$ \\
\hline N.sum[8] & $11,962.124$ & $1,148.504$ & $9,852.907$ & $11,156.776$ & $11,907.313$ & $12,721.277$ & $14,338.303$ \\
\hline N.sum[9] & $16,339.939$ & $1,385.372$ & $13,804.781$ & $15,408.282$ & $16,244.228$ & $17,220.989$ & $19,275.731$ \\
\hline N.sum[10] & $19,780.932$ & $1,676.010$ & $16,734.048$ & $18,635.660$ & $19,678.482$ & $20,833.972$ & $23,345.200$ \\
\hline N.sum[11] & $16,299.668$ & $1,468.737$ & $13,588.817$ & $15,253.396$ & $16,245.268$ & $17,263.452$ & $19,378.865$ \\
\hline N.sum[12] & $14,550.582$ & $1,271.487$ & $12,184.402$ & $13,654.989$ & $14,502.450$ & $15,378.443$ & $17,165.210$ \\
\hline N.sum[13] & $14,302.009$ & $1,244.775$ & $12,037.309$ & $13,443.486$ & $14,243.967$ & $15,085.311$ & $16,898.768$ \\
\hline N.sum[14] & $16,781.976$ & $1,388.744$ & $14,246.403$ & $15,819.471$ & $16,699.481$ & $17,689.562$ & $19,738.530$ \\
\hline N.sum[15] & $15,169.631$ & $1,398.356$ & $12,597.065$ & $14,199.792$ & $15,121.283$ & $16,074.261$ & $18,031.043$ \\
\hline N.sum[16] & $22,369.429$ & $1,840.063$ & $18,984.887$ & $21,089.134$ & $22,295.478$ & $23,574.384$ & $26,270.985$ \\
\hline N.sum[17] & $18,547.763$ & $1,606.158$ & $15,598.427$ & $17,411.189$ & $18,485.804$ & $19,596.456$ & $21,864.848$ \\
\hline N.sum[18] & $21,826.305$ & $1,794.308$ & $18,554.247$ & $20,581.479$ & $21,738.303$ & $22,996.292$ & $25,525.237$ \\
\hline N.sum[19] & $18,887.968$ & $1,721.043$ & $15,757.876$ & $17,681.071$ & $18,804.744$ & $19,983.130$ & $22,502.811$ \\
\hline N.sum[20] & $19,101.064$ & $1,701.755$ & $15,956.506$ & $17,939.901$ & $19,011.456$ & $20,176.182$ & $22,683.185$ \\
\hline
\end{tabular}




\begin{tabular}{|c|c|c|c|c|c|c|c|}
\hline N.sum[21] & $19,016.303$ & $1,686.309$ & $15,967.474$ & $17,858.585$ & $18,910.456$ & $20,094.730$ & $22,635.443$ \\
\hline N.sum[22] & $21,844.198$ & $1,924.742$ & $18,355.171$ & $20,494.271$ & $21,761.197$ & $23,060.075$ & $25,899.605$ \\
\hline N.sum[23] & $15,050.634$ & $1,426.188$ & $12,436.012$ & $14,060.884$ & $14,979.157$ & $15,954.104$ & $18,038.813$ \\
\hline N.sum[24] & $17,786.520$ & $1,590.591$ & $14,895.513$ & $16,667.353$ & $17,702.464$ & $18,829.744$ & $21,088.665$ \\
\hline N.sum[25] & $13,315.487$ & $1,214.459$ & $11,092.582$ & $12,475.663$ & $13,267.391$ & $14,081.979$ & $15,845.606$ \\
\hline N.sum2[1] & $5,636.634$ & $1,845.482$ & $2,826.882$ & $4,236.002$ & $5,375.227$ & $6,793.328$ & $9,871.087$ \\
\hline N.sum2[2] & $7,244.934$ & $1,541.261$ & $4,796.294$ & $6,128.731$ & $7,043.765$ & $8,157.739$ & $10,801.355$ \\
\hline N.sum2[3] & $9,310.841$ & $1,422.268$ & $6,935.925$ & $8,347.451$ & $9,153.648$ & $10,122.612$ & $12,502.290$ \\
\hline N.sum2[4] & $11,595.527$ & $1,949.634$ & $8,582.420$ & $10,222.845$ & $11,307.455$ & $12,653.605$ & $16,063.337$ \\
\hline N.sum2[5] & $13,621.813$ & $2,600.034$ & $9,862.112$ & $11,782.363$ & $13,152.114$ & $14,921.869$ & $19,956.235$ \\
\hline N.sum2[6] & $14,979.613$ & $2,818.354$ & $10,872.135$ & $12,985.994$ & $14,490.336$ & $16,410.050$ & $21,918.945$ \\
\hline N.sum2[7] & $15,621.209$ & $2,675.341$ & $11,661.290$ & $13,728.928$ & $15,176.692$ & $17,052.448$ & $21,996.711$ \\
\hline N.sum2[8] & $15,809.468$ & $2,516.304$ & $12,042.457$ & $14,039.111$ & $15,476.464$ & $17,095.556$ & $22,034.515$ \\
\hline N.sum2[9] & $15,875.074$ & $2,459.100$ & $11,854.242$ & $14,234.317$ & $15,579.808$ & $17,170.493$ & $21,490.902$ \\
\hline N.sum2[10] & $16,057.005$ & $2,404.692$ & $11,843.375$ & $14,490.020$ & $15,837.795$ & $17,377.623$ & $21,541.856$ \\
\hline N.sum2[11] & $16,470.052$ & $2,298.766$ & $12,397.924$ & $14,944.680$ & $16,287.184$ & $17,806.420$ & $21,518.190$ \\
\hline N.sum2[12] & $17,117.894$ & $2,188.280$ & $13,262.503$ & $15,595.715$ & $16,985.661$ & $18,486.005$ & $21,774.225$ \\
\hline N.sum2[13] & $17,917.471$ & $2,201.882$ & $14,209.548$ & $16,299.724$ & $17,777.059$ & $19,333.950$ & $22,642.240$ \\
\hline N.sum2[14] & $18,739.521$ & $2,437.723$ & $14,816.406$ & $17,069.378$ & $18,497.911$ & $19,990.811$ & $24,483.910$ \\
\hline N.sum2[15] & $19,462.433$ & $2,767.402$ & $15,280.375$ & $17,609.849$ & $19,088.257$ & $20,768.390$ & $26,416.991$ \\
\hline N.sum2[16] & $20,012.817$ & $2,933.071$ & $15,462.655$ & $18,056.947$ & $19,645.106$ & $21,446.208$ & $27,594.197$ \\
\hline N.sum2[17] & $20,385.210$ & $2,839.452$ & $15,533.931$ & $18,490.093$ & $20,070.604$ & $21,997.390$ & $26,909.212$ \\
\hline N.sum2[18] & $20,620.826$ & $2,690.925$ & $15,704.918$ & $18,778.684$ & $20,396.959$ & $22,288.012$ & $26,268.555$ \\
\hline N.sum2[19] & $20,740.399$ & $2,794.062$ & $15,997.596$ & $18,729.616$ & $20,440.120$ & $22,438.241$ & $26,904.586$ \\
\hline N.sum2[20] & $20,677.191$ & $3,076.295$ & $15,749.072$ & $18,545.711$ & $20,226.767$ & $22,414.426$ & $27,959.329$ \\
\hline N.sum2[21] & $20,293.663$ & $3,123.405$ & $14,983.489$ & $18,213.313$ & $19,921.853$ & $21,996.449$ & $27,706.757$ \\
\hline N.sum2[22] & $19,504.295$ & $2,760.124$ & $14,507.484$ & $17,638.330$ & $19,284.643$ & $21,103.703$ & $25,576.917$ \\
\hline N.sum2[23] & $18,405.496$ & $2,506.648$ & $14,046.722$ & $16,747.005$ & $18,155.642$ & $19,811.619$ & $24,203.954$ \\
\hline N.sum2[24] & $17,246.657$ & $3,135.390$ & $12,202.862$ & $15,093.911$ & $16,883.871$ & $18,981.935$ & $24,541.345$ \\
\hline N.sum2[25] & $16,260.671$ & $4,388.402$ & $8,924.095$ & $13,231.330$ & $15,847.049$ & $18,716.807$ & $26,603.599$ \\
\hline R.growth[1] & 0.282 & 0.149 & 0.059 & 0.166 & 0.257 & 0.387 & 0.611 \\
\hline R.growth[2] & 0.261 & 0.132 & 0.060 & 0.159 & 0.242 & 0.355 & 0.550 \\
\hline R.growth[3] & 0.217 & 0.099 & 0.058 & 0.139 & 0.208 & 0.288 & 0.435 \\
\hline R.growth[4] & 0.158 & 0.065 & 0.043 & 0.110 & 0.154 & 0.202 & 0.291 \\
\hline R.growth[5] & 0.095 & 0.054 & -0.018 & 0.064 & 0.095 & 0.128 & 0.202 \\
\hline R.growth[6] & 0.045 & 0.065 & -0.108 & 0.007 & 0.053 & 0.088 & 0.153 \\
\hline R.growth[7] & 0.014 & 0.071 & -0.150 & -0.028 & 0.027 & 0.064 & 0.127 \\
\hline R.growth[8] & 0.005 & 0.065 & -0.150 & -0.036 & 0.015 & 0.053 & 0.106 \\
\hline R.growth[9] & 0.012 & 0.056 & -0.109 & -0.024 & 0.019 & 0.051 & 0.107 \\
\hline R.growth[10] & 0.027 & 0.053 & -0.080 & -0.007 & 0.029 & 0.062 & 0.128 \\
\hline R.growth[11] & 0.040 & 0.055 & -0.064 & 0.006 & 0.037 & 0.070 & 0.170 \\
\hline R.growth[12] & 0.046 & 0.057 & -0.054 & 0.011 & 0.040 & 0.075 & 0.188 \\
\hline R.growth[13] & 0.044 & 0.056 & -0.062 & 0.011 & 0.040 & 0.072 & 0.183 \\
\hline R.growth[14] & 0.037 & 0.050 & -0.067 & 0.006 & 0.036 & 0.067 & 0.151 \\
\hline
\end{tabular}




\begin{tabular}{lrrrrrrr} 
R.growth[15] & 0.027 & 0.044 & -0.062 & -0.001 & 0.028 & 0.058 & 0.111 \\
R.growth[16] & 0.019 & 0.044 & -0.074 & -0.008 & 0.021 & 0.049 & 0.099 \\
R.growth[17] & 0.012 & 0.051 & -0.106 & -0.014 & 0.014 & 0.045 & 0.112 \\
R.growth[18] & 0.005 & 0.058 & -0.139 & -0.021 & 0.010 & 0.039 & 0.119 \\
R.growth[19] & -0.005 & 0.056 & -0.140 & -0.031 & -0.001 & 0.030 & 0.097 \\
R.growth[20] & -0.020 & 0.046 & -0.114 & -0.049 & -0.018 & 0.011 & 0.074 \\
R.growth[21] & -0.038 & 0.051 & -0.141 & -0.072 & -0.036 & -0.003 & 0.062 \\
R.growth[22] & -0.057 & 0.078 & -0.239 & -0.101 & -0.048 & -0.006 & 0.084 \\
R.growth[23] & -0.072 & 0.106 & -0.320 & -0.131 & -0.060 & -0.006 & 0.127 \\
R.growth[24] & -0.079 & 0.120 & -0.363 & -0.146 & -0.065 & -0.005 & 0.150 \\
Deviance & $41,463.463$ & 47.358 & $41,372.782$ & $41,431.734$ & $41,463.143$ & $41,494.556$ & $41,560.223$ \\
\hline
\end{tabular}


Tables A4.15. Bayesian generalized linear mixed model output for White-winged Scoter observed during aerial surveys 1992-2016 on the Arctic Coastal Plain, Alaska. We provide summaries including mean, SE, and several quantiles of the posterior distribution for model and derived parameters. See Table A4.4 for a key to parameter names.

\begin{tabular}{|c|c|c|c|c|c|c|c|}
\hline Parameter & Mean & SD & $2.5 \%$ & $25 \%$ & $50 \%$ & $75 \%$ & $97.5 \%$ \\
\hline MSE & 3.645 & 1.424 & 2.611 & 2.906 & 3.248 & 3.852 & 7.136 \\
\hline MSE.CV & 7.910 & 3.091 & 5.666 & 6.308 & 7.049 & 8.361 & 15.487 \\
\hline SSE & $10,868.093$ & $4,246.454$ & $7,785.235$ & $8,666.627$ & $9,685.490$ & $11,488.148$ & $21,279.073$ \\
\hline Trend & 1.116 & 0.036 & 1.051 & 1.092 & 1.114 & 1.138 & 1.191 \\
\hline R.trend & 0.109 & 0.032 & 0.050 & 0.088 & 0.108 & 0.129 & 0.175 \\
\hline M.dens & 0.334 & 0.069 & 0.226 & 0.286 & 0.325 & 0.372 & 0.490 \\
\hline M.y0 & -0.012 & 0.158 & -0.333 & -0.111 & -0.012 & 0.086 & 0.309 \\
\hline M.blyr & -2.028 & 0.207 & -2.441 & -2.164 & -2.023 & -1.889 & -1.638 \\
\hline M.cell & 0.000 & 0.013 & -0.029 & -0.006 & 0.000 & 0.005 & 0.028 \\
\hline R.mid & -1.978 & 0.088 & -2.150 & -2.037 & -1.977 & -1.919 & -1.806 \\
\hline b.ADOY & -1.863 & 0.189 & -2.256 & -1.987 & -1.857 & -1.731 & -1.514 \\
\hline b.ADOY.2 & -0.701 & 0.131 & -0.965 & -0.788 & -0.700 & -0.610 & -0.448 \\
\hline Sd.y0 & 0.763 & 0.186 & 0.452 & 0.631 & 0.743 & 0.873 & 1.179 \\
\hline SD.cell & 0.172 & 0.104 & 0.029 & 0.089 & 0.155 & 0.239 & 0.407 \\
\hline $\mathrm{y} 0[1]$ & 1.073 & 0.592 & -0.002 & 0.662 & 1.048 & 1.459 & 2.298 \\
\hline $\mathrm{y} 0[2]$ & -1.498 & 0.689 & -2.974 & -1.917 & -1.439 & -1.017 & -0.299 \\
\hline $\mathrm{y} 0[3]$ & 0.518 & 0.445 & -0.359 & 0.224 & 0.516 & 0.809 & 1.408 \\
\hline $\mathrm{y} 0[4]$ & -0.188 & 0.423 & -1.027 & -0.467 & -0.189 & 0.094 & 0.639 \\
\hline $\mathrm{y} 0[5]$ & -0.196 & 0.402 & -1.009 & -0.457 & -0.190 & 0.072 & 0.576 \\
\hline $\mathrm{y} 0[6]$ & 0.232 & 0.430 & -0.605 & -0.052 & 0.224 & 0.513 & 1.097 \\
\hline $\mathrm{y} 0[7]$ & -0.312 & 0.443 & -1.213 & -0.603 & -0.305 & -0.020 & 0.557 \\
\hline $\mathrm{y} 0[8]$ & 0.366 & 0.418 & -0.436 & 0.085 & 0.357 & 0.640 & 1.205 \\
\hline $\mathrm{y} 0[9]$ & 0.031 & 0.470 & -0.904 & -0.277 & 0.035 & 0.342 & 0.956 \\
\hline $\mathrm{y} 0[10]$ & -0.467 & 0.490 & -1.469 & -0.789 & -0.455 & -0.139 & 0.466 \\
\hline y0[11] & -0.556 & 0.447 & -1.448 & -0.849 & -0.552 & -0.250 & 0.298 \\
\hline y0[12] & 0.283 & 0.414 & -0.510 & -0.004 & 0.283 & 0.553 & 1.128 \\
\hline y0[13] & -0.124 & 0.473 & -1.041 & -0.438 & -0.124 & 0.188 & 0.824 \\
\hline $\mathrm{y} 0[14]$ & -0.106 & 0.410 & -0.893 & -0.379 & -0.109 & 0.162 & 0.719 \\
\hline $\mathrm{y} 0[15]$ & -0.660 & 0.470 & -1.586 & -0.974 & -0.656 & -0.338 & 0.239 \\
\hline y0[16] & 0.125 & 0.368 & -0.588 & -0.125 & 0.122 & 0.368 & 0.860 \\
\hline y0[17] & 0.530 & 0.367 & -0.169 & 0.282 & 0.524 & 0.770 & 1.275 \\
\hline y0[18] & -0.763 & 0.445 & -1.647 & -1.060 & -0.759 & -0.466 & 0.111 \\
\hline y0[19] & 0.820 & 0.372 & 0.109 & 0.570 & 0.810 & 1.061 & 1.574 \\
\hline y0[20] & -0.362 & 0.381 & -1.113 & -0.612 & -0.363 & -0.113 & 0.397 \\
\hline y0[21] & 0.709 & 0.377 & -0.008 & 0.456 & 0.698 & 0.956 & 1.478 \\
\hline $\mathrm{y} 0[22]$ & 0.753 & 0.429 & -0.039 & 0.461 & 0.737 & 1.025 & 1.644 \\
\hline $\mathrm{y} 0[23]$ & 0.488 & 0.408 & -0.291 & 0.215 & 0.480 & 0.752 & 1.316 \\
\hline $\mathrm{y} 0[24]$ & -0.415 & 0.425 & -1.257 & -0.695 & -0.415 & -0.138 & 0.422 \\
\hline $\mathrm{y} 0[25]$ & -0.589 & 0.457 & -1.492 & -0.886 & -0.586 & -0.289 & 0.304 \\
\hline SD.lam[1] & 0.543 & 0.215 & 0.262 & 0.402 & 0.500 & 0.625 & 1.152 \\
\hline SD.lam[2] & 3.764 & 1.905 & 1.336 & 2.339 & 3.277 & 4.754 & 8.670 \\
\hline
\end{tabular}




\begin{tabular}{|c|c|c|c|c|c|c|c|}
\hline SD.lam[3] & 4.176 & 2.181 & 1.151 & 2.450 & 3.709 & 5.575 & 9.193 \\
\hline SD.lam[4] & 2.971 & 2.084 & 0.124 & 1.418 & 2.538 & 4.084 & 8.211 \\
\hline SD.lam[5] & 4.512 & 2.789 & 0.239 & 2.124 & 4.276 & 6.788 & 9.631 \\
\hline SD.lam[6] & 2.735 & 0.896 & 1.666 & 2.149 & 2.537 & 3.047 & 5.060 \\
\hline SD.lam[7] & 5.289 & 2.269 & 2.123 & 3.364 & 4.601 & 7.284 & 9.604 \\
\hline SD.lam[8] & 1.181 & 1.368 & 0.081 & 0.357 & 0.703 & 1.456 & 5.215 \\
\hline N.sum[1] & $1,969.064$ & $1,307.754$ & 548.586 & $1,104.729$ & $1,633.498$ & $2,437.726$ & $5,427.412$ \\
\hline N.sum[2] & 170.352 & 132.087 & 25.927 & 80.461 & 135.674 & 221.290 & 513.814 \\
\hline N.sum[3] & $1,125.896$ & 546.451 & 442.484 & 743.602 & $1,007.354$ & $1,370.656$ & $2,476.059$ \\
\hline N.sum[4] & 573.799 & 252.858 & 240.594 & 400.190 & 524.402 & 689.088 & $1,202.281$ \\
\hline N.sum[5] & 601.306 & 252.131 & 251.801 & 422.904 & 553.066 & 725.591 & $1,221.177$ \\
\hline N.sum[6] & $1,010.335$ & 483.606 & 396.909 & 675.911 & 907.254 & $1,222.156$ & $2,233.103$ \\
\hline N.sum[7] & 645.516 & 327.734 & 228.490 & 417.600 & 575.752 & 790.392 & $1,449.592$ \\
\hline N.sum[8] & $1,372.963$ & 638.217 & 543.992 & 927.609 & $1,236.838$ & $1,662.445$ & $2,977.650$ \\
\hline N.sum[9] & $1,124.845$ & 594.089 & 372.345 & 714.935 & 997.884 & $1,387.934$ & $2,617.132$ \\
\hline N.sum[10] & 776.520 & 418.378 & 237.568 & 483.628 & 685.853 & 967.806 & $1,834.091$ \\
\hline N.sum[11] & 786.087 & 372.363 & 283.603 & 523.399 & 714.391 & 968.560 & $1,697.199$ \\
\hline N.sum[12] & $2,048.559$ & 893.260 & 833.271 & $1,412.971$ & $1,881.443$ & $2,488.479$ & $4,212.870$ \\
\hline N.sum[13] & $1,606.676$ & 811.244 & 568.320 & $1,047.406$ & $1,434.919$ & $1,962.976$ & $3,678.254$ \\
\hline N.sum[14] & $1,833.870$ & 794.917 & 741.137 & $1,274.497$ & $1,679.045$ & $2,226.536$ & $3,825.804$ \\
\hline N.sum[15] & $1,252.558$ & 638.484 & 432.422 & 811.316 & $1,117.000$ & $1,539.915$ & $2,860.466$ \\
\hline N.sum[16] & $2,988.636$ & $1,013.017$ & $1,550.875$ & $2,263.473$ & $2,817.891$ & $3,514.389$ & $5,465.198$ \\
\hline N.sum[17] & $5,187.216$ & $1,621.361$ & $2,821.026$ & $4,044.730$ & $4,908.459$ & $6,058.261$ & $9,119.999$ \\
\hline N.sum[18] & $1,739.053$ & 789.091 & 708.904 & $1,186.177$ & $1,574.519$ & $2,096.742$ & $3,733.035$ \\
\hline N.sum[19] & $9,424.111$ & $3,097.446$ & $5,026.746$ & $7,292.040$ & $8,906.626$ & $10,957.956$ & $16,906.811$ \\
\hline N.sum[20] & $3,345.398$ & $1,141.083$ & $1,678.861$ & $2,534.056$ & $3,155.204$ & $3,937.542$ & $6,126.667$ \\
\hline N.sum[21] & $10,916.963$ & $3,159.249$ & $6,267.743$ & $8,679.045$ & $10,409.342$ & $12,576.516$ & $18,422.078$ \\
\hline N.sum[22] & $13,237.514$ & $5,460.255$ & $6,044.489$ & $9,417.775$ & $12,125.005$ & $15,755.691$ & $27,098.525$ \\
\hline N.sum[23] & $11,006.090$ & $3,809.556$ & $5,674.811$ & $8,349.842$ & $10,306.180$ & $12,885.995$ & $20,408.531$ \\
\hline N.sum[24] & $4,922.924$ & $1,731.182$ & $2,489.686$ & $3,722.742$ & $4,605.375$ & $5,798.920$ & $9,022.623$ \\
\hline N.sum[25] & $4,545.058$ & $1,550.359$ & $2,361.306$ & $3,466.030$ & $4,267.287$ & $5,302.377$ & $8,390.257$ \\
\hline N.sum2[1] & 645.736 & 370.725 & 194.985 & 389.487 & 556.660 & 800.923 & $1,592.819$ \\
\hline N.sum2[2] & 646.612 & 329.130 & 225.176 & 416.374 & 573.918 & 792.141 & $1,484.711$ \\
\hline N.sum2[3] & 658.566 & 303.259 & 255.331 & 444.223 & 596.934 & 800.738 & $1,428.901$ \\
\hline N.sum2[4] & 681.695 & 288.661 & 288.261 & 476.729 & 625.210 & 821.059 & $1,410.439$ \\
\hline N.sum2[5] & 716.024 & 280.850 & 324.056 & 517.157 & 665.225 & 853.317 & $1,418.106$ \\
\hline N.sum2[6] & 762.343 & 277.722 & 358.605 & 564.543 & 715.263 & 905.122 & $1,444.553$ \\
\hline N.sum2[7] & 822.515 & 280.165 & 404.379 & 622.989 & 778.182 & 973.231 & $1,490.857$ \\
\hline N.sum2[8] & 899.522 & 289.138 & 448.652 & 695.547 & 858.466 & $1,062.127$ & $1,573.971$ \\
\hline N.sum2[9] & 997.082 & 304.323 & 508.930 & 784.882 & 959.611 & $1,167.433$ & $1,699.623$ \\
\hline N.sum2[10] & $1,119.199$ & 325.936 & 577.062 & 895.781 & $1,085.988$ & $1,305.853$ & $1,857.451$ \\
\hline N.sum2[11] & $1,269.588$ & 355.698 & 659.551 & $1,029.087$ & $1,240.573$ & $1,471.445$ & $2,062.644$ \\
\hline N.sum2[12] & $1,450.671$ & 394.494 & 759.870 & $1,191.186$ & $1,426.677$ & $1,673.438$ & $2,312.878$ \\
\hline N.sum2[13] & $1,662.964$ & 439.771 & 889.159 & $1,378.958$ & $1,634.689$ & $1,904.943$ & $2,612.129$ \\
\hline N.sum2[14] & $1,907.972$ & 488.989 & $1,065.018$ & $1,594.464$ & $1,866.461$ & $2,170.177$ & $3,003.502$ \\
\hline N.sum2[15] & $2,196.493$ & 547.441 & $1,284.878$ & $1,844.033$ & $2,137.714$ & $2,480.986$ & $3,451.700$ \\
\hline
\end{tabular}




\begin{tabular}{|c|c|c|c|c|c|c|c|}
\hline N.sum2[16] & $2,544.345$ & 626.800 & $1,534.881$ & $2,132.499$ & $2,462.287$ & $2,857.594$ & $4,029.902$ \\
\hline N.sum2[17] & $2,965.498$ & 737.454 & $1,833.060$ & $2,469.276$ & $2,857.337$ & $3,331.333$ & $4,765.885$ \\
\hline N.sum2[18] & $3,465.824$ & 884.718 & $2,126.859$ & $2,857.032$ & $3,329.304$ & $3,912.770$ & $5,644.982$ \\
\hline N.sum2[19] & $4,037.867$ & $1,069.338$ & $2,424.172$ & $3,293.750$ & $3,875.693$ & $4,590.014$ & $6,586.413$ \\
\hline N.sum2[20] & ,659.193 & $1,283.151$ & $2,720.272$ & $3,759.076$ & $4,466.291$ & $5,330.198$ & $7,675.188$ \\
\hline N.sum2[21] & ,303.192 & $1,509.811$ & $2,992.680$ & $4,240.685$ & $5,097.633$ & $6,117.264$ & $8,854.505$ \\
\hline N.sum2[22] & $5,957.608$ & $1,762.151$ & $3,195.035$ & $4,704.607$ & $5,718.388$ & $6,937.484$ & $10,017.359$ \\
\hline N.sum2[23] & $6,646.686$ & $2,142.295$ & $3,368.622$ & $5,136.171$ & $6,327.580$ & $7,821.060$ & $11,605.365$ \\
\hline N.sum2[24] & $7,439.202$ & $2,810.627$ & $3,425.814$ & $5,498.410$ & $6,956.386$ & $8,841.280$ & $14,242.373$ \\
\hline N.sum2[25] & $8,431.664$ & $3,900.588$ & $3,281.277$ & $5,775.800$ & $7,653.651$ & $10,178.222$ & $18,153.689$ \\
\hline R.growth[1] & 0.030 & 0.101 & -0.191 & -0.026 & 0.041 & 0.093 & 0.207 \\
\hline R.growth[2] & 0.039 & 0.094 & -0.164 & -0.016 & 0.049 & 0.097 & 0.202 \\
\hline R.growth[3] & 0.049 & 0.083 & -0.128 & -0.002 & 0.058 & 0.103 & 0.191 \\
\hline R.growth[4] & 0.060 & 0.073 & -0.096 & 0.014 & 0.068 & 0.109 & 0.184 \\
\hline R.growth[5] & 0.071 & 0.067 & -0.080 & 0.031 & 0.080 & 0.116 & 0.186 \\
\hline R.growth[6] & 0.083 & 0.065 & -0.065 & 0.046 & 0.090 & 0.125 & 0.193 \\
\hline R.growth[7] & 0.095 & 0.062 & -0.045 & 0.062 & 0.101 & 0.134 & 0.205 \\
\hline R.growth[8] & 0.107 & 0.061 & -0.024 & 0.074 & 0.111 & 0.144 & 0.218 \\
\hline R.growth[9] & 0.119 & 0.061 & -0.009 & 0.085 & 0.120 & 0.155 & 0.238 \\
\hline R.growth[10] & 0.129 & 0.062 & & 0.094 & 0.128 & & 0.258 \\
\hline R.growth[11] & 0.136 & 0.060 & 0.023 & 0.100 & 0.133 & 0.168 & 0.269 \\
\hline R.growth[12] & 0.139 & 0.056 & 0.037 & 0.105 & 0.135 & 0.168 & 0.267 \\
\hline R.growth[13] & 0.140 & 0.057 & 0.042 & 0.105 & 0.134 & 0.169 & 0.270 \\
\hline R.growth[14] & 0.143 & 0.061 & & 0.104 & 0.135 & 0.174 & 0.287 \\
\hline R.growth[15] & 0.148 & 0.065 & 0.042 & 0.107 & 0.139 & 0.180 & 0.305 \\
\hline R.growth[16] & 0.153 & 0.066 & 0.048 & 0.111 & 0.143 & 0.185 & 0.315 \\
\hline R.growth[17] & 0.154 & 0.065 & 0.051 & 0.112 & 0.145 & 0.187 & 0.313 \\
\hline R.growth[18] & 0.150 & 0.064 & 0.040 & 0.109 & 0.143 & 0.184 & 0.303 \\
\hline R.growth[19] & 0.140 & 0.064 & 0.022 & 0.101 & 0.136 & 0.176 & 0.280 \\
\hline R.growth[20] & 0.127 & 0.064 & -0.001 & 0.087 & 0.126 & 0.165 & 0.262 \\
\hline R.growth[21] & 0.113 & 0.071 & -0.033 & 0.068 & 0.113 & 0.157 & 0.254 \\
\hline R.growth[22] & 0.102 & 0.086 & -0.076 & 0.051 & 0.105 & 0.155 & 0.265 \\
\hline R.growth[23] & 0.097 & 0.101 & -0.117 & 0.040 & 0.102 & 0.158 & 0.285 \\
\hline R.growth[24] & 0.097 & 0.110 & -0.140 & 0.037 & 0.104 & 0.163 & 0.303 \\
\hline Deviance & $3,247.895$ & 12.804 & $3,223.167$ & $3,239.177$ & $3,247.774$ & $3,256.234$ & $3,273.567$ \\
\hline
\end{tabular}


Tables A4.16. Bayesian generalized linear mixed model output for Long-tailed Duck observed during aerial surveys 1992-2016 on the Arctic Coastal Plain, Alaska. We provide summaries including mean, SE, and several quantiles of the posterior distribution for model and derived parameters. See Table A4.4 for a key to parameter names.

\begin{tabular}{|c|c|c|c|c|c|c|c|}
\hline Parameter & Mean & SD & $2.50 \%$ & $25 \%$ & $50 \%$ & $75 \%$ & $97.50 \%$ \\
\hline MSE & 8.304 & 0.079 & 8.152 & 8.249 & 8.304 & 8.358 & 8.458 \\
\hline MSE.CV & 4.539 & 0.043 & 4.455 & 4.509 & 4.539 & 4.568 & 4.623 \\
\hline SSE & $216,902.8$ & $2,074.4$ & $212,919.6$ & $215,475.6$ & $216,896.7$ & $218,307.5$ & $220,912.2$ \\
\hline Trend & 0.993 & 0.004 & 0.986 & 0.990 & 0.993 & 0.995 & 1.000 \\
\hline R.trend & -0.007 & 0.004 & -0.015 & -0.010 & -0.007 & -0.005 & 0.000 \\
\hline M.dens & 0.814 & 0.011 & 0.792 & 0.806 & 0.814 & 0.821 & 0.835 \\
\hline M.y0 & 0.001 & 0.021 & -0.044 & -0.012 & 0.001 & 0.015 & 0.039 \\
\hline M.yz & -0.271 & 0.029 & -0.329 & -0.291 & -0.271 & -0.251 & -0.215 \\
\hline M.blyr & 0.316 & 0.024 & 0.271 & 0.298 & 0.315 & 0.332 & 0.365 \\
\hline M.cell & 0.000 & 0.005 & -0.010 & -0.003 & 0.000 & 0.003 & 0.010 \\
\hline M.psi & 0.449 & 0.004 & 0.442 & 0.447 & 0.449 & 0.451 & 0.456 \\
\hline R.mid & 2.451 & 0.055 & 2.343 & 2.414 & 2.450 & 2.487 & 2.562 \\
\hline b.ADOY & 0.008 & 0.015 & -0.020 & -0.002 & 0.008 & 0.018 & 0.037 \\
\hline b.ADOY.2 & -0.005 & 0.006 & -0.018 & -0.010 & -0.005 & -0.001 & 0.007 \\
\hline z.ADOY & 0.120 & 0.028 & 0.063 & 0.100 & 0.120 & 0.139 & 0.173 \\
\hline z.ADOY.2 & 0.038 & 0.013 & 0.012 & 0.029 & 0.038 & 0.047 & 0.065 \\
\hline SD.surv & 0.189 & 0.046 & 0.117 & 0.156 & 0.183 & 0.214 & 0.294 \\
\hline SD.surv.z & 0.394 & 0.094 & 0.250 & 0.328 & 0.382 & 0.447 & 0.608 \\
\hline SD.y0 & 0.102 & 0.019 & 0.072 & 0.089 & 0.100 & 0.113 & 0.144 \\
\hline SD.yz & 0.374 & 0.065 & 0.267 & 0.328 & 0.366 & 0.411 & 0.519 \\
\hline SD.cell & 0.209 & 0.010 & 0.191 & 0.202 & 0.209 & 0.216 & 0.228 \\
\hline SD.cell.z & 0.545 & 0.022 & 0.501 & 0.530 & 0.546 & 0.560 & 0.590 \\
\hline SD.lam[1] & 0.147 & 0.043 & 0.079 & 0.112 & 0.143 & 0.176 & 0.241 \\
\hline SD.lam[2] & 0.362 & 0.118 & 0.194 & 0.280 & 0.342 & 0.419 & 0.649 \\
\hline SD.lam[3] & 0.705 & 0.912 & 0.012 & 0.120 & 0.441 & 0.889 & 3.586 \\
\hline SD.lam[4] & 0.168 & 0.176 & 0.006 & 0.050 & 0.116 & 0.226 & 0.642 \\
\hline SD.lam[5] & 3.905 & 4.979 & 0.037 & 0.402 & 1.550 & 5.650 & 17.614 \\
\hline SD.lam[6] & 0.744 & 0.187 & 0.440 & 0.610 & 0.717 & 0.852 & 1.171 \\
\hline SD.lam[7] & 0.648 & 0.281 & 0.203 & 0.474 & 0.661 & 0.818 & 1.237 \\
\hline SD.lam[8] & 0.331 & 0.482 & 0.039 & 0.097 & 0.176 & 0.359 & 1.651 \\
\hline S.eff[1] & -0.033 & 0.062 & -0.155 & -0.074 & -0.032 & 0.008 & 0.090 \\
\hline S.eff[2] & 0.110 & 0.056 & -0.001 & 0.072 & 0.109 & 0.147 & 0.219 \\
\hline S.eff[3] & 0.107 & 0.055 & 0.000 & 0.069 & 0.107 & 0.144 & 0.217 \\
\hline S.eff[4] & 0.098 & 0.054 & -0.007 & 0.062 & 0.098 & 0.134 & 0.206 \\
\hline S.eff[5] & 0.116 & 0.053 & 0.014 & 0.081 & 0.116 & 0.151 & 0.221 \\
\hline S.eff[6] & 0.049 & 0.060 & -0.065 & 0.008 & 0.049 & 0.090 & 0.168 \\
\hline S.eff[7] & 0.118 & 0.059 & 0.001 & 0.078 & 0.118 & 0.159 & 0.232 \\
\hline S.eff[8] & 0.283 & 0.074 & 0.140 & 0.233 & 0.283 & 0.331 & 0.434 \\
\hline S.eff[9] & -0.241 & 0.071 & -0.382 & -0.289 & -0.242 & -0.193 & -0.101 \\
\hline
\end{tabular}




\begin{tabular}{|c|c|c|c|c|c|c|c|}
\hline S.eff[10] & 0.118 & 0.056 & 0.008 & 0.081 & 0.118 & 0.156 & 0.228 \\
\hline S.eff[11] & -0.029 & 0.053 & -0.132 & -0.064 & -0.030 & 0.007 & 0.077 \\
\hline S.eff[12] & 0.314 & 0.064 & 0.187 & 0.271 & 0.313 & 0.358 & 0.437 \\
\hline S.eff[13] & 0.244 & 0.061 & 0.123 & 0.202 & 0.245 & 0.285 & 0.365 \\
\hline S.eff[14] & 0.124 & 0.057 & 0.013 & 0.085 & 0.123 & 0.162 & 0.237 \\
\hline S.eff[15] & 0.076 & 0.059 & -0.038 & 0.036 & 0.075 & 0.116 & 0.193 \\
\hline S.eff.z[1] & 0.123 & 0.136 & -0.144 & 0.033 & 0.123 & 0.213 & 0.395 \\
\hline S.eff.z[2] & -0.086 & 0.129 & -0.339 & -0.173 & -0.087 & 0.002 & 0.168 \\
\hline S.eff.z[3] & 0.073 & 0.130 & -0.175 & -0.016 & 0.071 & 0.161 & 0.334 \\
\hline S.eff.z[4] & -0.087 & 0.130 & -0.342 & -0.175 & -0.088 & 0.002 & 0.167 \\
\hline S.eff.z[5] & -0.304 & 0.134 & -0.568 & -0.393 & -0.304 & -0.211 & -0.042 \\
\hline S.eff.z[6] & 0.109 & 0.140 & -0.165 & 0.013 & 0.106 & 0.203 & 0.386 \\
\hline S.eff.z[7] & 0.240 & 0.141 & -0.034 & 0.144 & 0.239 & 0.334 & 0.525 \\
\hline S.eff.z[8] & 0.470 & 0.154 & 0.177 & 0.368 & 0.468 & 0.571 & 0.781 \\
\hline S.eff.z[9] & 0.816 & 0.156 & 0.518 & 0.710 & 0.815 & 0.917 & 1.126 \\
\hline S.eff.z[10] & 0.297 & 0.135 & 0.036 & 0.206 & 0.296 & 0.386 & 0.562 \\
\hline S.eff.z[11] & 0.643 & 0.143 & 0.371 & 0.544 & 0.640 & 0.739 & 0.928 \\
\hline S.eff.z[12] & -0.145 & 0.134 & -0.406 & -0.235 & -0.145 & -0.055 & 0.121 \\
\hline S.eff.z[13] & -0.244 & 0.131 & -0.498 & -0.332 & -0.243 & -0.153 & 0.009 \\
\hline S.eff.z[14] & 0.165 & 0.132 & -0.081 & 0.076 & 0.164 & 0.255 & 0.425 \\
\hline S.eff.z[15] & 0.108 & 0.135 & -0.149 & 0.014 & 0.107 & 0.196 & 0.386 \\
\hline $\mathrm{y} 0[1]$ & 0.011 & 0.062 & -0.116 & -0.029 & 0.012 & 0.052 & 0.128 \\
\hline $\mathrm{y} 0[2]$ & 0.025 & 0.053 & -0.084 & -0.010 & 0.026 & 0.060 & 0.125 \\
\hline $\mathrm{y} 0[3]$ & -0.064 & 0.049 & -0.165 & -0.095 & -0.063 & -0.031 & 0.028 \\
\hline $\mathrm{y} 0[4]$ & 0.062 & 0.045 & -0.030 & 0.033 & 0.063 & 0.092 & 0.149 \\
\hline $\mathrm{y} 0[5]$ & 0.085 & 0.043 & -0.002 & 0.057 & 0.085 & 0.114 & 0.169 \\
\hline $\mathrm{y} 0[6]$ & -0.036 & 0.043 & -0.121 & -0.065 & -0.036 & -0.007 & 0.045 \\
\hline $\mathrm{y} 0[7]$ & -0.049 & 0.045 & -0.140 & -0.078 & -0.049 & -0.020 & 0.038 \\
\hline $\mathrm{y} 0[8]$ & -0.172 & 0.047 & -0.267 & -0.204 & -0.171 & -0.139 & -0.084 \\
\hline $\mathrm{y} 0[9]$ & 0.119 & 0.044 & 0.033 & 0.090 & 0.118 & 0.147 & 0.205 \\
\hline y0[10] & 0.084 & 0.042 & -0.001 & 0.056 & 0.083 & 0.112 & 0.169 \\
\hline y0[11] & 0.158 & 0.041 & 0.077 & 0.131 & 0.157 & 0.185 & 0.240 \\
\hline $\mathrm{y} 0[12]$ & -0.163 & 0.049 & -0.264 & -0.195 & -0.162 & -0.129 & -0.067 \\
\hline y0[13] & -0.129 & 0.048 & -0.225 & -0.160 & -0.128 & -0.097 & -0.035 \\
\hline y0[14] & -0.050 & 0.043 & -0.136 & -0.080 & -0.050 & -0.020 & 0.033 \\
\hline y0[15] & 0.022 & 0.046 & -0.069 & -0.008 & 0.022 & 0.052 & 0.111 \\
\hline y0[16] & 0.014 & 0.046 & -0.075 & -0.017 & 0.014 & 0.045 & 0.105 \\
\hline y0[17] & 0.032 & 0.042 & -0.053 & 0.003 & 0.032 & 0.061 & 0.113 \\
\hline y0[18] & 0.071 & 0.043 & -0.015 & 0.042 & 0.071 & 0.100 & 0.156 \\
\hline y0[19] & 0.022 & 0.045 & -0.066 & -0.007 & 0.023 & 0.052 & 0.110 \\
\hline y0[20] & 0.126 & 0.044 & 0.040 & 0.096 & 0.127 & 0.157 & 0.212 \\
\hline y0[21] & -0.047 & 0.049 & -0.142 & -0.080 & -0.047 & -0.015 & 0.047 \\
\hline y0[22] & -0.035 & 0.049 & -0.134 & -0.068 & -0.034 & -0.002 & 0.059 \\
\hline $\mathrm{y} 0[23]$ & -0.099 & 0.052 & -0.203 & -0.133 & -0.098 & -0.063 & -0.003 \\
\hline
\end{tabular}




\begin{tabular}{|c|c|c|c|c|c|c|c|}
\hline $\mathrm{y} 0[24]$ & 0.067 & 0.053 & -0.039 & 0.033 & 0.068 & 0.102 & 0.167 \\
\hline $\mathrm{y} 0[25]$ & -0.030 & 0.059 & -0.149 & -0.069 & -0.029 & 0.008 & 0.082 \\
\hline$y z[1]$ & -0.286 & 0.097 & -0.478 & -0.352 & -0.285 & -0.222 & -0.098 \\
\hline$y z[2]$ & -0.149 & 0.095 & -0.339 & -0.213 & -0.147 & -0.086 & 0.034 \\
\hline$y z[3]$ & -0.266 & 0.091 & -0.450 & -0.327 & -0.264 & -0.204 & -0.089 \\
\hline$y z[4]$ & -0.328 & 0.083 & -0.495 & -0.383 & -0.328 & -0.272 & -0.167 \\
\hline$y z[5]$ & -0.404 & 0.085 & -0.572 & -0.461 & -0.404 & -0.347 & -0.238 \\
\hline$y z[6]$ & -0.463 & 0.086 & -0.634 & -0.520 & -0.462 & -0.403 & -0.299 \\
\hline$y z[7]$ & -0.408 & 0.099 & -0.602 & -0.475 & -0.406 & -0.339 & -0.217 \\
\hline$y z[8]$ & -0.234 & 0.097 & -0.426 & -0.297 & -0.234 & -0.169 & -0.047 \\
\hline$y z[9]$ & -0.301 & 0.095 & -0.490 & -0.366 & -0.300 & -0.238 & -0.119 \\
\hline yz[10] & -0.421 & 0.095 & -0.612 & -0.484 & -0.420 & -0.358 & -0.239 \\
\hline$y z[11]$ & -0.668 & 0.105 & -0.875 & -0.737 & -0.666 & -0.597 & -0.464 \\
\hline yz[12] & 0.048 & 0.095 & -0.141 & -0.015 & 0.048 & 0.110 & 0.232 \\
\hline$y z[13]$ & 0.052 & 0.091 & -0.131 & -0.009 & 0.051 & 0.113 & 0.232 \\
\hline yz[14] & -0.227 & 0.090 & -0.406 & -0.288 & -0.226 & -0.165 & -0.054 \\
\hline yz[15] & -0.135 & 0.095 & -0.325 & -0.198 & -0.134 & -0.070 & 0.049 \\
\hline yz[16] & 0.054 & 0.083 & -0.112 & 0.000 & 0.055 & 0.110 & 0.216 \\
\hline yz[17] & -0.410 & 0.086 & -0.582 & -0.468 & -0.408 & -0.353 & -0.242 \\
\hline yz[18] & -0.414 & 0.087 & -0.590 & -0.473 & -0.414 & -0.354 & -0.244 \\
\hline yz[19] & -0.158 & 0.085 & -0.327 & -0.215 & -0.156 & -0.100 & 0.004 \\
\hline$y z[20]$ & -0.690 & 0.091 & -0.873 & -0.750 & -0.690 & -0.628 & -0.518 \\
\hline$y z[21]$ & 0.064 & 0.083 & -0.102 & 0.009 & 0.066 & 0.121 & 0.224 \\
\hline$y z[22]$ & -0.259 & 0.089 & -0.434 & -0.320 & -0.257 & -0.198 & -0.086 \\
\hline$y z[23]$ & -0.312 & 0.091 & -0.491 & -0.371 & -0.310 & -0.251 & -0.136 \\
\hline$y z[24]$ & -0.513 & 0.091 & -0.691 & -0.573 & -0.512 & -0.452 & -0.332 \\
\hline$y z[25]$ & 0.054 & 0.087 & -0.120 & -0.005 & 0.054 & 0.113 & 0.223 \\
\hline N.sum[1] & $45,940.904$ & $2,456.112$ & $41,355.745$ & $44,257.495$ & $45,875.766$ & $47,609.907$ & $50,807.665$ \\
\hline N.sum[2] & $43,881.349$ & $2,307.279$ & $39,435.606$ & $42,302.225$ & $43,841.012$ & $45,417.105$ & $48,546.992$ \\
\hline N.sum[3] & $42,035.808$ & $1,979.006$ & $38,363.811$ & $40,656.716$ & $41,985.506$ & $43,354.497$ & $46,022.924$ \\
\hline N.sum[4] & $48,769.173$ & $2,066.773$ & $44,774.021$ & $47,371.213$ & $48,738.306$ & $50,147.003$ & $52,899.610$ \\
\hline N.sum[5] & $51,281.620$ & $2,095.919$ & $47,308.367$ & $49,861.356$ & $51,258.481$ & $52,709.518$ & $55,436.248$ \\
\hline N.sum[6] & $46,320.677$ & $1,899.842$ & $42,574.943$ & $45,027.472$ & $46,287.985$ & $47,598.532$ & $50,065.634$ \\
\hline N.sum[7] & $44,701.466$ & $2,161.124$ & $40,508.317$ & $43,260.454$ & $44,634.258$ & $46,147.251$ & $49,026.481$ \\
\hline N.sum[8] & $36,844.809$ & $1,846.280$ & $33,254.258$ & $35,568.746$ & $36,819.325$ & $38,083.488$ & $40,474.639$ \\
\hline N.sum[9] & $50,461.149$ & $2,391.818$ & $45,929.688$ & $48,786.658$ & $50,415.623$ & $52,089.427$ & $55,194.432$ \\
\hline N.sum[10] & $50,861.717$ & $2,298.148$ & $46,483.174$ & $49,260.349$ & $50,838.305$ & $52,388.600$ & $55,480.654$ \\
\hline N.sum[11] & $59,453.275$ & $2,573.262$ & $54,527.450$ & $57,716.304$ & $59,444.475$ & $61,164.630$ & $64,610.733$ \\
\hline N.sum[12] & $32,517.942$ & $1,806.688$ & $29,141.704$ & $31,281.712$ & $32,469.583$ & $33,705.178$ & $36,140.240$ \\
\hline N.sum[13] & $33,547.703$ & $1,808.775$ & $30,101.318$ & $32,303.154$ & $33,529.448$ & $34,738.774$ & $37,173.966$ \\
\hline N.sum[14] & $41,008.332$ & $1,927.380$ & $37,287.621$ & $39,694.264$ & $41,000.781$ & $42,284.027$ & $44,876.560$ \\
\hline N.sum[15] & $42,404.958$ & $2,149.043$ & $38,242.968$ & $40,949.255$ & $42,369.351$ & $43,829.684$ & $46,742.387$ \\
\hline N.sum[16] & $38,664.962$ & $1,858.833$ & $35,147.014$ & $37,385.086$ & $38,634.218$ & $39,939.362$ & $42,372.648$ \\
\hline N.sum[17] & $47,845.033$ & $1,931.147$ & $44,132.824$ & $46,528.330$ & $47,827.433$ & $49,109.946$ & $51,751.275$ \\
\hline
\end{tabular}


N.sum[18]

N.sum[19]

N.sum[20]

N.sum[21]

N.sum[22]

N.sum[23]

N.sum[24]

N.sum[25]

N.sum2[1]

N.sum2[2]

N.sum2[3]

N.sum2[4]

N.sum2[5]

N.sum2[6]

N.sum2[7]

N.sum2[8]

N.sum2[9]

N.sum2[10]

N.sum2[11]

N.sum2[12]

N.sum2[13]

N.sum2[14]

N.sum2[15]

N.sum2[16]

N.sum2[17]

N.sum2[18]

N.sum2[19]

N.sum2[20]

N.sum2[21]

N.sum2[22]

N.sum2[23]

N.sum2[24]

N.sum2[25]

R.growth[1]

R.growth[2]

R.growth[3]

R.growth[4]

R.growth[5]

R.growth[6]

R.growth[7]

R.growth[8]

R.growth[9]

R.growth[10]

R.growth[11]
$49,854.135$

$42,869.270$

$57,817.925$

$36,143.115$

42,111.289

$40,350.184$

$51,368.665$

$37,058.388$

$45,742.432$

43,081.123

$45,113.928$

$46,140.067$

$47,402.608$

$48,337.335$

$47,248.888$

$44,024.580$

$45,090.937$

47,062.322

$51,093.171$

$38,495.916$

$38,384.539$

43,379.592

$41,744.606$

$38,365.001$

46,646.615

$46,721.739$

$42,182.638$

$51,277.702$

$38,132.663$

$43,891.572$

$44,842.050$

$48,360.355$

$38,452.175$

$-0.060$

0.046

0.023

0.027

0.020

$-0.023$

$-0.071$

0.024

0.043

0.082

$-0.283$
2,086.109

$1,945.238$

$2,223.323$

$1,756.668$

$1,919.436$

$1,835.374$

$2,156.982$

$1,832.282$

$2,842.617$

$2,465.330$

$2,286.366$

2,047.991

$1,998.057$

$1,919.913$

$2,034.213$

$1,990.346$

$1,948.848$

$1,929.510$

2,063.966

$1,883.405$

$1,830.702$

$1,858.452$

$1,914.211$

$1,714.514$

$1,821.302$

$1,846.409$

$1,821.083$

$1,991.797$

$1,815.631$

$2,057.821$

$2,189.456$

$2,423.989$

$2,248.314$

$$
0.056
$$

0.049

0.045

0.042

0.043

0.046

0.051

0.052

0.050

0.046

0.056
$45,842.956$

$39,153.973$

$53,572.814$

$32,814.021$

$38,417.122$

$36,834.712$

$47,272.873$

$33,544.605$

$40,435.588$

$38,471.666$

$40,813.587$

$42,232.704$

$43,622.892$

$44,682.835$

$43,342.338$

$40,151.666$

$41,376.706$

$43,283.901$

$46,955.143$

$34,847.767$

$34,727.109$

$39,773.925$

$38,121.893$

$35,080.225$

$43,148.668$

$43,177.471$

$38,728.099$

$47,440.082$

$34,704.293$

$40,020.486$

$40,808.122$

$43,830.447$

$34,132.970$

$-0.170$

$-0.050$

$-0.063$

$-0.054$

$-0.062$

$-0.114$

$-0.170$

$-0.075$

$-0.055$

$-0.009$

$-0.392$
$48,446.186$

$41,547.584$

$56,266.378$

$34,951.056$

$40,813.307$

$39,128.706$

$49,926.936$

$35,812.417$

$43,824.836$

$41,411.508$

$43,553.060$

$44,739.291$

$46,034.231$

$47,009.628$

$45,863.873$

$42,709.652$

$43,771.917$

$45,770.406$

$49,766.218$

$37,249.853$

$37,183.038$

$42,145.589$

$40,447.154$

$37,213.903$

$45,402.717$

$45,463.231$

$40,935.402$

$49,947.162$

$36,902.481$

$42,522.339$

$43,372.518$

$46,718.000$

$36,935.409$

$-0.098$

0.013

$-0.008$

$-0.001$

$-0.009$

$-0.053$

$-0.104$

$-0.010$

0.010

0.052

$-0.321$
$49,827.407$

$42,846.300$

$57,791.000$

$36,099.956$

$42,086.338$

$40,318.581$

$51,336.624$

$37,045.306$

$45,631.138$

$42,999.400$

$45,055.896$

$46,112.571$

$47,359.554$

$48,311.948$

$47,244.381$

$44,023.675$

$45,049.033$

$47,051.087$

$51,102.995$

$38,491.251$

$38,391.953$

$43,359.988$

$41,723.340$

$38,355.093$

$46,625.993$

$46,710.636$

$42,139.520$

$51,243.445$

$38,085.316$

$43,828.034$

$44,757.652$

$48,261.633$

$38,393.167$

$-0.059$

0.047

0.023

0.026

0.019

$-0.023$

$-0.071$

0.023

0.043

0.082

$-0.283$

Appendix 4 -Summary Tables 60

$51,231.218 \quad 53,965.540$

$44,167.279$

$46,760.131$

$59,300.330$

$37,299.105$

$43,389.676$

$41,535.339$

$52,794.712$

$38,251.429$

$47,523.082$

$44,707.736$

$46,584.162$

$47,469.437$

$48,746.746$

$49,629.786$

$48,629.485$

$45,310.159$

$46,373.672$

$48,360.560$

$52,463.893$ 


\begin{tabular}{lrrrrrrr} 
R.growth[12] & -0.003 & 0.061 & -0.120 & -0.045 & -0.003 & 0.038 & 0.119 \\
R.growth[13] & 0.123 & 0.056 & 0.016 & 0.084 & 0.122 & 0.161 & 0.233 \\
R.growth[14] & -0.039 & 0.055 & -0.145 & -0.075 & -0.038 & -0.003 & 0.070 \\
R.growth[15] & -0.084 & 0.056 & -0.195 & -0.122 & -0.084 & -0.046 & 0.023 \\
R.growth[16] & 0.196 & 0.049 & 0.098 & 0.163 & 0.195 & 0.229 & 0.293 \\
R.growth[17] & 0.002 & 0.044 & -0.085 & -0.028 & 0.002 & 0.032 & 0.087 \\
R.growth[18] & -0.102 & 0.048 & -0.196 & -0.135 & -0.102 & -0.069 & -0.009 \\
R.growth[19] & 0.195 & 0.045 & 0.108 & 0.164 & 0.196 & 0.226 & 0.285 \\
R.growth[20] & -0.297 & 0.049 & -0.391 & -0.329 & -0.296 & -0.264 & -0.202 \\
R.growth[21] & 0.141 & 0.053 & 0.038 & 0.105 & 0.140 & 0.176 & 0.246 \\
R.growth[22] & 0.021 & 0.049 & -0.075 & -0.011 & 0.021 & 0.054 & 0.122 \\
R.growth[23] & 0.075 & 0.047 & -0.018 & 0.044 & 0.076 & 0.107 & 0.167 \\
R.growth[24] & -0.230 & 0.052 & -0.329 & -0.265 & -0.229 & -0.196 & -0.128 \\
Deviance & $61,298.857$ & 224.283 & $60,869.105$ & $61,143.749$ & $61,296.687$ & $61,450.610$ & $61,746.722$ \\
\hline
\end{tabular}


Tables A4.17. Bayesian generalized linear mixed model output for Red-breasted Merganser observed during aerial surveys 1992-2016 on the Arctic Coastal Plain, Alaska. We provide summaries including mean, SE, and several quantiles of the posterior distribution for model and derived parameters. See Table A4.4 for a key to parameter names.

\begin{tabular}{|c|c|c|c|c|c|c|c|}
\hline Parameter & Mean & SD & $2.50 \%$ & $25 \%$ & $50 \%$ & $75 \%$ & $97.50 \%$ \\
\hline MSE & 0.484 & 0.011 & 0.473 & 0.477 & 0.481 & 0.487 & 0.510 \\
\hline MSE.CV & 2.772 & 0.061 & 2.713 & 2.736 & 2.756 & 2.789 & 2.923 \\
\hline SSE & $3,226.105$ & 70.918 & $3,157.545$ & $3,184.574$ & $3,208.099$ & $3,246.773$ & $3,401.791$ \\
\hline Trend & 1.038 & 0.016 & 1.009 & 1.028 & 1.038 & 1.049 & 1.072 \\
\hline R.trend & 0.038 & 0.015 & 0.009 & 0.027 & 0.037 & 0.047 & 0.070 \\
\hline M.dens & 0.085 & 0.009 & 0.069 & 0.079 & 0.084 & 0.090 & 0.103 \\
\hline М.y0 & -0.001 & 0.033 & -0.068 & -0.014 & 0.000 & 0.013 & 0.069 \\
\hline M.blyr & -2.649 & 0.105 & -2.857 & -2.720 & -2.649 & -2.578 & -2.443 \\
\hline M.cell & 0.000 & 0.005 & -0.010 & -0.002 & 0.000 & 0.002 & 0.010 \\
\hline R.mid & -2.694 & 0.076 & -2.844 & -2.745 & -2.694 & -2.643 & -2.546 \\
\hline b.ADOY & 0.024 & 0.107 & -0.196 & -0.047 & 0.028 & 0.099 & 0.219 \\
\hline b.ADOY.2 & -0.074 & 0.065 & -0.200 & -0.119 & -0.073 & -0.030 & 0.052 \\
\hline SD.surv & 0.998 & 0.292 & 0.533 & 0.796 & 0.960 & 1.162 & 1.695 \\
\hline SD.y0 & 0.137 & 0.090 & 0.011 & 0.064 & 0.126 & 0.195 & 0.340 \\
\hline SD.cell & 0.075 & 0.046 & 0.014 & 0.041 & 0.067 & 0.097 & 0.196 \\
\hline SD.lam[1] & 0.082 & 0.050 & 0.023 & 0.042 & 0.067 & 0.118 & 0.196 \\
\hline SD.lam[2] & 0.812 & 0.568 & 0.071 & 0.408 & 0.711 & 1.113 & 2.249 \\
\hline SD.lam[3] & 0.898 & 0.796 & 0.074 & 0.379 & 0.701 & 1.164 & 2.985 \\
\hline SD.lam[4] & 1.319 & 1.684 & 0.028 & 0.344 & 0.746 & 1.565 & 6.291 \\
\hline SD.lam[5] & 5.550 & 4.005 & 0.284 & 2.173 & 4.586 & 8.393 & 14.148 \\
\hline SD.lam[6] & 1.516 & 0.899 & 0.363 & 0.683 & 1.469 & 2.250 & 3.254 \\
\hline SD.lam[7] & 5.736 & 2.574 & 2.158 & 3.685 & 5.398 & 7.300 & 11.801 \\
\hline SD.lam[8] & 0.691 & 1.540 & 0.010 & 0.098 & 0.238 & 0.556 & 5.467 \\
\hline S.eff[1] & 0.321 & 0.505 & -0.629 & -0.021 & 0.315 & 0.653 & 1.328 \\
\hline S.eff[2] & 0.570 & 0.457 & -0.308 & 0.263 & 0.551 & 0.868 & 1.514 \\
\hline S.eff[3] & 0.442 & 0.456 & -0.408 & 0.137 & 0.426 & 0.737 & 1.391 \\
\hline S.eff[4] & 1.372 & 0.421 & 0.573 & 1.088 & 1.365 & 1.649 & 2.215 \\
\hline S.eff[5] & 0.957 & 0.451 & 0.107 & 0.650 & 0.943 & 1.249 & 1.877 \\
\hline S.eff[6] & -0.273 & 0.514 & -1.272 & -0.616 & -0.278 & 0.071 & 0.733 \\
\hline S.eff[7] & 1.302 & 0.469 & 0.433 & 0.979 & 1.291 & 1.608 & 2.279 \\
\hline S.eff[8] & 0.484 & 0.486 & -0.447 & 0.150 & 0.476 & 0.801 & 1.473 \\
\hline S.eff[9] & -1.251 & 0.642 & -2.600 & -1.667 & -1.216 & -0.801 & -0.095 \\
\hline S.eff[10] & 1.316 & 0.463 & 0.441 & 0.996 & 1.303 & 1.620 & 2.244 \\
\hline S.eff[11] & 0.516 & 0.401 & -0.241 & 0.241 & 0.511 & 0.779 & 1.325 \\
\hline S.eff[12] & 0.140 & 0.405 & -0.626 & -0.135 & 0.132 & 0.402 & 0.955 \\
\hline S.eff[13] & 0.024 & 0.427 & -0.788 & -0.259 & 0.011 & 0.306 & 0.881 \\
\hline S.eff[14] & 0.274 & 0.421 & -0.529 & -0.012 & 0.268 & 0.548 & 1.112 \\
\hline S.eff[15] & 0.719 & 0.400 & -0.034 & 0.442 & 0.711 & 0.985 & 1.527 \\
\hline $\mathrm{y} 0[1]$ & 0.008 & 0.152 & -0.296 & -0.061 & 0.002 & 0.068 & 0.355 \\
\hline
\end{tabular}




\begin{tabular}{|c|c|c|c|c|c|c|c|}
\hline $\mathrm{y} 0[2]$ & -0.030 & 0.149 & -0.371 & -0.095 & -0.013 & 0.041 & 0.264 \\
\hline $\mathrm{y} 0[3]$ & -0.086 & 0.165 & -0.510 & -0.159 & -0.043 & 0.009 & 0.168 \\
\hline $\mathrm{y} 0[4]$ & 0.027 & 0.144 & -0.262 & -0.043 & 0.012 & 0.091 & 0.356 \\
\hline $\mathrm{y} 0[5]$ & 0.153 & 0.182 & -0.072 & 0.014 & 0.101 & 0.249 & 0.609 \\
\hline $\mathrm{y} 0[6]$ & -0.043 & 0.151 & -0.405 & -0.107 & -0.019 & 0.033 & 0.228 \\
\hline $\mathrm{y} 0[7]$ & -0.052 & 0.151 & -0.414 & -0.116 & -0.024 & 0.026 & 0.215 \\
\hline $\mathrm{y} 0[8]$ & -0.015 & 0.143 & -0.332 & -0.075 & -0.005 & 0.051 & 0.275 \\
\hline $\mathrm{y} 0[9]$ & -0.019 & 0.141 & -0.343 & -0.082 & -0.008 & 0.047 & 0.269 \\
\hline y0[10] & -0.006 & 0.142 & -0.320 & -0.070 & -0.003 & 0.059 & 0.299 \\
\hline y0[11] & 0.008 & 0.140 & -0.285 & -0.054 & 0.003 & 0.070 & 0.318 \\
\hline y0[12] & -0.031 & 0.145 & -0.373 & -0.095 & -0.012 & 0.039 & 0.249 \\
\hline y0[13] & 0.033 & 0.141 & -0.242 & -0.037 & 0.014 & 0.097 & 0.368 \\
\hline y0[14] & 0.012 & 0.136 & -0.268 & -0.052 & 0.005 & 0.075 & 0.314 \\
\hline $\mathrm{y} 0[15]$ & 0.003 & 0.140 & -0.294 & -0.063 & 0.001 & 0.067 & 0.310 \\
\hline y0[16] & 0.024 & 0.137 & -0.249 & -0.041 & 0.009 & 0.086 & 0.337 \\
\hline y0[17] & 0.054 & 0.142 & -0.199 & -0.023 & 0.027 & 0.117 & 0.393 \\
\hline y0[18] & 0.015 & 0.137 & -0.265 & -0.050 & 0.006 & 0.074 & 0.320 \\
\hline y0[19] & -0.073 & 0.160 & -0.471 & -0.143 & -0.035 & 0.016 & 0.18 \\
\hline y0[20] & -0.024 & 0.145 & -0.356 & -0.089 & -0.011 & 0.043 & 0.25 \\
\hline y0[21] & -0.040 & 0.144 & -0.379 & -0.102 & -0.019 & 0.032 & 0.22 \\
\hline $\mathrm{y} 0[22]$ & 0.048 & 0.145 & -0.211 & -0.027 & 0.021 & 0.110 & 0.41 \\
\hline $\mathrm{y} 0[23]$ & 0.004 & 0.142 & -0.294 & -0.061 & 0.002 & 0.069 & 0.31 \\
\hline y0[24] & 0.045 & 0.144 & -0.210 & -0.030 & 0.020 & 0.108 & 0.39 \\
\hline y0[25] & -0.035 & 0.142 & -0.371 & -0.100 & -0.016 & 0.035 & 0.236 \\
\hline N.sum[1] & 751.890 & 261.999 & 346.034 & 573.294 & 715.279 & 889.701 & $1,354.379$ \\
\hline N.sum[2] & 688.674 & 195.843 & 373.022 & 550.737 & 666.338 & 805.552 & $1,141.154$ \\
\hline N.sum[3] & 646.040 & 164.421 & 349.779 & 533.549 & 634.225 & 747.084 & $1,011.528$ \\
\hline N.sum[4] & 735.508 & 169.173 & 457.817 & 617.459 & 712.983 & 832.718 & $1,114.869$ \\
\hline N.sum[5] & 870.389 & 216.114 & 550.388 & 719.158 & 833.572 & 982.576 & $1,399.202$ \\
\hline N.sum[6] & 745.677 & 164.303 & 461.182 & 631.152 & 734.433 & 842.060 & $1,113.525$ \\
\hline N.sum[7] & 773.539 & 171.048 & 461.654 & 658.803 & 764.701 & 876.435 & $1,137.327$ \\
\hline N.sum[8] & 834.398 & 175.043 & 532.846 & 715.197 & 820.217 & 934.667 & $1,218.611$ \\
\hline N.sum[9] & 864.677 & 170.621 & 564.812 & 748.778 & 853.358 & 970.530 & $1,238.454$ \\
\hline N.sum[10] & 918.437 & 188.739 & 593.254 & 791.876 & 902.731 & $1,027.099$ & $1,329.816$ \\
\hline N.sum[11] & 980.536 & 197.047 & 643.565 & 847.512 & 961.928 & $1,093.733$ & $1,421.651$ \\
\hline N.sum[12] & 995.693 & 193.709 & 647.210 & 865.469 & 983.034 & $1,107.202$ & $1,412.344$ \\
\hline N.sum[13] & $1,122.510$ & 233.935 & 753.468 & 964.854 & $1,091.990$ & $1,241.884$ & $1,679.499$ \\
\hline N.sum[14] & $1,151.628$ & 224.916 & 790.509 & 996.967 & $1,123.832$ & $1,276.347$ & $1,660.868$ \\
\hline N.sum[15] & $1,195.941$ & 238.966 & 805.045 & $1,034.049$ & $1,166.682$ & $1,330.531$ & $1,757.710$ \\
\hline N.sum[16] & $1,275.184$ & 242.824 & 891.869 & $1,112.154$ & $1,245.848$ & $1,405.700$ & $1,845.184$ \\
\hline N.sum[17] & $1,377.936$ & 261.284 & 967.989 & $1,195.567$ & $1,342.688$ & $1,516.134$ & $1,977.100$ \\
\hline N.sum[18] & $1,391.663$ & 257.192 & 960.917 & $1,220.893$ & $1,365.580$ & $1,534.793$ & $1,969.345$ \\
\hline N.sum[19] & $1,337.457$ & 257.382 & 841.515 & $1,168.418$ & $1,327.866$ & $1,495.171$ & $1,880.68$ \\
\hline N.sum[20] & $1,455.217$ & 268.491 & 986.796 & $1,273.778$ & $1,432.623$ & $1,603.573$ & $2,043.15$ \\
\hline
\end{tabular}




\begin{tabular}{|c|c|c|c|c|c|c|c|}
\hline N.sum[21] & $1,475.242$ & 272.408 & 988.912 & $1,295.720$ & $1,456.424$ & $1,633.771$ & .604 \\
\hline N.sum[22] & $1,656.017$ & 331.544 & $1,149.626$ & $1,430.794$ & $1,608.518$ & $1,826.008$ & $2,439.853$ \\
\hline N.sum[23] & ,628.706 & 22.080 & $1,102.309$ & $1,414.208$ & $1,593.400$ & $1,799.311$ & $2,380.774$ \\
\hline N.sum[24] & ,761.459 & 370.856 & $1,187.688$ & $1,509.833$ & $1,705.995$ & $1,950.716$ & $2,651.665$ \\
\hline N.sum[25] &, 717.467 & 385.548 & $1,081.410$ & $1,450.481$ & $1,679.410$ & $1,940.772$ & $2,600.91$ \\
\hline N.sum2[1] & 751.875 & 246.978 & 351.010 & 582.000 & 724.454 & 886.966 & $1,314.23$ \\
\hline N.sum2[2] & 712.575 & 180.495 & 414.775 & 584.278 & 693.581 & 818.437 & $1,118.86$ \\
\hline N.sum2[3] & 705.187 & 149.558 & 454.839 & 599.227 & 691.718 & 793.586 & $1,040.585$ \\
\hline N.sum2[4] & 720.250 & 142.785 & 482.748 & 619.346 & 707.563 & 804.800 & $1,037.36$ \\
\hline N.sum2[5] & 748.196 & 142.879 & 513.852 & 648.718 & 734.309 & 831.307 & $1,069.1 ?$ \\
\hline N.sum2[6] & 781.604 & 139.700 & 544.493 & 682.496 & 769.873 & 866.133 & $1,092.0$ \\
\hline N.sum2[7] & 816.021 & 136.499 & 574.251 & 719.634 & 805.636 & 903.441 & $1,104.65$ \\
\hline N.sum2[8] & 849.852 & 136.721 & 601.377 & 753.288 & 841.471 & 937.928 & $1,139.70$ \\
\hline N.sum2[9] & 885.881 & 139.050 & 635.252 & 788.854 & 879.964 & 976.453 & $1,180.61$ \\
\hline N.sum2[10] & 927.840 & 142.923 & 672.216 & 829.092 & 920.756 & $1,018.747$ & 1,2 \\
\hline N.sum2[11] & 977.559 & 150.622 & 715.091 & 875.022 & 966.704 & $1,066.580$ & $1,309.22$ \\
\hline N.sum2[12] & $1,033.391$ & 162.293 & 764.800 & 924.585 & $1,017.807$ & $1,124.311$ & $1,396.03$ \\
\hline N.sum2[13] & $1,090.471$ & 172.562 & 815.773 & 972.996 & $1,071.802$ & $1,184.118$ & $1,482.67$ \\
\hline N.sum2[14] & $1,144.557$ & 177.531 & 865.156 & $1,021.955$ & $1,124.618$ & $1,242.638$ & $1,551.69$ \\
\hline N.sum2[15] & $1,197.268$ & 180.442 & 911.436 & $1,073.308$ & $1,176.785$ & $1,298.252$ & $1,613.51($ \\
\hline N.sum2[16] & $1,252.873$ & 185.691 & 949.159 & $1,125.343$ & $1,233.336$ & $1,357.079$ & $1,671.167$ \\
\hline N.sum2[17] & $1,314.040$ & 192.477 & 983.963 & $1,184.010$ & $1,295.812$ & $1,421.856$ & $1,737.93$ \\
\hline N.sul & $1,379.556$ & 197.691 & $1,034.718$ & $1,245.136$ & $1,364.537$ & $1,496.910$ & 1,8 \\
\hline N.sum2[19] & $1,443.734$ & 201.789 & $1,094.022$ & $1,301.470$ & $1,428.428$ & $1,567.417$ & $1,880.95$ \\
\hline N.sum2[20] & $1,499.136$ & 208.753 & $1,143.138$ & $1,350.644$ & $1,482.848$ & $1,624.599$ & $1,962.8$ \\
\hline N.sum2[21] & $1,545.045$ & 220.910 & $1,174.043$ & $1,388.698$ & $1,525.418$ & $1,677.596$ & $2,035.93$ \\
\hline N.sum2[22] & $1,585.820$ & 234.476 & $1,192.804$ & $1,421.389$ & $1,563.094$ & $1,722.885$ & $2,112.42$ \\
\hline N.sum2[23] & $1,630.901$ & 252.507 & $1,208.286$ & & $1,605.245$ & $1,784.507$ & 2,2 \\
\hline N.sum2[24] & $1,695.821$ & 297.868 & $1,193.227$ & $1,487.080$ & $1,659.390$ & $1,872.745$ & 2,3 \\
\hline N.sum2[25] & $1,799.683$ & 394.987 & $1,143.800$ & $1,526.281$ & $1,758.157$ & $2,029.750$ & $2,700.87$ \\
\hline R.growth[1] & -0.032 & 0.114 & -0.243 & -0.092 & -0.035 & 0.015 & 0.26 \\
\hline R.growth[2] & -0.001 & 0.092 & -0.159 & -0.051 & -0.007 & 0.033 & 0.25 \\
\hline R.growth[3] & 0.024 & 0.065 & -0.087 & -0.012 & 0.019 & 0.050 & 0.19 \\
\hline R.growth[4] & 0.040 & 0.043 & -0.042 & 0.014 & 0.038 & 0.063 & 0.13 \\
\hline R.growth[5] & 0.046 & 0.040 & -0.046 & 0.025 & 0.047 & 0.069 & 0.12 \\
\hline R.growth[6] & 0.045 & 0.044 & -0.070 & 0.027 & 0.049 & 0.070 & 0.12 \\
\hline R.growth[7] & 0.042 & 0.043 & -0.069 & 0.025 & 0.045 & 0.065 & 0.11 \\
\hline R.growth[8] & 0.042 & 0.039 & -0.046 & 0.024 & 0.042 & 0.061 & 0.11 \\
\hline R.growth[9] & 0.047 & 0.038 & -0.022 & 0.026 & 0.043 & 0.064 & 0.13 \\
\hline R.growth[10] & 0.052 & 0.040 & -0.010 & 0.029 & 0.046 & 0.069 & 0.15 \\
\hline R.growth[11] & 0.055 & 0.039 & -0.003 & 0.031 & 0.049 & 0.072 & 0.15 \\
\hline R.growth[12] & 0.054 & 0.035 & -0.003 & 0.032 & 0.049 & 0.069 & 0.14 \\
\hline R.growth[13] & 0.049 & 0.035 & -0.015 & 0.030 & 0.047 & 0.065 & 0.12 \\
\hline R.growth[14] & 0.046 & 0.039 & -0.037 & 0.029 & 0.047 & 0.064 & 0.11 \\
\hline
\end{tabular}




\begin{tabular}{lrrrrrrr} 
R.growth[15] & 0.046 & 0.040 & -0.049 & 0.029 & 0.049 & 0.067 & 0.117 \\
R.growth[16] & 0.048 & 0.037 & -0.037 & 0.030 & 0.050 & 0.070 & 0.116 \\
R.growth[17] & 0.049 & 0.035 & -0.027 & 0.029 & 0.050 & 0.071 & 0.116 \\
R.growth[18] & 0.046 & 0.037 & -0.033 & 0.026 & 0.046 & 0.067 & 0.120 \\
R.growth[19] & 0.038 & 0.040 & -0.041 & 0.017 & 0.038 & 0.057 & 0.121 \\
R.growth[20] & 0.030 & 0.039 & -0.044 & 0.008 & 0.030 & 0.050 & 0.113 \\
R.growth[21] & 0.025 & 0.038 & -0.052 & 0.002 & 0.027 & 0.049 & 0.099 \\
R.growth[22] & 0.027 & 0.046 & -0.074 & 0.000 & 0.031 & 0.057 & 0.113 \\
R.growth[23] & 0.036 & 0.062 & -0.106 & 0.003 & 0.040 & 0.073 & 0.149 \\
R.growth[24] & 0.051 & 0.077 & -0.122 & 0.012 & 0.053 & 0.094 & 0.206 \\
Deviance & $5,313.262$ & 12.987 & $5,287.470$ & $5,304.646$ & $5,313.559$ & $5,322.097$ & $5,338.074$ \\
\hline
\end{tabular}


Tables A4.18. Bayesian generalized linear mixed model output for Jaeger (Pomarine, Parasitic, and Longtailed combined) observed during aerial surveys 1992-2016 on the Arctic Coastal Plain, Alaska. We provide summaries including mean, SE, and several quantiles of the posterior distribution for model and derived parameters. See Table A4.4 for a key to parameter names.

\begin{tabular}{|c|c|c|c|c|c|c|c|}
\hline Parameter & Mean & SD & $2.50 \%$ & $25 \%$ & $50 \%$ & $75 \%$ & $97.50 \%$ \\
\hline MSE & 0.424 & 0.001 & 0.422 & 0.423 & 0.424 & 0.425 & 0.427 \\
\hline MSE.CV & 1.468 & 0.005 & 1.460 & 1.465 & 1.468 & 1.471 & 1.477 \\
\hline SSE & $10,570.015$ & 32.637 & $10,507.649$ & $10,547.745$ & $10,569.196$ & $10,592.371$ & $10,634.587$ \\
\hline Trend & 0.999 & 0.012 & 0.975 & 0.992 & 0.999 & 1.007 & 1.023 \\
\hline R.trend & -0.001 & 0.012 & -0.025 & -0.008 & -0.001 & 0.007 & 0.023 \\
\hline M.dens & 0.163 & 0.006 & 0.153 & 0.158 & 0.162 & 0.166 & 0.177 \\
\hline М.y0 & -0.005 & 0.076 & -0.161 & -0.053 & -0.004 & 0.044 & 0.144 \\
\hline M.blyr & -1.978 & 0.080 & -2.135 & -2.030 & -1.978 & -1.925 & -1.818 \\
\hline M.cell & 0.000 & 0.006 & -0.012 & -0.004 & 0.000 & 0.004 & 0.012 \\
\hline R.mid & 0.929 & 0.099 & 0.747 & 0.860 & 0.926 & 0.993 & 1.134 \\
\hline b.ADOY & 0.065 & 0.032 & 0.003 & 0.043 & 0.064 & 0.086 & 0.128 \\
\hline b.ADOY.2 & -0.013 & 0.014 & -0.039 & -0.022 & -0.013 & -0.003 & 0.015 \\
\hline SD.surv & 0.605 & 0.140 & 0.389 & 0.507 & 0.584 & 0.683 & 0.926 \\
\hline SD.y0 & 0.385 & 0.067 & 0.279 & 0.337 & 0.377 & 0.424 & 0.537 \\
\hline SD.cell & 0.234 & 0.024 & 0.184 & 0.218 & 0.235 & 0.252 & 0.278 \\
\hline SD.lam[1] & 0.336 & 0.084 & 0.196 & 0.279 & 0.325 & 0.382 & 0.524 \\
\hline SD.lam[2] & 0.706 & 0.244 & 0.366 & 0.543 & 0.664 & 0.821 & 1.307 \\
\hline SD.lam[3] & 1.585 & 1.834 & 0.083 & 0.573 & 1.063 & 1.899 & 7.026 \\
\hline SD.lam[4] & 1.782 & 1.648 & 0.081 & 0.568 & 1.331 & 2.501 & 6.129 \\
\hline SD.lam[5] & 4.894 & 4.049 & 0.161 & 1.456 & 3.788 & 7.746 & 13.958 \\
\hline SD.lam[6] & 1.580 & 0.361 & 1.009 & 1.281 & 1.556 & 1.847 & 2.312 \\
\hline SD.lam[7] & 3.841 & 1.423 & 1.939 & 2.855 & 3.543 & 4.479 & 7.517 \\
\hline SD.lam[8] & 0.440 & 0.617 & 0.006 & 0.117 & 0.229 & 0.477 & 2.337 \\
\hline S.eff[1] & 0.145 & 0.165 & -0.181 & 0.033 & 0.145 & 0.257 & 0.472 \\
\hline S.eff[2] & -0.414 & 0.120 & -0.653 & -0.494 & -0.413 & -0.335 & -0.182 \\
\hline S.eff[3] & -0.482 & 0.150 & -0.777 & -0.582 & -0.481 & -0.380 & -0.193 \\
\hline S.eff[4] & -1.195 & 0.145 & -1.485 & -1.292 & -1.191 & -1.095 & -0.916 \\
\hline S.eff[5] & -0.347 & 0.131 & -0.606 & -0.438 & -0.345 & -0.256 & -0.099 \\
\hline S.eff[6] & -0.302 & 0.147 & -0.589 & -0.401 & -0.300 & -0.206 & -0.016 \\
\hline S.eff[7] & -0.400 & 0.153 & -0.710 & -0.503 & -0.399 & -0.298 & -0.103 \\
\hline S.eff[8] & -0.619 & 0.156 & -0.927 & -0.723 & -0.616 & -0.514 & -0.320 \\
\hline S.eff[9] & -0.926 & 0.153 & -1.232 & -1.028 & -0.923 & -0.825 & -0.624 \\
\hline S.eff[10] & -0.664 & 0.139 & -0.931 & -0.757 & -0.664 & -0.570 & -0.391 \\
\hline S.eff[11] & -0.217 & 0.158 & -0.530 & -0.325 & -0.215 & -0.108 & 0.084 \\
\hline S.eff[12] & -0.112 & 0.145 & -0.395 & -0.211 & -0.110 & -0.013 & 0.171 \\
\hline S.eff[13] & -0.267 & 0.153 & -0.568 & -0.370 & -0.271 & -0.166 & 0.036 \\
\hline S.eff[14] & 0.073 & 0.142 & -0.209 & -0.022 & 0.074 & 0.168 & 0.350 \\
\hline S.eff[15] & -0.516 & 0.110 & -0.730 & -0.590 & -0.516 & -0.441 & -0.304 \\
\hline y0[1] & -0.652 & 0.243 & -1.123 & -0.810 & -0.656 & -0.499 & -0.171 \\
\hline
\end{tabular}




\begin{tabular}{|c|c|c|c|c|c|c|c|}
\hline $\mathrm{y} 0[2]$ & 0.425 & 0.193 & 0.031 & 0.300 & 0.426 & 0.550 & 0.815 \\
\hline $\mathrm{y} 0[3]$ & -0.205 & 0.170 & -0.549 & -0.312 & -0.201 & -0.092 & 0.125 \\
\hline $\mathrm{y} 0[4]$ & 0.567 & 0.149 & 0.266 & 0.469 & 0.568 & 0.669 & 0.851 \\
\hline $\mathrm{y} 0[5]$ & 0.055 & 0.148 & -0.241 & -0.042 & 0.056 & 0.151 & 0.347 \\
\hline y0[6] & -0.212 & 0.148 & -0.502 & -0.311 & -0.214 & -0.115 & 0.080 \\
\hline $\mathrm{y} 0[7]$ & -0.272 & 0.152 & -0.583 & -0.371 & -0.270 & -0.170 & 0.023 \\
\hline $\mathrm{y} 0[8]$ & 0.191 & 0.146 & -0.093 & 0.094 & 0.192 & 0.288 & 0.478 \\
\hline $\mathrm{y} 0[9]$ & 0.338 & 0.148 & 0.049 & 0.240 & 0.335 & 0.437 & 0.630 \\
\hline y0[10] & 0.185 & 0.156 & -0.114 & 0.081 & 0.182 & 0.287 & 0.510 \\
\hline $\mathrm{y} 0[11]$ & -0.453 & 0.174 & -0.790 & -0.570 & -0.457 & -0.342 & -0.097 \\
\hline y0[12] & -0.205 & 0.172 & -0.522 & -0.323 & -0.213 & -0.094 & 0.156 \\
\hline y0[13] & -0.377 & 0.167 & -0.694 & -0.489 & -0.383 & -0.268 & -0.040 \\
\hline y0[14] & -0.438 & 0.155 & -0.737 & -0.540 & -0.438 & -0.338 & -0.130 \\
\hline y0[15] & 0.786 & 0.139 & 0.519 & 0.691 & 0.785 & 0.879 & 1.062 \\
\hline y0[16] & 0.259 & 0.145 & -0.034 & 0.163 & 0.264 & 0.357 & 0.535 \\
\hline y0[17] & 0.039 & 0.159 & -0.305 & -0.063 & 0.053 & 0.151 & 0.323 \\
\hline y0[18] & -0.017 & 0.168 & -0.379 & -0.123 & -0.004 & 0.099 & 0.289 \\
\hline y0[19] & -0.106 & 0.167 & -0.460 & -0.212 & -0.097 & 0.008 & 0.200 \\
\hline y0[20] & 0.078 & 0.158 & -0.236 & -0.026 & 0.082 & 0.183 & 0.387 \\
\hline $\mathrm{y} 0[21]$ & 0.013 & 0.155 & -0.300 & -0.087 & 0.016 & 0.115 & 0.309 \\
\hline y0[22] & -0.045 & 0.158 & -0.355 & -0.149 & -0.048 & 0.058 & 0.274 \\
\hline y0[23] & 0.120 & 0.164 & -0.193 & 0.012 & 0.116 & 0.226 & 0.456 \\
\hline y0[24] & 0.057 & 0.189 & -0.298 & -0.071 & 0.052 & 0.174 & 0.455 \\
\hline $\mathrm{y} 0[25]$ & -0.251 & 0.226 & -0.669 & -0.403 & -0.266 & -0.107 & 0.227 \\
\hline N.sum[1] & $3,724.701$ & 456.676 & $2,891.294$ & $3,402.027$ & $3,703.140$ & $4,020.028$ & $4,682.679$ \\
\hline N.sum[2] & $10,995.367$ & 902.390 & $9,318.913$ & $10,374.183$ & $10,964.005$ & $11,586.757$ & $12,853.059$ \\
\hline N.sum[3] & $5,979.861$ & 543.912 & $4,962.322$ & $5,606.064$ & $5,965.219$ & $6,340.455$ & $7,099.496$ \\
\hline N.sum[4] & $13,266.393$ & 826.058 & $11,696.862$ & $12,706.709$ & $13,235.091$ & $13,811.088$ & $14,933.805$ \\
\hline N.sum[5] & $8,185.139$ & 595.120 & $7,080.313$ & $7,771.829$ & $8,172.615$ & $8,575.969$ & $9,404.327$ \\
\hline N.sum[6] & $6,393.075$ & 510.564 & $5,438.628$ & $6,041.704$ & $6,372.476$ & $6,729.713$ & $7,432.972$ \\
\hline N.sum[7] & $6,070.700$ & 613.886 & $4,929.481$ & $5,651.801$ & $6,043.086$ & $6,471.032$ & $7,352.617$ \\
\hline N.sum[8] & $9,522.061$ & 794.027 & $8,052.980$ & $8,971.580$ & $9,492.214$ & $10,048.370$ & $11,149.255$ \\
\hline N.sum[9] & $10,793.182$ & 884.222 & $9,102.785$ & $10,192.629$ & $10,770.522$ & $11,370.332$ & $12,606.375$ \\
\hline N.sum[10] & $9,053.820$ & 760.939 & $7,675.414$ & $8,521.217$ & $9,028.880$ & $9,547.253$ & $10,641.116$ \\
\hline N.sum[11] & $4,712.086$ & 521.170 & $3,764.837$ & $4,351.261$ & $4,688.082$ & $5,052.131$ & $5,783.249$ \\
\hline N.sum[12] & $6,005.840$ & 598.219 & $4,899.067$ & $5,585.157$ & $5,983.656$ & $6,403.193$ & $7,238.314$ \\
\hline N.sum[13] & $5,097.686$ & 522.256 & $4,155.405$ & $4,737.783$ & $5,072.316$ & $5,435.560$ & $6,194.274$ \\
\hline N.sum[14] & $4,885.962$ & 489.737 & $3,982.978$ & $4,548.908$ & $4,871.281$ & $5,203.314$ & $5,897.266$ \\
\hline N.sum[15] & $16,984.451$ & $1,151.380$ & $14,857.860$ & $16,188.215$ & $16,941.377$ & $17,730.584$ & $19,350.472$ \\
\hline N.sum[16] & $10,303.005$ & 700.268 & $8,966.244$ & $9,819.898$ & $10,289.788$ & $10,762.670$ & $11,715.179$ \\
\hline N.sum[17] & $8,452.123$ & 589.160 & $7,343.937$ & $8,043.059$ & $8,441.686$ & $8,842.325$ & $9,640.667$ \\
\hline N.sum[18] & $8,081.647$ & 596.727 & $6,973.838$ & $7,667.624$ & $8,053.667$ & $8,486.492$ & $9,283.702$ \\
\hline N.sum[19] & $7,371.163$ & 569.032 & $6,307.651$ & $6,978.886$ & $7,356.661$ & $7,741.476$ & $8,551.403$ \\
\hline N.sum[20] & $8,683.898$ & 629.534 & $7,515.555$ & $8,240.535$ & $8,668.987$ & $9,094.364$ & $9,963.580$ \\
\hline
\end{tabular}




\begin{tabular}{|c|c|c|c|c|c|c|c|}
\hline N.sum[21] & $7,886.450$ & 575.573 & $6,816.099$ & $7,493.311$ & $7,862.158$ & $8,263.050$ & $9,052.213$ \\
\hline N.sum[22] & $7,185.901$ & 572.715 & $6,131.154$ & $6,785.158$ & $7,169.539$ & $7,560.949$ & $8,373.449$ \\
\hline N.sum[23] & $8,201.001$ & 619.396 & $7,042.762$ & $7,768.020$ & $8,185.309$ & $8,611.801$ & $9,466.778$ \\
\hline N.sum[24] & $7,509.084$ & 582.280 & $6,421.200$ & $7,107.988$ & $7,496.956$ & $7,894.432$ & $8,690.592$ \\
\hline N.sum[25] & $5,427.088$ & 495.829 & $4,510.889$ & $5,087.088$ & $5,403.395$ & $5,752.515$ & $6,459.682$ \\
\hline N.sum2[1] & $7,812.676$ & $1,752.848$ & $4,925.040$ & $6,605.424$ & $7,641.301$ & $8,826.584$ & $11,540.362$ \\
\hline N.sum2[2] & $7,812.412$ & $1,384.055$ & $5,492.285$ & $6,869.229$ & $7,674.729$ & $8,610.115$ & $10,718.432$ \\
\hline N.sum2[3] & $7,931.956$ & $1,154.483$ & $5,935.498$ & $7,144.505$ & $7,850.622$ & $8,604.811$ & $10,505.874$ \\
\hline N.sum2[4] & $8,135.302$ & $1,057.422$ & $6,282.851$ & $7,388.784$ & $8,062.369$ & $8,756.216$ & $10,508.506$ \\
\hline N.sum2[5] & $8,357.950$ & $1,024.281$ & $6,568.176$ & $7,656.887$ & $8,269.311$ & $8,958.015$ & $10,697.639$ \\
\hline N.sum2[6] & $8,523.739$ & $1,000.460$ & $6,715.352$ & $7,847.823$ & $8,460.987$ & $9,101.431$ & $10,761.020$ \\
\hline N.sum2[7] & $8,569.490$ & 982.671 & $6,777.775$ & $7,913.575$ & $8,493.887$ & $9,139.818$ & $10,762.736$ \\
\hline N.sum2[8] & $8,477.832$ & 973.620 & $6,776.796$ & $7,833.179$ & $8,380.219$ & $9,044.718$ & $10,677.555$ \\
\hline N.sum2[9] & $8,299.347$ & 975.685 & $6,520.418$ & $7,666.544$ & $8,233.426$ & $8,870.959$ & $10,473.953$ \\
\hline N.sum2[10] & $8,113.706$ & $1,007.976$ & $6,168.144$ & $7,477.859$ & $8,099.992$ & $8,708.920$ & $10,259.947$ \\
\hline N.sum2[11] & $7,987.950$ & $1,063.274$ & $5,874.643$ & $7,315.242$ & $8,019.948$ & $8,624.139$ & $10,189.437$ \\
\hline N.sum2[12] & $7,954.392$ & $1,088.344$ & $5,777.172$ & $7,284.551$ & $8,005.187$ & $8,603.241$ & $10,173.566$ \\
\hline N.sum2[13] & $8,011.236$ & $1,035.249$ & $5,931.895$ & $7,391.302$ & $8,051.638$ & $8,619.298$ & $10,080.085$ \\
\hline N.sum2[14] & $8,141.620$ & 917.156 & $6,377.803$ & $7,570.196$ & $8,134.124$ & $8,675.436$ & $10,035.188$ \\
\hline N.sum2[15] & $8,337.700$ & 851.212 & $6,718.092$ & $7,797.392$ & $8,272.819$ & $8,849.347$ & $10,153.642$ \\
\hline N.sum2[16] & $8,572.279$ & 969.897 & $7,004.783$ & $7,905.521$ & $8,428.517$ & $9,118.512$ & $10,787.002$ \\
\hline N.sum2[17] & $8,783.084$ & $1,178.681$ & $7,120.512$ & $7,963.688$ & $8,515.167$ & $9,346.628$ & $11,713.635$ \\
\hline N.sum2[18] & $8,892.792$ & $1,283.442$ & $7,182.705$ & $7,985.711$ & $8,584.158$ & $9,511.189$ & $12,052.209$ \\
\hline N.sum2[19] & $8,851.695$ & $1,222.562$ & $7,067.985$ & $7,975.197$ & $8,596.860$ & $9,515.185$ & $11,839.467$ \\
\hline N.sum2[20] & $8,666.149$ & $1,088.098$ & $6,920.292$ & $7,912.656$ & $8,533.652$ & $9,261.403$ & $11,132.305$ \\
\hline N.sum2[21] & $8,395.147$ & $1,000.876$ & $6,702.273$ & $7,744.954$ & $8,281.658$ & $8,919.113$ & $10,654.141$ \\
\hline N.sum2[22] & $8,109.849$ & 999.920 & $6,410.534$ & $7,438.894$ & $8,005.497$ & $8,689.359$ & $10,338.570$ \\
\hline N.sum2[23] & $7,868.296$ & $1,097.179$ & $5,912.654$ & $7,134.560$ & $7,806.733$ & $8,522.765$ & $10,214.696$ \\
\hline N.sum2[24] & $7,707.852$ & $1,316.608$ & $5,359.354$ & $6,817.794$ & $7,659.702$ & $8,495.201$ & $10,566.089$ \\
\hline N.sum2[25] & $7,640.947$ & $1,647.685$ & $4,630.983$ & $6,533.970$ & $7,573.820$ & $8,594.900$ & $11,302.675$ \\
\hline R.growth[1] & 0.009 & 0.069 & -0.114 & -0.036 & 0.001 & 0.052 & 0.159 \\
\hline R.growth[2] & 0.020 & 0.063 & -0.091 & -0.022 & 0.012 & 0.059 & 0.157 \\
\hline R.growth[3] & 0.027 & 0.053 & -0.066 & -0.008 & 0.022 & 0.061 & 0.140 \\
\hline R.growth[4] & 0.028 & 0.044 & -0.054 & -0.001 & 0.024 & 0.055 & 0.122 \\
\hline R.growth[5] & 0.020 & 0.040 & -0.057 & -0.005 & 0.017 & 0.043 & 0.109 \\
\hline R.growth[6] & 0.006 & 0.040 & -0.080 & -0.016 & 0.006 & 0.029 & 0.084 \\
\hline R.growth[7] & -0.011 & 0.040 & -0.106 & -0.031 & -0.004 & 0.014 & 0.063 \\
\hline R.growth[8] & -0.022 & 0.042 & -0.119 & -0.046 & -0.013 & 0.005 & 0.050 \\
\hline R.growth[9] & -0.024 & 0.044 & -0.119 & -0.050 & -0.015 & 0.005 & 0.053 \\
\hline R.growth[10] & -0.017 & 0.041 & -0.114 & -0.037 & -0.010 & 0.010 & 0.053 \\
\hline R.growth[11] & -0.005 & 0.035 & -0.086 & -0.022 & 0.000 & 0.016 & 0.059 \\
\hline R.growth[12] & 0.008 & 0.034 & -0.053 & -0.012 & 0.005 & 0.026 & 0.083 \\
\hline R.growth[13] & 0.018 & 0.045 & -0.052 & -0.011 & 0.008 & 0.041 & 0.129 \\
\hline R.growth[14] & 0.025 & 0.055 & -0.056 & -0.011 & 0.008 & 0.053 & 0.161 \\
\hline
\end{tabular}




\begin{tabular}{lrrrrrrr} 
R.growth[15] & 0.027 & 0.056 & -0.054 & -0.010 & 0.009 & 0.057 & 0.173 \\
R.growth[16] & 0.022 & 0.048 & -0.055 & -0.008 & 0.011 & 0.048 & 0.135 \\
R.growth[17] & 0.011 & 0.037 & -0.058 & -0.010 & 0.007 & 0.031 & 0.086 \\
R.growth[18] & -0.004 & 0.037 & -0.078 & -0.026 & -0.003 & 0.016 & 0.077 \\
R.growth[19] & -0.020 & 0.045 & -0.128 & -0.042 & -0.011 & 0.009 & 0.058 \\
R.growth[20] & -0.031 & 0.050 & -0.155 & -0.055 & -0.019 & 0.002 & 0.043 \\
R.growth[21] & -0.035 & 0.050 & -0.152 & -0.065 & -0.024 & 0.000 & 0.045 \\
R.growth[22] & -0.032 & 0.055 & -0.164 & -0.064 & -0.025 & 0.004 & 0.065 \\
R.growth[23] & -0.026 & 0.063 & -0.183 & -0.057 & -0.017 & 0.012 & 0.090 \\
R.growth[24] & -0.018 & 0.068 & -0.192 & -0.049 & -0.009 & 0.022 & 0.108 \\
Deviance & $32,950.909$ & 53.702 & $32,849.548$ & $32,913.169$ & $32,950.326$ & $32,987.358$ & $33,058.950$ \\
\hline
\end{tabular}


Tables A4.19. Bayesian generalized linear mixed model output for Sabine's Gull observed during aerial surveys 1992-2016 on the Arctic Coastal Plain, Alaska. We provide summaries including mean, SE, and several quantiles of the posterior distribution for model and derived parameters. See Table A4.4 for a key to parameter names.

\begin{tabular}{|c|c|c|c|c|c|c|c|}
\hline Parameter & Mean & SD & $2.50 \%$ & $25 \%$ & $50 \%$ & $75 \%$ & $97.50 \%$ \\
\hline MSE & 1.019 & 0.006 & 1.008 & 1.015 & 1.019 & 1.023 & 1.031 \\
\hline MSE.CV & 2.288 & 0.013 & 2.263 & 2.279 & 2.287 & 2.296 & 2.314 \\
\hline SSE & $18,718.627$ & 106.035 & $18,520.182$ & $18,645.922$ & $18,713.725$ & $18,787.933$ & $18,935.536$ \\
\hline Trend & 1.026 & 0.009 & 1.010 & 1.020 & 1.026 & 1.032 & 1.047 \\
\hline R.trend & 0.026 & 0.009 & 0.010 & 0.020 & 0.025 & 0.032 & 0.046 \\
\hline M.dens & 0.189 & 0.006 & 0.179 & 0.185 & 0.189 & 0.193 & 0.201 \\
\hline M.y0 & -0.001 & 0.045 & -0.090 & -0.030 & 0.000 & 0.028 & 0.088 \\
\hline M.blyr & -2.019 & 0.057 & -2.129 & -2.057 & -2.019 & -1.981 & -1.906 \\
\hline M.cell & 0.000 & 0.019 & -0.036 & -0.013 & 0.000 & 0.012 & 0.036 \\
\hline R.mid & -0.214 & 0.046 & -0.303 & -0.245 & -0.213 & -0.183 & -0.124 \\
\hline b.ADOY & -0.156 & 0.034 & -0.222 & -0.180 & -0.157 & -0.133 & -0.089 \\
\hline b.ADOY.2 & 0.029 & 0.016 & -0.003 & 0.018 & 0.029 & 0.040 & 0.061 \\
\hline SD.surv & 0.384 & 0.107 & 0.219 & 0.309 & 0.369 & 0.442 & 0.629 \\
\hline SD.y0 & 0.241 & 0.049 & 0.160 & 0.206 & 0.235 & 0.269 & 0.351 \\
\hline SD.cell & 0.563 & 0.024 & 0.518 & 0.547 & 0.563 & 0.579 & 0.612 \\
\hline SD.lam[1] & 0.252 & 0.065 & 0.147 & 0.203 & 0.246 & 0.293 & 0.393 \\
\hline SD.lam[2] & 1.725 & 0.619 & 0.886 & 1.289 & 1.612 & 2.021 & 3.293 \\
\hline SD.lam[3] & 0.878 & 1.168 & 0.084 & 0.330 & 0.547 & 0.928 & 4.887 \\
\hline SD.lam[4] & 1.605 & 1.529 & 0.076 & 0.642 & 1.203 & 2.062 & 5.591 \\
\hline SD.lam[5] & 4.566 & 3.944 & 0.132 & 1.330 & 3.312 & 7.054 & 13.866 \\
\hline SD.lam[6] & 1.462 & 0.385 & 0.878 & 1.178 & 1.391 & 1.707 & 2.339 \\
\hline SD.lam[7] & 1.728 & 1.346 & 0.454 & 0.628 & 1.354 & 2.517 & 4.895 \\
\hline SD.lam[8] & 0.263 & 0.503 & 0.001 & 0.026 & 0.082 & 0.256 & 1.676 \\
\hline S.eff[1] & -0.320 & 0.186 & -0.698 & -0.444 & -0.317 & -0.191 & 0.032 \\
\hline S.eff[2] & -0.188 & 0.160 & -0.514 & -0.292 & -0.185 & -0.079 & 0.125 \\
\hline S.eff[3] & -0.060 & 0.156 & -0.377 & -0.163 & -0.057 & 0.044 & 0.244 \\
\hline S.eff[4] & -0.175 & 0.152 & -0.474 & -0.276 & -0.173 & -0.072 & 0.121 \\
\hline S.eff[5] & -0.262 & 0.167 & -0.599 & -0.372 & -0.257 & -0.147 & 0.054 \\
\hline S.eff[6] & -0.534 & 0.182 & -0.896 & -0.655 & -0.529 & -0.408 & -0.191 \\
\hline S.eff[7] & 0.159 & 0.178 & -0.192 & 0.039 & 0.158 & 0.275 & 0.518 \\
\hline S.eff[8] & -0.587 & 0.240 & -1.083 & -0.742 & -0.580 & -0.418 & -0.148 \\
\hline S.eff[9] & 0.266 & 0.155 & -0.037 & 0.161 & 0.267 & 0.371 & 0.561 \\
\hline S.eff[10] & 0.080 & 0.147 & -0.208 & -0.019 & 0.079 & 0.178 & 0.367 \\
\hline S.eff[11] & -0.308 & 0.154 & -0.614 & -0.410 & -0.307 & -0.203 & -0.011 \\
\hline S.eff[12] & 0.184 & 0.149 & -0.112 & 0.085 & 0.184 & 0.285 & 0.476 \\
\hline S.eff[13] & -0.379 & 0.165 & -0.714 & -0.488 & -0.374 & -0.268 & -0.055 \\
\hline S.eff[14] & 0.266 & 0.137 & -0.004 & 0.174 & 0.265 & 0.358 & 0.536 \\
\hline S.eff[15] & -0.395 & 0.165 & -0.725 & -0.506 & -0.393 & -0.282 & -0.085 \\
\hline $\mathrm{y} 0[1]$ & -0.107 & 0.158 & -0.426 & -0.210 & -0.105 & -0.002 & 0.197 \\
\hline
\end{tabular}




\begin{tabular}{|c|c|c|c|c|c|c|c|}
\hline $\mathrm{y} 0[2]$ & 0.013 & 0.135 & -0.244 & -0.077 & 0.013 & 0.101 & 0.287 \\
\hline $\mathrm{y} 0[3]$ & 0.008 & 0.124 & -0.233 & -0.076 & 0.008 & 0.091 & 0.252 \\
\hline $\mathrm{y} 0[4]$ & 0.312 & 0.121 & 0.075 & 0.230 & 0.313 & 0.393 & 0.555 \\
\hline $\mathrm{y} 0[5]$ & 0.103 & 0.117 & -0.129 & 0.027 & 0.105 & 0.182 & 0.330 \\
\hline $\mathrm{y} 0[6]$ & 0.297 & 0.115 & 0.071 & 0.223 & 0.295 & 0.372 & 0.524 \\
\hline $\mathrm{y} 0[7]$ & -0.580 & 0.135 & -0.859 & -0.670 & -0.575 & -0.489 & -0.326 \\
\hline $\mathrm{y} 0[8]$ & -0.363 & 0.134 & -0.630 & -0.451 & -0.361 & -0.271 & -0.105 \\
\hline $\mathrm{y} 0[9]$ & 0.072 & 0.128 & -0.172 & -0.017 & 0.069 & 0.158 & 0.329 \\
\hline y0[10] & 0.075 & 0.129 & -0.171 & -0.011 & 0.072 & 0.160 & 0.337 \\
\hline y0[11] & 0.128 & 0.125 & -0.113 & 0.045 & 0.124 & 0.210 & 0.385 \\
\hline y0[12] & -0.096 & 0.127 & -0.342 & -0.180 & -0.095 & -0.010 & 0.153 \\
\hline y0[13] & -0.066 & 0.117 & -0.292 & -0.145 & -0.066 & 0.012 & 0.164 \\
\hline y0[14] & -0.092 & 0.116 & -0.320 & -0.169 & -0.091 & -0.013 & 0.132 \\
\hline y0[15] & -0.063 & 0.117 & -0.296 & -0.143 & -0.060 & 0.018 & 0.160 \\
\hline y0[16] & 0.181 & 0.118 & -0.050 & 0.102 & 0.183 & 0.260 & 0.415 \\
\hline y0[17] & 0.064 & 0.120 & -0.172 & -0.014 & 0.065 & 0.142 & 0.305 \\
\hline y0[18] & 0.143 & 0.121 & -0.092 & 0.063 & 0.145 & 0.221 & 0.381 \\
\hline y0[19] & 0.180 & 0.122 & -0.059 & 0.098 & 0.180 & 0.259 & 0.421 \\
\hline y0[20] & -0.159 & 0.119 & -0.389 & -0.239 & -0.159 & -0.079 & 0.074 \\
\hline $\mathrm{y} 0[21]$ & -0.035 & 0.120 & -0.268 & -0.117 & -0.035 & 0.045 & 0.201 \\
\hline y0[22] & 0.079 & 0.121 & -0.161 & -0.001 & 0.081 & 0.160 & 0.319 \\
\hline y0[23] & 0.106 & 0.119 & -0.129 & 0.027 & 0.107 & 0.183 & 0.343 \\
\hline y0[24] & 0.001 & 0.134 & -0.266 & -0.090 & 0.002 & 0.093 & 0.262 \\
\hline $\mathrm{y} 0[25]$ & -0.223 & 0.168 & -0.554 & -0.336 & -0.222 & -0.109 & 0.101 \\
\hline N.sum[1] & $3,742.783$ & 382.730 & $3,048.369$ & $3,480.108$ & $3,725.329$ & $3,987.894$ & $4,536.632$ \\
\hline N.sum[2] & $4,314.812$ & 448.916 & $3,510.059$ & $3,999.504$ & $4,291.325$ & $4,603.076$ & $5,251.926$ \\
\hline N.sum[3] & $4,369.563$ & 421.778 & $3,601.896$ & $4,072.341$ & $4,350.355$ & $4,634.989$ & $5,261.861$ \\
\hline N.sum[4] & $5,987.075$ & 519.710 & $5,055.718$ & $5,615.083$ & $5,954.203$ & $6,331.383$ & $7,028.872$ \\
\hline N.sum[5] & $4,870.679$ & 421.751 & $4,100.335$ & $4,577.308$ & $4,854.943$ & $5,145.607$ & $5,757.856$ \\
\hline N.sum[6] & $5,889.545$ & 494.880 & $4,986.368$ & $5,551.652$ & $5,864.087$ & $6,205.054$ & $6,917.426$ \\
\hline N.sum[7] & $2,447.976$ & 287.643 & $1,915.105$ & $2,252.519$ & $2,435.219$ & $2,633.946$ & $3,056.913$ \\
\hline N.sum[8] & $3,054.259$ & 326.035 & $2,457.276$ & $2,821.129$ & $3,037.841$ & $3,272.227$ & $3,735.137$ \\
\hline N.sum[9] & $4,806.665$ & 436.335 & $4,005.904$ & $4,507.132$ & $4,788.063$ & $5,092.727$ & $5,710.376$ \\
\hline N.sum[10] & $5,014.522$ & 451.049 & $4,185.404$ & $4,699.100$ & $5,000.860$ & $5,311.006$ & $5,941.181$ \\
\hline N.sum[11] & $5,600.321$ & 497.837 & $4,681.968$ & $5,254.685$ & $5,575.288$ & $5,916.870$ & $6,648.383$ \\
\hline N.sum[12] & $4,803.689$ & 447.070 & $3,969.911$ & $4,500.288$ & $4,785.954$ & $5,088.351$ & $5,736.597$ \\
\hline N.sum[13] & $5,340.929$ & 470.701 & $4,465.783$ & $5,015.041$ & $5,324.558$ & $5,645.347$ & $6,311.781$ \\
\hline N.sum[14] & $5,604.364$ & 477.717 & $4,718.315$ & $5,271.686$ & $5,586.840$ & $5,919.607$ & $6,589.311$ \\
\hline N.sum[15] & $6,180.382$ & 529.087 & $5,206.232$ & $5,812.373$ & $6,160.710$ & $6,526.577$ & $7,262.816$ \\
\hline N.sum[16] & $8,384.357$ & 641.764 & $7,206.227$ & $7,941.035$ & $8,367.839$ & $8,808.993$ & $9,730.796$ \\
\hline N.sum[17] & $7,865.333$ & 618.799 & $6,715.667$ & $7,442.601$ & $7,843.106$ & $8,267.519$ & $9,169.377$ \\
\hline N.sum[18] & $8,890.077$ & 671.986 & $7,625.746$ & $8,425.822$ & $8,868.087$ & $9,323.323$ & $10,272.468$ \\
\hline N.sum[19] & $9,560.334$ & 750.850 & $8,183.326$ & $9,033.475$ & $9,528.320$ & $10,055.949$ & $11,092.853$ \\
\hline N.sum[20] & $6,978.400$ & 579.990 & $5,903.753$ & $6,581.000$ & $6,963.370$ & $7,354.833$ & $8,171.469$ \\
\hline
\end{tabular}




\begin{tabular}{|c|c|c|c|c|c|c|c|}
\hline N.sum[21] & $7,977.210$ & 638.362 & $6,806.997$ & $7,534.427$ & $7,944.160$ & $8,392.225$ & 9,326 \\
\hline N.sum[22] & $3,928.534$ & 730.263 & $7,575.328$ & $8,419.708$ & $8,903.775$ & $9,414.838$ & $10,423.358$ \\
\hline N.sum[23] & ,036.988 & 702.394 & $7,728.225$ &, 553.176 & $9,011.468$ & $9,490.805$ & $10,494.063$ \\
\hline N.sum[24] & ,969.724 & 637.646 &, 778.923 & $7,526.524$ & $7,946.345$ & $8,388.233$ & $9,278.746$ \\
\hline N.sum[25] & $6,214.610$ & 522.585 & $5,239.854$ & $5,855.406$ & $6,197.418$ & $6,548.911$ & 7,308.192 \\
\hline N.sum2[1] &, 313.553 & 638.684 & $3,222.569$ & $3,857.600$ & $4,267.917$ & $4,690.966$ & $5,721.873$ \\
\hline N.sum2[2] & $4,391.135$ & 514.301 & $3,463.920$ & $4,025.538$ & $4,367.796$ & $4,715.657$ & $5,474.519$ \\
\hline N.sum2[3] & $4,469.154$ & 472.629 & $3,623.454$ & $4,133.323$ & $4,444.508$ & $4,771.121$ & $5,470.308$ \\
\hline N.sum2[4] & $4,519.280$ & 469.059 & $3,722.491$ & $4,196.841$ & $4,470.768$ & $4,805.106$ & $5,546.450$ \\
\hline N.sum2[5] & $4,527.197$ & 440.381 & $3,782.727$ & $4,227.759$ & $4,486.570$ & $4,787.169$ & $5,483.134$ \\
\hline N.sum2[6] & $4,506.049$ & 395.525 & $3,796.402$ & $4,232.243$ & $4,481.579$ & $4,748.814$ & $5,349.504$ \\
\hline N.sum2[7] & $4,489.574$ & 390.338 & $3,772.238$ & $4,212.470$ & $4,474.412$ & $4,752.062$ & $5,297.119$ \\
\hline N.sum2[8] & $4,515.180$ & 435.086 & $3,686.886$ & $4,202.939$ & $4,510.969$ & $4,818.795$ & $5,370.486$ \\
\hline N.sum2[9] & $4,612.370$ & 486.697 & $3,667.587$ & $4,264.794$ & $4,611.082$ & $4,956.923$ & $5,543.295$ \\
\hline N.sum2[10] & $4,798.775$ & 514.090 & $3,789.639$ & $4,436.834$ & $4,802.280$ & $5,164.669$ & $5,770.681$ \\
\hline N.sum2[11] & $5,079.234$ & 513.060 & $4,080.037$ & $4,721.945$ & $5,086.202$ & $5,439.234$ & $6,065.587$ \\
\hline N.sum2[12] & $5,444.265$ & 497.463 & $4,452.433$ & $5,109.645$ & $5,451.219$ & $5,771.335$ & $6,420.906$ \\
\hline N.sum2[13] & $5,869.788$ & 488.343 & $4,891.241$ & $5,557.572$ & $5,873.439$ & $6,165.902$ & $6,872.791$ \\
\hline N.sum2[14] & $6,323.175$ & 503.259 & $5,380.637$ & $5,998.534$ & $6,301.600$ & $6,614.585$ & $7,431.289$ \\
\hline N.sum2[15] & $6,775.695$ & 550.719 & $5,827.328$ & $6,396.411$ & $6,719.695$ & $7,104.123$ & $8,029.127$ \\
\hline N.sum2[16] & $7,206.521$ & 620.883 & $6,220.205$ & $6,752.011$ & $7,132.015$ & $7,574.470$ & $8,617.257$ \\
\hline N.sum2[17] & $7,601.030$ & 683.983 & $6,513.893$ & $7,097.901$ & $7,530.435$ & $8,002.610$ & $9,148.063$ \\
\hline N.sum2[18] & $7,946.585$ & 716.544 & $6,766.348$ & $7,426.208$ & $7,888.789$ & $8,377.038$ & $9,533.761$ \\
\hline N.sum2[19] & $8,227.959$ & 730.619 & $6,971.510$ & $7,694.614$ & $8,177.986$ & $8,691.411$ & $9,826.132$ \\
\hline N.sum2[20] & $8,424.847$ & 754.284 & $7,122.169$ & $7,886.089$ & $8,357.153$ & $8,892.325$ & $10,074.519$ \\
\hline N.sum2[21] & $8,517.616$ & 775.780 & $7,199.946$ & 85.556 & $8,435.372$ & 1.245 & $10,285.478$ \\
\hline N.sum2[22] & $8,498.038$ & 768.700 & $7,182.535$ & $7,963.341$ & $8,422.828$ & $8,951.307$ & $10,224.442$ \\
\hline N.sum2[23] & $8,385.360$ & 797.861 & $6,988.173$ & $7,830.521$ & $8,307.906$ & $8,882.266$ & $10,122.535$ \\
\hline N.sum2[24] & $8,227.508$ & 985.629 & $6,541.946$ & $7,531.107$ & $8,138.619$ & 4.507 & $10,382.068$ \\
\hline N.sum2[25] & $8,078.468$ & $1,316.697$ & $5,851.769$ & $7,124.297$ & $7,961.400$ & $8,920.693$ & $10,929.191$ \\
\hline R.growth[1] & 0.022 & 0.055 & -0.083 & -0.012 & 0.019 & 0.049 & 0.150 \\
\hline R.growth[2] & 0.019 & 0.048 & -0.075 & -0.010 & 0.018 & 0.044 & 0.124 \\
\hline R.growth[3] & 0.011 & 0.035 & -0.063 & -0.011 & 0.013 & 0.034 & 0.081 \\
\hline R.growth[4] & 0.002 & 0.029 & -0.057 & -0.018 & 0.004 & 0.024 & 0.054 \\
\hline R.growth[5] & -0.004 & 0.036 & -0.084 & -0.026 & 0.000 & 0.023 & 0.053 \\
\hline R.growth[6] & -0.004 & 0.041 & -0.100 & -0.026 & 0.004 & 0.027 & 0.057 \\
\hline R.growth[7] & 0.005 & 0.038 & -0.088 & -0.016 & 0.013 & 0.032 & 0.063 \\
\hline R.growth[8] & 0.020 & 0.030 & -0.050 & 0.003 & 0.025 & 0.041 & 0.071 \\
\hline R.growth[9] & 0.039 & 0.026 & -0.019 & 0.025 & 0.041 & 0.056 & 0.088 \\
\hline R.growth[10] & 0.057 & 0.029 & 0.005 & 0.038 & 0.055 & 0.075 & 0.121 \\
\hline R.growth[11] & 0.070 & 0.033 & 0.018 & 0.046 & 0.066 & 0.089 & 0.146 \\
\hline R.growth[12] & 0.076 & 0.034 & 0.022 & 0.050 & 0.072 & 0.096 & 0.155 \\
\hline R.growth[13] & 0.075 & 0.034 & 0.022 & 0.048 & 0.071 & 0.097 & 0.150 \\
\hline R.growth[14] & 0.069 & 0.034 & 0.015 & 0.044 & 0.065 & 0.090 & 0.143 \\
\hline
\end{tabular}




\begin{tabular}{lrrrrrrr} 
R.growth[15] & 0.061 & 0.031 & 0.008 & 0.040 & 0.058 & 0.080 & 0.131 \\
R.growth[16] & 0.053 & 0.028 & 0.005 & 0.035 & 0.050 & 0.068 & 0.120 \\
R.growth[17] & 0.044 & 0.027 & -0.002 & 0.027 & 0.042 & 0.059 & 0.106 \\
R.growth[18] & 0.035 & 0.029 & -0.020 & 0.017 & 0.034 & 0.051 & 0.103 \\
R.growth[19] & 0.024 & 0.031 & -0.040 & 0.005 & 0.025 & 0.041 & 0.088 \\
R.growth[20] & 0.011 & 0.030 & -0.052 & -0.007 & 0.013 & 0.031 & 0.065 \\
R.growth[21] & -0.002 & 0.032 & -0.066 & -0.024 & -0.002 & 0.022 & 0.056 \\
R.growth[22] & -0.014 & 0.042 & -0.100 & -0.042 & -0.012 & 0.019 & 0.059 \\
R.growth[23] & -0.022 & 0.054 & -0.147 & -0.055 & -0.016 & 0.019 & 0.066 \\
R.growth[24] & -0.024 & 0.061 & -0.172 & -0.061 & -0.018 & 0.021 & 0.073 \\
Deviance & $29,493.584$ & 44.060 & $29,408.552$ & $29,463.989$ & $29,492.695$ & $29,522.343$ & $29,583.442$ \\
\hline
\end{tabular}


Tables A4.20. Bayesian generalized linear mixed model output for Glaucous Gull observed during aerial surveys 1992-2016 on the Arctic Coastal Plain, Alaska. We provide summaries including mean, SE, and several quantiles of the posterior distribution for model and derived parameters. See Table A4.4 for a key to parameter names.

\begin{tabular}{|c|c|c|c|c|c|c|c|}
\hline Parameter & Mean & SD & $2.50 \%$ & $25 \%$ & $50 \%$ & $75 \%$ & $97.50 \%$ \\
\hline MSE & 1.044 & 0.003 & 1.038 & 1.042 & 1.044 & 1.046 & 1.051 \\
\hline MSE.CV & 1.780 & 0.006 & 1.769 & 1.776 & 1.779 & 1.783 & 1.791 \\
\hline SSE & $25,608.022$ & 80.576 & $25,452.623$ & $25,553.257$ & $25,606.669$ & $25,661.018$ & $25,772.080$ \\
\hline Trend & 1.018 & 0.006 & 1.007 & 1.014 & 1.018 & 1.022 & 1.031 \\
\hline R.trend & 0.018 & 0.006 & 0.007 & 0.014 & 0.017 & 0.022 & 0.030 \\
\hline M.dens & 0.260 & 0.004 & 0.252 & 0.257 & 0.260 & 0.263 & 0.269 \\
\hline M.y0 & 0.001 & 0.030 & -0.058 & -0.019 & 0.000 & 0.020 & 0.060 \\
\hline M.blyr & -1.503 & 0.036 & -1.574 & -1.527 & -1.503 & -1.479 & -1.432 \\
\hline M.cell & 0.000 & 0.010 & -0.020 & -0.007 & 0.000 & 0.007 & 0.020 \\
\hline R.mid & 0.758 & 0.052 & 0.658 & 0.723 & 0.758 & 0.793 & 0.860 \\
\hline b.ADOY & -0.061 & 0.020 & -0.101 & -0.075 & -0.061 & -0.048 & -0.025 \\
\hline b.ADOY.2 & -0.003 & 0.010 & -0.023 & -0.010 & -0.003 & 0.003 & 0.016 \\
\hline SD.surv & 0.134 & 0.047 & 0.052 & 0.102 & 0.129 & 0.161 & 0.236 \\
\hline SD.y0 & 0.151 & 0.029 & 0.104 & 0.131 & 0.148 & 0.169 & 0.216 \\
\hline SD.cell & 0.390 & 0.016 & 0.359 & 0.379 & 0.390 & 0.401 & 0.421 \\
\hline SD.lam[1] & 0.235 & 0.051 & 0.150 & 0.197 & 0.232 & 0.269 & 0.340 \\
\hline SD.lam[2] & 1.242 & 0.499 & 0.621 & 0.902 & 1.128 & 1.457 & 2.557 \\
\hline SD.lam[3] & 0.822 & 0.738 & 0.065 & 0.346 & 0.616 & 1.027 & 2.889 \\
\hline SD.lam[4] & 0.924 & 0.901 & 0.032 & 0.331 & 0.658 & 1.206 & 3.481 \\
\hline SD.lam[5] & 4.311 & 3.953 & 0.105 & 1.109 & 2.948 & 6.663 & 13.722 \\
\hline SD.lam[6] & 0.601 & 0.275 & 0.280 & 0.402 & 0.513 & 0.737 & 1.299 \\
\hline SD.lam[7] & 1.109 & 0.871 & 0.254 & 0.391 & 0.689 & 1.776 & 3.083 \\
\hline SD.lam[8] & 0.345 & 0.483 & 0.004 & 0.086 & 0.189 & 0.398 & 1.808 \\
\hline S.eff[1] & -0.118 & 0.086 & -0.297 & -0.173 & -0.114 & -0.058 & 0.039 \\
\hline S.eff[2] & -0.120 & 0.090 & -0.308 & -0.179 & -0.117 & -0.056 & 0.041 \\
\hline S.eff[3] & -0.051 & 0.082 & -0.216 & -0.105 & -0.049 & 0.004 & 0.106 \\
\hline S.eff[4] & 0.095 & 0.084 & -0.058 & 0.037 & 0.092 & 0.150 & 0.270 \\
\hline S.eff[5] & -0.009 & 0.079 & -0.164 & -0.061 & -0.009 & 0.042 & 0.152 \\
\hline S.eff[6] & 0.081 & 0.087 & -0.080 & 0.022 & 0.077 & 0.137 & 0.264 \\
\hline S.eff[7] & 0.020 & 0.081 & -0.139 & -0.034 & 0.019 & 0.073 & 0.184 \\
\hline S.eff[8] & 0.171 & 0.101 & -0.008 & 0.097 & 0.167 & 0.237 & 0.379 \\
\hline S.eff[9] & 0.142 & 0.091 & -0.021 & 0.077 & 0.138 & 0.202 & 0.331 \\
\hline S.eff[10] & 0.014 & 0.081 & -0.145 & -0.039 & 0.014 & 0.067 & 0.178 \\
\hline S.eff[11] & 0.068 & 0.082 & -0.089 & 0.013 & 0.065 & 0.122 & 0.231 \\
\hline S.eff[12] & 0.042 & 0.078 & -0.107 & -0.011 & 0.040 & 0.093 & 0.205 \\
\hline S.eff[13] & -0.080 & 0.082 & -0.247 & -0.133 & -0.077 & -0.024 & 0.075 \\
\hline S.eff[14] & 0.088 & 0.080 & -0.061 & 0.032 & 0.084 & 0.141 & 0.253 \\
\hline S.eff[15] & -0.133 & 0.084 & -0.303 & -0.189 & -0.131 & -0.073 & 0.021 \\
\hline $\mathrm{y} 0[1]$ & 0.175 & 0.102 & -0.021 & 0.108 & 0.173 & 0.241 & 0.380 \\
\hline
\end{tabular}




\begin{tabular}{|c|c|c|c|c|c|c|c|}
\hline $\mathrm{y} 0[2]$ & -0.159 & 0.089 & -0.341 & -0.217 & -0.159 & -0.101 & 0.014 \\
\hline $\mathrm{y} 0[3]$ & -0.048 & 0.079 & -0.204 & -0.100 & -0.047 & 0.004 & 0.108 \\
\hline $\mathrm{y} 0[4]$ & -0.041 & 0.074 & -0.194 & -0.088 & -0.040 & 0.009 & 0.099 \\
\hline $\mathrm{y} 0[5]$ & 0.170 & 0.070 & 0.030 & 0.125 & 0.170 & 0.216 & 0.310 \\
\hline $\mathrm{y} 0[6]$ & -0.046 & 0.075 & -0.195 & -0.097 & -0.046 & 0.004 & 0.101 \\
\hline y0[7] & 0.024 & 0.076 & -0.122 & -0.027 & 0.023 & 0.073 & 0.179 \\
\hline $\mathrm{y} 0[8]$ & -0.087 & 0.078 & -0.235 & -0.139 & -0.087 & -0.035 & 0.069 \\
\hline $\mathrm{y} 0[9]$ & 0.041 & 0.080 & -0.111 & -0.013 & 0.040 & 0.093 & 0.206 \\
\hline y0[10] & -0.071 & 0.081 & -0.228 & -0.124 & -0.072 & -0.017 & 0.093 \\
\hline y0[11] & -0.086 & 0.080 & -0.241 & -0.138 & -0.087 & -0.033 & 0.073 \\
\hline y0[12] & 0.006 & 0.075 & -0.139 & -0.045 & 0.004 & 0.056 & 0.155 \\
\hline y0[13] & -0.105 & 0.074 & -0.254 & -0.153 & -0.104 & -0.056 & 0.035 \\
\hline y0[14] & -0.085 & 0.073 & -0.231 & -0.132 & -0.083 & -0.036 & 0.054 \\
\hline y0[15] & 0.035 & 0.076 & -0.117 & -0.016 & 0.035 & 0.086 & 0.187 \\
\hline y0[16] & 0.329 & 0.082 & 0.164 & 0.272 & 0.330 & 0.386 & 0.485 \\
\hline y0[17] & 0.158 & 0.083 & -0.008 & 0.103 & 0.159 & 0.214 & 0.316 \\
\hline y0[18] & -0.030 & 0.080 & -0.189 & -0.083 & -0.028 & 0.025 & 0.125 \\
\hline y0[19] & -0.003 & 0.074 & -0.144 & -0.052 & -0.003 & 0.047 & 0.145 \\
\hline y0[20] & -0.232 & 0.075 & -0.385 & -0.280 & -0.231 & -0.181 & -0.087 \\
\hline $\mathrm{y} 0[21]$ & 0.063 & 0.075 & -0.084 & 0.012 & 0.063 & 0.112 & 0.211 \\
\hline y0[22] & -0.018 & 0.077 & -0.164 & -0.070 & -0.019 & 0.033 & 0.136 \\
\hline $\mathrm{y} 0[23]$ & 0.003 & 0.078 & -0.149 & -0.050 & 0.002 & 0.054 & 0.158 \\
\hline y0[24] & 0.154 & 0.083 & -0.007 & 0.099 & 0.154 & 0.209 & 0.319 \\
\hline $\mathrm{y} 0[25]$ & -0.124 & 0.097 & -0.322 & -0.186 & -0.123 & -0.060 & 0.061 \\
\hline N.sum[1] & $11,918.695$ & 766.964 & $10,510.324$ & $11,383.067$ & $11,901.198$ & $12,405.464$ & $13,504.797$ \\
\hline N.sum[2] & $8,505.453$ & 568.535 & $7,446.600$ & $8,117.881$ & $8,483.019$ & $8,877.441$ & $9,664.176$ \\
\hline N.sum[3] & $9,490.077$ & 573.136 & $8,402.831$ & $9,092.044$ & $9,485.608$ & $9,860.587$ & $10,636.906$ \\
\hline N.sum[4] & $9,564.852$ & 537.291 & $8,551.628$ & $9,202.480$ & $9,552.027$ & $9,909.329$ & $10,645.704$ \\
\hline N.sum[5] & $11,858.282$ & 617.163 & $10,713.475$ & $11,435.146$ & $11,845.785$ & $12,271.294$ & $13,108.730$ \\
\hline N.sum[6] & $9,614.377$ & 550.256 & $8,579.748$ & $9,232.884$ & $9,594.252$ & $9,974.511$ & $10,744.254$ \\
\hline N.sum[7] & $10,413.351$ & 630.007 & $9,239.208$ & $9,982.434$ & $10,391.388$ & $10,821.984$ & $11,714.511$ \\
\hline N.sum[8] & $9,442.770$ & 584.058 & $8,338.745$ & $9,044.408$ & $9,430.751$ & $9,835.037$ & $10,636.227$ \\
\hline N.sum[9] & $10,924.531$ & 662.217 & $9,672.946$ & $10,470.041$ & $10,903.461$ & $11,359.446$ & $12,296.112$ \\
\hline N.sum[10] & $10,007.003$ & 585.031 & $8,883.027$ & $9,617.453$ & $9,991.796$ & $10,387.758$ & $11,197.125$ \\
\hline N.sum[11] & $10,175.640$ & 607.650 & $9,017.959$ & $9,758.066$ & $10,163.980$ & $10,569.258$ & $11,388.646$ \\
\hline N.sum[12] & $11,590.113$ & 645.977 & $10,352.455$ & $11,149.931$ & $11,588.056$ & $12,012.563$ & $12,904.280$ \\
\hline N.sum[13] & $10,855.223$ & 612.314 & $9,676.546$ & $10,433.356$ & $10,843.476$ & $11,264.353$ & $12,116.597$ \\
\hline N.sum[14] & $11,599.837$ & 642.024 & $10,403.918$ & $11,161.571$ & $11,582.250$ & $12,025.084$ & $12,916.220$ \\
\hline N.sum[15] & $13,653.679$ & 762.188 & $12,253.357$ & $13,134.740$ & $13,629.005$ & $14,150.064$ & $15,220.712$ \\
\hline N.sum[16] & $19,001.043$ & 942.511 & $17,229.357$ & $18,344.432$ & $18,989.049$ & $19,634.330$ & $20,874.943$ \\
\hline N.sum[17] & $16,445.063$ & 823.828 & $14,882.955$ & $15,883.937$ & $16,428.936$ & $16,993.387$ & $18,089.685$ \\
\hline N.sum[18] & $13,842.745$ & 734.779 & $12,430.207$ & $13,351.770$ & $13,834.388$ & $14,327.347$ & $15,312.582$ \\
\hline N.sum[19] & $14,308.147$ & 757.391 & $12,885.273$ & $13,795.368$ & $14,286.212$ & $14,806.241$ & $15,844.235$ \\
\hline N.sum[20] & $11,393.206$ & 674.155 & $10,136.570$ & $10,928.322$ & $11,366.832$ & $11,847.144$ & $12,767.084$ \\
\hline
\end{tabular}




\begin{tabular}{|c|c|c|c|c|c|c|c|}
\hline N.sum[21] & $5,301.506$ & 796.173 & 1.495 & 2.736 & 3.614 & 30 & 16 \\
\hline N.sum[22] & $4,201.761$ & 786.319 & $12,750.176$ & $13,653.556$ & $14,185.339$ & $14,726.481$ & 30.081 \\
\hline J.sum[23] & $4,692.396$ & 88.299 & $13,179.537$ & $14,159.618$ & $4,675.960$ & $15,213.327$ & $16,318.014$ \\
\hline [.sum[24] & $7,439.186$ & 89.717 & $15,716.164$ & $16,836.889$ & $7,428.625$ & $18,013.692$ & $19,239.978$ \\
\hline N.sum[25] & $3,527.851$ & 35.403 & $12,147.636$ & $13,011.478$ & $3,510.904$ & $14,004.888$ & $15,018.416$ \\
\hline [.sum2[1] & $0,156.330$ & 76.939 & $8,388.710$ & $9,485.017$ &, 113.062 & $10,765.528$ & 94.600 \\
\hline J.sum2[2] &, 111.519 & 769.240 & $8,685.514$ & $9,596.562$ & $10,091.615$ & $10,609.543$ & $11,738.423$ \\
\hline J.sum2[3] &, 089.402 & 632.705 & $8,881.843$ & $9,662.474$ & $10,079.882$ & $10,494.358$ & 1.137 \\
\hline I.sum2[4] & $0,095.661$ & 568.446 & $9,043.622$ & $9,706.034$ & $10,078.847$ & 458.084 & $11,272.983$ \\
\hline I.sum2[5] & $0,131.935$ & 553.349 & $9,127.835$ & $9,746.296$ & $10,111.700$ & 79.843 & $11,286.112$ \\
\hline. $\operatorname{sum} 2[6]$ & $0,198.018$ & 568.356 & $9,101.201$ & $9,816.366$ & $10,192.471$ & 3.494 & 9.778 \\
\hline. $\operatorname{sum} 2[7]$ & $10,295.402$ & 601.066 & $9,087.264$ & $9,907.754$ & $10,298.258$ & 86.663 & 86.513 \\
\hline .sum2[8] & $10,431.081$ & 635.227 & $9,125.553$ & $10,020.338$ & $10,446.334$ & $10,862.750$ & $11,645.220$ \\
\hline. $\operatorname{sum} 2[9]$ & $10,619.274$ & 656.854 & $9,288.912$ & $10,187.237$ & $10,643.385$ & 0.116 & $11,825.904$ \\
\hline .sum2[10] & $10,879.344$ & 661.035 & $9,551.452$ & $10,427.541$ & $10,914.625$ & 3.166 & 76.789 \\
\hline .sum2[11] & $11,228.895$ & 647.043 & $9,919.272$ & $10,789.366$ & $11,272.594$ & 8.906 & 36.564 \\
\hline .sum2[12] & $11,674.533$ & 617.089 & $10,397.557$ & $11,280.995$ & $11,707.071$ & $12,084.065$ & $12,836.140$ \\
\hline J.sum2[13] & $12,203.948$ & 593.106 & $11,061.624$ & $11,834.100$ & $12,191.062$ & 9.346 & 7.837 \\
\hline .sum2[14] & $12,782.709$ & 629.516 & $11,723.348$ & $12,363.764$ & $12,709.411$ & 315 & 2.703 \\
\hline [.sum2[15] & $13,357.761$ & 751.577 & $12,177.288$ & $12,806.404$ & $13,255.167$ & 692 & 6.965 \\
\hline .sum2[16] & 2.060 & 887.370 & $12,477.156$ & 4.734 & $13,736.669$ & 32 & 4.491 \\
\hline J.sum2[17] & 4.593 & 935.742 & 6.510 & 9.249 & 7.929 & 754 & 7.532 \\
\hline 18] & $14,449.255$ & 862.310 & $12,996.502$ & 5.091 & $14,383.965$ & 764 & 0.685 \\
\hline 19] & $14,530.625$ & 745.342 & $13,179.816$ & .547 & $14,503.815$ & 352 & 1.162 \\
\hline 20] & 2.868 & 718.439 & 2.770 & 6.556 & 14.009 & 951 & 3.184 \\
\hline. $\operatorname{sum} 2[21]$ & .614 & 787.530 & 8.235 & 818 & 31.672 & 374 & 2.685 \\
\hline .sum2[22] & .308 & 852.135 & 5.950 & .089 & 7.398 & 71 & 8.274 \\
\hline & 7.819 & 904.678 & .328 & 1.246 & 4.276 & 00 & 4.431 \\
\hline & $15,155.247$ & ,071.995 & & $14,427.595$ & $15,129.160$ & & 5.367 \\
\hline & $15,552.925$ & $1,456.851$ & $12,950.746$ & $14,598.385$ & $15,477.479$ & 0.192 & $18,847.666$ \\
\hline growth[1] & -0.003 & 0.029 & -0.061 & -0.022 & 0.000 & & 0.052 \\
\hline growth[2] & -0.001 & 0.027 & -0.054 & -0.019 & 0.001 & 0.017 & 0.049 \\
\hline growth[3] & 0.001 & 0.023 & -0.045 & -0.014 & 0.002 & 0.017 & 0.044 \\
\hline R.growth[4] & 0.004 & 0.020 & -0.040 & -0.009 & 0.005 & 0.018 & 0.040 \\
\hline growth[5] & 0.006 & 0.019 & -0.034 & -0.006 & 0.009 & 0.019 & 0.040 \\
\hline R.growth[6] & 0.009 & 0.018 & -0.030 & -0.001 & 0.012 & 0.022 & 0.040 \\
\hline R.growth[7] & 0.013 & 0.016 & -0.023 & 0.003 & 0.015 & 0.024 & 0.042 \\
\hline R.growth[8] & 0.018 & 0.016 & -0.019 & 0.009 & 0.019 & 0.028 & 0.046 \\
\hline R.growth[9] & 0.024 & 0.017 & -0.013 & 0.015 & 0.024 & 0.034 & 0.057 \\
\hline R.growth[10] & 0.032 & 0.018 & -0.001 & 0.020 & 0.030 & 0.043 & 0.072 \\
\hline R.growth[11] & 0.039 & 0.019 & 0.009 & 0.025 & 0.036 & 0.051 & 0.083 \\
\hline R.growth[12] & 0.045 & 0.022 & 0.012 & 0.027 & 0.041 & 0.059 & 0.094 \\
\hline R.growth[13] & 0.046 & 0.025 & 0.011 & 0.027 & 0.042 & 0.063 & 0.104 \\
\hline R.growth[14] & 0.044 & 0.025 & 0.009 & 0.025 & 0.039 & 0.059 & 0.103 \\
\hline
\end{tabular}




\begin{tabular}{lrrrrrrr} 
R.growth[15] & 0.037 & 0.020 & 0.006 & 0.022 & 0.033 & 0.049 & 0.085 \\
R.growth[16] & 0.026 & 0.015 & -0.003 & 0.017 & 0.026 & 0.035 & 0.059 \\
R.growth[17] & 0.015 & 0.017 & -0.025 & 0.007 & 0.018 & 0.026 & 0.045 \\
R.growth[18] & 0.006 & 0.023 & -0.055 & -0.006 & 0.011 & 0.022 & 0.041 \\
R.growth[19] & 0.001 & 0.026 & -0.065 & -0.013 & 0.006 & 0.019 & 0.039 \\
R.growth[20] & 0.001 & 0.023 & -0.051 & -0.013 & 0.005 & 0.018 & 0.036 \\
R.growth[21] & 0.006 & 0.019 & -0.033 & -0.007 & 0.008 & 0.019 & 0.040 \\
R.growth[22] & 0.013 & 0.023 & -0.031 & -0.001 & 0.013 & 0.025 & 0.065 \\
R.growth[23] & 0.020 & 0.031 & -0.035 & 0.003 & 0.018 & 0.032 & 0.104 \\
R.growth[24] & 0.024 & 0.036 & -0.037 & 0.004 & 0.021 & 0.037 & 0.127 \\
Deviance & $49,495.674$ & 53.963 & $49,392.595$ & $49,458.900$ & $49,495.658$ & $49,531.701$ & $49,602.443$ \\
\hline
\end{tabular}


Tables A4.21. Bayesian generalized linear mixed model output for Arctic Tern observed during aerial surveys 1992-2016 on the Arctic Coastal Plain, Alaska. We provide summaries including mean, SE, and several quantiles of the posterior distribution for model and derived parameters. See Table A4.4 for a key to parameter names.

\begin{tabular}{|c|c|c|c|c|c|c|c|}
\hline Parameter & Mean & SD & $2.50 \%$ & $25 \%$ & $50 \%$ & $75 \%$ & $97.50 \%$ \\
\hline MSE & 1.533 & 0.008 & 1.519 & 1.527 & 1.532 & 1.538 & 1.549 \\
\hline MSE.CV & 2.142 & 0.011 & 2.122 & 2.134 & 2.141 & 2.149 & 2.164 \\
\hline SSE & $33,219.883$ & 168.147 & $32,910.883$ & $33,102.978$ & $33,212.144$ & $33,330.945$ & $33,564.706$ \\
\hline Trend & 1.014 & 0.006 & 1.002 & 1.010 & 1.014 & 1.018 & 1.026 \\
\hline R.trend & 0.014 & 0.006 & 0.002 & 0.010 & 0.014 & 0.018 & 0.026 \\
\hline M.dens & 0.333 & 0.007 & 0.319 & 0.328 & 0.333 & 0.338 & 0.348 \\
\hline М.y0 & 0.001 & 0.027 & -0.053 & -0.016 & 0.001 & 0.019 & 0.054 \\
\hline M.blyr & -1.453 & 0.037 & -1.525 & -1.478 & -1.452 & -1.428 & -1.380 \\
\hline M.cell & 0.000 & 0.014 & -0.028 & -0.010 & 0.000 & 0.009 & 0.027 \\
\hline R.mid & 0.329 & 0.038 & 0.255 & 0.303 & 0.329 & 0.354 & 0.402 \\
\hline b.ADOY & -0.077 & 0.028 & -0.132 & -0.096 & -0.078 & -0.059 & -0.022 \\
\hline b.ADOY.2 & -0.012 & 0.013 & -0.037 & -0.021 & -0.012 & -0.003 & 0.012 \\
\hline SD.surv & 0.326 & 0.078 & 0.208 & 0.272 & 0.315 & 0.366 & 0.517 \\
\hline SD.y0 & 0.136 & 0.029 & 0.089 & 0.116 & 0.133 & 0.153 & 0.201 \\
\hline SD.cell & 0.506 & 0.018 & 0.471 & 0.494 & 0.506 & 0.518 & 0.542 \\
\hline SD.lam[1] & 0.550 & 0.112 & 0.377 & 0.467 & 0.535 & 0.615 & 0.809 \\
\hline SD.lam[2] & 1.328 & 0.503 & 0.719 & 0.980 & 1.203 & 1.542 & 2.652 \\
\hline SD.lam[3] & 0.711 & 0.780 & 0.016 & 0.151 & 0.498 & 0.961 & 2.871 \\
\hline SD.lam[4] & 1.068 & 0.704 & 0.327 & 0.621 & 0.883 & 1.288 & 2.983 \\
\hline SD.lam[5] & 4.531 & 4.095 & 0.122 & 1.178 & 3.084 & 7.104 & 14.021 \\
\hline SD.lam[6] & 2.582 & 1.031 & 1.131 & 1.677 & 2.486 & 3.294 & 4.782 \\
\hline SD.lam[7] & 1.067 & 0.497 & 0.405 & 0.597 & 1.068 & 1.430 & 2.092 \\
\hline SD.lam[8] & 0.430 & 1.401 & 0.006 & 0.059 & 0.135 & 0.303 & 2.154 \\
\hline S.eff[1] & 0.021 & 0.123 & -0.221 & -0.063 & 0.021 & 0.103 & 0.263 \\
\hline S.eff[2] & -0.157 & 0.118 & -0.388 & -0.234 & -0.158 & -0.077 & 0.070 \\
\hline S.eff[3] & -0.224 & 0.115 & -0.446 & -0.301 & -0.224 & -0.146 & 0.002 \\
\hline S.eff[4] & 0.232 & 0.105 & 0.027 & 0.161 & 0.232 & 0.301 & 0.433 \\
\hline S.eff[5] & -0.030 & 0.106 & -0.237 & -0.102 & -0.031 & 0.041 & 0.180 \\
\hline S.eff[6] & -0.247 & 0.116 & -0.475 & -0.325 & -0.247 & -0.168 & -0.022 \\
\hline S.eff[7] & -0.019 & 0.107 & -0.234 & -0.091 & -0.019 & 0.053 & 0.189 \\
\hline S.eff[8] & -0.025 & 0.122 & -0.267 & -0.108 & -0.025 & 0.056 & 0.215 \\
\hline S.eff[9] & -0.564 & 0.124 & -0.805 & -0.650 & -0.564 & -0.480 & -0.324 \\
\hline S.eff[10] & -0.303 & 0.103 & -0.506 & -0.374 & -0.302 & -0.234 & -0.101 \\
\hline S.eff[11] & -0.557 & 0.116 & -0.786 & -0.635 & -0.555 & -0.478 & -0.335 \\
\hline S.eff[12] & 0.106 & 0.100 & -0.088 & 0.038 & 0.106 & 0.174 & 0.303 \\
\hline S.eff[13] & -0.173 & 0.102 & -0.375 & -0.242 & -0.172 & -0.105 & 0.028 \\
\hline S.eff[14] & 0.403 & 0.100 & 0.209 & 0.335 & 0.402 & 0.470 & 0.601 \\
\hline S.eff[15] & -0.207 & 0.108 & -0.419 & -0.279 & -0.206 & -0.133 & 0.002 \\
\hline $\mathrm{y} 0[1]$ & -0.086 & 0.107 & -0.304 & -0.154 & -0.083 & -0.013 & 0.116 \\
\hline
\end{tabular}




\begin{tabular}{|c|c|c|c|c|c|c|c|}
\hline $\mathrm{y} 0[2]$ & 0.008 & 0.090 & -0.171 & -0.051 & 0.009 & 0.068 & 0.181 \\
\hline $\mathrm{y} 0[3]$ & 0.074 & 0.081 & -0.084 & 0.020 & 0.073 & 0.127 & 0.234 \\
\hline $\mathrm{y} 0[4]$ & -0.072 & 0.076 & -0.223 & -0.123 & -0.071 & -0.021 & 0.077 \\
\hline $\mathrm{y} 0[5]$ & 0.025 & 0.074 & -0.119 & -0.024 & 0.025 & 0.075 & 0.172 \\
\hline $\mathrm{y} 0[6]$ & 0.020 & 0.076 & -0.132 & -0.031 & 0.021 & 0.071 & 0.171 \\
\hline $\mathrm{y} 0[7]$ & 0.047 & 0.078 & -0.105 & -0.005 & 0.046 & 0.097 & 0.205 \\
\hline $\mathrm{y} 0[8]$ & -0.141 & 0.079 & -0.301 & -0.191 & -0.140 & -0.088 & 0.011 \\
\hline $\mathrm{y} 0[9]$ & 0.146 & 0.080 & -0.009 & 0.093 & 0.144 & 0.198 & 0.302 \\
\hline y0[10] & 0.154 & 0.075 & 0.014 & 0.102 & 0.152 & 0.204 & 0.304 \\
\hline y0[11] & -0.102 & 0.075 & -0.248 & -0.152 & -0.101 & -0.051 & 0.043 \\
\hline y0[12] & -0.048 & 0.076 & -0.191 & -0.099 & -0.048 & 0.002 & 0.103 \\
\hline y0[13] & 0.003 & 0.077 & -0.145 & -0.048 & 0.002 & 0.054 & 0.162 \\
\hline y0[14] & -0.155 & 0.079 & -0.315 & -0.207 & -0.153 & -0.101 & -0.004 \\
\hline y0[15] & -0.073 & 0.078 & -0.228 & -0.125 & -0.072 & -0.020 & 0.080 \\
\hline y0[16] & 0.222 & 0.075 & 0.081 & 0.172 & 0.220 & 0.270 & 0.378 \\
\hline y0[17] & 0.116 & 0.073 & -0.025 & 0.068 & 0.114 & 0.164 & 0.266 \\
\hline y0[18] & 0.066 & 0.073 & -0.074 & 0.017 & 0.066 & 0.115 & 0.210 \\
\hline y0[19] & 0.014 & 0.075 & -0.132 & -0.035 & 0.014 & 0.063 & 0.162 \\
\hline y0[20] & -0.254 & 0.080 & -0.418 & -0.306 & -0.250 & -0.200 & -0.106 \\
\hline $\mathrm{y} 0[21]$ & 0.047 & 0.077 & -0.106 & -0.004 & 0.047 & 0.098 & 0.198 \\
\hline y0[22] & 0.013 & 0.078 & -0.145 & -0.037 & 0.013 & 0.064 & 0.167 \\
\hline $\mathrm{y} 0[23]$ & 0.006 & 0.080 & -0.152 & -0.046 & 0.008 & 0.061 & 0.159 \\
\hline y0[24] & 0.047 & 0.085 & -0.122 & -0.008 & 0.047 & 0.103 & 0.212 \\
\hline $\mathrm{y} 0[25]$ & -0.050 & 0.098 & -0.255 & -0.113 & -0.048 & 0.015 & 0.138 \\
\hline N.sum[1] & $8,636.725$ & 752.378 & $7,262.314$ & $8,126.423$ & $8,598.980$ & $9,122.064$ & $10,178.143$ \\
\hline N.sum[2] & $10,239.809$ & 854.802 & $8,630.276$ & $9,650.954$ & $10,206.486$ & $10,790.862$ & $12,006.800$ \\
\hline N.sum[3] & $11,813.940$ & 869.308 & $10,207.214$ & $11,212.215$ & $11,768.670$ & $12,390.048$ & $13,620.752$ \\
\hline N.sum[4] & $11,016.458$ & 714.083 & $9,677.026$ & $10,524.382$ & $10,999.585$ & $11,478.090$ & $12,473.270$ \\
\hline N.sum[5] & $13,060.442$ & 818.846 & $11,552.055$ & $12,500.236$ & $13,038.117$ & $13,607.130$ & $14,762.196$ \\
\hline N.sum[6] & $13,894.297$ & 893.645 & $12,179.315$ & $13,282.694$ & $13,875.363$ & $14,482.821$ & $15,703.256$ \\
\hline N.sum[7] & $15,128.716$ & $1,100.165$ & $13,115.597$ & $14,352.627$ & $15,085.391$ & $15,851.740$ & $17,465.730$ \\
\hline N.sum[8] & $13,137.323$ & 940.746 & $11,387.693$ & $12,492.695$ & $13,104.815$ & $13,749.235$ & $15,078.658$ \\
\hline N.sum[9] & $18,155.296$ & $1,243.243$ & $15,873.757$ & $17,291.352$ & $18,093.506$ & $18,966.442$ & $20,797.321$ \\
\hline N.sum[10] & $18,821.861$ & $1,199.837$ & $16,579.076$ & $17,994.026$ & $18,747.715$ & $19,616.846$ & $21,297.235$ \\
\hline N.sum[11] & $14,927.456$ & $1,018.829$ & $13,053.774$ & $14,213.060$ & $14,879.418$ & $15,607.258$ & $16,996.291$ \\
\hline N.sum[12] & $16,071.959$ & $1,059.696$ & $14,085.027$ & $15,348.722$ & $16,045.115$ & $16,763.762$ & $18,238.536$ \\
\hline N.sum[13] & $17,238.914$ & $1,120.981$ & $15,103.673$ & $16,469.084$ & $17,212.640$ & $17,982.616$ & $19,509.358$ \\
\hline N.sum[14] & $14,977.053$ & $1,022.086$ & $13,080.705$ & $14,273.838$ & $14,950.313$ & $15,638.488$ & $17,040.022$ \\
\hline N.sum[15] & $16,478.126$ & $1,109.205$ & $14,385.527$ & $15,718.611$ & $16,443.088$ & $17,205.311$ & $18,708.339$ \\
\hline N.sum[16] & $22,296.956$ & $1,236.160$ & $19,987.125$ & $21,445.123$ & $22,246.165$ & $23,100.837$ & $24,800.901$ \\
\hline N.sum[17] & $20,009.122$ & $1,068.378$ & $17,998.852$ & $19,267.864$ & $19,974.114$ & $20,706.319$ & $22,199.871$ \\
\hline N.sum[18] & $18,771.026$ & $1,038.565$ & $16,831.975$ & $18,065.950$ & $18,742.473$ & $19,429.627$ & $20,895.476$ \\
\hline N.sum[19] & $17,359.992$ & 999.353 & $15,498.086$ & $16,669.153$ & $17,334.858$ & $18,021.974$ & $19,390.130$ \\
\hline N.sum[20] & $12,807.399$ & 852.297 & $11,188.855$ & $12,211.938$ & $12,785.485$ & $13,375.913$ & $14,511.695$ \\
\hline
\end{tabular}




\begin{tabular}{|c|c|c|c|c|c|c|c|}
\hline N.sum[21] & $16,536.178$ & 940.945 & $14,761.023$ & $15,890.286$ & 9.281 & $17,161.477$ & 18 \\
\hline N.sum[22] & $5,243.435$ & 927.716 & $13,477.915$ & $14,609.981$ & $15,213.979$ & $15,849.908$ & 9.618 \\
\hline. $\operatorname{sum}[23]$ & $4,425.606$ & 886.428 & $12,736.040$ & $13,812.007$ & $14,401.995$ & $15,014.431$ & $16,243.219$ \\
\hline. $\operatorname{sum}[24]$ & $4,335.266$ & 75.330 & $12,656.994$ & $13,734.673$ & $14,318.577$ & $14,901.214$ & $16,140.152$ \\
\hline J.sum[25] & $12,426.124$ & 815.284 & $10,908.953$ & $11,865.688$ & $12,399.018$ & $12,963.022$ & $14,085.580$ \\
\hline. $\operatorname{sum} 2[1]$ & $9,531.480$ & 035.214 & $7,694.284$ & $8,817.153$ & $9,475.655$ & $10,181.594$ & $11,724.649$ \\
\hline J.sum2[2] & $10,272.857$ & 879.225 & $8,711.040$ & $9,676.730$ & $10,227.580$ & $10,813.170$ & .764 \\
\hline N.sum2[3] & $11,089.996$ & 784.544 & $9,614.408$ & $10,559.772$ & $11,068.598$ & $11,589.033$ & 6.863 \\
\hline J.sum2[4] & $11,967.827$ & 757.725 & $10,541.314$ & $11,463.125$ & $11,942.712$ & $12,460.647$ & $13,529.471$ \\
\hline J.sum2[5] & $12,873.813$ & 766.393 & $11,472.497$ & $12,344.362$ & $12,843.957$ & $13,371.765$ & $14,466.387$ \\
\hline N.sum2[6] & $13,762.088$ & 800.742 & $12,308.494$ & $13,205.419$ & $13,728.566$ & $14,282.949$ & $15,424.594$ \\
\hline N.sum2[7] & $14,580.362$ & 868.547 & $13,018.076$ & $13,989.778$ & $14,534.200$ & $15,129.323$ & $16,399.263$ \\
\hline I.sum2[8] & $15,283.348$ & 935.827 & $13,620.344$ & $14,649.029$ & $15,242.289$ & $15,861.794$ & $17,244.703$ \\
\hline. $\operatorname{sum} 2[9]$ & $15,854.782$ & 951.787 & $14,135.208$ & $15,219.725$ & $15,813.684$ & $16,451.298$ & $17,802.519$ \\
\hline. $\operatorname{sum} 2[10]$ & $16,311.049$ & 922.252 & $14,601.607$ & $15,673.011$ & $16,283.158$ & 1.789 & 6.126 \\
\hline J.sum2[11] & $16,690.971$ & 910.149 & $14,961.011$ & 4.217 & $16,674.478$ & $17,287.898$ & 5.760 \\
\hline J.sum2[12] & $17,034.181$ & 946.756 & $15,258.520$ & $16,382.792$ & $17,010.848$ & $17,654.239$ & $18,976.302$ \\
\hline J.sum2[13] & $17,361.340$ & 988.743 & $15,468.181$ & $16,712.033$ & $17,339.350$ & $17,993.481$ & $19,360.921$ \\
\hline J.sum2[14] & $17,665.525$ & 993.646 & 8.320 & $17,008.578$ & 9.709 & 17.001 & 5.028 \\
\hline. $\operatorname{sum} 2[15]$ & $17,911.587$ & 976.073 & $16,034.403$ & $17,260.465$ & $17,885.538$ & $18,542.422$ & .617 \\
\hline. $\operatorname{sum} 2[16]$ & $18,044.838$ & 972.916 & 5.807 & .350 & 0.679 & 0.753 & 366 \\
\hline $2[17]$ & $18,006.359$ & 975.823 & 0.659 & .965 & .821 & 138 & 515 \\
\hline [18] & $17,756.906$ & 947.243 & $16,000.673$ & $17,105.254$ & $17,709.648$ & 5.007 & 7.791 \\
\hline [19] & $17,297.534$ & 897.518 & 7.038 & $16,680.785$ & $17,257.745$ & 6.604 & 9.383 \\
\hline $2[20]$ & $16,672.130$ & 878.602 & 3.755 & 2.157 & 4.125 & .891 & 7.293 \\
\hline. $\operatorname{sum} 2[21]$ & 2.413 & 893.032 & 1.489 & 3.344 & 6.293 & 708 & 3.696 \\
\hline. $\operatorname{sum} 2[22]$ & .353 & 900.254 & 2.720 & .000 & 6.329 & .420 & 3.474 \\
\hline & 2.361 & & .402 & .579 & 3.964 & 85 & 3.240 \\
\hline & $13,834.223$ & & 52.388 & .889 & & & 5.655 \\
\hline & $13,240.862$ & $1,278.014$ & $10,978.337$ & $12,362.371$ & $13,169.426$ & $14,012.236$ & $15,974.144$ \\
\hline growth[1] & 0.077 & & 0.014 & 0.054 & 0.075 & 0.098 & 0.154 \\
\hline .growth[2] & 0.078 & 0.031 & 0.021 & 0.057 & 0.076 & 0.096 & 0.145 \\
\hline growth[3] & 0.077 & 0.025 & 0.029 & 0.060 & 0.076 & 0.092 & 0.130 \\
\hline growth[4] & 0.073 & 0.021 & 0.032 & 0.059 & 0.073 & 0.087 & 0.116 \\
\hline growth[5] & 0.067 & 0.021 & 0.027 & 0.053 & 0.066 & 0.080 & 0.112 \\
\hline .growth[6] & 0.058 & 0.021 & 0.018 & 0.045 & 0.057 & 0.070 & 0.101 \\
\hline R.growth[7] & 0.047 & 0.018 & 0.013 & 0.035 & 0.047 & 0.058 & 0.083 \\
\hline R.growth[8] & 0.037 & 0.016 & 0.004 & 0.026 & 0.037 & 0.048 & 0.067 \\
\hline R.growth[9] & 0.029 & 0.019 & -0.011 & 0.018 & 0.029 & 0.041 & 0.062 \\
\hline R.growth[10] & 0.023 & 0.021 & -0.023 & 0.012 & 0.024 & 0.036 & 0.062 \\
\hline R.growth[11] & 0.020 & 0.021 & -0.025 & 0.009 & 0.021 & 0.033 & 0.058 \\
\hline R.growth[12] & 0.019 & 0.018 & -0.019 & 0.009 & 0.019 & 0.030 & 0.053 \\
\hline R.growth[13] & 0.017 & 0.017 & -0.015 & 0.006 & 0.017 & 0.029 & 0.052 \\
\hline R.growth[14] & 0.014 & 0.019 & -0.023 & 0.002 & 0.013 & 0.026 & 0.055 \\
\hline
\end{tabular}




\begin{tabular}{lrrrrrrr} 
R.growth[15] & 0.007 & 0.020 & -0.031 & -0.005 & 0.007 & 0.019 & 0.049 \\
R.growth[16] & -0.002 & 0.019 & -0.039 & -0.014 & -0.002 & 0.009 & 0.036 \\
R.growth[17] & -0.014 & 0.017 & -0.049 & -0.025 & -0.014 & -0.003 & 0.021 \\
R.growth[18] & -0.026 & 0.019 & -0.063 & -0.039 & -0.026 & -0.014 & 0.012 \\
R.growth[19] & -0.037 & 0.021 & -0.078 & -0.051 & -0.037 & -0.023 & 0.005 \\
R.growth[20] & -0.044 & 0.020 & -0.086 & -0.057 & -0.044 & -0.031 & -0.004 \\
R.growth[21] & -0.048 & 0.019 & -0.087 & -0.061 & -0.047 & -0.035 & -0.010 \\
R.growth[22] & -0.048 & 0.023 & -0.095 & -0.064 & -0.048 & -0.033 & -0.003 \\
R.growth[23] & -0.047 & 0.030 & -0.109 & -0.065 & -0.047 & -0.029 & 0.010 \\
R.growth[24] & -0.046 & 0.033 & -0.115 & -0.065 & -0.045 & -0.025 & 0.020 \\
Deviance & $45,223.268$ & 52.528 & $45,122.394$ & $45,187.118$ & $45,222.125$ & $45,258.552$ & $45,326.912$ \\
\hline
\end{tabular}


Tables A4.22. Bayesian generalized linear mixed model output for Red-throated Loon observed during aerial surveys 1992-2016 on the Arctic Coastal Plain, Alaska. We provide summaries including mean, SE, and several quantiles of the posterior distribution for model and derived parameters. See Table A4.4 for a key to parameter names.

\begin{tabular}{|c|c|c|c|c|c|c|c|}
\hline Parameter & Mean & SD & $2.50 \%$ & $25 \%$ & $50 \%$ & $75 \%$ & $97.50 \%$ \\
\hline MSE & 0.373 & 0.001 & 0.370 & 0.372 & 0.373 & 0.374 & 0.375 \\
\hline MSE.CV & 1.762 & 0.006 & 1.751 & 1.758 & 1.762 & 1.766 & 1.774 \\
\hline SSE & $6,344.404$ & 20.574 & $6,304.360$ & $6,330.587$ & $6,344.278$ & $6,358.066$ & $6,384.904$ \\
\hline Trend & 0.966 & 0.011 & 0.944 & 0.958 & 0.966 & 0.973 & 0.987 \\
\hline R.trend & -0.035 & 0.011 & -0.058 & -0.042 & -0.035 & -0.028 & -0.013 \\
\hline M.dens & 0.093 & 0.005 & 0.085 & 0.090 & 0.093 & 0.096 & 0.104 \\
\hline M.y0 & 0.001 & 0.068 & -0.132 & -0.042 & 0.001 & 0.043 & 0.142 \\
\hline M.blyr & -2.610 & 0.079 & -2.770 & -2.659 & -2.609 & -2.559 & -2.454 \\
\hline M.cell & 0.000 & 0.008 & -0.016 & -0.005 & 0.000 & 0.005 & 0.017 \\
\hline R.mid & -1.061 & 0.057 & -1.171 & -1.100 & -1.062 & -1.023 & -0.952 \\
\hline b.ADOY & -0.126 & 0.044 & -0.213 & -0.156 & -0.126 & -0.096 & -0.042 \\
\hline b.ADOY.2 & 0.014 & 0.019 & -0.023 & 0.000 & 0.013 & 0.027 & 0.052 \\
\hline SD.surv & 0.758 & 0.171 & 0.497 & 0.637 & 0.734 & 0.853 & 1.157 \\
\hline SD.y0 & 0.342 & 0.069 & 0.228 & 0.293 & 0.335 & 0.383 & 0.499 \\
\hline SD.cell & 0.243 & 0.049 & 0.142 & 0.209 & 0.245 & 0.275 & 0.336 \\
\hline SD.lam[1] & 0.414 & 0.160 & 0.155 & 0.298 & 0.408 & 0.514 & 0.757 \\
\hline SD.lam[2] & 1.806 & 0.712 & 0.872 & 1.322 & 1.662 & 2.125 & 3.606 \\
\hline SD.lam[3] & 1.098 & 1.432 & 0.066 & 0.377 & 0.716 & 1.273 & 4.474 \\
\hline SD.lam[4] & 1.666 & 1.842 & 0.021 & 0.407 & 1.062 & 2.277 & 6.796 \\
\hline SD.lam[5] & 5.420 & 4.056 & 0.287 & 2.030 & 4.370 & 8.294 & 14.160 \\
\hline SD.lam[6] & 1.240 & 0.416 & 0.687 & 0.911 & 1.147 & 1.508 & 2.201 \\
\hline SD.lam[7] & 3.552 & 2.380 & 0.977 & 1.679 & 2.997 & 4.542 & 9.739 \\
\hline SD.lam[8] & 0.490 & 0.678 & 0.024 & 0.131 & 0.264 & 0.538 & 2.748 \\
\hline S.eff[1] & -0.831 & 0.227 & -1.295 & -0.984 & -0.831 & -0.678 & -0.393 \\
\hline S.eff[2] & -0.758 & 0.231 & -1.223 & -0.913 & -0.754 & -0.602 & -0.304 \\
\hline S.eff[3] & -0.633 & 0.212 & -1.053 & -0.775 & -0.636 & -0.488 & -0.222 \\
\hline S.eff[4] & -0.462 & 0.218 & -0.887 & -0.612 & -0.459 & -0.314 & -0.042 \\
\hline S.eff[5] & 0.078 & 0.205 & -0.319 & -0.060 & 0.078 & 0.217 & 0.481 \\
\hline S.eff[6] & -0.334 & 0.246 & -0.838 & -0.497 & -0.331 & -0.166 & 0.140 \\
\hline S.eff[7] & 0.636 & 0.237 & 0.172 & 0.475 & 0.638 & 0.795 & 1.108 \\
\hline S.eff[8] & 1.009 & 0.251 & 0.521 & 0.837 & 1.011 & 1.181 & 1.490 \\
\hline S.eff[9] & 0.544 & 0.212 & 0.127 & 0.401 & 0.541 & 0.685 & 0.967 \\
\hline S.eff[10] & 0.736 & 0.192 & 0.361 & 0.606 & 0.737 & 0.865 & 1.114 \\
\hline S.eff[11] & -0.022 & 0.210 & -0.436 & -0.160 & -0.020 & 0.117 & 0.392 \\
\hline S.eff[12] & 0.613 & 0.216 & 0.182 & 0.465 & 0.615 & 0.755 & 1.037 \\
\hline S.eff[13] & 0.710 & 0.200 & 0.323 & 0.573 & 0.710 & 0.848 & 1.098 \\
\hline S.eff[14] & 0.453 & 0.210 & 0.048 & 0.311 & 0.448 & 0.594 & 0.866 \\
\hline S.eff[15] & 1.087 & 0.223 & 0.648 & 0.939 & 1.083 & 1.237 & 1.531 \\
\hline $\mathrm{y} 0[1]$ & 0.184 & 0.249 & -0.322 & 0.024 & 0.189 & 0.348 & 0.673 \\
\hline
\end{tabular}




\begin{tabular}{|c|c|c|c|c|c|c|c|}
\hline $\mathrm{y} 0[2]$ & -0.098 & 0.200 & -0.497 & -0.228 & -0.095 & 0.036 & 0.283 \\
\hline $\mathrm{y} 0[3]$ & 0.158 & 0.173 & -0.178 & 0.044 & 0.159 & 0.270 & 0.496 \\
\hline $\mathrm{y} 0[4]$ & 0.133 & 0.160 & -0.182 & 0.027 & 0.133 & 0.240 & 0.444 \\
\hline $\mathrm{y} 0[5]$ & 0.192 & 0.153 & -0.104 & 0.090 & 0.193 & 0.292 & 0.501 \\
\hline $\mathrm{y} 0[6]$ & 0.114 & 0.163 & -0.196 & 0.001 & 0.110 & 0.218 & 0.446 \\
\hline $\mathrm{y} 0[7]$ & -0.654 & 0.193 & -1.037 & -0.782 & -0.652 & -0.524 & -0.285 \\
\hline $\mathrm{y} 0[8]$ & -0.523 & 0.191 & -0.893 & -0.653 & -0.523 & -0.392 & -0.146 \\
\hline $\mathrm{y} 0[9]$ & 0.156 & 0.172 & -0.166 & 0.041 & 0.149 & 0.267 & 0.518 \\
\hline y0[10] & 0.168 & 0.174 & -0.164 & 0.051 & 0.163 & 0.281 & 0.534 \\
\hline y0[11] & 0.200 & 0.169 & -0.124 & 0.084 & 0.198 & 0.313 & 0.534 \\
\hline y0[12] & -0.067 & 0.175 & -0.405 & -0.182 & -0.067 & 0.049 & 0.283 \\
\hline y0[13] & -0.044 & 0.171 & -0.385 & -0.155 & -0.046 & 0.068 & 0.296 \\
\hline y0[14] & -0.030 & 0.171 & -0.375 & -0.142 & -0.028 & 0.086 & 0.304 \\
\hline y0[15] & -0.318 & 0.186 & -0.695 & -0.437 & -0.314 & -0.195 & 0.030 \\
\hline y0[16] & 0.522 & 0.168 & 0.188 & 0.411 & 0.524 & 0.633 & 0.848 \\
\hline y0[17] & 0.071 & 0.169 & -0.269 & -0.038 & 0.070 & 0.184 & 0.399 \\
\hline y0[18] & 0.287 & 0.161 & -0.031 & 0.183 & 0.286 & 0.394 & 0.612 \\
\hline y0[19] & -0.013 & 0.173 & -0.358 & -0.127 & -0.013 & 0.101 & 0.326 \\
\hline y0[20] & -0.234 & 0.182 & -0.594 & -0.353 & -0.231 & -0.111 & 0.120 \\
\hline $\mathrm{y} 0[21]$ & -0.039 & 0.178 & -0.393 & -0.158 & -0.039 & 0.079 & 0.316 \\
\hline y0[22] & -0.215 & 0.193 & -0.599 & -0.343 & -0.213 & -0.084 & 0.163 \\
\hline y0[23] & -0.432 & 0.199 & -0.826 & -0.564 & -0.431 & -0.298 & -0.041 \\
\hline y0[24] & 0.307 & 0.201 & -0.069 & 0.169 & 0.307 & 0.441 & 0.711 \\
\hline $\mathrm{y} 0[25]$ & 0.207 & 0.228 & -0.242 & 0.053 & 0.210 & 0.363 & 0.645 \\
\hline N.sum[1] & $5,464.960$ & 744.487 & $4,168.211$ & $4,941.950$ & $5,410.200$ & $5,932.741$ & $7,085.118$ \\
\hline N.sum[2] & $3,780.500$ & 499.965 & $2,910.894$ & $3,422.587$ & $3,752.494$ & $4,095.278$ & $4,824.659$ \\
\hline N.sum[3] & $4,497.124$ & 523.273 & $3,554.439$ & $4,137.478$ & $4,467.987$ & $4,828.381$ & $5,611.301$ \\
\hline N.sum[4] & $4,065.339$ & 457.306 & $3,250.230$ & $3,748.014$ & $4,032.696$ & $4,357.722$ & $5,028.214$ \\
\hline N.sum[5] & $4,018.481$ & 449.933 & $3,213.439$ & $3,702.714$ & $3,993.646$ & $4,305.456$ & $4,957.009$ \\
\hline N.sum[6] & $3,475.950$ & 430.489 & $2,712.870$ & $3,172.276$ & $3,446.502$ & $3,748.009$ & $4,381.369$ \\
\hline N.sum[7] & $1,523.541$ & 252.210 & $1,080.270$ & $1,348.355$ & $1,509.567$ & $1,679.726$ & $2,058.934$ \\
\hline N.sum[8] & $1,639.486$ & 258.229 & $1,184.290$ & $1,456.483$ & $1,623.094$ & $1,809.090$ & $2,186.056$ \\
\hline N.sum[9] & $3,076.720$ & 397.198 & $2,367.669$ & $2,798.545$ & $3,051.106$ & $3,334.143$ & $3,919.117$ \\
\hline N.sum[10] & $3,018.566$ & 395.112 & $2,315.372$ & $2,743.062$ & $2,993.479$ & $3,255.527$ & $3,895.240$ \\
\hline N.sum[11] & $3,065.486$ & 396.069 & $2,368.550$ & $2,789.786$ & $3,036.499$ & $3,315.119$ & $3,912.483$ \\
\hline N.sum[12] & $2,331.838$ & 329.890 & $1,759.738$ & $2,093.199$ & $2,305.069$ & $2,543.757$ & $3,041.984$ \\
\hline N.sum[13] & $2,369.912$ & 323.234 & $1,797.003$ & $2,138.414$ & $2,348.004$ & $2,575.668$ & $3,056.341$ \\
\hline N.sum[14] & $2,376.531$ & 320.439 & $1,813.480$ & $2,153.194$ & $2,354.759$ & $2,581.454$ & $3,064.473$ \\
\hline N.sum[15] & $1,750.806$ & 281.454 & $1,253.868$ & $1,556.220$ & $1,729.851$ & $1,927.750$ & $2,369.043$ \\
\hline N.sum[16] & $3,908.877$ & 460.412 & $3,091.725$ & $3,581.261$ & $3,873.998$ & $4,210.648$ & $4,875.183$ \\
\hline N.sum[17] & $2,394.549$ & 312.982 & $1,836.161$ & $2,180.912$ & $2,378.913$ & $2,588.863$ & $3,058.034$ \\
\hline N.sum[18] & $2,834.859$ & 349.759 & $2,208.702$ & $2,593.523$ & $2,809.019$ & $3,057.429$ & $3,591.860$ \\
\hline N.sum[19] & $2,006.693$ & 289.584 & $1,479.967$ & $1,803.248$ & $1,994.247$ & $2,190.449$ & $2,624.989$ \\
\hline N.sum[20] & $1,544.124$ & 240.516 & $1,116.026$ & $1,374.285$ & $1,529.195$ & $1,697.419$ & $2,066.383$ \\
\hline
\end{tabular}




\begin{tabular}{|c|c|c|c|c|c|c|c|}
\hline N.sum[21] & $1,813.780$ & 262.946 & $1,347.849$ & $1,632.471$ & $1,799.260$ & $1,977.520$ & $2,378.030$ \\
\hline N.sum[22] & $1,501.618$ & 240.186 & $1,077.367$ & $1,330.722$ & $1,488.943$ & $1,651.684$ & $2,022.624$ \\
\hline N.sum[23] &, 215.546 & 206.463 & 854.866 & $1,069.032$ & $1,200.353$ & $1,347.137$ & $1,660.142$ \\
\hline J.sum[24] &, 580.947 & 347.544 & $1,970.437$ & $2,342.805$ & $2,556.718$ & $2,794.185$ & $3,344.374$ \\
\hline N.sum[25] &, 411.295 & 344.138 & $1,813.721$ & $2,167.295$ & $2,389.044$ & $2,628.319$ & $3,140.356$ \\
\hline N.sum2[1] & $4,918.532$ &, 153.183 & $3,203.306$ & $4,112.417$ & $4,714.853$ & $5,538.549$ & $7,773.093$ \\
\hline N.sum2[2] & $4,467.916$ & 812.726 & $3,158.353$ & $3,904.311$ & $4,358.012$ & $4,929.154$ & $6,398.334$ \\
\hline N.sum2[3] & $4,102.201$ & 613.248 & $3,095.262$ & $3,685.459$ & $4,031.091$ & $4,441.240$ & $5,504.774$ \\
\hline N.sum2[4] & $3,796.175$ & 500.092 & $2,957.050$ & $3,452.085$ & $3,746.184$ & $4,084.172$ & $4,921.754$ \\
\hline N.sum2[5] & $3,531.791$ & 431.873 & $2,737.737$ & $3,242.695$ & $3,513.484$ & $3,796.990$ & $4,461.219$ \\
\hline N.sum2[6] & $3,300.475$ & 396.905 & $2,532.371$ & $3,037.615$ & $3,298.451$ & $3,551.329$ & $4,104.638$ \\
\hline N.sum2[7] & $3,099.832$ & 385.957 & $2,335.035$ & $2,847.448$ & $3,106.588$ & $3,353.974$ & $3,856.252$ \\
\hline N.sum2[8] & $2,931.574$ & 380.776 & $2,182.840$ & $2,682.100$ & $2,950.731$ & $3,195.299$ & $3,647.003$ \\
\hline N.sum2[9] & $2,802.383$ & 367.829 & $2,055.096$ & $2,558.262$ & $2,825.736$ & $3,058.646$ & $3,472.376$ \\
\hline N.sum2[10] & $2,715.384$ & 344.439 & $2,025.036$ & $2,486.996$ & $2,737.312$ & $2,948.818$ & $3,355.231$ \\
\hline N.sum2[11] & $2,666.626$ & 318.751 & $2,040.937$ & $2,457.029$ & $2,678.234$ & $2,866.715$ & $3,315.888$ \\
\hline N.sum2[12] & $2,643.953$ & 304.423 & $2,082.813$ & $2,453.426$ & $2,639.680$ & $2,813.859$ & $3,321.205$ \\
\hline N.sum2[13] & $2,628.163$ & 306.174 & $2,105.580$ & $2,437.513$ & $2,600.432$ & $2,777.084$ & $3,330.300$ \\
\hline N.sum2[14] & $2,599.529$ & 313.549 & $2,091.232$ & $2,399.614$ & $2,556.330$ & $2,745.736$ & $3,393.527$ \\
\hline N.sum2[15] & $2,546.711$ & 314.565 & $2,054.537$ & $2,343.402$ & $2,492.894$ & $2,683.064$ & $3,377.123$ \\
\hline N.sum2[16] & $2,467.671$ & 303.374 & $2,001.380$ & $2,272.253$ & $2,421.862$ & $2,598.422$ & $3,249.480$ \\
\hline N.sum2[17] & $2,368.078$ & 279.269 & $1,925.724$ & $2,189.304$ & $2,336.888$ & $2,497.155$ & $3,037.048$ \\
\hline N.sum2[18] & $2,259.163$ & 250.869 & $1,825.175$ & $2,100.084$ & $2,238.220$ & $2,392.194$ & 7.848 \\
\hline N.sum2[19] & $2,154.089$ & 235.528 & $1,724.146$ & $1,997.993$ & $2,144.329$ & $2,298.534$ & $2,651.759$ \\
\hline N.sum2[20] & $2,064.702$ & 238.325 & $1,604.059$ & $1,906.064$ & $2,060.182$ & $2,220.284$ & $2,552.526$ \\
\hline N.sum2[21] & $2,002.012$ & 246.502 & $1,529.991$ & $1,835.325$ & $1,998.099$ & $2,165.371$ & $2,503.814$ \\
\hline N.sum2[22] & $1,973.022$ & 252.516 & $1,494.328$ & $1,801.943$ & $1,966.400$ & $2,134.810$ & 5.708 \\
\hline N.sum2[23] & $1,981.023$ & 268.797 & $1,505.453$ & $1,795.071$ & $1,965.471$ & $2,152.370$ & 9.185 \\
\hline N.sum2[24] & $2,026.615$ & 324.590 & $1,482.212$ & $1,797.059$ & $1,994.352$ & $2,226.942$ & 7.945 \\
\hline N.sum2[25] & $2,107.662$ & 439.870 & $1,429.704$ & $1,798.064$ & $2,044.392$ & $2,354.677$ & $3,138.962$ \\
\hline R.growth[1] & -0.086 & 0.068 & -0.257 & -0.121 & -0.070 & -0.040 & 0.011 \\
\hline R.growth[2] & -0.080 & 0.062 & -0.236 & -0.113 & -0.065 & -0.037 & 0.010 \\
\hline R.growth[3] & -0.075 & 0.053 & -0.203 & -0.106 & -0.065 & -0.036 & 0.004 \\
\hline R.growth[4] & -0.071 & 0.047 & -0.178 & -0.099 & -0.063 & -0.036 & 0.000 \\
\hline R.growth[5] & -0.068 & 0.046 & -0.175 & -0.092 & -0.059 & -0.035 & 0.000 \\
\hline R.growth[6] & -0.063 & 0.044 & -0.170 & -0.085 & -0.055 & -0.034 & 0.005 \\
\hline R.growth[7] & -0.057 & 0.040 & -0.147 & -0.077 & -0.051 & -0.033 & 0.017 \\
\hline R.growth[8] & -0.045 & 0.035 & -0.115 & -0.066 & -0.045 & -0.028 & 0.036 \\
\hline R.growth[9] & -0.031 & 0.036 & -0.093 & -0.052 & -0.036 & -0.015 & 0.057 \\
\hline R.growth[10] & -0.017 & 0.042 & -0.079 & -0.044 & -0.027 & 0.002 & 0.089 \\
\hline R.growth[11] & -0.008 & 0.045 & -0.068 & -0.039 & -0.021 & 0.013 & 0.107 \\
\hline R.growth[12] & -0.006 & 0.045 & -0.066 & -0.037 & -0.019 & 0.017 & 0.108 \\
\hline R.growth[13] & -0.011 & 0.042 & -0.074 & -0.038 & -0.021 & 0.009 & 0.095 \\
\hline R.growth[14] & -0.021 & 0.038 & -0.094 & -0.041 & -0.025 & -0.004 & 0.073 \\
\hline
\end{tabular}




\begin{tabular}{lrrrrrrr} 
R.growth[15] & -0.031 & 0.036 & -0.115 & -0.048 & -0.030 & -0.014 & 0.043 \\
R.growth[16] & -0.041 & 0.038 & -0.133 & -0.057 & -0.033 & -0.017 & 0.019 \\
R.growth[17] & -0.046 & 0.041 & -0.153 & -0.066 & -0.036 & -0.019 & 0.013 \\
R.growth[18] & -0.048 & 0.044 & -0.160 & -0.067 & -0.037 & -0.019 & 0.015 \\
R.growth[19] & -0.043 & 0.042 & -0.143 & -0.061 & -0.036 & -0.018 & 0.023 \\
R.growth[20] & -0.032 & 0.037 & -0.112 & -0.050 & -0.030 & -0.011 & 0.037 \\
R.growth[21] & -0.015 & 0.037 & -0.084 & -0.038 & -0.020 & 0.007 & 0.067 \\
R.growth[22] & 0.003 & 0.048 & -0.073 & -0.031 & -0.007 & 0.031 & 0.117 \\
R.growth[23] & 0.019 & 0.062 & -0.071 & -0.025 & 0.005 & 0.055 & 0.171 \\
R.growth[24] & 0.031 & 0.069 & -0.069 & -0.018 & 0.014 & 0.070 & 0.203 \\
Deviance & $17,910.782$ & 39.820 & $17,832.574$ & $17,882.962$ & $17,910.414$ & $17,938.906$ & $17,987.837$ \\
\hline
\end{tabular}


Tables A4.23. Bayesian generalized linear mixed model output for Pacific Loon observed during aerial surveys 1992-2016 on the Arctic Coastal Plain, Alaska. We provide summaries including mean, SE, and several quantiles of the posterior distribution for model and derived parameters. See Table A4.4 for a key to parameter names.

\begin{tabular}{|c|c|c|c|c|c|c|c|}
\hline Parameter & Mean & SD & $2.5 \%$ & $25 \%$ & $50 \%$ & $75 \%$ & $97.5 \%$ \\
\hline MSE & 2.639 & 0.011 & 2.618 & 2.631 & 2.639 & 2.646 & 2.661 \\
\hline MSE.CV & 2.071 & 0.009 & 2.055 & 2.065 & 2.071 & 2.076 & 2.088 \\
\hline SSE & $67,583.267$ & 281.653 & $67,056.840$ & $67,392.351$ & $67,576.025$ & $67,761.293$ & $68,153.355$ \\
\hline Trend & 1.001 & 0.006 & 0.990 & 0.997 & 1.000 & 1.004 & 1.013 \\
\hline R.trend & 0.001 & 0.006 & -0.010 & -0.003 & 0.000 & 0.004 & 0.013 \\
\hline M.dens & 0.561 & 0.010 & 0.542 & 0.554 & 0.560 & 0.567 & 0.582 \\
\hline M.y0 & 0.002 & 0.052 & -0.102 & -0.033 & 0.004 & 0.037 & 0.099 \\
\hline M.blyr & -0.807 & 0.055 & -0.912 & -0.845 & -0.810 & -0.769 & -0.694 \\
\hline M.cell & 0.000 & 0.012 & -0.022 & -0.008 & 0.000 & 0.008 & 0.023 \\
\hline R.mid & 0.595 & 0.029 & 0.540 & 0.576 & 0.595 & 0.614 & 0.652 \\
\hline b.ADOY & 0.044 & 0.022 & 0.001 & 0.029 & 0.044 & 0.059 & 0.089 \\
\hline b.ADOY.2 & 0.001 & 0.009 & -0.015 & -0.004 & 0.001 & 0.007 & 0.018 \\
\hline SD.surv & 0.354 & 0.079 & 0.232 & 0.298 & 0.344 & 0.398 & 0.537 \\
\hline SD.y0 & 0.245 & 0.040 & 0.181 & 0.217 & 0.241 & 0.270 & 0.335 \\
\hline SD.cell & 0.462 & 0.014 & 0.435 & 0.452 & 0.462 & 0.471 & 0.490 \\
\hline SD.lam[1] & 0.480 & 0.103 & 0.328 & 0.405 & 0.462 & 0.539 & 0.724 \\
\hline SD.lam[2] & 1.329 & 0.377 & 0.822 & 1.073 & 1.261 & 1.500 & 2.212 \\
\hline SD.lam[3] & 1.150 & 1.088 & 0.169 & 0.537 & 0.835 & 1.327 & 4.533 \\
\hline SD.lam[4] & 0.391 & 0.419 & 0.012 & 0.121 & 0.264 & 0.501 & 1.571 \\
\hline SD.lam[5] & 3.585 & 3.884 & 0.044 & 0.580 & 1.924 & 5.599 & 13.584 \\
\hline SD.lam[6] & 1.348 & 0.418 & 0.645 & 1.030 & 1.350 & 1.614 & 2.240 \\
\hline SD.lam[7] & 0.743 & 0.448 & 0.284 & 0.394 & 0.505 & 1.094 & 1.750 \\
\hline SD.lam[8] & 0.503 & 0.874 & 0.039 & 0.122 & 0.231 & 0.499 & 3.105 \\
\hline S.eff[1] & -0.213 & 0.087 & -0.388 & -0.270 & -0.213 & -0.154 & -0.046 \\
\hline S.eff[2] & -0.085 & 0.081 & -0.243 & -0.140 & -0.086 & -0.030 & 0.072 \\
\hline S.eff[3] & -0.387 & 0.081 & -0.547 & -0.442 & -0.388 & -0.331 & -0.228 \\
\hline S.eff[4] & -0.151 & 0.077 & -0.304 & -0.202 & -0.150 & -0.098 & 0.000 \\
\hline S.eff[5] & -0.215 & 0.080 & -0.371 & -0.268 & -0.214 & -0.161 & -0.058 \\
\hline S.eff[6] & -0.245 & 0.083 & -0.408 & -0.302 & -0.244 & -0.189 & -0.084 \\
\hline S.eff[7] & 0.335 & 0.084 & 0.170 & 0.278 & 0.335 & 0.392 & 0.502 \\
\hline S.eff[8] & -0.047 & 0.090 & -0.224 & -0.107 & -0.046 & 0.014 & 0.128 \\
\hline S.eff[9] & -0.747 & 0.093 & -0.929 & -0.809 & -0.744 & -0.683 & -0.569 \\
\hline S.eff[10] & -0.380 & 0.083 & -0.543 & -0.435 & -0.380 & -0.324 & -0.217 \\
\hline S.eff[11] & -0.437 & 0.081 & -0.595 & -0.493 & -0.437 & -0.382 & -0.280 \\
\hline S.eff[12] & -0.279 & 0.080 & -0.438 & -0.332 & -0.279 & -0.224 & -0.123 \\
\hline S.eff[13] & -0.196 & 0.081 & -0.357 & -0.252 & -0.195 & -0.140 & -0.040 \\
\hline S.eff[14] & -0.162 & 0.080 & -0.319 & -0.216 & -0.164 & -0.108 & -0.006 \\
\hline S.eff[15] & -0.041 & 0.083 & -0.206 & -0.097 & -0.041 & 0.015 & 0.120 \\
\hline y0[1] & -0.238 & 0.117 & -0.470 & -0.314 & -0.238 & -0.161 & -0.008 \\
\hline
\end{tabular}




\begin{tabular}{|c|c|c|c|c|c|c|c|}
\hline $\mathrm{y} 0[2]$ & -0.001 & 0.103 & -0.203 & -0.068 & -0.004 & 0.067 & 0.205 \\
\hline $\mathrm{y} 0[3]$ & 0.122 & 0.093 & -0.066 & 0.061 & 0.120 & 0.183 & 0.309 \\
\hline $\mathrm{y} 0[4]$ & 0.243 & 0.087 & 0.068 & 0.185 & 0.242 & 0.303 & 0.413 \\
\hline $\mathrm{y} 0[5]$ & 0.154 & 0.083 & -0.011 & 0.099 & 0.155 & 0.210 & 0.316 \\
\hline $\mathrm{y} 0[6]$ & 0.086 & 0.083 & -0.076 & 0.029 & 0.085 & 0.141 & 0.248 \\
\hline y0[7] & -0.422 & 0.085 & -0.593 & -0.479 & -0.421 & -0.365 & -0.255 \\
\hline $\mathrm{y} 0[8]$ & -0.073 & 0.082 & -0.232 & -0.129 & -0.072 & -0.018 & 0.087 \\
\hline $\mathrm{y} 0[9]$ & 0.167 & 0.080 & 0.012 & 0.112 & 0.166 & 0.222 & 0.325 \\
\hline y0[10] & -0.021 & 0.081 & -0.181 & -0.077 & -0.020 & 0.035 & 0.136 \\
\hline y0[11] & 0.078 & 0.081 & -0.081 & 0.022 & 0.076 & 0.133 & 0.235 \\
\hline y0[12] & -0.003 & 0.081 & -0.162 & -0.057 & -0.002 & 0.052 & 0.151 \\
\hline y0[13] & -0.126 & 0.081 & -0.284 & -0.181 & -0.125 & -0.069 & 0.028 \\
\hline y0[14] & -0.078 & 0.081 & -0.238 & -0.133 & -0.077 & -0.022 & 0.076 \\
\hline y0[15] & -0.162 & 0.083 & -0.325 & -0.218 & -0.162 & -0.103 & -0.003 \\
\hline y0[16] & 0.379 & 0.079 & 0.222 & 0.325 & 0.380 & 0.433 & 0.529 \\
\hline y0[17] & 0.056 & 0.080 & -0.100 & 0.002 & 0.056 & 0.111 & 0.211 \\
\hline y0[18] & 0.236 & 0.081 & 0.075 & 0.182 & 0.236 & 0.289 & 0.397 \\
\hline y0[19] & -0.161 & 0.085 & -0.329 & -0.218 & -0.159 & -0.105 & 0.008 \\
\hline y0[20] & -0.198 & 0.088 & -0.377 & -0.256 & -0.197 & -0.139 & -0.026 \\
\hline $\mathrm{y} 0[21]$ & -0.264 & 0.090 & -0.445 & -0.323 & -0.262 & -0.202 & -0.091 \\
\hline y0[22] & 0.202 & 0.092 & 0.019 & 0.141 & 0.204 & 0.263 & 0.380 \\
\hline $\mathrm{y} 0[23]$ & 0.108 & 0.096 & -0.087 & 0.046 & 0.110 & 0.173 & 0.294 \\
\hline y0[24] & 0.391 & 0.101 & 0.183 & 0.325 & 0.391 & 0.457 & 0.588 \\
\hline $\mathrm{y} 0[25]$ & -0.436 & 0.114 & -0.668 & -0.509 & -0.434 & -0.360 & -0.214 \\
\hline N.sum[1] & $22,402.290$ & $1,242.853$ & $20,105.401$ & $21,528.467$ & $22,368.757$ & $23,218.983$ & $24,957.587$ \\
\hline N.sum[2] & $28,690.546$ & $1,669.219$ & $25,597.349$ & $27,530.710$ & $28,631.257$ & $29,767.989$ & $32,153.930$ \\
\hline N.sum[3] & $32,779.138$ & $1,669.293$ & $29,627.162$ & $31,616.838$ & $32,748.335$ & $33,881.955$ & $36,155.117$ \\
\hline N.sum[4] & $37,349.973$ & $1,722.447$ & $34,032.657$ & $36,157.973$ & $37,329.165$ & $38,446.327$ & $40,857.413$ \\
\hline N.sum[5] & $34,463.273$ & $1,522.008$ & $31,612.082$ & $33,403.823$ & $34,438.621$ & $35,481.232$ & $37,567.494$ \\
\hline N.sum[6] & $32,395.801$ & $1,462.004$ & $29,646.397$ & $31,373.471$ & $32,359.987$ & $33,358.667$ & $35,361.204$ \\
\hline N.sum[7] & $19,594.650$ & $1,160.167$ & $17,413.890$ & $18,806.330$ & $19,570.800$ & $20,355.180$ & $21,938.836$ \\
\hline N.sum[8] & $27,815.236$ & $1,424.817$ & $25,089.827$ & $26,837.291$ & $27,781.512$ & $28,757.519$ & $30,688.739$ \\
\hline N.sum[9] & $35,400.586$ & $1,684.937$ & $32,208.527$ & $34,244.180$ & $35,366.878$ & $36,529.418$ & $38,830.026$ \\
\hline N.sum[10] & $29,384.222$ & $1,459.182$ & $26,605.570$ & $28,382.849$ & 29,337.919 & $30,365.681$ & $32,385.878$ \\
\hline N.sum[11] & $32,435.656$ & $1,662.018$ & $29,295.363$ & $31,281.227$ & $32,397.498$ & $33,536.380$ & $35,809.421$ \\
\hline N.sum[12] & $29,915.289$ & $1,457.159$ & $27,107.770$ & $28,927.585$ & $29,874.367$ & $30,912.600$ & $32,871.685$ \\
\hline N.sum[13] & $26,441.665$ & $1,316.256$ & $23,963.777$ & $25,548.028$ & $26,415.203$ & $27,297.254$ & $29,114.185$ \\
\hline N.sum[14] & $27,672.082$ & $1,342.410$ & $25,116.874$ & $26,772.337$ & $27,650.009$ & $28,564.315$ & $30,381.062$ \\
\hline N.sum[15] & $25,388.602$ & $1,370.636$ & $22,794.745$ & $24,443.462$ & $25,347.456$ & $26,302.291$ & $28,173.353$ \\
\hline N.sum[16] & $43,391.941$ & $1,789.782$ & $40,031.962$ & $42,145.209$ & $43,366.423$ & $44,558.620$ & $47,003.842$ \\
\hline N.sum[17] & $31,283.349$ & $1,379.553$ & $28,690.027$ & $30,329.896$ & $31,234.211$ & $32,183.945$ & $34,081.989$ \\
\hline N.sum[18] & $37,282.569$ & $1,591.455$ & $34,300.475$ & $36,198.113$ & $37,244.281$ & $38,359.581$ & $40,474.692$ \\
\hline N.sum[19] & $24,973.310$ & $1,184.381$ & $22,717.014$ & $24,151.571$ & $24,942.205$ & $25,763.713$ & $27,330.837$ \\
\hline N.sum[20] & $23,955.364$ & $1,195.016$ & $21,696.626$ & $23,134.113$ & $23,925.990$ & $24,747.033$ & $26,367.818$ \\
\hline
\end{tabular}


N.sum[21]

N.sum[22]

N.sum[23]

N.sum[24]

N.sum[25]

N.sum2[1]

N.sum2[2]

N.sum2[3]

N.sum2[4]

N.sum2[5]

N.sum2[6]

N.sum2[7]

N.sum2[8]

N.sum2[9]

N.sum2[10]

N.sum2[11]

N.sum2[12]

N.sum2[13]

N.sum2[14]

N.sum2[15]

N.sum2[16]

N.sum2[17]

N.sum2[18]

N.sum2[19]

N.sum2[20]

N.sum2[21]

N.sum2[22]

N.sum2[23]

N.sum2[24]

N.sum2[25]

R.growth[1]

R.growth[2]

R.growth[3]

R.growth[4]

R.growth[5]

R.growth[6]

R.growth[7]

R.growth[8]

R.growth[9]

R.growth[10]

R.growth[11]

R.growth[12]

R.growth[13]

R.growth[14]
$22,352.674$

$35,472.793$

$32,207.992$

$42,597.240$

$18,616.155$

$29,443.570$

$29,717.892$

$30,014.071$

$30,297.902$

$30,539.616$

$30,723.574$

$30,847.993$

$30,920.982$

$30,961.107$

$30,984.341$

$30,997.098$

$30,995.266$

$30,968.642$

$30,909.207$

$30,816.202$

$30,697.659$

$30,565.659$

$30,431.214$

$30,301.520$

$30,180.350$

$30,072.162$

29,979.180

$29,903.121$

$29,847.846$

$29,818.388$

0.010

0.011

0.010

0.008

0.006

0.004

0.002

0.001

0.001

0.000

0.000

$-0.001$

$-0.002$

$-0.003$
$1,074.146$

$1,614.857$

$1,502.928$

$1,823.505$

992.531

$2,871.548$

$2,504.926$

$2,215.748$

$1,990.654$

$1,806.028$

$1,668.980$

$1,600.506$

$1,584.146$

$1,573.701$

$1,544.298$

$1,506.445$

$1,476.284$

$1,448.718$

$1,412.697$

$1,382.408$

$1,386.860$

$1,429.962$

$1,491.171$

$1,564.026$

$1,662.661$

$1,795.068$

$1,957.360$

$2,159.021$

$2,427.310$

$2,776.776$

0.020

0.019

0.017

0.015

0.015

0.015

0.014

0.012

0.012

0.013

0.013

0.013

0.013

0.014
$20,253.747$

$32,403.307$

$29,309.073$

$39,157.173$

$16,721.518$

$24,039.526$

$24,967.411$

$25,848.071$

$26,565.222$

$27,190.432$

$27,665.153$

$27,931.006$

$27,964.125$

$28,137.648$

$28,239.950$

$28,271.439$

$28,307.990$

$28,318.367$

$28,376.154$

$28,345.928$

$28,182.397$

$27,969.702$

$27,670.900$

$27,421.857$

$27,174.829$

$26,873.879$

$26,501.733$

$26,027.841$

$25,523.274$

$24,793.120$

$-0.026$

$-0.024$

$-0.022$

$-0.023$

$-0.027$

$-0.029$

$-0.027$

$-0.024$

$-0.025$

$-0.025$

$-0.025$

$-0.027$

$-0.028$

$-0.031$
$21,637.317$

$34,377.652$

$31,189.596$

$41,342.110$

$17,936.076$

$27,514.690$

$28,037.585$

$28,540.443$

$28,969.730$

$29,326.174$

$29,605.278$

$29,778.193$

$29,883.549$

$29,936.419$

$29,985.878$

$30,031.227$

$30,059.127$

$30,077.021$

$30,025.875$

$29,940.723$

$29,808.520$

$29,620.087$

$29,431.477$

$29,243.020$

$29,034.613$

$28,828.517$

$28,650.149$

$28,447.652$

$28,196.270$

$27,905.510$

$-0.002$

0.000

0.000

$-0.001$

$-0.002$

$-0.004$

$-0.005$

$-0.006$

$-0.006$

$-0.007$

$-0.007$

$-0.007$

$-0.009$

$-0.010$
$22,334.840$

$35,444.534$

$32,179.722$

$42,535.777$

$18,598.562$

$29,307.678$

$29,575.068$

$29,889.492$

$30,212.041$

$30,462.264$

$30,629.044$

$30,736.335$

$30,795.943$

$30,817.307$

$30,822.406$

$30,833.188$

$30,850.306$

$30,817.219$

$30,769.409$

$30,689.349$

$30,586.851$

$30,482.702$

$30,387.580$

$30,276.165$

$30,147.670$

$30,013.185$

$29,890.749$

$29,820.911$

$29,755.637$

$29,688.427$

0.007

0.008

0.008

0.007

0.006

0.004

0.003

0.002

0.001

0.000

$-0.001$

$-0.001$

$-0.002$

$-0.002$

$23,045.444$

$36,528.851$

$33,204.158$

$43,788.674$

$19,269.340$

$31,217.354$

$31,297.087$

$31,352.252$

$31,528.446$

$31,643.944$

$31,761.135$

$31,819.357$

$31,853.542$

$31,877.113$

$31,856.064$

$31,845.189$

$31,833.586$

$31,764.475$

$31,651.933$

$31,570.459$

$31,504.319$

$31,391.619$ 


\begin{tabular}{lrrrrrrr} 
R.growth[15] & -0.004 & 0.014 & -0.033 & -0.011 & -0.003 & 0.004 & 0.024 \\
R.growth[16] & -0.004 & 0.014 & -0.036 & -0.011 & -0.003 & 0.003 & 0.022 \\
R.growth[17] & -0.005 & 0.014 & -0.035 & -0.011 & -0.003 & 0.003 & 0.021 \\
R.growth[18] & -0.004 & 0.014 & -0.033 & -0.012 & -0.004 & 0.003 & 0.022 \\
R.growth[19] & -0.004 & 0.014 & -0.036 & -0.011 & -0.003 & 0.004 & 0.023 \\
R.growth[20] & -0.004 & 0.014 & -0.035 & -0.011 & -0.003 & 0.004 & 0.024 \\
R.growth[21] & -0.003 & 0.014 & -0.034 & -0.012 & -0.003 & 0.005 & 0.026 \\
R.growth[22] & -0.003 & 0.016 & -0.036 & -0.012 & -0.003 & 0.006 & 0.031 \\
R.growth[23] & -0.003 & 0.018 & -0.039 & -0.012 & -0.002 & 0.007 & 0.036 \\
R.growth[24] & -0.002 & 0.019 & -0.043 & -0.012 & -0.002 & 0.008 & 0.039 \\
Deviance & $74,913.174$ & 59.400 & $74,799.244$ & $74,872.776$ & $74,912.251$ & $74,953.482$ & $75,031.893$ \\
\hline
\end{tabular}


Tables A4.24. Bayesian generalized linear mixed model output for Yellow-billed Loon observed during aerial surveys 1992-2016 on the Arctic Coastal Plain, Alaska. We provide summaries including mean, SE, and several quantiles of the posterior distribution for model and derived parameters. See Table A4.4 for a key to parameter names.

\begin{tabular}{|c|c|c|c|c|c|c|c|}
\hline Parameter & Mean & SD & $2.5 \%$ & $25.0 \%$ & $50.0 \%$ & $75.0 \%$ & $97.5 \%$ \\
\hline MSE & 0.262 & 0.002 & 0.258 & 0.260 & 0.261 & 0.262 & 0.266 \\
\hline MSE.CV & 1.586 & 0.011 & 1.567 & 1.579 & 1.585 & 1.591 & 1.610 \\
\hline SSE & $2,532.328$ & 17.269 & $2,502.153$ & $2,521.731$ & $2,530.556$ & $2,541.130$ & $2,571.068$ \\
\hline Trend & 1.044 & 0.012 & 1.021 & 1.036 & 1.044 & 1.053 & 1.069 \\
\hline R.trend & 0.043 & 0.012 & 0.021 & 0.035 & 0.043 & 0.051 & 0.067 \\
\hline M.dens & 0.090 & 0.006 & 0.079 & 0.086 & 0.089 & 0.094 & 0.103 \\
\hline M.y0 & -0.005 & 0.067 & -0.143 & -0.048 & -0.004 & 0.039 & 0.123 \\
\hline M.blyr & -2.725 & 0.083 & -2.887 & -2.780 & -2.726 & -2.669 & -2.561 \\
\hline M.cell & 0.000 & 0.006 & -0.013 & -0.002 & 0.000 & 0.002 & 0.013 \\
\hline R.mid & -0.556 & 0.103 & -0.751 & -0.625 & -0.559 & -0.489 & -0.347 \\
\hline b.ADOY & -0.977 & 0.065 & -1.107 & -1.020 & -0.975 & -0.934 & -0.850 \\
\hline b.ADOY.2 & -0.306 & 0.047 & -0.400 & -0.337 & -0.306 & -0.274 & -0.215 \\
\hline SD.y0 & 0.331 & 0.074 & 0.208 & 0.280 & 0.323 & 0.375 & 0.495 \\
\hline SD.cell & 0.101 & 0.077 & 0.011 & 0.026 & 0.095 & 0.154 & 0.272 \\
\hline SD.lam[1] & 0.735 & 0.195 & 0.450 & 0.598 & 0.703 & 0.839 & 1.200 \\
\hline SD.lam[2] & 1.230 & 0.544 & 0.384 & 0.865 & 1.168 & 1.508 & 2.547 \\
\hline SD.lam[3] & 5.079 & 2.159 & 1.736 & 3.380 & 4.767 & 6.610 & 9.534 \\
\hline SD.lam[4] & 1.500 & 1.273 & 0.065 & 0.647 & 1.178 & 1.973 & 4.977 \\
\hline SD.lam[5] & 3.635 & 2.731 & 0.127 & 1.312 & 2.968 & 5.608 & 9.419 \\
\hline SD.lam[6] & 1.856 & 0.545 & 1.086 & 1.429 & 1.745 & 2.226 & 3.066 \\
\hline SD.lam[7] & 1.205 & 0.475 & 0.527 & 0.827 & 1.180 & 1.489 & 2.268 \\
\hline SD.lam[8] & 0.453 & 0.926 & 0.000 & 0.071 & 0.183 & 0.428 & 3.115 \\
\hline $\mathrm{y} 0[1]$ & 0.610 & 0.250 & 0.142 & 0.440 & 0.605 & 0.774 & 1.112 \\
\hline $\mathrm{y} 0[2]$ & -0.298 & 0.205 & -0.717 & -0.434 & -0.287 & -0.160 & 0.085 \\
\hline $\mathrm{y} 0[3]$ & -0.429 & 0.199 & -0.846 & -0.552 & -0.423 & -0.294 & -0.057 \\
\hline $\mathrm{y} 0[4]$ & -0.001 & 0.174 & -0.348 & -0.116 & 0.000 & 0.116 & 0.335 \\
\hline $\mathrm{y} 0[5]$ & 0.182 & 0.171 & -0.142 & 0.069 & 0.178 & 0.294 & 0.519 \\
\hline $\mathrm{y} 0[6]$ & 0.155 & 0.184 & -0.206 & 0.034 & 0.156 & 0.275 & 0.512 \\
\hline $\mathrm{y} 0[7]$ & -0.252 & 0.189 & -0.630 & -0.379 & -0.250 & -0.123 & 0.109 \\
\hline $\mathrm{y} 0[8]$ & -0.049 & 0.191 & -0.422 & -0.177 & -0.048 & 0.078 & 0.334 \\
\hline $\mathrm{y} 0[9]$ & -0.272 & 0.203 & -0.680 & -0.407 & -0.269 & -0.134 & 0.116 \\
\hline y0[10] & -0.046 & 0.186 & -0.408 & -0.170 & -0.047 & 0.079 & 0.316 \\
\hline $\mathrm{y} 0[11]$ & 0.006 & 0.180 & -0.352 & -0.113 & 0.005 & 0.125 & 0.366 \\
\hline y0[12] & 0.309 & 0.184 & -0.045 & 0.183 & 0.306 & 0.429 & 0.679 \\
\hline y0[13] & -0.335 & 0.194 & -0.729 & -0.459 & -0.332 & -0.208 & 0.041 \\
\hline y0[14] & -0.017 & 0.178 & -0.383 & -0.133 & -0.015 & 0.102 & 0.329 \\
\hline $\mathrm{y} 0[15]$ & -0.156 & 0.179 & -0.525 & -0.271 & -0.152 & -0.037 & 0.186 \\
\hline y0[16] & 0.376 & 0.183 & 0.024 & 0.252 & 0.376 & 0.498 & 0.735 \\
\hline y0[17] & 0.023 & 0.176 & -0.324 & -0.093 & 0.023 & 0.140 & 0.363 \\
\hline
\end{tabular}




\begin{tabular}{|c|c|c|c|c|c|c|c|}
\hline y0[18] & 0.175 & 0.168 & -0.154 & 0.063 & 0.175 & 0.286 & 0.503 \\
\hline y0[19] & 0.143 & 0.175 & -0.199 & 0.025 & 0.142 & 0.257 & 0.493 \\
\hline y0[20] & -0.233 & 0.179 & -0.589 & -0.348 & -0.232 & -0.113 & 0.111 \\
\hline y0[21] & 0.072 & 0.173 & -0.274 & -0.043 & 0.069 & 0.186 & 0.416 \\
\hline y0[22] & 0.074 & 0.179 & -0.280 & -0.046 & 0.072 & 0.196 & 0.425 \\
\hline y0[23] & -0.184 & 0.188 & -0.564 & -0.305 & -0.181 & -0.060 & 0.181 \\
\hline y0[24] & 0.364 & 0.194 & -0.007 & 0.235 & 0.362 & 0.487 & 0.762 \\
\hline $\mathrm{y} 0[25]$ & -0.351 & 0.219 & -0.794 & -0.495 & -0.345 & -0.203 & 0.067 \\
\hline N.sum[1] & $1,682.124$ & 356.145 & $1,093.897$ & $1,432.978$ & $1,646.039$ & $1,891.245$ & $2,491.650$ \\
\hline N.sum[2] & 663.348 & 127.405 & 445.965 & 573.485 & 653.901 & 741.041 & 943.489 \\
\hline N.sum[3] & 574.077 & 109.266 & 384.021 & 497.196 & 564.838 & 643.311 & 809.088 \\
\hline N.sum[4] & 869.498 & 131.787 & 635.819 & 778.514 & 861.458 & 951.602 & $1,149.982$ \\
\hline N.sum[5] & $1,045.019$ & 146.850 & 789.041 & 942.511 & $1,034.170$ & $1,134.577$ & $1,364.410$ \\
\hline N.sum[6] & $1,034.986$ & 167.970 & 743.094 & 918.058 & $1,019.990$ & $1,139.858$ & $1,393.639$ \\
\hline N.sum[7] & 708.889 & 128.614 & 485.486 & 618.506 & 698.555 & 786.985 & 989.510 \\
\hline N.sum[8] & 901.765 & 158.276 & 626.838 & 788.532 & 889.567 & $1,000.620$ & $1,243.237$ \\
\hline N.sum[9] & 760.175 & 144.174 & 508.961 & 660.781 & 745.748 & 848.887 & $1,078.852$ \\
\hline N.sum[10] & $1,009.700$ & 167.311 & 724.853 & 890.766 & 995.811 & $1,112.614$ & $1,366.359$ \\
\hline N.sum[11] & $1,142.752$ & 178.379 & 828.377 & $1,016.796$ & $1,130.348$ & $1,253.596$ & $1,528.764$ \\
\hline N.sum[12] & $1,682.554$ & 278.018 & $1,197.320$ & $1,483.343$ & $1,659.289$ & $1,860.334$ & $2,271.863$ \\
\hline N.sum[13] & 968.219 & 175.949 & 659.053 & 846.172 & 956.024 & $1,076.974$ & $1,352.523$ \\
\hline N.sum[14] & $1,450.264$ & 232.434 & $1,051.370$ & $1,284.121$ & $1,430.879$ & $1,595.392$ & $1,960.843$ \\
\hline N.sum[15] & $1,378.518$ & 223.185 & 984.899 & $1,221.509$ & $1,365.327$ & $1,519.968$ & $1,858.176$ \\
\hline N.sum[16] & $2,543.579$ & 397.354 & $1,849.060$ & $2,259.742$ & $2,512.703$ & $2,792.616$ & $3,398.140$ \\
\hline N.sum[17] & $1,918.077$ & 281.484 & $1,426.188$ & $1,715.710$ & $1,900.156$ & $2,095.430$ & $2,522.582$ \\
\hline N.sum[18] & $2,366.827$ & 302.960 & $1,837.817$ & $2,153.394$ & $2,345.009$ & $2,555.928$ & $3,025.813$ \\
\hline N.sum[19] & $2,416.781$ & 363.133 & $1,786.140$ & $2,160.047$ & $2,390.864$ & $2,641.899$ & $3,212.084$ \\
\hline N.sum[20] & $1,729.758$ & 269.160 & $1,267.550$ & $1,538.970$ & $1,712.333$ & $1,895.475$ & $2,320.715$ \\
\hline N.sum[21] & $2,423.109$ & 359.816 & $1,792.689$ & $2,169.485$ & $2,395.550$ & $2,644.641$ & $3,224.697$ \\
\hline N.sum[22] & $2,503.639$ & 382.707 & $1,849.858$ & $2,231.195$ & $2,469.448$ & $2,741.839$ & $3,340.904$ \\
\hline N.sum[23] & $1,993.324$ & 300.850 & $1,468.474$ & $1,782.642$ & $1,972.212$ & $2,174.165$ & $2,642.305$ \\
\hline N.sum[24] & $3,550.766$ & 493.246 & $2,684.086$ & $3,202.033$ & $3,519.592$ & $3,860.606$ & $4,604.461$ \\
\hline N.sum[25] & $1,806.132$ & 300.530 & $1,282.689$ & $1,594.881$ & $1,784.694$ & $1,994.156$ & $2,454.696$ \\
\hline N.sum2[1] & 964.786 & 219.005 & 635.962 & 809.395 & 931.112 & $1,080.154$ & $1,474.960$ \\
\hline N.sum2[2] & 939.362 & 170.850 & 663.637 & 819.920 & 918.987 & $1,034.984$ & $1,326.999$ \\
\hline N.sum2[3] & 922.819 & 143.546 & 682.673 & 823.114 & 907.684 & $1,005.295$ & $1,247.679$ \\
\hline N.sum2[4] & 915.442 & 131.251 & 692.869 & 823.776 & 904.028 & 994.100 & $1,203.721$ \\
\hline N.sum2[5] & 917.626 & 126.037 & 691.576 & 829.255 & 909.676 & 995.032 & $1,185.968$ \\
\hline N.sum2[6] & 930.365 & 125.743 & 701.577 & 842.874 & 925.760 & $1,009.322$ & $1,191.953$ \\
\hline N.sum2[7] & 954.902 & 130.968 & 715.254 & 865.659 & 949.466 & $1,040.241$ & $1,226.171$ \\
\hline N.sum2[8] & 992.262 & 139.723 & 731.708 & 895.055 & 987.217 & $1,085.516$ & $1,277.039$ \\
\hline N.sum2[9] & $1,043.521$ & 148.340 & 762.488 & 940.564 & $1,040.577$ & $1,143.563$ & $1,337.053$ \\
\hline N.sum2[10] & $1,110.099$ & 154.885 & 815.574 & $1,004.447$ & $1,105.343$ & $1,216.451$ & $1,415.389$ \\
\hline N.sum2[11] & $1,193.620$ & 160.181 & 886.688 & $1,085.233$ & $1,190.917$ & $1,303.886$ & $1,511.113$ \\
\hline
\end{tabular}




\begin{tabular}{|c|c|c|c|c|c|c|c|}
\hline N.sum2[12] & $1,295.021$ & 166.384 & 977.386 & $1,184.590$ & $1,291.639$ & $1,401.903$ & $1,639.005$ \\
\hline N.sum2[13] & $1,413.448$ & 175.164 & $1,091.722$ & $1,297.449$ & $1,408.181$ & $1,516.074$ & $1,795.980$ \\
\hline N.sum2[14] & $1,545.845$ & 187.684 & $1,218.479$ & $1,421.121$ & $1,534.711$ & $1,647.684$ & $1,967.415$ \\
\hline N.sum2[15] & $1,687.435$ & 205.566 & $1,347.874$ & $1,550.834$ & $1,666.107$ & $1,794.518$ & $2,173.856$ \\
\hline N.sum2[16] & $1,831.364$ & 228.354 & $1,467.737$ & $1,675.637$ & $1,801.893$ & $1,952.365$ & $2,369.514$ \\
\hline N.sum2[17] & $1,969.127$ & 249.866 & $1,572.360$ & $1,794.513$ & $1,933.937$ & $2,106.454$ & $2,551.962$ \\
\hline N.sum2[18] & $2,092.940$ & 262.589 & $1,677.016$ & $1,906.095$ & $2,060.747$ & $2,245.949$ & $2,691.130$ \\
\hline N.sum2[19] & $2,198.973$ & 267.895 & $1,763.372$ & $2,006.677$ & $2,168.338$ & $2,356.406$ & $2,813.074$ \\
\hline N.sum2[20] & $2,288.718$ & 276.906 & $1,828.845$ & $2,095.166$ & $2,259.854$ & $2,449.853$ & $2,910.474$ \\
\hline N.sum2[21] & $2,367.754$ & 297.619 & $1,858.372$ & $2,165.247$ & $2,336.014$ & $2,539.906$ & $3,028.850$ \\
\hline N.sum2[22] & $2,443.141$ & 329.444 & $1,876.669$ & $2,220.325$ & $2,410.870$ & $2,635.161$ & $3,174.553$ \\
\hline N.sum2[23] & $2,522.338$ & 375.717 & $1,892.007$ & $2,266.279$ & $2,485.456$ & $2,735.737$ & $3,355.505$ \\
\hline N.sum2[24] & $2,612.629$ & 449.268 & $1,872.808$ & $2,307.762$ & $2,562.860$ & $2,859.691$ & $3,582.595$ \\
\hline N.sum2[25] & $2,719.303$ & 559.329 & $1,806.944$ & $2,341.265$ & $2,655.918$ & $3,024.212$ & $3,979.237$ \\
\hline R.growth[1] & -0.019 & 0.065 & -0.169 & -0.055 & -0.011 & 0.028 & 0.086 \\
\hline R.growth[2] & -0.014 & 0.059 & -0.153 & -0.047 & -0.007 & 0.030 & 0.077 \\
\hline R.growth[3] & -0.006 & 0.049 & -0.121 & -0.035 & -0.002 & 0.030 & 0.069 \\
\hline R.growth[4] & 0.003 & 0.042 & -0.092 & -0.023 & 0.007 & 0.035 & 0.071 \\
\hline R.growth[5] & 0.014 & 0.041 & -0.078 & -0.010 & 0.019 & 0.043 & 0.080 \\
\hline R.growth[6] & 0.026 & 0.041 & -0.069 & 0.003 & 0.031 & 0.052 & 0.096 \\
\hline R.growth[7] & 0.038 & 0.038 & -0.047 & 0.018 & 0.041 & 0.060 & 0.110 \\
\hline R.growth[8] & 0.050 & 0.034 & -0.022 & 0.031 & 0.050 & 0.069 & 0.122 \\
\hline R.growth[9] & 0.062 & 0.034 & -0.004 & 0.043 & 0.060 & 0.080 & 0.137 \\
\hline R.growth[10] & 0.073 & 0.037 & 0.011 & 0.050 & 0.068 & 0.092 & 0.160 \\
\hline R.growth[11] & 0.082 & 0.039 & 0.023 & 0.056 & 0.076 & 0.103 & 0.180 \\
\hline R.growth[12] & 0.088 & 0.039 & 0.031 & 0.060 & 0.082 & 0.109 & 0.185 \\
\hline R.growth[13] & 0.090 & 0.040 & 0.030 & 0.061 & 0.084 & 0.112 & 0.184 \\
\hline R.growth[14] & 0.088 & 0.040 & 0.025 & 0.059 & 0.081 & 0.111 & 0.185 \\
\hline R.growth[15] & 0.082 & 0.038 & 0.015 & 0.056 & 0.077 & 0.103 & 0.172 \\
\hline R.growth[16] & 0.072 & 0.034 & 0.004 & 0.051 & 0.070 & 0.092 & 0.147 \\
\hline R.growth[17] & 0.061 & 0.032 & -0.008 & 0.043 & 0.061 & 0.081 & 0.124 \\
\hline R.growth[18] & 0.050 & 0.035 & -0.029 & 0.031 & 0.052 & 0.071 & 0.114 \\
\hline R.growth[19] & 0.040 & 0.039 & -0.049 & 0.019 & 0.044 & 0.064 & 0.111 \\
\hline R.growth[20] & 0.033 & 0.039 & -0.053 & 0.010 & 0.037 & 0.058 & 0.104 \\
\hline R.growth[21] & 0.030 & 0.038 & -0.050 & 0.007 & 0.033 & 0.056 & 0.099 \\
\hline R.growth[22] & 0.030 & 0.042 & -0.058 & 0.004 & 0.033 & 0.058 & 0.107 \\
\hline R.growth[23] & 0.032 & 0.049 & -0.074 & 0.002 & 0.035 & 0.063 & 0.125 \\
\hline R.growth[24] & 0.034 & 0.054 & -0.083 & 0.002 & 0.037 & 0.068 & 0.140 \\
\hline Deviance & $8,228.017$ & 22.695 & $8,175.305$ & $8,214.354$ & $8,231.969$ & $8,244.350$ & $8,263.594$ \\
\hline
\end{tabular}


Appendix 5. Density $\left(\mathrm{km}^{2}\right)$ maps

\section{Spatio-temporal population change of arctic-breeding waterbirds}

We mapped the relative density $\left(\mathrm{km}^{2}\right)$ within approximately $36 \mathrm{~km}^{2}$ (6 $\mathrm{km}$ by $6 \mathrm{~km}$ ) cells to 20 waterbird species observed during the Arctic Coastal Plain Breeding Waterfowl Survey, Alaska, 1992-2016. We estimated density using hierarchical generalized linear mixed models (see main text, Methods). We present maps in taxonomic order (Chesser et al. 2018) for Lesser Snow Goose, Greater White-fronted Goose, Black Brant, Cackling Goose, Tundra Swan, Northern Pintail, Scaup (Lesser and Greater combined), Steller's Eider, Spectacled Eider, King Eider, Whitewinged Scoter, Long-tailed Duck, Red-breasted Merganser, Jaeger (Pomarine, Parasitic, and Long-tailed combined), Sabine's Gull, Glaucous Gull, Arctic Tern, Red-throated Loon, Pacific Loon, and Yellow-billed Loon.

Chesser, R. T., K. J. Burns, C. Cicero, J. L. Dunn, A. W. Kratter, I. J. Lovette, P. C. Rasmussen, J. V. Remsen, Jr., J. D. Rising, D. F. Stotz, and K. Winker. 2018. Fifty-ninth supplement to the American Ornithological Society's check-list of North American birds. The Auk: Ornithological Advances 135:798-813. 
Figure A5.1. Lesser Snow Goose density $\left(\mathrm{km}^{2}\right)$ across the Arctic Coastal Plain, Alaska 1992-2016.

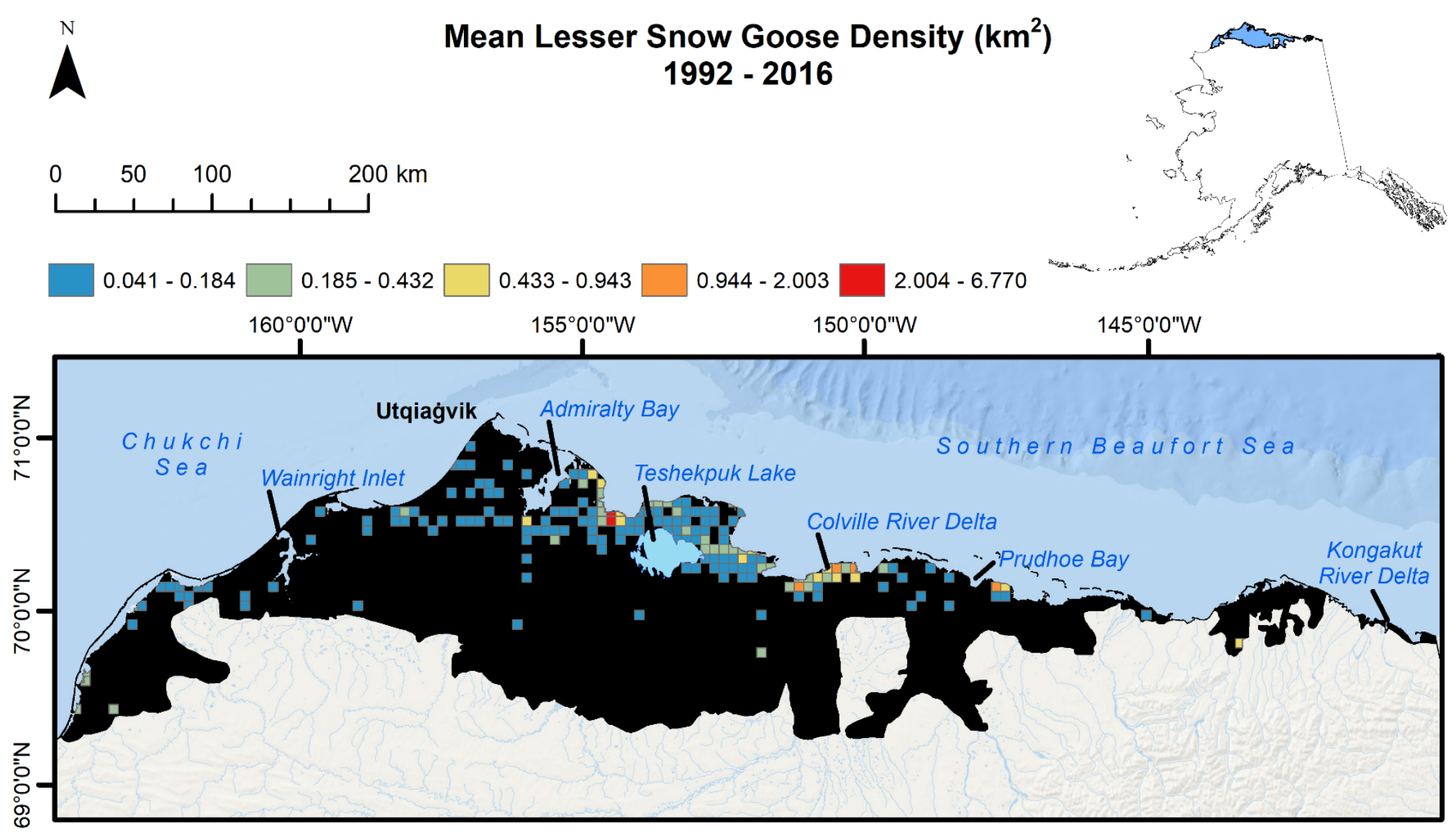


Figure A5.2. Greater White-fronted Goose density $\left(\mathrm{km}^{2}\right)$ across the Arctic Coastal Plain, Alaska 1992-2016.

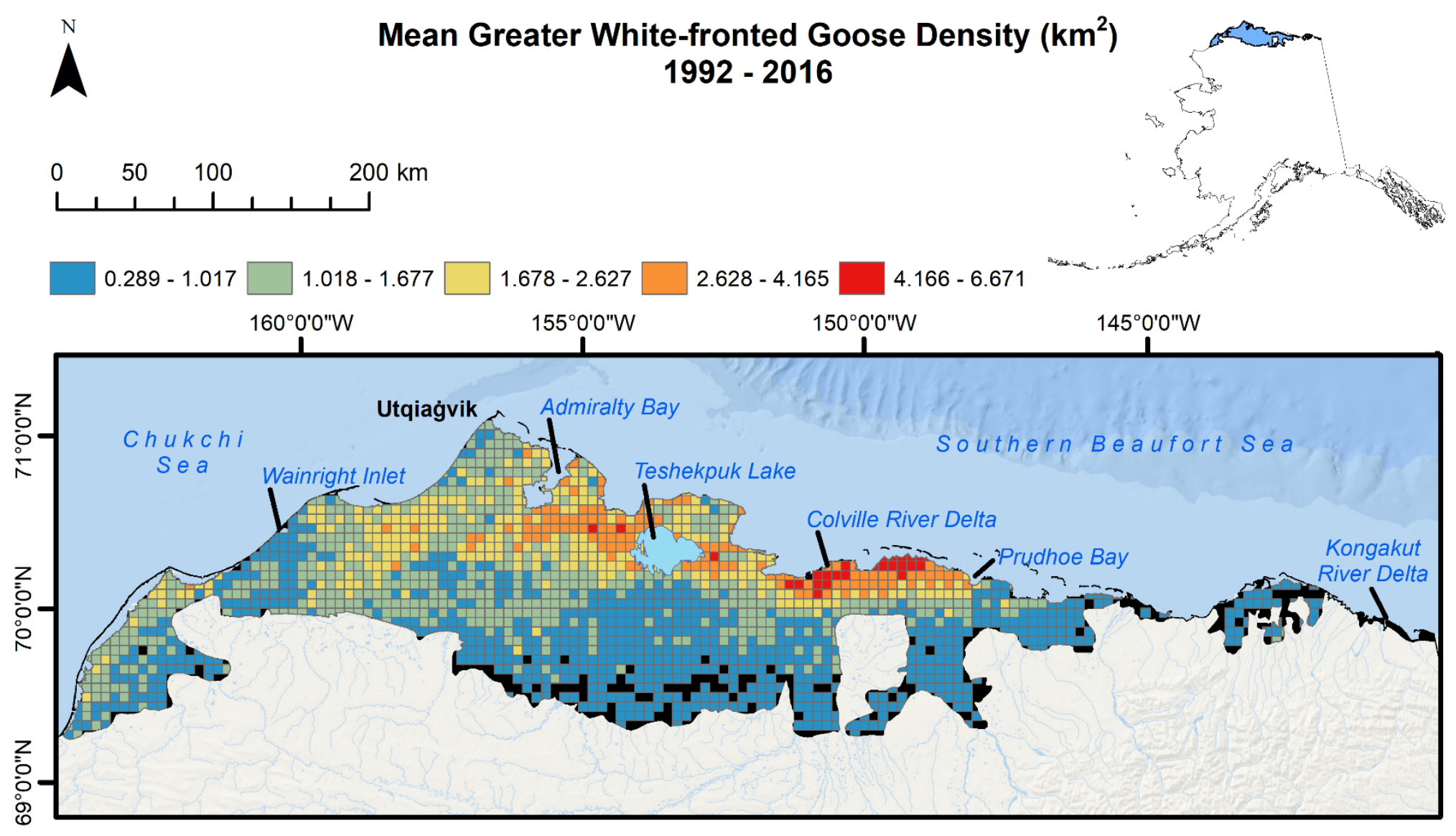


Figure A5.3. Black Brant density $\left(\mathrm{km}^{2}\right)$ across the Arctic Coastal Plain, Alaska 1992-2016.

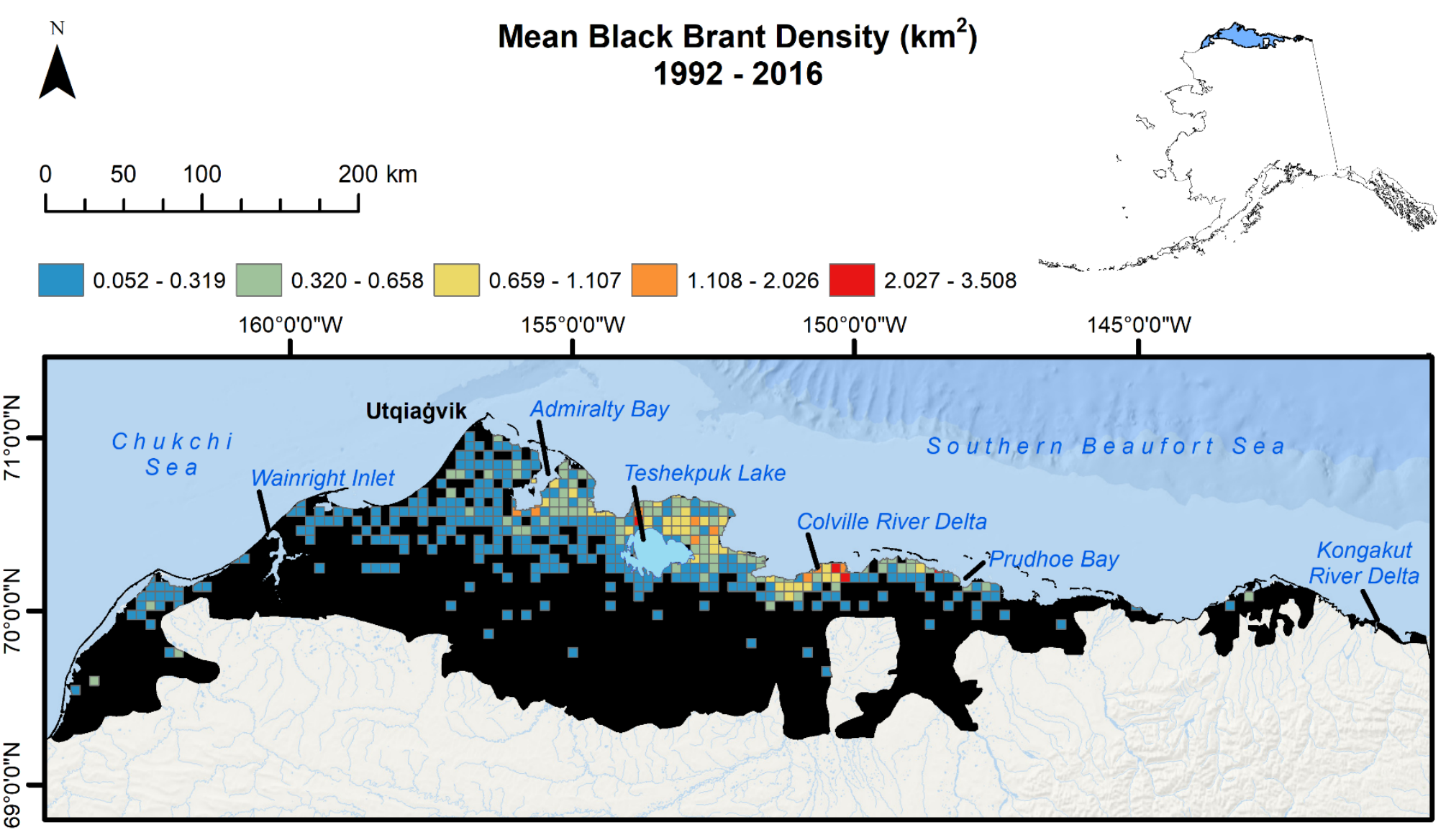


Figure A5.4. Cackling Goose density $\left(\mathrm{km}^{2}\right)$ across the Arctic Coastal Plain, Alaska 1992-2016.

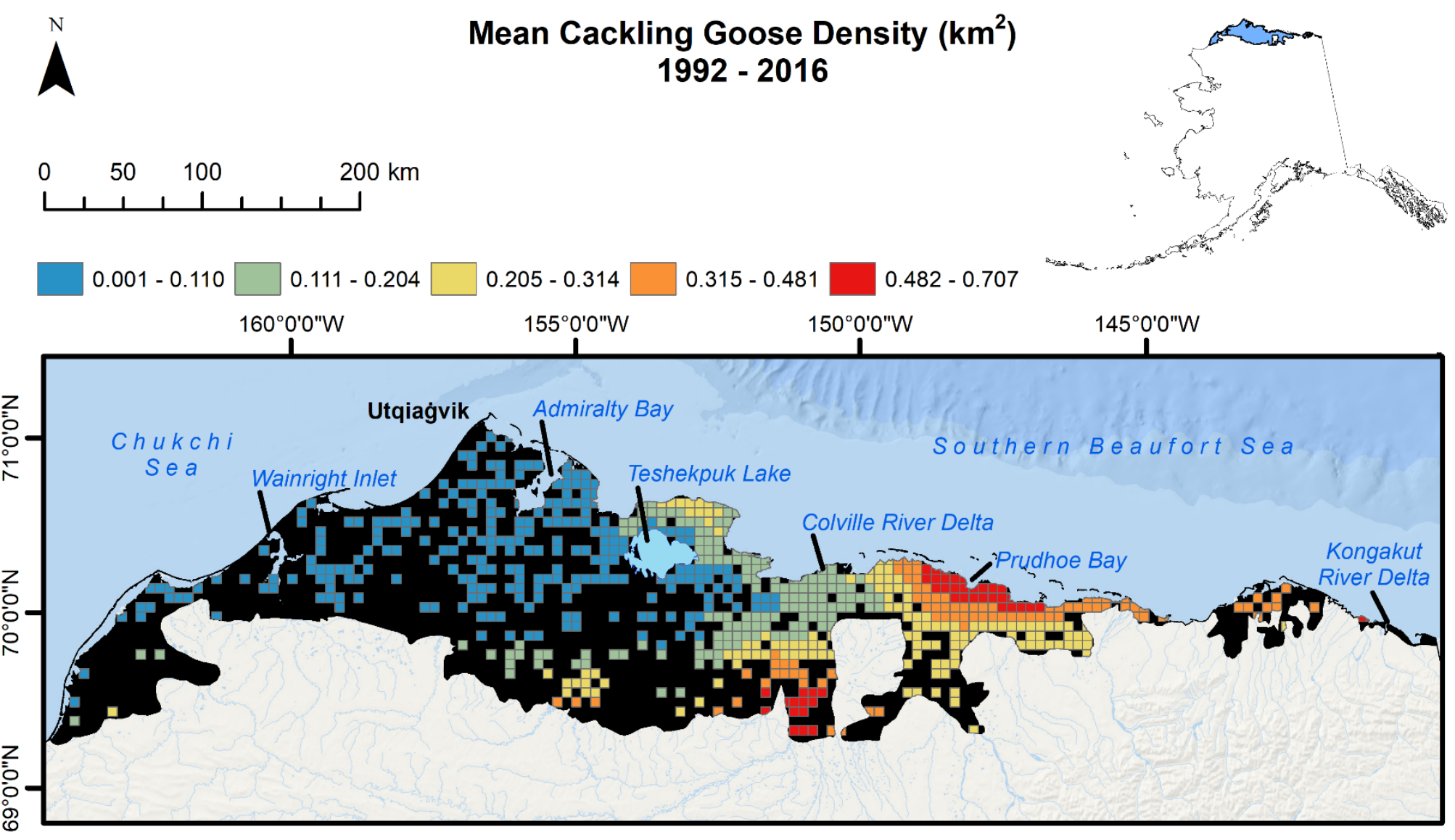


Figure A5.5. Tundra Swan density $\left(\mathrm{km}^{2}\right)$ across the Arctic Coastal Plain, Alaska 1992-2016.

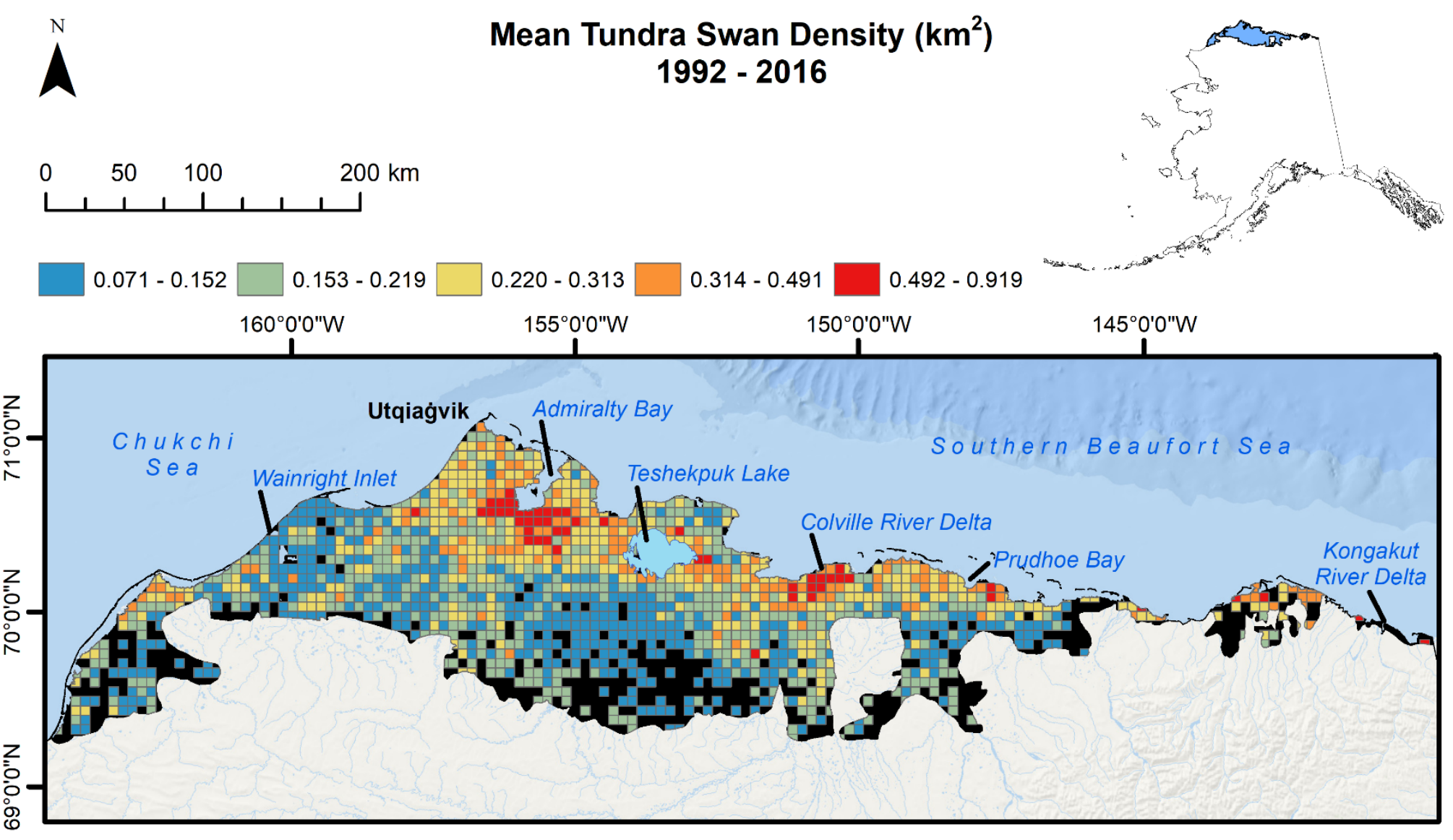


Figure A5.6. Northern Pintail density $\left(\mathrm{km}^{2}\right)$ across the Arctic Coastal Plain, Alaska 1992-2016.

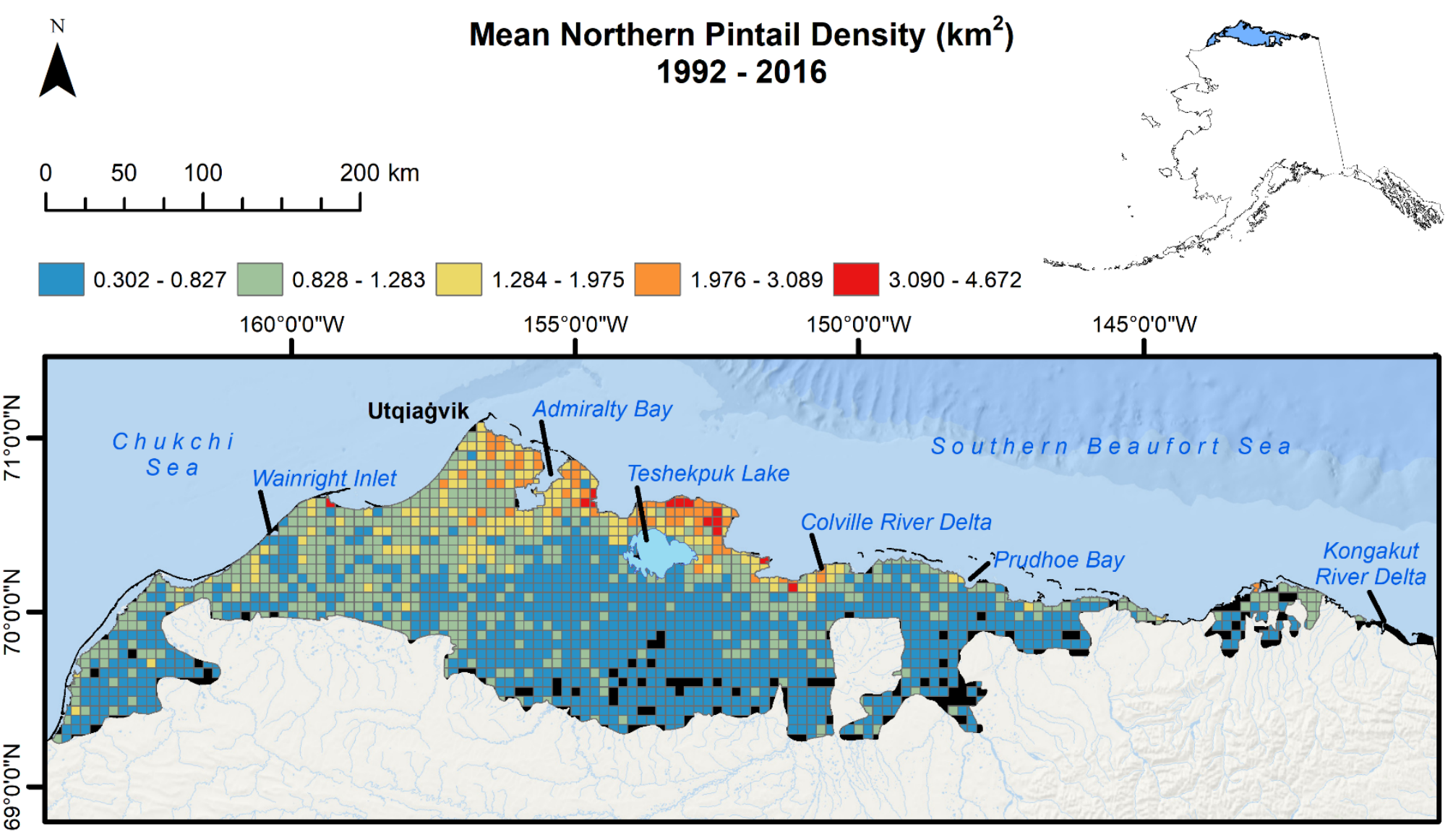


Figure A5.7. Scaup sp. (Lesser and Greater combined) density $\left(\mathrm{km}^{2}\right)$ across the Arctic Coastal Plain, Alaska $1992-2016$.

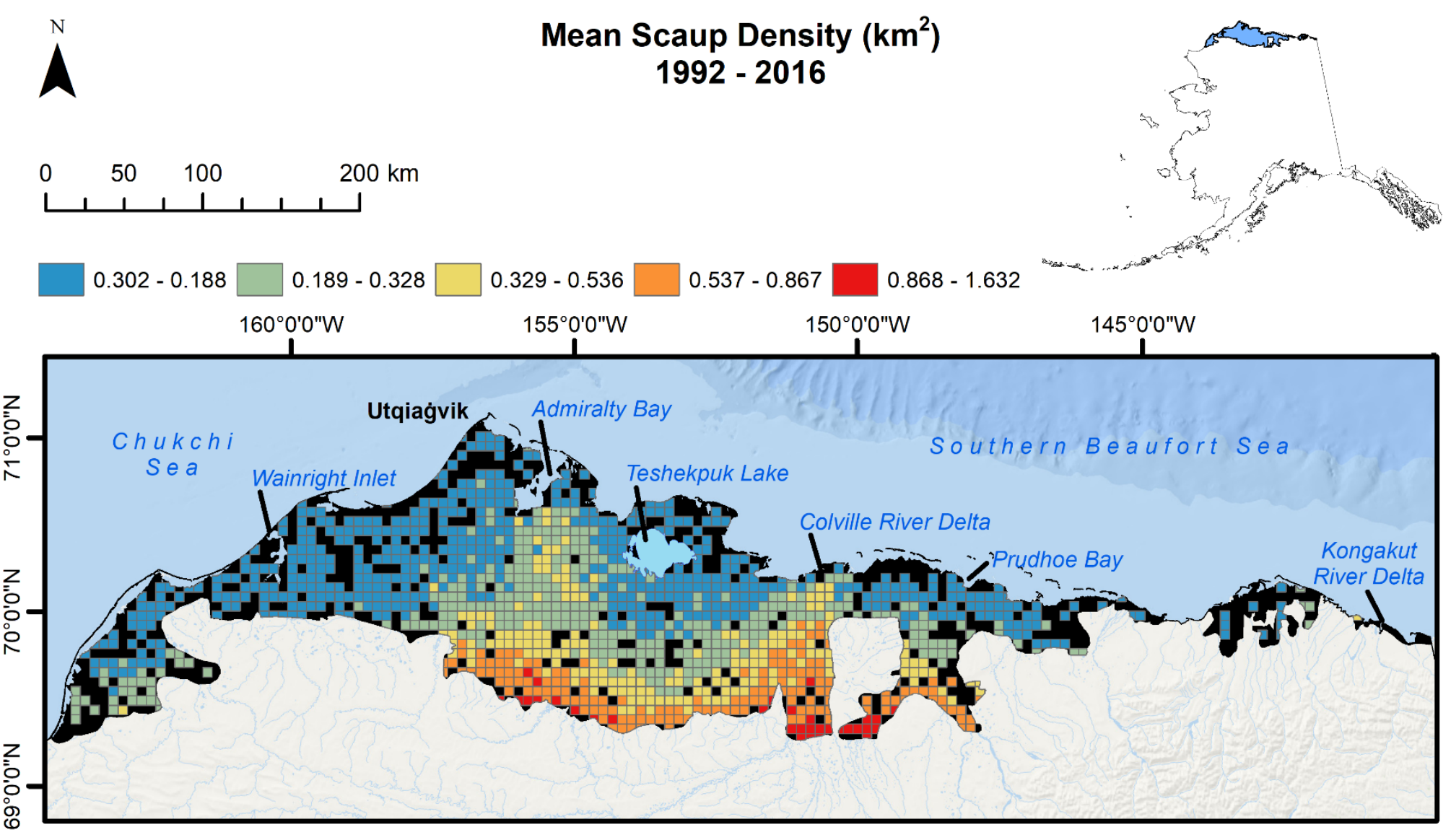


Figure A5.8. Steller's Eider density $\left(\mathrm{km}^{2}\right)$ across the Arctic Coastal Plain, Alaska 1992-2016.

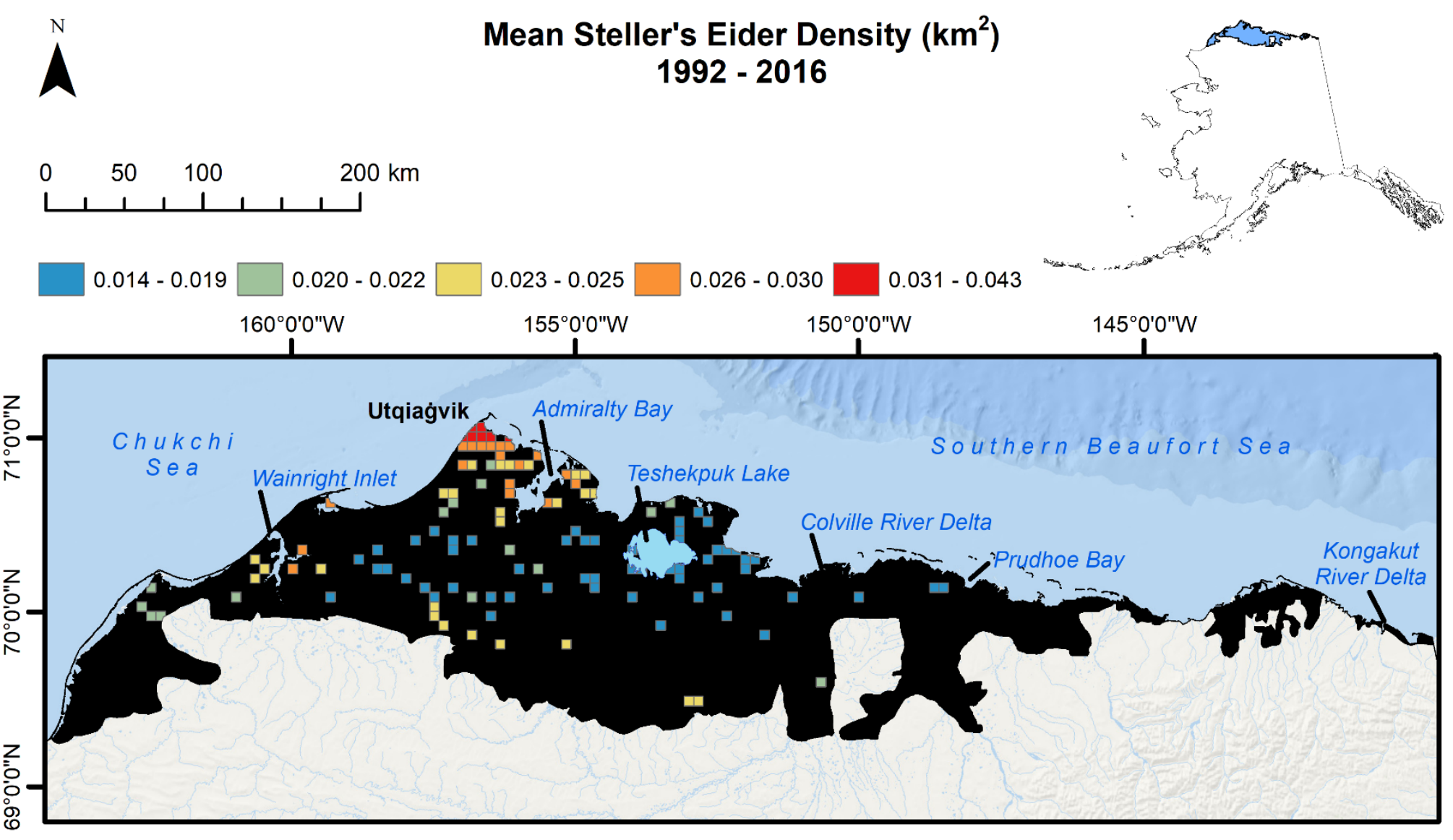


Figure A5.9. Spectacled Eider density $\left(\mathrm{km}^{2}\right)$ across the Arctic Coastal Plain, Alaska 1992-2016.

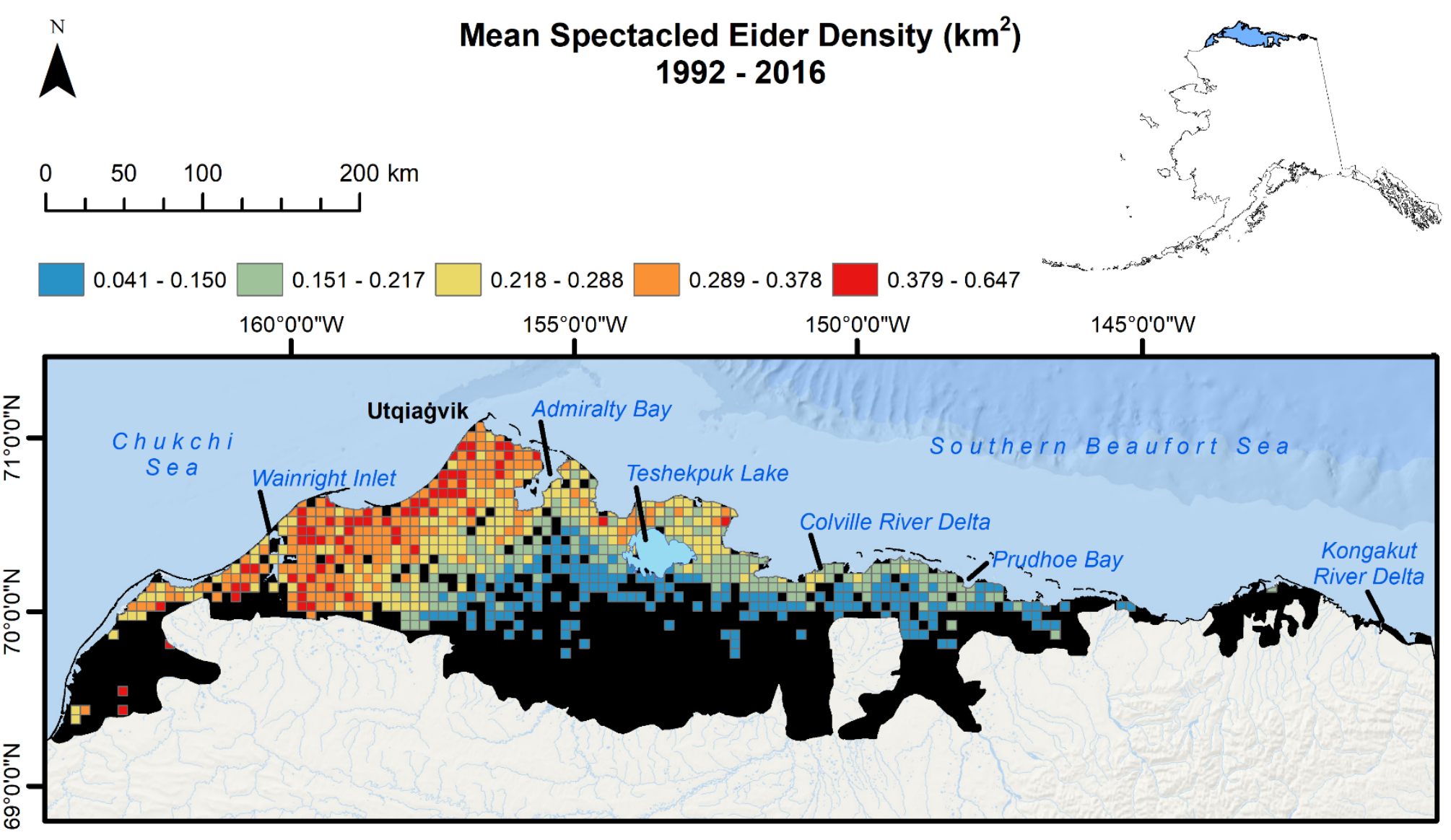


Figure A5.10. King Eider density $\left(\mathrm{km}^{2}\right)$ across the Arctic Coastal Plain, Alaska 1992-2016.

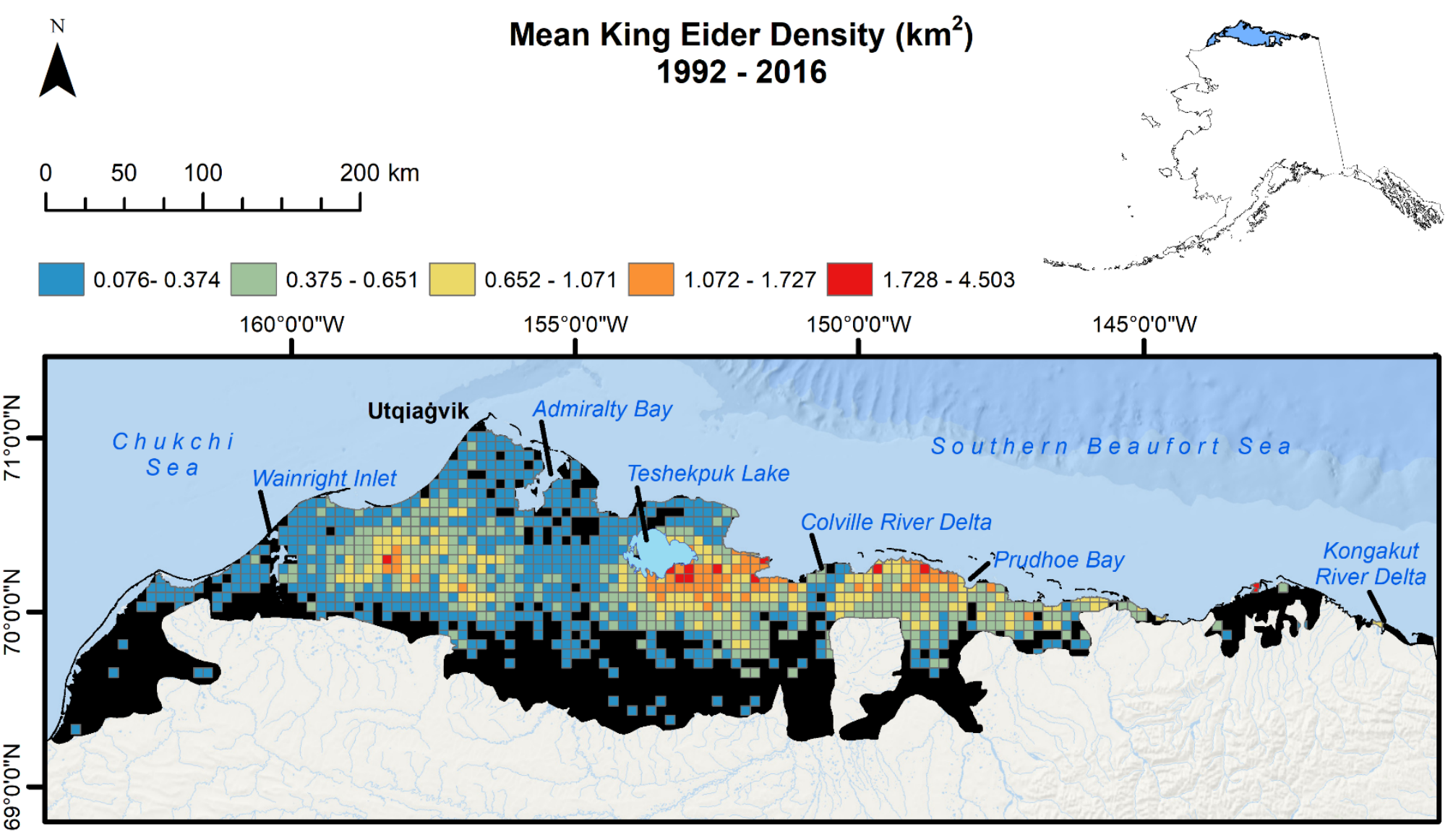


Figure A5.11. White-winged Scoter $\left(\mathrm{km}^{2}\right)$ across the Arctic Coastal Plain, Alaska 1992-2016.

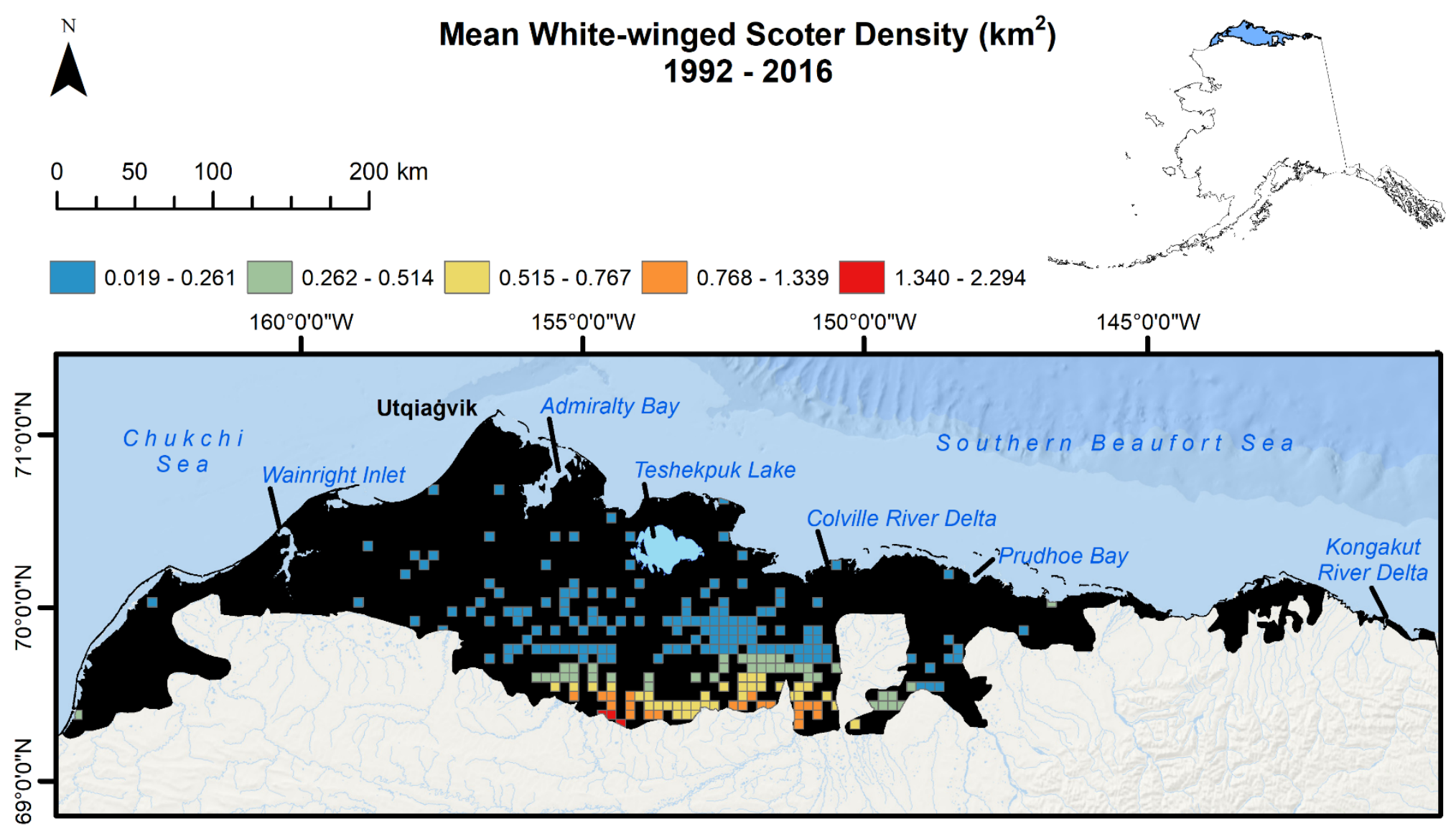


Figure A5.12. Long-tailed Duck density $\left(\mathrm{km}^{2}\right)$ across the Arctic Coastal Plain, Alaska 1992-2016.

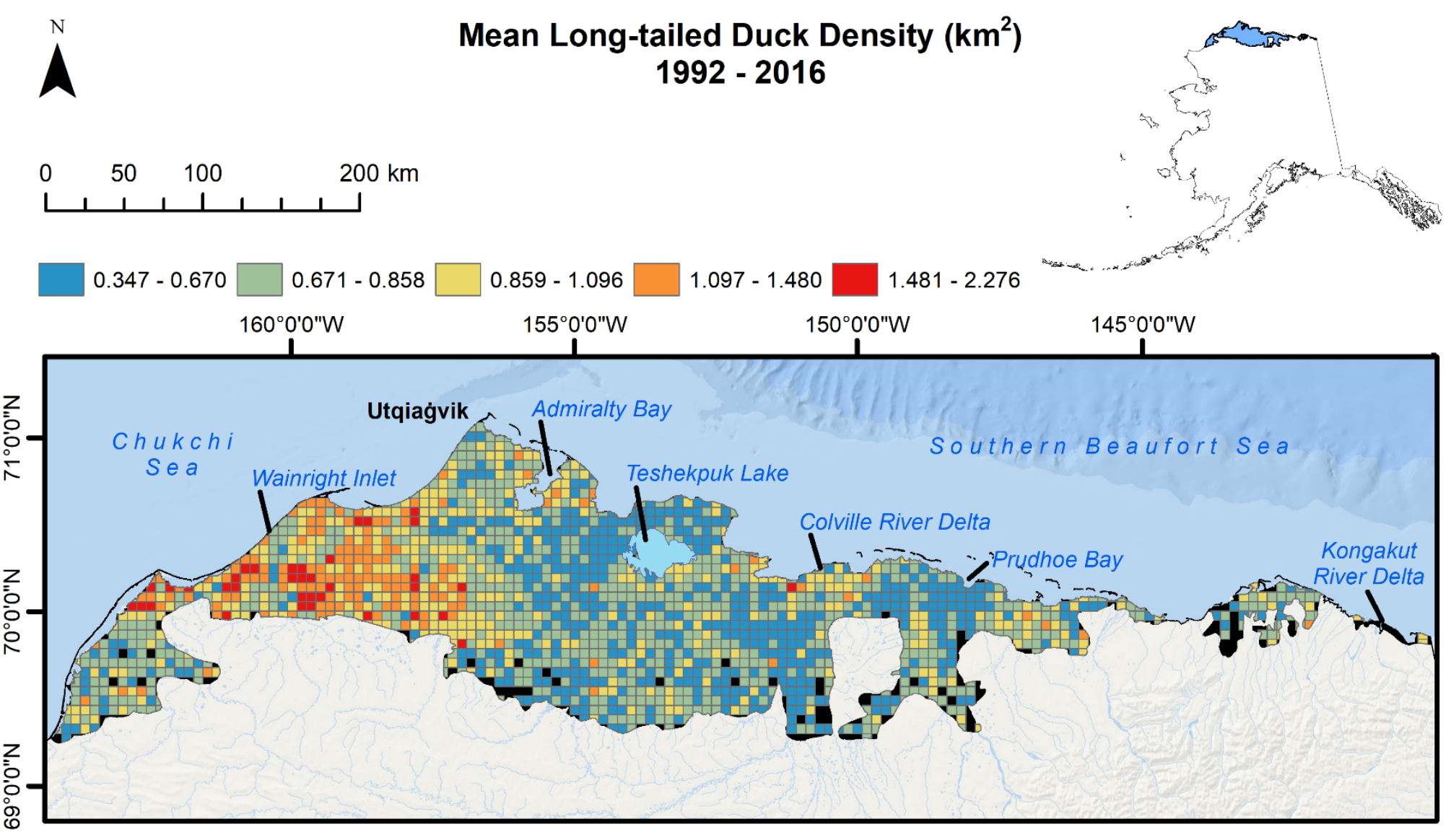


Figure A5.13. Red-breasted Merganser density $\left(\mathrm{km}^{2}\right)$ across the Arctic Coastal Plain, Alaska 1992-2016.

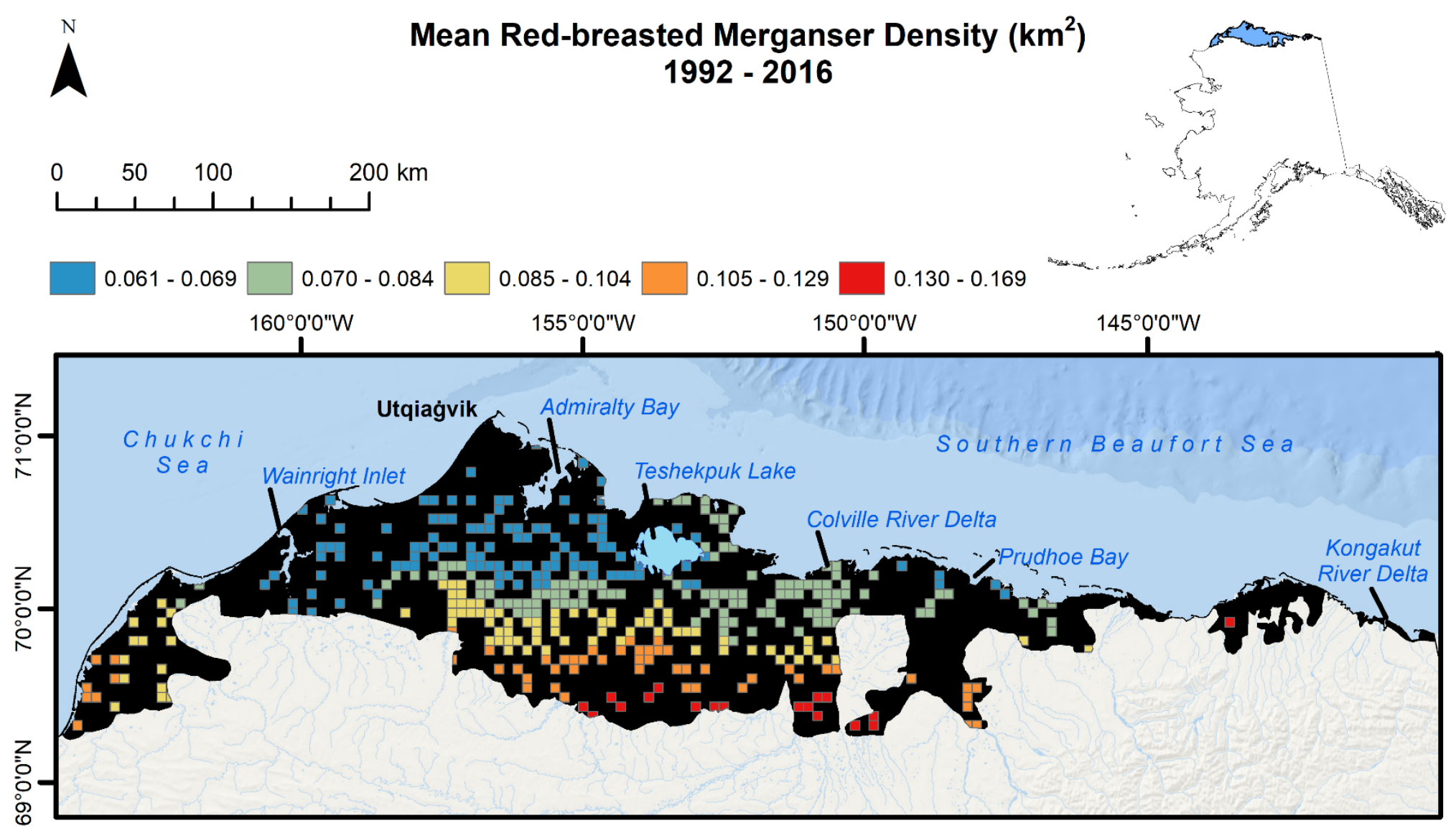


Figure A5.14. Jaeger (Pomarine, Parasitic and Long-tailed combined) density $\left(\mathrm{km}^{2}\right)$ across the Arctic Coastal Plain, Alaska 19922016.

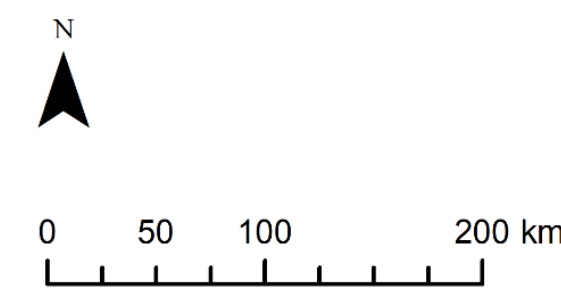

\section{Mean Jaeger Density $\left(\mathrm{km}^{2}\right)$ 1992 - 2016}

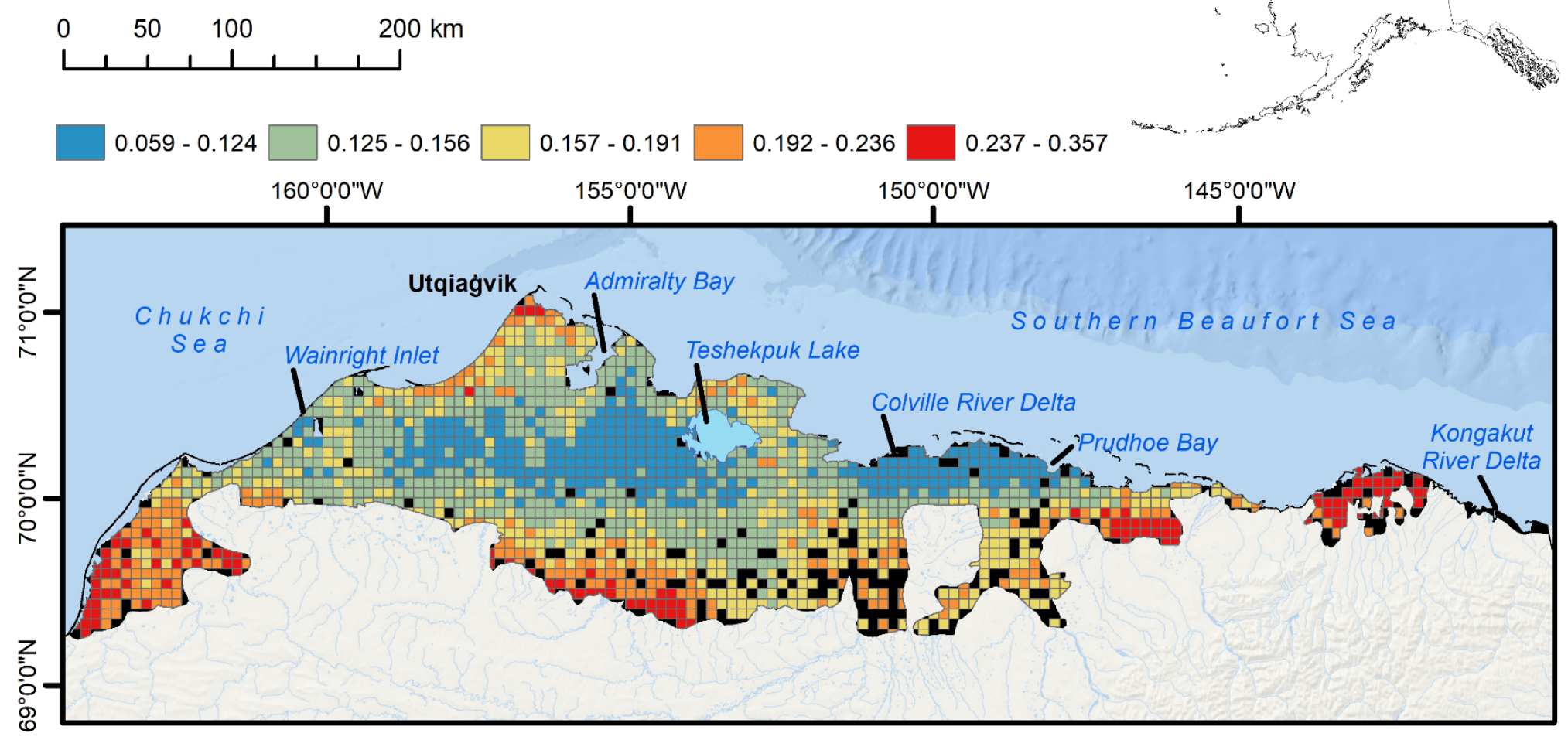


Figure A5.15. Sabine's Gull density $\left(\mathrm{km}^{2}\right)$ across the Arctic Coastal Plain, Alaska 1992-2016.

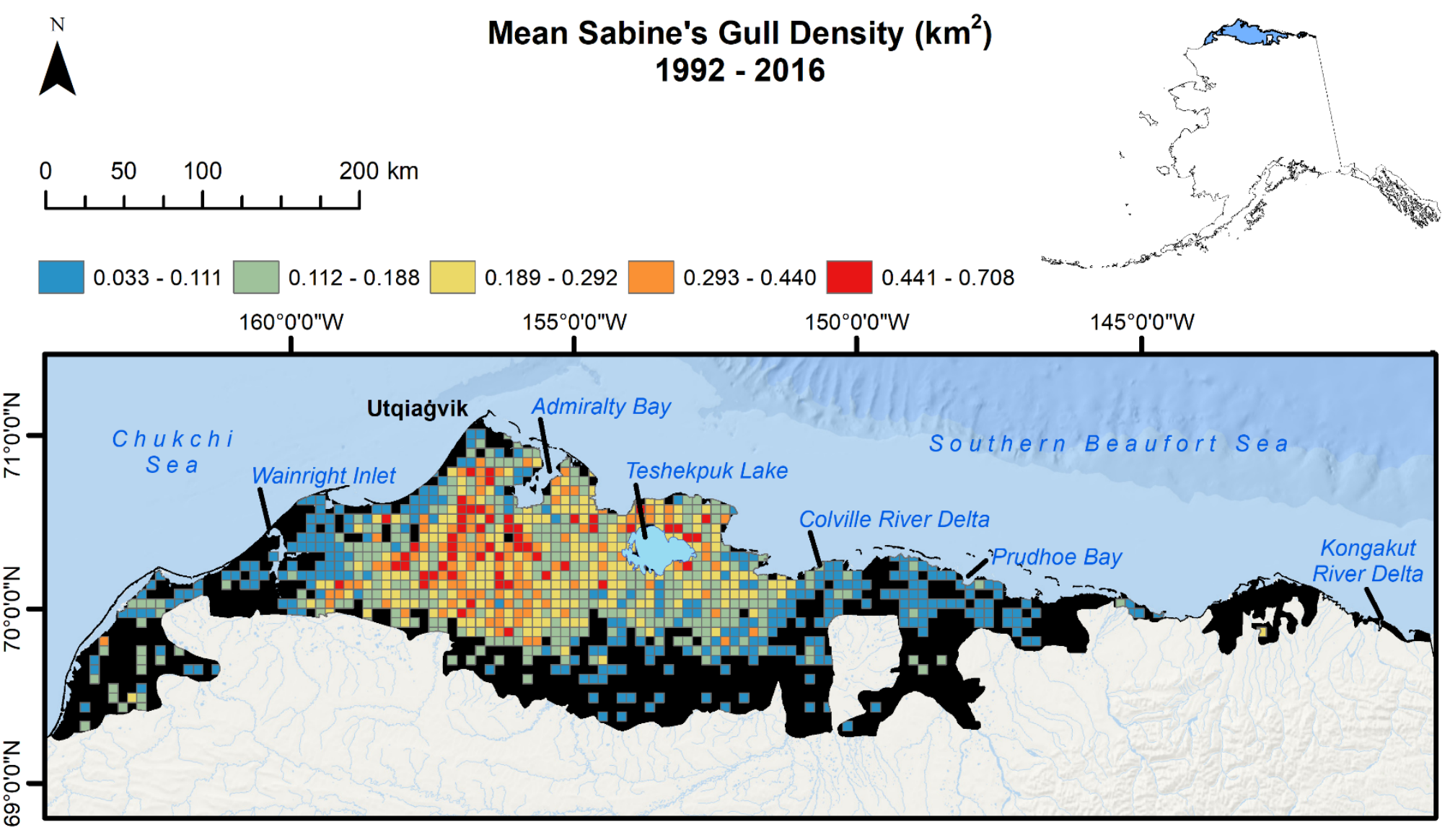


Figure A5.16. Glaucous Gull density $\left(\mathrm{km}^{2}\right)$ across the Arctic Coastal Plain, Alaska 1992-2016.

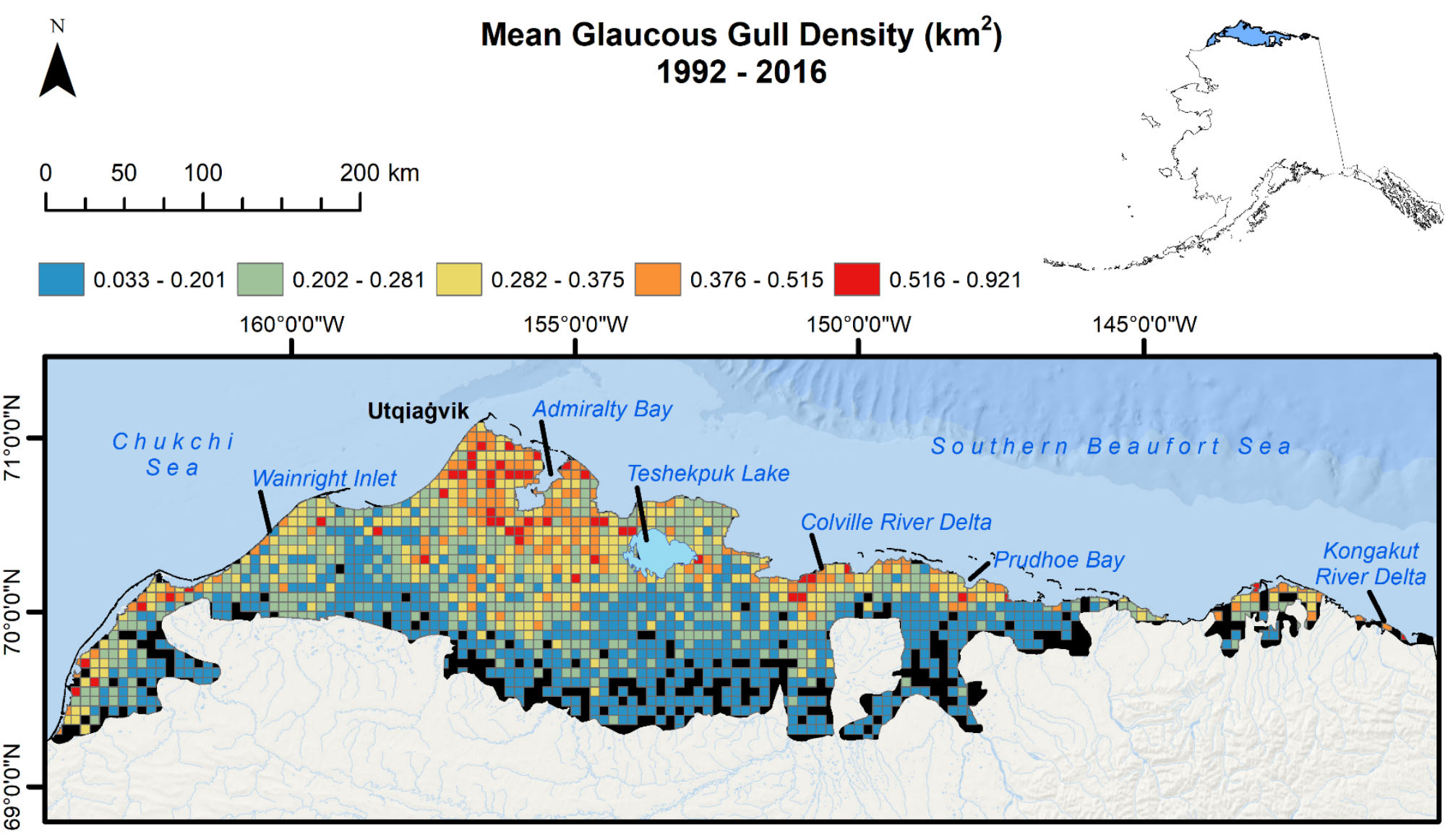


Figure A5.17. Arctic Tern density $\left(\mathrm{km}^{2}\right)$ across the Arctic Coastal Plain, Alaska 1992-2016.

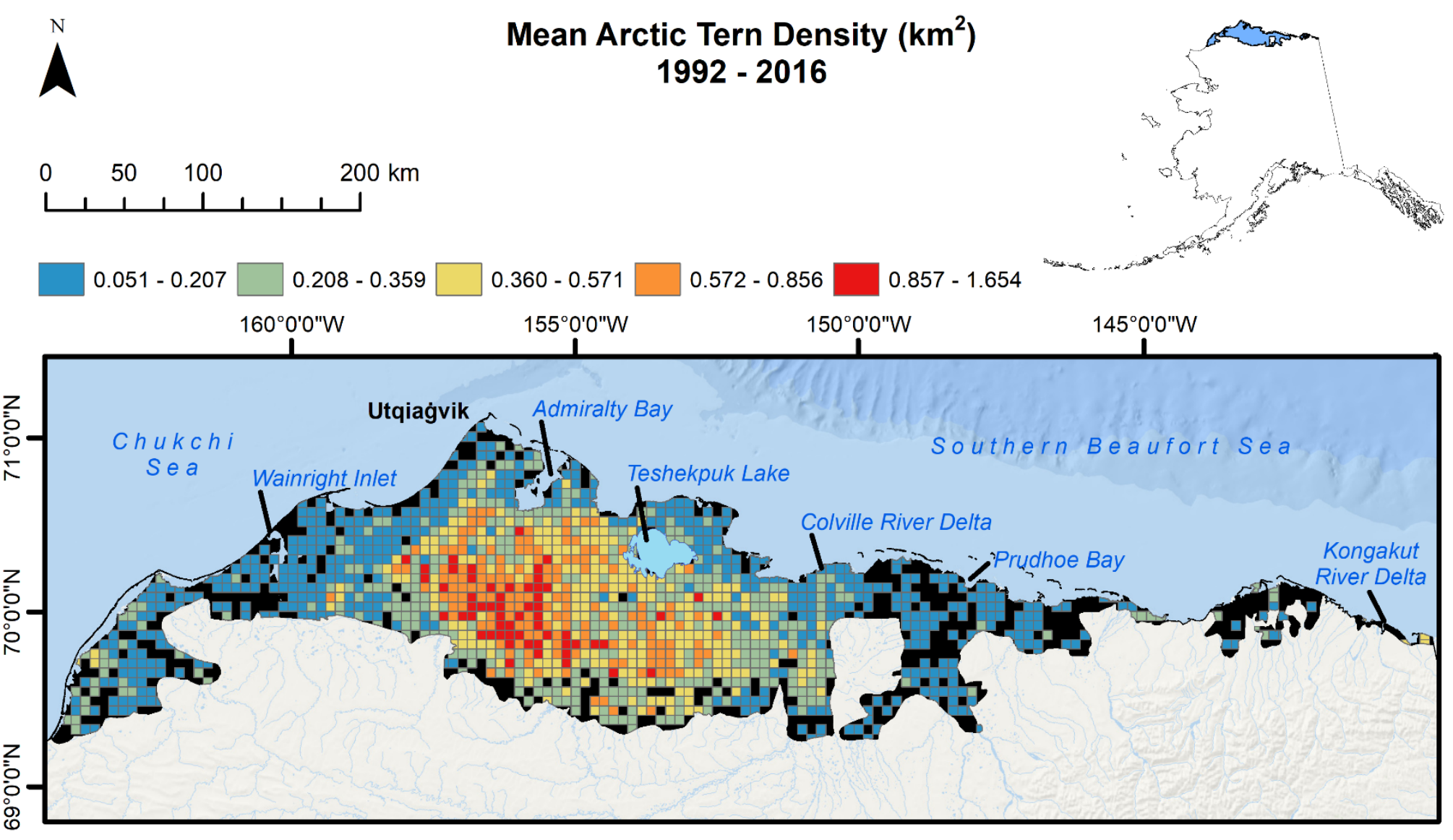


Figure A5.18. Red-throated Loon density $\left(\mathrm{km}^{2}\right)$ across the Arctic Coastal Plain, Alaska 1992-2016.

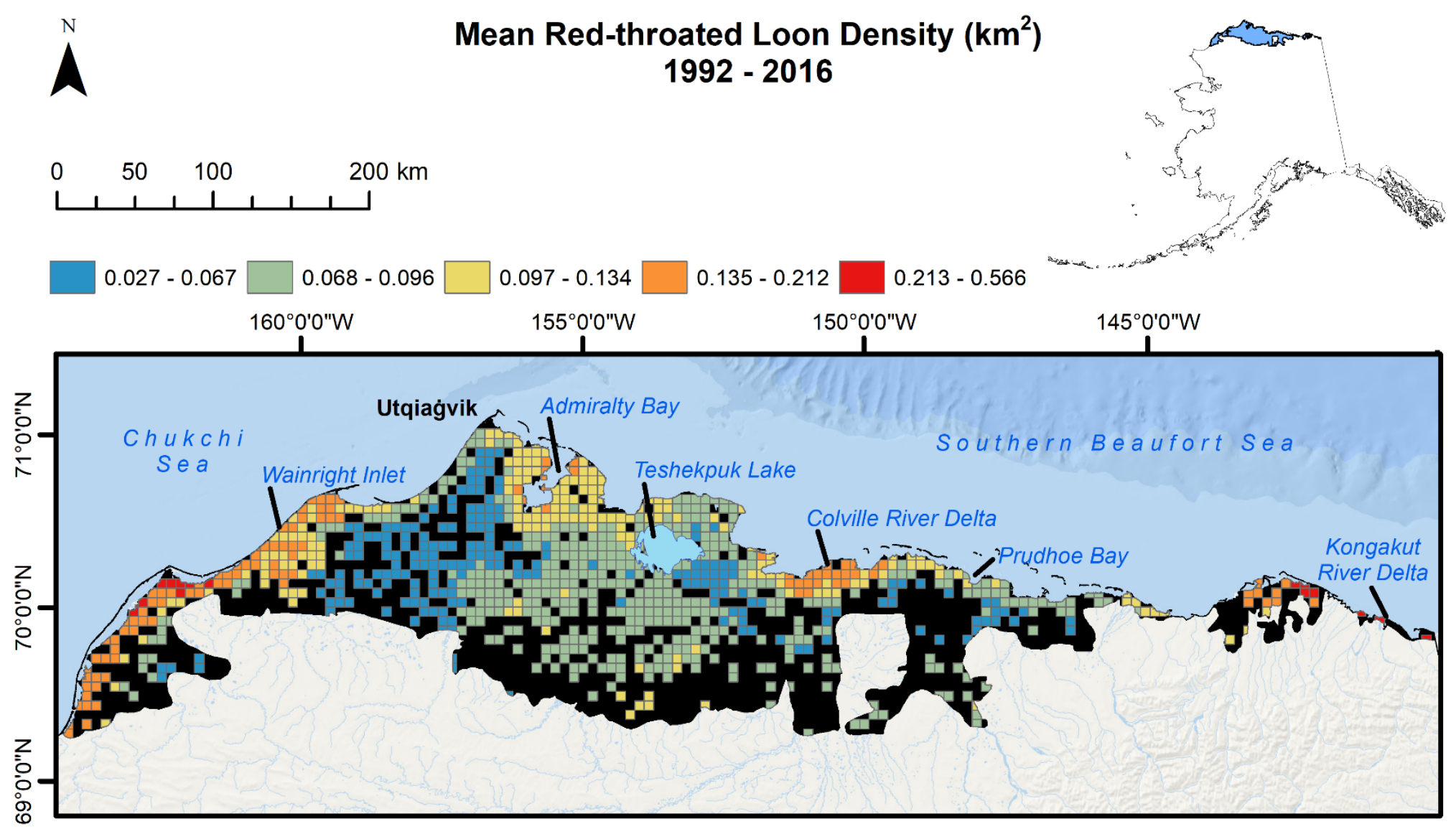


Figure A5.19. Pacific Loon density $\left(\mathrm{km}^{2}\right)$ across the Arctic Coastal Plain, Alaska 1992-2016.

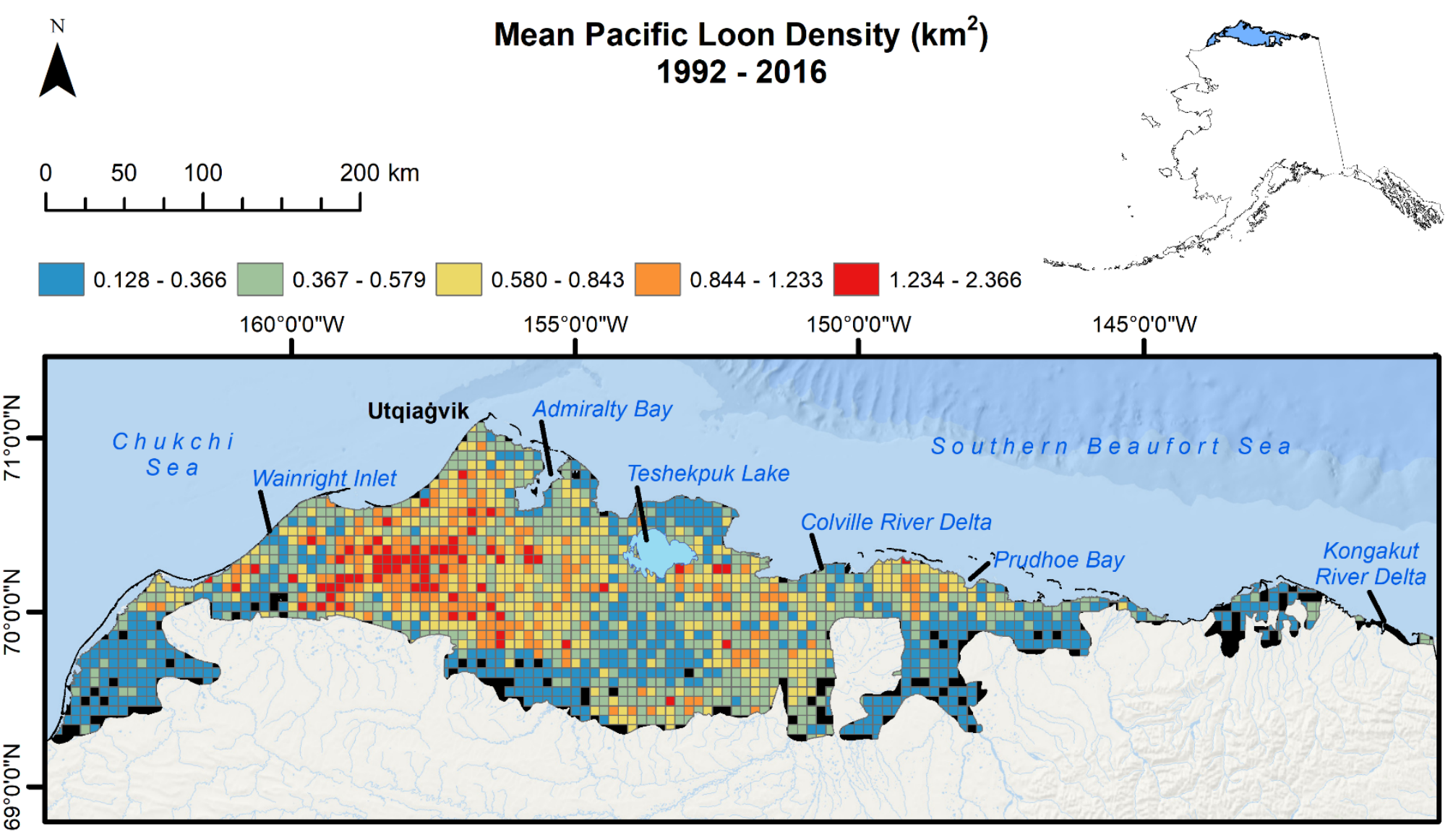


Figure A5.20. Yellow-billed Loon density $\left(\mathrm{km}^{2}\right)$ across the Arctic Coastal Plain, Alaska 1992-2016.

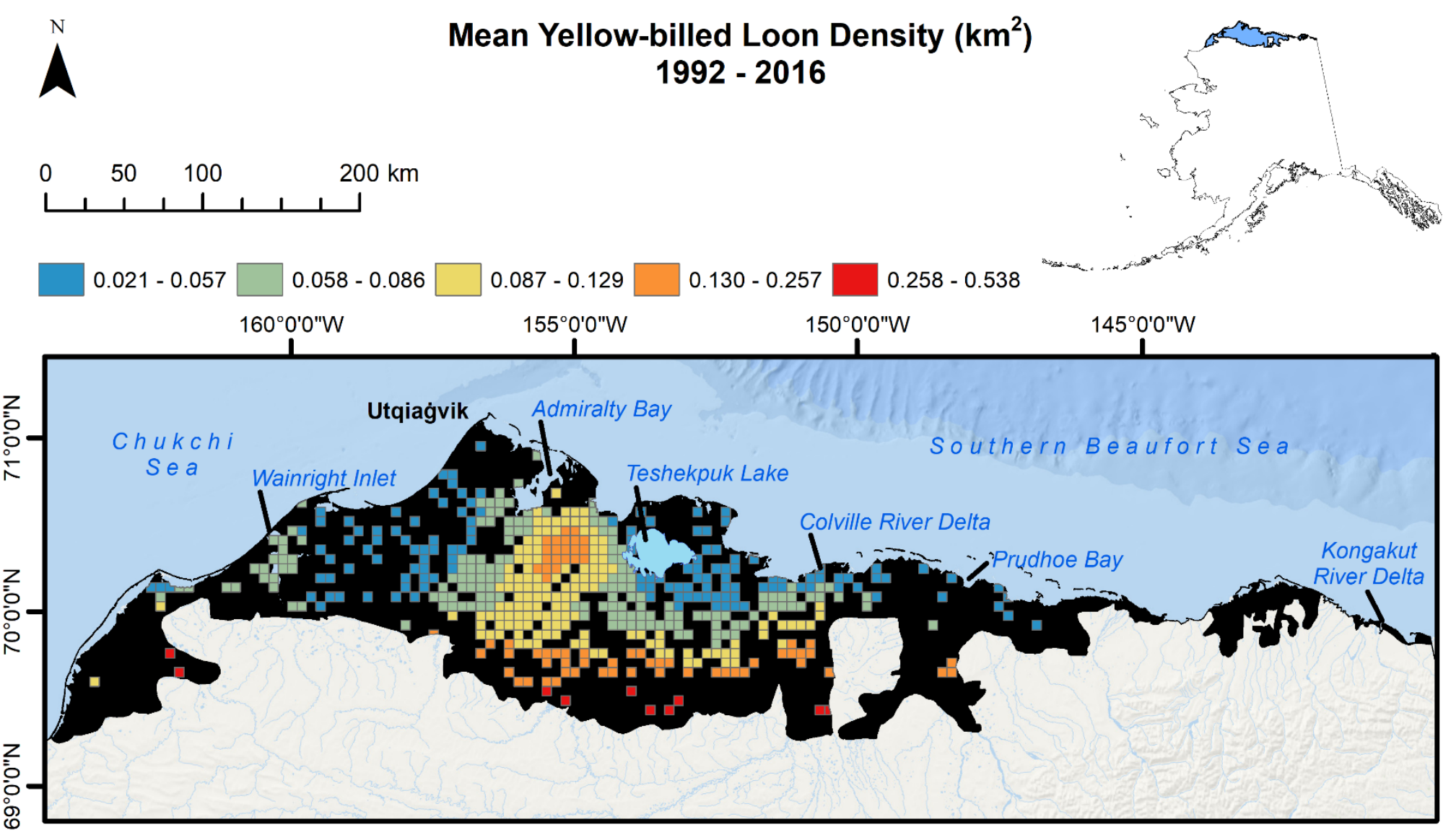


Appendix 6. Population trend

\section{Spatio-temporal population change of arctic-breeding waterbirds}

We mapped population change from 1992 to $2016(\%)$ within approximately $36 \mathrm{~km}^{2}$ (6 $\mathrm{km}$ by 6 $\mathrm{km})$ cells for 20 waterbird species observed during the Arctic Coastal Plain Breeding Waterfowl Survey, Alaska. We estimated population trends using hierarchical generalized linear mixed models (see main text, Methods). We present maps in taxonomic order (Chesser et al. 2018) for Lesser Snow Goose, Greater White-fronted Goose, Black Brant, Cackling Goose, Tundra Swan, Northern Pintail, Scaup (Lesser and Greater combined), Steller's Eider, Spectacled Eider, King Eider, White-winged Scoter, Long-tailed Duck, Red-breasted Merganser, Jaeger (Pomarine, Parasitic, and Long-tailed combined), Sabine's Gull, Glaucous Gull, Arctic Tern, Red-throated Loon, Pacific Loon, and Yellow-billed Loon. Color shades represent approximately 1 SD change in trend.

Chesser, R. T., K. J. Burns, C. Cicero, J. L. Dunn, A. W. Kratter, I. J. Lovette, P. C. Rasmussen, J. V. Remsen, Jr., J. D. Rising, D. F. Stotz, and K. Winker. 2018. Fifty-ninth supplement to the American Ornithological Society's check-list of North American birds. The Auk:

Ornithological Advances 135:798-813. 
Figure A6.1. Lesser Snow Goose population trend (\%) across the Arctic Coastal Plain, Alaska 1992-2016. We present trends for cells with $\geq 3$ nonzero counts.

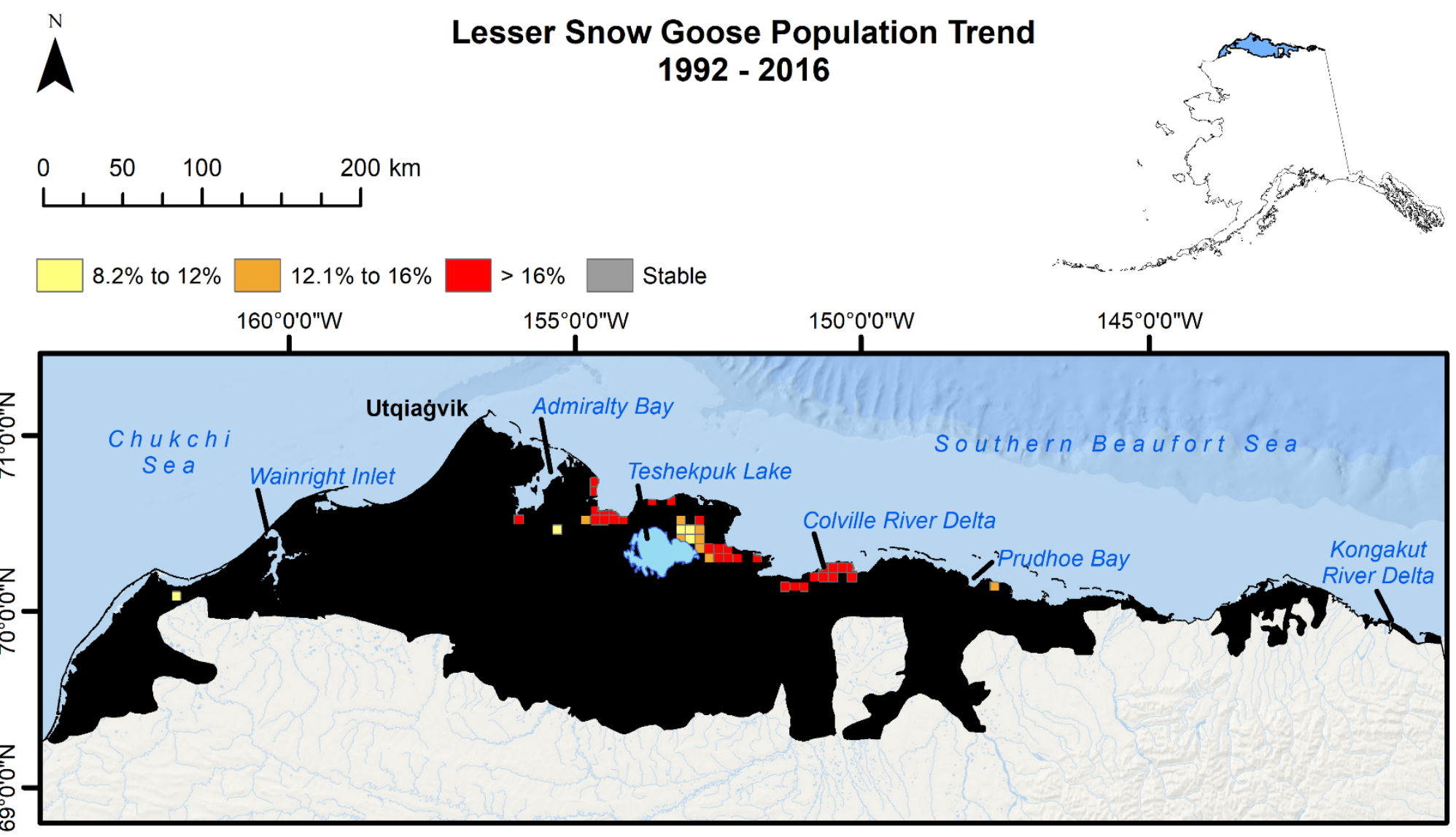


Figure A6.2. Greater White-fronted Goose population trend (\%) across the Arctic Coastal Plain, Alaska 1992-2016. We present trends for cells with $\geq 3$ nonzero counts.

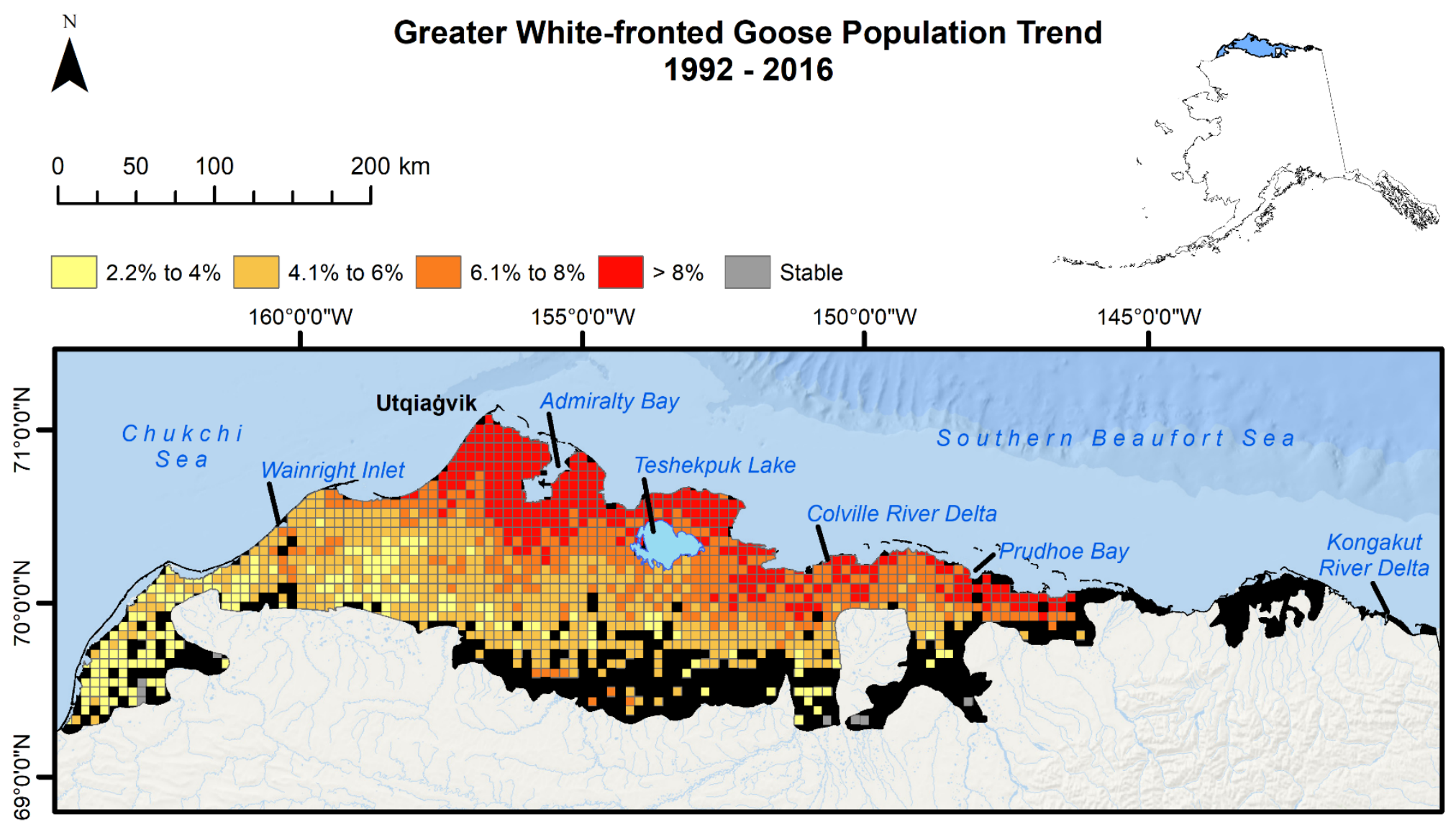


Figure A6.3. Black Brant population trend (\%) across the Arctic Coastal Plain, Alaska 1992-2016. We present trends for cells with $\geq$ 3 nonzero counts.

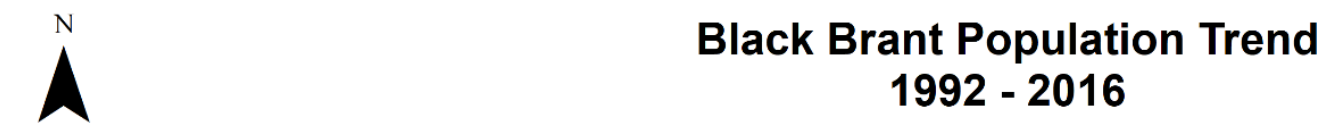
1992 - 2016

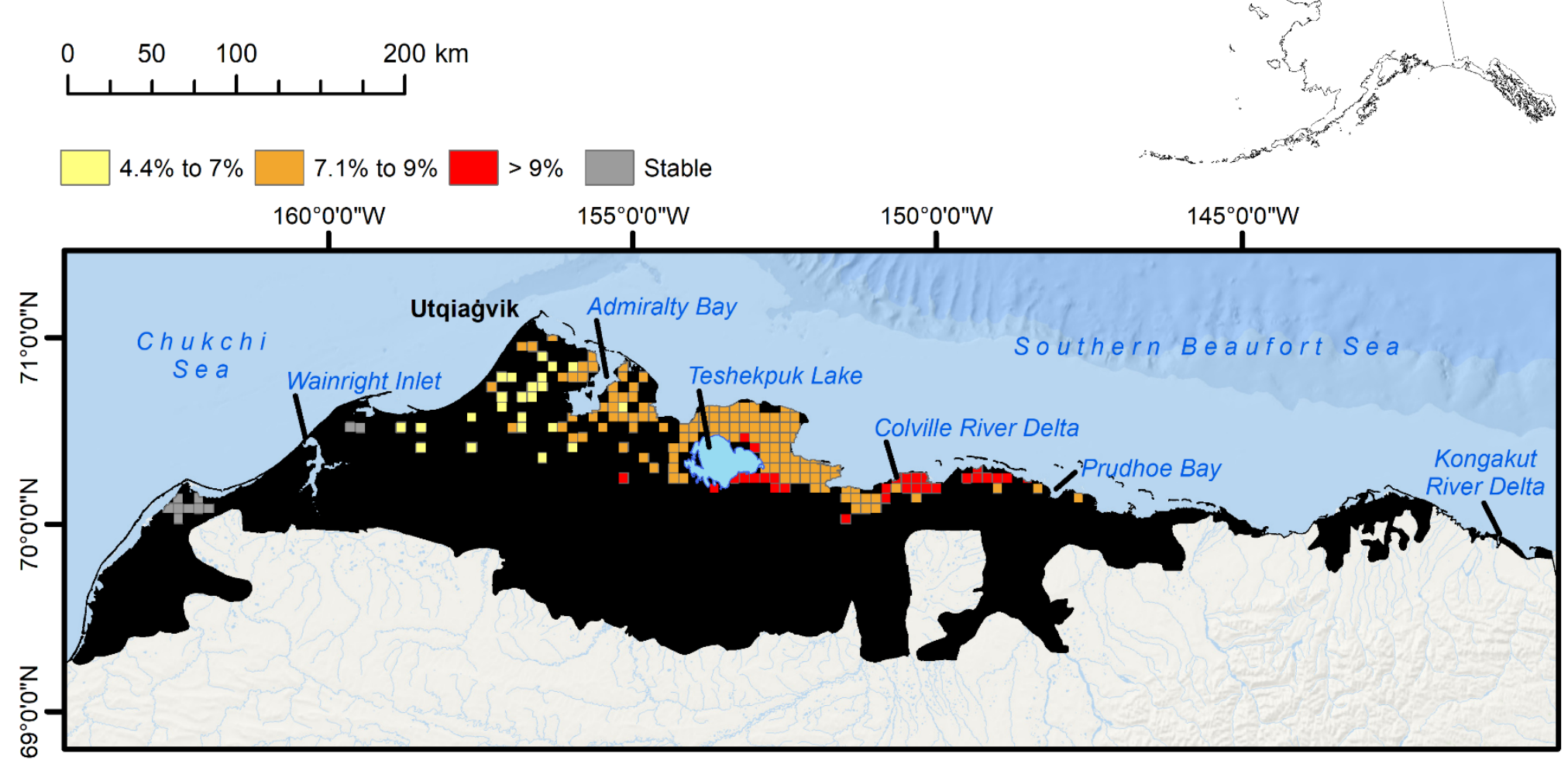


Figure A6.4. Cackling Goose population trend (\%) across the Arctic Coastal Plain, Alaska 1992-2016. We present trends for cells with $\geq 3$ nonzero counts.

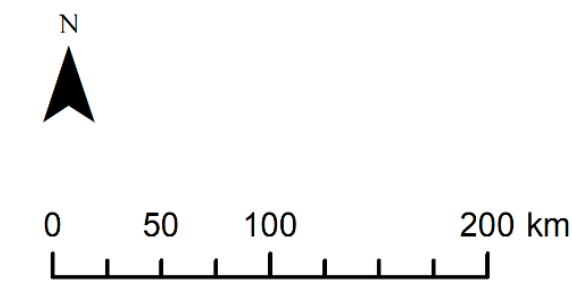

\section{Cackling Goose Population Trend} 1992 - 2016

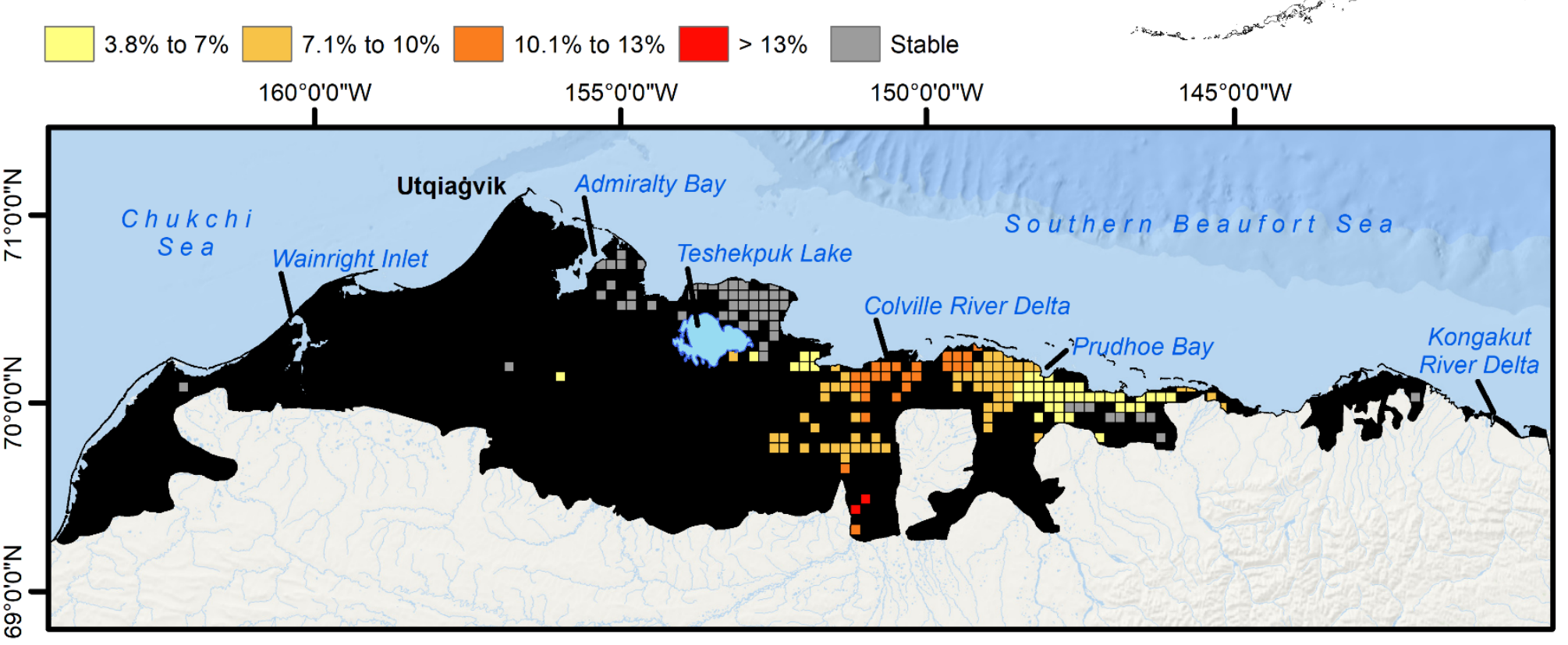


Figure A6.5. Tundra Swan population trend (\%) across the Arctic Coastal Plain, Alaska 1992-2016. We present trends for cells with $\geq 3$ nonzero counts.

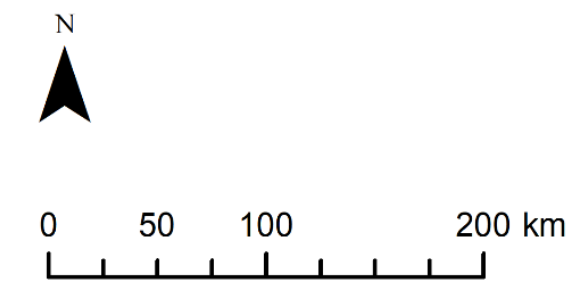

\section{Tundra Swan Population Trend 1992 - 2016}

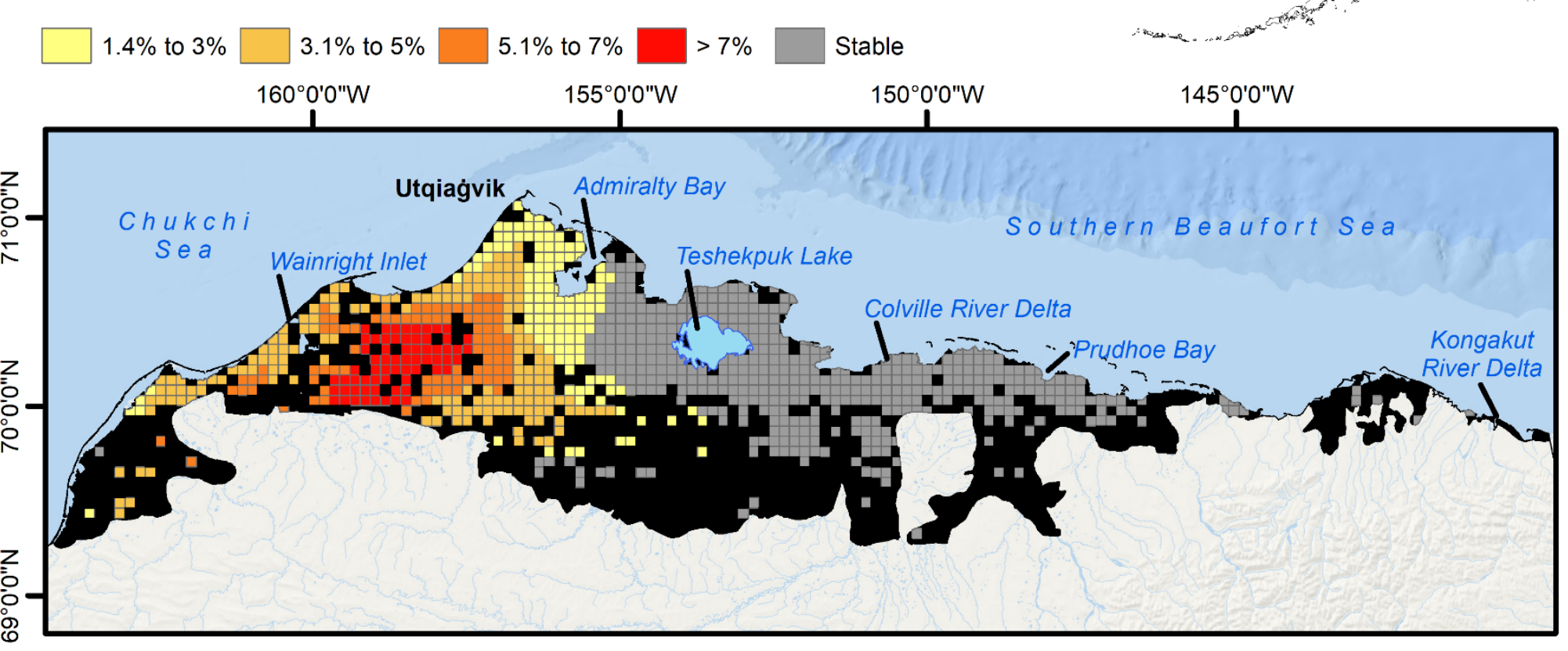


Figure A6.6. Northern Pintail population trend (\%) across the Arctic Coastal Plain, Alaska 1992-2016. We present trends for cells with $\geq 3$ nonzero counts.

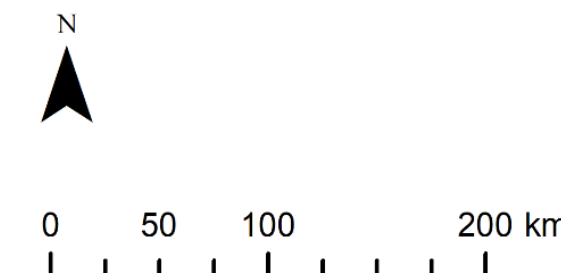

\section{Northern Pintail Population Trend} 1992 - 2016

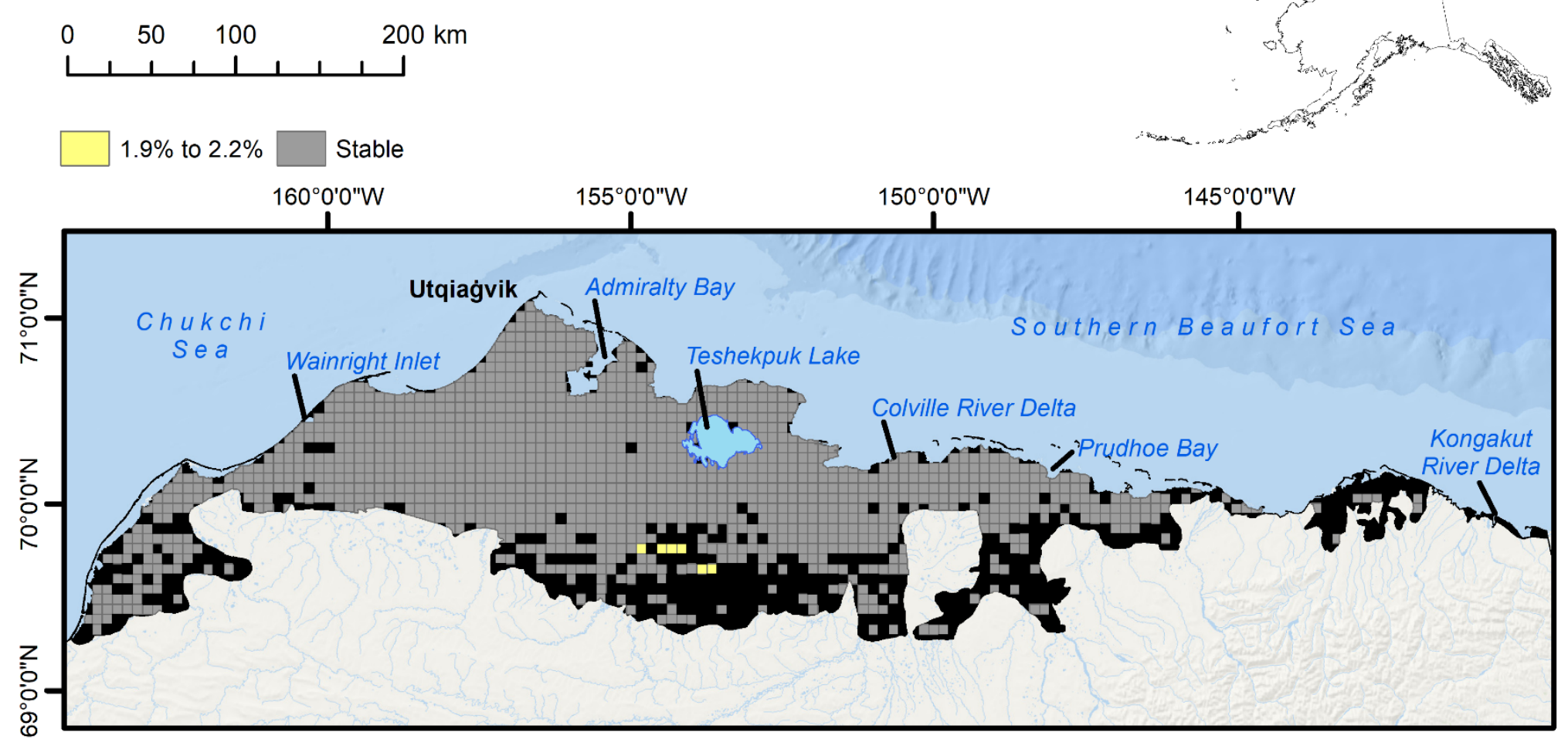


Figure A6.7. Scaup (Lesser and Greater combined) population trend (\%) across the Arctic Coastal Plain, Alaska 1992-2016. We present trends for cells with $\geq 3$ nonzero counts.

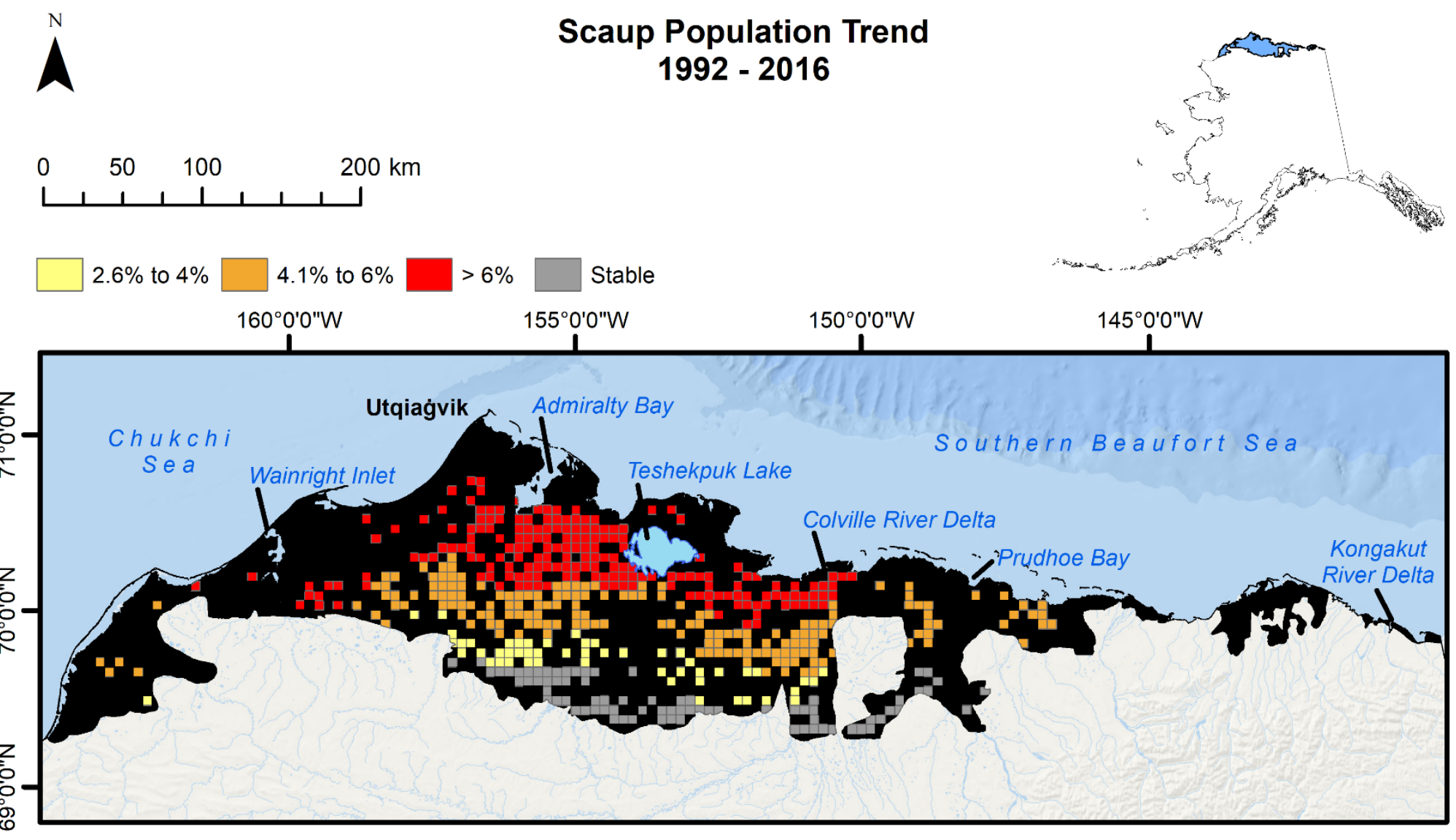


Figure A6.8. Steller's Eider population trend (\%) across the Arctic Coastal Plain, Alaska 1992-2016. We present trends for cells with $\geq 3$ nonzero counts.

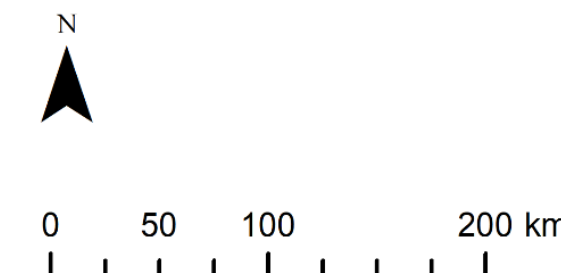

\section{Steller's Eider Population Trend}

1992 - 2016

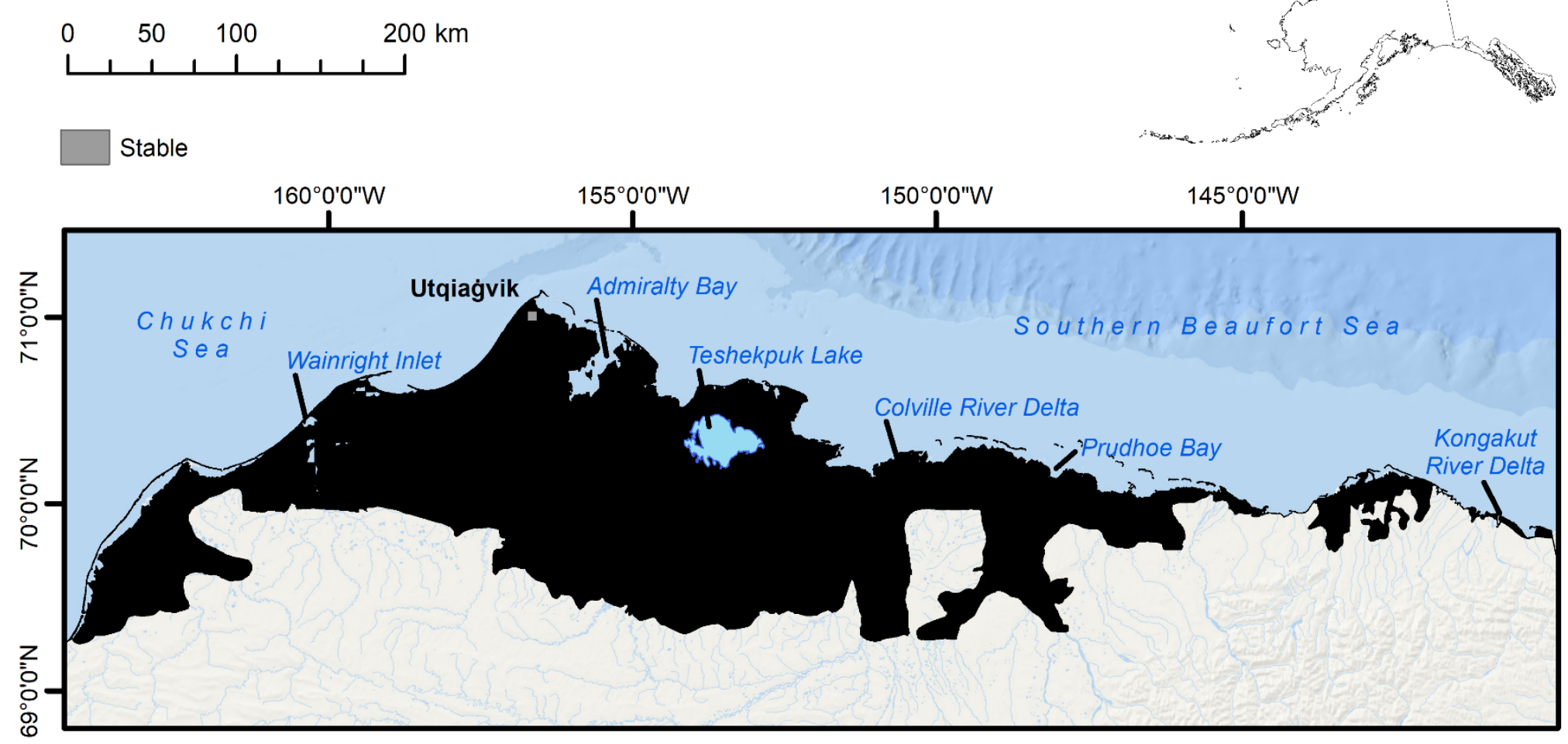


Figure A6.9. Spectacled Edier population trend (\%) across the Arctic Coastal Plain, Alaska 1992-2016. We present trends for cells with $\geq 3$ nonzero counts.

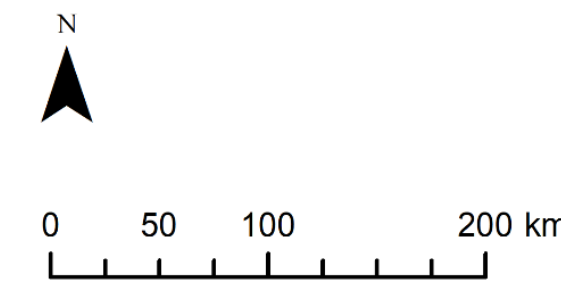

\section{Spectacled Eider Population Trend} 1992 - 2016

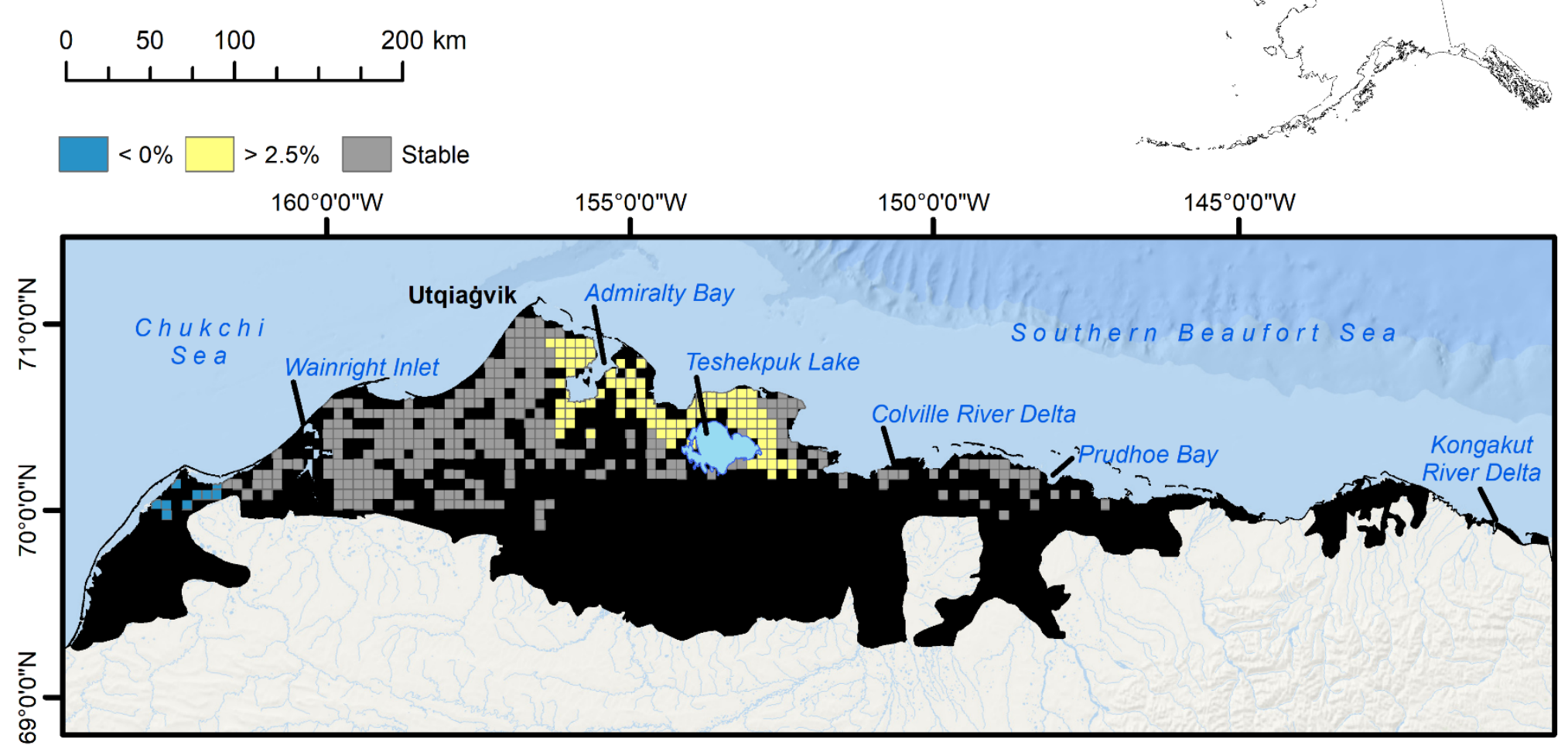


Figure A6.10. King Eider population trend (\%) across the Arctic Coastal Plain, Alaska 1992-2016. We present trends for cells with $\geq$ 3 nonzero counts.
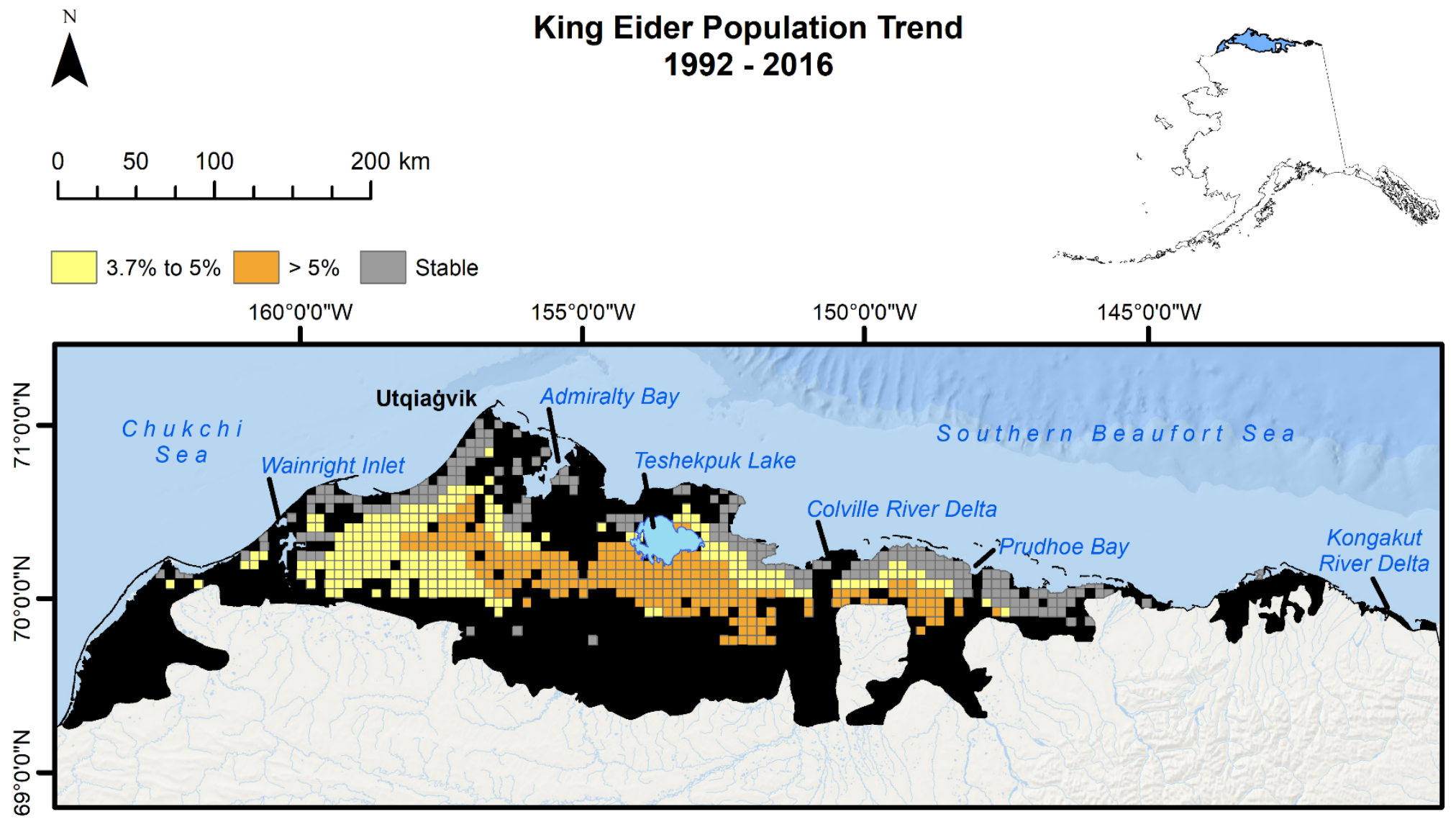
Figure A6.11. White-winged Scoter population trend (\%) across the Arctic Coastal Plain, Alaska 1992-2016. We present trends for cells with $\geq 3$ nonzero counts.

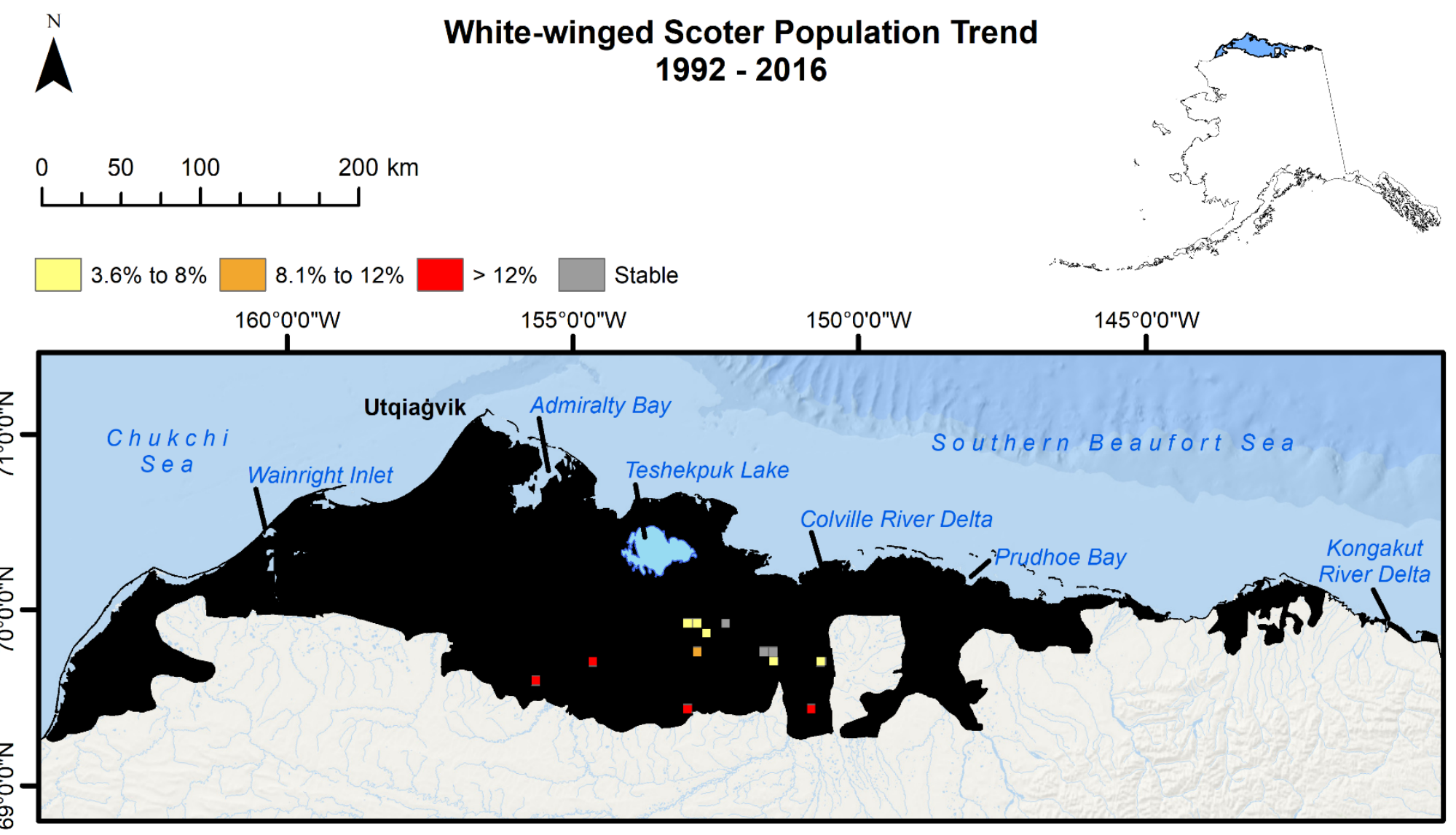


Figure A6.12. Long-tailed Duck population trend (\%) across the Arctic Coastal Plain, Alaska 1992-2016. We present trends for cells with $\geq 3$ nonzero counts.

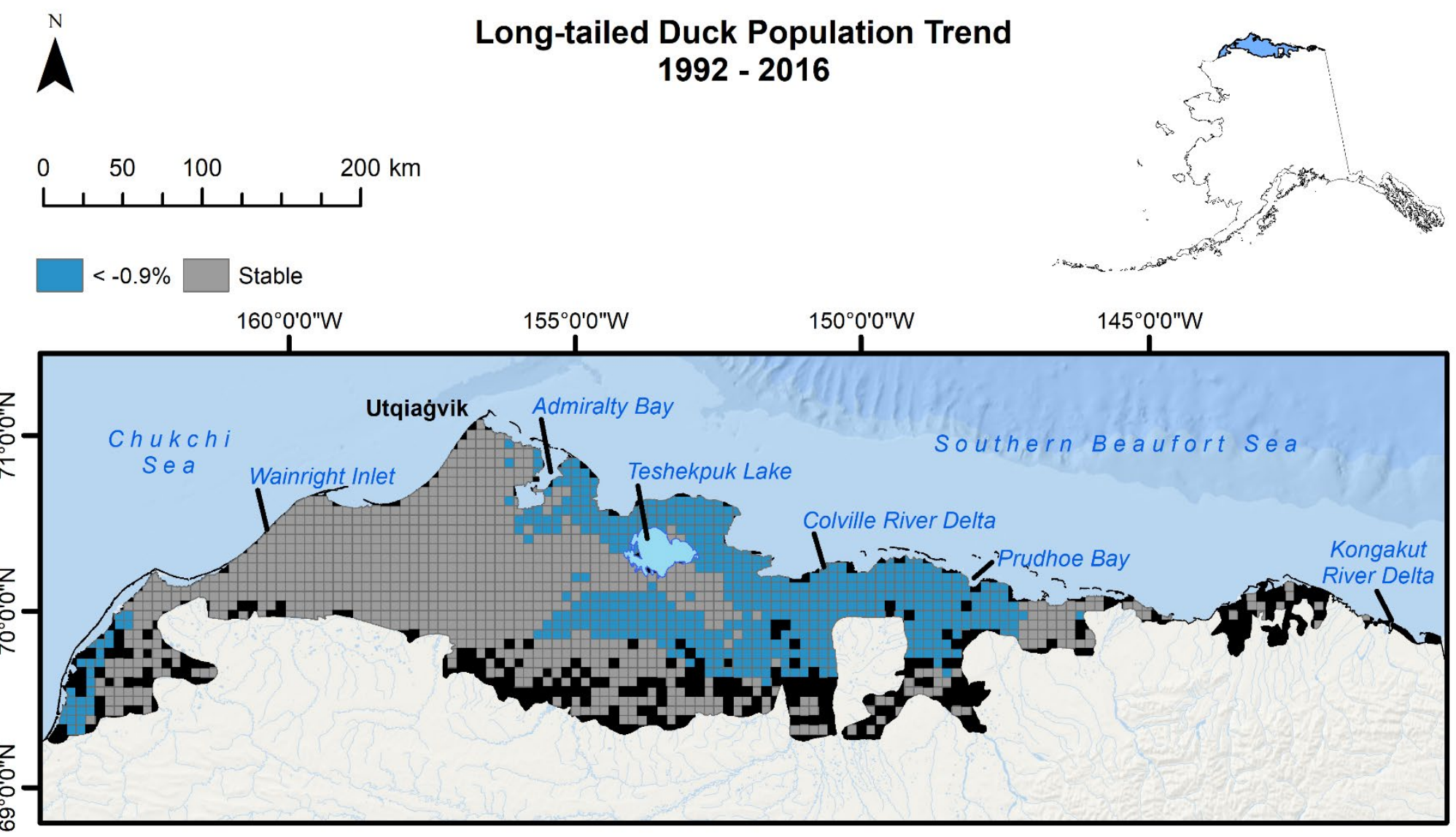


Figure A6.13. Red-breasted Merganser population trend (\%) across the Arctic Coastal Plain, Alaska 1992-2016. We present trends for cells with $\geq 3$ nonzero counts.

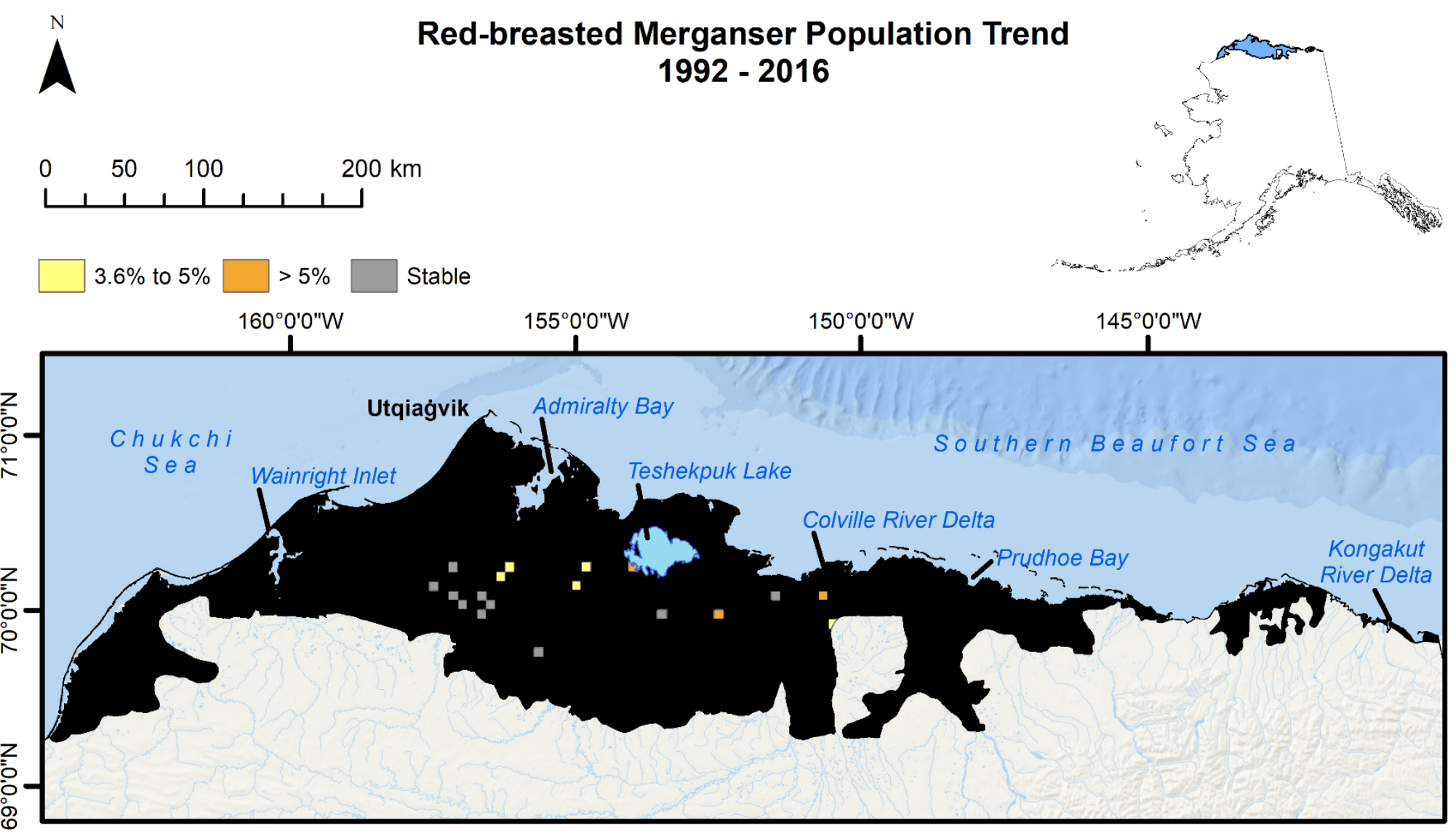


Figure A6.14. Jaeger (Pomarine, Parasitic and Long-tailed combined) population trend (\%) across the Arctic Coastal Plain, Alaska 1992-2016. We present trends for cells with $\geq 3$ nonzero counts.

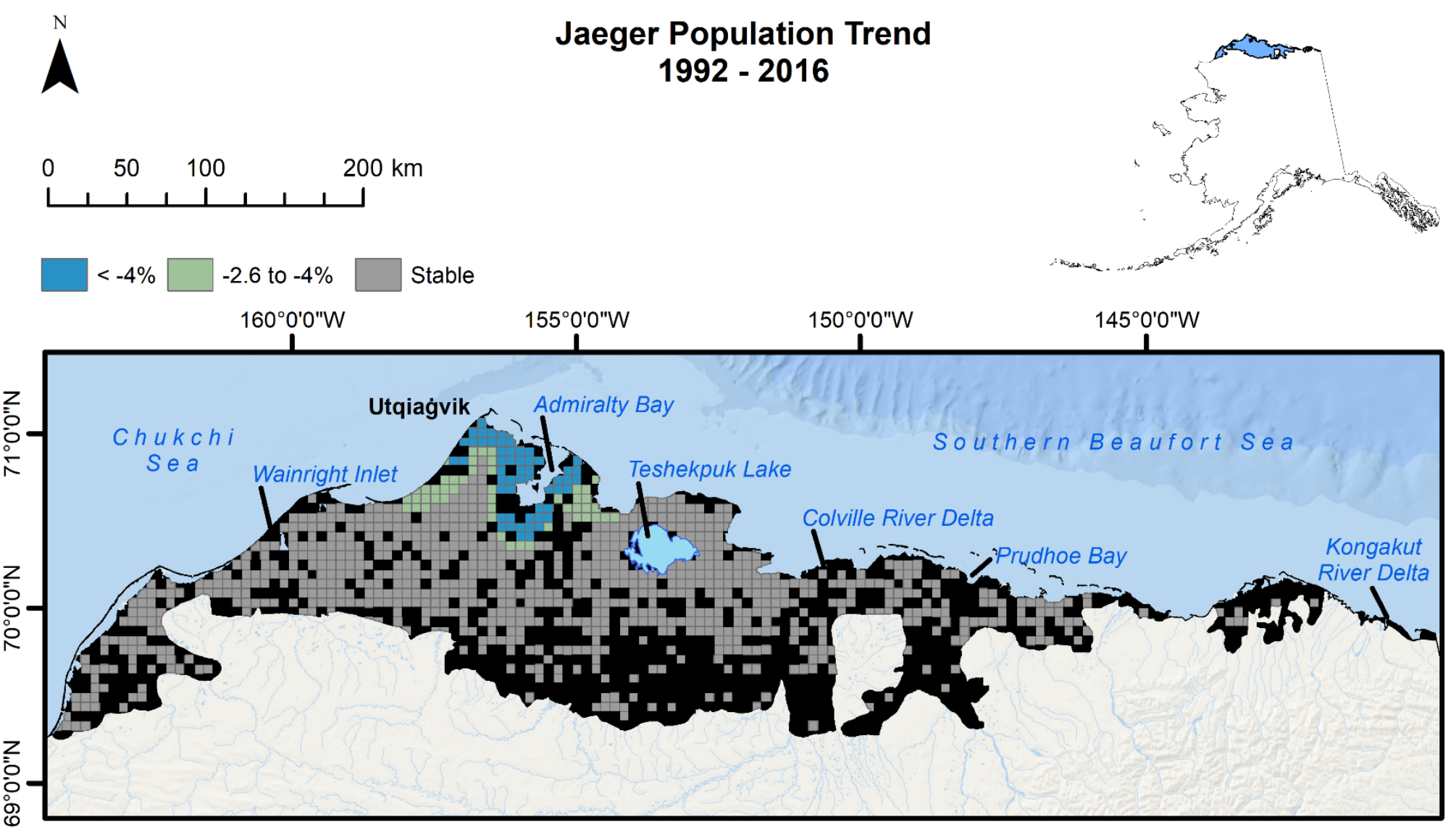


Figure A6.15. Sabine's Gull population trend (\%) across the Arctic Coastal Plain, Alaska 1992-2016. We present trends for cells with $\geq 3$ nonzero counts.
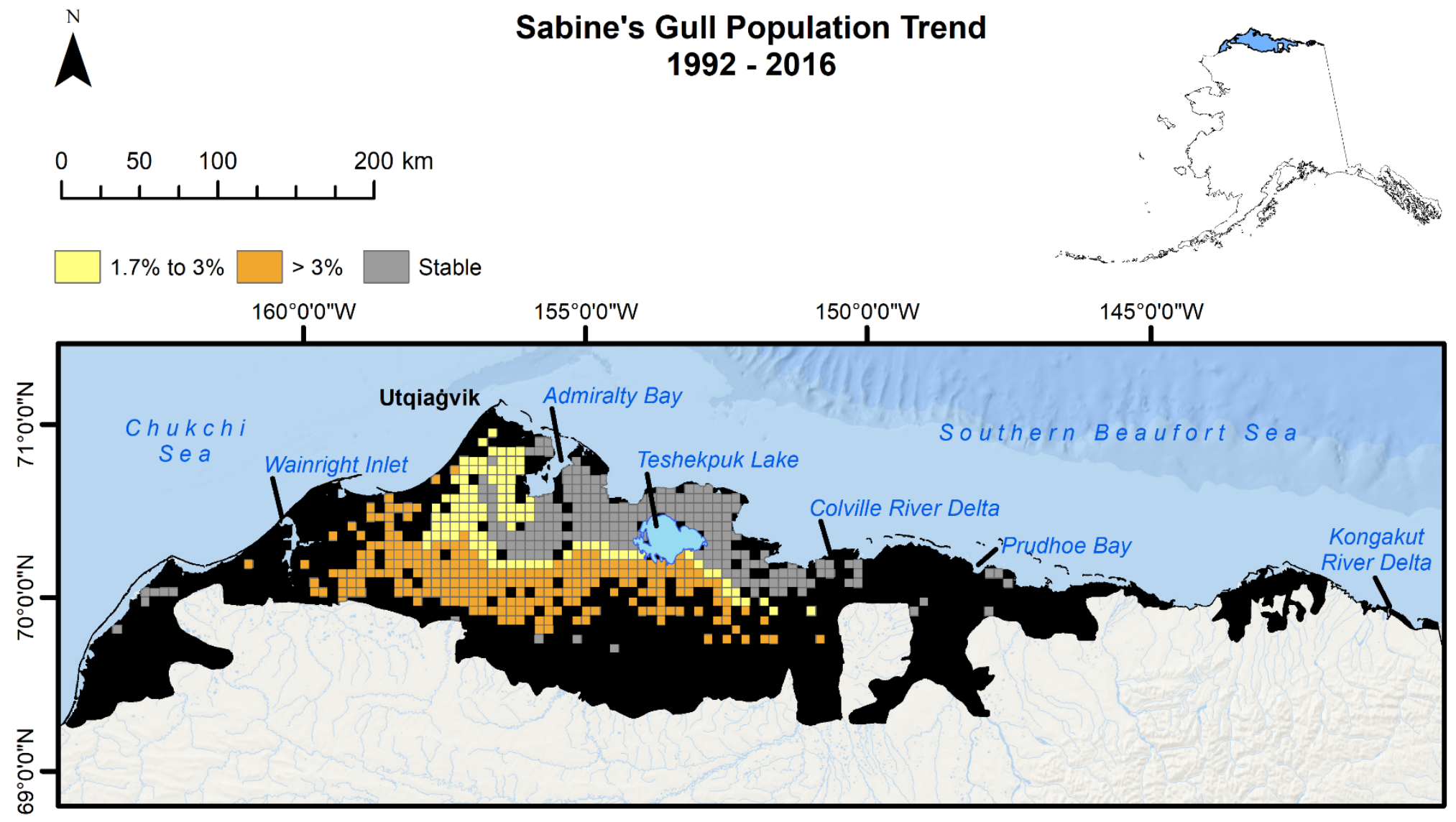
Figure A6.16. Glaucous Gull population trend (\%) across the Arctic Coastal Plain, Alaska 1992-2016. We present trends for cells with $\geq 3$ nonzero counts.

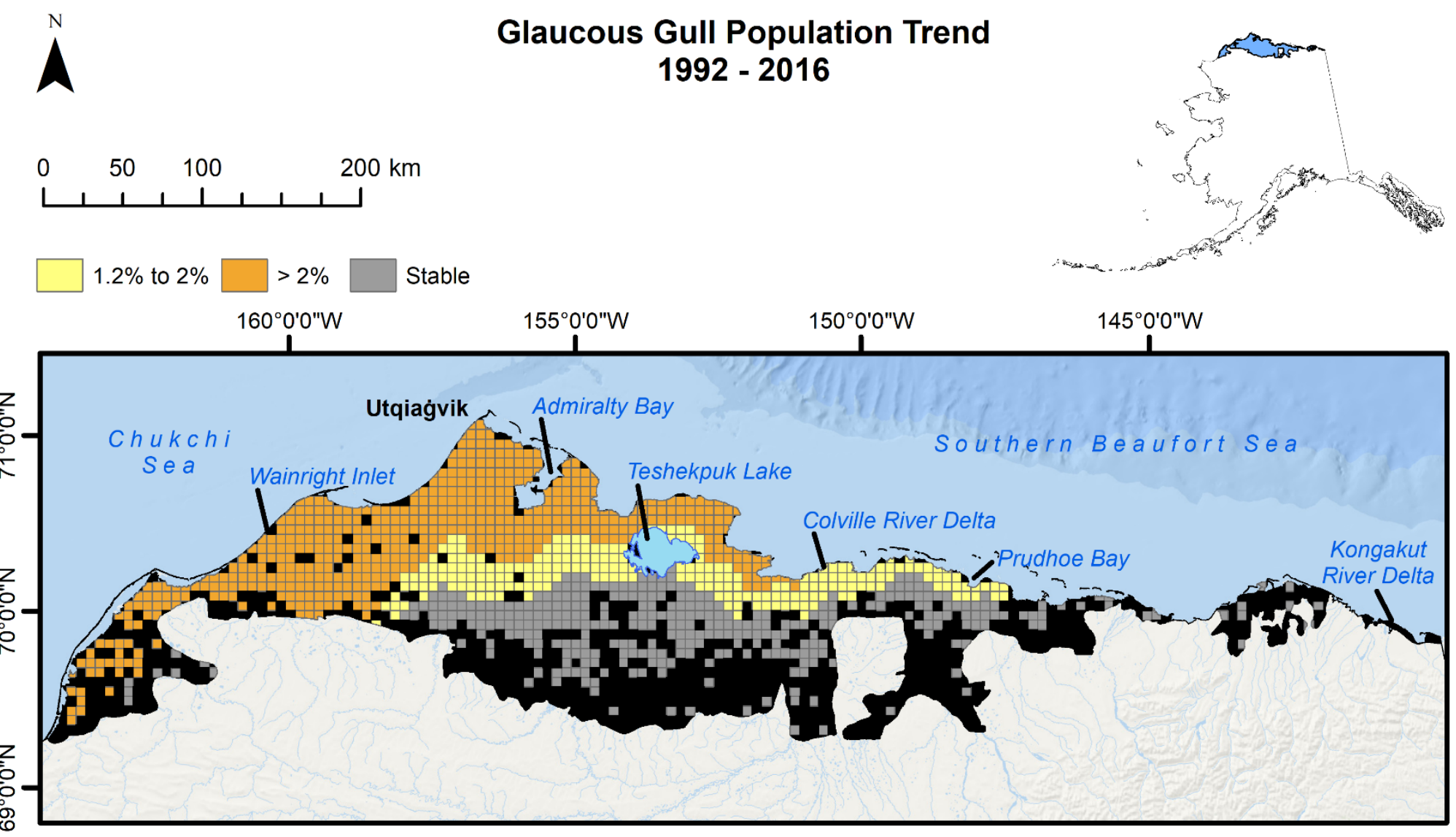


Figure A6.17. Arctic Tern population trend (\%) across the Arctic Coastal Plain, Alaska 1992-2016. We present trends for cells with $\geq$ 3 nonzero counts.
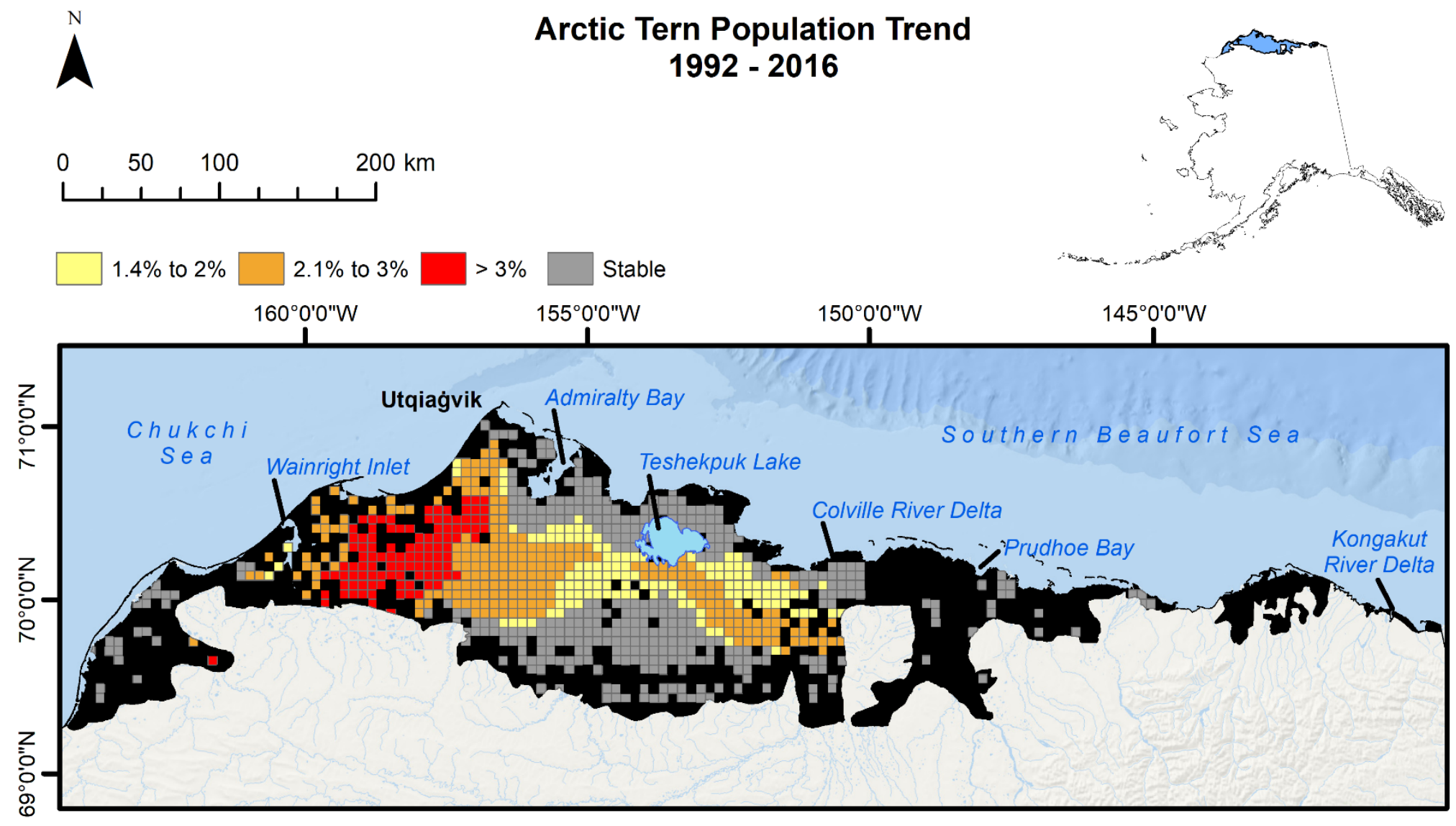
Figure A6.18. Red-throated Loon population trend (\%) across the Arctic Coastal Plain, Alaska 1992-2016. We present trends for cells with $\geq 3$ nonzero counts.

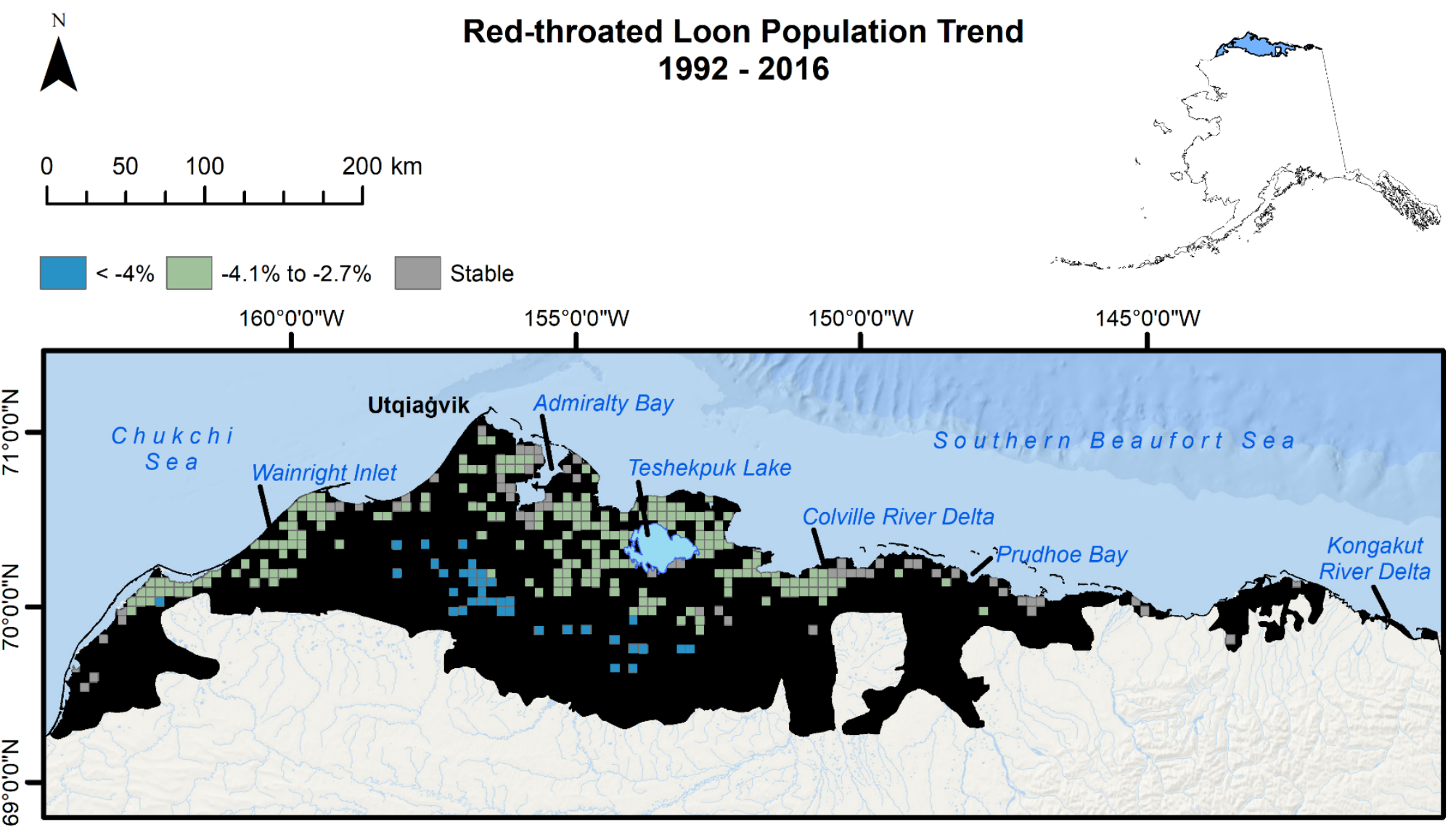


Figure A6.19. Pacific Loon population trend (\%) across the Arctic Coastal Plain, Alaska 1992-2016. We present trends for cells with $\geq 3$ nonzero counts.

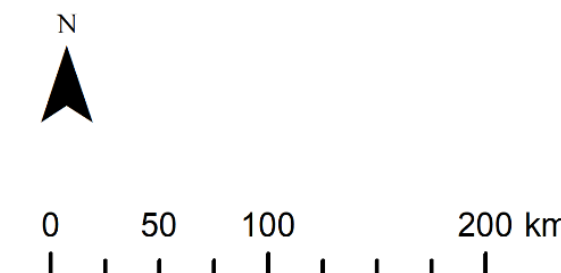

\section{Pacific Loon Population Trend} 1992 - 2016

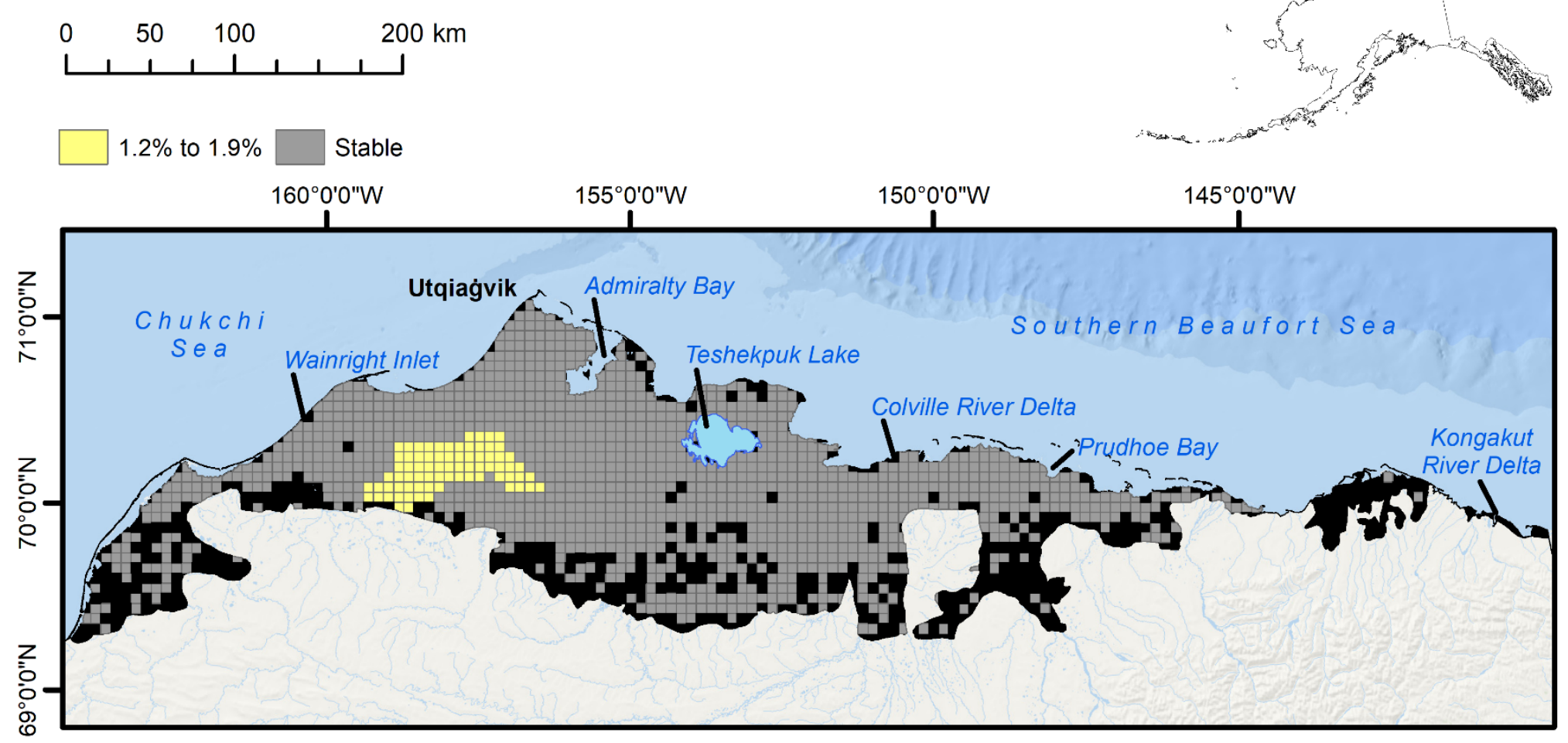


Figure A6.20. Yellow-billed Loon population trend (\%) across the Arctic Coastal Plain, Alaska 1992-2016. We present trends for cells with $\geq 3$ nonzero counts.

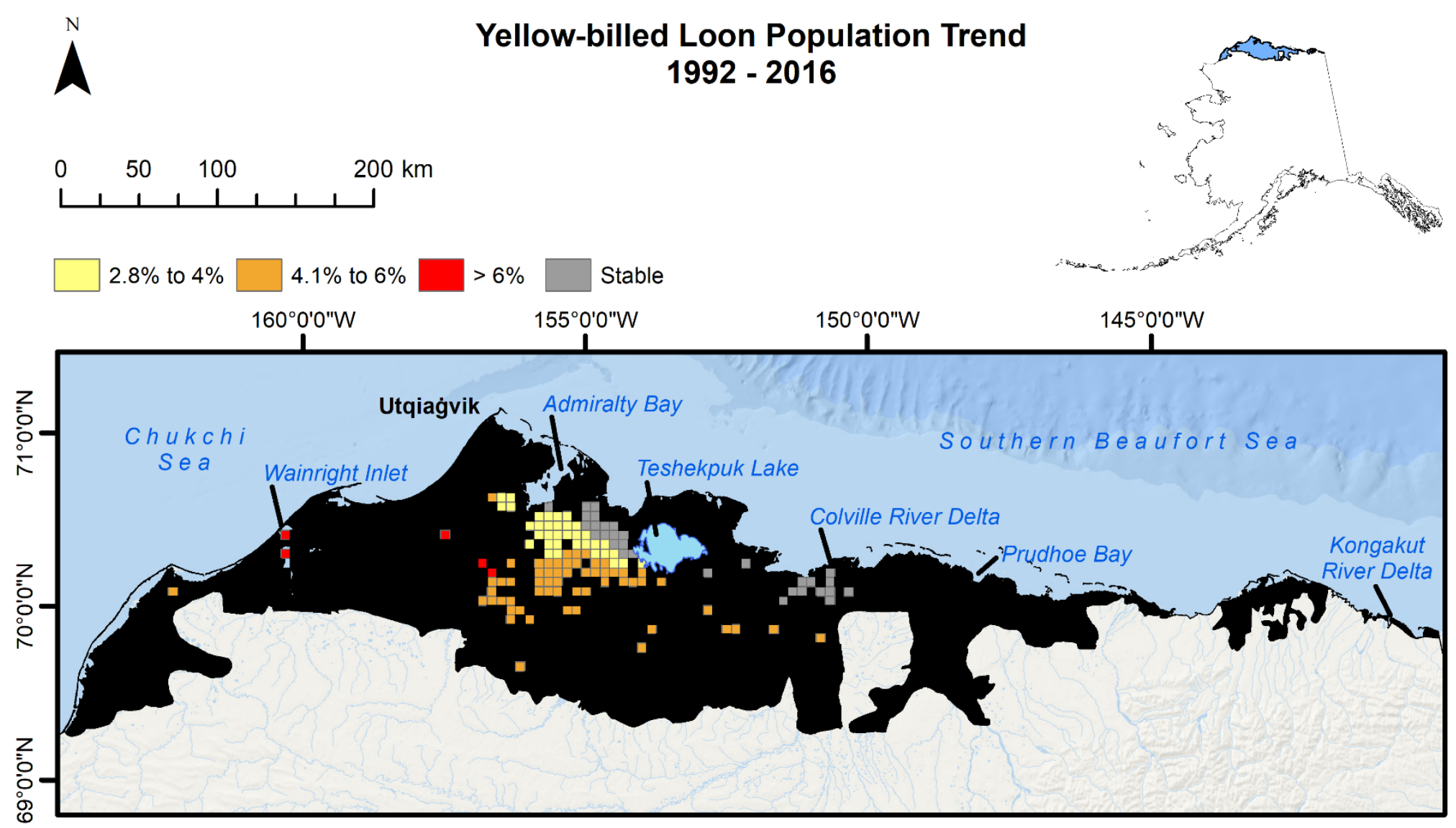


Appendix 7. Guild importance

\section{Spatio-temporal population change of arctic-breeding waterbirds}

We mapped the relative importance of approximately $36 \mathrm{~km}^{2}(6 \mathrm{~km}$ by $6 \mathrm{~km})$ cells to five guilds of waterbirds (herbivores, aquatic omnivores, invertivores, generalist predators, and piscivores) observed during the Arctic Coastal Plain Breeding Waterfowl Survey, Alaska, 1992-2016. We defined cells as important, unimportant, or average for each cell-species with values $1,-1$, and 0 , respectively. We then summed species scores across species within guilds to determine overall relative importance of each cell. See the Methods section of the main text for more detail on importance scores. 
Figure A7.1. Relative importance of cells for avian herbivores across the Arctic Coastal Plain, Alaska 1992-2016. Herbivores include: Lesser Snow Goose, Greater White-fronted Goose, Black Brant and Cackling Goose.

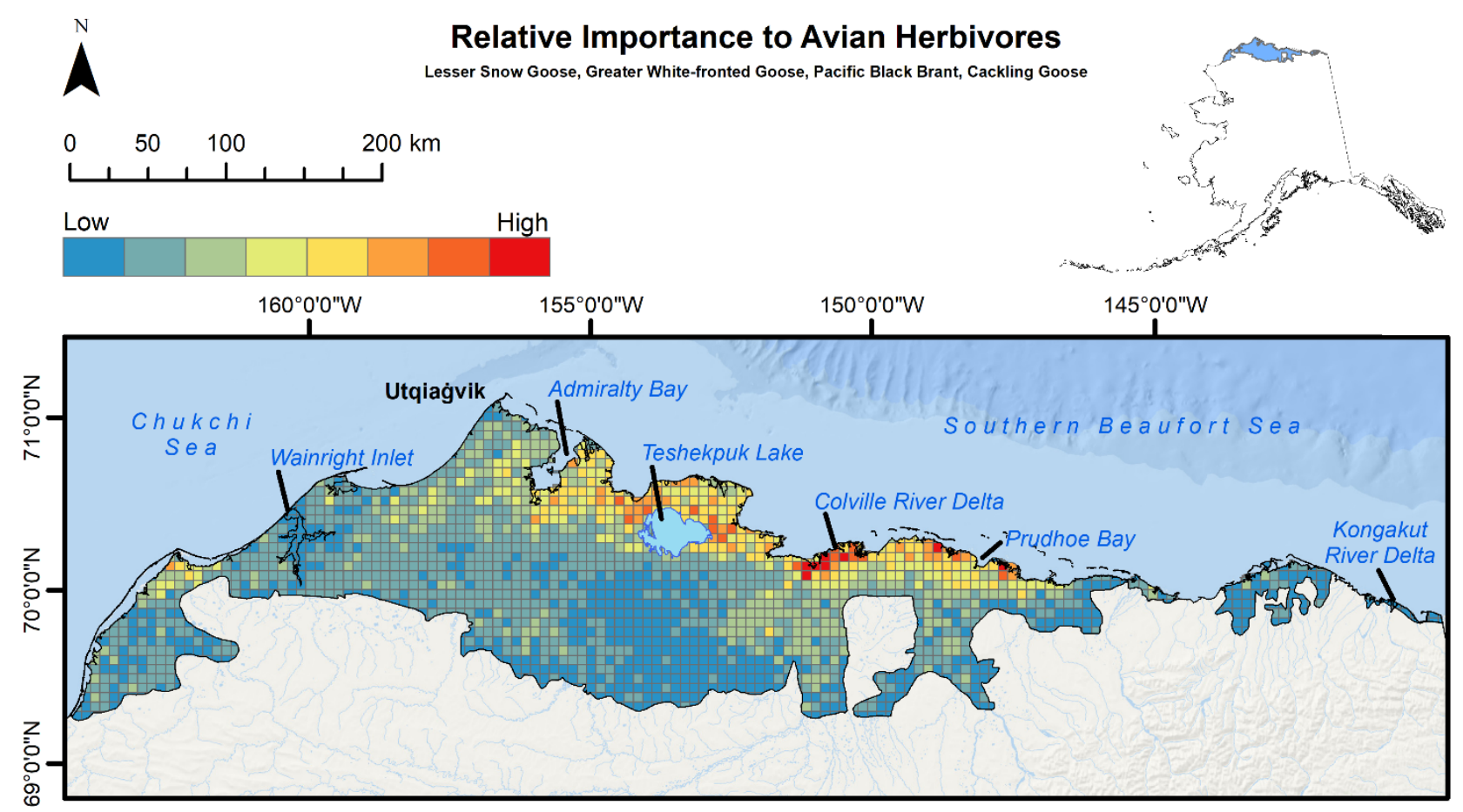


Figure A7.2. Relative importance of cells for avian omnivores across the Arctic Coastal Plain, Alaska 1992-2016. Omnivores include: Northern Pintail and Scaup sp. (Lesser and Greater combined).

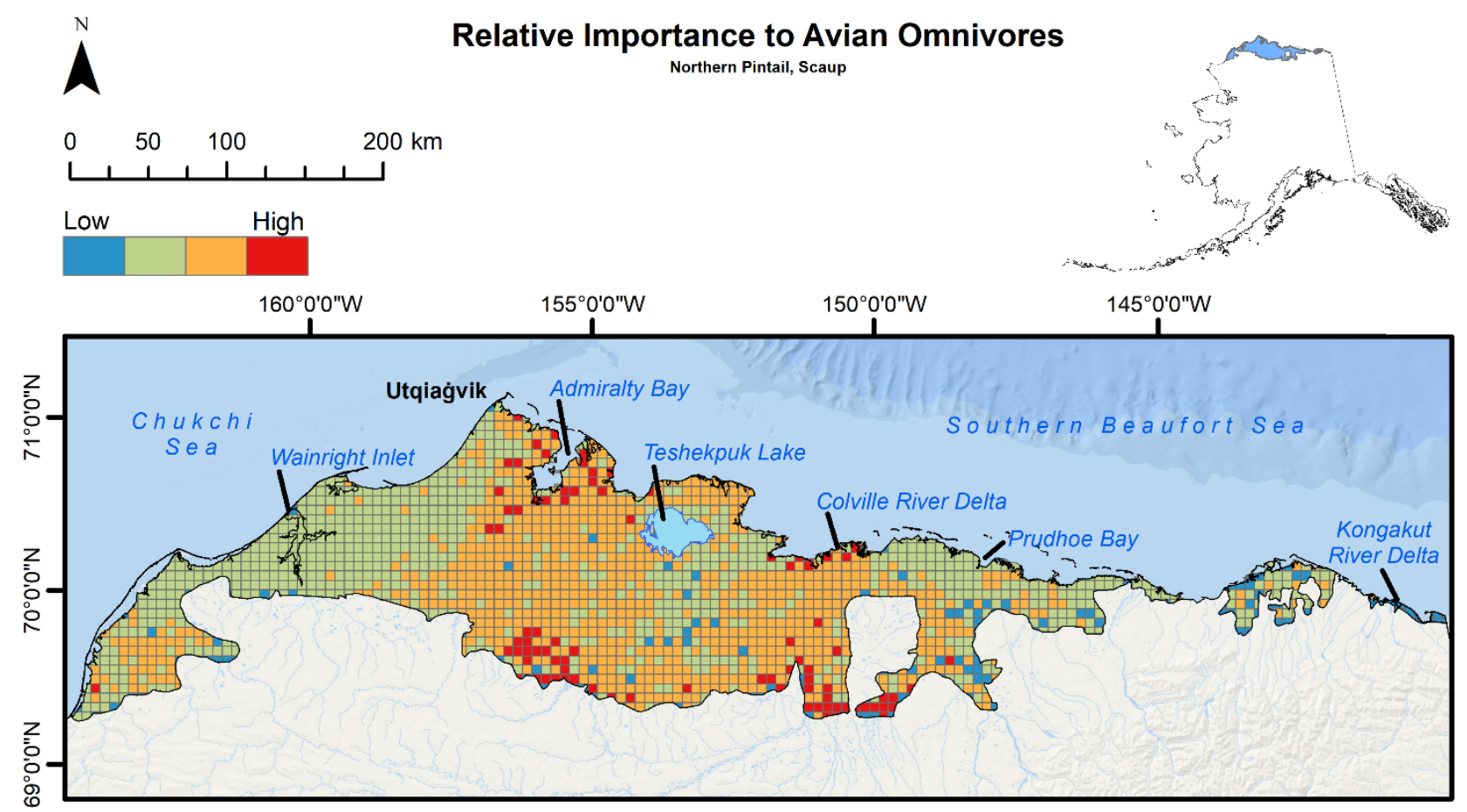


Figure A7.3. Relative importance of cells for avian invertivores across the Arctic Coastal Plain, Alaska 1992-2016. Invertivores include: Steller's Eider, Spectacled Eider, King Eider, White-winged Scoter and Long-tailed Duck.

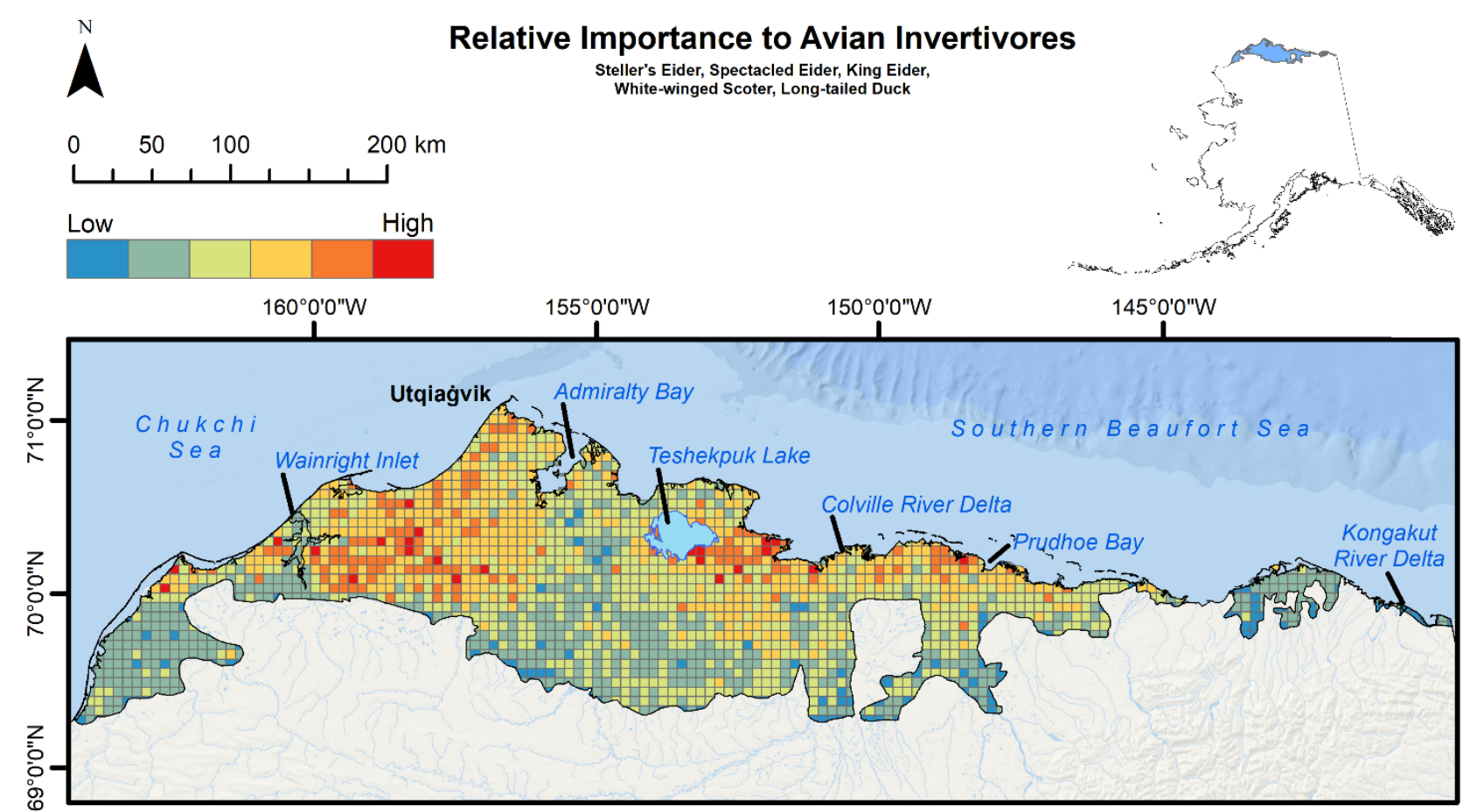


Figure A7.4. Relative importance of cells for avian generalist predators across the Arctic Coastal Plain, Alaska 1992-2016. Generalist predators include: Jaeger (Pomarine, Parasitic and Long-tailed combined), Sabine's Gull, Glaucous Gull and Arctic Tern.

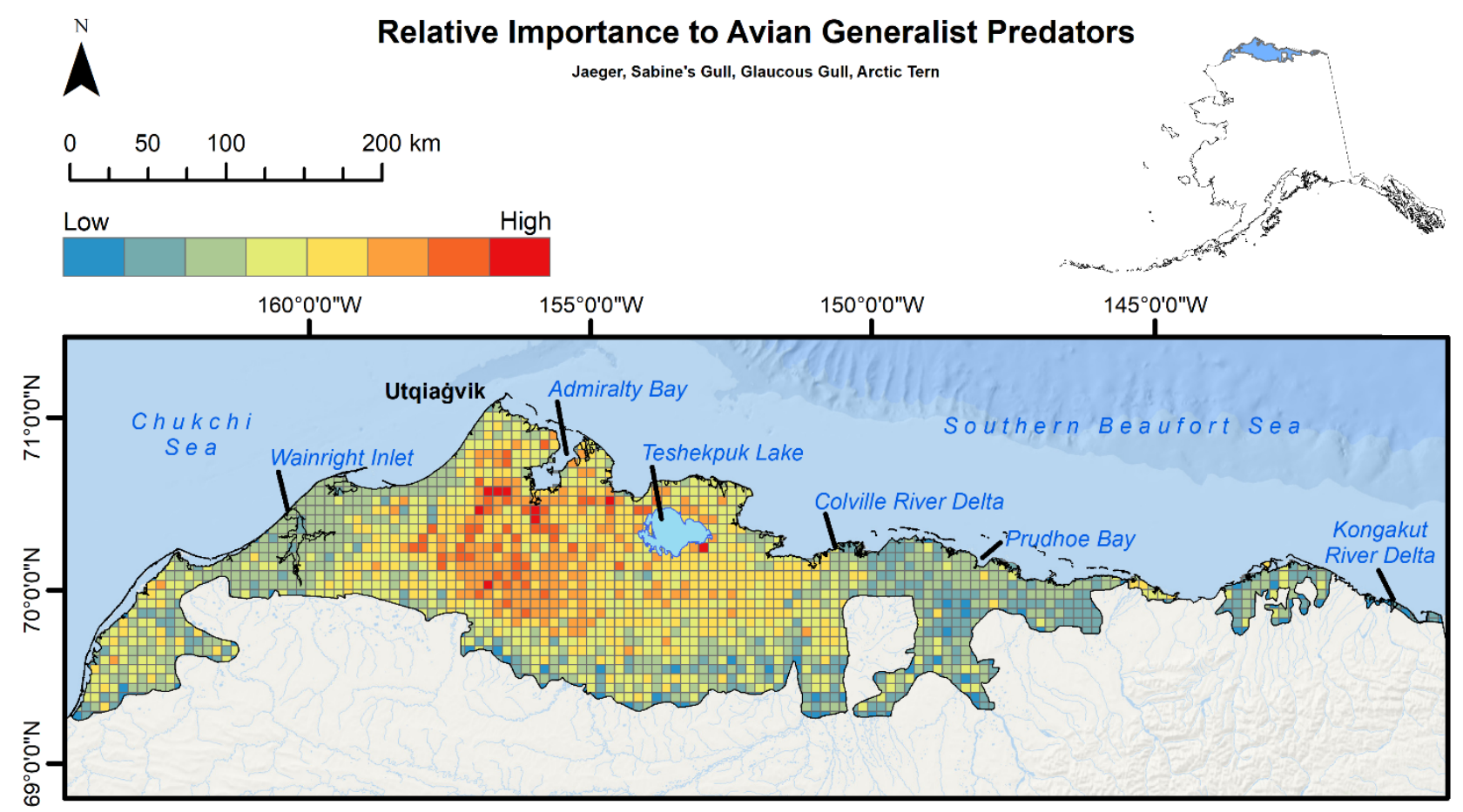


Figure A7.5. Relative importance of cells for avian piscivores across the Arctic Coastal Plain, Alaska 1992-2016. Piscivores include: Red-breasted Merganser, Red-throated Loon, Pacific Loon and Yellow-billed Loon.

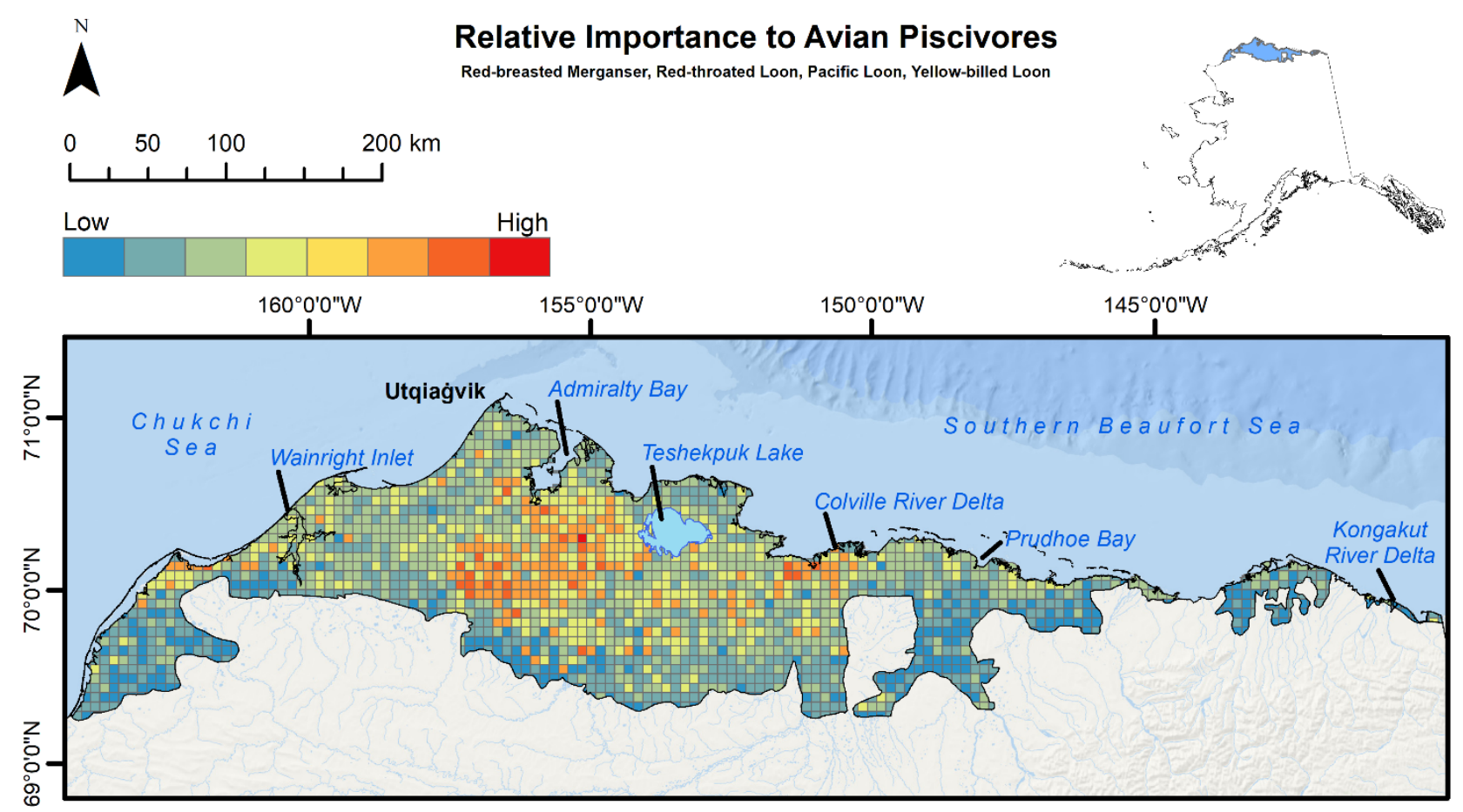


Appendix 8. Species importance

\section{Spatio-temporal population change of arctic-breeding waterbirds}

We mapped the relative importance of approximately $36 \mathrm{~km}^{2}(6 \mathrm{~km}$ by $6 \mathrm{~km})$ cells to 20 waterbird species observed during the Arctic Coastal Plain Breeding Waterfowl Survey, Alaska, 1992-2016. We defined cells as important, unimportant, or average for each cell-species. See the Methods section of the main text for more detail on importance scores. We present maps in taxonomic order (Chesser et al. 2018) for Lesser Snow Goose, Greater White-fronted Goose, Black Brant, Cackling Goose, Tundra Swan, Northern Pintail, Scaup (Lesser and Greater combined), Steller's Eider, Spectacled Eider, King Eider, White-winged Scoter, Long-tailed Duck, Red-breasted Merganser, Jaeger (Pomarine, Parasitic, and Long-tailed combined), Sabine's Gull, Glaucous Gull, Arctic Tern, Red-throated Loon, Pacific Loon, and Yellow-billed Loon.

Chesser, R. T., K. J. Burns, C. Cicero, J. L. Dunn, A. W. Kratter, I. J. Lovette, P. C. Rasmussen, J. V. Remsen, Jr., J. D. Rising, D. F. Stotz, and K. Winker. 2018. Fifty-ninth supplement to the American Ornithological Society's check-list of North American birds. The Auk: Ornithological Advances 135:798-813. 
Figure A8.1. Relative importance of cells for Lesser Snow Goose across the Arctic Coastal Plain, Alaska $1992-2016$.

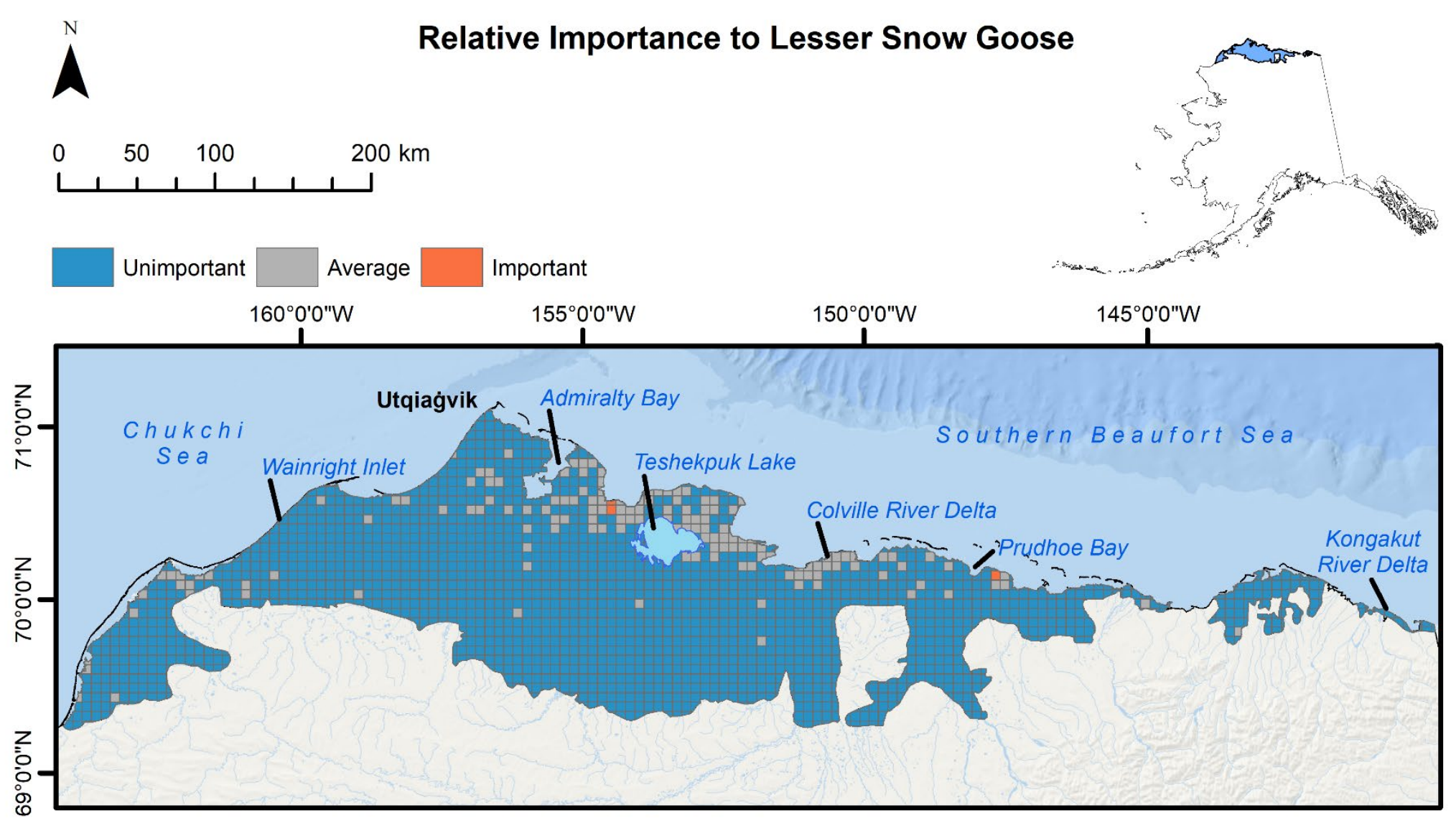


Figure A8.2. Relative importance of cells for Greater White-fronted Goose across the Arctic Coastal Plain, Alaska $1992-2016$.

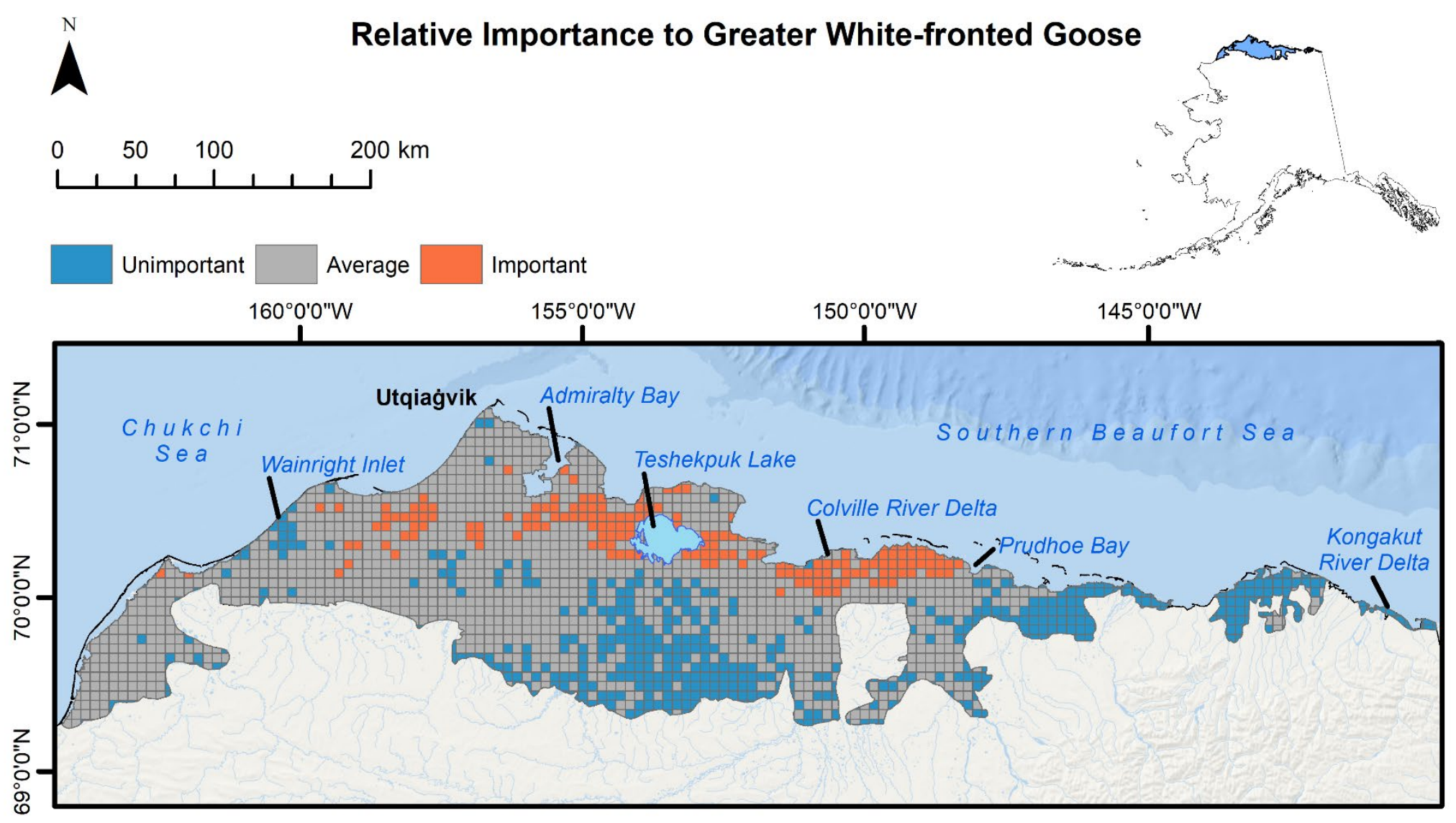


Figure A8.3. Relative importance of cells for Black Brant across the Arctic Coastal Plain, Alaska 1992-2016.

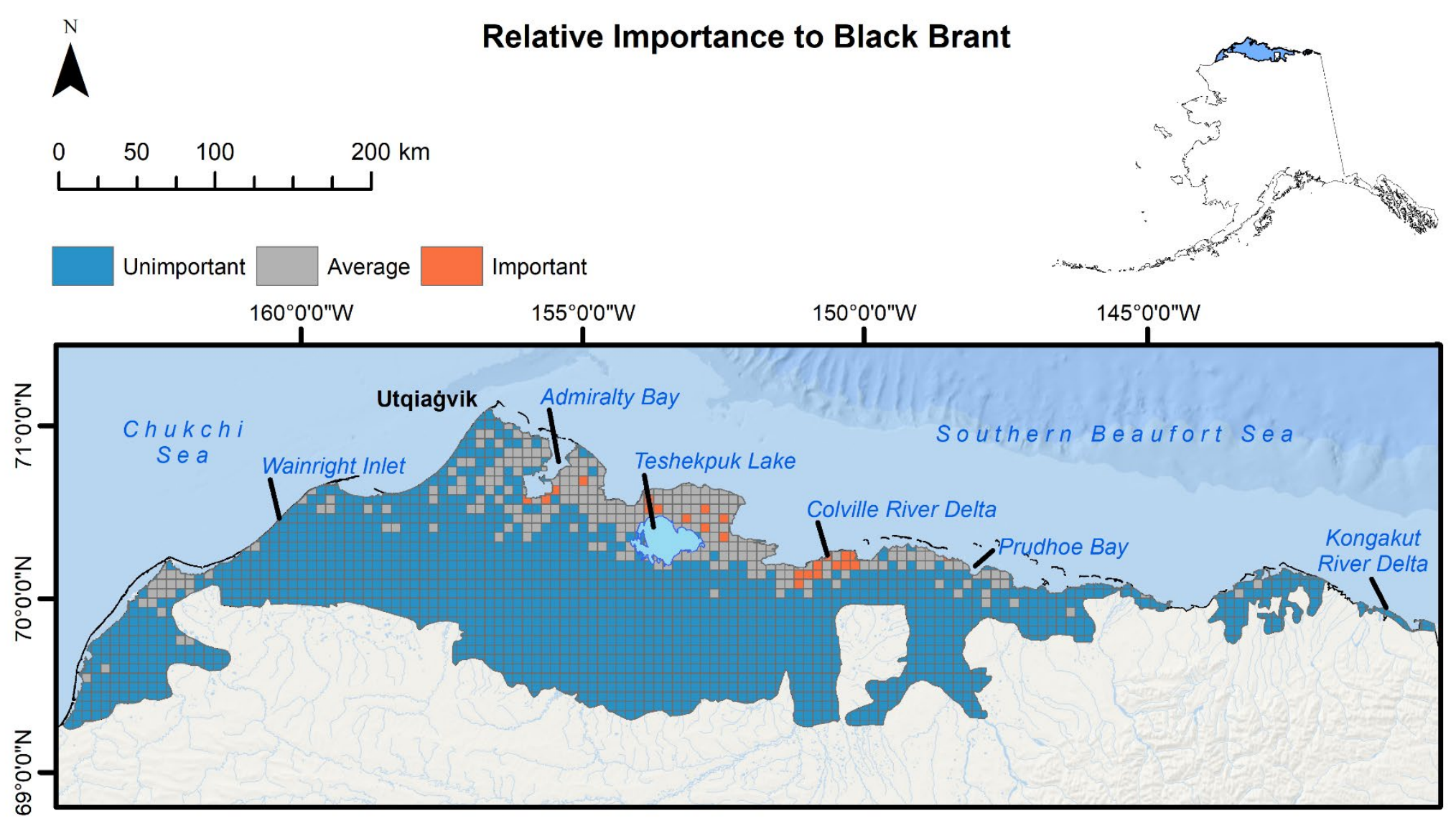


Figure A8.4. Relative importance of cells for Cackling Goose across the Arctic Coastal Plain, Alaska 1992-2016.

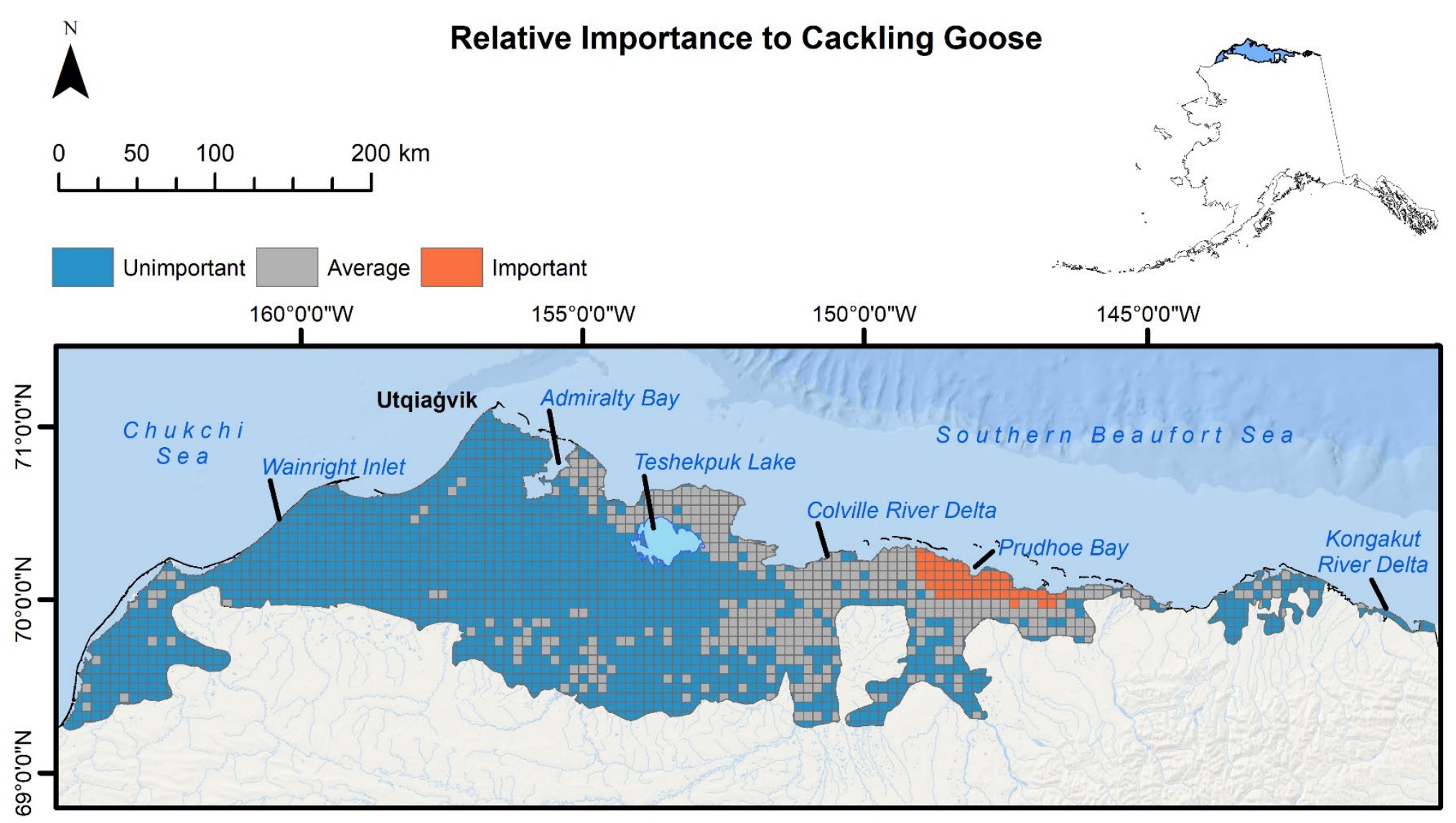


Figure A8.5. Relative importance of cells for Tundra Swan across the Arctic Coastal Plain, Alaska 1992-2016.

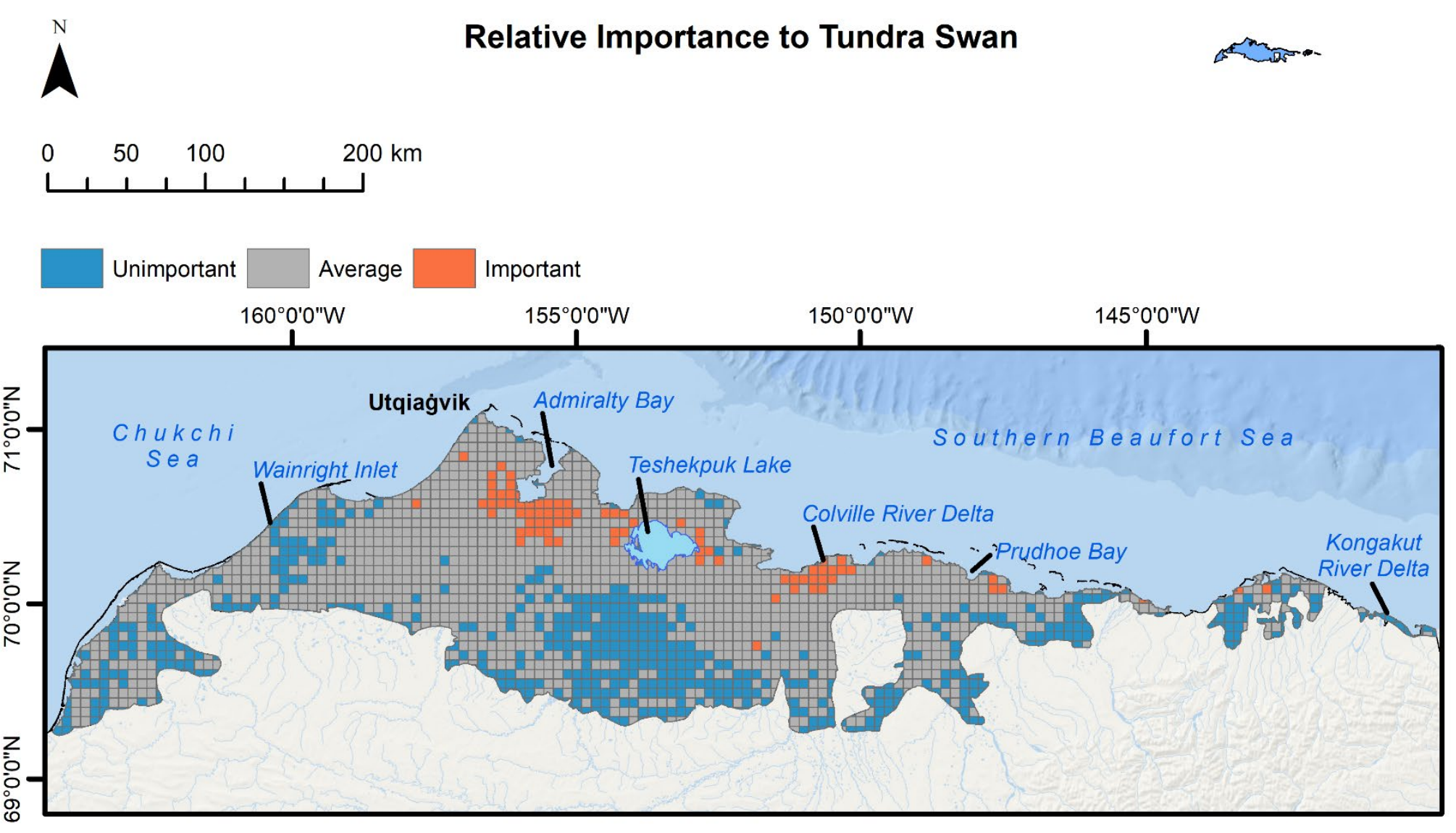


Figure A8.6. Relative importance of cells for Northern Pintail across the Arctic Coastal Plain, Alaska 1992-2016.

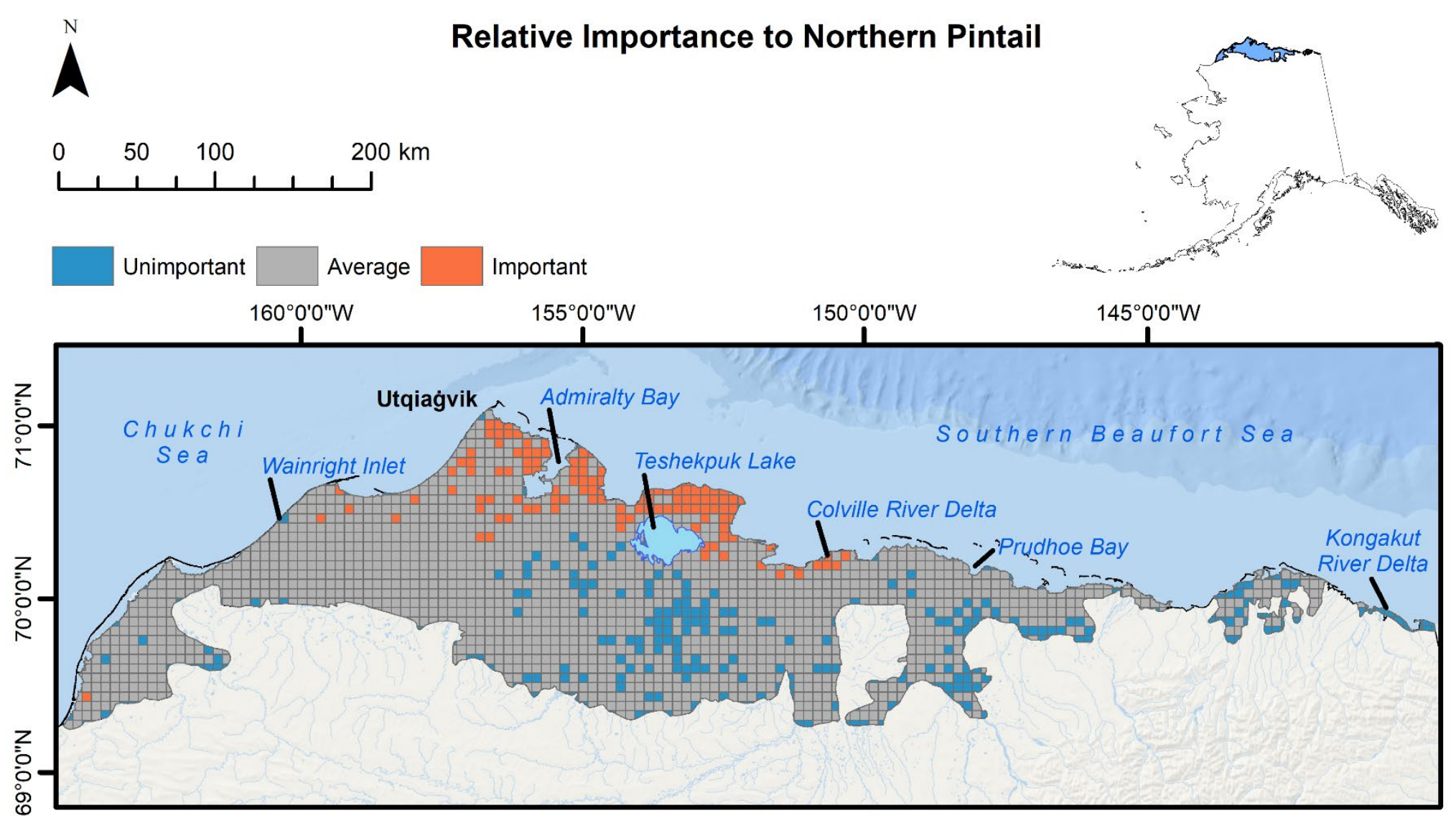


Figure A8.7. Relative importance of cells for Scaup sp. (Lesser and Greater combined) across the Arctic Coastal Plain, Alaska 19922016.
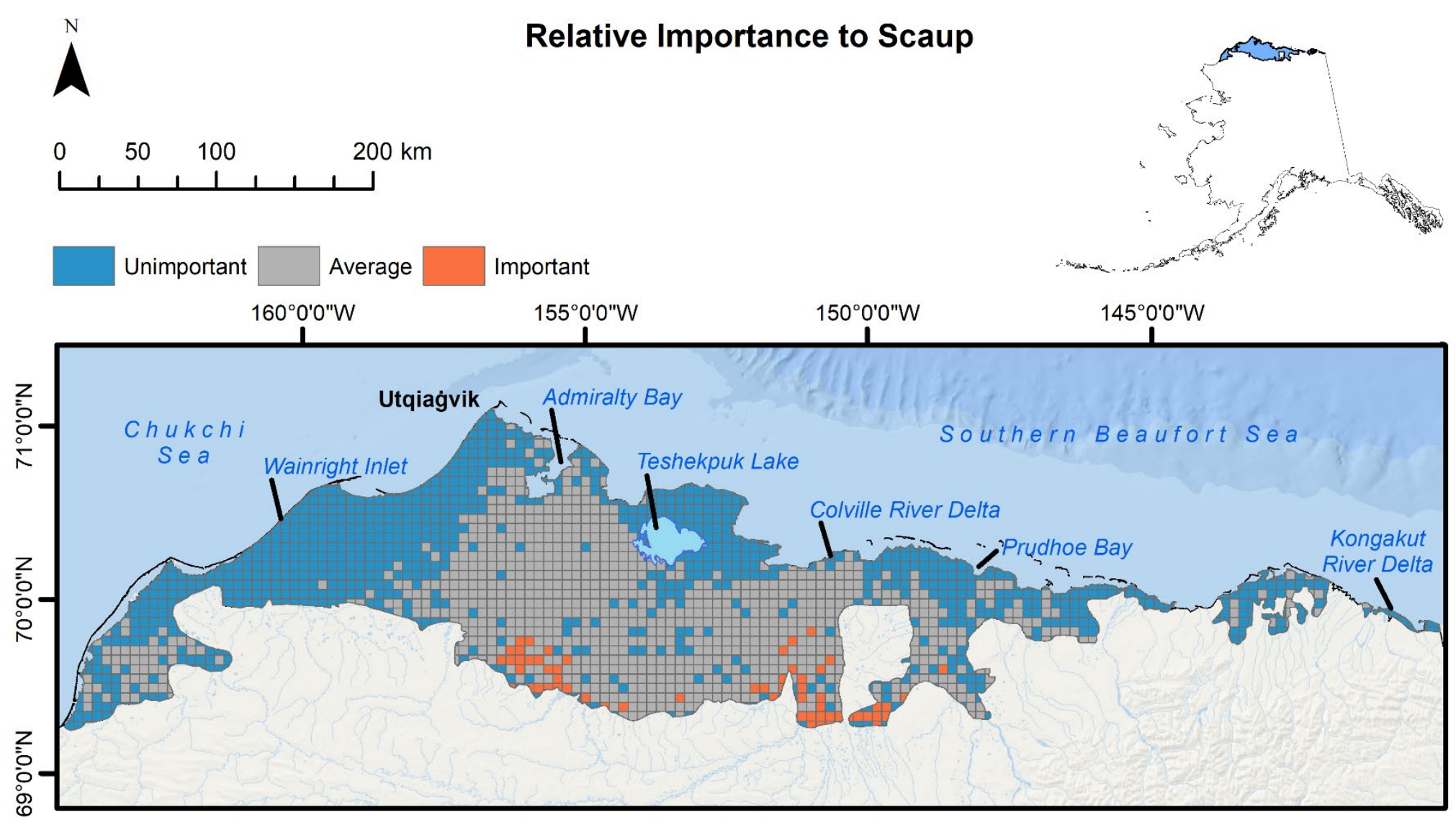
Figure A8.8. Relative importance of cells for Steller's Eider across the Arctic Coastal Plain, Alaska 1992-2016.

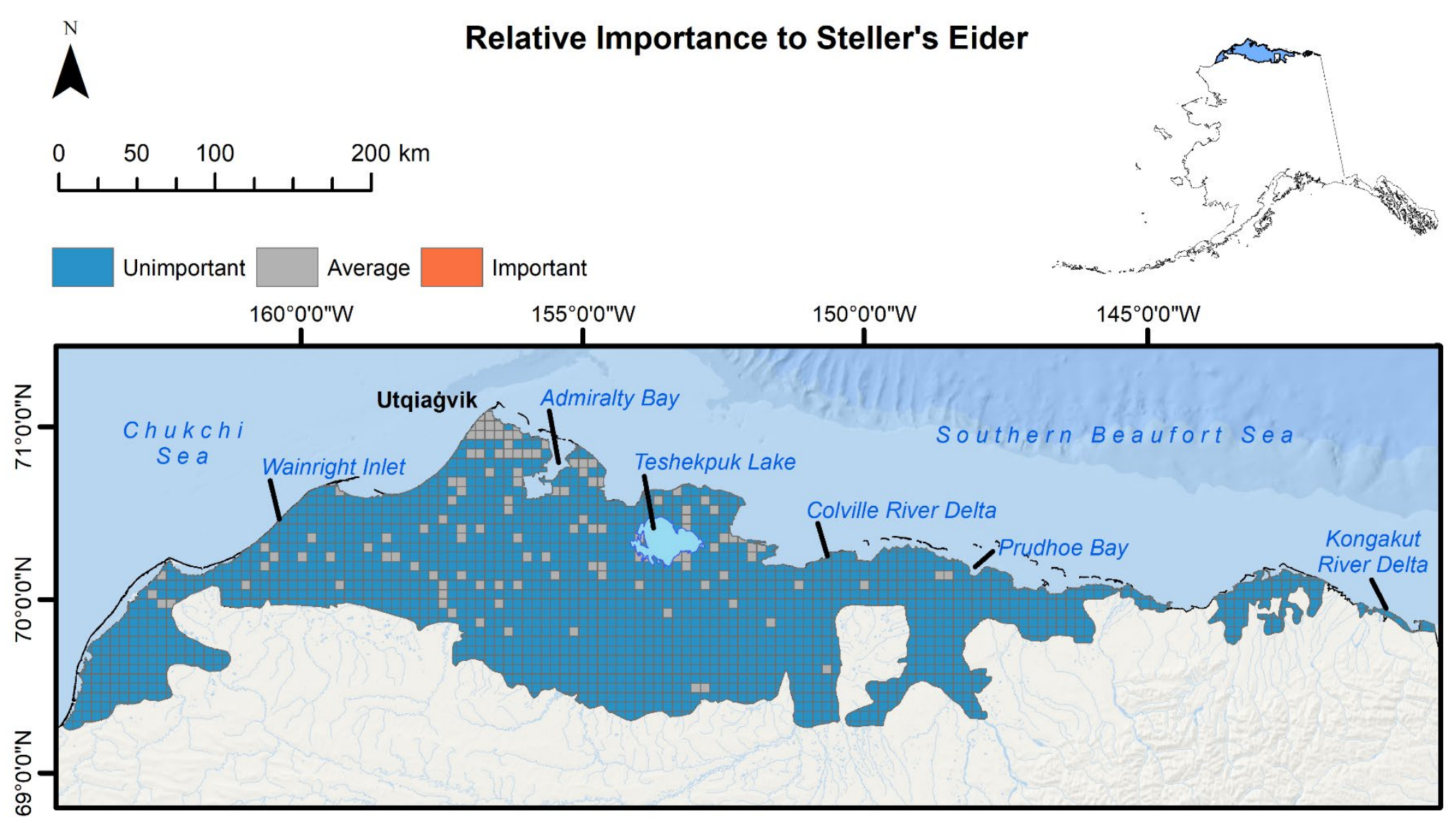


Figure A8.9. Relative importance of cells for Spectacled Eider across the Arctic Coastal Plain, Alaska 1992-2016.

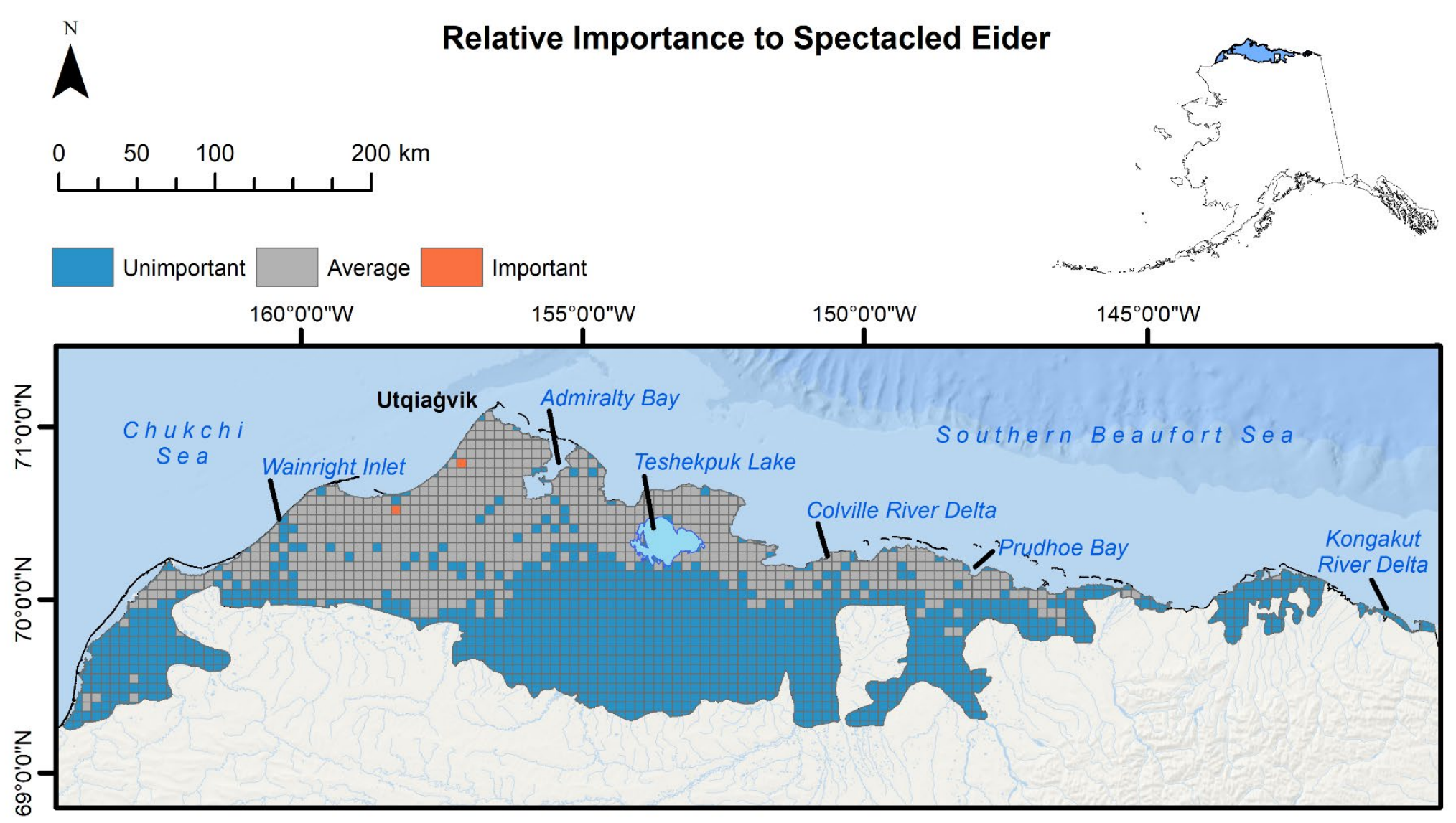


Figure A8.10. Relative importance of cells for King Eider across the Arctic Coastal Plain, Alaska 1992-2016.

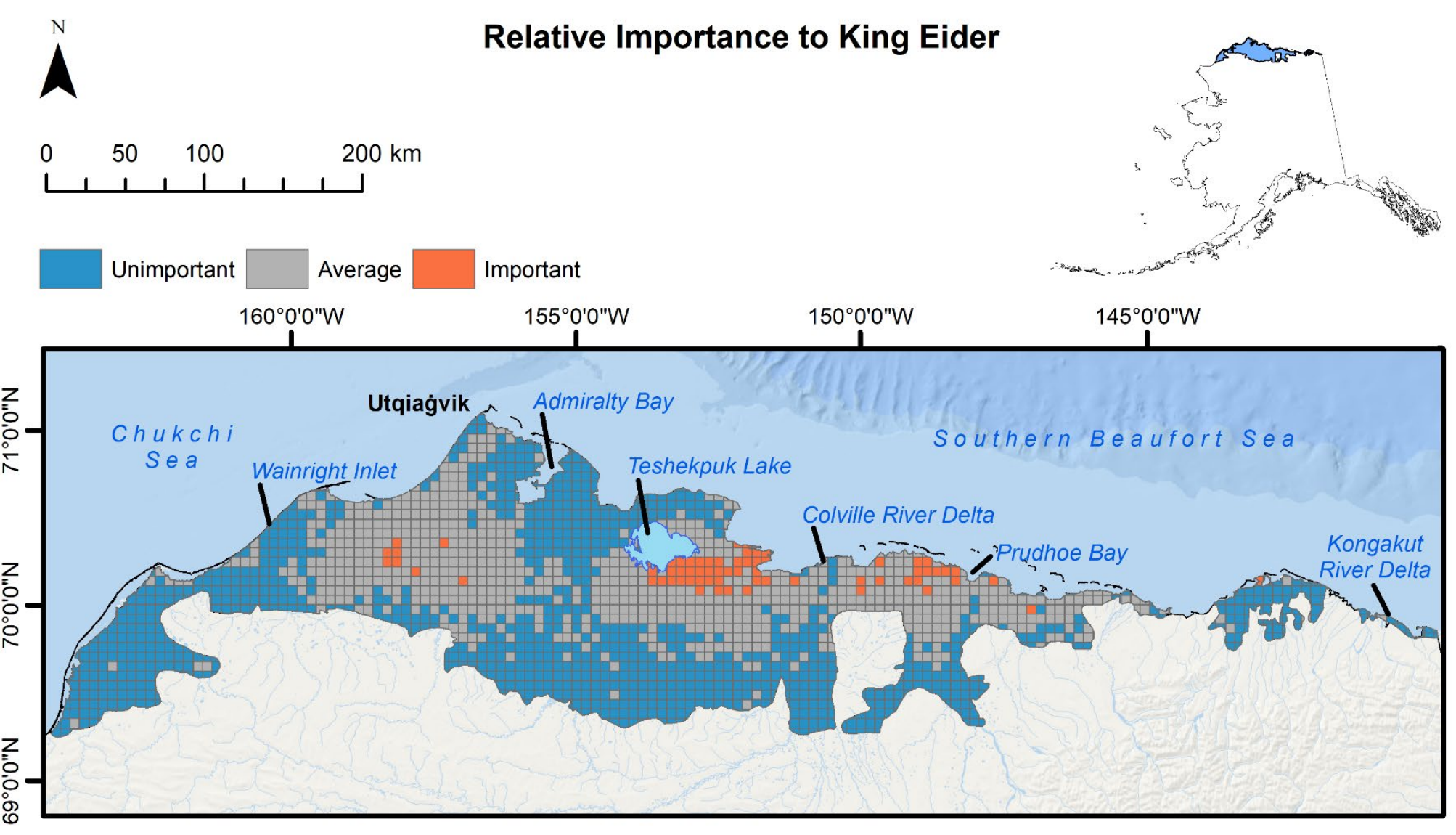


Figure A8.11. Relative importance of cells for White-winged Scoter across the Arctic Coastal Plain, Alaska $1992-2016$.

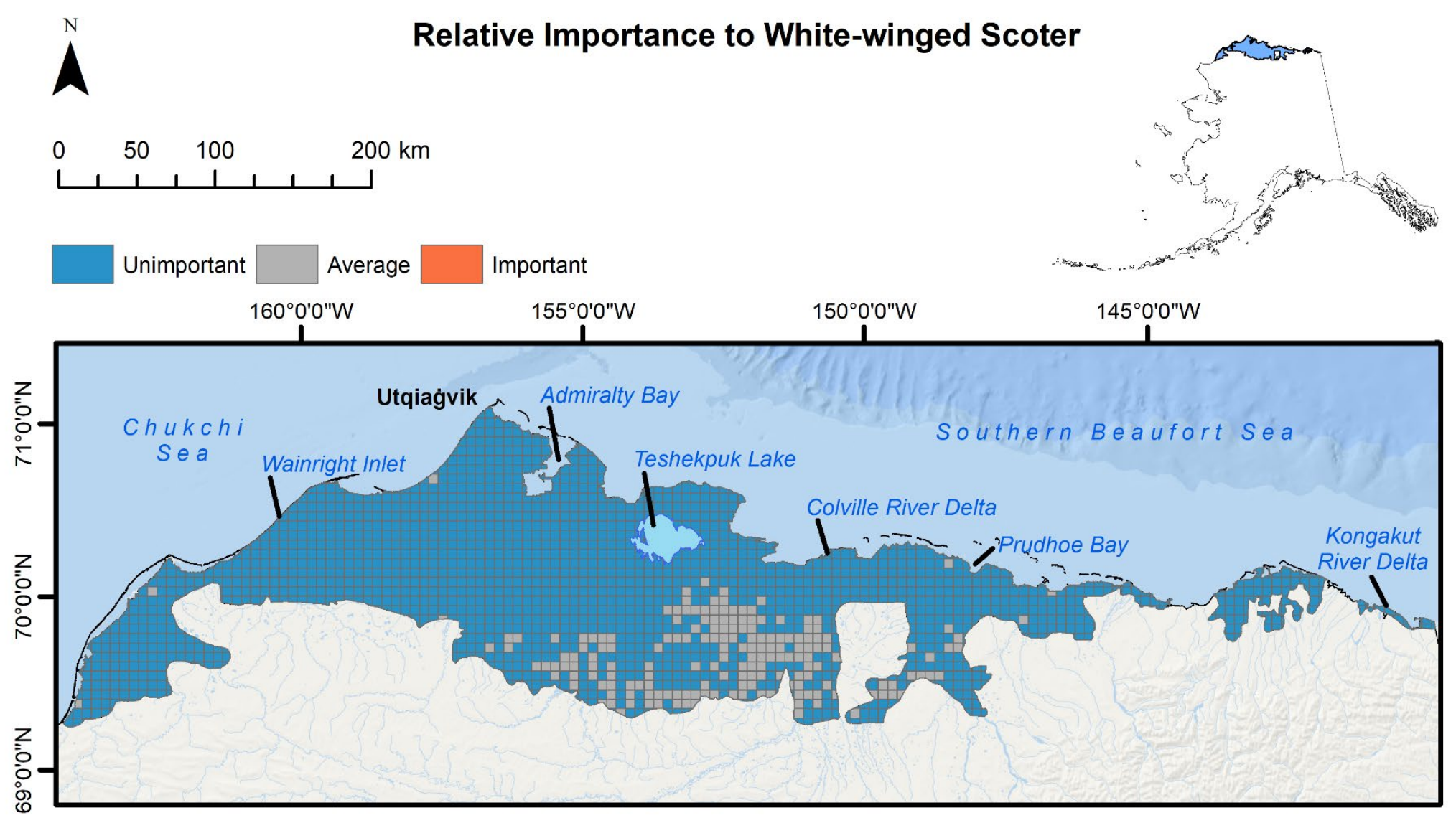


Figure A8.12. Relative importance of cells for Long-tailed Duck across the Arctic Coastal Plain, Alaska $1992-2016$.

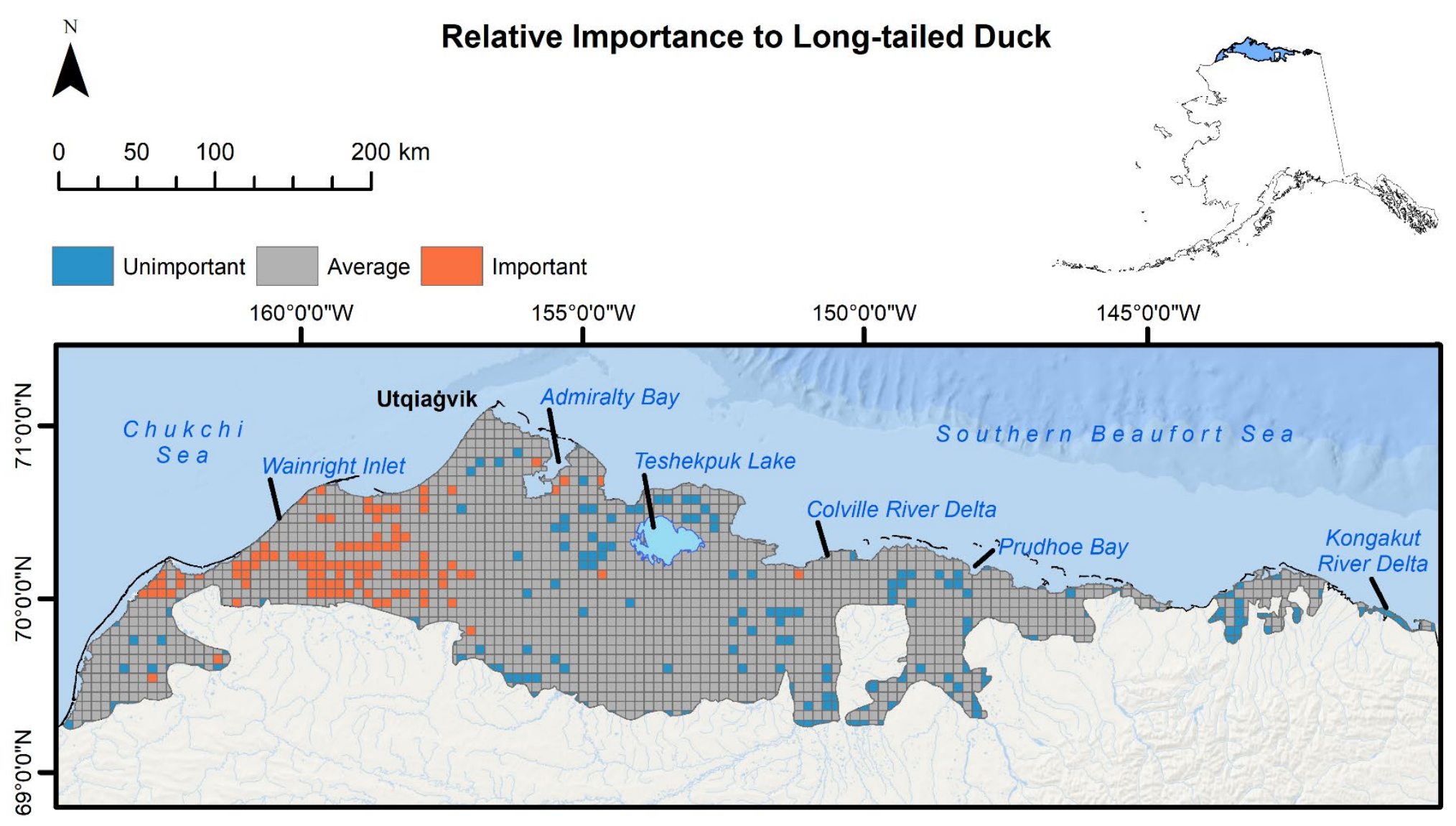


Figure A8.13. Relative importance of cells for Red-breasted Merganser across the Arctic Coastal Plain, Alaska $1992-2016$.

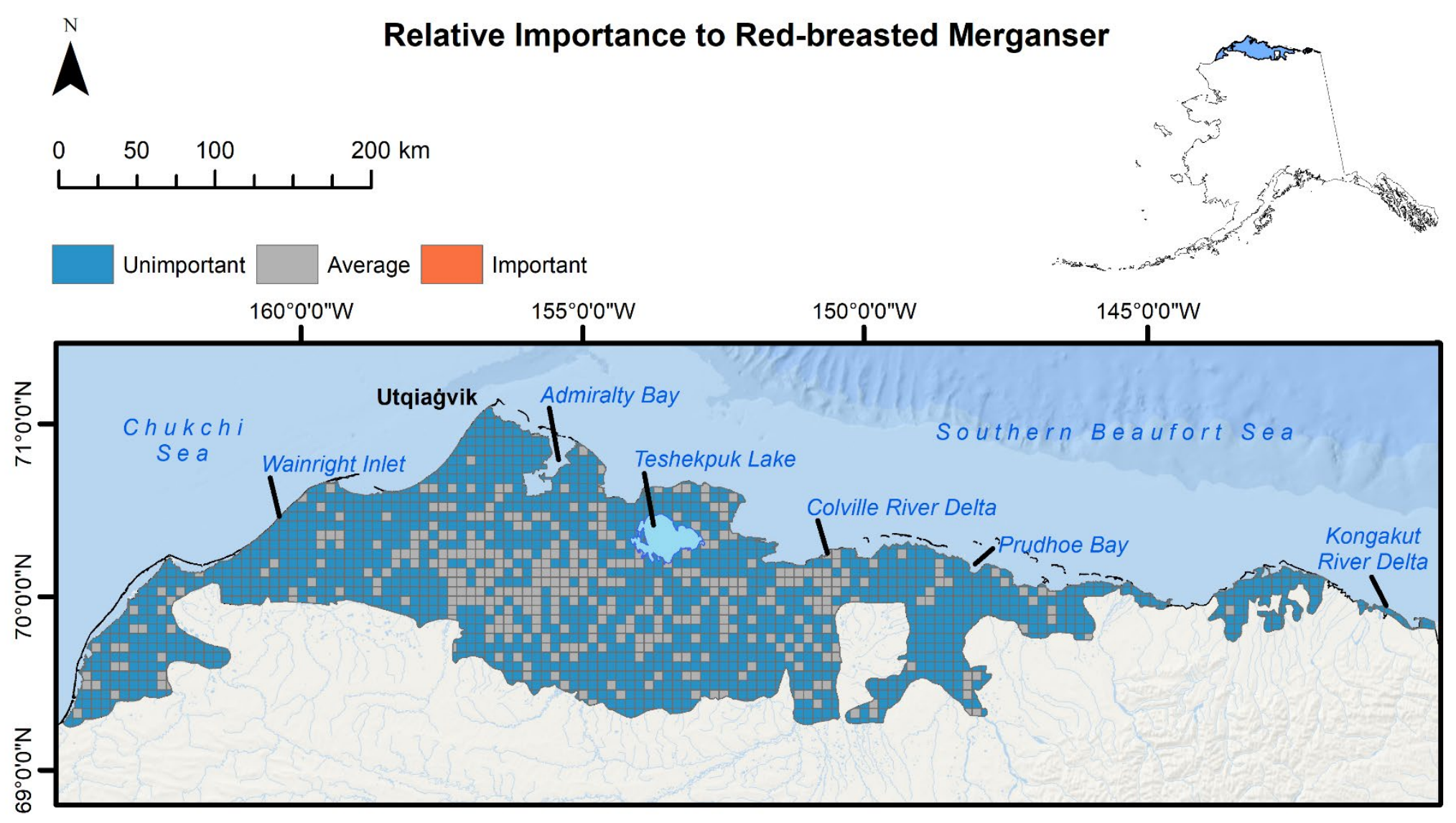


Figure A8.14. Relative importance of cells for Jaeger (Pomarine, Parasitic and Long-tailed combined) across the Arctic Coastal Plain, Alaska 1992-2016.

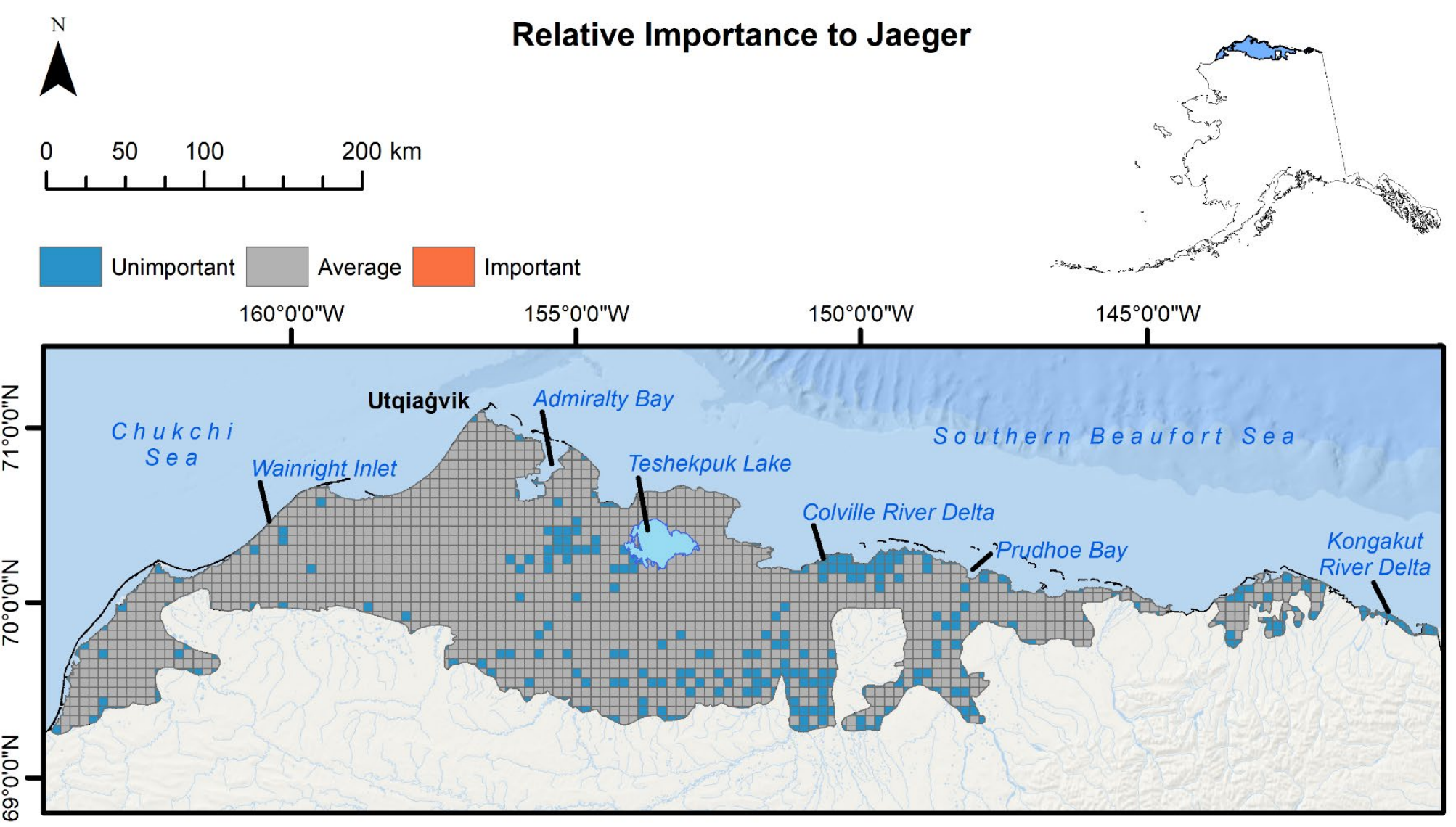


Figure A8.15. Relative importance of cells for Sabine's Gull across the Arctic Coastal Plain, Alaska 1992-2016.

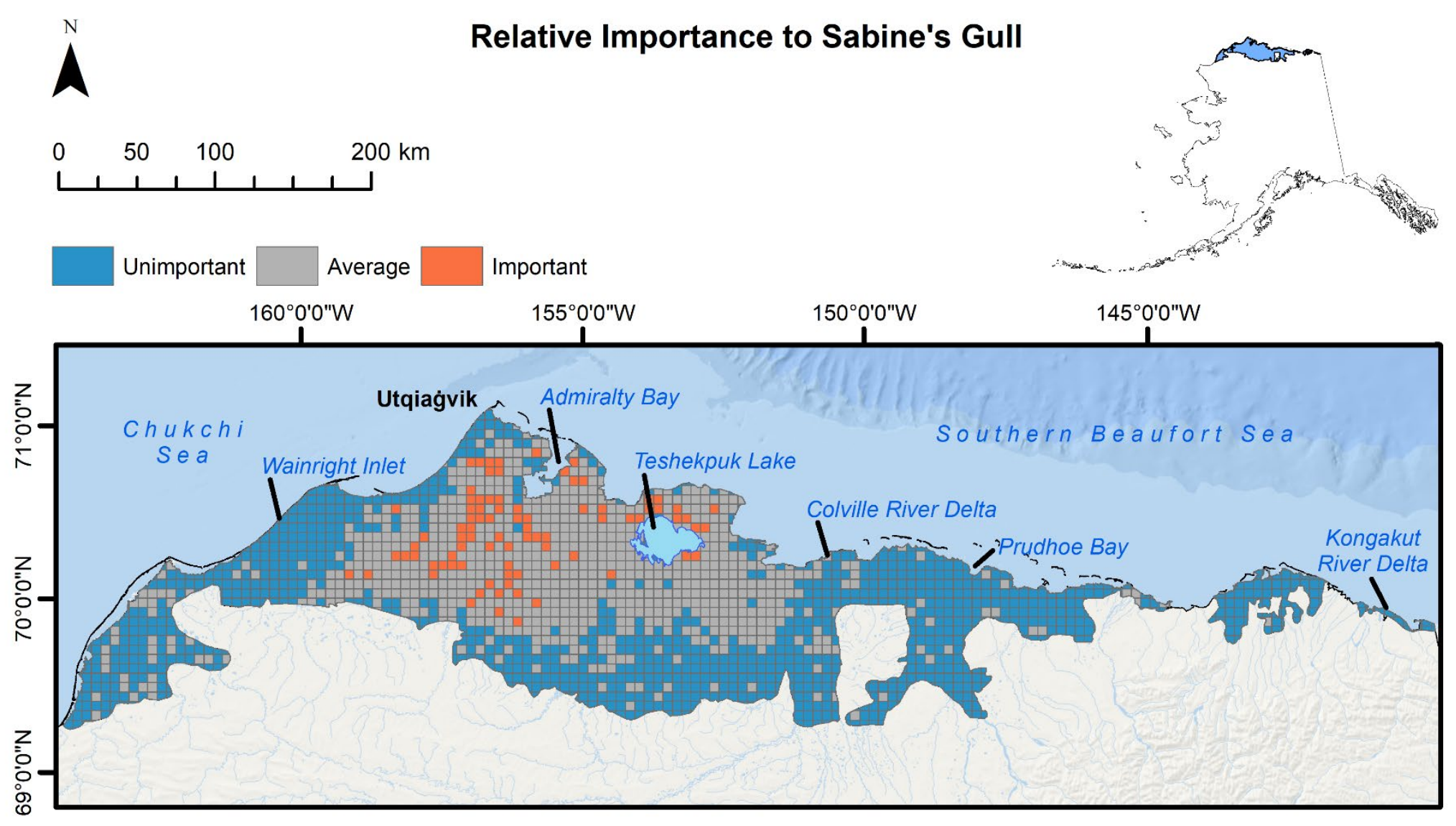


Figure A8.16. Relative importance of cells for Glaucous Gull across the Arctic Coastal Plain, Alaska 1992-2016.

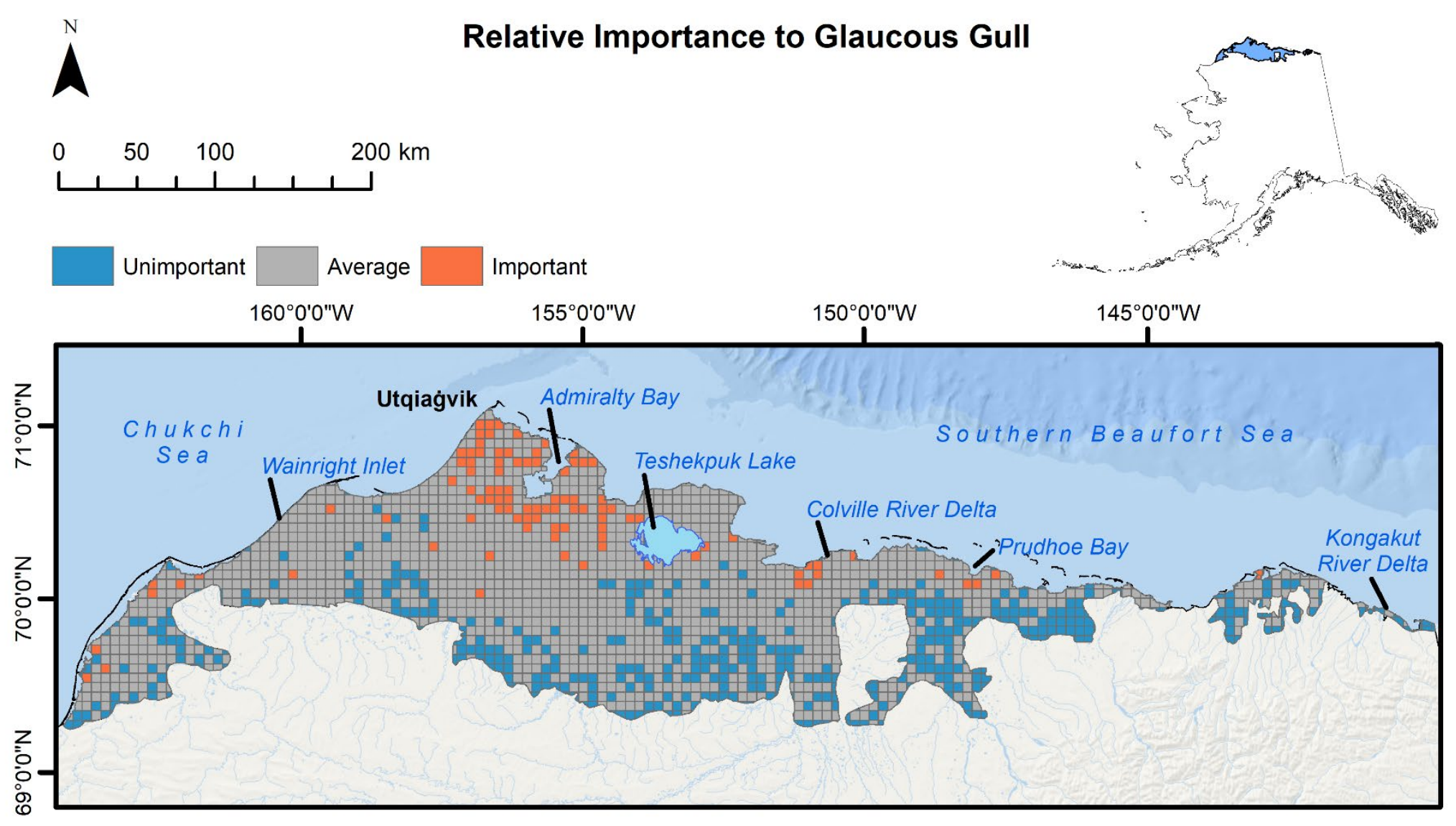


Figure A8.17. Relative importance of cells for Arctic Tern across the Arctic Coastal Plain, Alaska 1992-2016.

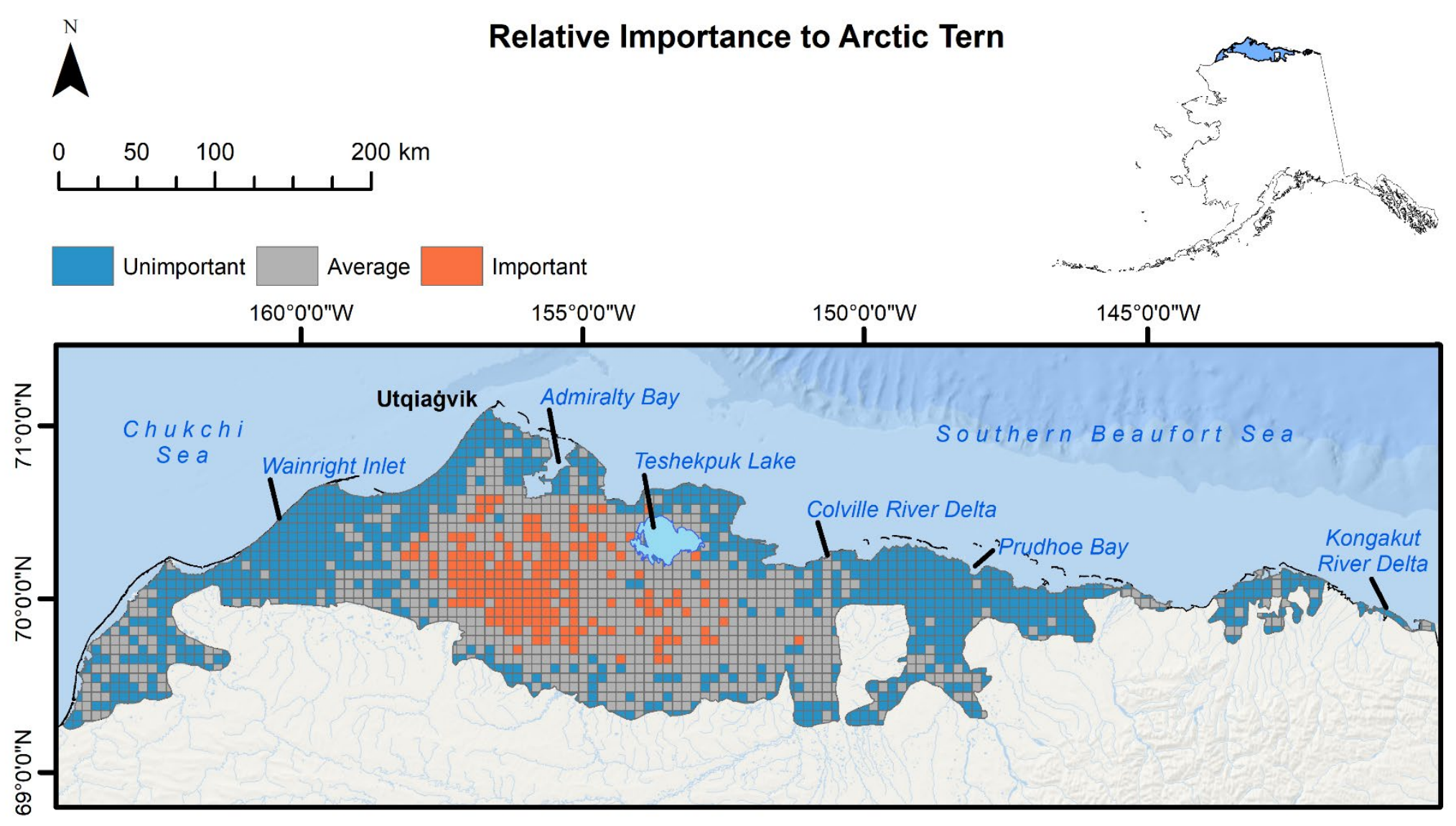


Figure A8.18. Relative importance of cells for Red-throated Loon across the Arctic Coastal Plain, Alaska 1992-2016.

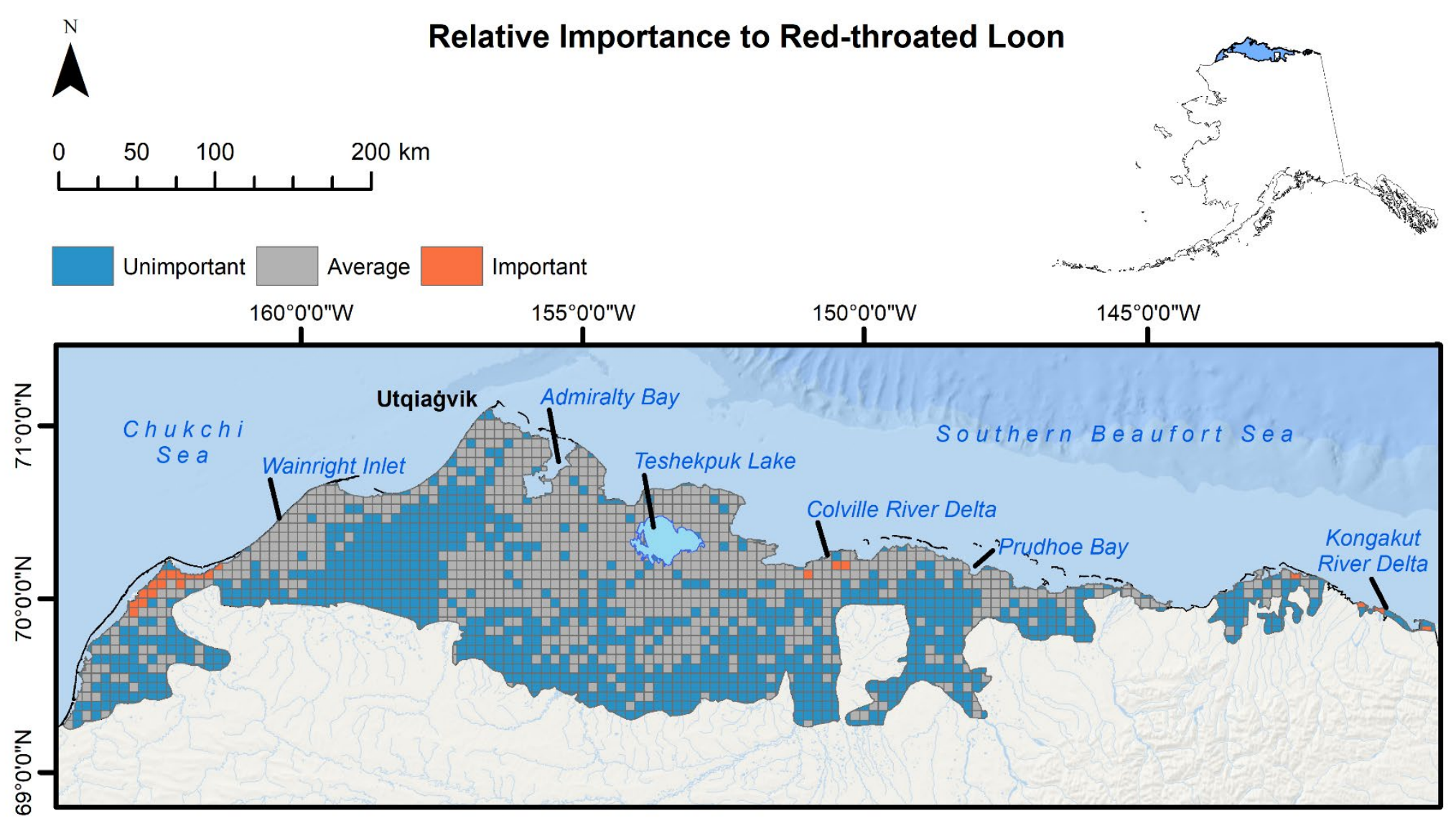


Figure A8.19. Relative importance of cells for Pacific Loon across the Arctic Coastal Plain, Alaska 1992-2016.

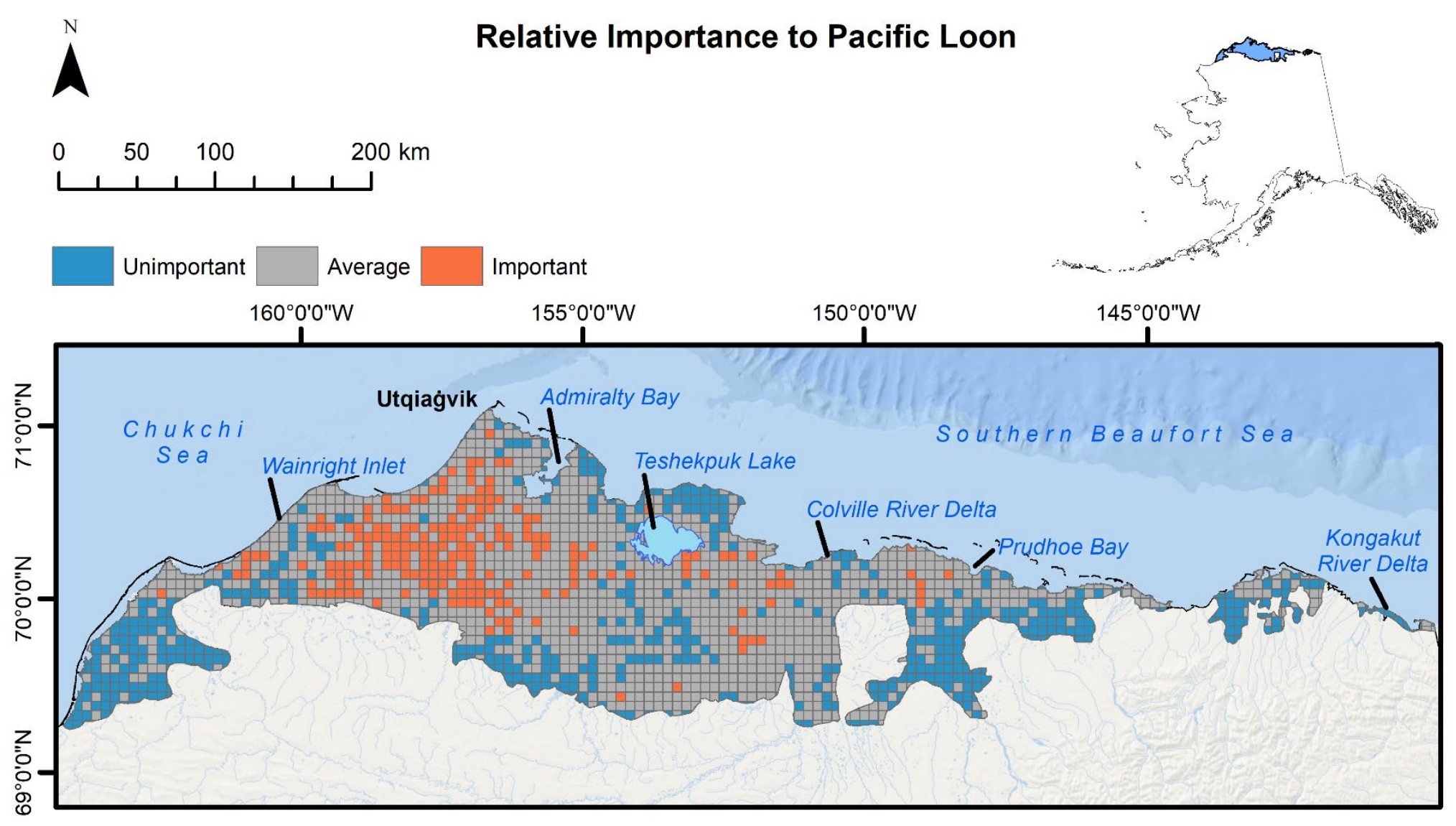


Figure A8.20. Relative importance of cells for Yellow-billed Loon across the Arctic Coastal Plain, Alaska $1992-2016$.

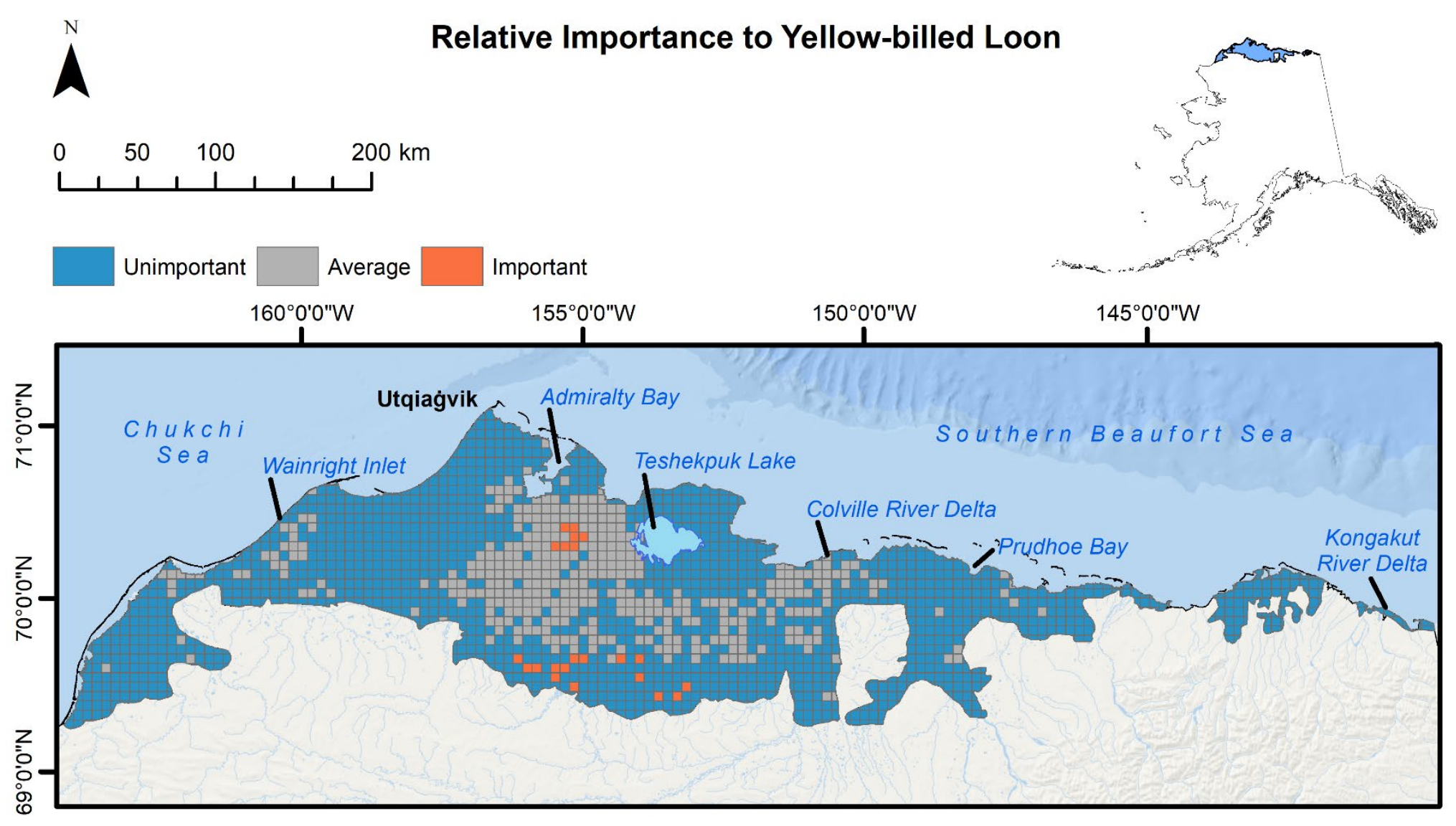


Appendix 9. Trend comparison

\section{Spatio-temporal population change of arctic-breeding waterbirds}

Table A9.1. Breeding population trends estimated for 20 waterbird species in our study compared to examples of other studies in both the Arctic Coastal Plain, Alaska (ACP) or elsewhere in Alaska, the Arctic, or North America. We provide trend direction as increasing (+), decreasing $(-)$ or stable $(\mathrm{O})$ with references as footnotes that include the date range examined bolded in parentheses. This list is not meant to be exhaustive, but rather showcase agreement or disagreement in population trends for each species.

\begin{tabular}{|c|c|c|c|}
\hline Species & Our Study & $\mathrm{ACP}$ & Elsewhere \\
\hline $\begin{array}{l}\text { Lesser Snow Goose (Anser caerulescens } \\
\text { caerulescens) }\end{array}$ & + & $+4,19$ & $+^{1,9,13,18}$ \\
\hline Greater White-fronted Goose (A. alibfrons) & + & $++^{19}$ & $+{ }^{17,18}$ \\
\hline Black Brant (Branta bernicla nigricans) & + & $+^{19}$ & $+^{17}$ \\
\hline Cackling Goose $(B . \text { hutchinsii })^{\mathrm{a}}$ & + & $++^{19}$ & $\mathrm{O}^{13,17,18}$ \\
\hline Tundra Swan (Cygnus columbianus) & + & $+{ }^{19}$ & $+^{11,17,18}$ \\
\hline Northern Pintail (A. acuta) & $\mathrm{O}$ & $+{ }^{19}$ & $-16,18$ \\
\hline Scaup (Lesser and Greater combined) (Aythya sp.) & + & $+{ }^{19}$ & $-{ }^{18}$ to $\mathrm{O}^{16}$ \\
\hline Steller's Eider (Polysticta stelleri) & $\mathrm{O}$ & $\mathrm{O}^{19}$ & \\
\hline Spectacled Eider (Somateria fischeri) & $\mathrm{O}$ & $\mathrm{O}^{19}$ & $+{ }^{16}$ \\
\hline King Eider (S. spectabilis) & + & $t^{2,19}$ & -15 \\
\hline White-winged Scoter (Melanitta perspicillata) & + & $+{ }^{19}$ & \\
\hline Long-tailed Duck (Clangula hyemalis) & $\mathrm{O}$ & $\mathrm{O}^{19}$ & $-{ }^{3}$ to $\mathrm{O}^{16}$ \\
\hline Red-breasted Merganser (Mergus serrator) & + & $+{ }^{19}$ & $\mathrm{O}^{3}$ to $+{ }^{14,16}$ \\
\hline $\begin{array}{l}\text { Jaeger (Pomarine, Parasitic and Long-tailed } \\
\text { combined) (Stercorarius sp.) }\end{array}$ & $\mathrm{O}$ & $\mathrm{O}^{19}$ & $-{ }^{16}$ \\
\hline Sabine's Gull (Xema sabini) & + & $+{ }^{19}$ & $+{ }^{16}$ \\
\hline Glaucous Gull (Larus hyperboreus) & + & $+{ }^{19}$ & $-7,12$ to $\mathrm{O}^{6,16}$ \\
\hline Arctic Tern (Sterna paradisaea) & + & $+{ }^{19}$ & $-7,10$ \\
\hline Red-throated Loon (Gavia stellata) & - & $\mathrm{O}^{19}$ & $-{ }^{8}$ to $\mathrm{O}^{16}$ \\
\hline Pacific Loon (G. pacifica) & $\mathrm{O}$ & $\mathrm{O}^{5,19}$ & $\mathrm{O}^{8,16}$ \\
\hline Yellow-billed Loon (G. adamsii) & + & $\mathrm{O}^{5}$ to $+{ }^{19}$ & \\
\hline
\end{tabular}

a Trend results for Taverner's goose $(B . h$. taverneri) where applicable.

1. Alisauskas, R. T., R. F. Rockwell, K. W. Dufour, E. G. Cooch, G. Zimmerman, K. L. Drake, J. O. Leafloor, T. J. Moser, and E. T. Reed. 2011. Harvest, survival, and abundance of Midcontinent Lesser Snow Geese relative to population reduction efforts. Wildlife Monographs 179:1-42. (1971 - 2006) 
2. Bentzen, R. L., and A. N. Powell. 2012. Population dynamics of King Eiders breeding in northern Alaska. Journal of Wildlife Management 76:1011-1020 (2001-2010)

3. Bowman, T. D., E. D. Silverman, S. G. Gilliland, and J. B. Leirness. 2015. Status and trends of North American sea ducks: reinforcing the need for better monitoring. Pages 128 in J-P. L. Savard, D. V. Derksen, D. Esler, and J. M. Eadie, editors. Ecology and conservation of North American sea ducks. Studies in Avian Biology 46. (1975 - 2012 for $L T D U$, recent 10-20 years, RBME)

4. Burgess, R. M., R. J. Ritchie, B. T. Person, R. S. Suydam, J. E. Shook, A. K. Prichard, and T. Obritschkewitsch. 2017. Rapid growth of a nesting colony of Lesser Snow Geese (Chen caerulescens caerulescens) on the Ikpikpuk River Delta, North Slope, Alaska, USA. Waterbirds 40:11-23. (1995 - 2015)

5. Earnst, S. L. 2004. Status assessment and conservation plan for the Yellow-billed Loon (Gavia adamsii). U. S. Geological Survey Scientific Investigations Report 2004-5258, Department of the Interior, Washington D. C., USA. (1986- 2003)

6. Gaston, A. J., M. L. Mallory, and H. G. Gilchrist. 2012. Populations and trends of Canadian Arctic seabirds. Polar Biology 35:1221-1232. (various time periods)

7. Gilchrist, H. G., and G. J. Robertson. 1999. Population trends of gulls and arctic terns nesting in the Belcher Islands, Nunavut. Arctic 52:325-331. (1980s - 1997)

8. Groves, D. J., B. Conant, R. J. King, J. I. Hodges, J. G. King, 1996. Status and trends of loon populations summering in Alaska, 1971-1993. The Condor 98:189-195. (1971 1993)

9. Jefferies, R. L., A. P. Jano, and K. F. Abraham. 2006. A biotic agent promotes large-scale catastrophic change in the coastal marshes of Hudson Bay. Journal of Ecology 94:234242. (1963 - 1997)

10. Maftei, M., S. E. Davis, and M. L. Mallory. 2015. Assessing regional populations of ground-nesting marine birds in the Canadian High Arctic. Polar Research 34: doi:10.3402/polar.v34.25055. (2002 - 2013)

11. Pacific Flyway Council. 2001. Pacific Flyway management plan for the western population of Tundra Swans. Pacific Flyway Study Committee, Subcommittee on Tundra Swans. U. S. Fish and Wildlife Service, Unpublished Report, Migratory Birds, Portland, Oregon, USA. (1964- 2001) https://ecos.fws.gov/ServCat/DownloadFile/36959?Reference=36942 (19FEB19)

12. Petersen, A., D. B. Irons, H. G. Gilchrist, G. J. Robertson, D. Boertmann, H. Strøm, M. Gavrilo, Y. Artukhin, D. S. Clausen, K. J. Kuletz, and M. L. Mallory. 2015. The status of Glaucous Gulls Larus hyperboreus in the circumpolar Arctic. Arctic 68:107-120. (Various time periods)

13. Reiter, M. E., D. E. Andersen, A. H. Raedeke, and D. D. Humburg. 2013. Species associations and habitat influence the range-wide distribution of breeding Canada geese (Branta canadensis interior) on Western Hudson Bay. Waterbirds 36:20-33. (19872008)

14. Sauer, J. R., D. K. Niven, J. E. Hines, D. J. Ziolkowski, Jr., K. L. Pardieck, J. E. Fallon, and W. A. Link 2017. The North American breeding bird survey, results and analysis 1966-2015. Version 2.07.2017. U. S. Geological Survey, Patuxent Wildlife Research Center, Laurel, Maryland, USA. (1966 - 2015) 
15. Suydam, R. S., D. L. Dickson, J. B. Fadely, and L. T. Quakenbush. 2000. Population declines of King and Common Eiders of the Beaufort Sea. The Condor 102:219-222. (between 1960 and 1994)

16. Swaim, M. A. 2017. Abundance and trend of waterbird populations on the YukonKuskokwim Delta, Alaska, 1988-2016. U.S. Fish and Wildlife Service, Region 7, Migratory Birds, Anchorage, Alaska, USA. (1988 - 2016 for NOPI, SCAU, SPEI, LTDU, RBME; 1992 - 2016 for JAEG, SAGU, GLGU; 1989 - 2016 for RTLO, PALO) https://www.fws.gov/alaska/mbsp/mbm/waterfowl/surveys/pdf/20152016_YKD_Coastal_Waterbird_Survey.pdf (19FEB19)

17. Swaim, M. A., and H. M. Wilson. 2018. 2018 Yukon-Kuskokwim Delta Coastal Zone Survey of Geese, Swans, and Sandhill Cranes. U.S. Fish and Wildlife Service, Region 7, Migratory Birds, Anchorage, Alaska, USA. (1985 - 2018)

https://www.fws.gov/alaska/mbsp/mbm/waterfowl/surveys/pdf/2018_YKD_Goose_Swan Crane_Survey.pdf (19FEB19)

18. U. S. Fish and Wildlife Service (USFWS). 2018. Waterfowl population status, 2018. Department of the Interior, Washington, D. C., USA. (1970 - 2018 for GWFG, 2009 2018 for CAGO, 1985 - 2018 for TUSC; 1955 - 2018 for NOPI, SCAU)

https://www.fws.gov/migratorybirds/pdf/surveys-and-data/Populationstatus/Waterfowl/WaterfowlPopulationStatusReport18.pdf (19FEB19)

19. Wilson, H. M., W. W. Larned, and M. A. Swaim. 2018. Aerial survey indices of waterbird populations on the Arctic Coastal Plain, 1986-2017. U.S. Fish and Wildlife Service, Region 7, Migratory Birds, Anchorage, Alaska, USA. (1986 - 2017) https://www.fws.gov/alaska/mbsp/mbm/waterfowl/surveys/pdf/20142017_ACP_Breeding_Pair.pdf (19FEB19) 